\title{
THE EFFECT OF CORROSION ON SHEAR BEHAVIOUR OF SELF-CONSOLIDATING CONCRETE BEAMS
}

\author{
By: Nabil Al-Bayati \\ Bachelor of Engineering in Irrigation and Drainage \\ College of Engineering \\ University of Baghdad, 1984
}

A thesis presented to Ryerson University In partial fulfillment of the requirements For the degree of Master of Applied Science In the program of Civil Engineering

Toronto, Canada, 2009

CNabil Al-Bayati, 2009 


\section{AUTHOR'S DECLARATIONS}

I herby declare that I am the sole author of this thesis.

I authorize Ryerson University to lend this thesis to other institutions or individuals for the purpose of scholarly research.

Nabil Al-Bayati

I further authorize Ryerson University to reproduce this thesis by photocopying or by other means, in total or in part, at the request of other institutions or individuals for the purpose of scholarly research.

Nabil Al-Bayati 


\section{BORROWERS PAGE}

Ryerson University requires the signatures of all persons using or photocopying this thesis.

Please sign below and provide address and date.

\begin{tabular}{|l|l|l|l|}
\hline Name & Address & Signature & Date \\
\hline & & & \\
\hline & & & \\
\hline & & & \\
\hline & & & \\
\hline & & & \\
\hline & & & \\
\hline & & & \\
\hline & & & \\
\hline & & & \\
\hline & & & \\
\hline & & & \\
\hline & & & \\
\hline & & & \\
\hline & & & \\
\hline & & & \\
\hline & & & \\
\hline & & & \\
\hline & & & \\
\hline & & & \\
\hline & & & \\
\hline & & & \\
\hline & & & \\
\hline & & & \\
\hline & & & \\
\hline & & & \\
\hline & & & \\
\hline & & & \\
\hline & & & \\
\hline & & & \\
\hline & & & \\
\hline & & & \\
\hline & & & \\
\hline & & & \\
\hline & & & \\
\hline & & & \\
\hline & & & \\
\hline
\end{tabular}




\title{
ABSTRACT
}

Thesis Title:

The Effect of Corrosion on Shear Behaviour

of Self-consolidating Concrete Beams

Degree: $\quad$ Master of Applied Science

Year of Convocation: 2009

Name:

Nabil Al-Bayati

Program:

Civil Engineering

University:

\author{
Ryerson University
}

The objectives of this research are to compare the effect of corrosion on shear behavior in particular, and the overall structural response in both NC and SCC beams in general. Twenty reinforced concrete beams were used, with ten specimens cast using normal concrete $(\mathrm{NC})$, and the other ten were cast using self-consolidating concrete (SCC). The dimensions for each beam were $150 \mathrm{~mm} \times 220 \mathrm{~mm} \times 1400 \mathrm{~mm}$. Using accelerated corrosion through the application of a constant current of one ampere, four stages of corrosion were established at $5 \%, 10 \%, 15 \%$, and $20 \%$ of mass loss. Simply supported beams were loaded with two concentrated loads, and a four-point loading test was applied to the reinforced concrete (RC) beams. If (a) is the distance from the concentrated load to the reaction, and (d) is the distance from the center of the tensile flexural rebars to the top of the concrete beam, then $\mathrm{a} / \mathrm{d}=2.5$ was applied to assure the highest probability of shear failure mode. The data collected from load cell, LVDTs, corrosion crack patterns and loading cracks patterns were used to study the effects of multiple stages of corrosion on the shear behaviour of reinforced NC and SCC concrete beams. The corroded rebars were then retrieved rand cleaned to compare the calculated mass loss with real mass loss. The results showed high correlation between the calculated mass loss (according to Faraday law) and real mass loss. The accelerated corrosion resulted in a corrosion crack pattern, which was documented and analyzed.

In this research, the use of NC and SCC showed minor influences on failure mode, while the different stages of corrosion showed a higher degree of influence on 
failure mode and the structural capacity of beams made from both types of concrete. The apparent changes in failure mode were associated with the increased corrosion stage. 


\section{TABLE OF CONTENTS}

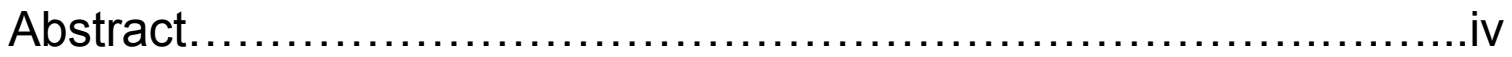

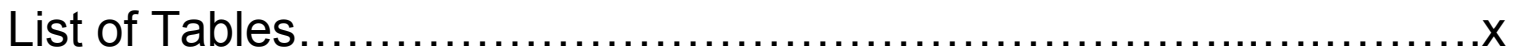

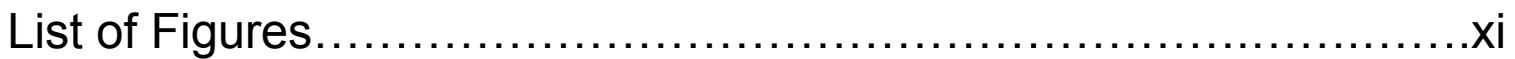

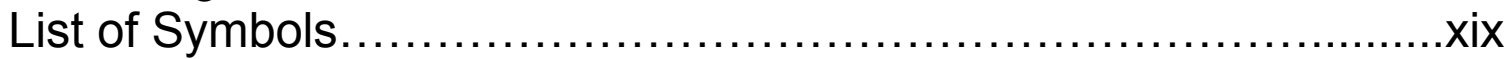

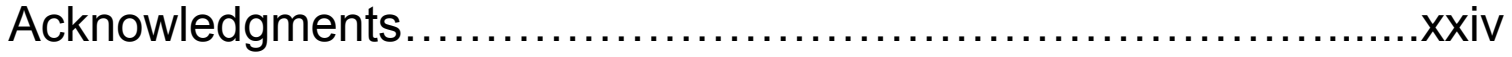

\section{Chapter 1}

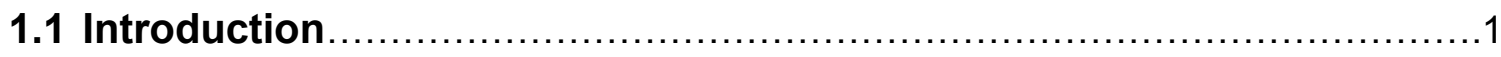

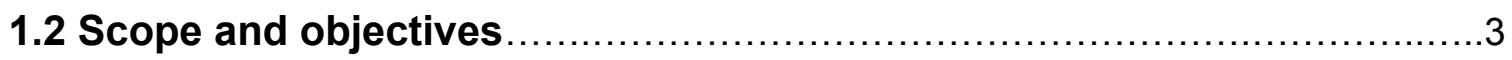

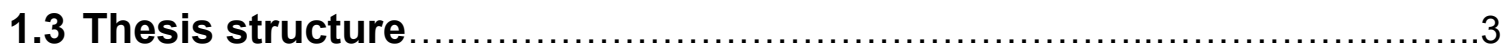

\section{Chapter 2}

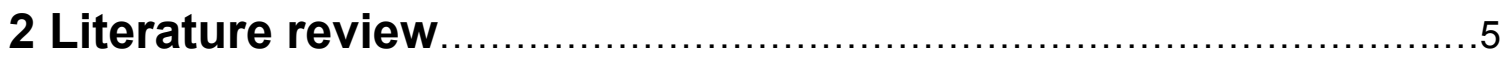

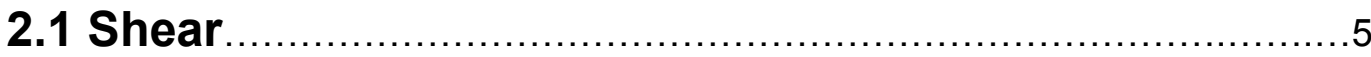

2.1.1 Brief historical review for shear studies and researches.....5

2.1.2 Compression Field Theory (CFT) ............................10

2.1.3 Modified Compression Field Theory (MCFT) ....................15

2.1.4 Simplified Modified Compression Field Theory (SMCFT) .....23

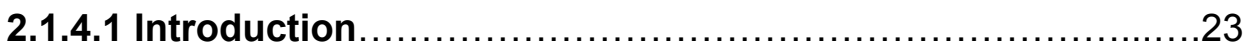

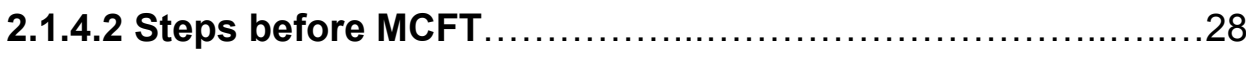

2.1.4.3 Derivation of Simplified (MCFT) ................................. 32

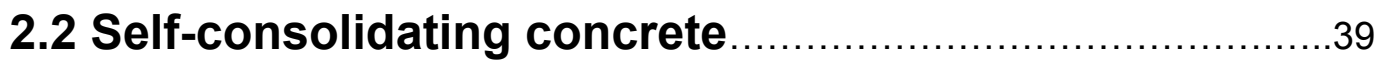

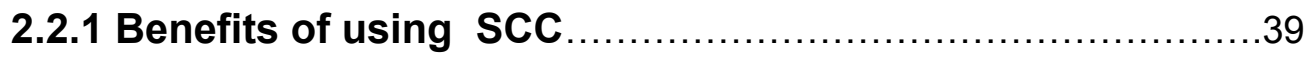

2.2.2 Difficulties facing the use of SCC ..............................40

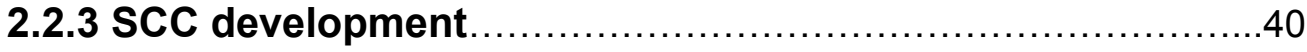

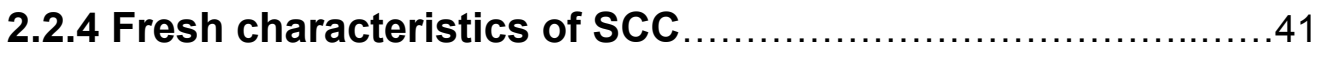

2.2.5 Testing SCC fresh properties .................................42

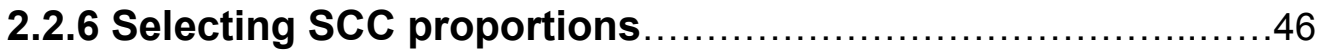

2.2.6.1 Finely divided powders and supplementary cementitious

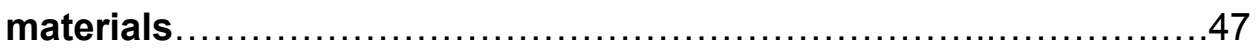

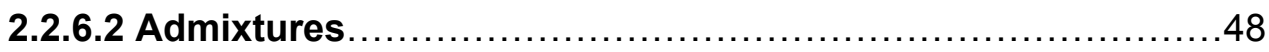




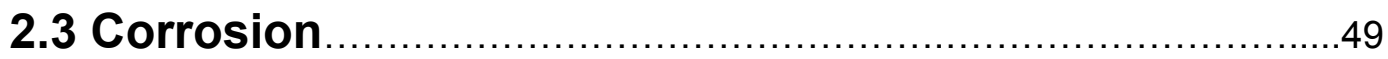

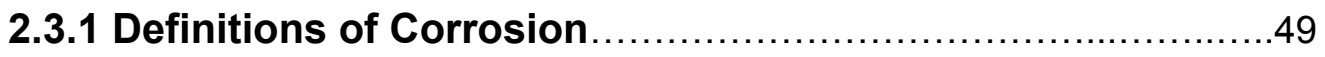

2.3.2 Fundamentals and concepts of corrosion of reinforcing steel

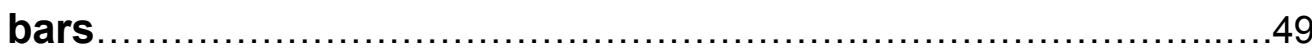

2.3.3 Corrosion electrochemical reactions ...........................50

2.3.4 Hypothetical calculation of corrosion mass loss ................52

2.3.5 Stages of corrosion deterioration................................54

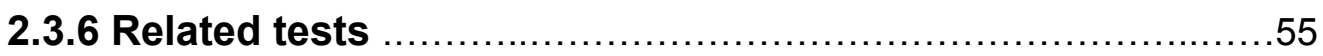

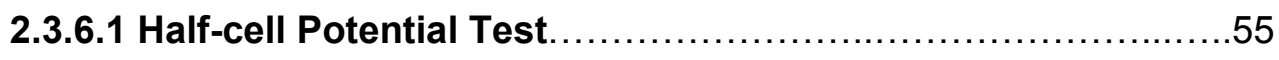

2.3.6.2 Rapid Chloride Permeability Test................................60

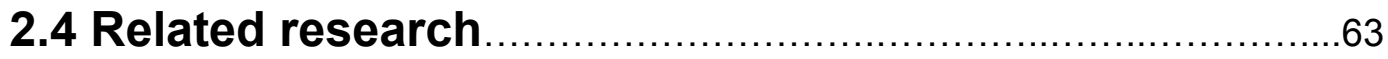

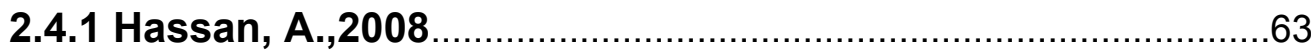

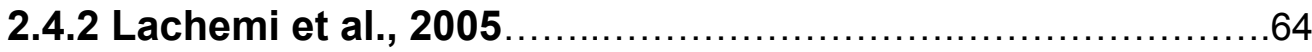

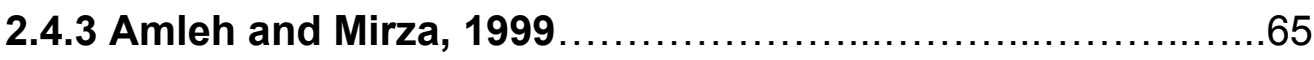

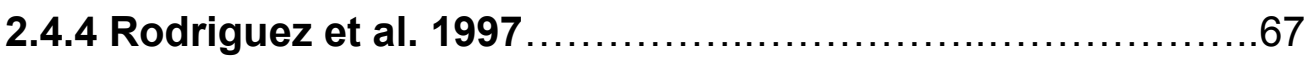

\section{Chapter 3}

3 Experimental program.......................................................... 68

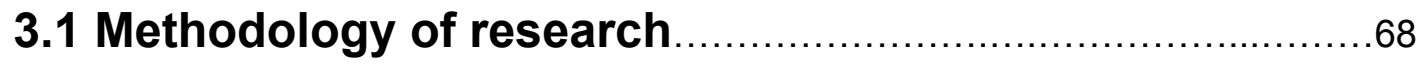

3.1.1. RC beams, categorizing and preparations ..................68

3.1.2. Mass loss calculations ................................................ 70

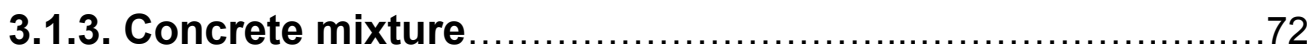

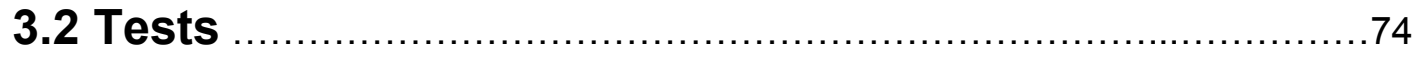

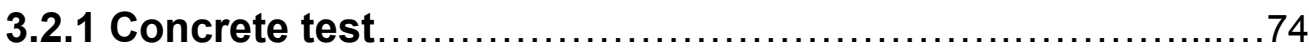

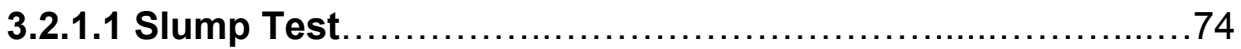

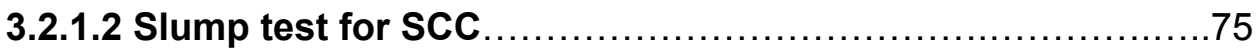

3.2.1.3 Compression test ............................................. 76

3.2.1.4 Split cylinder test.............................................. 77

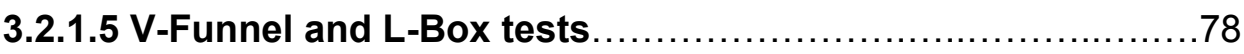

3.2.2. Half-cell potential test and rapid chloride ion test..............79 
3.3 Accelerated Corrosion ........................................................

3.3.1 Electrolyte cell setup and voltage daily monitoring ............79

3.3.2 Drawing corrosion crack patterns .................................... 80

3.4 Loading test and LVDTs setting ....................... 82

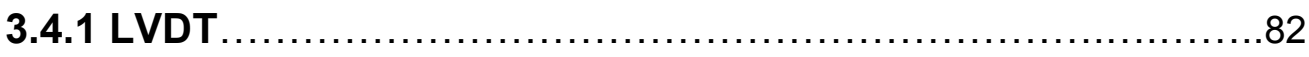

3.4.2 LVDTs setting .................................................. 82

3.5 The loading crack patterns ....................................

3.6 Actual mass loss measuring ....................................90

\section{Chapter 4}

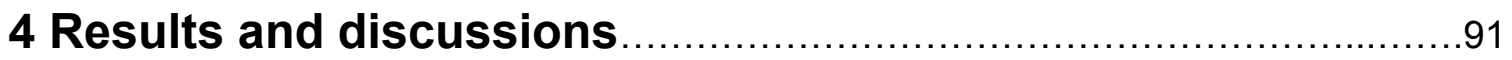

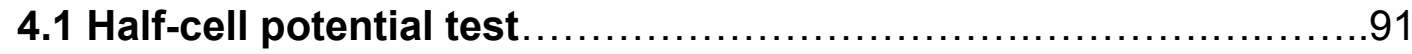

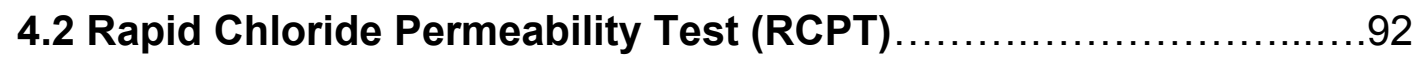

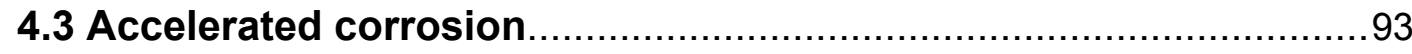

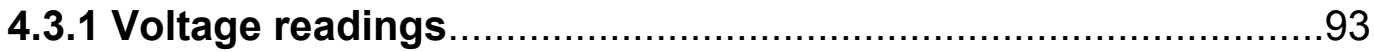

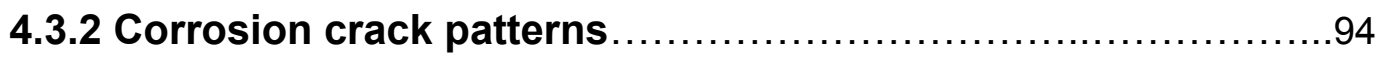

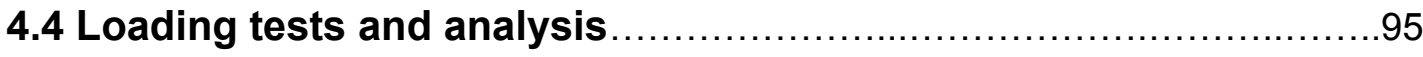

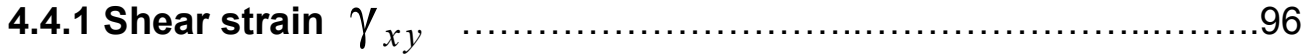

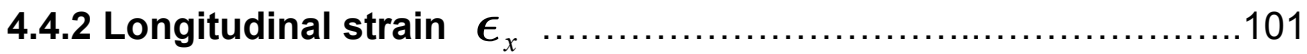

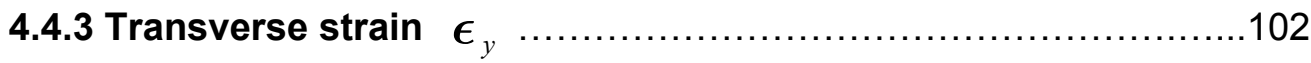

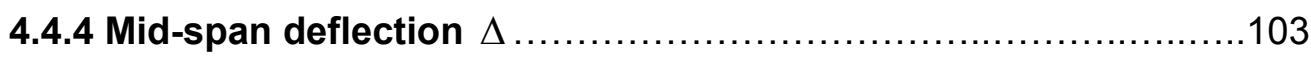

4.4.5 Deflection along the beam........................................ 104

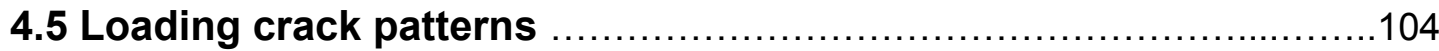

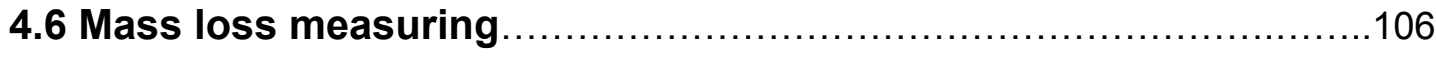

\section{Chapter 5}

Conclusions and recommendations ..................................117

5.1 Summary. 


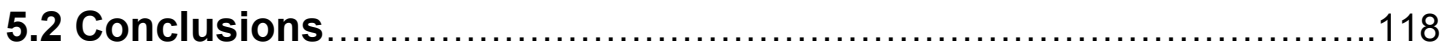

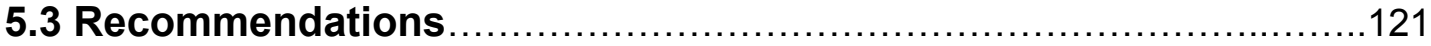

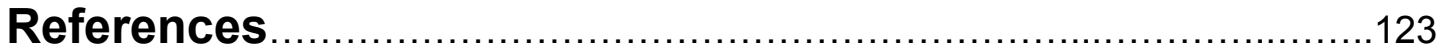

\section{Appendices}

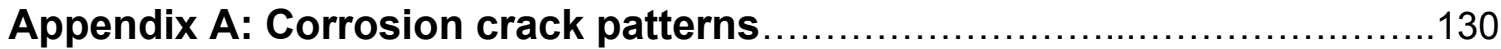

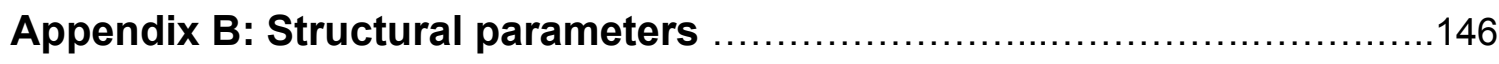

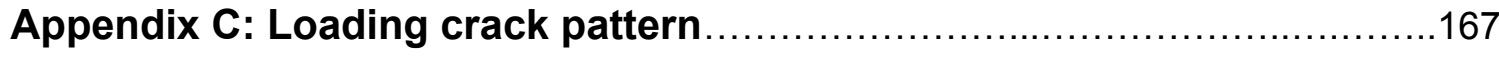

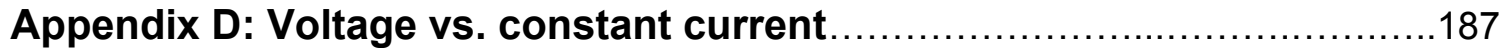

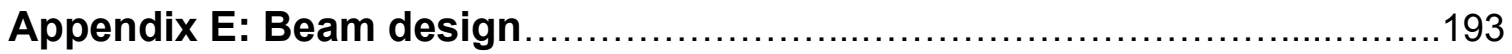




\section{List of Tables}

Table (2-1): Values of $\beta$ and $\theta$ for sections without transverse reinforcement. (From A23.3 Table11-1) (MacGregor and Bartlett, 2000).....................26

Table (2-2): Values of $\beta$ and $\theta$ for sections with transverse reinforcement. (From A23.3 Table11-1) (MacGregor \& Bartlett, 2000)........................27

Table (2-3): Chloride ion penetrability based on charge passed (ASTM C1202-97) .....61

Table (3-1): Weights of rebars in NC beams, stirrups and flexural....................71

Table (3-2): Weights of self-consolidating concrete beams, stirrups and flexural.........71

Table (3-3): The mixture proportions for NC and SCC mixtures $\ldots \ldots \ldots \ldots \ldots \ldots \ldots \ldots \ldots \ldots \ldots \ldots \ldots \ldots \ldots$

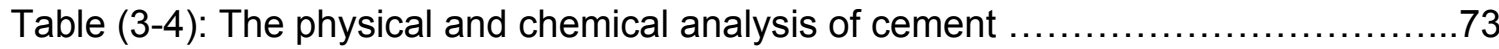

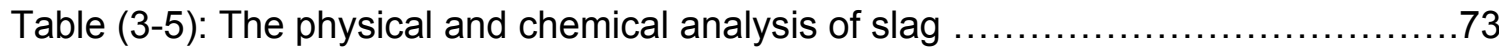

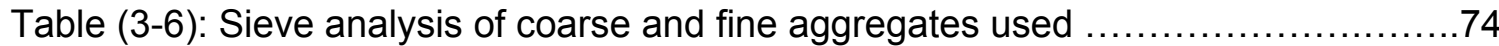

Table (3-7): The fresh properties of NC and SCC used in this research................78

Table (3-8): Compressive and split strength results for NC and SCC $\ldots \ldots \ldots \ldots \ldots \ldots \ldots .78$

Table (4-1): Half-cell potential test readings for NC and SCC beams. .................108

Table (4-2): Categories of half-cell potential test results (half-cell potential test manual, 2003) 108

Table (4-3): Categories of corrosion conditions of copper/copper sulphate half-cell vs. voltmeter reading ranges, ASTM C 876 - 91 (Reapproved 1999) 109

Table (4-4): Max crack width on NC and SCC beams at multi-stages of corrosion......111

Table (4-5): Loads at failure, at first flexural crack, and at first diagonal crack with the effect corrosion level on $\mathrm{P}_{\mathrm{f}} / \mathrm{P}_{\mathrm{u}}$ and $\mathrm{P}_{\mathrm{d}} / \mathrm{P}_{\mathrm{u}}$

Table (4-6): The weight of steel components of the steel cage for each NC and SCC beams, before and after corrosion.

Table (4-7): The initial and final weight of the steel subjected to corrosion, the remaining percentage of steel and the actual mass loss after finalizing the corrosion

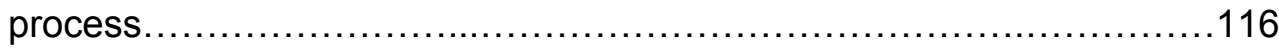

Table (D-1): Voltage readings for the NC beams at different stages of corrosion........187 Table (D-2): Voltage readings for the SCC beams at different stages of corrosion.....190 


\section{List of Figures}

Figure (1-1): Corrosion damage in concrete beams and columns.......................

Figure (2-1): Jourawski's model, the simplest case of a rectangular cantilever loaded at the free end (Timoshenko, 1983) .......................................... 6

Figure (2-2): American specification for shear design (1920-1951) based on ACl Standard No. 23, 1920 (ACl 445R-99) ..................................

Figure (2-3): Shear failure of roof beams in Air Force warehouse, Wilkins Air Force Depot, Shelby, Ohio (Collins and Mitchell, 1991)..........................9

Figure (2-4): Frame structure containing $B$ regions and $D$ regions, its static system, and bending moments (Schlaich et al. 1987) (ACl 445R-99) ...................10

Figure (2-5): Compatibility conditions for cracked web element, (ACI 445R-99)..........11

Figure (2-6): Influence of $\theta$ on reinforcement strain (Collins and Mitchell, 1991) ..........11

Figure (2-7): Compression Field Theory for prestressed beams subjected to shear (Mitchell and Collins, 1974)

Figure (2-8): Comparison of conditions in a cylinder and in diagonally cracked concrete (Collins and Mitchell, 1991). 13

Figure (2-9): Reinforced concrete element failing in shear (Vecchio and Collins, 1982).

Figure (2-10): Compressive stress-strain relationship for cracked concrete (Collins and Mitchell, 1991). 15

Figure (2-11): Stress field in web of reinforced concrete beam (Collins and Mitchell, 1991) 16

Figure (2-12): Equilibrium conditions of modified compression field theory (Collins and Mitchell, 1991). 16

Figure (2-13): Tensile stress-strain relationship for diagonally cracked concrete (Vecchio and Collins, 1986). 18

Figure (2-14): Transmitting forces across cracks (Vecchio and Collins, 1986)...........19

Figure (2-15): Spacing of inclined cracks (Collins and Mitchell, 1991) ...................21

Figure (2-16): Definition of $A_{c e f}$ (Collins and Mitchell, 1991)...........................22

Figure (2-17): Parameters influencing crack spacing (Collins and Mitchell, 1991)........23 Figure (2- 18): Predicting shear strength of reinforced concrete beams (Bentz et al. 2006). .24

Figure (2-19): Values of $\theta$ and $\beta$ for members without stirrups (Collins, 1993).......29 
Figure (2-20): Values of $\theta$ and $\beta$ for members with stirrups (Collins, 1993)..........30

Figure (2-21): Equations of modified compression field theory (Bentz et al. 2006)........31

Figure (2-22): Comparison of predicted and observed shear stress shear strain response of six elements (Bentz et al. 2006).................................. 31

Figure (2-23): Transmission of forces across cracks (Bentz et al. 2006)..................33

Figure (2-24): Determination of beta and theta values for elements not containing transverse reinforcement (Bentz et al. 2006). 34

Figure (2-25): Comparison of values for $\theta$ and $\beta$ given by simple equations with values determined from MCFT for elements without transverse reinforcement (Bentz et al. 2006). .36

Figure (2-26): Comparison of values for $\theta$ and $\beta$ given by simple equations with values determined from MCFT for elements with transverse reinforcement (Bentz et al. 2006). 37

Figure (2-27): Relationship between shear stress and shear rate (Korradi, 2003).......41

Figure (2-28): Tangential components of gravity force (Korradi, 2003).................41

Figure (2-29): SCC slump flow (ACI 237R-07) ...............................43

Figure (2-30): J-Ring apparatus (ACI 237R-07) ............................... 43

Figure (2-31): Typical L-Box arrangement to measure flow of SCC around rebar obstructions. The left part of the image is adopted from EFNARC (2002); and the right from Groth (2000).

Figure (2-32): Schematic of V-funnel apparatus (Khayat, 2004). Right-hand photo is from Abdul Hameed (2005)..........................................45

Figure (2-33): Schematic of U-box test (Khayat, 2004)............................46

Figure (2-34): Corrosion of steel bar embedded in concrete (iron is dissolved at anode and precipitates as rust at cathode) ( $\mathrm{ACl} 228.2 \mathrm{R}-98)$ .51

Figure (3-35): The relative volumes of iron and its reaction product $(\mathrm{ACl} 222 \mathrm{R}-01) \ldots \ldots 52$

Figure (2-36): Four stages in the corrosion deterioration of CRC bridge elements. Additional stages are possible when deterioration is more severe (Higgins et al. 2003) 54

Figure (2-37): Apparatus for half-cell potential method described in ASTM C 876 (ACI 228.2R-98). .56

Figure (2-38): Sectional view of a copper-copper sulphate half-cell (ASTM C 876 91). .57 
Figure (2-39): Equipotential contours from survey data of bridge deck at grid spacing of $0.76 \mathrm{~m}$ (only contours less than $-0.30 \mathrm{~V}$ are shown and contour interval is

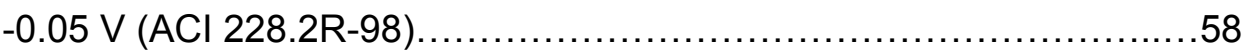

Figure (2-40): Cumulative frequency diagram (Sharp,2004) ............................59

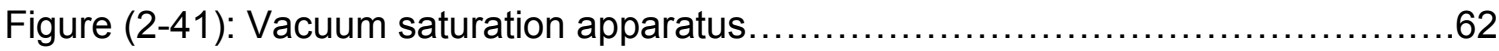

Figure (2-42): Rapid Chloride Permeability Test cell. Image on left adopted from Abdul Hameed (2005), and image on right shows cell ready for test. 62

Figure (2-43): Influence of coarse aggregate on crack shear plane (a) $19 \mathrm{~mm}$ aggregate (S19), and (b) $12 \mathrm{~mm}$ aggregate (N12) (Lachemi et al., 2005). 64

Figure (2-44): The the graph on the left side refers to relative bond stress versus level of corrosion. The graph on the right refers to relative bond stress versus average crack spacing (Amleh and Mirza, 1999) .66

Figure (2-45): Scheme of beams types 11 and 31 (Rodriguez et al. 1997). .67

Figure (3-1): The distribution of the beams using NC and SCC with the stages of corrosion based on mass loss percentage 68

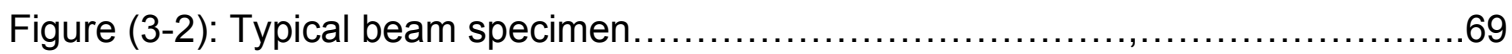

Figure (3-3): Cross-sectional details and beam dimensions...............................69

Figure (3-4): Mold for slump test (ASTM C 143 / C 143M) ...............................

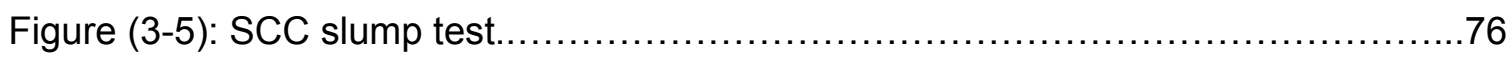

Figure (3-6): Split cylinder test (MacGregor and Bartlett, 2000) ........................77

Figure (3-7): Electrolyte cell components (power suppliers, concrete beams as an anode, metal mesh as a cathode, saline solution, plastic basins, and connecting wires) ...................................................... 79

Figure (3-8): Measuring the crack widths on the corrosion crack pattern..................80

Figure (3-9): Spalling of concrete from the bottom of specimen NC B-8 .................81

Figure (3-10): The effects of shear stress on two elements located on the concrete beam. The left element represents Case \#1, and the right element

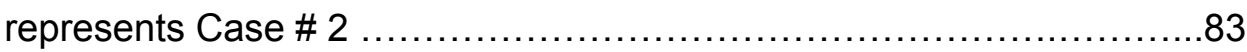

Figure (3-11): Arbitrarily oriented LVDTs in a biaxial strain field (Rosettes strain gauge measurements, 2000)

Figure (3-12): Defining $X$ axis as bisector of angle between gage axes (Rosettes strain gauge measurements, 2000). $\gamma_{x y}=\frac{\boldsymbol{\epsilon}_{1}-\boldsymbol{\epsilon}_{2}}{\operatorname{Sin} 2 \alpha}$. .85

Figure (3-13): $45^{\circ}$ rosette LVDTs (Rosettes strain gauge measurements, 2000).........87 
Figure (3-14): The LVDTs numbers and locations on the beam.

Figure (3-15): Deflection behavior for a reinforced concrete beam: a) beam elevation, b) bending moment diagram, c) flexural stiffness, d) bending moment deflection at the mid-span (Brzev and Pao, 2006). 89

Figure (4-1): Half-cell potential test applied to NC and SCC beams numbered. 310. 107

Figure (4-2): The RCPT results at age of 64 days for NC samples, and 57 days for SCC samples. 109

Figure (4-3): The RCPT results at age of 137 days for NC samples, and 130 days for SCC samples. 110

Figure (4-4): Electrical resistance values for both NC and NCC beams measured by (ohms) vs. elapsed time by (days).

Figure (4-5): The effects of corrosion level on load at failure, load at first flexural crack, and load at first diagonal crack

Figure (4-6): Effects of corrosion level on the percentage of $P_{f} / P_{u}$ and $P_{d} / P_{u}$ 113

Figure (4-7): Effects of stages of corrosion measured by mass loss on ratios of $\mathrm{P}_{\mathrm{fl}} / \mathrm{P}_{\mathrm{u}}$ and $\mathrm{P}_{\mathrm{d}} / \mathrm{P}_{\mathrm{u}}$ 114

Figure (4-8): Strut and tie model shows the tension and compression zones in the beam (Russo et al. 2005)............................................114

Figure (4-9): Calculation of longitudinal strain (Collins et al. 2007)..................114

Figure (A-1): The crack pattern of NC beam no. 3. $5 \%$ mass loss .................... 130

Figure (A-2): The crack pattern of NC beam no. 9. 5\% mass loss............................131

Figure (A-3): The crack pattern of SCC beam no. 4. 5\% mass loss. ...................132

Figure (A-4): The crack pattern of SCC beam no.10. 5\% mass loss ..................133

Figure (A-5): The crack pattern of NC beam no. $7.10 \%$ mass loss ....................134

Figure (A-6): The crack pattern of NC beam no. 10. 10\% mass loss...................135

Figure (A-7): The crack pattern of SCC beam no. 5. 10\% mass loss ..................136

Figure (A-8): The crack pattern of SCC beam no.8 10\% mass loss .....................137

Figure (A-9): The crack pattern of NC beam no. 4. 15\% mass loss....................138

Figure (A-10): The crack pattern of NC beam no. 5. 15\% mass los...................139

Figure (A-11): The crack pattern of SCC beam no. 6. 15\% mass loss ..................140

Figure (A-12): The crack pattern of SCC beam no. 7. 15\% mass loss ..................141

Figure (A-13): The crack pattern of NC beam no. $6.20 \%$ mass loss...................142

Figure (A-14): The crack pattern of NC beam no. 8. 20\% mass loss....................143 
Figure (A-15): The crack pattern of SCC beam no. 3. 20\% mass loss................ 144

Figure (A-16): The crack pattern of SCC beam no. 9. 20\% mass loss................ 145

Figure (B1- 1): Shear strain for the left and right sides of the NC and SCC control beams, with zero mass loss

Figure (B1-2): Shear strain for left and right sides of the $5 \%$ mass loss NC and SCC beams. 146

Figure (B1-3): Shear strain of left and right sides of the $10 \%$ mass loss NC and SCC beams. 147

Figure (B1-4): Shear strain for the left and right sides of the $15 \%$ mass loss NC and SCC beams 147

Figure (B1-5): Shear strain for left and right sides of the $20 \%$ mass loss NC and SCC beams.

Figure (B2-1): $\quad \boldsymbol{\epsilon}_{x \text { left }}$ and $\boldsymbol{\epsilon}_{x \text { right }}$ for the NC and SCC control beams with zero mass loss.

Figure (B2-2): $\boldsymbol{\epsilon}_{x \text { left }}$ and $\boldsymbol{\epsilon}_{x \text { right }}$ for the $5 \%$ mass loss $\mathrm{NC}$ and SCC

Figure (B2-3): $\quad \boldsymbol{\epsilon}_{x}$ left and $\boldsymbol{\epsilon}_{x \text { right }}$ for the $10 \%$ mass loss $\mathrm{NC}$ and $\mathrm{SCC}$ beams. 150

Figure (B2-4): $\quad \boldsymbol{\epsilon}_{x \text { left }}$ and $\boldsymbol{\epsilon}_{x \text { right }}$ for the $15 \%$ mass loss NC and SCC beams. .150

Figure (B2-5): $\quad \boldsymbol{\epsilon}_{x \text { left }}$ and $\boldsymbol{\epsilon}_{x \text { right }}$ for the $20 \%$ mass loss NC and SCC beams.

Figure (B3-1): $\quad \boldsymbol{\epsilon}_{y}$ left and $\boldsymbol{\epsilon}_{y \text { right }}$ for the control NC and SCC beams with zero mass loss 152

Figure (B3-2): $\quad \boldsymbol{\epsilon}_{y \text { left }}$ and $\boldsymbol{\epsilon}_{y \text { right }}$ for the $5 \%$ mass loss NC and SCC beams. 152

Figure (B3-3): $\quad \boldsymbol{\epsilon}_{y}$ left and $\boldsymbol{\epsilon}_{y \text { right }}$ for the $10 \%$ mass loss NC and SCC beams. .153

Figure (B3-4): $\quad \boldsymbol{\epsilon}_{y \text { left }}$ and $\boldsymbol{\epsilon}_{y \text { right }}$ for the $15 \%$ mass loss NC and SCC beams 
Figure (B3-5): $\quad \epsilon_{y}$ left and $\boldsymbol{\epsilon}_{y \text { right }}$ for the $20 \%$ mass loss NC and SCC beams. 154

Figure (B4-1): Mid-span deflection $\Delta$ vs. load for the control NC and SCC beams with zero mass loss.

Figure (B4-2): Mid-span deflection $\Delta$ vs. load for the $5 \%$ mass loss NC and SCC beams.

Figure (B4-3): Mid-span deflection $\Delta$ vs. load for the $10 \%$ mass loss NC and SCC beams.

Figure (B4-4): Mid-span deflection $\Delta$ vs. load for the $15 \%$ mass loss NC and SCC beams. 156

Figure (B4-5): Mid-span deflection $\Delta$ vs. load for the $20 \%$ mass loss NC and SCC beams. 157

Figure (B5-1): The deflection along the beam for different values of loading for NC; B-2 (zero mass loss)..... 157

Figure (B5-2): The deflection along the beam for different values of loading for SCC; B-1 (zero mass loss). 158

Figure (B5-3): The deflection along the beam for different values of loading for SCC; B-2 (zero mass loss). 158

Figure (B5-4): The deflection along the beam for different values of loading for NC; B-3 (5\% mass loss). 159

Figure (B5-5): The deflection along the beam for different values of loading for NC; B-9 (5\% mass loss). 159

Figure (B5-6): The deflection along the beam for different values of loading for SCC; B-4 (5\% mass loss). 160

Figure (B5-7): The deflection along the beam for different values of loading for SCC; B10 (5\% mass loss). 160

Figure (B5-8): The deflection along the beam for different values of loading for NC; B-7 (10\% mass loss). 161

Figure (B5-9): The deflection along the beam for different values of loading for NC; B-10 (10\% mass loss). 161

Figure (B5-10): The deflection along the beam for different values of loading for SCC; B$5(10 \%$ mass loss $)$ 162 
Figure (B5-11): The deflection along the beam for different values of loading for SCC; B-

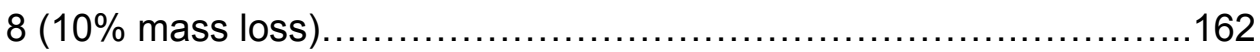

Figure (B5-12): The deflection along the beam for different values of loading for NC; B-4

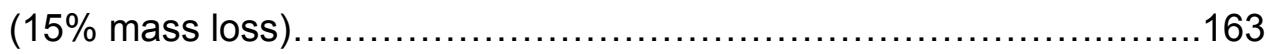

Figure (B5-13): The deflection along the beam for different values of loading for NC; B-5

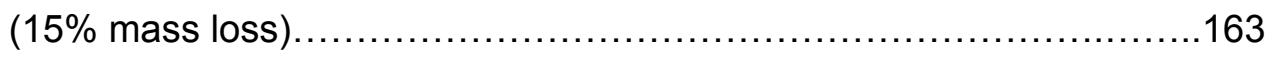

Figure (B5-14): The deflection along the beam for different values of loading for SCC; B-

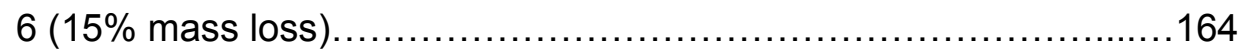

Figure (B5-15): The deflection along the beam for different values of loading for SCC; B-

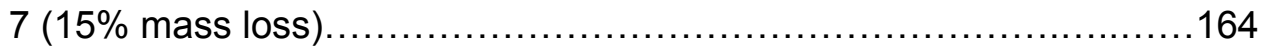

Figure (B5-16): The deflection along the beam for different values of loading for NC; B-6

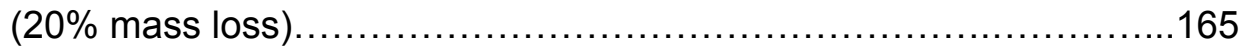

Figure (B5-17): The deflection along the beam for different values of loading for NC; B-8

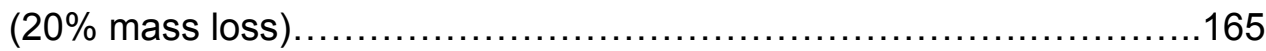

Figure (B5-18): The deflection along the beam for different values of loading for SCC; B-

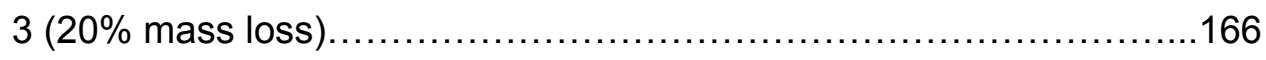

Figure (B5-19): The deflection along the beam for different values of loading for SCC; B-

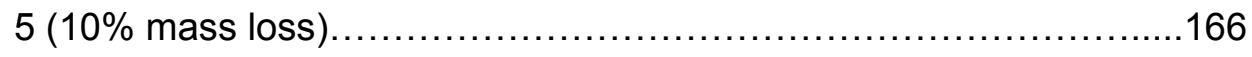

Figure (C-1): Loading crack pattern for NC beam no. 1 with zero mass loss............167

Figure (C-2): Loading crack pattern for NCbeam no. 2 with zero mass loss.............168

Figure (C-3): Loading crack pattern for SCCbeam no. 1 with zero mass loss............169

Figure (C-4): Loading crack pattern for SCC beam no. 2 with zero mass loss...........170

Figure (C-5): Loading crack pattern for NC beam no. 3 with $5 \%$ mass loss.............171

Figure (C-6): Loading crack pattern for NC beam no. 9 with $5 \%$ mass loss...............172

Figure (C-7): Loading crack pattern for SCC beam no. 4 with $5 \%$ mass loss.............173

Figure (C-8): Loading crack pattern for SCC beam no. 10 with $5 \%$ mass loss...........174

Figure (C-9): Loading crack pattern for NC beam no. 7 with $10 \%$ mass loss............175

Figure (C-10): Loading crack pattern for NC beam no. 10 with $10 \%$ mass loss..........176

Figure (C-11): Loading crack pattern for SCC beam no. 5 with $10 \%$ mass loss.........177

Figure (C-12): Loading crack pattern for SCC beam no. 8 with 10\% mass loss.........178

Figure (C-13): Loading crack pattern for NC beam no. 4 with $15 \%$ mass loss...........179

Figure (C-14): Loading crack pattern for NC beam no. 5 with $15 \%$ mass loss...........180

Figure (C-15): Loading crack pattern for SCC beam no. 6 with 15\% mass loss.........181

Figure (C-16): Loading crack pattern for SCC beam no. 7 with 15\% mass loss..........182 
Figure (C-17): Loading crack pattern for NC beam no. 6 with $20 \%$ mass loss...........183

Figure (C-18): Loading crack pattern for NC beam no. 8 with $20 \%$ mass loss..........184

Figure (C-19): Loading crack pattern for SCC beam no. 3 with 20\% mass loss.........185

Figure (C-20): Loading cracks pattern for SCC beam no. 7 with $20 \%$ mass loss........186

Figure $(E-1)$ : Effect of a/d ratio on the shear strength of beams without stirrups (MacGregor and Bartlett, 2000).....................................193

Figure (E-2): Shear failure in short beams, $a / b=1-2.5$ (MacGregor and Bartlett,

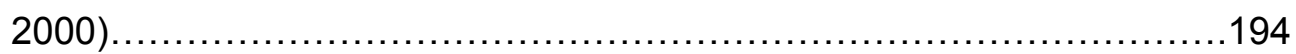

Figure (E-3): Modes of failure in deep beams, a/d = 0.5 to 2 (MacGregor and Bartlett,

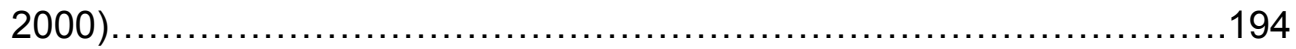

Figure (E-4) Shear force diagram and bending moment diagram...................196 


\section{List of Symbols}

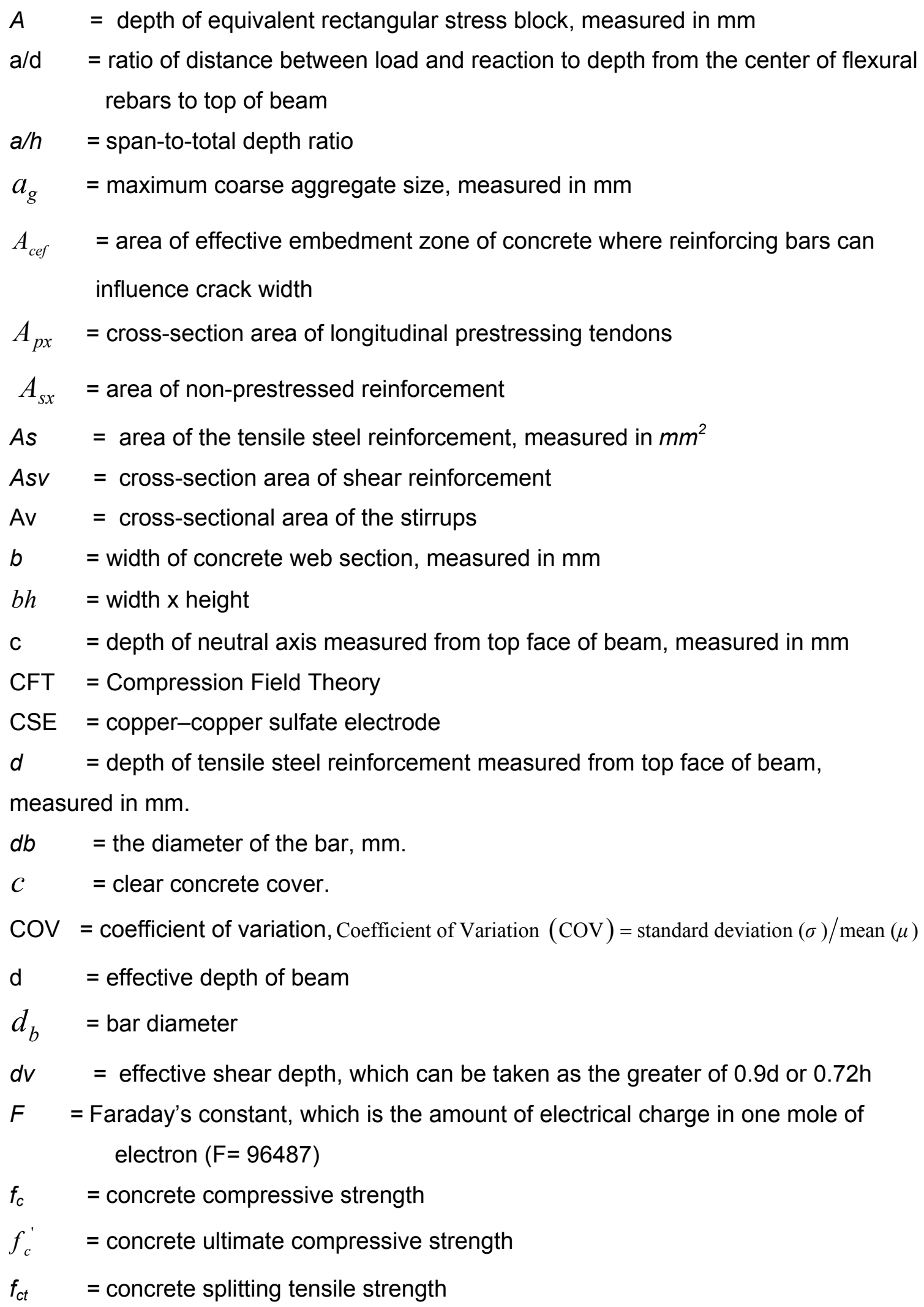




$$
\begin{aligned}
& f_{l} \quad=\text { average stresses in longitudinal reinforcing bars } \\
& f_{p} \quad=\text { average stresses in longitudinal prestressing steel } \\
& f_{x} \quad=\text { stress in longitudinal bars } \\
& f_{v} \quad=\text { stress in stirrups } \\
& f_{y} \quad=\text { yield stress of stirups } \\
& f_{2}=\text { diagonal compressive stress in concrete } \\
& F_{v} \quad=\text { allowable tensile stress in shear reinforcement } \\
& f_{z} \quad=\text { clamping stresses } \\
& \mathrm{G} \quad=\text {-weight of bar after removal of corrosion products, } \mathrm{N} \\
& \text { GGBFS = ground-granulated blast furnace slag } \\
& \mathrm{H} \quad=\text { height of section } \\
& \mathrm{HPC}=\text { high-performance concrete } \\
& \text { HRWR = high-range water reducers } \\
& \text { HRWRA = high-range water reducer admixtures } \\
& \mathrm{HWC}=\text { high workability concrete } \\
& i=\text { current passed, measured in amperes } \\
& \text { I = moment of inertia } \\
& I_{c r} \quad=\text { moment of inertia transformed from }\left(I_{g}\right) \text { to the cracked transformed section } \\
& I_{g} \quad=\text { gross transformed moment of inertia } \\
& j d=\text { the beam's lever arm approximately equal } 0.875 d \\
& \boldsymbol{k}=\text { relative beam stiffness (i.e. slope of load-deflection curve), measured in \% } \\
& L \quad=\text { effective beam span } \\
& \text { Id = developmental length of deformed bar } \\
& \text { LVDT = Linear Variable Displacement Transducers } \\
& \text { I = bond length, measured in } \mathrm{mm} \\
& k_{1}=\text { coefficient that characterizes bond properties of bars } \\
& L \quad=\text { length of specimen, measured in } \mathrm{mm} \\
& m \quad=\text { discharged mass at an electrode } \\
& \mathrm{M} \quad=\text { applied bending moment at specific cross section } \\
& M \quad=\text { atomic weight of iron }(55.847 \mathrm{~g} / \mathrm{mol})
\end{aligned}
$$




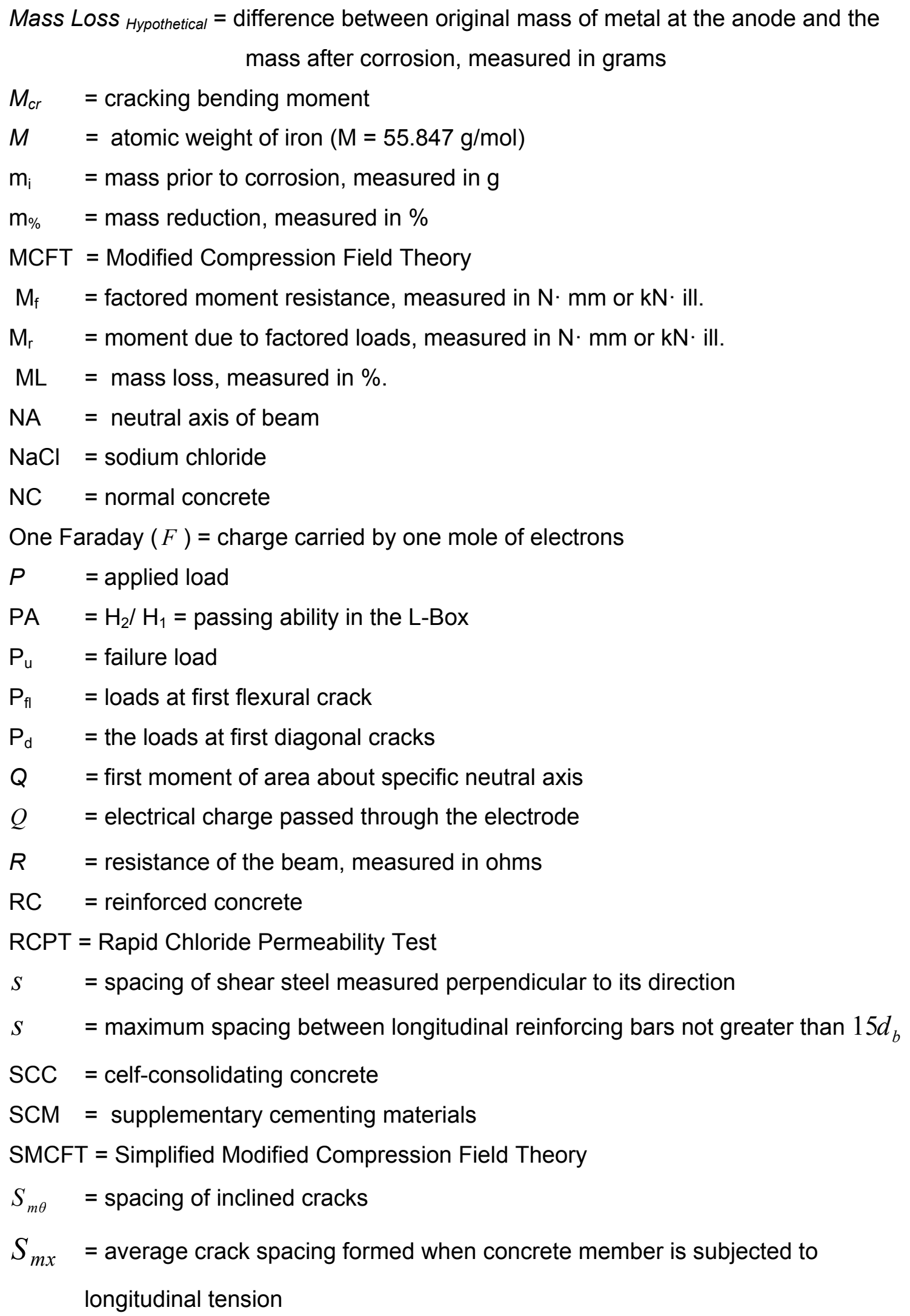




$$
\begin{array}{ll}
S_{m v}= & \text { average crack spacing formed when concrete member is subjected to } \\
& \text { transverse tension } \\
S V & =\text { stirrup spacing } \\
S_{x e}= & \text { crack spacing parameter dependent on crack control characteristics of } \\
& \text { longitudinal reinforcement } \\
S_{z} \quad= & \text { horizontal spacing between vertical bars in the } z \text {-direction } \\
t & =\text { time passed, measured in seconds } \\
T & =\text { tension force acting on a specific section } \\
V & =\text { potential difference across the beam, measured in volts. } \\
V & =\text { applied shear force at specific cross section } \\
V & =\text { total shear minus } 0.02 f_{c}^{\prime} b j d \text { (or } 0.03 f_{c}^{\prime} b j d \text { with special anchorage) } \\
U & =\text { dynamic Poisson's ratio } \\
V & =\text { shear stress } \\
V a & =
\end{array}
$$




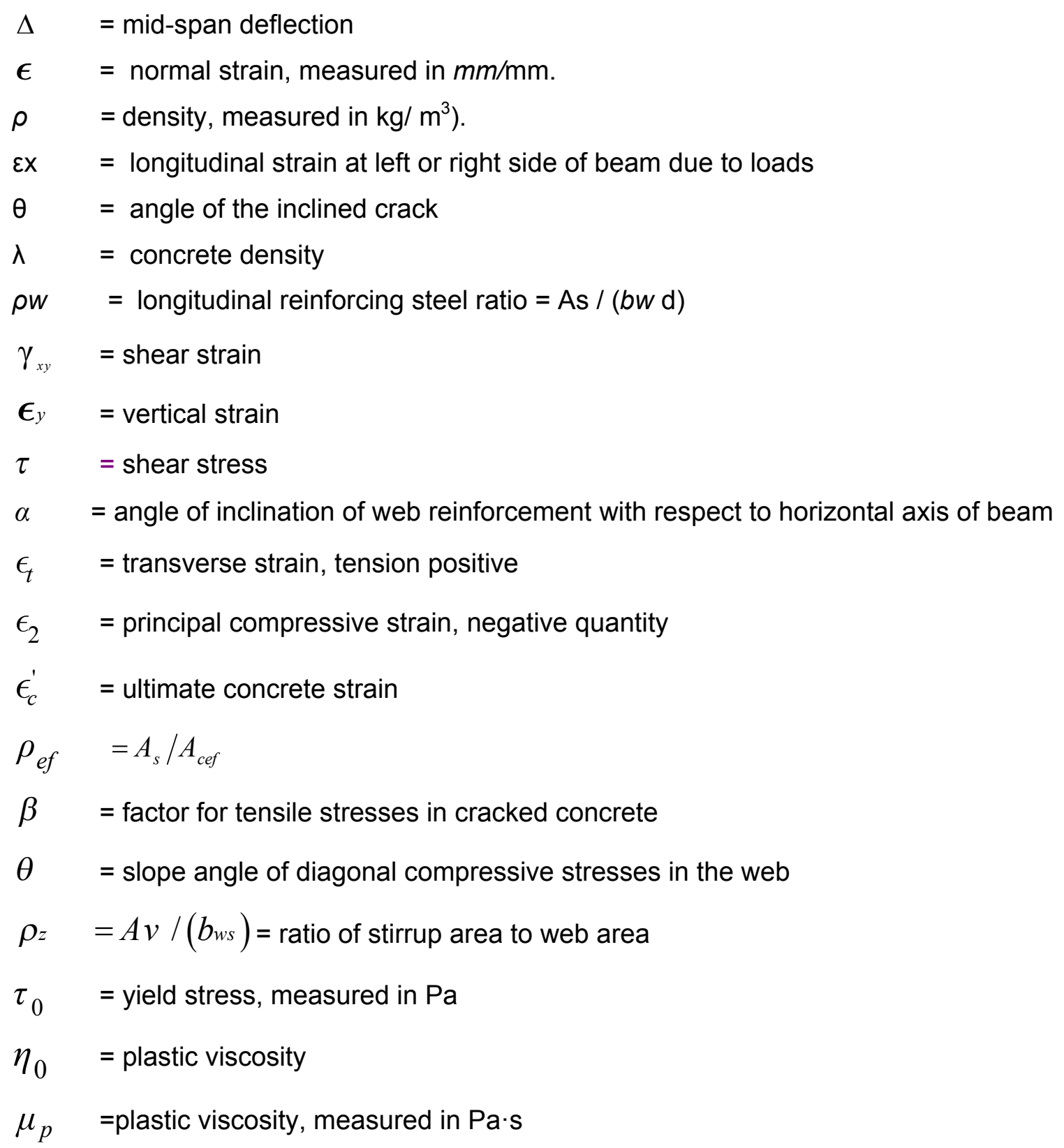




\section{Acknowledgements}

First and foremost, praises to ALLAH with all my thanks and gratitude for the blessings and grace surrounding my life, and for giving me the ability to complete this research.

It is an honour to express my warmest appreciation to my supervisors, Dr. Lamya Amleh and Dr. Mohamed Lachemi, for their scientific, academic and financial support. I would like also to express my gratitude to Dr. Anwar Hossain and Dr. Medhat Shehata, who have enriched the scientific contents of this thesis with their valuable comments and discussion during the defence.

I also owe my deepest gratitude to my wife, Abeer, for her endless support and patience. I would like to thank my parents, extended family, friends and every person who prayed for me to succeed in life and especially in this research.

I am grateful to Dr. Assem Hassan for his wonderful scientific consultations, recommendations and support. I would like to thank Dr. Mustafa Sahmaran and his team of undergraduate students Bo Sung Hwang, Ying Eric Liu, and Shahid Neaz for their help in casting the concrete beams, performing the concrete tests, and demolishing the beams to retrieve the rebars. Dr. Sahmaran's efforts in accelerating the loading tests are also greatly appreciated. I would also like to thank PhD candidate Ramy Zahran for his help in the RCPT test in the concrete lab and NDT lab.

In addition, I would like to thank my friend Tahrir Abdul Razak for his continuous computer support.

My thanks to structural lab staff Nidal Jaalouck and Mohamad Aldardari for the wonderful help in the practical part of my research, and to Alan Machin from the Mechanical Department for his sincere early efforts to make the bending jig.

Finally, I would like to thank Dufferin concrete for providing the normal concrete and self-consolidating concrete used in this research. 


\section{To}

My Mother...my first and closest teacher.

My Father...an icon of dedication in scientific research.

My wife...the angel that makes everything easy in my life.

My daughter...my spring of happiness. 


\section{Chapter 1}

\subsection{Introduction}

This research study investigates the effects of corrosion on shear behaviour using two different types of concrete, normal concrete (NC), and self-consolidating concrete (SCC).

SCC is a relatively new type of concrete that attracts both construction companies and civil engineers for a number of reasons. It offers a more environmentally friendly alternative, is more economical, and offers practical ease of use in construction sites. This new high-performance concrete is known for its excellent deformability and high resistance to segregation and bleeding. Not only is SCC easy to cast without vibration, it is also a suitable material to cast when congested reinforcement is used. Its usage has been limited due to a lack of information about some of its fresh and hardened characteristics. In Japan's 1998 Akashi-Kaikyo suspension bridge project, the SCC expedited pouring concrete from higher free falls, when the free fall was more than $3 \mathrm{~m}$ high, and was finished without segregation (Avery, 2004). Lachemi et al. (2005) mentioned that there is some concern among researchers and designers that SCC may not be strong enough in shear. This concern is due to some uncertainties in mechanisms resisting shear, especially the aggregate interlock mechanism, because of comparatively smaller amounts of coarse aggregates in SCC.

Shear behaviour has been studied for more than 100 years. However, predicting shear strength of RC beams still has no definitive answer. The approach taken by current codes in calculating shear strengths for a particular beam section can vary by factors of more than two. The current design codes are $\mathrm{ACl}$ 318-05 and Commentary 318R-05, AASHTO LRFD, BS EN 1992-1-1:2004 Euro code, CSA - A23.3-04, and JSCE Japan Society of Civil Engineers. The flexural strengths predicted by these codes are unlikely to vary by more than $10 \%$ (Bentz et al. 2006). The current design codes do not agree on a basis for a logical theory, and unlike pure flexure, experiments cannot be directed on reinforced concrete beams subjected to pure shear. In the normal four-points loading test for concrete beams, the area between the two point loads is under pure flexure, while the shear span of the beam, located from support to the load, is under the influence of constant shear and linearly varying moment. It is not easy to predict the shear behavior in general, because of the changing response from section to section along the shear span. Therefore, because shear strain is varied along both longitudinal 
and vertical directions of the concrete beams, at clamping stresses, and near the point loads, the reactions cause stirrup strains in these locations to be close to zero (Collins et al. 2006). One of the latest and most accurate theories is the modified compression field theory (MCFT), which has been followed by the simplified MCFT to ease the calculations required for analysis and design.

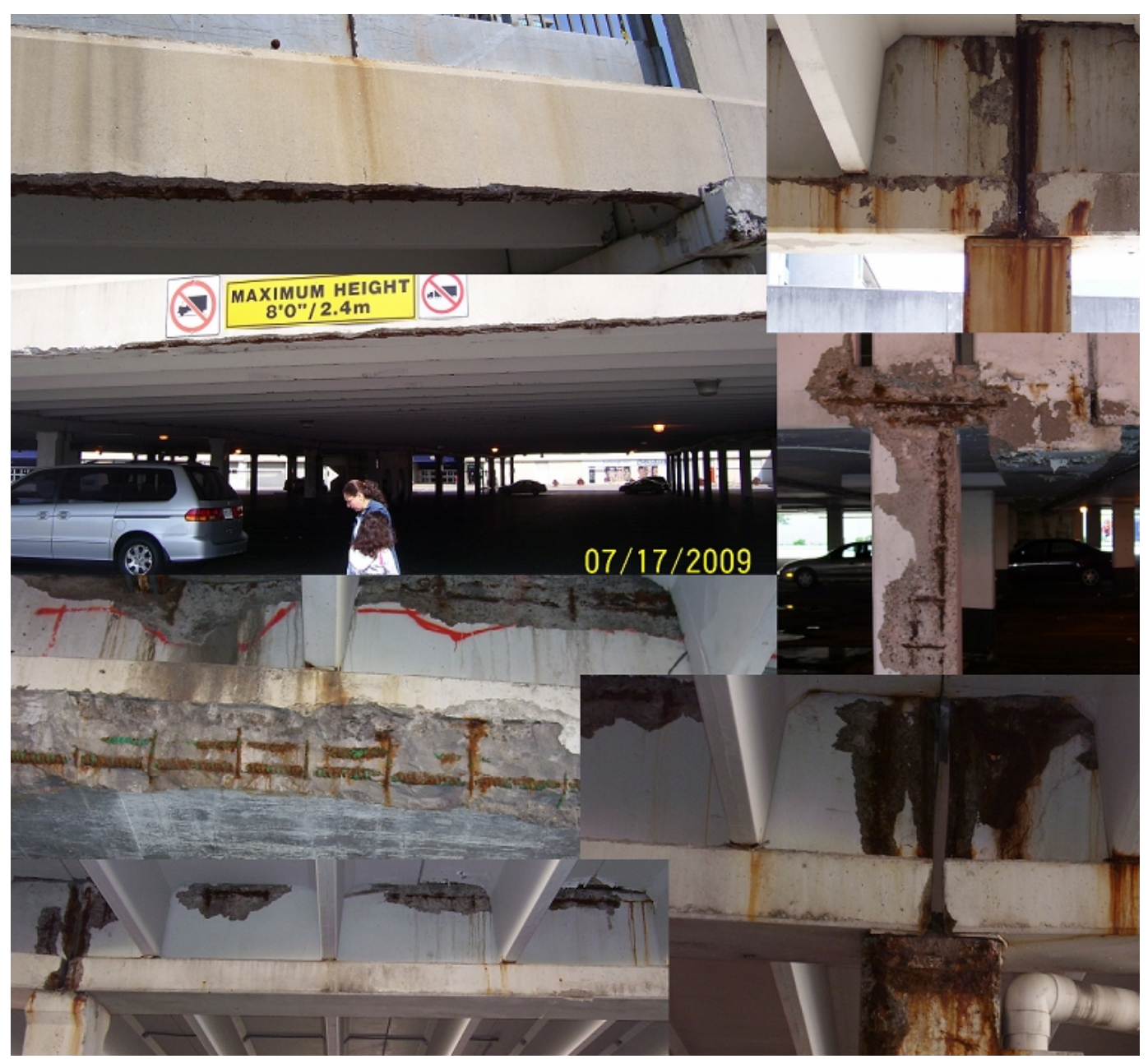

Figure (1-1): Corrosion damage in concrete beams and columns.

Rebar corrosion is a critical problem that affects both the structural safety and the economic value of corroded concrete structures and their surrounding environment. Globally, rehabilitation plans for concrete structures affected by corrosion cost billions of dollars every year. Figure (1-1) shows a number of critical corrosion attacks on the concrete elements of the parking lot at Scarborough Town Centre. Although the corrosion process is well known, further study of bond deterioration between rebars and 
the concrete resulting from corrosion is needed. Moreover, we need to know more about the effect of corrosion on structural response, and on the remaining structural capacity of the corroded elements. Corrosion is the root cause of most bridge failures; therefore, we need to develop effective corrosion protection techniques to ensure safer concrete structures, extend the life of future concrete structures, and to decrease the huge budgets required for proper rehabilitation.

\subsection{Scope and objectives}

The main purpose of this research is to gain a better understanding of the effects of corrosion on the shear behavior of self-consolidating concrete (SCC) beams. Most of the previous research that investigated shear resistance of SCC used SCC beams without stirrups; this research test SCCs beams with stirrups. The loading test has to provide an $\mathrm{a} / \mathrm{d}$ ratio of $2.5 \mathrm{a} / \mathrm{d}=$ the ratio of distance between load and reaction to the depth from the center of flexural rebars to the top of the beam) to assure the highest probability of shear failure. The main objectives of the research are:

- To study shear behaviour on beams made of different types of concrete and at different stages of corrosion by comparing the values of shear strain, $\gamma_{x y}$, the strain in x-direction, $\boldsymbol{\epsilon}_{x}$, the strain in y-direction, $\boldsymbol{\epsilon}_{y}$, the mid-span deflection, and the shape of deflection along the beams with loading variations for both NC and SCC beams.

- To study the effects of different stages of corrosion on the failure modes of NC and SCC beams, and on their loading crack patterns.

\subsection{Thesis structure}

This thesis contains five chapters:

Chapter 1: Introduces the research, scope and objective, and the thesis structure.

Chapter 2: Contains a literature review, including an overview of the development of shear research from the early stages to the latest attempts to predict the closest precise approach to analysis and design in shear. It includes a detailed review of SCC fundamentals and concepts of corrosion of reinforcing steel bars. Finally, it reviews some of the previous research. 
Chapter 3: Presents the experimental program, starting with the methodology of research including mass loss calculations, concrete mixtures, and tests required for fresh and hardened concrete.

Chapter 4: Demonstrates the results of all of the tests and explains the changes in the results across the NC and SCC beams. It summarizes the tests results of NC and SCC for fresh and hard concrete, including the Rapid Chloride Permeability Test (RCPT), and the half-cell potential test, and also includes the corrosion crack patterns.

Chapter 5: Contains a summary of this study, the conclusions drawn from this research investigation, and some future recommendations. 


\section{Chapter 2}

\section{Literature review}

\subsection{Shear}

Beams subjected to shear may develop diagonal cracks. Without providing the proper amount of reinforcement in the web and longitudinal direction, those cracks can lead to premature or sudden beam failure (CSA-A23.3 2004).

The priority in the design of any reinforced concrete beam is to determine the applied moment magnitude followed by the calculations of the beam dimensions, which leads to finding the area of the flexural steel reinforcement that can resist the moment. The design should ensure gradual failure, when the moment increases and exceeds the designed moment. The second major requirement is to supply shear reinforcement. In some cases, the reinforced concrete beams will need to be designed for torsion as well.

Shear failures are sudden and brittle. Because of this, designing for shear reinforcement must ensure that the shear strength covers or exceeds the flexural strength that leads to flexural failure before shear failure. Shear failure may happen in different ways, depending on beam shape, size, loading and properties of members. For this reason the shear stress behaviour depends on whether the member is slender or short and deep, whether the section is rectangular or has a different shape, whether it is a beam, footing, column or slab, whether the loading is normal or cyclic, or there is any chance of seismic effects. For the different cases mentioned above, there is no unique way to design for shear.

\subsubsection{Brief historical review for shear studies and researches}

French scientist Saint-Venant held one of the earliest trials to find a precise solution for the problem of shearing stresses in beams. His solution was outlined in his famous paper "Mémoire sur la Flexion" (Timoshenko, 1983). It covered only the simplest cases; engineers who faced cases that are more complicated were, until recently, forced to use an approximate elementary solution presented by Jourawski (1821-1891) (Timoshenko, 1983). Jourawski developed the approximate theory for shear stresses in beams, now widely used. In 1844, two years after graduating from the Institute of Engineers of Ways of Communication in St. Petersburg, he was asked to design and construct a major bridge on the first railway line from Moscow to St. Petersburg. He noticed that some of the large timber beams split longitudinally in the center of the cross 
sections, where he knew the bending stresses were zero. The wooden beams were very weak in shear along the fibers, and Jourawski correctly concluded that shearing stresses in such beams were important and could not be neglected (Timoshenko, 1983). He started with the simplest case of a rectangular cantilever loaded at the free end as shown in Figure (2-1), and considered the neutral plane $O O$, concluding that the normal stresses that are distributed over the cross section $m n$ at the built-in end have a tendency to produce shear in the plane OO (Timoshenko, 1983). Jourawski drew freebody diagrams and quickly discovered the existence of horizontal shear stresses in the beams. He derived the shear formula and applied his theory to various shapes of beams (Gere and Timoshenko, 1997).

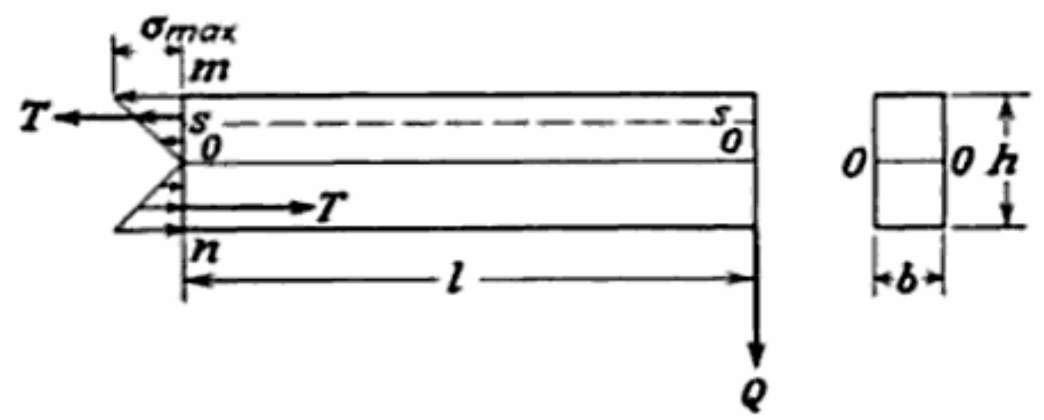

Figure (2-1): Jourawski's model, the simplest case of a rectangular cantilever loaded at the free end (Timoshenko, 1983).

The magnitude of the shearing force $T$ is (Timoshenko, 1983)

$$
T=\frac{\sigma_{\max } b h}{4}=\frac{3 Q l}{2 h}
$$

And the corresponding shear stress, which is uniformly distributed over the neutral plane OO, is (Timoshenko, 1983)

$$
\tau=\frac{T}{l b}=\frac{3}{2} \frac{Q}{b h}
$$

$Q=$ First moment about the centroidal axis of the part of the cross sectional-area lying farther from the centroidal axis than the point where the shear stresses are being calculated.

$\tau=$ shear stress $;=$ span of the beam; $b h=$ width $\mathrm{x}$ height

In the early 1900s, truss models were used in the analysis and design of reinforced concrete beams. Ritter (1899) supposed that beam cracks resulting from diagonal 
tension stresses could give a parallel chord truss with compression diagonals inclined at $45^{\circ}$ with respect to the longitudinal axis of the beam. Mörsch $(1920,1922)$ used the truss models for torsion, and he neglected the contribution of the concrete in tension $(\mathrm{ACl}$ 445R-99).

The concrete contribution $\mathrm{V}_{\mathrm{C}}$ had previously been used in the United States to add the $45^{\circ}$ sectional truss model. ACl Standard Specification No. 23 (1920) allowed the shear stress of $0.025 f_{c}^{\prime}<0.41 \mathrm{MPa}$ for beams without web reinforcement, and with longitudinal reinforcement with no mechanical anchorage. When longitudinal reinforcement was hooks of $180^{\circ}$, permissible shear stress increased to $0.03 f_{c}^{\prime}<0.62$ MPa, see Figure (2-2). Web reinforcement designed by Equation (2-3)

$$
A_{v} F_{v}=V^{\prime} s \sin \alpha / j d
$$

Where:

$A_{v}=$ area of shear reinforcement within s;

$F_{v}=$ allowable tensile stress in the shear reinforcement;

$j d=$ flexural lever arm;

$V^{\prime}=$ total shear minus $0.02 f_{c}^{\prime} b j d$ (or $0.03 f_{c}^{\prime} b j d$ with special anchorage);

$b=$ width of the web;

$s=$ spacing of shear steel measured perpendicular to its direction; and

$\alpha=$ angle of inclination of the web reinforcement with respect to the horizontal axis of the beam.

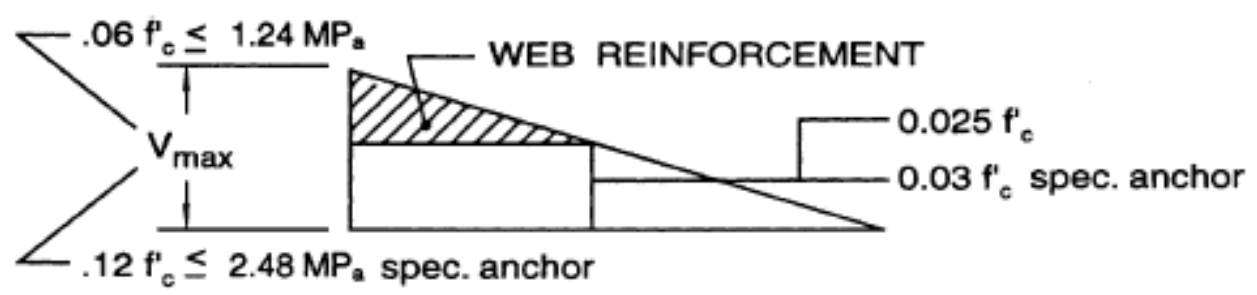

Figure (2-2): American specification for shear design (1920-1951) based on ACl Standard No. 23, 1920 (ACl 445R-99).

The shear stresses at service loads were limited to $0.06 f_{c}^{\prime}<1.24 \mathrm{MPa}$, or with anchorage of longitudinal steel, $0.12 f_{c}^{\prime}<2.48 \mathrm{MPa}$. This shear stress was designed to 
stop diagonal crushing failures of the web concrete before yielding of the stirrups. According to these specifications, the nominal shear stress as $v=V / b j d$, forming the basis for future $\mathrm{ACl}$ codes from 1921 to 1951 (ACl 445R-99).

In 1951, the updated requirement showed that all plain bars must be hooked, and deformed bars must meet ASTM A 305, and so the maximum allowable shear stress on the concrete for beams without web reinforcement $(\mathrm{ACl} 318-51)$ was $0.03 f_{c}^{\prime}$, and the maximum allowable shear stress for beams with web reinforcement was $0.12 f_{c}^{\prime}$. The $45^{\circ}$ truss analogy was the foundation for the shear reinforcement calculation that had to be designed to carry the difference between total shear and the shear assumed to be carried by the concrete ( $\mathrm{ACl} 445 \mathrm{R}-99)$.

The well-known August 1955 shear failure of beams in a warehouse at Wilkins Air Force Depot in Shelby, Ohio, raised concerns about traditional $\mathrm{ACl}$ shear design procedures; for details see Figure (2-3). This tragic focused attention onto finding a better understanding of shear and diagonal tension, and determined the need to study the problem further by taking some forgotten fundamentals into account (ACI 445R-99).

Talbot (1909), based on his tests of 106 beams, indicated the wrong procedures about the failure of beams without web reinforcement, deciding that the value of $v$ [shear stress at failure] will vary according to three factors: the amount of reinforcement, the relative length of the beam, and other factors which affect stiffness. Unfortunately, Talbot's conclusions regarding the effect of the percentage of flexural reinforcement and the length-to-depth ratio $(a / d)$ were not considered in design equations until the later decades of the twentieth century (ACl 445R-99).

Research studies launched as a result of the 1956 Wilkins warehouse failures took Talbot's conclusions into consideration. Since then, many design procedures have been improved to optimize the design of the stirrup amount. Researchers took two approaches: in the first, they added a concrete contribution term to the shear reinforcement capacity obtained, assuming a 45-degree truss (one of these approaches is $\mathrm{ACl}$ 318-95). In the second approach, they used a truss with a variable angle of inclination of the diagonals; the slope of the truss diagonals is permitted to change from $45^{\circ}$ within certain limits suggested based on the theory of plasticity ( $\left.\mathrm{ACl} 445 \mathrm{R}-99\right)$. 


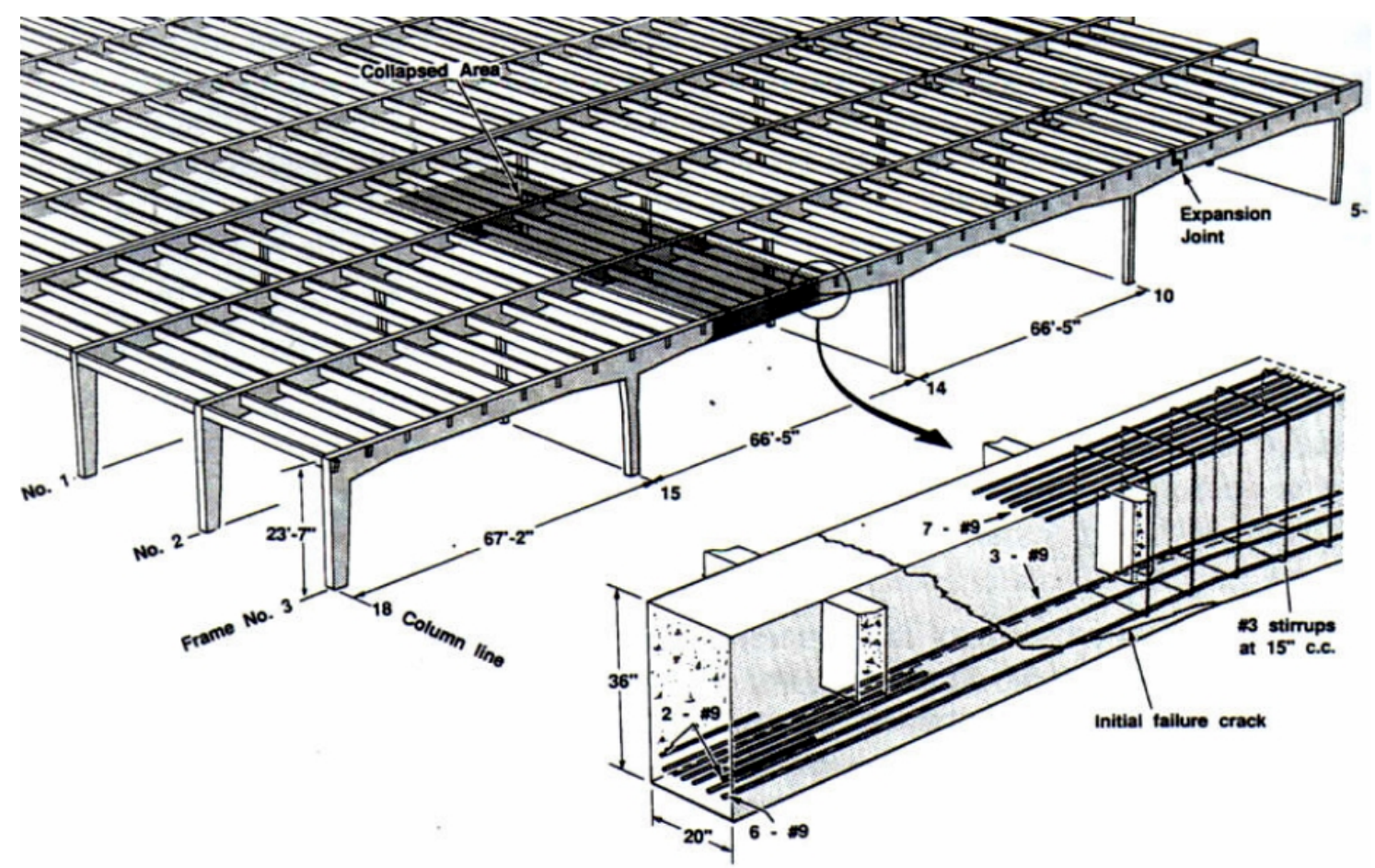

Figure (2-3): Shear failure of roof beams in Air Force warehouse, Wilkins Air Force Depot, Shelby, Ohio (Collins and Mitchell, 1991).

The tension force that came from longitudinal reinforcement can be calculated from equilibrium conditions of the truss model as $V \cot \theta$, where $\theta$ is the angle of inclination of the truss diagonals and $V=$ the applied shear force at specific cross section. Because early efforts by Ritter and Mörsch stimulated research from the 1960s to the 1980s, modified truss models were used in the relatively new design codes. Researchers concentrated on the truss model with a variable angle of inclination as a practical model for shear and torsion in reinforced and prestressed concrete beams (Kupfer 1964; Caflisch et al. 1971; Lampert and Thurlimann 1971; Thurlimann et al. 1983) (ACl 445R-99).

Nielsen and Braestrup (1975), Muller (1978), and Marti (1980) extended the applicability of the model to nonyielding domains as an additional development of plasticity theories. In the concept of $D$ and $B$ regions, introduced by Schlaich et al. (1987), where D stands for "discontinuity" or "disturbed," and B stands for "beam" or "Bernoulli," the distribution of strains is nonlinear in D regions, but the distribution is linear in B regions, as illustrated in Figure (2-4) (Schlaich et al. 1987) .

According to St. Venant's principle, the local disturbance will dissipate within a beam depth from the point at which it is applied (MacGregor and Bartlett, 2000). 
Therefore, $\mathrm{D}$ regions extend a distance equal to the member depth away from any discontinuity. For slender members, the portions of the structure or member between $D$ regions are $B$ regions, as shown in Figure (2-4).
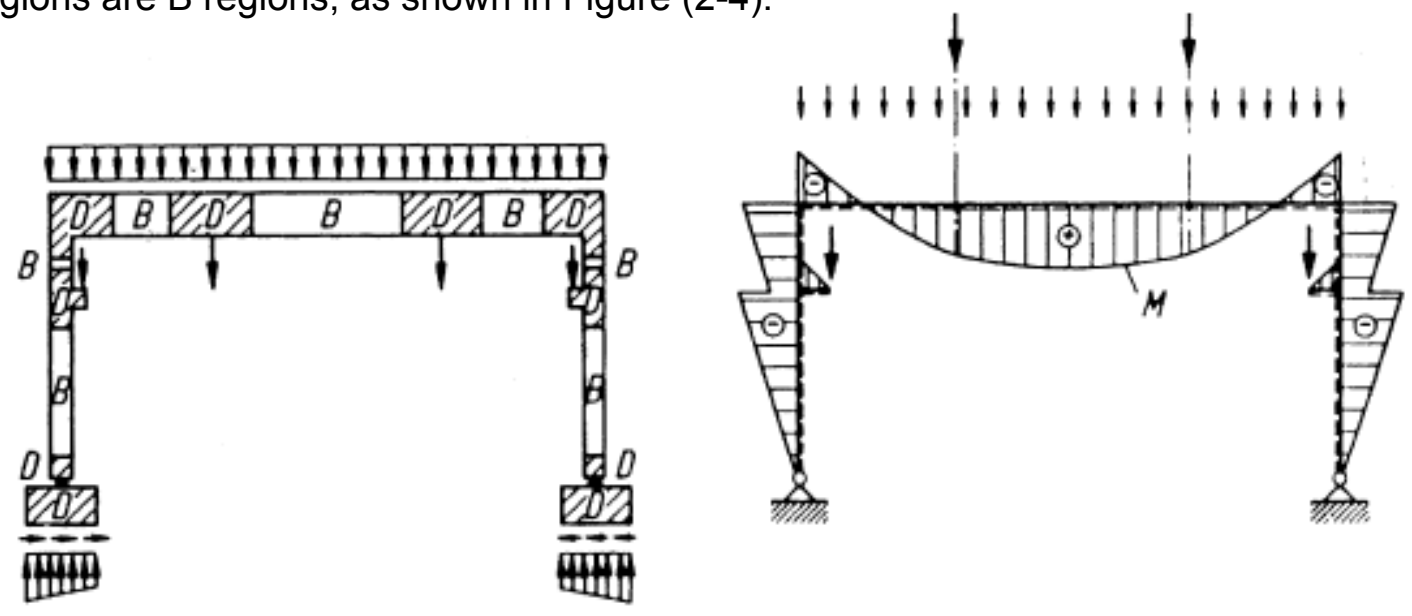

Figure (2-4): Frame structure containing $B$ regions and $D$ regions, its static system, and bending moments (Schlaich et al. 1987) (ACl 445R-99).

\subsubsection{Compression Field Theory (CFT)}

To apply the equilibrium equations of the variable-angle truss model in designing a member for shear, the crack's slope - represented by angle $\theta$ - must be known. A similar problem was encountered by Wagner in 1929 when he was studying postbuckling shear resistance of thin-webbed metal girders. He considered that after buckling or bending, thin webs would not resist compression, and that the shear would be carried by a field of diagonal tension. Wagner's assumption was that the angle of inclination of the diagonal tensile stresses would be compatible with the angle of inclination of the principal tensile strains. This approach is known as Tension Field Theory (Collins and Mitchell, 1991).

By applying Wagner's approximation to reinforced concrete that carries no tension after cracking, with shear that is carried by a field of diagonal compression, one arrives at the following equation for the angle of inclination of the diagonal compression (Collins and Mitchell, 1991)

$$
\tan ^{2} \theta=\frac{\epsilon_{x}-\epsilon_{2}}{\epsilon_{t}-\epsilon_{2}}
$$

Where $\epsilon_{x}=$ longitudinal strain of web, tension positive 


$$
\begin{aligned}
& \epsilon_{t}=\text { transverse strain, tension positive } \\
& \epsilon_{2}=\text { principal compressive strain, negative quantity }
\end{aligned}
$$

With a known value of $\theta$, Equation (2-4) can be considered as a compatibility condition relating the three strains $\epsilon_{2}, \epsilon_{x}$ and $\epsilon_{t}$. When these strains are known, we can find the strain in any other direction by using the geometry of the Mohr circle of strain, as shown in Figure (2-5a and b). The term "average strain" describes the compatibility relationships of cracked concrete; the measuring strains must occur over a long enough area to include many expected cracks, as shown in Figure (2-5a).

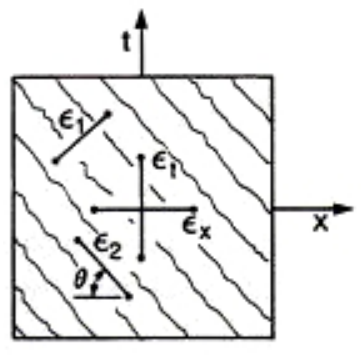

(a) Average strains in cracked element

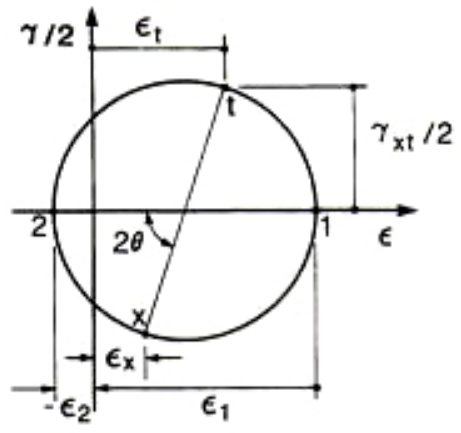

(b) Mohr's circle for average strains

Figure (2-5): Compatibility conditions for cracked web element, (ACI 445R-99).

In addition, the principal tensile strain in the web can be obtained by using Mohr's circle (Collins and Mitchell, 1991)

$$
\epsilon_{1}=\epsilon_{x}+\epsilon_{t}-\epsilon_{2}
$$

The shear strain $\gamma_{x y}$ in the web is

$$
\gamma_{x y}=2\left(\epsilon_{x}-\epsilon_{2}\right) \cot \theta
$$

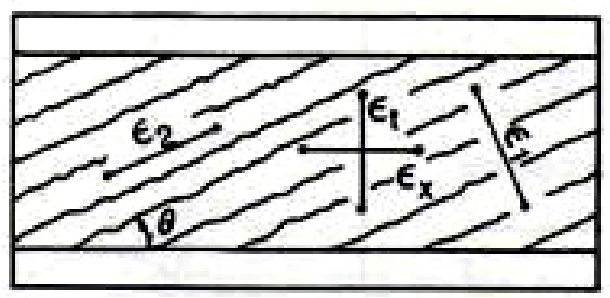

(a) Strains in beam-small $\theta$

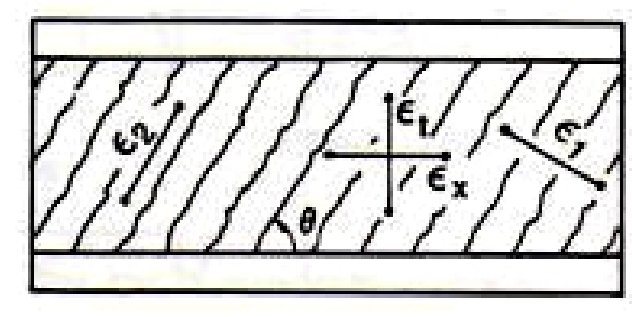

(b) Strains in beam - large $\theta$

Figure (2-6): Influence of $\theta$ on reinforcement strain (Collins and Mitchell, 1991). 
For low values of $\theta$, as shown in Figure (2-6a), the web reinforcement will be highly strained. For the high values of $\theta$, as shown in Figure (2-6b), the longitudinal reinforcement will be highly strained.

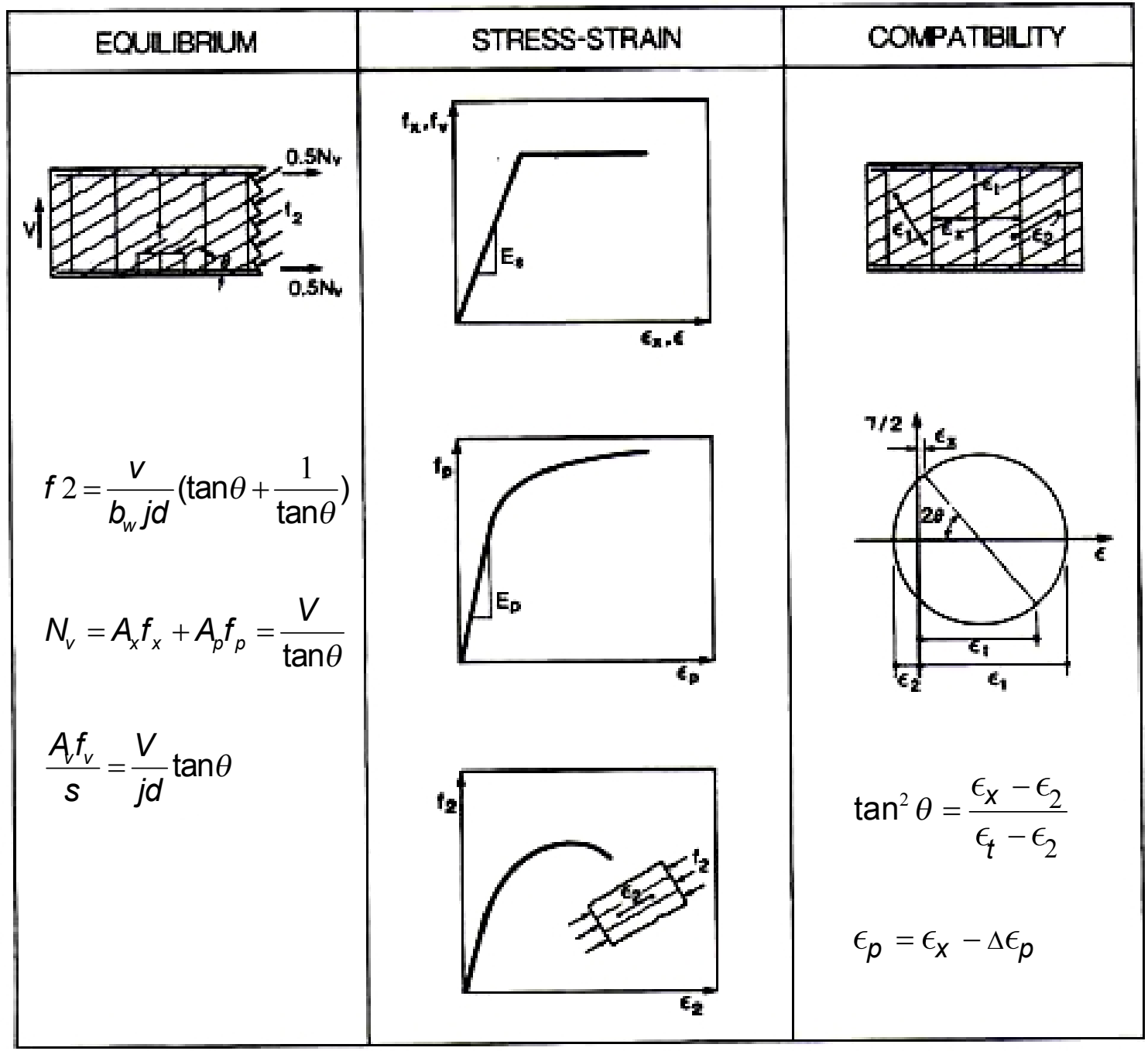

Figure (2-7): Compression Field Theory for prestressed beam subjected to shear (Mitchell and Collins, 1974).

In the case of symmetrically reinforced concrete beams with longitudinal prestressing and subjected to shear, there are five unknowns (Collins and Mitchell, 1991):

1. The stress in the longitudinal bars, $f_{x}$.

2. The stress in the longitudinal prestressing tendons, $f_{p}$.

3. The stress in the stirrups, $f_{v}$.

4. The diagonal compressive stress in the concrete, $f_{2}$. 
5. The inclination, $\theta$, of these diagonal compressive stresses.

Figure (2-7) shows three equilibrium equations, two compatibility equations, and the fundamental relationships for the stresses and strains.

The load deformation response of a beam subjected to shear can be determined by using the equilibrium, compatibility and stress-strain relationships. This approach has become known as Compression Field Theory (Mitchell and Collins, 1974, 1980).
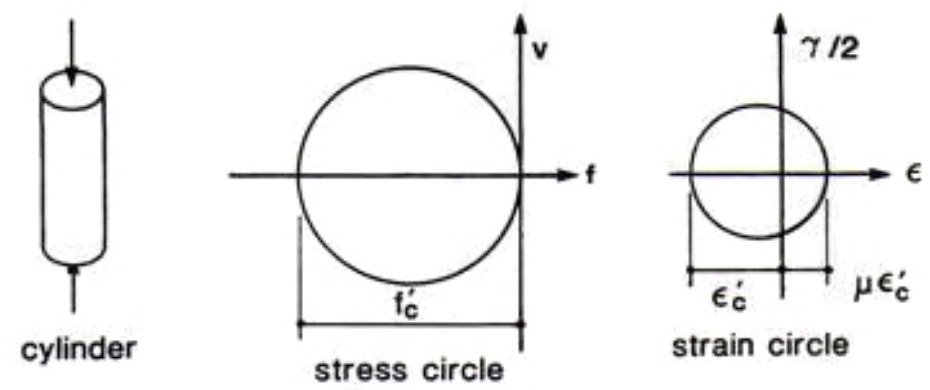

(a) Stress and strain conditions for a concrete cylinder

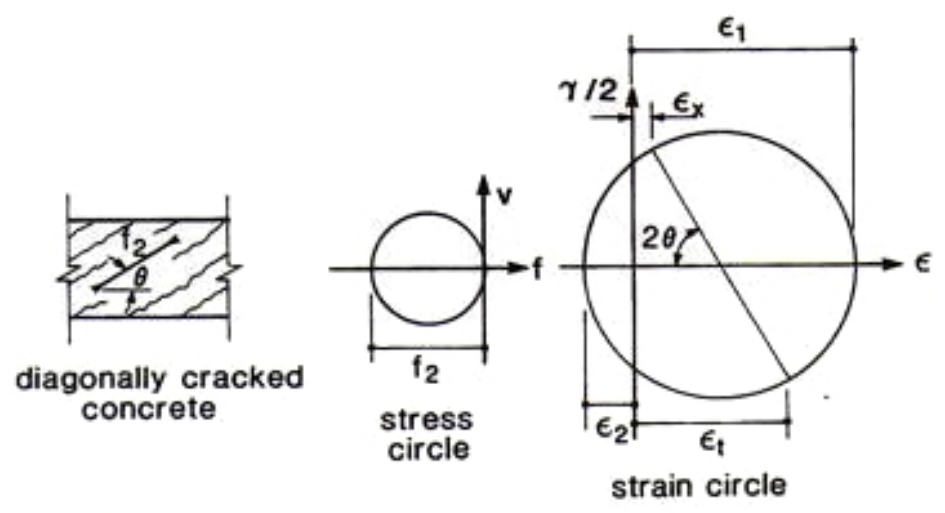

(b) Stress and strain conditions for diagonally cracked web

Figure (2-8): Comparison of conditions in a cylinder and in diagonally cracked concrete (Collins and Mitchell, 1991).

Concrete cylinders under compressive force are subjected to small tensile strains because of Poisson's effect. However, in the diagonally cracked web, the concrete is under the influence of very considerable tensile strains, and the compressive stress, $f_{2}$ may need to be transmitted across earlier formed cracks; hence, the concrete in a diagonally cracked web is weaker and softer than the concrete in a cylinder, as shown in Figure (2-8), (Collins and Mitchell, 1991). 
Vecchio and Collins (1982) investigated the stress-strain properties of diagonally cracked concrete by testing reinforced concrete elements in pure shear, as shown in Figure (2-9). As a result of these tests, they found that $f_{2}$ (the main diagonal compressive stress in the concrete) is a function not only of $\epsilon_{2}$ (the main compressive strain), but also of $\epsilon_{1}$ (the coexisting main tensile strain).

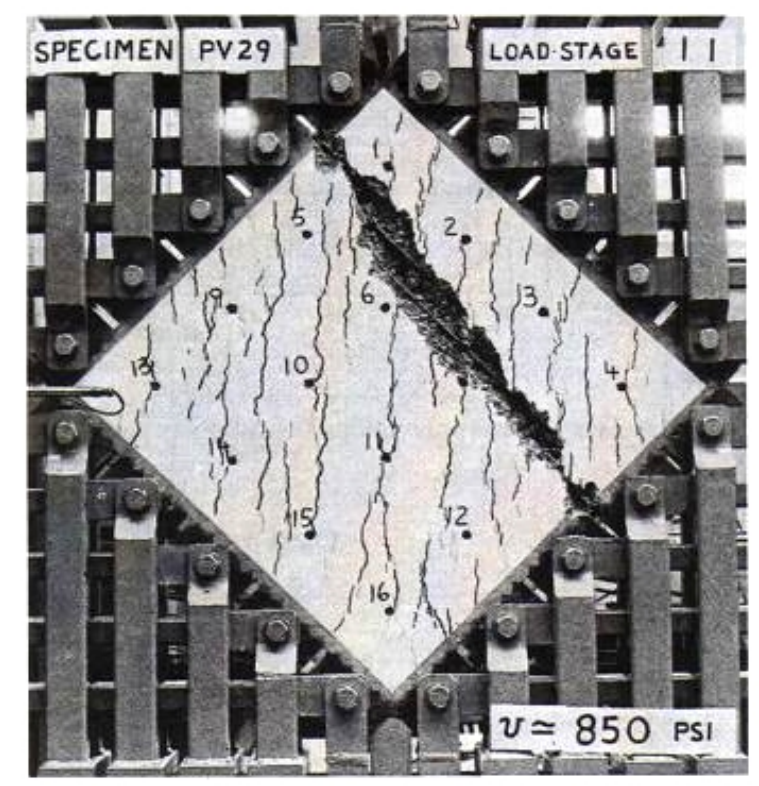

Figure (2-9): Reinforced concrete element failing in shear (Vecchio and Collins, 1982).

They suggested the following stress-strain relationship (Vecchio and Collins, 1986)

$$
f_{2}=f_{2 \max }\left[2\left(\frac{\epsilon_{2}}{\epsilon_{c}^{\prime}}\right)-\left(\frac{\epsilon_{2}}{\epsilon_{c}^{\prime}}\right)^{2}\right]
$$

Where

$$
\frac{f_{2 \max }}{f_{c}^{\prime}}=\frac{1}{0.8+170 \epsilon_{1}} \leq 1.0
$$

The increasing of $\epsilon_{1}$ in the denominator will reduce $f_{2 \max } / f_{c}^{\prime}$, which is explained in Figure (2-10).

Since Compression Field Theory ignores the contribution of tensile stresses in cracked concrete, it overestimates deformations and gives conservative estimates of 
strength. The Modified Compression Field Theory took in the consideration the effects of tension in the concrete (Collins and Mitchell, 1991).

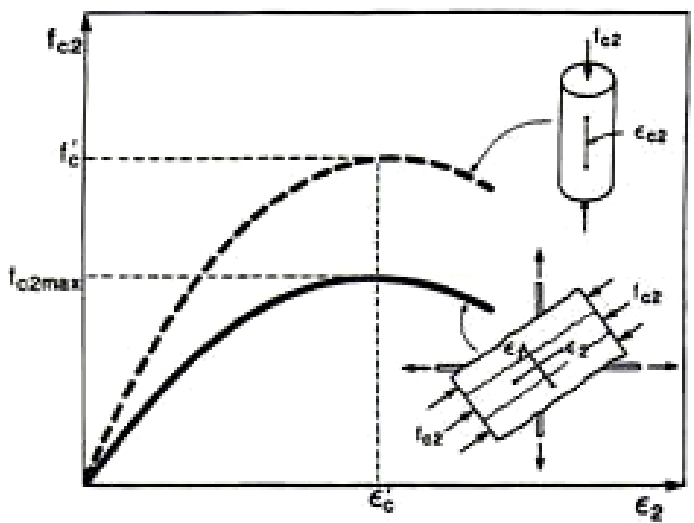

(a) Stress-strain relationship for cracked concrete in compression

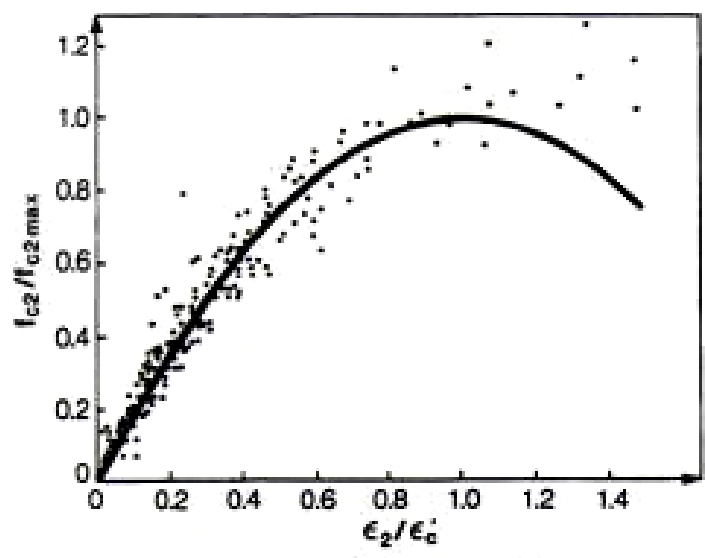

(c) Correlation of test data for cracked concrete in compression

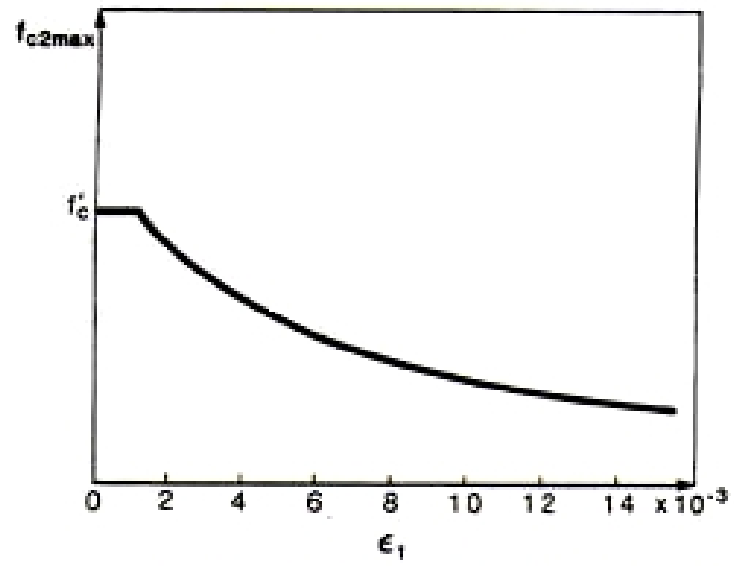

(b) Proposed relationship for maximum compressive stress

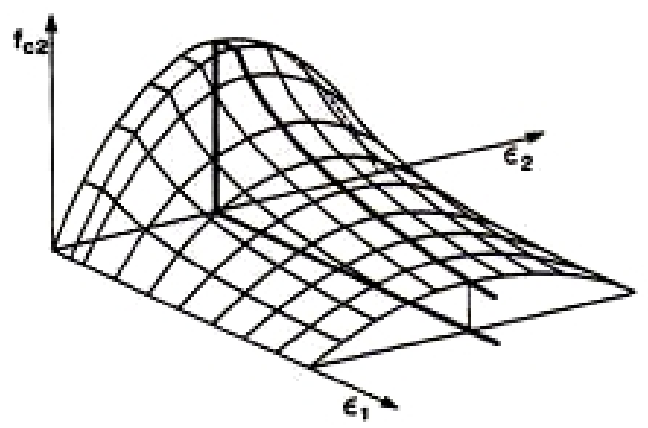

(d) Three-dimensional representation of cornoressive stress-strain relationshio

Figure (2-10): Compressive stress-strain relationship for cracked concrete (Collins and Mitchell, 1991).

\subsubsection{Modified Compression Field Theory (MCFT)}

In the web, before cracking, shear stress is distributed equally by diagonal tensile and diagonal compressive stresses at $45^{\circ}$, but when diagonal cracks appear, the tensile stresses in the concrete are significantly reduced. In Compression Field Theory, the assumption was that the principal tensile stress, $f_{1}=0$, occurred after the cracking of the concrete, but the Modified Compression Field Theory considered the contribution of the tensile stresses in the concrete between the cracks, as shown in Figure (2-11), (Collins and Mitchell, 1991). 


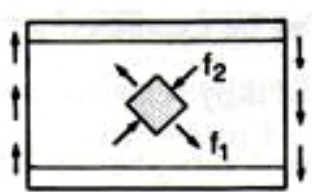

(a) Before cracking $f_{1}=f_{2}, \theta=45^{\circ}$

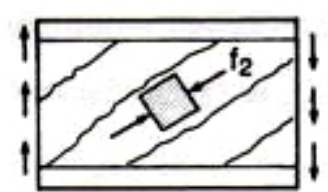

(b) Compression field theory, $f_{1}=0$

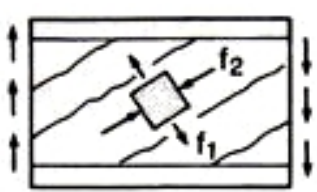

(c) Modified compression field theory, $f_{1} \neq 0$

Figure (2-11): Stress field in web of reinforced concrete beam,

(Collins and Mitchell, 1991).

The symmetrical cross section subjected to pure shear is used to present the equilibrium conditions for Modified Compression Field Theory, as shown in Figure (212), (Collins and Mitchell, 1991).

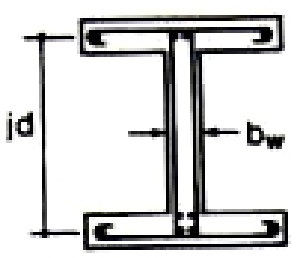

(a) Cross section
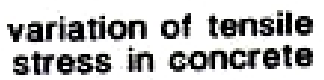

zero moment section

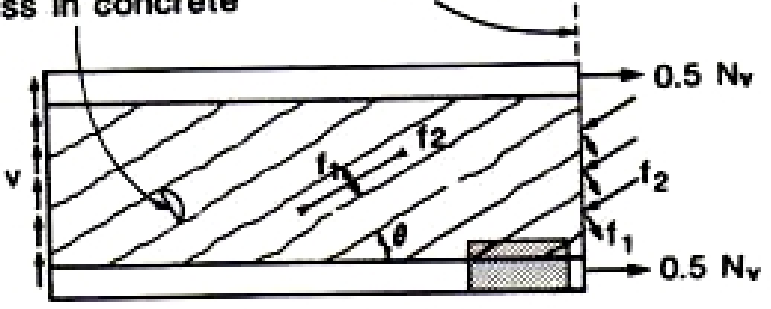

(b) Principal stresses in concrete
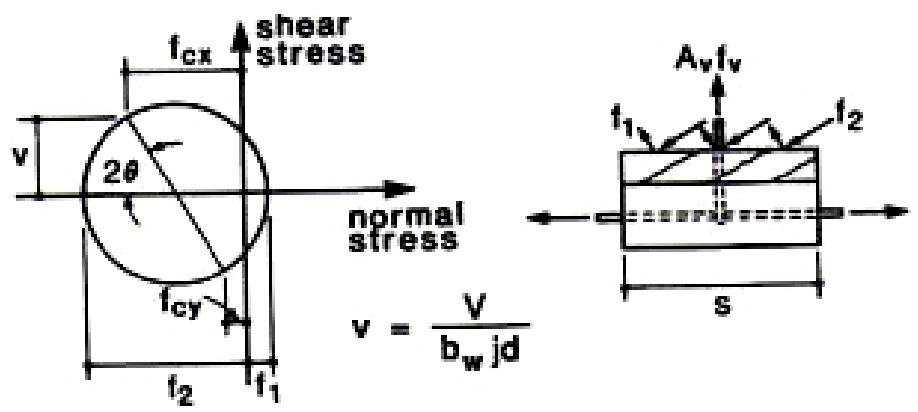

(c) Average concrete

(d) Tension in web reinforcement stresses

Figure (2-12): Equilibrium conditions of Modified Compression Field Theory (Collins and Mitchell, 1991). 
Both the diagonal compressive stresses, $f_{2}$, and the diagonal tensile stresses, $f_{1}$, resist shear. Figure (2-12b) shows that $f_{1}$ varies from zero at the crack location to peak values between the cracks. The equilibrium equations are obtained by integrating the stresses over the cross section; therefore, it is suitable to use the average value of the tensile stresses when developing these equilibrium equations. Using Mohr's stress circle in Figure (2-12c), $f_{2}$ can be derived as follows: (Collins and Mitchell, 1991)

$$
f_{2}=(\tan \theta+\cot \theta) v-f_{1}
$$

Where

$$
v=\frac{V}{b_{w} j d}
$$

$f_{2}$, Push apart the flanges of the beam while $f_{1}$ pull them together (see figure 2-12). The tensile stress in stirrups can rebalance the unbalanced component in the web; the equilibrium requirement can be expressed as (Collins and Mitchell, 1991)

$$
A_{v} f_{v}=\left(f_{2} \sin ^{2} \theta-f_{1} \cos ^{2} \theta\right) b_{w} s
$$

Where $f_{v}=$ average stress in the stirrups. Substituting for $f_{2}$ from Equation (2-8) gives (Collins and Mitchell, 1991)

$$
V=f_{1} b_{w} j d \cot \theta+\frac{A_{v} f_{v}}{s} j d \cot \theta
$$

Equation (2-9) shows the shear resistance of a concrete member as the sum of the concrete tensile stresses and the stirrups tensile stresses, which is the same form as $\mathrm{ACl}$ shear equation $V_{c}+V_{s}$ (Collins and Mitchell, 1991).

In beams with axial load = zero, the tensile stresses in the longitudinal reinforcement must provide equilibrium to the unbalanced longitudinal component of the diagonal concrete. This longitudinal equilibrium requirement can be expressed as

$$
A_{s x} f_{l}+A_{p x} f_{p}=\left(f_{2} \cos ^{2} \theta-f_{1} \sin ^{2} \theta\right) b_{w} j d
$$

Where $A_{p x}$ is the cross sectional area of longitudinal prestressing tendons, $A_{s x}$ is the area of non-prestressed reinforcement, and $f_{l}$ and $f_{p}$ are the average stresses in the longitudinal reinforcing bars and longitudinal prestressing steel. Substituting for $f_{2}$ from Equation (2-8) gives (Collins and Mitchell, 1991) 


$$
A_{s x} f_{l}+A_{p x} f_{p}=V \cot \theta-f_{1} b_{w} j d
$$

Vecchio and Collins (1986), in their testing of reinforced concrete panels in pure shear, recommended the average tensile stress vs. average tensile strain relationship shown in Figure (2-13).

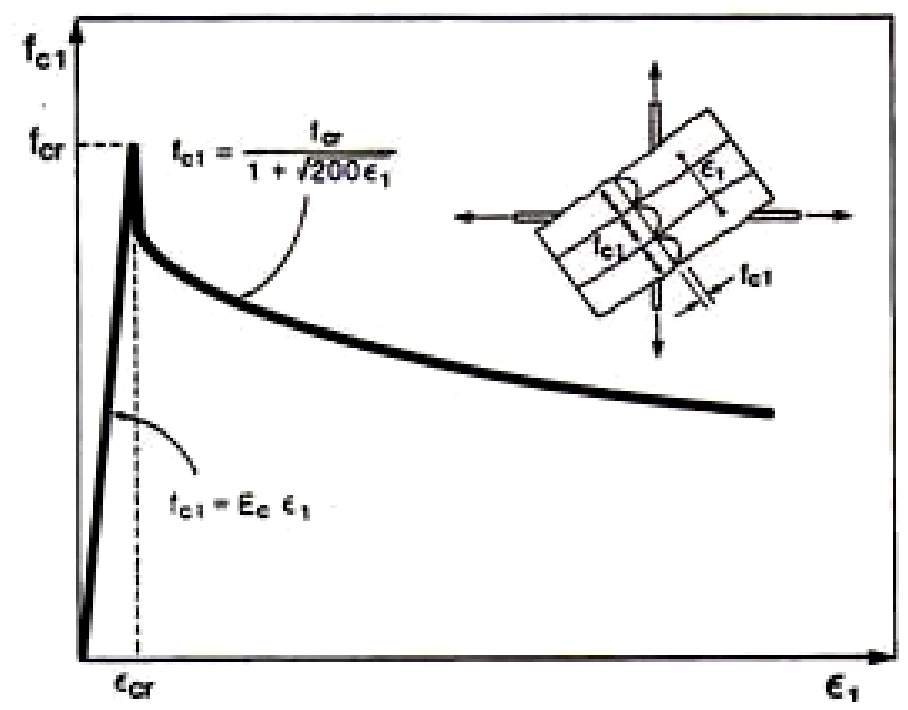

(a) Average stress-strain relationship for cracked concrete in tension

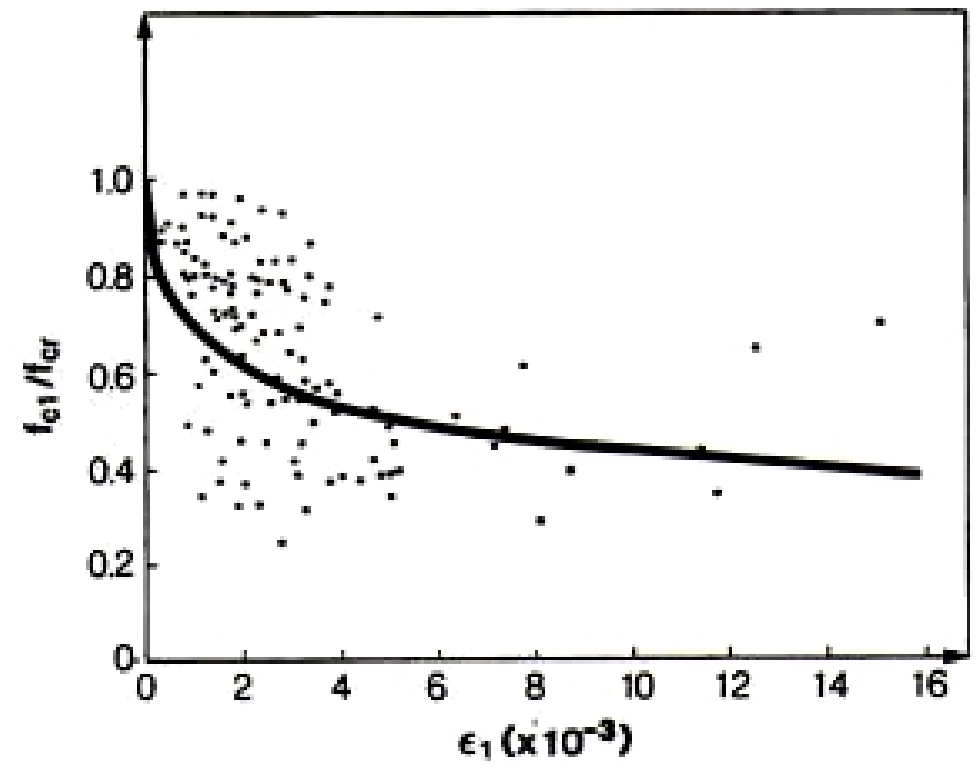

(b) Correlation of test data for cracked concrete in tension

Figure (2-13): Tensile stress-strain relationship for diagonally cracked concrete (Vecchio and Collins, 1986) 
They also recommend using the following relationships (Collins and Mitchell, 1991):

$$
\begin{aligned}
& \text { If } \epsilon_{1} \leq \epsilon_{c r} \text { then } f_{1}=E_{c} \epsilon_{1} \\
& \epsilon_{1}>\epsilon_{c r} \text { then } f_{1}=\frac{\alpha 1 \alpha 2 f_{c r}}{1+\sqrt{500 \epsilon_{1}}}
\end{aligned}
$$

Where $\quad \alpha 1=$ factor accounting for bond characteristics of reinforcement

$\alpha 1=1.0$ for deformed reinforcing bars

$\alpha 1=0.7$ for plain bars, wires, or bonded strands

$\alpha 1=0$ for unbonded reinforcement

$\alpha 2=$ factor accounting for sustained or repeated loading

$\alpha 2=1.0$ for short-term monotonic loading

$\alpha 2=0.7$ sustained and/or repeated loads

The exact values of stresses at the crack spots are different from the calculated average values, as shown in Figure (2-14). The tensile stress in the concrete at crack is zero, but it increases in the reinforcement, and the ability of the member to transmit stresses across the crack indicates its capacity for shear (Collins and Mitchell, 1991).

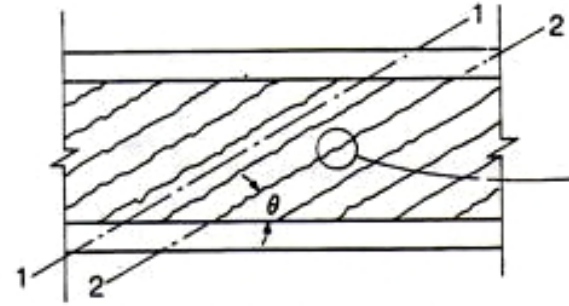

(a) Beam loaded in shear

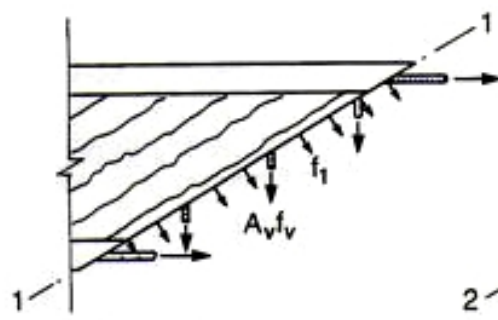

(b) Calculated average stresses

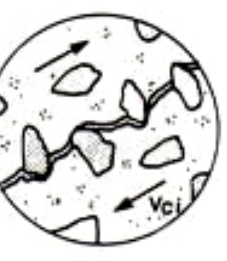

detail at crack

Figure (2-14): Transmitting forces across cracks (Vecchio and Collins, 1986) 
For relatively low shear stresses, tension can be transmitted through the crack via local increases in reinforcement stresses. Because of the increase in shear force, the web reinforcement stress will reach yield at cracks. For higher shear stresses, transmitting tension through the crack requires local shear stresses, $v_{c i}$, on the surface of the crack, as shown in Figure (2-14c). The interface of the crack is sometimes capable, of transmitting shear stresses depending on the crack width $w$. As recommended, the limiting value of the shear stresses, $v_{c i}$ is taken as (Vecchio and Collins, 1986)

$$
v_{c i}=\frac{0.18 \sqrt{f_{c}^{\prime}}}{0.3+\frac{24 w}{a+16}} \quad M P a \text { and } \mathrm{mm}
$$

Where: $a$ is the maximum aggregate size.

Vecchio and Collins (1986) used the experimental data from Walraven (1981). Equation (2-13) shows that the useful influences of the local compressive stresses through the crack have been ignored (Collins and Mitchell, 1991).

Figures $(2-14 b$ and $c)$ show that two different sets of stresses must be statically equivalent. Vecchio and Collins refer to the requirement that makes the two sets of stresses produce the same vertical force as

$$
A_{v} f_{v}\left(\frac{j d}{s \tan \theta}\right)+f_{1} \frac{b_{w} j d}{\sin \theta} \cos \theta=A_{v} f_{v y}\left(\frac{j d}{s \tan \theta}\right)+v_{c i} b_{w} j d
$$

Therefore, to keep this equity, $f_{1}$ must be limited to (Collins and Mitchell, 1991)

$$
f_{1}=v_{c i} \tan \theta+\frac{A_{v}}{s b_{w}}\left(f_{v y}-f_{v}\right)
$$

where ; $v_{c i}$ is given by Equation (2-13)

$w$, the crack width in Equation (2-13), is the product of the principal tensile strain, $\epsilon_{1}$, and the average spacing of the diagonal cracks, so (Collins and Mitchell, 1991)

$$
w=\epsilon_{1} S_{m \theta}
$$

$S_{m \theta}$ is the spacing of the inclined cracks, and it is affected by both the longitudinal and transverse reinforcement. It is recommended that the spacing be taken as

$$
S_{m \theta}=1 /\left(\frac{\sin \theta}{S_{m x}}+\frac{\cos \theta}{S_{m v}}\right)
$$




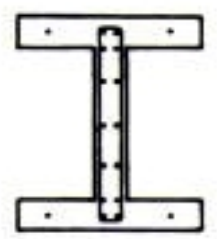

(a) Cross section

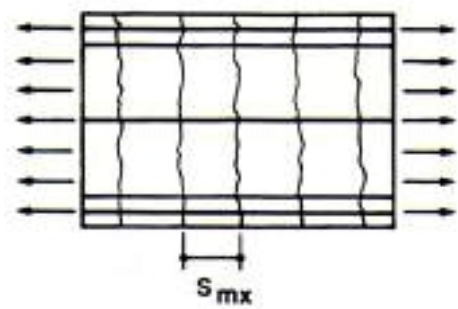

(c) Vertical cracks due to axial tension

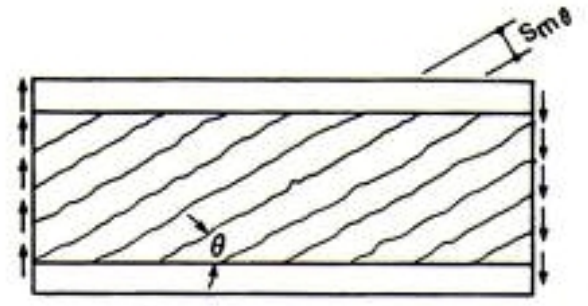

(b) Inclined cracks due to shear

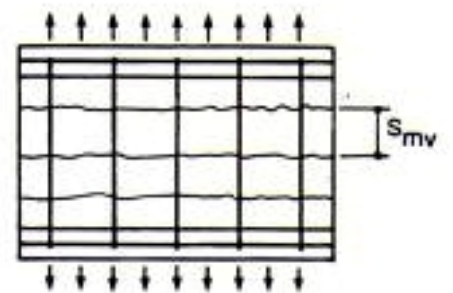

(d) Horizontal cracks due to transverse tension

Figure (2-15): Spacing of inclined cracks (Collins and Mitchell, 1991).

Figure (2-15) illustrates both $S_{m x}$ and $S_{m v}$ as the crack spacings that express the crack control characteristics of the longitudinal and transverse reinforcements respectively. Therefore,

$S_{m x}=$ average crack spacing formed when the concrete member was subjected to longitudinal tension.

$S_{m v}=$ average crack spacing formed when the concrete member was subjected to transverse tension.

The crack spacings can be approximately calculated from the CEB-FIP Code, such as the following expression (Collins and Mitchell, 1991)

$$
s_{m}=2\left(c+\frac{s}{10}\right)+k_{1} k_{2} \frac{d_{b}}{\rho_{e f}}
$$

where $\quad c=$ clear concrete cover

$S=$ maximum spacing between longitudinal reinforcing bars but shall not be taken greater than $15 d_{b}$

$d_{b}=$ bar diameter

$\rho_{e f}=A_{s} / A_{c e f}$

$A_{s}=$ area of steel considered to be effectively bonded to the concrete 


$$
\begin{aligned}
& A_{c e f}=\text { area of effective embedment zone } \\
& \text { of the concrete where the reinforcing } \\
& \text { bars can influence the crack widths, } \\
& \text { as shown in Figure }(2-16) \\
& k_{1}=\text { coefficient that characterizes bond } \\
& \text { properties of bars } \\
& k_{1}=0.4 \text { for deformed bars } \\
& k_{1}=0.8 \text { for plain bars } \\
& k_{2}=\text { coefficient to account for strain gradient } \\
& k_{2}=0.25\left(\epsilon_{1}+\epsilon_{2}\right) / 2 \epsilon_{1}
\end{aligned}
$$

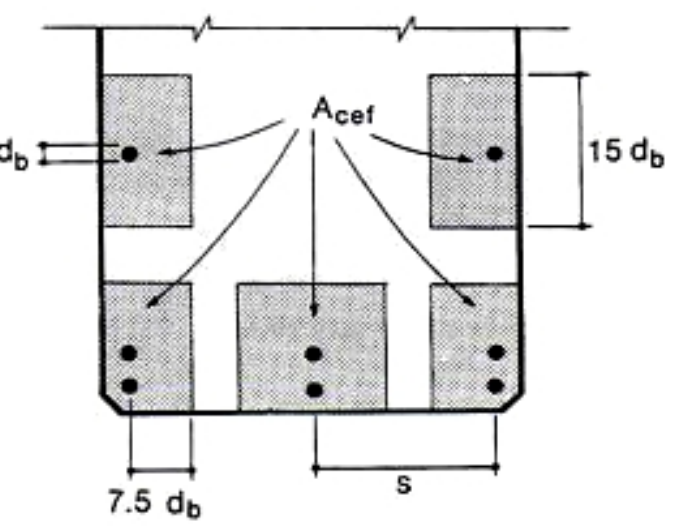

where $\left(\epsilon_{1}\right.$ and $\epsilon_{2}=$ the largest and the smallest tensile strains in the effective embedment zone)

The CEB equation is designed to calculate the crack spacings on the surface of the concrete member, while Equation (2-13) is used to measure crack spacings in the shear area of the beam of the greatest interest. To explain the increase of the crack spacings away from the reinforcement, Figure (2-17) illustrates that for uniform tensile straining (i.e., $k_{2}=0.25$ ), Equation (2-17) becomes (Collins and Mitchell, 1991)

$$
\begin{aligned}
& s_{m x}=2\left(c_{x}+\frac{s_{x}}{10}\right)+0.25 k_{1} \frac{d_{b x}}{\rho_{x}} \\
& s_{m v}=2\left(c_{v}+\frac{s}{10}\right)+0.25 k_{1} \frac{d_{b v}}{\rho_{v}}
\end{aligned}
$$

Where $\rho_{v}=A v /\left(b_{w} s\right)$ and $\rho_{x}=\left(A_{s x}+A_{p x}\right) / A_{c}$, and $k_{1}$ is 0.4 for deformed bars or 0.8 for plain bars or bonded strands.

At the cracking spots, there is a limited value of transmitted concrete tension due to the yielding of the longitudinal reinforcement. In Figure (2-14), the requirement that the two sets of stresses produce the same horizontal force will be satisfied if

$$
A_{s x} f_{y}+A_{p x} f_{p s} \geq A_{s x} f_{s x}+A_{p x} f_{p x}+f_{1} b_{w} j d+\left[f_{1}-\frac{A_{v}}{b_{w} s}\left(f_{v y}-f_{v}\right)\right] b_{w} j d \cot ^{2} \theta
$$

(Collins and Mitchell, 1991) 


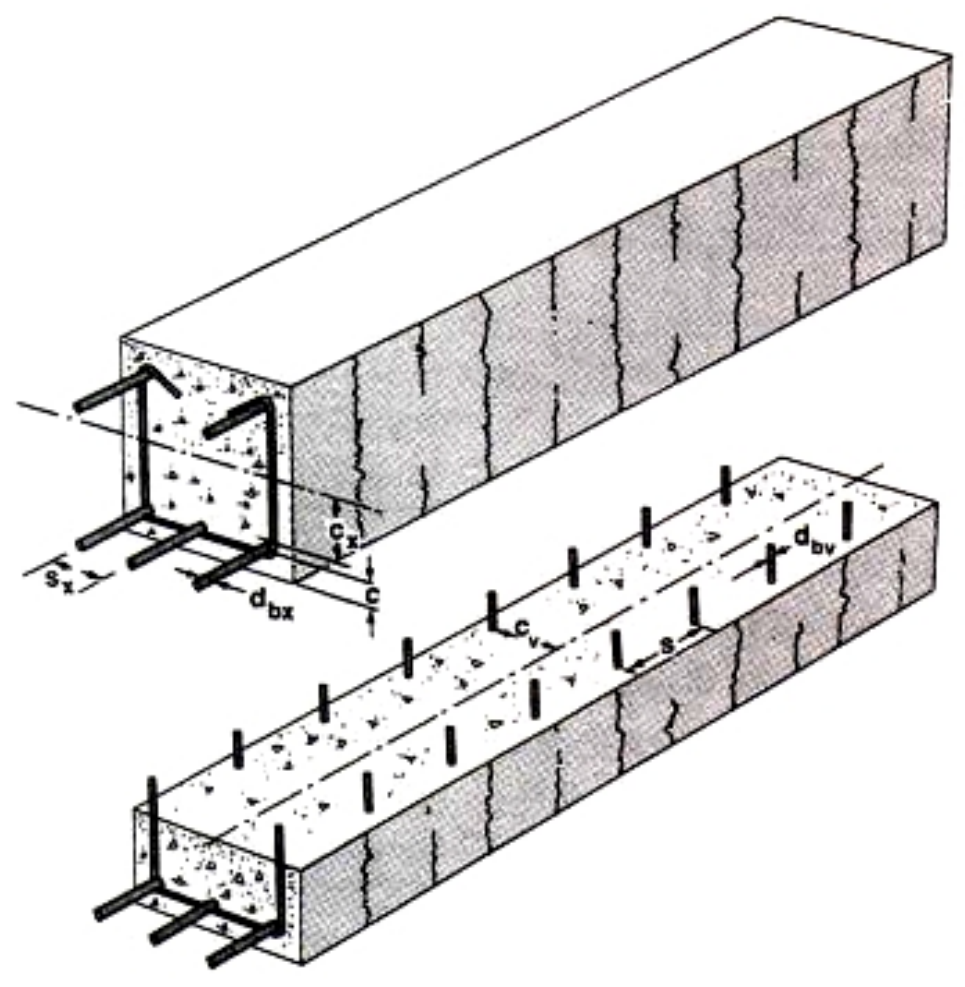

Figure (2-17): Parameters influencing crack spacing (Collins and Mitchell, 1991)

Vecchio and Collins (1986) and Collins and Mitchell (1991) presented 17 to 21 general steps for a solution technique to predict shear response. The MCFT's equations tend to have analytical capability to predict the shear response of a particular section of a prestressed concrete member that is subjected to shear, moment, and axial load. Collins and Mitchell (1991) developed design technique procedures with additional equations derived from previous equations presented in the review of the MCFT. A computer program "RESPONSE" was developed by Benz and Collins to get an easy way to evaluate and predict structural response of concrete members, and especially shear response.

\subsubsection{Simplified Modified Compression Field Theory (SMCFT)}

\subsubsection{Introduction}

After working on over 100 pure shear tests on reinforced concrete panels, Bentz, Vecchio and Collins (2006), summarized their efforts and experiences by working on a simplified MCFT that targets a better understanding of shear behaviour and enhanced simplicity of its calculations. According to the ACl approach, shear strength is the sum of 
a diagonal cracking load. A $45^{\circ}$ truss model predicts the strength of these panels poorly, with an average experimental-over-predicted shear strength ratio of 1.40 with a coefficient of variation of $46.7 \%$. Based on a subset of these experiments, the MCFT developed in 1980s is able to predict full load deformation relationships. The accuracy of shear strength prediction, according to the MCFT, was 1.01 with a coefficient of variation $(\mathrm{COV})^{*}$ of $12.2 \%$. The new simplified analysis method gives an average shear strength ratio as 1.11 with a COV of $13.0 \%$. The authors describe the ease of applying the simplified approach as a method suitable for "back of the envelope" calculations.

The behaviour of reinforced concrete in shear has been studied for more than 100 years, but the problem of determining shear strength of reinforced concrete beams still remains open to discussion. Therefore, shear strengths predicted by different current design codes for a particular beam section can vary by factors of more than two, while the flexural strengths predicted by the same codes are unlikely to vary by more than 10\% (Bentz et al. 2006).
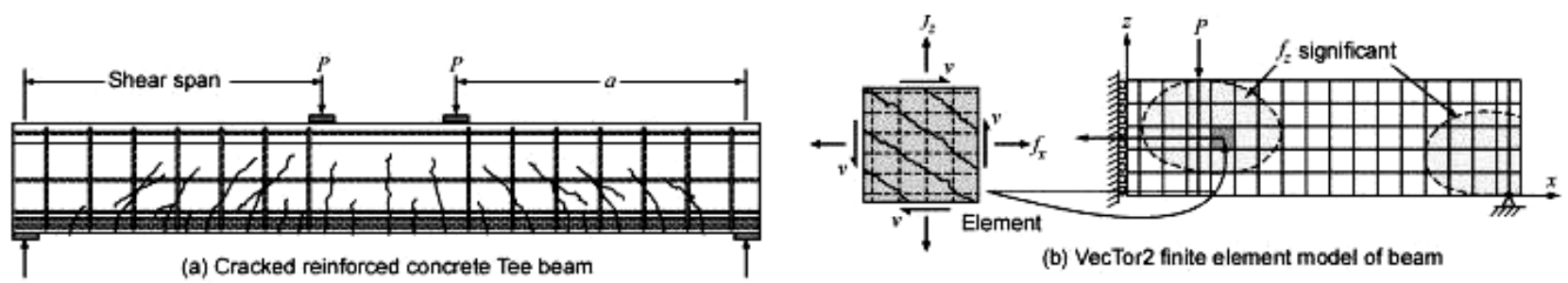

(a) Cracked reinforced concrete Tee beam

(b) VecTor2 finite element model of beam

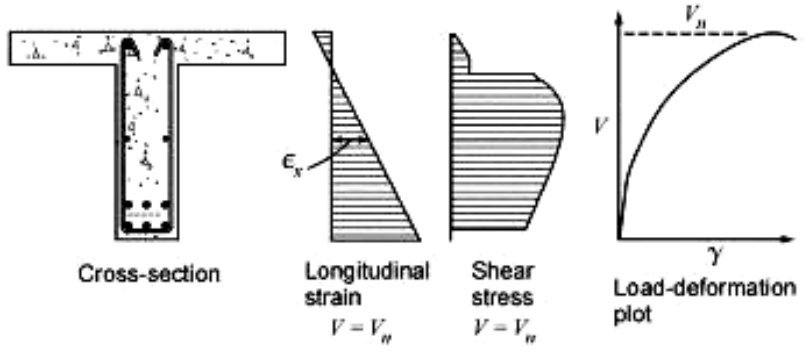

(c) Response-2000 model of beam section

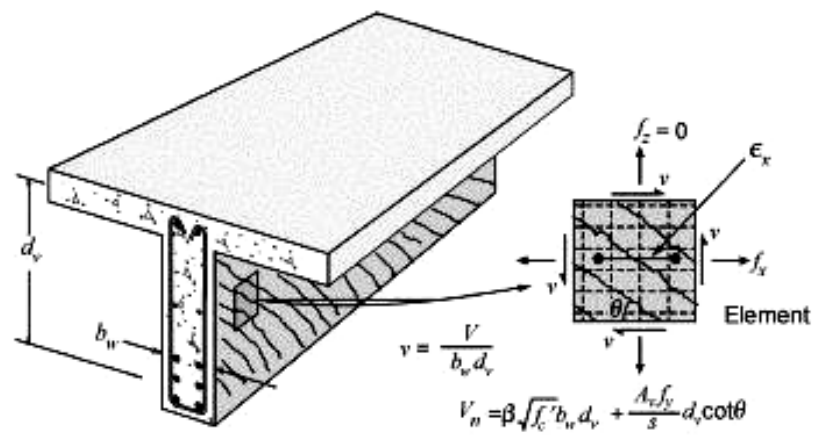

(d) AASHTO LRFD model of section of beam

Figure (2-18): Predicting shear strength of reinforced concrete beams (Bentz et al. 2006).

* Coefficient of Variation $(\mathrm{COV})=\operatorname{standard}$ deviation $(\sigma) /$ mean $(\mu)$ 
Figure (2-18) describes a traditional shear test on a reinforced concrete beam. According to the shear force diagram and the bending moment diagram, the region of the beam between the two point loads is subjected to pure flexure, whereas the shear spans are subjected to constant shear and linearly varying moment. The behaviour of this member changes from section to section along the shear span, so it is difficult to use the test results to develop a general theory for shear behaviour. Therefore, the relationship between the magnitude of the shear force and the strains in the stirrups shows that the strains are different for every stirrup and differ over the height of each stirrup. In addition, the clamping stresses $f_{z}$ (representing the high net vertical compressive stresses) near the point loads and reactions cause stirrup strains in these locations to be close to zero. Watching and studying the response of a large number of reinforced concrete elements loaded in pure shear or in shear combined with axial stress led to the development of the MCFT. Despite the difficulties involved in managing such tests, they offered experimental results that clearly illustrate the fundamental behaviour of reinforced concrete in shear. The MCFT attempted to predict the relationships between the axial and shear stresses applied to a membrane element, such as that shown in Figure (2-18b), along with the resulting axial and shear strains. If the MCFT approach is a more accurate approach to understanding the behaviour of these elements, it could be used as the fundamental technique for many analytical models. Despite the complexity of arranging the concrete structure as an array of biaxial elements, some studies (Vecchio, 1989) used the information in a nonlinear finite element analysis in a computer program (Wong, 2002), which led to very precise predictions, as shown in Figure (2-18b). The model showed precise results in both areas of pure flexural regions and the areas of mixed constant shear and variable flexure regions where (clamping stresses $f_{z}$ ) can meaningfully increase the shear strength. The basis of program Response-2000 (Bentz, 2000) was to assume that plane sections remain plane and that the vertical clamping stresses are negligibly small, which makes the program capable of predicting shear stress distribution over the height of the beam, and the shear force/shear deformation relationship of the section, as shown in Figure (218c). If just one biaxial element within the web of the section is considered and the shear stress is assumed to remain constant over the depth of the web, then simple expressions for the shear strength of a section can be derived. This is the basis of the sectional design model for shear (Collins et al. 1996) included in the AASHTO LRFD Bridge Design Specifications (AASHTO LRFD, 2004), as shown in Figure (2-18d). 
$\beta$ and $\theta$ are the two main parameters that influence the shear strength of a section in the AASHTO LRFD shear design method. Where $\theta$ is the slope angle of the diagonal compressive stresses in the web, and $\beta$ is the factor for tensile stresses in the cracked concrete, the longitudinal straining of the web $\epsilon_{x}$ is influencing both $\beta$ and $\theta$. For beams without stirrups, $\beta$ and $\theta$ values obtained from the MCFT are given as functions of $\epsilon_{x}$ and the crack spacing $S_{x e}$, as shown in Table (2-1).

Table (2-1): Values of $\beta$ and $\theta$ for sections without transverse reinforcement (from A23.3, Table11-1) (MacGregor and Bartlett, 2000).

\begin{tabular}{|c|c|c|c|c|c|c|c|}
\hline & & \multicolumn{6}{|c|}{ Longitudinal strain, $\epsilon_{x}$} \\
\hline $\begin{array}{l}S_{x e} \\
m m\end{array}$ & & $\begin{array}{l}\leq \\
0.0000\end{array}$ & $\begin{array}{l}\leq \\
0.00025\end{array}$ & $\begin{array}{l}\leq \\
0.0005\end{array}$ & $\begin{array}{l}\leq \\
0.0010\end{array}$ & $\begin{array}{l}\leq \\
0.0015\end{array}$ & $\begin{array}{l}\leq \\
0.0020\end{array}$ \\
\hline$\leq 125$ & $\beta$ & $\begin{array}{l}0.406 \\
27.0^{\circ}\end{array}$ & $\begin{array}{l}0.309 \\
29.0^{\circ}\end{array}$ & $\begin{array}{l}0.263 \\
32.0^{\circ}\end{array}$ & $\begin{array}{l}0.214 \\
34.0^{\circ}\end{array}$ & $\begin{array}{l}0.183 \\
36.0^{\circ}\end{array}$ & $\begin{array}{l}0.161 \\
38.0^{\circ}\end{array}$ \\
\hline$\leq 250$ & $\beta$ & $\begin{array}{l}0.384 \\
30.0^{\circ}\end{array}$ & $\begin{array}{l}0.283 \\
34.0^{\circ}\end{array}$ & $\begin{array}{l}0.235 \\
37.0^{\circ}\end{array}$ & $\begin{array}{l}0.183 \\
41.0^{\circ}\end{array}$ & $\begin{array}{l}0.156 \\
43.0^{\circ}\end{array}$ & $\begin{array}{l}0.138 \\
45.0^{\circ}\end{array}$ \\
\hline$\leq 500$ & $\beta$ & $\begin{array}{l}0.359 \\
34.0^{\circ}\end{array}$ & $\begin{array}{l}0.248 \\
39.0^{\circ}\end{array}$ & $\begin{array}{l}0.201 \\
43.0^{\circ}\end{array}$ & $\begin{array}{l}0.153 \\
48.0^{\circ}\end{array}$ & $\begin{array}{l}0.127 \\
51.0^{\circ}\end{array}$ & $\begin{array}{l}0.108 \\
54.0^{\circ}\end{array}$ \\
\hline$\leq 1000$ & $\beta$ & $\begin{array}{l}0.335 \\
37.0^{\circ}\end{array}$ & $\begin{array}{l}0.212 \\
45.0^{\circ}\end{array}$ & $\begin{array}{l}0.163 \\
51.0^{\circ}\end{array}$ & $\begin{array}{l}0.118 \\
56.0^{\circ}\end{array}$ & $\begin{array}{l}0.095 \\
60.0^{\circ}\end{array}$ & $\begin{array}{l}0.080 \\
63.0^{\circ}\end{array}$ \\
\hline$\leq 2000$ & $\beta$ & $\begin{array}{l}0.306 \\
41.0^{\circ}\end{array}$ & $\begin{array}{l}0.171 \\
53.0^{\circ}\end{array}$ & $\begin{array}{l}0.126 \\
59.0^{\circ}\end{array}$ & $\begin{array}{l}0.084 \\
66.0^{\circ}\end{array}$ & $\begin{array}{l}0.064 \\
69.0^{\circ}\end{array}$ & $\begin{array}{l}0.052 \\
72.0^{\circ}\end{array}$ \\
\hline
\end{tabular}

A separate table is given for $\beta$ and $\theta$ values for members with transverse reinforcement, as shown in Table (2-2). Simplifying the shear design procedures is needed for better understanding and easier use. An experienced engineer should be able to perform preliminary calculations easily. Although the AASHTO LRFD shear design method is straightforward, it is simply impossible to remember all the values in the tables. Most 
engineers would rather deal with simple equations than tables because they provide direct results and are more convenient for spreadsheet calculations. The Simplified MCFT targets simple equations for $\beta$ and $\theta$, and is determined from the basic expressions of the MCFT (Bentz et al. 2006).

Table (2-2): Values of $\beta$ and $\theta$ for sections with transverse reinforcement (from A23.3 Table11-1)(MacGregor and Bartlett, 2000).

\begin{tabular}{|l|l|l|l|l|l|l|l|l|}
\hline \multicolumn{2}{|c|}{} & \multicolumn{7}{|c|}{ Longitudinal strain, $\epsilon_{x}$} \\
\hline$\frac{v_{f}}{\lambda \phi_{f f_{c}^{\prime}}}$ & & $\leq$ & $\leq$ & $\leq$ & $\leq$ & $\leq$ & $\leq$ & $\leq$ \\
\hline$\leq 0.050$ & $\beta$ & 0.405 & 0.290 & 0.208 & 0.197 & 0.185 & 0.185 & 0.162 \\
& $\theta$ & $27.0^{\circ}$ & $28.5^{\circ}$ & $29.0^{\circ}$ & $33.0^{\circ}$ & $36.0^{\circ}$ & $41.0^{\circ}$ & $43.0^{\circ}$ \\
\hline$\leq 0.075$ & $\beta$ & 0.405 & 0.250 & 0.205 & 0.194 & 0.197 & 0.158 & 0.137 \\
& $\theta$ & $27.0^{\circ}$ & $27.5^{\circ}$ & $30.0^{\circ}$ & $33.0^{\circ}$ & $36.0^{\circ}$ & $40.0^{\circ}$ & $42.0^{\circ}$ \\
\hline$\leq 0.100$ & $\beta$ & 0.271 & 0.211 & 0.200 & 0.189 & 0.174 & 0.143 & 0.120 \\
& $\theta$ & $23.5^{\circ}$ & $26.5^{\circ}$ & $30.5^{\circ}$ & $34.0^{\circ}$ & $36.0^{\circ}$ & $38.0^{\circ}$ & $39.0^{\circ}$ \\
\hline$\leq 0.125$ & $\beta$ & 0.216 & 0.208 & 0.197 & 0.181 & 0.167 & 0.133 & 0.112 \\
& $\theta$ & $23.5^{\circ}$ & $28.0^{\circ}$ & $31.5^{\circ}$ & $34.0^{\circ}$ & $36.0^{\circ}$ & $37.0^{\circ}$ & $38.0^{\circ}$ \\
\hline$\leq 0.150$ & $\beta$ & 0.212 & 0.203 & 0.189 & 0.171 & 0.160 & 0.125 & 0.103 \\
& $\theta$ & $25.0^{\circ}$ & $29.0^{\circ}$ & $32.0^{\circ}$ & $34.0^{\circ}$ & $36.0^{\circ}$ & $36.0^{\circ}$ & $37.0^{\circ}$ \\
\hline$\leq 0.200$ & $\beta$ & 0.203 & 0.194 & 0.174 & 0.151 & 0.131 & 0.100 & 0.038 \\
& $\theta$ & $27.0^{\circ}$ & $31.0^{\circ}$ & $33.0^{\circ}$ & $34.0^{\circ}$ & $34.5^{\circ}$ & $35.0^{\circ}$ & $36.0^{\circ}$ \\
\hline$\leq 0.250$ & $\beta$ & 0.191 & 0.167 & 0.136 & 0.126 & 0.116 & 0.108 & 0.104 \\
& $\theta$ & $30.0^{\circ}$ & $32.0^{\circ}$ & $33.0^{\circ}$ & $34.0^{\circ}$ & $35.5^{\circ}$ & $38.5^{\circ}$ & $41.5^{\circ}$ \\
\hline
\end{tabular}




\subsubsection{Steps before MCFT}

The first step in achieving the shear design procedures performed by Ritter in 1899 and Mörsch in 1922 was developed over 100 years ago. They assumed that cracked concrete in the web of a beam, resisted shear stress $v$ only by diagonal compressive stresses $f 2$ and that these stresses were inclined at an angle of $45^{\circ}$ to the longitudinal axis of the member (Bentz et al. 2006).

$f_{2}$ push apart the flanges of the beam, resulting in tension in the stirrups; after the stirrups yield, the beam is able to resist a shear stress of $\rho_{z} f_{y}$, where $\rho_{z}$ is the ratio of the stirrup area to the web area, $\rho_{z}=A v /\left(b_{w s}\right)$, and $f_{y}$ is the yield stress of the stirrups. The $45^{\circ}$ truss model provides a very conservative approach to shear strength for a member with a small number of stirrups because it ignores the effects of concrete on tensile strength. For this reason, the $\mathrm{ACl}$ specifications ( $\mathrm{ACl} 318-63$ and $\mathrm{ACl} 318-05)$, count $\rho_{z} f_{y}+v_{c}$ as the shear stress at which diagonal cracks form $v_{c r}$. The prestressing increases the diagonal shear stress $v_{c r}$, while the axial tension decreases it. European researchers in the 1970 s and 1980 s observed the fact that, in general, $\theta$ is not $45^{\circ}$. The truss model with $\theta$ angle of inclination predicted the shear stress capacity of a web to be $\rho_{z} f_{y} \cot \theta$. However, it is difficult to determine the proper value of $\theta$. Models developed based on the theory of plasticity ${ }^{(66)} \&(67)$ were allowed the engineer to select the value of $\theta$. To avoid unsafe predictions and limit brittle concrete shear failures, it was important to limit the values of $\theta$ and $f_{2}$ (for example, $\theta>30^{\circ}$ ) and $\left(f_{2}<0.6 f_{c}^{\prime}\right)$ (Bentz et al. 2006).

The next important step towards a more rational theory for shear was the development of the Compression Field Theory (Collins and Mitchell, 1974), (Collins, 1978). Vecchio and Collins (1986) achieved the equilibrium, geometric, and fundamental relationships of the MCFT by adjusting the CFT relationships to explain the average principal tensile stresses in the cracked concrete $f_{1}$. Figure (2-21) shows the 15 equations used in the MCFT (Collins and Mitchell, 1991). 


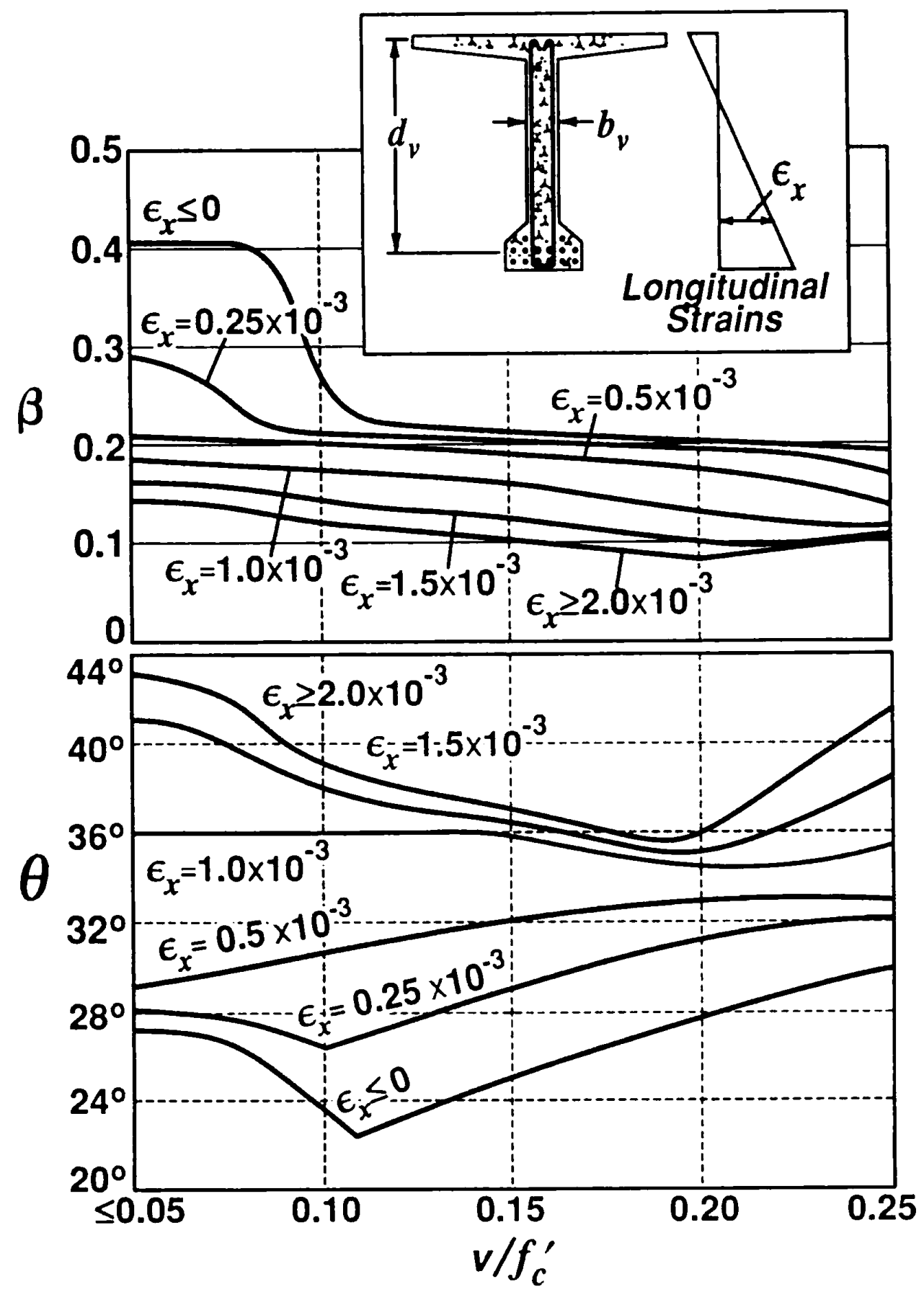

Figure (2-19): Values of $\theta$ and $\beta$ for members without stirrups (Collins, 1993). 


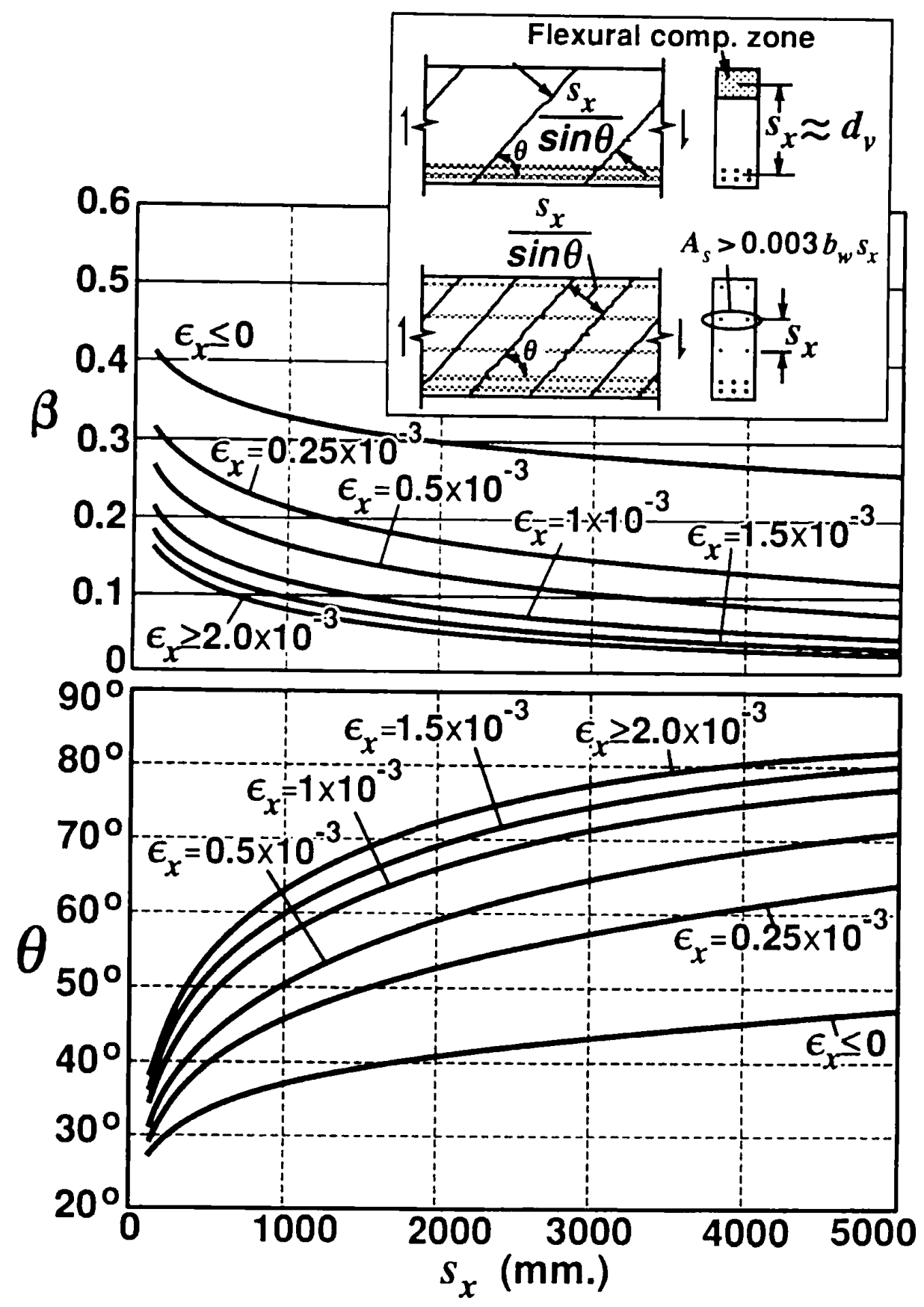

Figure (2-20): Values of $\theta$ and $\beta$ for members with stirrups (Collins, 1993). 


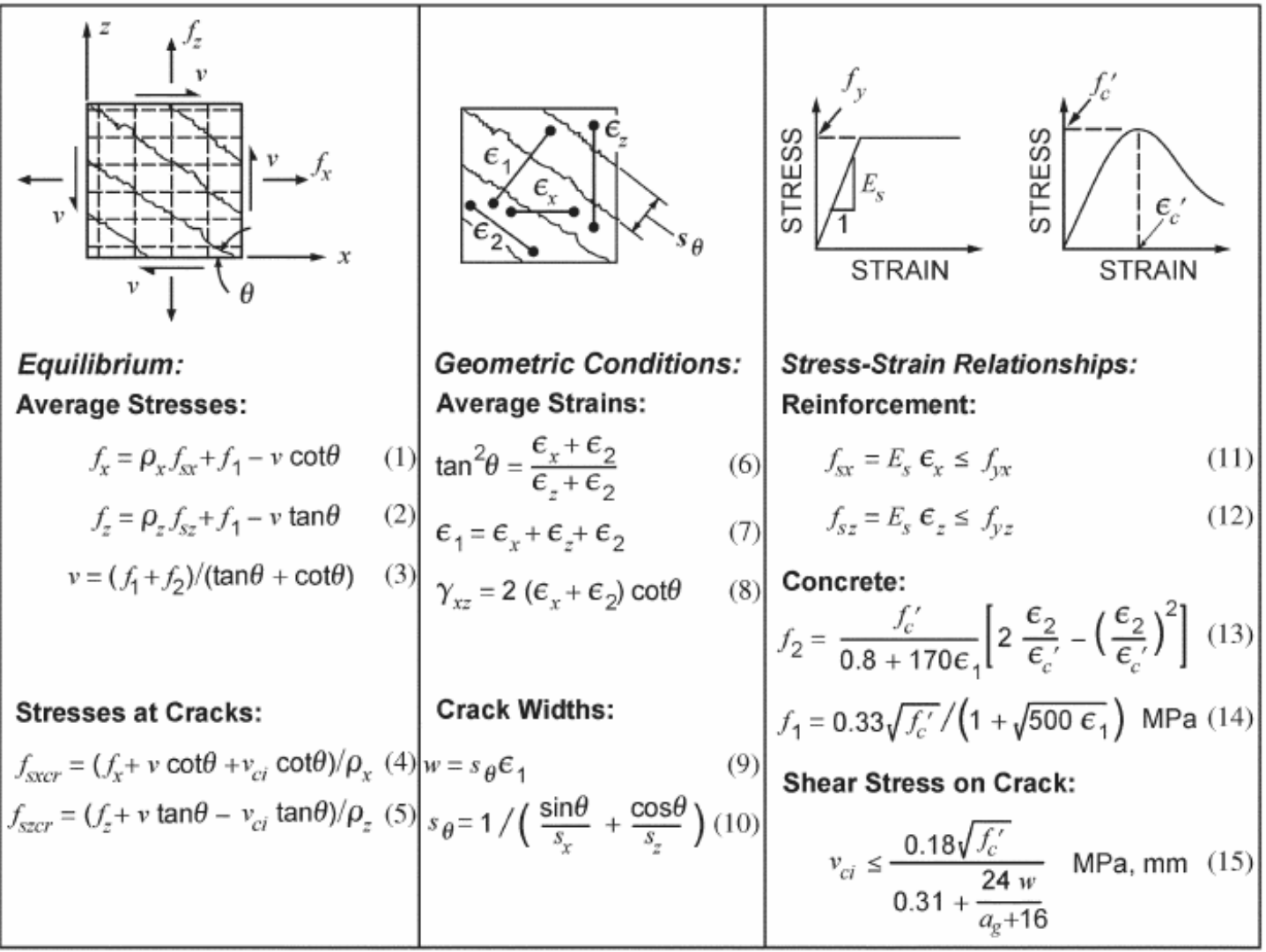

Figure (2-21): Equations of modified compression field theory (Bentz et al. 2006).

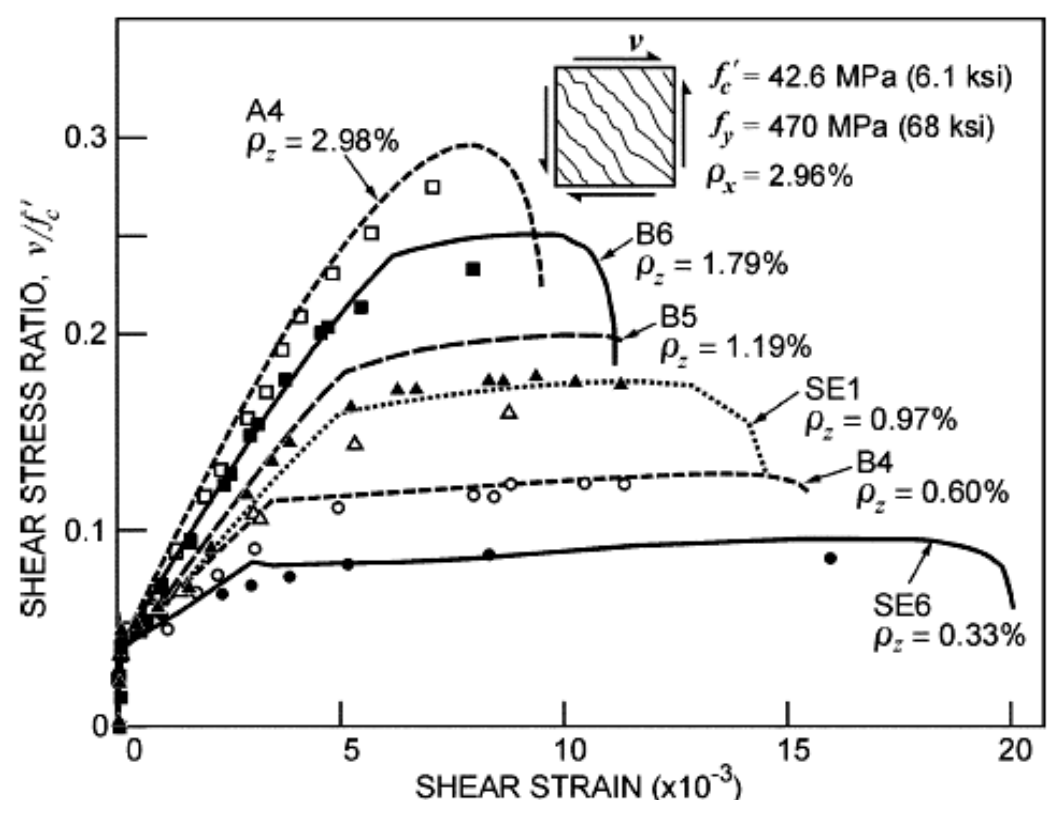

Figure (2-22): Comparison of predicted and observed shear stress shear strain response of six elements (Bentz et al. 2006). 
Note that, average strains related to strains measured over base lengths are at least equal to the crack spacing, while average stresses are calculated considering effects both at the cracks and between the cracks and are different from stresses calculated at cracks. Therefore, it takes too long to solve the 15 equations of the MCFT manually. Membrane-2000 (Bentz, 2000) is a suitable computer program that predicts the loaddeformation response of reinforced concrete membrane elements as shown in Figure (222). Of particular note is the outstanding agreement between the MCFT results and the points of the real experimental data (Bentz et al. 2006).

\subsubsection{Derivation of Simplified MCFT}

To simplify the calculations required to determine the shear strength of an element, neglect the clamping stresses $f_{z}$, as shown in Figure (2-18d). To make the transverse reinforcement yield at failure, the following have to be achieved: $\epsilon_{z}>0.002$ and $\epsilon_{2} \approx 0.002$. If $\epsilon_{x}=0.002$ at failure, Equations (3), (6), (7), (13) and (14) predict that the maximum shear stress will be approximately $0.28 f_{c}^{\prime}$. For very low values of $\epsilon_{x}$, however, the shear stress at failure is predicted to reach approximately $0.32 f_{c}^{\prime}$. To simplify the MCFT for failure occurring before yielding of the transverse reinforcement, the failure shear stress will be $0.25 f_{c}^{\prime}$. For failures occurring when shear stress $<0.25 f_{c}^{\prime}$, at failure both $f_{s z}$ and $f_{s z c r}$ are equal to the yield stress of the transverse reinforcement, which will be called $f_{y}$. Equation (5) from Figure (2-21) can be derived by summing up the forces in the z-direction for the free body diagram shown in Figure (2-23). For $f_{z}=0$ and $f_{s z c r}=f_{y}$, this equation can be rearranged to give, (Bentz et al. 2006).

$$
v=v_{c i}+\rho_{z} f_{y} \cot \theta
$$

In a similar fashion, Equation (2) from Figure (2-21) can be rearranged to give

$$
v=f_{1} \cot \theta+\rho_{z} f_{y} \cot \theta
$$

Both of these equations can be written as

$$
v=v_{c}+v_{s}=\beta \sqrt{f_{c}^{\prime}}+\rho_{z} f_{y} \cot \theta
$$

From Equations (14), (17) and (18) of Figure (2-21), the value of $\beta$ is given by

$$
\beta=\frac{0.33 \cot \theta}{1+\sqrt{500 \epsilon_{1}}}
$$


Similarly, in Equations (15), (16) and (18) of Figure (2-21), the value of $\beta$ must fulfill (Bentz et al. 2006)

$\beta \leq \frac{0.18}{0.31+24 w\left(a_{g}+16\right)}$

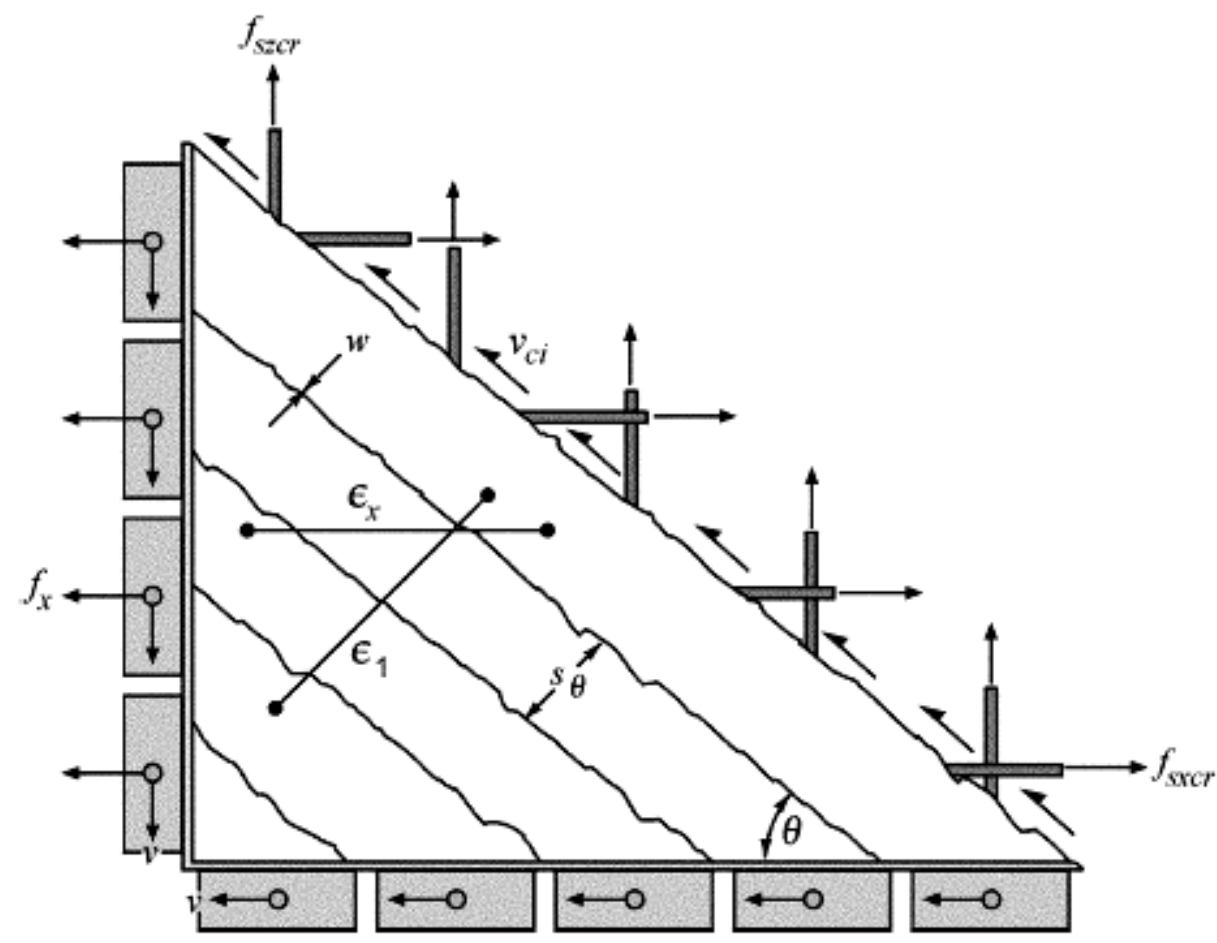

Figure (2-23): Transmission of forces across cracks (Bentz et al. 2006).

The crack width $w$ is obtained as the result of the crack spacing $S_{\theta}$ and the principal tensile strain $\epsilon_{1}$. $a_{g}$ refers to the maximum coarse aggregate size in $\mathrm{mm}$.

$S_{x}$ is the crack spacing in $x$-direction, and it depends on the properties of the reinforcement in that direction. The same applies in z-direction, which is expressed by $S_{z}$ Equation (10) of Figure (2-21). In a direct expression, $S_{x}=$ the vertical distance between bars in x-direction, and $S_{z}=$ the horizontal spacing between vertical bars in the z-direction.

For concrete elements with no transverse reinforcement, $S_{\theta}=S_{x} / \sin \theta$ and Equation (20) of Figure (2-21) can be written as (Bentz et al. 2006) 


$$
\beta \leq \frac{0.18}{0.31+0.686 S_{x e} \epsilon_{1} / \sin \theta}
$$

Where

$$
s x e=\frac{35 s x}{a g+16}
$$

$a_{g}=0$ with elements of high-strength concrete $\left(f_{c}^{\prime}>70 \mathrm{MPa}\right)$, because the cracks will go through the aggregate instead of passing around them.

The maximum value of $\beta$ and maximum post-cracking shear capacity will occur when Equations (24) and (26) from Figure (2-21) give the same value of $\beta$ (Adebar and Collins,1996). This requirement result in the following equation (Bentz et al. 2006)

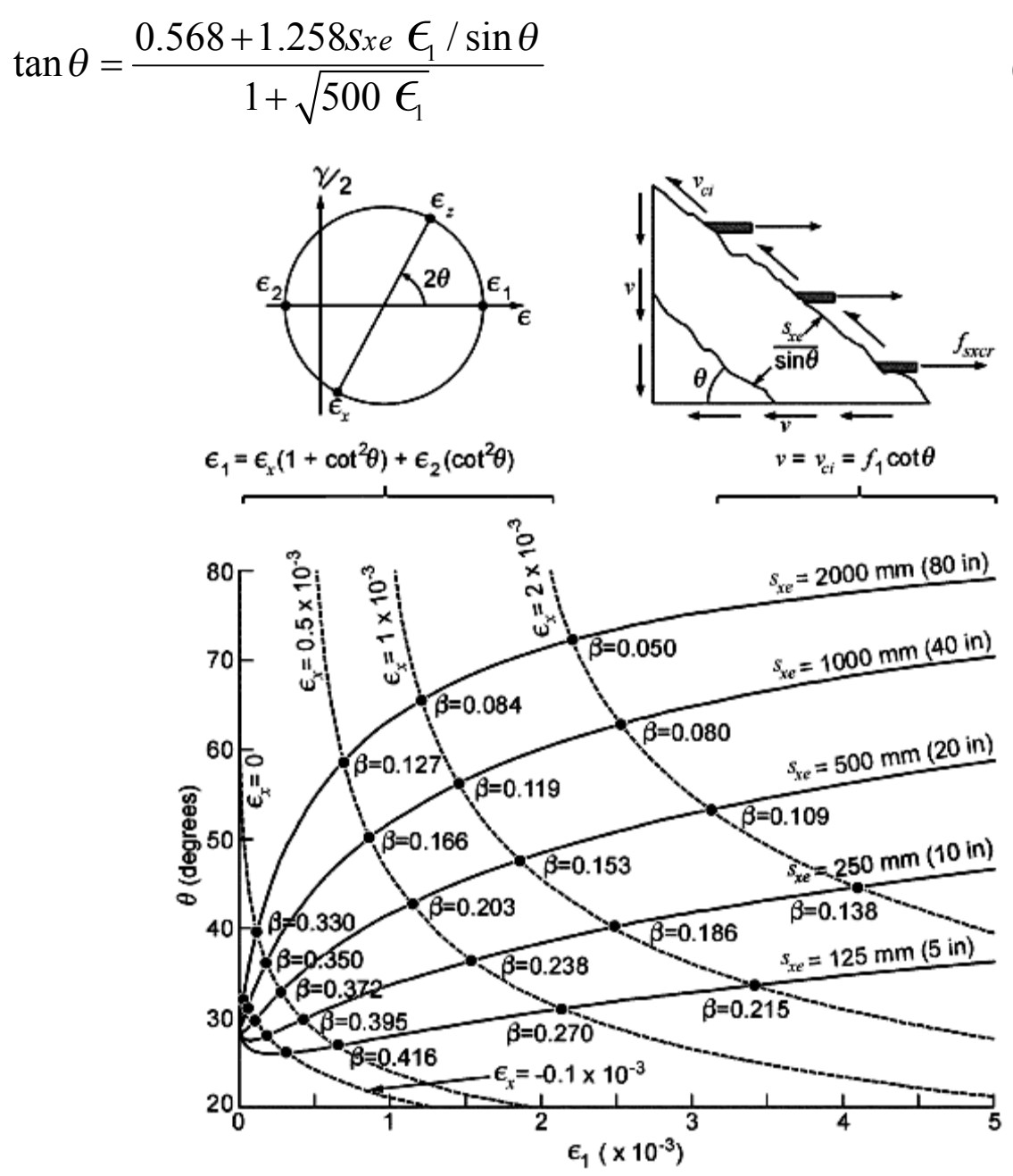

Figure (2-24): Determination of beta and theta values for elements not containing transverse reinforcement (Bentz et al. 2006). 
Figure (2-24) shows how Equation (2-28) connects the diagonal compressive stresses $\theta$ with the principal tensile strain $\epsilon_{1}$ for different values of the crack spacing parameter $S_{x e}$. To connect the longitudinal strain $\epsilon_{x}$ to $\epsilon_{1}$, Equations (6) and (7) from Figure (2-21) can be reorganized to give (Bentz et al. 2006)

$$
\epsilon_{1}=\epsilon_{x}\left(1+\cot ^{2} \theta\right)+\epsilon_{2} \cot ^{2} \theta
$$

The principal compressive stress $f_{2}$ produces the principal compressive strain $\epsilon_{2}$, so when $\rho_{z}$ and $f_{z}$ are zero, Equations (2) and (3) of Figure (2-21) can be reorganized to produce (Bentz et al. 2006)

$$
f_{2}=f_{1} \cot ^{2} \theta
$$

For the small $f_{2}$ of these elements, the assumption of $\epsilon_{2}=f_{2} / E_{c}$ is close to be accurate; and $E_{c}=4950 \sqrt{f_{c}^{\prime}} \mathrm{MPa}$. Then Equation (2-29) will turn into (Bentz et al. 2006)

$$
\epsilon_{1}=\epsilon_{x}\left(1+\cot ^{2} \theta\right)+\frac{\cot ^{4} \theta}{15000\left(1+\sqrt{500 \epsilon_{1}}\right)}
$$

Figure (2-24) shows how this geometric equation connects $\epsilon_{1}$ and $\theta$ for a variable value of $\epsilon_{x}$; so by knowing $\epsilon_{x}$ and $S_{x e}$, we can get $\epsilon_{1}$ and $\theta$, which will simultaneously solve Equations (2-28) and (2-31). Figure (2-24) also shows similar values of $\beta$ that are a result of Equation (2-24). The increased crack spacing $S_{x e}$ is accompanied by a decrease in $\beta$ values and shear strengths.

Kani (1967) and Lubell (2004) showed that large reinforced beams with no stirrups fail at lower shear stresses than geometrically similar smaller beams, and outcome which is known as the size effect in shear. It is important to note that one of the expectations of the MCFT is to prove that the size effect is related to the distance between the layers of longitudinal reinforcement instead of the overall size of the element. This is in agreement with the results of the extensive experimental studies on size effect conducted by Collins and Kuchma (1999). The "strain effect factor" $\epsilon_{x}$ and the "size effect factor" $S_{x e}$ are not completely independent, but in the simplified version of the MCFT, the influence of these two factors on each other is neglected and is 
supposed that $\beta$ is a product of strain factor and size factor. Equation (2-32) shows a new proposal for $\beta ; \beta$-values resulting from this equation are compared to the values from the MCFT in Figure (2-25). It is obvious that the simple equation produces conservative values in general, but the values are not only conservative with very small values of $\epsilon_{x}$ jointed with small values of $S_{x e}$, (Bentz et al. 2006)

$$
\beta=\frac{0.4}{1+1500 \epsilon_{x}} \cdot \frac{1300}{1000+S_{x e}}
$$
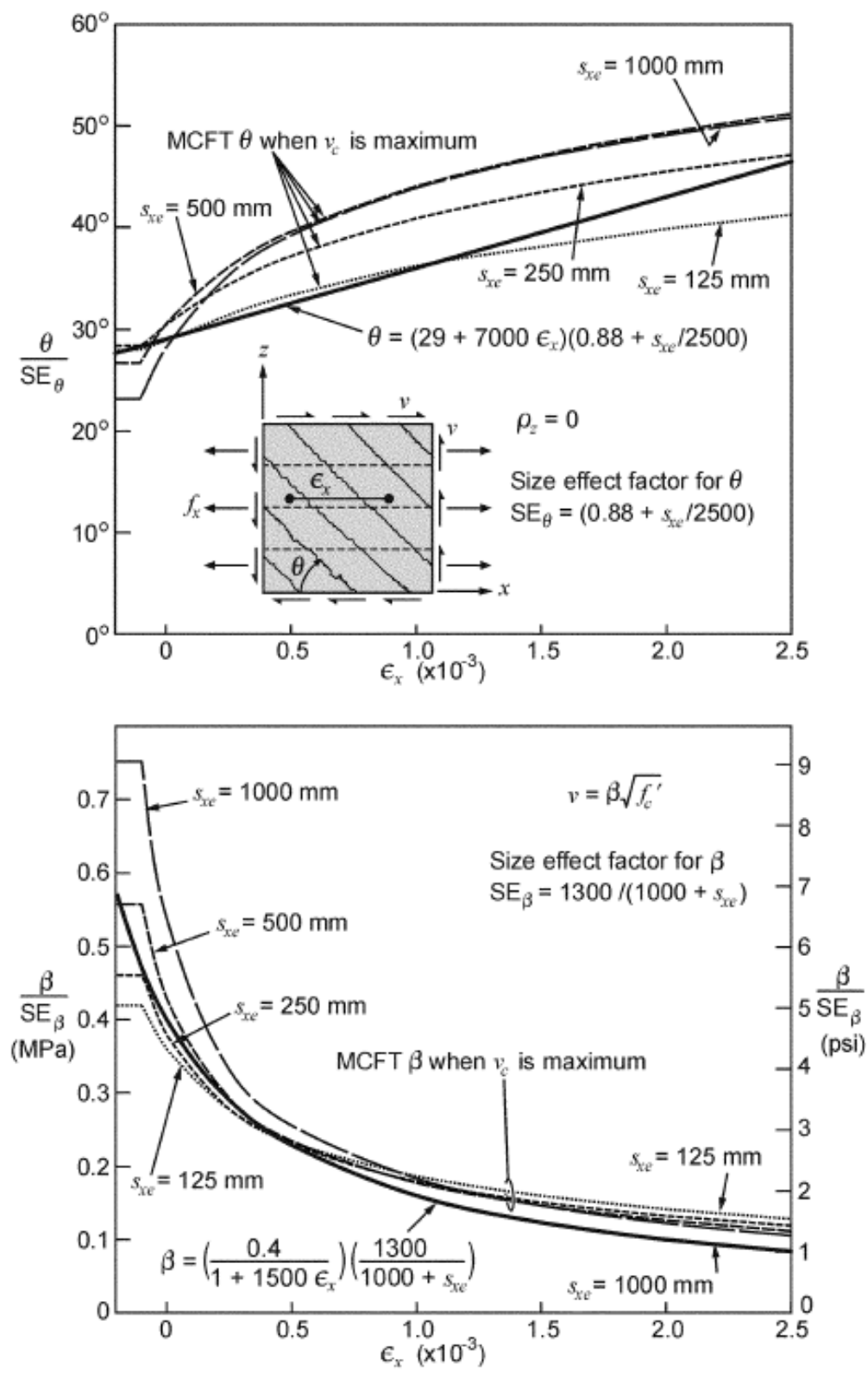

Figure (2-25): Comparison of values for $\theta$ and $\beta$ given by simple equations with values determined from MCFT for elements without transverse reinforcement (Bentz et al. 2006) 
Equation (2-32) is to be used with a concrete strength, in MPa andSxe, in millimetres. The simplified MCFT uses the following expression for the angle of inclination $\theta$ (Bentz et al. 2006)

$$
\theta=\left(29^{\circ}+7000 \epsilon_{x}\right)\left(0.88+\frac{s_{x e}}{2500}\right) \leq 75^{\circ}
$$
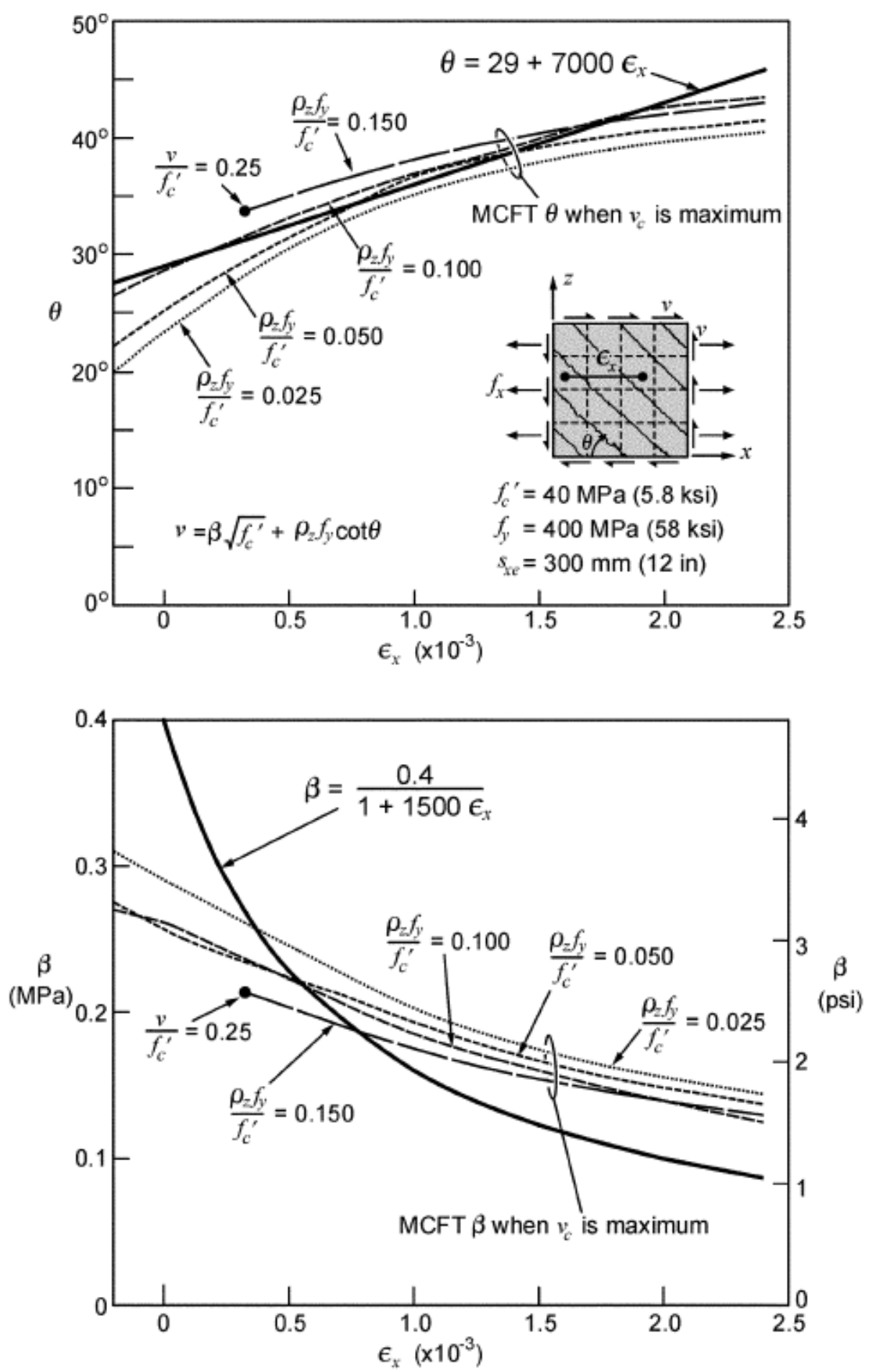

Figure (2-26): Comparison of values for $\theta$ and $\beta$ given by simple equations with values determined from MCFT for elements with transverse reinforcement (Bentz et al. 2006) 
It seems that $\theta$ is a function of $\epsilon_{x}$ and $S_{x e}$, and the results of this equation can be compared with the MCFT results, shown in Figure (2-25). By examining those results, as well as those for beams without stirrups, it is conservative to underestimate $\theta$, and the calculated stress in the longitudinal reinforcement will be increased, as shown in Figure (2-25). Note the difference between the results of the elements without transverse reinforcement and those with transverse reinforcement. A conservative approach for determining $\theta$ for the simplified MCFT is to take into account the calculated value of $\theta$ when $v_{c}$ at the maximum level of contribution to the strength. It is also useful to calculate $\theta$ for the members with and without transverse reinforcement within the same calculation. Therefore, Figure (2-26) shows a comparison between the values of $\theta$ collaborating with maximum $V_{c}$ with $\theta$ resulting from Equation (2-33), and it shows that the concurrence is logical. For the elements with longitudinal and transverse reinforcement, large $\theta$ is conservative because it reduces $\nu_{s}$. It is also conservative to take $s_{x e}=300 \mathrm{~mm}$ in Equations (2-32) and (2-33) because the cracking of the diagonal cracks will typically be $<300 \mathrm{~mm}$. Figure (2-26) shows a comparison of the similar values of $\beta$ predicted by the MCFT with the other values of $\beta$ produced by Equation (2-32). It is clear that the values of $\beta$ calculated by the simple equations are conservative over large number of possible $\epsilon_{x}$-values. When $\epsilon_{x}$ values are very low, they tend to be slightly unconservative; for these value of $\epsilon_{x}$, the unconservative results for $\beta$ will be partly balanced by the conservative estimate for $\theta$ (Bentz et al. 2006). 


\subsection{Self-consolidating concrete}

SCC stands for Self-Consolidating Concrete and Self-Compacted Concrete; some publications refer to it as self-levelling concrete. All of these names refer to the type of high-performance concrete that takes the shape of its form and achieves the required compaction under its own weight without the need for mechanical interferences to move, compact it or vibrate the concrete. The main difference between SCC and NC is evident during placing time. At the time of pouring, SCC must flow for specific distances, filling all the small places and passing through congested rebars cages without the segregation of coarse aggregate from the paste that results directly in high-quality finishing.

\subsubsection{Benefits of using SCC}

1. SCC is easier to pour and cast without vibration, and has higher workability than NC. This characteristic allows it show almost no repair to the outer face of the concrete.

2. SCC is excellent in flow through restricted shaped areas or congested rebars. This feature offers more flexibility in retrofit applications or in maintaining old or damaged concrete structures. SCC can be pumped through an open end of the formwork until it is filled and comes out the other far end opening. It offers noticeably improved pump ability, which is ideal for architects developing the shapes and finishing of concrete panels.

3. SCC offers remarkable cost savings through reduced labour during casting. It also extends the life of machinery and equipment used for placing and vibrating concrete, since form moulds last longer without vibration.

4. SCC is less harmful to the environment than conventional concrete. It consumes less energy to reach optimum strength and durability. SCC also offers improved consistency; homogeneous vibration cannot be guaranteed throughout the concrete structure, but the SCC does not require uniform vibration.

5. The noise of the vibration applied to the normal concrete is eliminated with the SCC. Reducing noise in construction sites also reduces the premiums paid to insurance agencies responsible for eventual treatment of hearing-impaired workers (Jacobs and Hunkeler, 2001).

6. Using SCC is safer than using $\mathrm{NC}$; shorter labour time and less machinery lowers the probability of accidents and injuries. 
7. With SCC, there is no need to use underlay materials such as carpeting and tile, where allowed by building regulations.

Although SCC is a relatively new product, its many advantages make it very attractive to engineers and construction companies. However, in order to increase the safe application of SCC, engineers must have a clear understanding of the benefits, but also the difficulties it may face in the future.

\subsubsection{Difficulties facing the use of SCC}

1. Producing SCC is more difficult than producing NC because it requires special additives and higher quality control.

2. There is a lack of information about SCC in the current codes and standards, compared with the available information about NC. This gap is slowly narrowing, and one of the main objectives of this research is to develop a new understanding of the shear behaviour of SCC beams affected by corrosion.

3. The long-term performance of SCC cannot be judged until many decades have passed and precise monitoring and testing to the concrete structures built with the SCC has been performed.

4. When using SCC, the formwork must be strengthened more than when using NC due to hydrostatic pressure.

\subsubsection{SCC development}

In Japan in the 1980s, SCC was found to be a suitable solution to two issues: a) the durability of concrete consolidating through the highly congested rebars structures was more resistant to earthquake damage, and b) SCC's reduced labour requirements was an answer to the labour shortage in the Japanese construction industry. ACI 237R-07 contains many references to examples of the early use of SCC in different kinds of structures, and includes Tanaka et al. (1993); Hayakawa et al. (1993, 1995); Miura et al. (1993); Okamura and Ozawa (1994); Takeuchi et al. (1994); Izumi et al. (1995); Fukute et al. (1995); Kitamura et al. (1996); and Ushijima et al. (1995). New projects in Canada and Switzerland used SCC successfully in repair applications for a variety of concrete structures; it proved sufficient in the filling of confined areas and offered very good surface quality (Jacobs and Hunkeler, 2001), (Khayat and Morin, 2002). In addition, SCC 
is suitable for both cast-in-place and precast units (RILEM, 2000), (Khayat and Aïtcin, 1998), (Skarendahl, 2001), (Walraven, 2001), (Ouchi, 2001).

\subsubsection{Fresh characteristics of SCC}

SCC has the following fresh characteristics:

1. Rheology: as a study of deformation and flow of matter, SCC rheology is described by a low yield stress to ensure high deformability and moderate plastic viscosity to maintain homogeneous suspension of solids, and reduced interparticle collision, segregation, and flow blockage (ACI 237R-07).

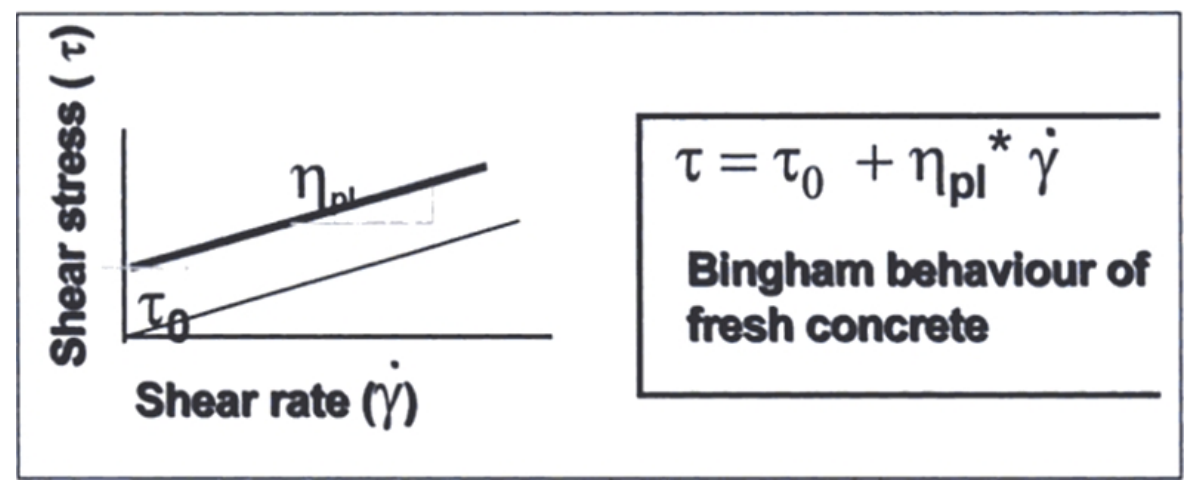

Figure (2-27): Relationship between shear stress and shear rate (Korradi, 2003).

With fresh SCC, a force has to be applied to start the flow; it is called the yield stress $\tau_{0}$. Resistance to flow will increase with the increase in rate of flow; the slope of the line represents the resistance to increase in flow rate or plastic viscosity $\eta_{0}$. Figure (2-27) shows the relationship between the shear stress and shear rate (Korradi, 2003).

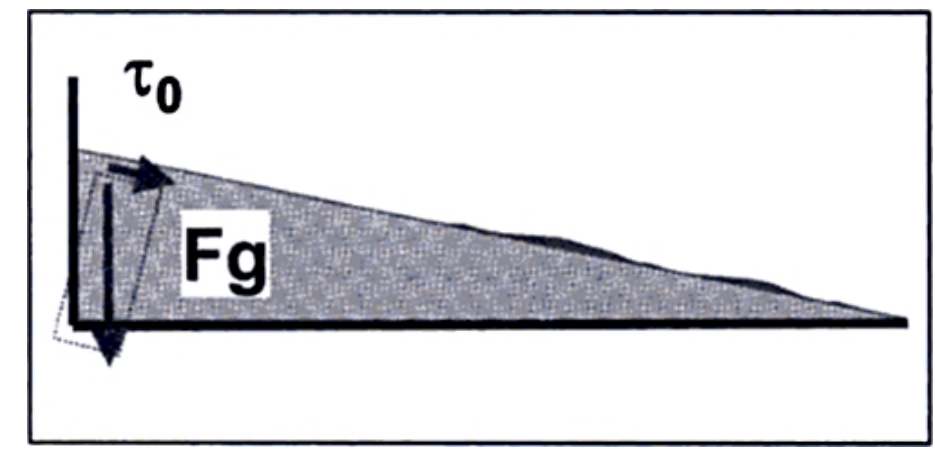

Figure (2-28): Tangential component of the gravity force (Korradi, 2003). 
In SCC, the tangential component of the gravity force deforms the SCC paste and makes it flow, as shown in Figure (2-28). The SCC will flow like a fluid only when the tangential component of the gravity force exceeds the yield stress $\tau_{0}$. It is important to note that the high probability of segregation is accompanied by low plastic viscosity. With high plastic viscosity, the flow of concrete will be very slow and the self-consolidation time will be very long, which may minimize the advantages of SCC (Korradi, 2003). (ACl 237R-07) refers to Equation (2-34):

$$
\tau=\tau_{0}+\mu_{p} \cdot \gamma
$$

Where: $\tau=$ shear stress $(\mathrm{Pa}) ; \tau_{0}=$ yield stress $(\mathrm{Pa})$ (minimum shear stress required to initiate flow); $\mu_{p}=$ plastic viscosity (Pa.s); $\gamma=$ shear rate.

2. Workability: refers to the ease of mixing, placing, consolidating and finishing the concrete. ACI 237R-07 expresses precisely how SCC shows high filling ability, passing ability, and stability.

3. Filling ability: refers to the capability of SCC to flow and fill all spaces and corners within the formwork by its own gravitational force (ACI 237R-07).

4. Passing ability: the ability of SCC to flow by its weight only through obstacles and spaces congested with many rebars, without blockage.

5. Stability: refers to the ability of SCC to retains its homogeneous ingredients during its flow and setting. Dynamic stability occurs when there is no separation of SCC mixture ingredients because of flow through obstacles or congested rebars. Static stability refers to the resistance of SCC, when it is in a plastic state, to bleeding, segregation, and surface settlement (ACl 237R-07).

\subsubsection{Testing SCC fresh properties}

1. Slump flow test: A suitable way to evaluate filling ability, this test is performed in a similar way to the conventional slump test using the standard ASTM C 143/C 143M slump cone. According to ASTM C, 1611/C 1611M, instead of measuring the vertical collapse of the concrete, the average measurement of the diameter of the circular shape of SCC represents SCC slump flow. The visual stability index (VSI) is evaluated by assessing the stability of the slump flow patty. Figure (2-29) shows the SCC slump flow test measuring (Daczko and Kurtz, 2001). 


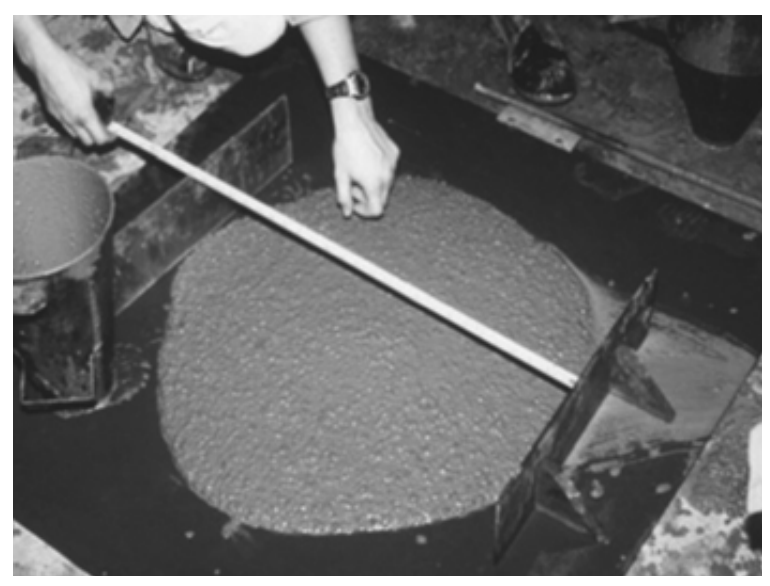

Figure (2-29): SCC slump flow (ACI 237R-07).

2. J-ring: This apparatus is designed to measure passing ability. It is made of a ring reinforcing bar that fits around the base of a standard ASTM C 143/C 143M slump cone, as shown in Figure (2-30). The slump cone in the J-ring is filled with concrete and then lifted, and the final spread of the concrete is measured and compared to the value of the conventional slump ( $\mathrm{ACl} 237 \mathrm{R}-07)$.

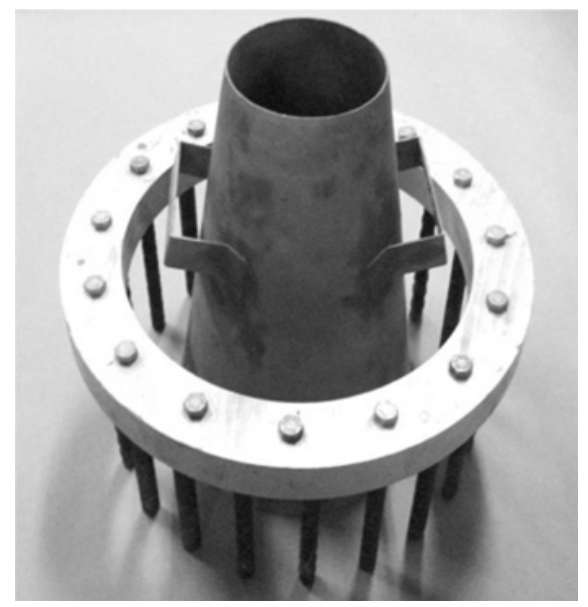

Figure (2-30): J-Ring apparatus (ACI 237R-07).

3. L-Box: This is another apparatus to measure the passing ability. It is L-shaped, and is made up of two vertical and horizontal parts with a sliding door located between the parts, as illustrated in Figure (2-31). It contains three rebars, located in the horizontal part beside the sliding door, to obstruct SCC flow from the vertical part to the horizontal part. The L-Box test is a good indicator of blocking and stability of SCC. The acceptable range for the ratio of pass ability is $\boldsymbol{P A}=\boldsymbol{H}_{\mathbf{2}} / \boldsymbol{H}_{\mathbf{1}}=\mathbf{0 . 8} \mathbf{- 0 . 8 5}$, however, EFNARC 
suggested the range of the ratio $\boldsymbol{H}_{2} / \boldsymbol{H}_{\mathbf{1}}$ to be from $\mathbf{0 . 8}-\mathbf{1 . 0}$ (EFNARC, 2002), (Khayat et al. 2004) and (Groth, 2000).
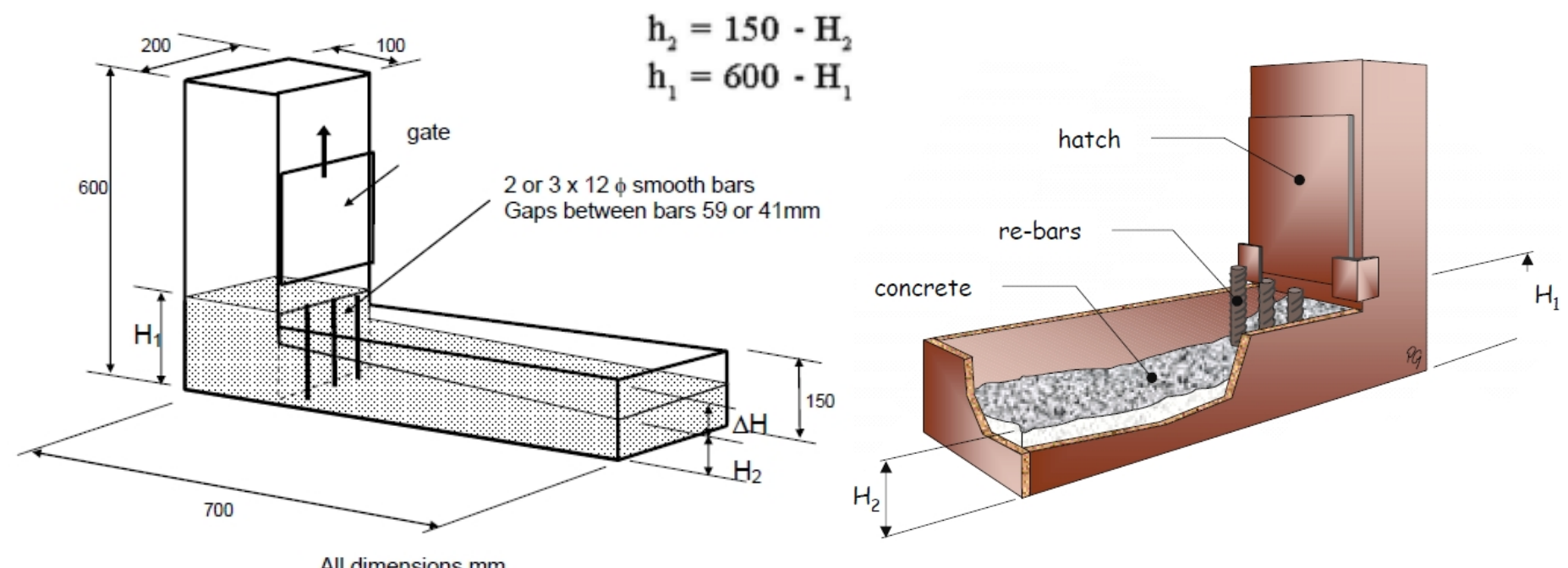

Figure (2-31): Typical L-Box arrangement to measure flow of SCC around rebar obstructions. The left part of the image is adopted from EFNARC (2002), and the right part from Groth (2000).

The L-Box test is carried out by filling the vertical part with SCC, then opening the sliding door to let the SCC flow into the horizontal part to calculate the blockage ratio $h_{2} / h_{1}$, as illustrated in Figure (2-31) (Khayat et al. 2004).

4. V-funnel flow time test: Flow is tested using a v-shaped container, illustrated in Figure (2-32). The model suggested by Ozawa, Sakata, and Okamura (with an outlet of $65 \times 75 \mathrm{~mm}$ ) has been modified to an outlet of $75 \times 75 \mathrm{~mm}$. In the test, the container is filled with SCC. After one minute, the bottom gate is opened and the time required for the SCC to pass through the tapered outlet is monitored (Ozawa et al. 1994). The test evaluates SCC flow through a confined section without segregation and blockage. To consider a concrete mix to be SCC, the time measured should be less than 6 seconds (Khayat and Manai, 1996). A time of less than 6 seconds of V-funnel flow shows a good relative signal of plastic viscosity with a lack of SCC blocking that must be monitored during the test (Lachemi et al. 2004). Several other studies expand the allowable time of 
V-funnel flow from 6-12 seconds (Abdul Hameed, 2005), (EFNARC, 2002) and (Khayat, 2004).
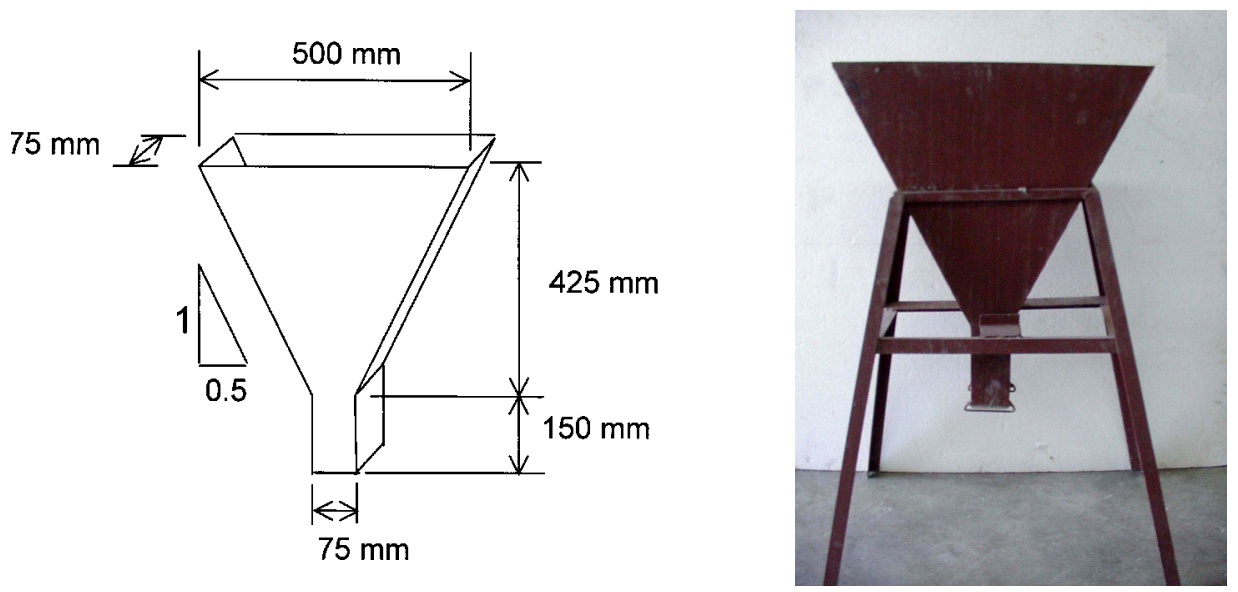

Figure (2-32): Schematic of V-funnel apparatus (Khayat, 2004).

Photo on right (Abdul Hameed, 2005).

5. U-box test: This test is designed to evaluate the ability of SCC to pass through a narrow opening. Figure (2-33) illustrates the U-shape apparatus (Khayat, 2004). One minute after filling compartment $A$ with concrete, the bottom gate is lifted up to allow the concrete to flow into compartment $B$ through congested rebars at the bottom. Three bars of $12 \mathrm{~mm}$ diameter with $40 \mathrm{~mm}$ clear spacing were used to be similar to the confined flow area. When the SCC stops flowing, the height of the concrete in compartment B is measured from the bottom of the apparatus to the top surface. The filling height $B_{h}$ refers to the passing ability and non-blocking behaviour of the SCC; the maximum $B_{h}$ value is $338 \mathrm{~mm}$. The time required to complete the flow into compartment $\mathrm{B}$ is also monitored to evaluate the rate of deformability of the SCC (Khayat, 2004). 


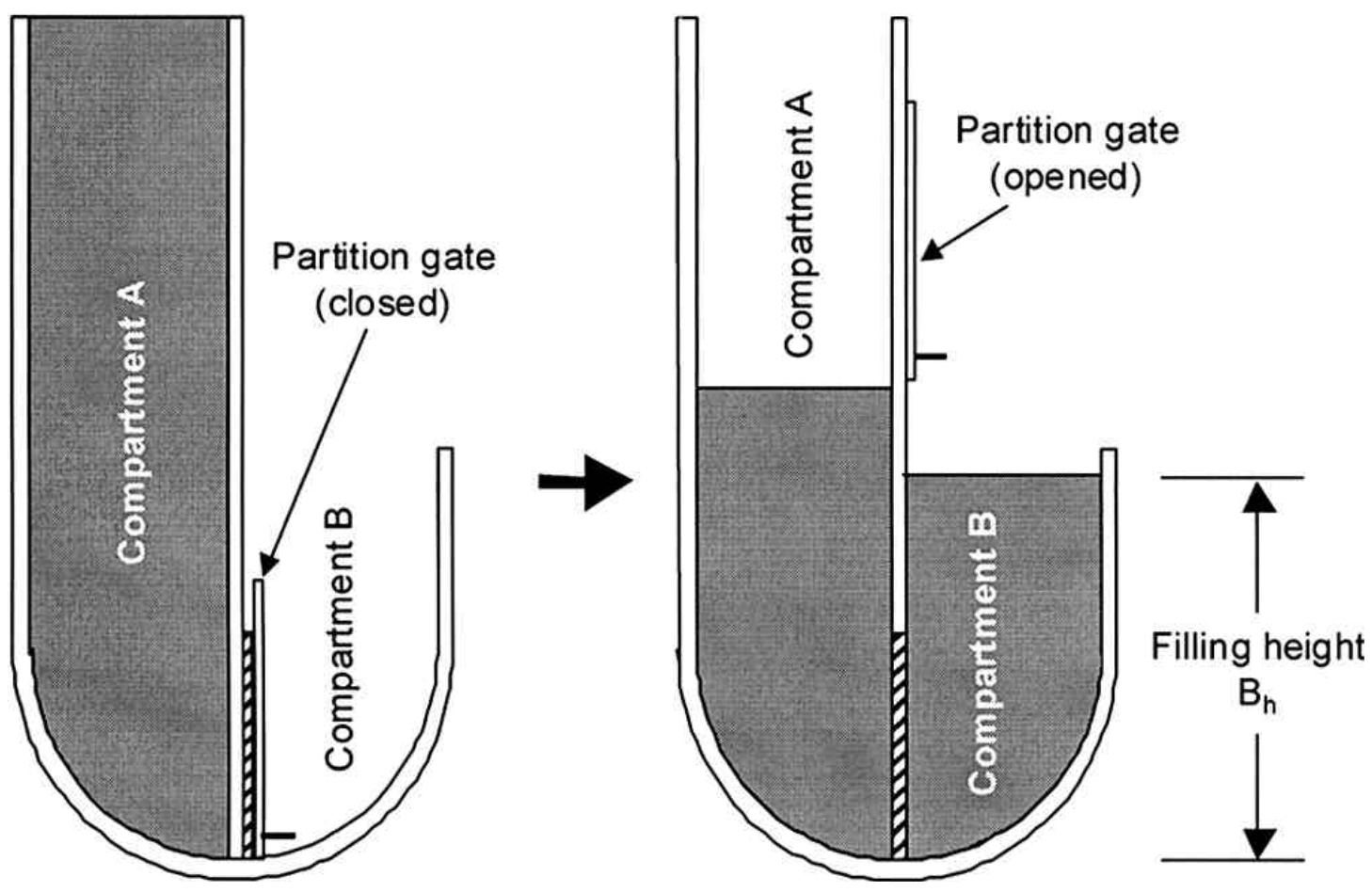

Figure (2-33): Schematic of U-box test (Khayat, 2004).

\subsubsection{Selecting SCC proportions}

To achieve the expected higher workability of SCC, the SCC mixture should be proportioned carefully, in consideration of the application and placement methods. SCC ingredients - coarse and fine aggregates, cementitious materials, and additives - should be balanced to fulfill the desired fresh and hardened characteristics (ACI 237R-07). To determine the required filling ability, passing ability, and stability, the quantity of SCC has to be determined as well as the size of the, the concrete member, the rebars congestion, and the method and timing of SCC delivery. For the mixture proportion, the ACI 237R-07 refers to three basic mixture-proportioning approaches for SCC:

- High powder content and high-range water reducer admixtures (HRWRA);

- Low powder content, HRWRA, and viscosity modifying admixtures (VMA); and

- Moderate powder content, HRWRA, and moderate VMA dose (stability can be controlled by blending aggregates, lowering water content, or using a VMA) (ACI 237R-07). 
In some applications, VMAs or higher powder contents can be used to assist stability. Decreasing the free water and increasing the volume of powder increases the stability of SCC. Inert filler, obtained from powdering limestone or siliceous aggregates, can be used to improve the Portland cement particle-size distribution and achieve a better filling density. The powder fragments can fill the gap between the sand and Portland cement. Replacing part of cement with limestone powder filler improves filling ability and stability without affecting the 1-day compressive strength (ACI 237R-07).

\subsubsection{Finely divided powders and supplementary cementitious materials}

The inert filler, produced by grinding limestone or siliceous aggregates, improves Portland cement particle-size distribution and obtains a better packing density. The fine segments of the fillers develop the surface of the mixture, but the coarse segments can fill the empty spaces between the sand and Portland cement. The part substitution of cement with finely ground limestone filler is proven to increase the values of filling ability and stability without influencing the 1-day compressive strength. However, at 28 days, the concrete showed $10 \%$ less strength than the concrete without filler. Suitable ground filler can improve the packing density of solid particles and enable the reduction of water or HRWRA demand required to achieve high filling ability (ACl 237R-07) (Ghezal and Khayat, 2002). It is an advantage to substitute part of the cement with a less reactive powder, especially with relatively large concrete members or, in general, wherever project requirements limit the heat of hydration. The finely divided powders and supplementary cementitious materials are as follows:

1. Silica fume: It is applied to enhance the mixture stability of SCC mixtures by reducing the mobility of the water through the concrete form. With a substitution of $5 \%$ or less, it reduces the viscosity of SCC. Silica fume's small size and rounded shape work as a lubricant to reduce the friction between larger cement particles (ACI 237R-07).

2. Fly ash: The circular shape and smooth surface of its particles work as a ball bearing inside the paste of the SCC mixture; therefore, it may increase the workability and slump flow of SCC. Fang et al. (1999) refers to the increased slump flow with substitution rates between 20 and $40 \%$ Portland cement. The most efficient replacement value, however, is affected by job specifications, material compatibility, and cost. The fly ash, with its relatively low density, makes the SCC paste larger and more stable (ACI 237R-07). 
3. Ground-granulated blast furnace slag: GGBFS meeting ASTM C 989 is hydraulic cement and can be used to replace a portion of the Portland cement. The most efficient replacement values will be decided by the nature of the job and the general compatibility with the cementitious system (ACl 237R-07).

\subsubsection{Admixtures}

There are many commercial names for HRWRAs and VMAs used in the SCC mixture. Most of the HRWRAs are polycarboxylate- based, used for developing and proportioning the SCC and the VMAs, are useful for modifying the viscosity and stability of SCC (ACl 237R-07).

The HRWRAs increase fluidity and maintain viscosity while VMAs increase viscosity. Using admixtures together with other proportioning techniques such as combined gradation of aggregates, increasing fine aggregate content, powder content, or both, are managed to increase stability. Therefore, adding the VMAs to the HRWRAs into the SCC mixtures is expected to develop flowability, viscosity and stability. The maximum benefits can be obtained from the VMA when it is used with a mixture of lower powder contents and gap graded. Increased passing ability resulting from adding lower powder content to VMA has to be evaluated before applying it in applications with dense reinforcement. It is important to take into consideration the specifications, dosage, and timing of use that is recommended by the admixture producers (ACI 237R-07).

To control the trial batch of SCC production, it is recommended to do an initial slump test before adding the HRWRA. The first indicator is the amount of water used to produce an initial slump (less than $200 \mathrm{~mm}$ ). When managing trial batches, the influence of water of the mixture should be recognized by monitoring concrete proportions and adding sequential amounts of water to the mixture, then recording the stability level after each water addition. At the level that the mixture starts to be unstable, the amount of water added limits the water mixture's sensitivity (ACl 237R-07). 


\subsection{Corrosion}

The Latin word corrodere, which means to eat away or destroy, forms the basic concept of the corrosion process (Baeckmann et al. 1997) (Leierzapf, 1985). Davy (1824) refers to corrosion in translation from French in the manufacture of white lead in 1785. It is also mentioned in 1836 in the translation of an English paper by Davy on the cathodic protection of iron in seawater.

\subsubsection{Definitions of Corrosion}

1. "The term corrosion is used to describe the reaction of a material with its surroundings that produces measurable changes and can lead to damage. With metallic materials and aqueous solutions, the reactions are in general of an electrochemical nature. However, in addition, pure chemical reactions or entirely physical processes can also be occurring. Not every process necessarily leads to damage. This is a question of the extent of the reaction and the demands on the function of material or medium, which should always be considered together. Damage is said to occur when this function is impaired. Corrosion protection is designed to prevent such detrimental action." (Baeckmann et al. 1997).

2. "Corrosion, by its simplest definition, is the process of a metal returning to the material's thermodynamic state. For most materials, this means the formation of the oxides or sulphides from which they originally started when they were taken from the earth, before being refined into useful engineering materials. Most corrosion processes are electrochemical in nature, consisting of two or more electrode reactions: the oxidation of a metal (anodic partial reaction) and the reduction of an oxidizing agent (cathodic partial reaction). The study of electrochemical thermodynamics and electrochemical kinetics is necessary to understand corrosion reactions." (Schweitzer, 2007).

\subsubsection{Fundamentals and concepts of corrosion of reinforcing steel bars}

Based on the above definitions, corrosion is a chemical reaction that may happen in nature or in the laboratory under certain conditions. Corrosion is always accompanied with a gradual reduction in the structural characteristics of the reinforcing steel bars. It is a normal behaviour of materials to return to their thermodynamically stable state. To stimulate corrosion in the lab or to force it through the reinforcing steel bars, the 
following materials must be present: electrolyte (usually saline water of concentration of $5 \%$ ), cathode and power supply to provide the electrical current that will accelerate the process of corrosion in the lab or any experimental environment. The reinforcing steel bars embedded inside the concrete should act as anode in the corrosion cell (Baeckmann et al. 1997).

The alkaline complex of the concrete causes the formation of strong sticky layer, which passivates the reinforcing steel and protects it from corrosion. The concrete cover above the reinforcing steel bars can contribute positively in protecting the steel bars. The thicker and denser concrete cover results in making the concrete more resistant to the electrical current, which needs more energy to induce the same amount of corrosion measured. Adding fine materials to the mix of the concrete, supplying the ultimate vibration to the concrete (in a case where SCC is not used) and mixing with the minimum possible (water/cement) or (water/binder) ratio will minimize the permeability of the concrete. There are some other ways to protect against corrosion, such as using epoxy-coated, galvanized steel (achieved by dipping the steel in hot tin to make a protective layer) and by using stainless steel bars. Designers who decide to use epoxycoated or stainless steel reinforcing bars have to consider the extra cost, structural efficiency, maintenance costs for the lifetime of the structure, and other factors depending on the type of structure, its function and its surrounding environment.

\subsubsection{Corrosion Electrochemical Reactions}

In the electrochemical reaction that causes corrosion, electrons move from the anode to the cathode in the presence of both humidity and oxygen. The anodic reaction is represented in Equation (2-35) as follows (Roberge, 2000)

$$
\text { Anode } \mathrm{Fe} \Rightarrow \mathrm{Fe}^{2+}+2 e^{-}
$$

On the other side, the cathodic reaction seems as it is in Equation (2-36)

$$
\text { Cathode } \quad 1 / 2 \mathrm{O}_{2}+\mathrm{H}_{2} \mathrm{O}+2 e^{-} \Rightarrow 2(\mathrm{OH})^{-}
$$

The anodic and cathodic reactions are illustrated in Figure (2-34). Melted oxygen reacts with hydrogen atoms adsorbed at random on the iron surface, independent of the presence or absence of impurities in the metal. 


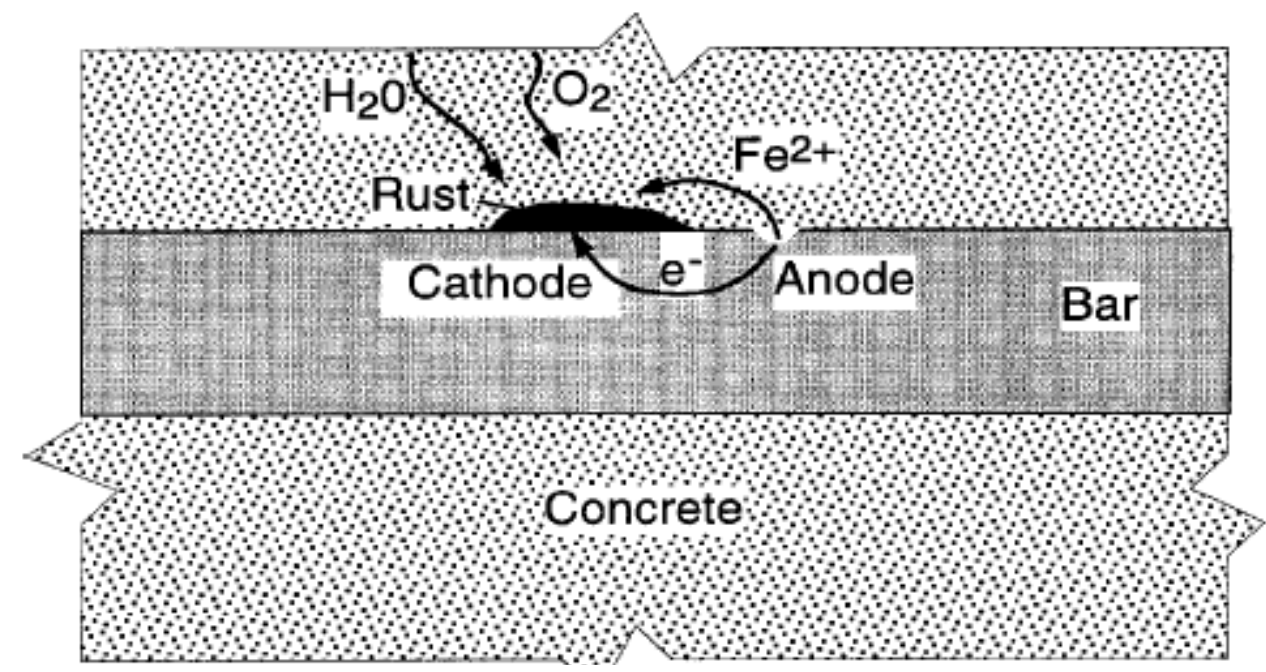

Figure (2-34): Corrosion of steel bar embedded in concrete (iron is dissolved at anode and precipitates as rust at cathode) (ACl 228.2R-98).

The oxidation reaction proceeds as rapidly as oxygen reaches the metal surface, (Roberge, 2000). By adding Equation (2-35) to (2-36), and taking advantage of reaction $\mathrm{H}_{2} \mathrm{O} \Leftrightarrow \mathrm{H}^{+}+\mathrm{OH}^{-}$results to (Roberge, 2000)

Ferrous hydroxide $\quad 2 \mathrm{Fe}+2 \mathrm{H}_{2} \mathrm{O}+\mathrm{O}_{2} \Rightarrow 2 \mathrm{Fe}(\mathrm{OH})_{2}$

Ferrous hydroxide composes the scattering-obstacle layer next to the iron surface through which $\mathrm{O}_{2}$ must scatter. The surface of corroded iron in aerated water is alkaline because the $\mathrm{pH}$ of the saturated $\mathrm{Fe}(\mathrm{OH})_{2}$ is around 9.5. The color of $\mathrm{Fe}(\mathrm{OH})_{2}$ is white when the material is pure, but commonly it turns green to greenish black due to starting oxidation by air. The thin layer of ferrous oxide $\mathrm{Fe}(\mathrm{OH})_{2}$ converts to ferric oxide or ferric hydroxide by reacting with the dissolved oxygen as follows (Roberge, 2000)

Ferric hydroxide $4 \mathrm{Fe}(\mathrm{OH})_{2}+2 \mathrm{H}_{2} \mathrm{O}+\mathrm{O}_{2} \Rightarrow 4 \mathrm{Fe}(\mathrm{OH})_{3}$

The color of ferric hydroxide is orange to red-brown and it forms most ordinary rust. In nature it is either nonmagnetic $\alpha \mathrm{Fe}_{2} \mathrm{O}_{3}$ (hematite) or magnetic $\alpha \mathrm{Fe}_{2} \mathrm{O}_{3}$, as shown in Equation (2-39) (Roberge, 2000)

Hydrated ferric oxide (Rust) $2 \mathrm{Fe}(\mathrm{OH})_{3} \Rightarrow \mathrm{Fe}_{2} \mathrm{O}_{3} \cdot \mathrm{H}_{2} \mathrm{O}+2 \mathrm{H}_{2} \mathrm{O}$ 
Saturated $\mathrm{Fe}(\mathrm{OH})_{3}$ is almost neutral in $\mathrm{pH}$. A magnetic hydrous ferrous ferrite, $\mathrm{Fe}_{3} \mathrm{O}_{4} \cdot n \mathrm{H}_{2} \mathrm{O}$ often forms a black layer between hydrous $\alpha \mathrm{Fe}_{2} \mathrm{O}_{3}$ and $\mathrm{FeO}$. Therefore, rust films normally consist of three layers of iron oxides in different states of oxidation (Roberge, 2000).

The corrosion production may expand up to 6.5 times the original size of the steel. Figure (2-35) shows the details of the change in size as a result of a specific chemical reaction.

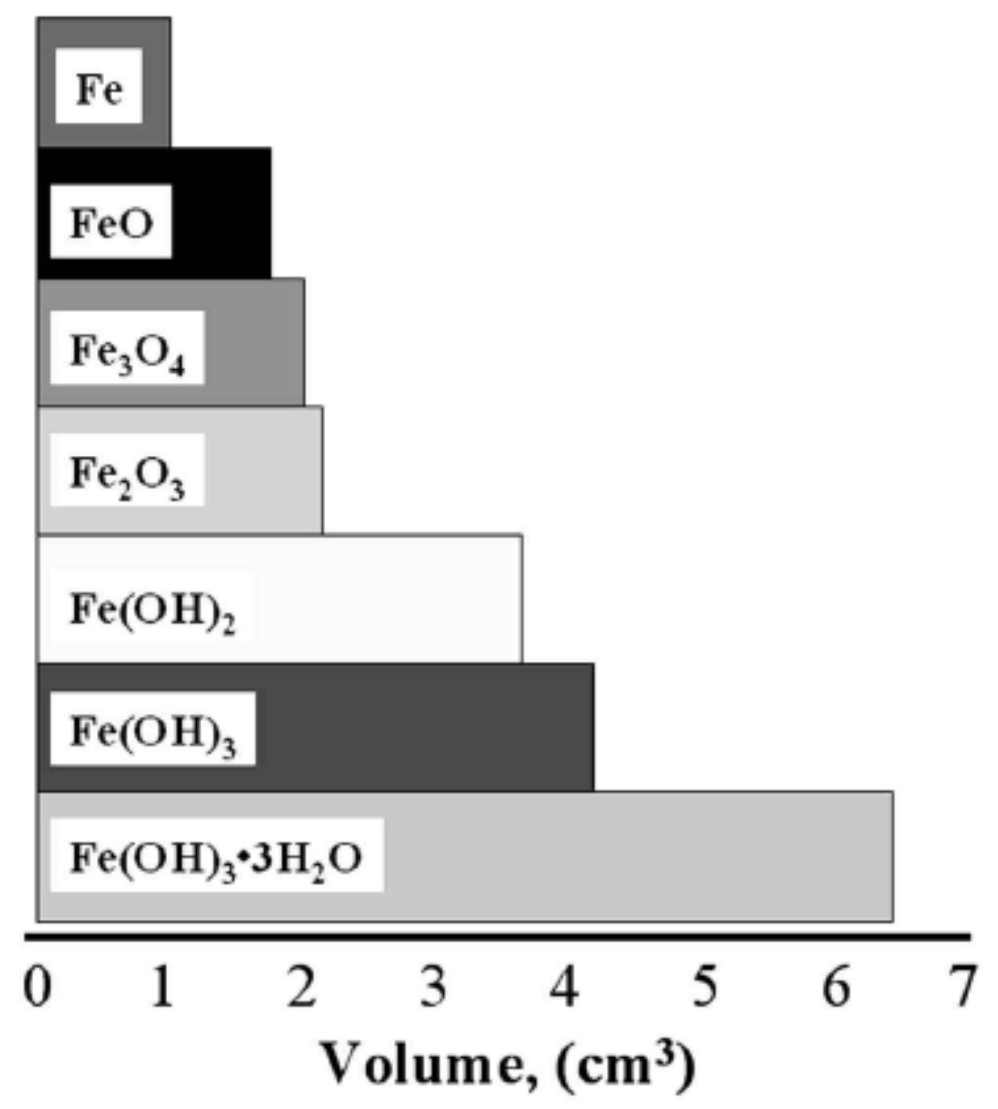

Figure (3-35): The relative volumes of iron and its reaction product $(A C l 222 R-01)$.

\subsubsection{Hypothetical calculation of corrosion mass loss}

Michael Faraday (1791-1867) was an English chemist and physicist who contributed to the fields of electromagnetism and electrochemistry. Faraday performed an impressive number of experimental studies on electrochemical reactions in the early 1800s. He described the correlation between the results of the electrochemical reactions and the normal chemical stoichiometric relations (calculation of the quantities of 
elements in Chemistry), and certain stoichiometric rules related to charge. These additional rules are now known as Faraday's laws. They can be expressed as follows:

Faraday's First Law: The discharged mass, $m$, at an electrode is proportional to the electrical charge, $Q$, passed through the electrode (Kelly et al. 2003).

Faraday's Second Law: If the same electrical charge, $Q$,passed through several electrodes, the mass, $m$, of an element discharged at each will be directly proportional to both the atomic mass of the element and the number of moles of electrons, $z$, required to discharge one mole of the element from whatever material is being discharged at the electrode (Kelly et al. 2003).

The second law means that the masses of the substances reacting at the electrodes are in direct ratio to their equivalent masses.

One Faraday $(F)=$ the charge carried by one mole of electrons. The Faraday is related to other electrical units because the charge on a single electron $\mathrm{S} 1.6 \times 10^{-19} \mathrm{C} /$ electron. Multiplying the electronic charge by the Avogadro number $6.02 \times 10^{23}$ electrons/mole electrons tells us that $1 \mathrm{~F}=96,485 \mathrm{C}$ (Kelly et al. 2003). Some other references refer to the same value as $1 \mathrm{~F}=96,487 \mathrm{C}$ for greater calculation accuracy.

The preceding empirical laws are very important to corrosion for their link between electrical quantities (charge and current, its time derivative) and mass loss rate. These laws come with basics that may help predict and calculate mass loss. Faraday's laws of electrolysis can be written in one equation by joining the principles of Faraday with an electrochemical reaction of known stoichiometry as follows:

$$
\text { Mass } \text { Loss }_{\text {Hypothetical }}=\frac{t \cdot i \cdot M}{z \cdot F}
$$

Where: Mass Loss Hypothetical $=$ the difference between the original mass of the metal at the anode and the mass after corrosion, measured in grams.

$$
\begin{aligned}
& t=\text { time }(\mathrm{sec}) \\
& i=\text { current }(\text { Amperes }) \\
& M=\text { atomic weight of iron }(55.847 \mathrm{~g} / \mathrm{mol}) \\
& \left.\mathrm{z}=\text { ion charge (Meta valency assumed } 2 \text { for } \mathrm{Fe} \rightarrow \mathrm{Fe}^{2+}+2 e^{-}\right) \\
& F=\text { Faraday's constant }(96,487 \mathrm{Amp} . \mathrm{sec}) .
\end{aligned}
$$




\subsubsection{Stages of corrosion deterioration}

There are four main stages of corrosion deterioration of reinforcing concrete structures in general and reinforcing concrete beams in particular. The stages are:

Stage I

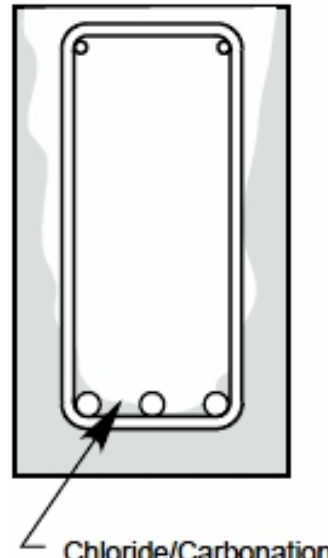

Penetration at Threshold Level
Stage II

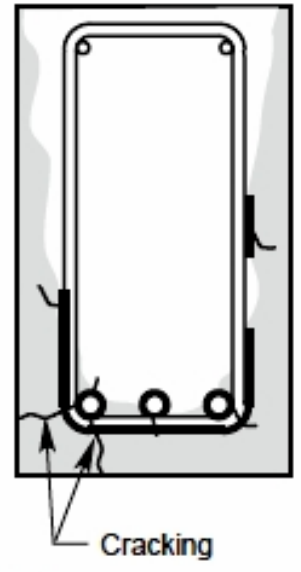

Stage III

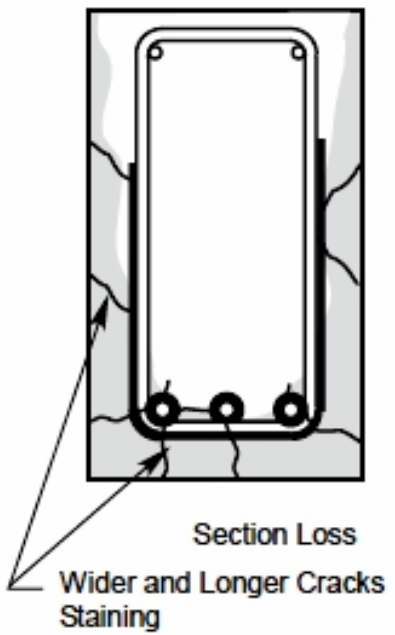

Stage IV

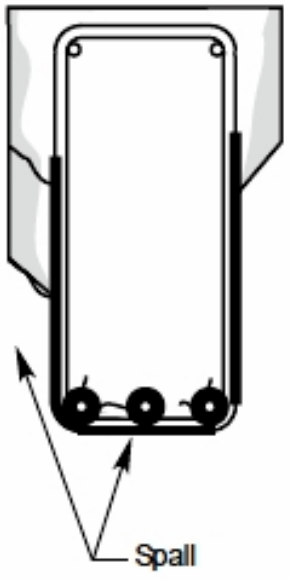

Figure (2-36): Four stages in the corrosion deterioration of CRC bridge elements. Additional stages are possible when the deterioration is more severe (Higgins et al. 2003).

Stage I: Starting the penetration of the chloride through the concrete cover, and the beginning of the electrochemical process of corrosion (Higgins et al. 2003).

Stage II: Extending the corrosion that results in the beginning of corrosion features like initial cracks and rust spots on the concrete surface (Higgins et al. 2003).

Stage III: Developing the influence of corrosion in its advanced stages into structural deterioration such as wider cracks, more concrete delamination, less bond, fewer cross-sectional areas and lower structural characteristics of the rebars, all resulting from increasing exposure to moisture and chloride ions (Higgins et al. 2003).

Stage IV: Spalling of the concrete is the main phenomenon that leaves reinforcing steel without any protection from the direct attack of corrosive agents; Figure (2-36) illustrates the four stages of corrosion deterioration (Higgins et al. 2003).

The advanced sequences of corrosion end in a weaker bond between the reinforcing steel and the core concrete, with cracks in the concrete core and failure of the reinforcing steel bars. 


\subsubsection{Related tests}

\subsubsection{Half-cell Potential Test}

According to ASTM C 876 - 91 (Reapproved 1999) the half-cell potential test is designed to estimate the expected level of the corrosion activity of the uncoated reinforcing steel bars embedded in the concrete. In many cases, additional data such as chloride contents, depth of carbonation, delamination survey findings, rate of corrosion results, environmental exposure conditions, and half-cell potential measurements is required to formulate conclusions concerning corrosion activity of embedded steel and its probable effect on the service life of a structure (ASTM C $876-91$ ).

The half-cell potential test is a non-destructive-test to predict future corrosion through detecting the areas of the most probable corrosion activity in reinforced concrete structures.

For a better understanding of the principle of the half-cell potential test, a brief review of the basic information about half-cell reaction in reinforcing steel bars is useful. The electrochemical process of corrosion with the flow of electrons and ions shown in Figure (2-34) is an example of a corroding rebar embedded in concrete. The anode is the active part of the rebars where the iron atoms lose electrons and move into the surrounding concrete as ferrous ions; this chemical activity is called half-cell oxidizing reaction, or the anodic reaction, and Equation (2-41) is symbolizes it ( $\mathrm{ACl} 228.2 \mathrm{R}-98)$

$$
\mathrm{Fe} \rightarrow \mathrm{Fe}^{2+}+2 \mathrm{e}^{-}
$$

The electrons in the rebar move to the cathode, where they combine with water and oxygen in the concrete. The reaction at the cathode is called a reduction reaction and is represented as in Equation (2-42) (ACl 228.2R-98)

$$
2 \mathrm{H}_{2} \mathrm{O}+\mathrm{O}_{2}+4 \mathrm{e}^{-} \rightarrow 4 \mathrm{OH}^{-}
$$

For sustainable electrical neutrality, the ferrous ions (with their positive charge) move through the concrete to the cathodic places on the rebars, where they combine to form hydrated iron oxide, or rust. 


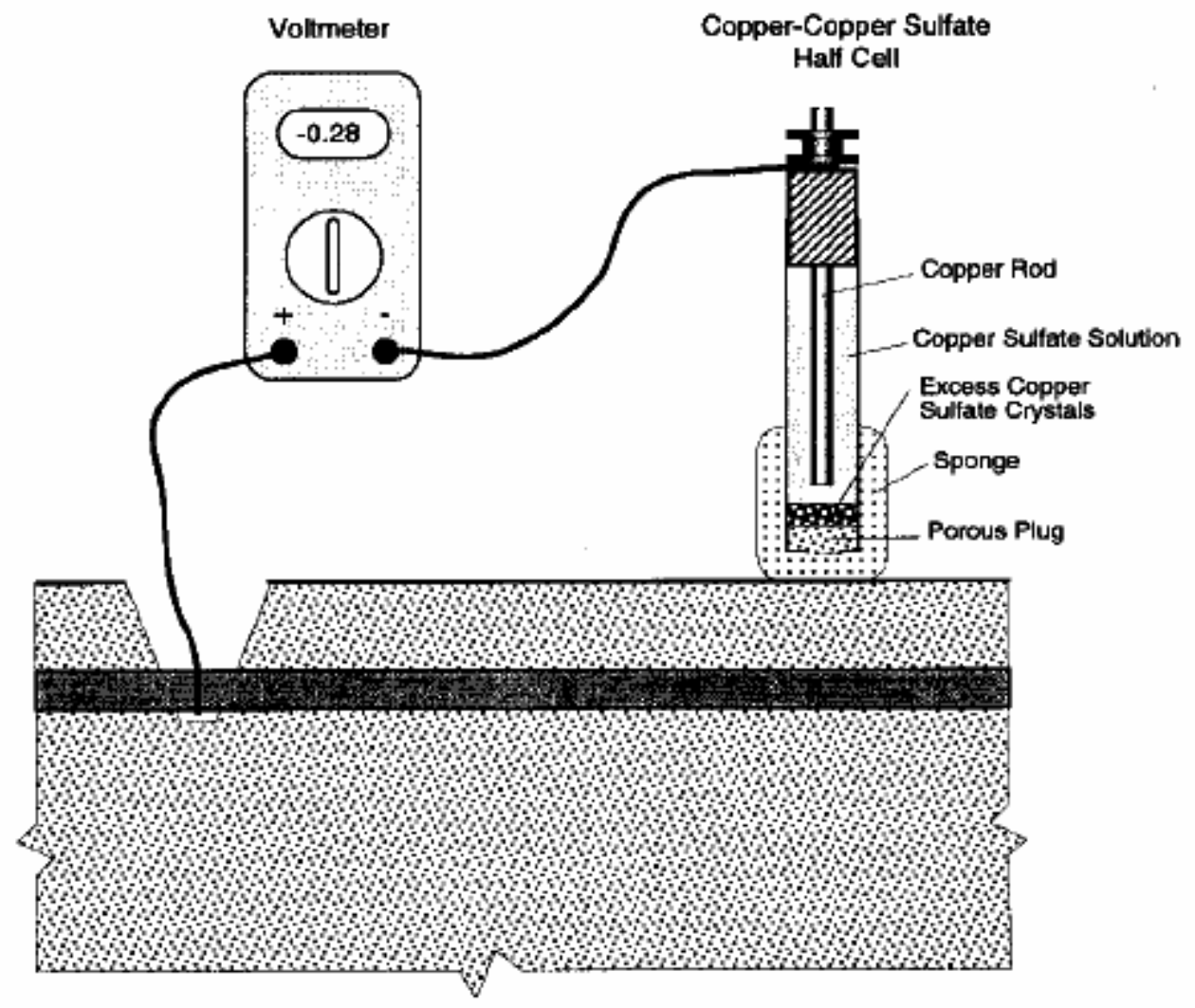

Figure (2-37): Apparatus for half-cell potential method described in ASTM C 876 (ACI 228.2R-98).

According to this understanding, in the corroding rebars, electrons move through the rebars and ions move through the concrete, but when rebars are not in the corroding phase the electrons and ions do not move. The half-cell potential method is designed to discover the remaining negative charge in the corroding rebars.

The device used in the half-cell potential test is composed of two main parts; a probe and a high-impedance voltmeter with connecting wires. The probe includes a copper-copper sulphate (or electrically similar) half-cell, as illustrated in Figures (2-37) and (2-38) from ASTM-C876. This half-cell is made of a copper bar submerged in a saturated copper sulphate solution. Many other half-cells can be used as a reference to measure the degree of possibility of future corrosive activity in embedded rebars (ASTM C $876-91)$. 


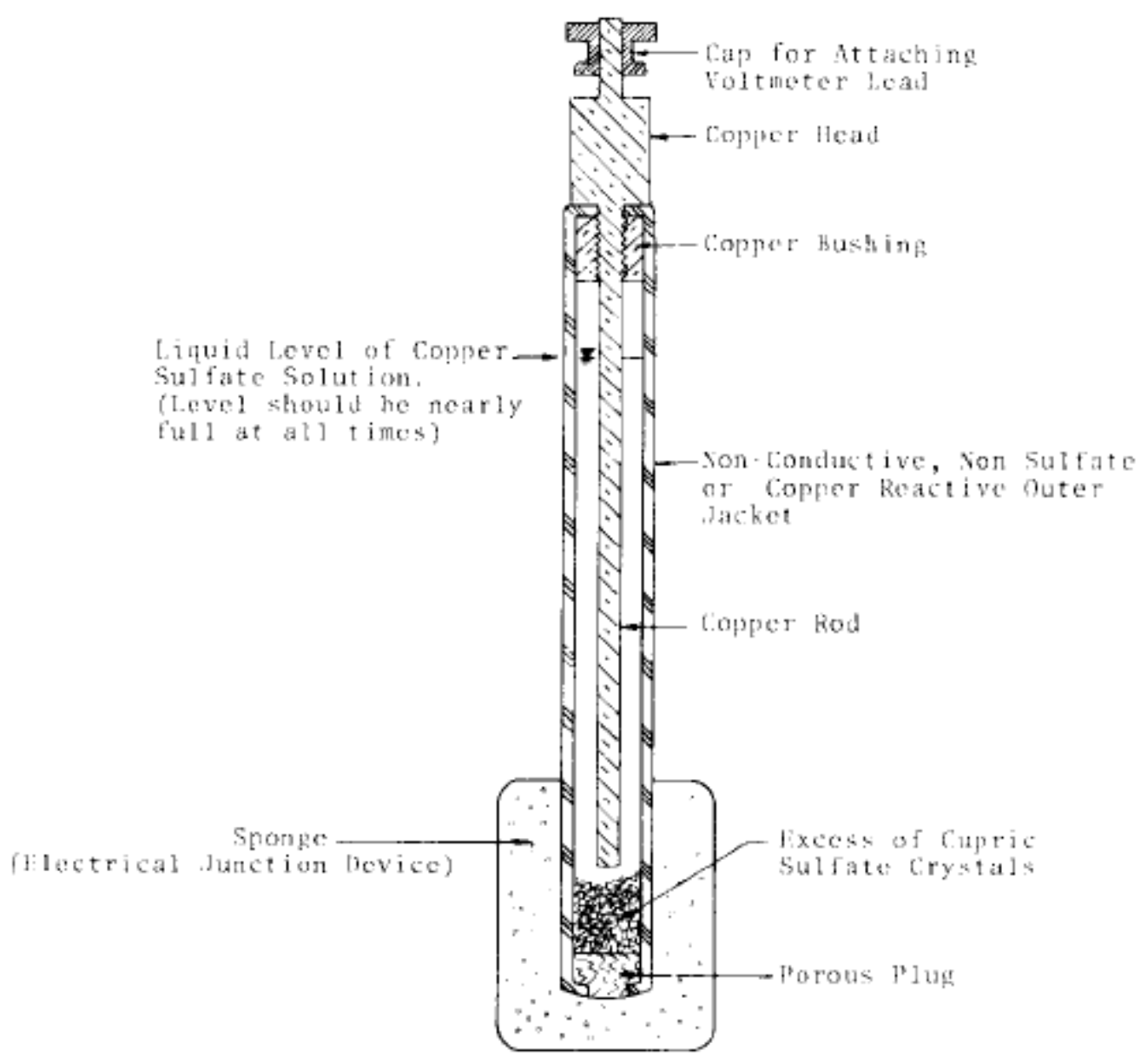

Figure (2-38): Sectional view of a copper-copper sulphate half-cell (ASTM C $876-91$ ).

With the use of high-impedance voltmeter, a very small amount of current flows through the circuit. After installing and connecting the probe to the voltmeter, the positive terminal of the voltmeter is attached to the reinforcement and the negative terminal is attached to the copper-copper sulfate half-cell, as shown in Figure (2-37). According to this connection, the half-cell makes electrical contact with the concrete through the porous plug and moistened sponge.

The half-cell potential readings will be more accurate only when the reinforcing steel bars are electrically connected. For this reason, readings should be taken between the widely separated rebars (ASTM C876). The half-cell potential method will not work with structures that have no access to its rebars, nor will it work when the rebars are epoxy-coated. The readings are normally taken from points on the structure organized in a grid. The spacing between the network of reading points depends on the shape of the 
structure, reinforcing steel bar details, and the budget assigned for the test in a specific project. The accuracy of the results affected by the distance between the reading points and the potential targeted rebars, however, and ASTM C 876 recommends a spacing of $1.2 \mathrm{~m}$. If the differences in voltage between adjacent points exceed $150 \mathrm{mV}$, a closer spacing between the reading points is preferred. According to ASTM C 876, the concrete must be sufficiently moist but there should be no free surface water between test points. If the condition of steady moisture is not present, then the electrical current will be scattered and the half-cell potential method should not be used. To ensure sensitivity of the test to moisture, a correction factor must be applied to the voltage measurement when the test is done out of a temperature range of 17 to $28 \mathrm{C}^{\circ}$ ( $\left.\mathrm{ACl} 228.2 \mathrm{R}-98\right)$.

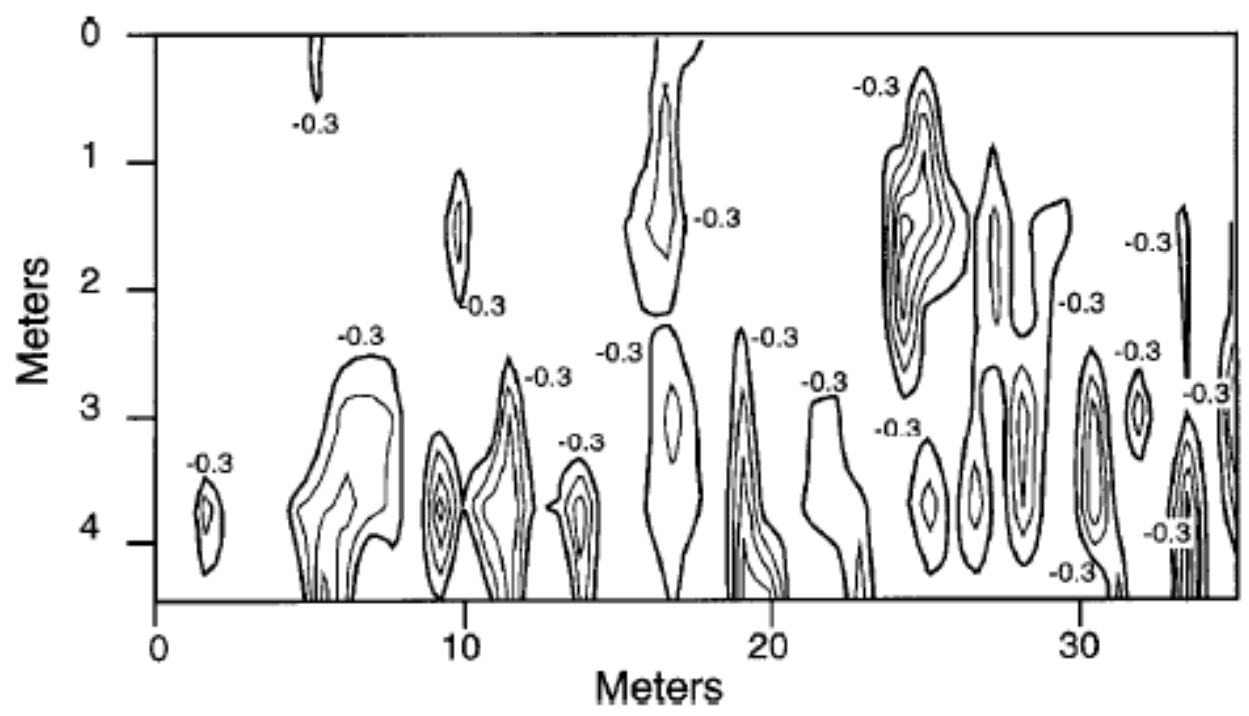

Figure (2-39): Equipotential contours from survey data of bridge deck at grid spacing of $0.76 \mathrm{~m}$ (only contours less than $-0.30 \mathrm{~V}$ are shown and contour interval is $-0.05 \mathrm{~V}(\mathrm{ACl} 228.2 \mathrm{R}-98)$. 


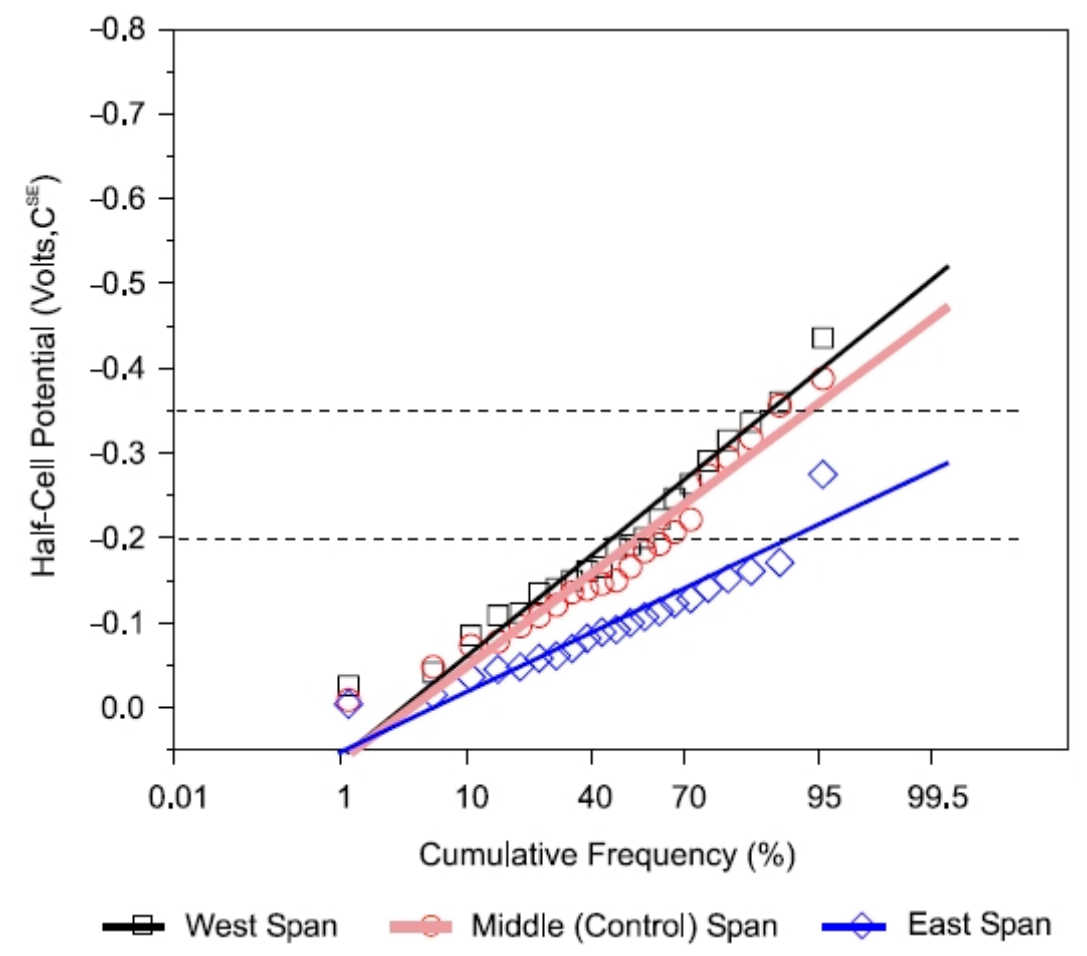

Figure (2-40): Cumulative frequency diagram (Sharp, 2004).

There are two ways to display the results from the half-cell potential test; using the equipotential contour map in Figure (2-39), or using a cumulative frequency diagram, as shown in Figure (2-40). The equipotential contour line is the line that connects the equal voltage reading points. The displayed result must be drawn on a scaled area that is similar to the original testing area. Thn the half-cell potential voltage readings are then marked on its location, and the final step is connect the equal voltage points in one line or contour, as illustrated in Figure (2-39). In this case, the equipotential contour lines are drawn from test points on a 0.76-m spacing (Clemena et al. 1992). The cumulative frequency diagram shows the size of the affected area of the concrete member. ASTM C 876 explains the technique of making a plot of the data on normal probability paper, as shown in Figure (2-40). The two techniques have different ways of expressing the degree of corrosion activity. In the potential difference method, the spots with corrosion activity are shown based on the corrosion gradients. However, with the method of equipotential contour plot, the areas with high probability of corrosion are represented by areas with close spacing of the voltage contours. Figure (2-39) illustrates the difference between the areas with highl corrosion activity and those with less corrosion activity by 
monitoring the spacing between contour lines. The closer contours refer to a higher probability of corrosion (ASTM C 876).

\subsubsection{Rapid Chloride Permeability Test}

The Rapid Chloride Permeability Test (RCPT), according to ASTM C 1202-97, provides rapid signals of concrete resistance to the penetration of chloride ions. Concrete without protection from salt contact is under the influence of chloride penetration, causing comprehensive deterioration, early signs of weakness, and in some severe cases may cause early failure of the affected concrete structures. Working to lower concrete permeability is one of the preventative measures that can minimize the intrusion of the deteriorating solutions.

The RCPT was first created for the Federal Highway Administration (FHWA) by the Portland Cement Association (Whiting, 1981). Previous studies using the RCPT showed results with a high degree of correlation with those obtained from commonly accepted ponding test AASHTO-T 259. The operating time of ponding test AASHTO-T 259 is about 90 days while RCPT integrates current passing through a disk of saturated concrete over a 6-hour period, resulting in an electrical charge measurement expressed numerically in coulombs' (Hoppe et al. 2007).

This test monitors the amount of electrical current moved through 51-mm thick slices of 102-mm diameter cylinders within a 6-hour period. A potential difference of $60 \mathrm{~V}$ dc is provided across the ends of the specimen; one of the ends is immersed in a sodium chloride solution, the other in a sodium hydroxide solution. The resistance of the specimen to chloride ion penetration was found to be related to the passing total charge (ASTM C1202-97) .

ASTM C 1202-97 refers to the significance and use of the test as follows:

- It provides a fast evaluation of the concrete specimen's resistance to chloride ion penetration. Therefore, it is useful for estimating material proportions for design purposes, research and development. Table (2-3) shows the classification of charge passed in coulombs vs. the chloride ion penetrability. 
Table (2-3): Chloride ion penetrability based on charge passed (ASTM C1202-97).

\begin{tabular}{|l|l|}
\hline Charge Passed (coulombs) & Chloride Ion Penetrability \\
\hline$>4000$ & High \\
$2000-4000$ & Moderate \\
$1000-2000$ & Low \\
$100-1000$ & Very Low \\
$<100$ & Negligible \\
\hline
\end{tabular}

- The description of the test method dealt with $102 \mathrm{~mm}$ diameter specimens. The same tools can be used for specimen sizes from 95-102 mm. Other smaller or bigger diameter specimens may be tested with proper adjustments in the applied voltage cell design.

- The age of the specimens, type of concrete, and the curing procedure affect the test results. Older age and proper curing result in less permeability (ASTM C1202-97).

The apparatus used to achieve the RCPT is composed of many parts. The main parts are vacuum saturation apparatus including separator funnel, beaker of $1000 \mathrm{~mL}$ or larger, $250 \mathrm{~mm}$ vacuum desiccator, vacuum pump, and vacuum gauge or manometer, as shown in Figure (2-41). The reagents, materials and test cell are composed of specimen-cell sealant, as show in in Figure (2-42), sodium chloride solution-3.0 \% by mass (reagent grade) in distilled water, sodium hydroxide solution- $0.3 \mathrm{~N}$ (reagent grade) in distilled water, applied voltage cell, and voltage application and data readout apparatus (ASTM C1202-97).

The RCPT was used to examine the effect of mineral admixtures on the resistance of chloride ion penetration (Li and Roy, 1986), (Wee et al. 2000). Yang and Su (2002) and Wee et al. (1999) used the RCPT to examine the effect of aggregate fraction, while Aldea et al. (2000) used it to investigate curing condition. Aldea et al. (2000) and Yang, (2004) also used the RCPT to examine the effect of pore size on the penetration of chloride ions.

The importance of the RCPT in this research came from the strong correlation between corrosion resistance of the concrete and the amount of electrical resistivity of the same concrete. 


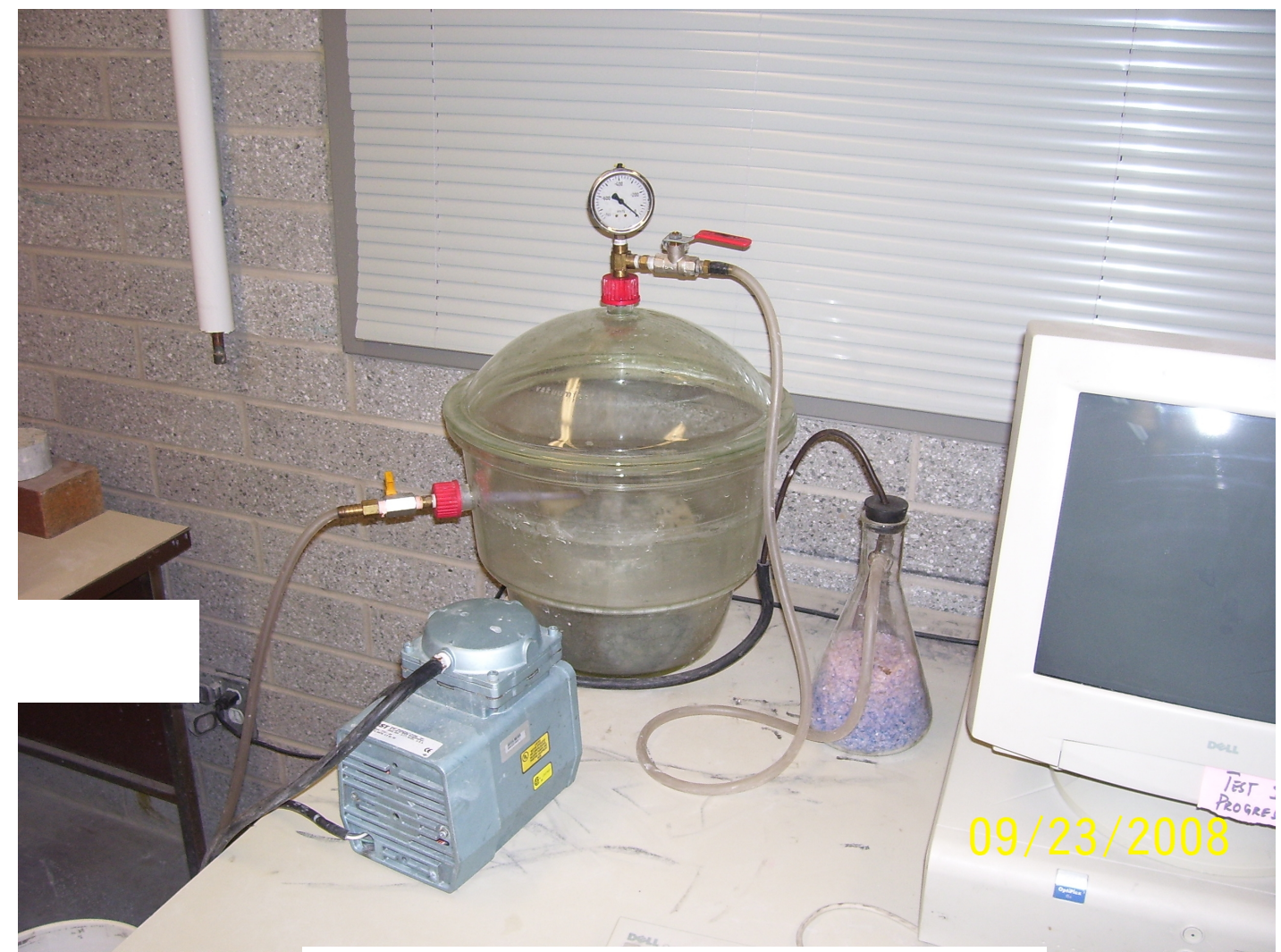

Figure (2-41): Vacuum saturation apparatus.

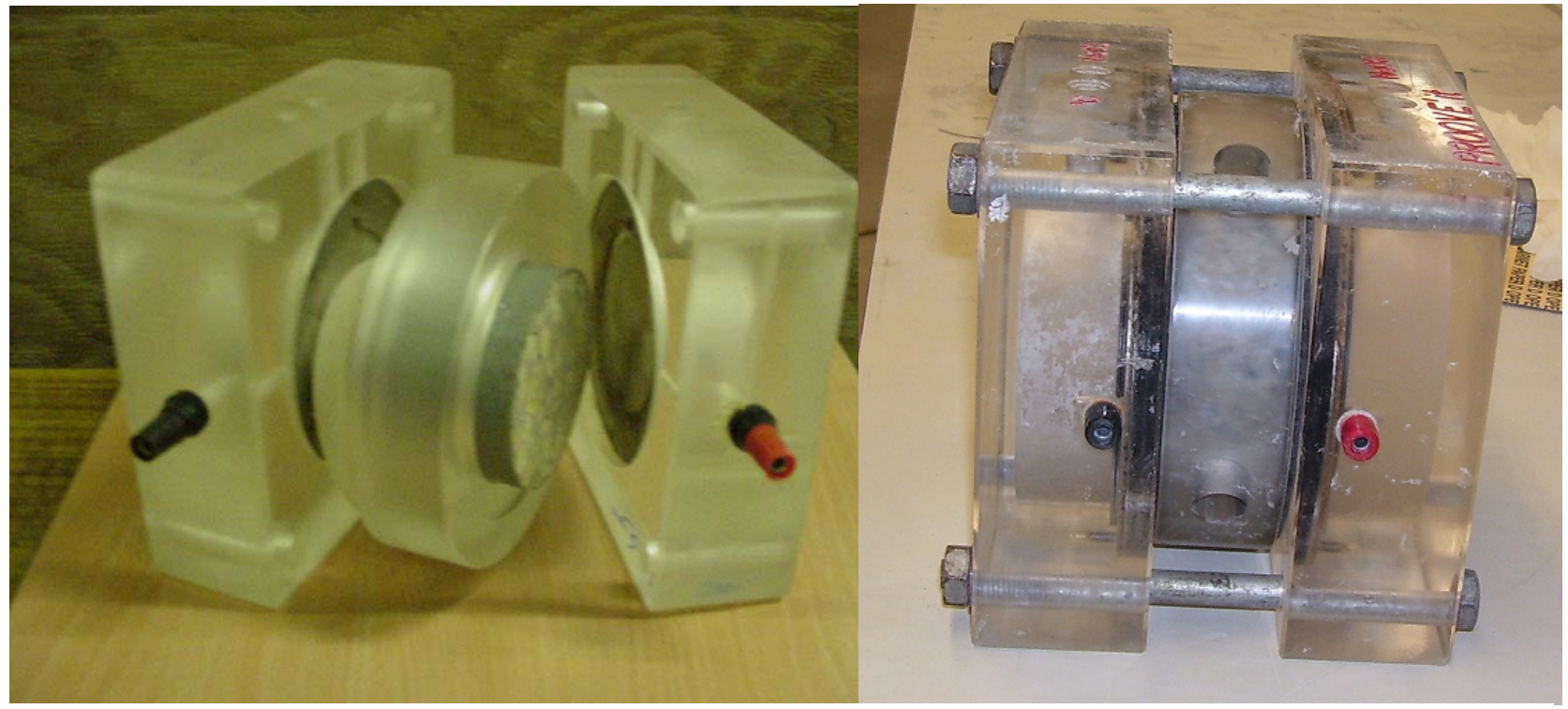

Figure (2-42): Rapid Chloride Permeability Test cell. Left side image adopted from Abdul Hameed (2005). Right side shows cell ready for test. 


\subsection{Related Research}

\subsubsection{Hassan, A., 2008}

Hassan's research investigated the shear resistance, bond characteristics, and corrosion performance of SCC compared to those of NC. Full-scale beams were used to test the shear strength, cracking behavior, and deflection characteristics. Twenty RC beams without stirrups were tested under concentrated load in the mid-span until shear failure occurred. The four variables of concrete type, coarse aggregate content, beam depth and longitudinal reinforcing steel ratio were subjected to testing. Beam depth varied from 150 to $750 \mathrm{~mm}$, while the shear span-to-depth ratio (a/d) was kept constant in all beams, and the two steel ratios of $1 \%$ and $2 \%$ were used for longitudinal reinforcement.

The comparison between the SCC and NC beams was through the results of crack pattern, crack width, load at the first flexure/diagonal cracking, ultimate shear resistance, post-cracking shear resistance/ductility, load-deflection response, failure mode, first flexural cracking moment/load, and ultimate shear resistance, as well as simulated loaddeflection response. The bond stress was tested for bars located at three different heights from the bottom of the beam at different concrete ages. The corrosion of rebars used in SCC beams was investigated and compared to that used in NC beams. The corrosion performance of $400 \mathrm{~mm}$ width $\times 363 \mathrm{~mm}$ depth $\times 2340 \mathrm{~mm}$ length beams containing epoxy and non-epoxy coated stirrups was monitored by partial immersion in a sodium chloride solution and an impressed current. Half-cell potential tests were implemented to evaluate the probability of steel corrosion along the beam length/perimeter, and the chloride ion content near the bar surface was measured to study the variation of the chloride-ion penetrability along the beam length/perimeter. The mass loss and bar diameter degradation of each bar were investigated at the end of the test. Small-scale cylinder specimens made of NC and SCC with centrally located embedded reinforcing bars were also tested to investigate the effect of segregation and bleeding on corrosion performance. The corrosion influence on different types of SCC according to the amount of high range water reducers (HRWRs) were also investigated with small-scale cylinder specimens. The variation of the structural capacity and cracking behavior after the effects of corrosion was studies on both SCC and NC beams.

The ultimate shear strength of SCC beams was found to be slightly lower than that of NC beams; the difference was more considerable with the reduction of longitudinal steel reinforcement and an increase in beam depth. As expected, the SCC showed high 
performance during casting time. Bond stress was somewhat higher in SCC beams than in NC beams and the difference was more significant in the top bars and at 28 days of testing. The corrosion investigation in this research showed that SCC beams had better performance compared to their NC equivalents in terms of corrosion cracking, corrosion rate, half-cell potential, time of corrosion initiation, rebar mass loss and rebar diameter reduction. The SCC beams showed severe localized corrosion of stirrups and longitudinal rebars at the far end of the beam (away from the casting point), and that may have caused, in some cases, spalling of the concrete cover at the corners due to inadequate local compaction and distribution of concrete. The results of calculated mass loss and real mass loss were very close on the corroded rebars using Faraday's law. In terms of corrosion resistance, SCC mixtures showed higher performance in comparison to NC mixtures, only in large-scale beams. The types of HRWR used have no influence on corrosion performance.

\subsubsection{Lachemi et al., 2005}

Lack of information regarding fresh properties of SCC and structural performance at the hardening phase is one of the main obstacles to its growth in the construction industry. The issue that most influenced the undertaking of this research is the concern of researchers and designers about the shear strength of SCC due to the ambiguity in mechanisms resisting shear, especially the aggregate interlock mechanism.
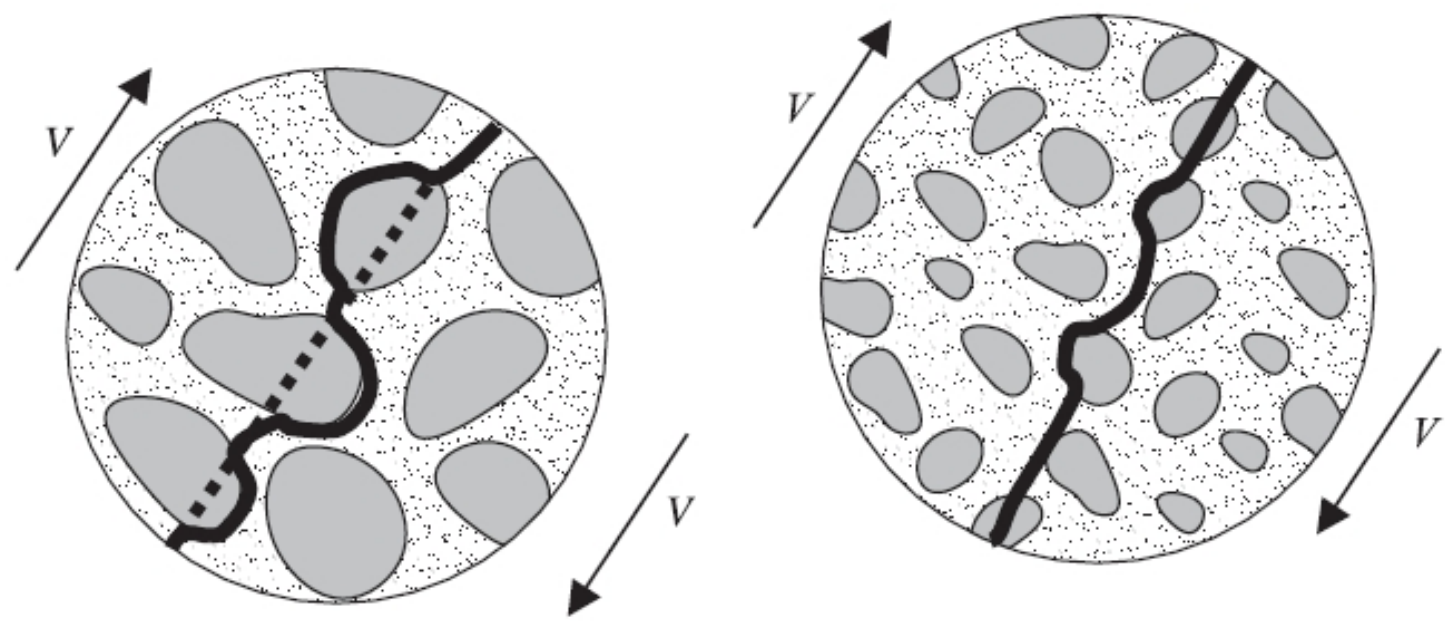

Figure (2-43): Influence of coarse aggregate on crack shear plane (a) $19 \mathrm{~mm}$ aggregate (S19), and (b) $12 \mathrm{~mm}$ aggregate (N12) (Lachemi et al, 2005). 
SCC has a noticeably smaller amount of coarse aggregates, as shown in Figure (243), and therefore the friction resulting from the aggregate interlock between fracture surfaces is less in SCC than in NC. The research compared the shear resistance of SCC and NC beams by using the results of 18 beams with flexural reinforcement and without shear reinforcement. The variables examined were concrete type, maximum size of coarse aggregate, coarse aggregate content, and beam shear span-to-depth ratio. All the experimental beams were compared to analyze the shear resistance mechanisms of SCC and NC beams in both pre- and post-cracking stages with relation to shear strength, shear ductility, crack patterns, and failure modes.

Based on the results of this research, it is useful to take advantage of the following conclusions:

- An increase in the size of coarse aggregate from 12 to $19 \mathrm{~mm}$ in SCC decreases the shear capacity of concrete (shear load at first shear crack) and increases the ultimate shear resistance (at failure). The large size and large amount of coarse aggregate improves the post-cracking shear transfer mechanisms and enhances the ultimate shear resistance of SCC beams. Therefore, the existence of large coarse aggregate is useful for relatively deeper SCC beams with low shear span-to-depth ratio.

- Generally, SCC beams with the same maximum size of coarse aggregate, but with a lower coarse aggregate content, showed similar concrete shear resistance characteristics in pre-cracking stage to NC. The research also showed the development of lower post-cracking shear resistance in SCC because of lesser aggregate interlock and dowel action due to the presence of lower quantity of coarse aggregate compared with NC. Designers of SCC beams should take the effects of post-cracking shear transfer mechanisms into consideration.

\subsubsection{Amleh and Mirza, 1999}

This study investigated the effect of corrosion on bond between reinforcing steel and concrete by using an introductory series of tests on 14 tension specimens. Each specimen was $100 \mathrm{~mm}$ in diameter and $1 \mathrm{~m}$ long, and was reinforced with one No. 20 bar. Accelerated corrosion was applied to the specimens by submerging them in a $5 \%$ $\mathrm{NaCl}$ solution under the influence of a voltage of 5 volts. Depending on the information of the transverse and longitudinal splitting cracks, the relative bond influence was decided. 
Bond strength decreased rapidly with an increase in corrosion level (measured by loss of weight), as shown in Figure (2-44) (Amleh and Mirza, 1999) .

The bond decreases under corrosion due to a decline of the ribs in the deformed rebars, and reduced adhesion and cohesion at the reinforcing bar surface. For advanced levels of corrosion, cracks may appear as a result of the pressure of corrosion production. The results of the study found two conclusions:

1. As it appears in Figure (2-44), 4\% of corrosion weight loss (accompanied by transverse cracks) pointed to a $9 \%$ loss of bond strength, but a $17.5 \%$ weight loss (with no transverse cracks before yielding of the bar), resulting in $92 \%$ loss of bond between the steel and the surrounding concrete.
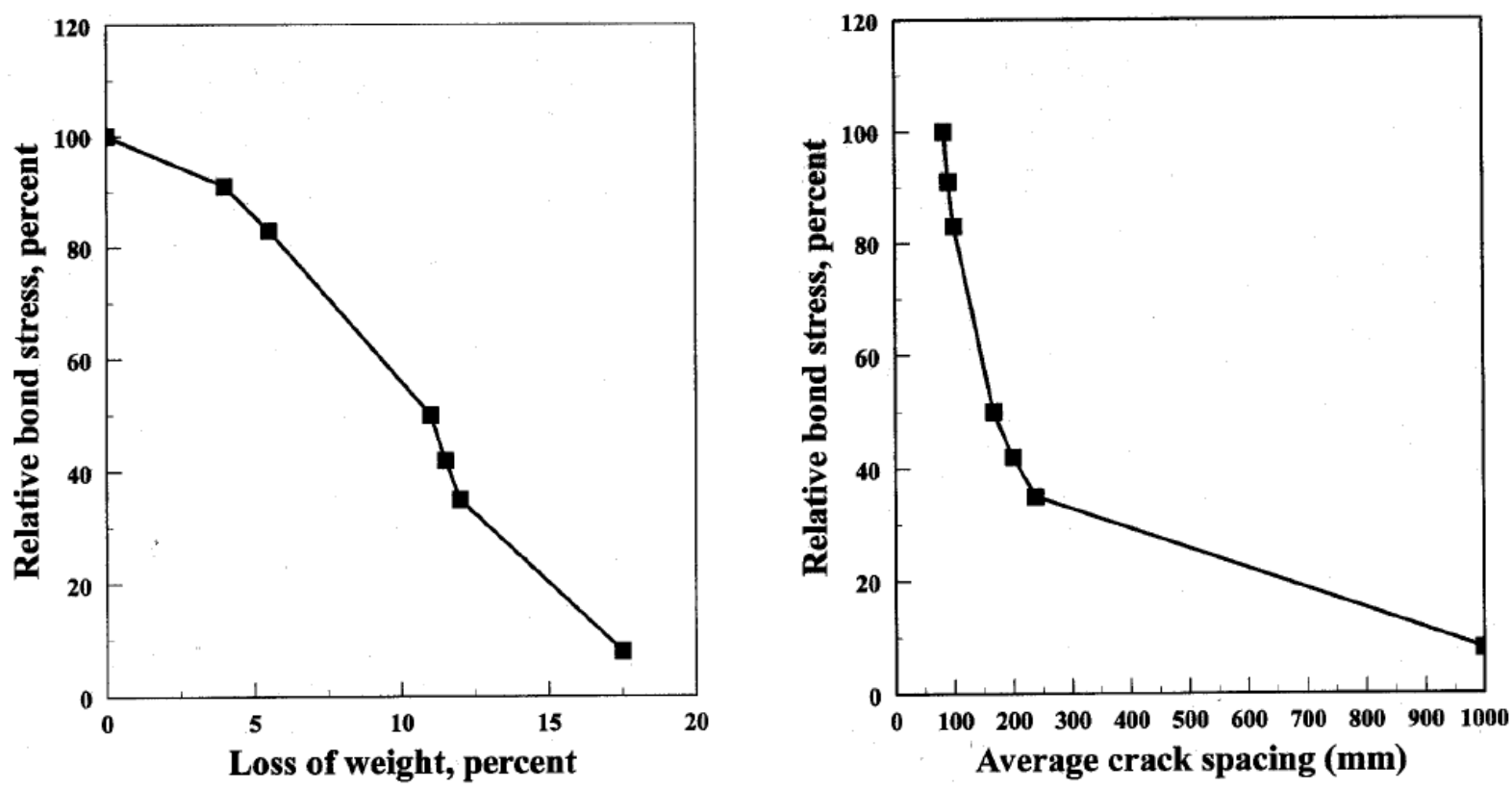

Figure (2-44): The graph on the left side refers to relative bond stress vs. level of corrosion. The graph on the right refers to relative bond stress vs. average crack spacing (Amleh and Mirza, 1999).

2. Severely localized corrosion will deteriorate the essential mechanism of the bond in deformed bars, which is the transfer of forces by mechanical interlocking of the ribs, so that it results in a meaningful decline in bond strength. With increasing corrosion level, tension stiffening decreases, which indicates the beginning of bond breakdown. The bond collapses due to surface conditions of the level of its adhesion and cohesion to the surrounding concrete. 


\subsubsection{Rodriguez et al., 1997}

Using 31 beams with dimensions of $200 \times 150 \times 2300 \mathrm{~mm}$ and $200 \times 150 \times 2050 \mathrm{~mm}$, this study targeted the effects of corrosion on loading. The beam reinforcement was variable, as shown in Figure (2-45), as was the rate of corrosion. The addition of $3 \%$ calcium chloride to the mixing water and application of a current density of $100 \mu \mathrm{A} / \mathrm{cm}^{2}$ for 100 - 200 days resulted in increased corrosion intensity $I_{\text {corr }}$ to ten times. The results showed that the effects of corrosion were increased deflection, increased crack width at the service load. In addition, the strength at the ultimate load decreases, and for bond deterioration, the transverse cracking width and spacing increases. In addition corrosion changes the failure behaviour in concrete beams with regular ratios of reinforcement.
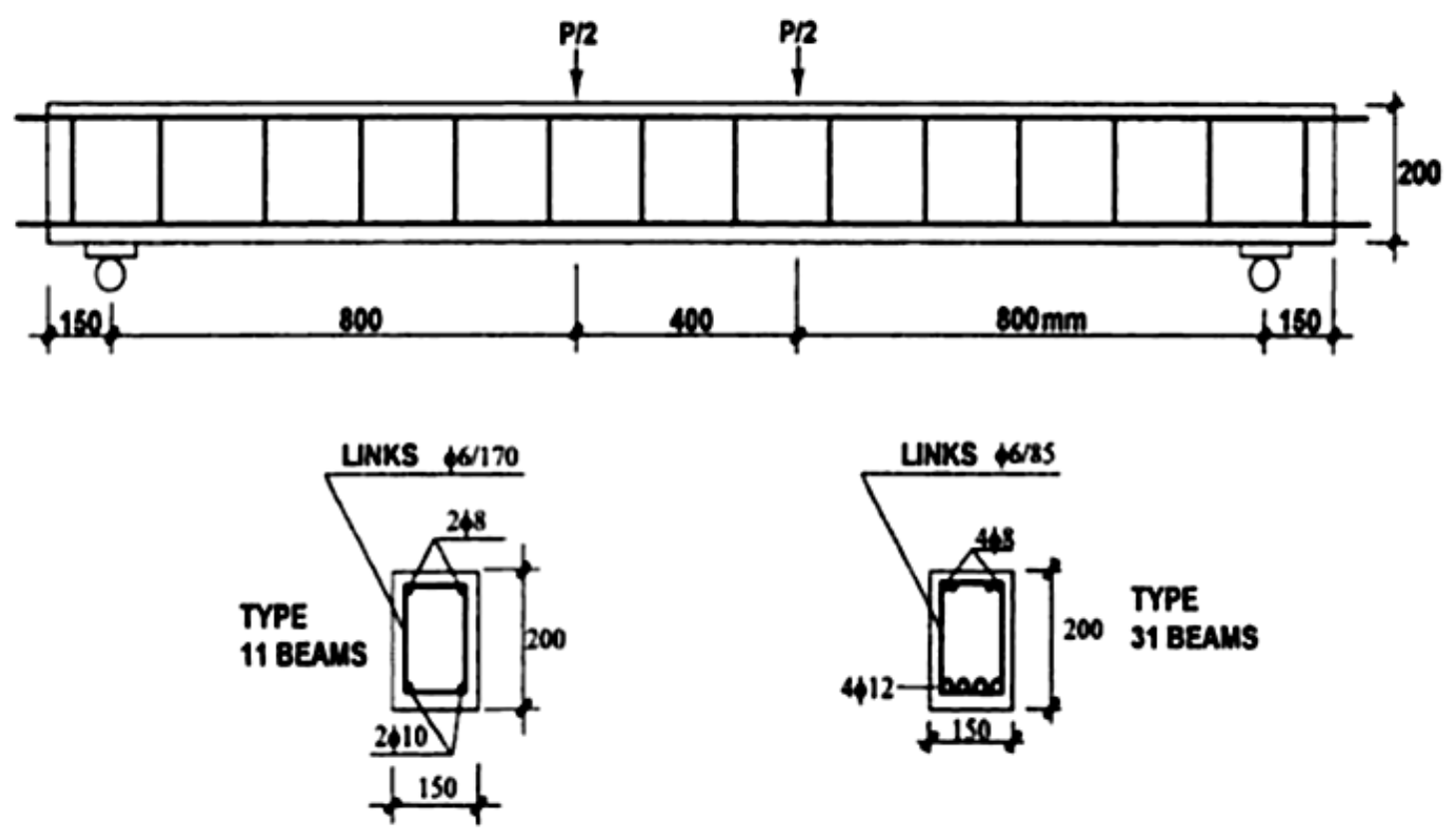

Figure (2-45): Scheme of beams types 11 and 31 (Rodriguez et al. 1997).

The uncorroded beams showed bending failure but the corroded beams failed in shear. Rodriguez et al. (1997) noticed that the failure response of the corroded beams is premature brittle concrete cracking. They referred to the concrete deterioration produced by corrosion, but did not mention the decrease in the bond between the corroded rebars and concrete. Furthermore, they inferred that the reduced sections of steel and concrete of conventional RC models, in keeping with the values of bending moment and shearing force, can be designed for corroded beams (Rodriguez et al. 1997). 


\section{Chapter 3}

\section{Experimental Program}

\subsection{Methodology of Research}

\subsubsection{RC beams, categorizing and preparations}

To achieve the objectives of this research, 20 reinforced beams built using two types of concrete, normal Portland cement and SCC, as illustrated in Figure (3-1). Mass loss of reinforcing steel due to corrosion was used to identify the different stages of corrosion. Figure (3-1) illustrates the distribution of the specimens under different stages of corrosion.

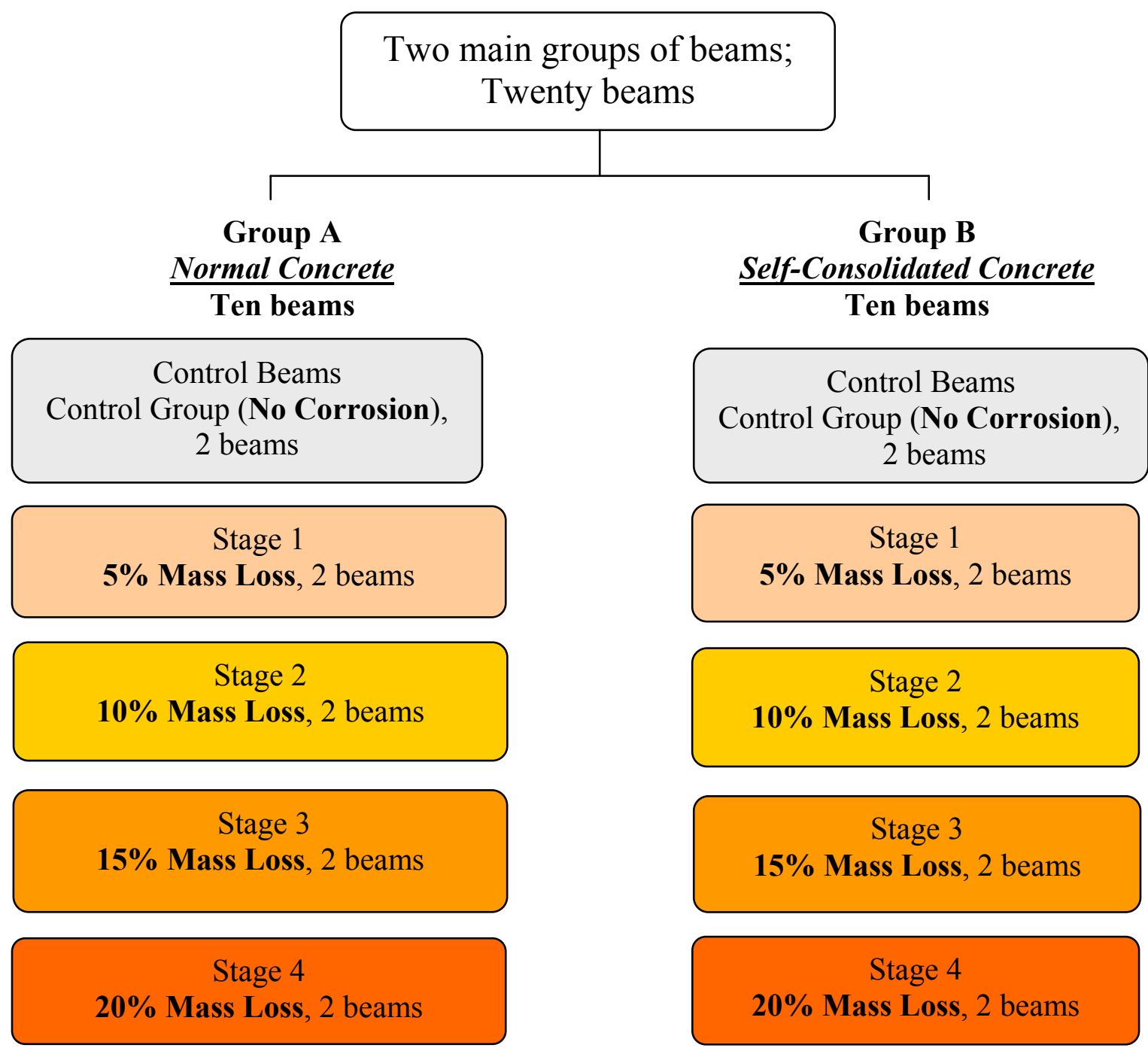

Figure (3-1): The distribution of the beams using NC and SCC with the stages of corrosion based on mass loss percentage. 
The beam design and approach to choosing a/d $=2.5$ is located in Appendix $E$.

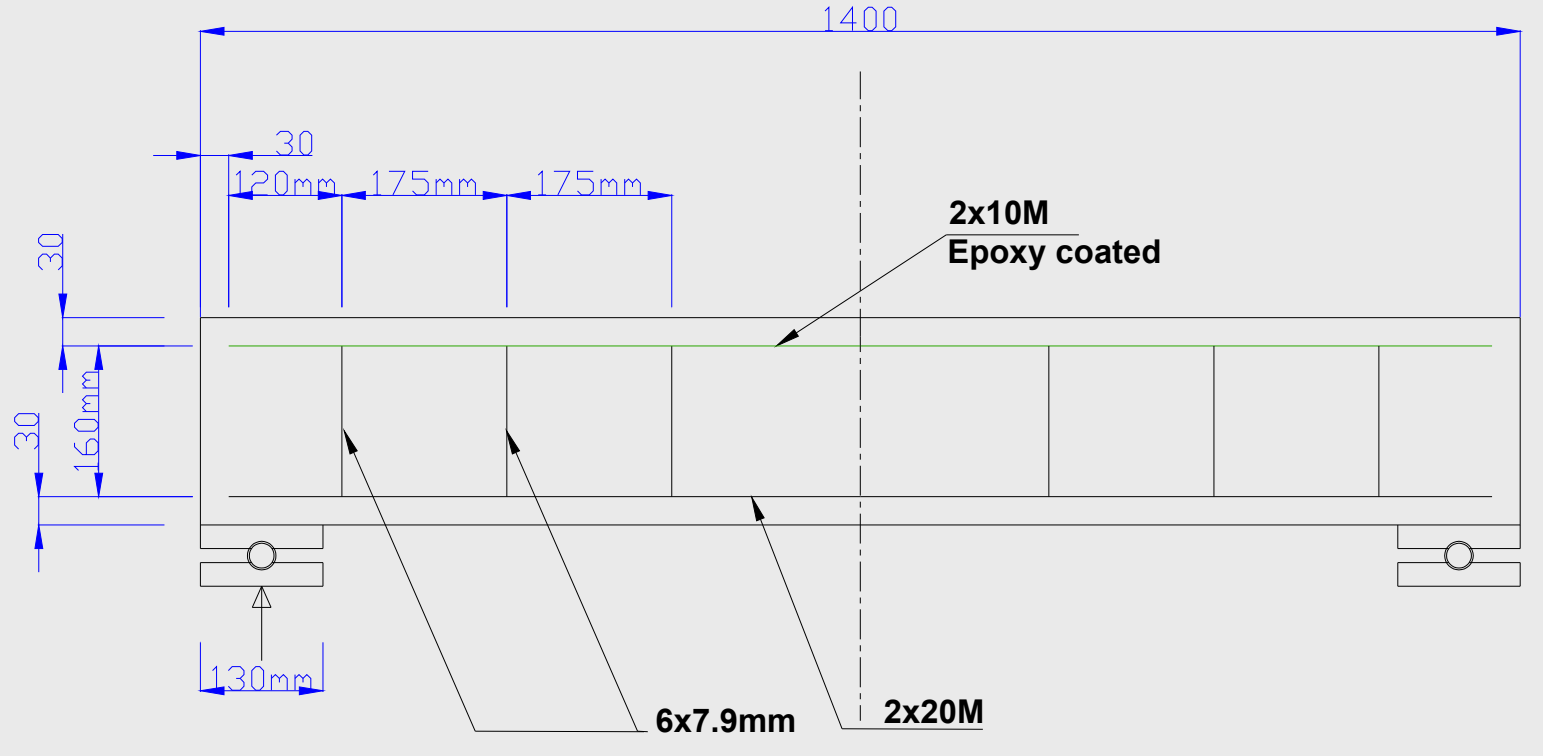

Figure (3-2): Typical beam specimen.

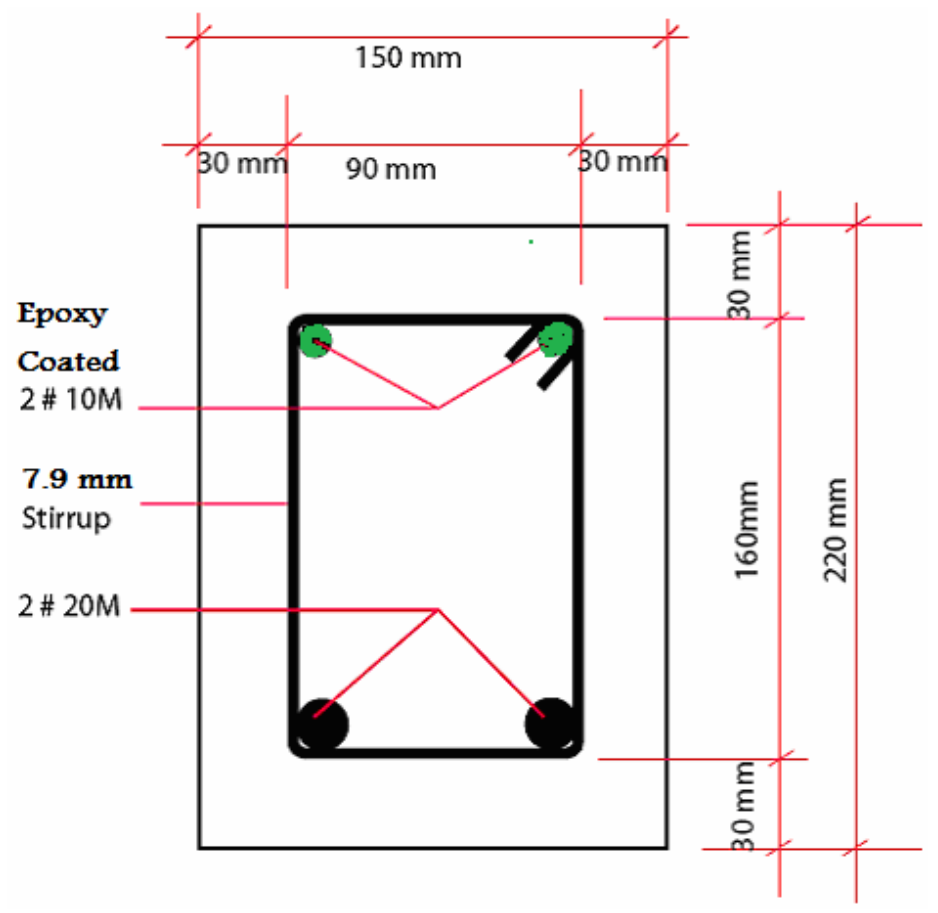

Figure (3-3): Cross-sectional details and dimensions.

Figures (3-2) and (3-3) show the longitudinal and cross-sectional details respectively, with dimensions and reinforcement details. 


\subsubsection{Mass loss calculations}

As discussed in Chapter 2, the amount of mass loss resulting from corrosion, through electrochemical process, is highly correlated to the electrical energy consumed. Electrical energy consumed is related directly to electrical current, Ampere, voltage potential difference, Volt, and the time elapsed for applying the electrical current through the anode, the reinforced concrete beam. The mass loss resulting from corrosion can be predicted by using the equation below, which is reproduced from Faraday's law.

Mass Loss $_{\text {Hypothetical }}=\frac{t \cdot i \cdot M}{z \cdot F}$

To use Equation (2-40) in this research, the hypothetical mass loss targeting 5\%, 10\%, $15 \%$, and $20 \%$ must be calculated from the reinforcing bars. A current of one ampere was used through all corrosion stages in this research. This is not a random value; it was chosen after testing both devices used in the research (power supplies and wiring connections) and the initial electrical resistivity of the concrete beams through $(\mathrm{NaCl})$ electrolyte solution of $5 \%$ by weight. To estimate the value of the electrical current used to stimulate the corrosion process in the RC beams, several trials were performed to predict the suitable electrical current (measured in amperes). The factors that influenced the estimation are the initial resistance of the RC beams, the limitations of the power output for the power supply, and the electrolyte $\mathrm{NaCl}$ concentration. By applying Faraday's equation for the known value of mass loss (measured in grams) and the electrical current (measured in amperes), the only remaining unknown in Equation (2-40) is time (measured by seconds). For this reason, it is easy to calculate the time required to target $5 \%, 10 \%, 15 \%$ and $20 \%$ of the known initial rebars mass.

Each of the reinforcing steel bars used in the beams was weighed; Table (3-1) shows the weight of every piece and its number and location on each beam. From Table (3-1), the average of the rebars subjected to corrosion in the NC beams is 7501.544 grams and in SCC beams is 7508.79 grams.

Total Average Weight of Rebars $=7505.115 \mathrm{~g}$

$5 \%$ Mass Loss $=375.26 \mathrm{~g} ; 10 \%$ Mass Loss $=750.51 \mathrm{~g}$

$15 \%$ Mass Loss $=1125.77 \mathrm{~g} ; 20 \%$ Mass Loss $=1501.02 \mathrm{~g}$ 
Table (3-1): Weights of rebars in NC beams, stirrups and flexural.

Normal Concrete Beams
weight unit is gram
\begin{tabular}{|l|l|l|l|l|l|l|l|l|l|}
\hline Beam \# & Stirrup\#1-Long & S2 & S3 & S4 & S5 & S6 & $\begin{array}{l}\text { Left-Bottom- } \\
\text { Flex }\end{array}$ & $\begin{array}{l}\text { Right-Bottom- } \\
\text { Flex }\end{array}$ & Sum \\
\hline B1 & 291.6 & 219.63 & 219.98 & 220.67 & 221.06 & 221.86 & 3091 & 3042 & 7527.8 \\
\hline B2 & 291.86 & 221.97 & 221.06 & 219.7 & 221.4 & 221.53 & 3026 & 3089 & 7512.52 \\
\hline B3 & 291.89 & 220.63 & 221.06 & 221.08 & 221.07 & 221.43 & 3081 & 3061 & 7539.16 \\
\hline B4 & 292.02 & 220.07 & 219.54 & 220.19 & 221.61 & 220.75 & 3035 & 3046 & 7475.18 \\
\hline B5 & 290.87 & 220.97 & 221.55 & 221.39 & 221.32 & 221.4 & 3093 & 3037 & 7527.5 \\
\hline B6 & 291 & 221.45 & 220.61 & 221.91 & 221.15 & 220.5 & 3014 & 3081 & 7491.62 \\
\hline B7 & 291.47 & 220.95 & 221.18 & 221.02 & 220.95 & 220.95 & 3051 & 3086 & 7533.52 \\
\hline B8 & 290.6 & 221.36 & 221.58 & 221.22 & 220.99 & 221.32 & 3036 & 3052 & 7485.07 \\
\hline B9 & 291.5 & 220.88 & 221.18 & 220.4 & 220.94 & 221.79 & 3023 & 3030 & 7449.69 \\
\hline B10 & 291.08 & 220.94 & 220.42221 .62 & 221.27 & 222.05 & 3005 & 3071 & 7473.38 \\
\hline & & 7 & & Average $=$ & 7501.544 \\
\hline
\end{tabular}

Table (3-2): Weights of self-consolidating concrete beams, stirrups and flexural.

\begin{tabular}{|c|c|c|c|c|c|c|c|c|c|}
\hline \multicolumn{10}{|c|}{ S.C.C Beams } \\
\hline Beam \# & Stirrup\#1-Long & S2 & S3 & S4 & S5 & S6 & $\begin{array}{l}\text { Left-Bottom- } \\
\text { Flex }\end{array}$ & \begin{tabular}{|l|} 
Right-Bottom- \\
Flex
\end{tabular} & Sum \\
\hline B1 & 291.31 & 220.55 & 221.61 & 221.14 & 223.32 & 221.22 & 3044 & 3042 & 7485.15 \\
\hline $\mathrm{B} 2$ & 291.38 & 221.11 & 222.47 & 221.08 & 221.38 & 221.52 & 3042 & 3084 & 7524.94 \\
\hline B3 & 291.17 & 221.34 & 220.55 & 220.92 & 221.85 & 222.06 & 3050 & 3040 & 7487.89 \\
\hline B4 & 290.47 & 220.73 & 220.66 & 221.21 & 221.12 & 220.28 & 3046 & 3094 & 7534.47 \\
\hline B5 & 291.52 & 222.05 & 221.36 & 221.3 & 221.18 & 221.13 & 3091 & 3099 & 7588.54 \\
\hline B6 & 291.22 & 221.65 & 221.3 & 220.26 & 221.76 & 221.46 & 3042 & 3030 & 7469.65 \\
\hline B7 & 291.1 & 221.06 & 221.21 & 221.43 & 220.98 & 221.05 & 3080 & 3040 & 7516.83 \\
\hline B8 & 292.04 & 221.47 & 221.26 & 220.5 & 221.26 & 221.05 & 3038 & 3042 & 7477.58 \\
\hline B9 & 290.78 & 221.51 & 220.75 & 221.22 & 220.98 & 221.08 & 3042 & 3096 & 7534.32 \\
\hline B10 & 291.44 & 220.22 & 221.34 & 220.91 & 221.31 & 221.31 & 3038 & 3034 & 7468.53 \\
\hline & & & & & & & & Average $=$ & 7508.79 \\
\hline
\end{tabular}

Using Faraday's mass loss equation for the masses targeted above, the time required to achieve the mentioned mass losses is calculated as follows: 
$375.26_{\text {gram }}=\frac{t_{\text {sec }} \times 1_{\text {Ampere }} \times 55.847}{2 \times 96487}$

Since it is a hypothetical calculation, the number of days needed to achieve $5 \%$ of mass loss from the initial mass is 15 days. The time required for $10 \%$ of mass loss is 30 days, $15 \%$ is 45 days and $20 \%$ (the last targeted stage of corrosion) is 60 days.

To verify the predicted time values, the embedded reinforcement steel bars were retrieved and weighed after corrosion to calculate the real mass loss compared with hypothetical mass loss.

\subsubsection{Concrete mixture}

The NC and the SCC mixtures were similar to the mixes used by previous research conducted at Ryerson University (Hassan, 2008). The SCC mixture was adopted from the same mixture delivered to the Pearson Airport project in Toronto, Canada in 2000 (Lessard et al., 2002). The two concrete mixture proportions are summarized in Table (3-3).

Two cementitious materials were used in both NC and SCC. The first one is type GU Canadian cement, which is similar to ASTM type I, and the second is slag cement (ground granulated blast furnace slag). Tables (3-4) and (3-5) show the chemical and physical properties of cement and slag respectively. Sieve analysis of the coarse and fine aggregates used is shown in Table (3-6). High-range water reducer admixture, similar to Type F of ASTM C 494, and water reducer (WR), similar to Type A of ASTM C 494 , were used to adjust the flowability of SCC and NC mixtures, respectively.

Table (3-3): The mixture proportions for NC and SCC mixtures.

\begin{tabular}{|c|c|c|c|c|c|c|c|}
\hline $\begin{array}{l}\text { Concrete } \\
\text { Type }\end{array}$ & $\begin{array}{l}\text { Cement } \\
\text { Type } \\
\text { GU } \\
\left(\mathrm{Kg} / \mathrm{m}^{3}\right)\end{array}$ & $\left(\mathrm{Kg} / \mathrm{m}^{3}\right)$ & $\begin{array}{l}10 \mathrm{~mm} \\
\text { Coarse } \\
\text { Aggregate } \\
\left(\mathrm{Kg} / \mathrm{m}^{3}\right)\end{array}$ & $\begin{array}{l}\text { Fine } \\
\text { Aggregate } \\
\left(\mathrm{Kg} / \mathrm{m}^{3}\right)\end{array}$ & $\left(\mathrm{Kg} / \mathrm{m}^{3}\right)$ & $\begin{array}{l}\text { HRWR } \\
\mathrm{mL} / 100 \mathrm{kkg} \\
\text { of binder }\end{array}$ & $\begin{array}{l}\text { WR } \\
\mathrm{mL} / 100 \mathrm{kkg} \\
\text { of binder }\end{array}$ \\
\hline $\mathrm{NC}$ & 300 & 100 & 1130 & 725 & 160 & 0 & 300 \\
\hline SCC & 315 & 135 & 900 & 930 & 180 & Variable & 0 \\
\hline
\end{tabular}


Table (3-4): The physical and chemical analysis of cement.

\begin{tabular}{|lr|lr|}
\hline Chemical Analysis ( \% ) & & Physical Analysis & \\
\hline LOI & 2.05 & Residue 45um (\%) & 8.42 \\
SiO2 & 19.64 & Blaine (m2/kg) & 410 \\
Al2O3 & 5.48 & Air Content (\%) & 7.78 \\
Fe2O3 & 2.38 & Initial Set (mins.) & 103 \\
CaO & 62.44 & Auto. Exp. (\%) & 0.14 \\
MgO & 2.48 & Sulf. Exp. (\%) & 0.013 \\
SO3 & 4.32 & & \\
Total Alkali & 0.97 & Compressive Strength (MPa) & \\
Free Lime & 1.03 & 1 day & \\
Insol. & 0.6 & 3 days & \\
C3S & 52.34 & 7 days & 29 \\
C2S & 16.83 & 28 days & \\
C3A & 10.5 & & 41 \\
C4AF & 7.24 & & \\
\hline
\end{tabular}

Table (3-5): The physical and chemical analysis of slag.

\begin{tabular}{|lc|lc|}
\hline Chemical Analysis ( \% ) & & Physical Analysis & \\
\hline $\mathrm{LOI}$ & 0.65 & Residue 45um (\%) & 3.14 \\
$\mathrm{SiO} 2$ & 40.3 & Blaine $(\mathrm{m} 2 / \mathrm{kg})$ & 422 \\
$\mathrm{Al} 2 \mathrm{O} 3$ & 8.4 & & \\
$\mathrm{Fe} 2 \mathrm{O} 3$ & 0.5 & Compressive Strength (MPa) & \\
$\mathrm{CaO}$ & 38.71 & (50:50 cement: slag) & \\
$\mathrm{MgO}$ & 11.06 & 7 days & 24 \\
$\mathrm{SO} 3$ & 2.31 & 28 days & 42 \\
$\mathrm{~K} 2 \mathrm{O}$ & 0.57 & Slag Activity Index & \\
$\mathrm{Na} 2 \mathrm{O}$ & 0.37 & (\% of 28 day control) & 99.9 \\
\hline
\end{tabular}


Table (3-6): Sieve analysis of the coarse and fine aggregates used.

\begin{tabular}{|c|c|c|c|c|c|c|c|c|c|c|}
\hline \multicolumn{2}{|l|}{ Sieve \# } & $9.5 \mathrm{~mm}$ & $6.7 \mathrm{~mm}$ & $\begin{array}{l}\text { No. } \\
4\end{array}$ & $\begin{array}{l}\text { No. } \\
8\end{array}$ & $\begin{array}{l}\text { No. } \\
16\end{array}$ & $\begin{array}{l}\text { No. } \\
30\end{array}$ & $\begin{array}{l}\text { No. } \\
50\end{array}$ & $\begin{array}{l}\text { No. } \\
100\end{array}$ & $\begin{array}{l}\text { No. } \\
200\end{array}$ \\
\hline \multicolumn{2}{|l|}{ Opening(mm) } & 9.5 & 6.7 & 4.75 & 2.36 & 1.18 & 0.6 & 0.3 & 0.15 & 0.075 \\
\hline \multicolumn{2}{|l|}{$\%$ of passing } & 100 & 99.8 & 96.5 & 82.8 & 69.8 & 51.8 & 22.1 & 6 & 1.5 \\
\hline \multirow{2}{*}{$\begin{array}{l}\text { Standard } \\
\text { Specifications }\end{array}$} & Min. & 100 & & 95 & 80 & 50 & 25 & 10 & & \\
\hline & Max. & 100 & & 100 & 100 & 85 & 60 & 30 & 10 & 3 \\
\hline
\end{tabular}

\subsection{Tests}

\subsubsection{Concrete tests}

\subsubsection{Slump Test}

This test is used to determine the collapse (sudden change in height) of fresh normal concrete of hydraulic-cement concrete. A sample of fresh concrete is poured in the mold, shaped like truncated cone, as shown in Figure (3-4), then compacted by using a rod. The mold is lifted, and the concrete is allowed to collapse. The measured distance of the drop in the concrete, which is the difference between the original and dropped elevation, represents the slump value of that sample (ASTM C 143 / C 143M). 


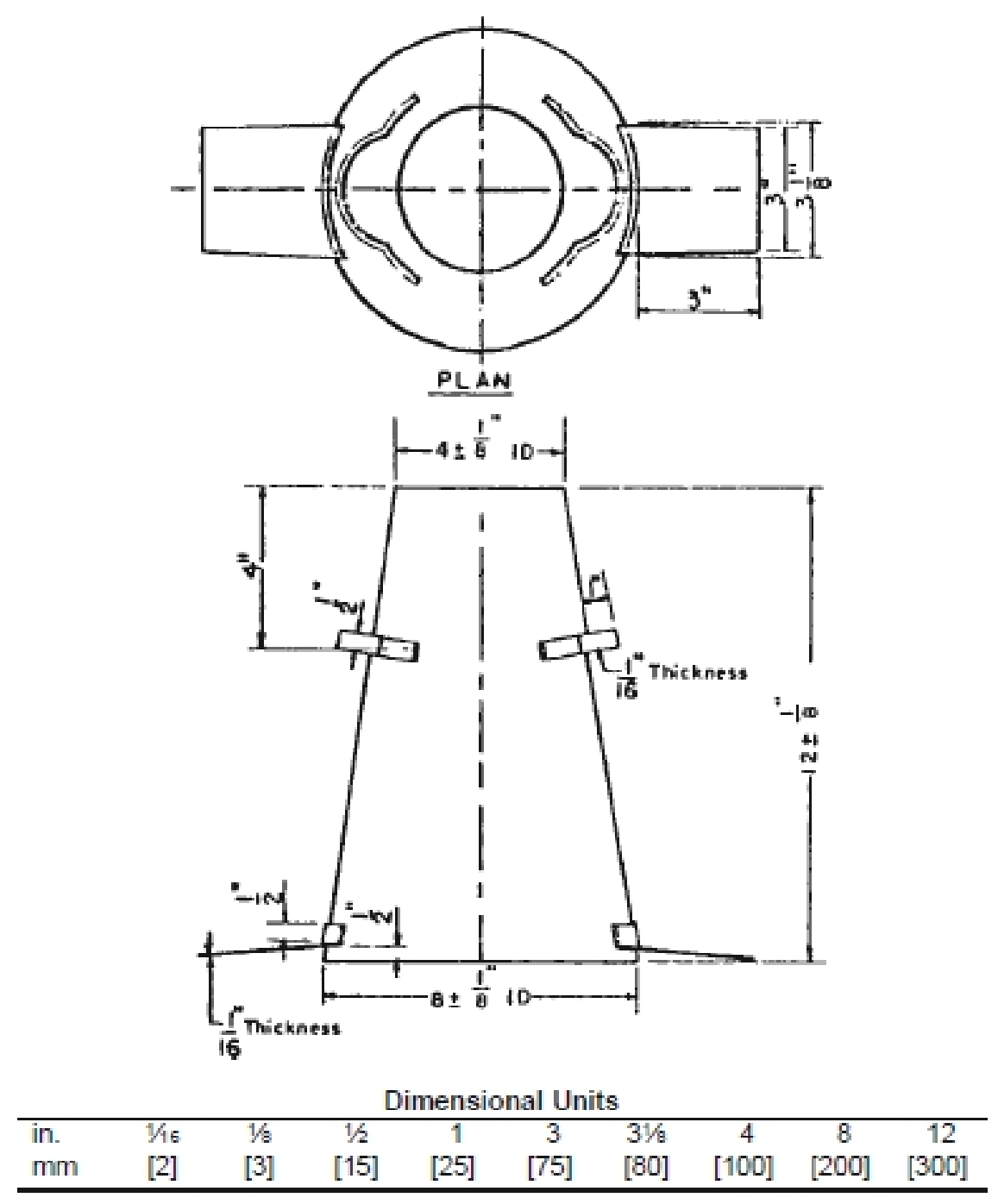

Figure (3-4): Mold for slump test (ASTM C 143 / C 143M).

\subsubsection{Slump test for SCC}

According to ASTM C 1611/C 1611M, freshly mixed concrete is poured in a slump mold in the inverted position, without tamping or vibration. The mold is raised, allowing the concrete to spread. The diameter of the spread concrete is measured in two perpendicular directions, and the slump flow is the average of these two diameters. Figure (3-5) shows the SCC spread on the base plate, after placing the fresh SCC mix in the inverted cone.

This test gives a clear indication of the degree of flowability; it also monitors the consistency of fresh SCC and its flow potential. Equation (3-1) is used to calculate SCC slump flow as follows 


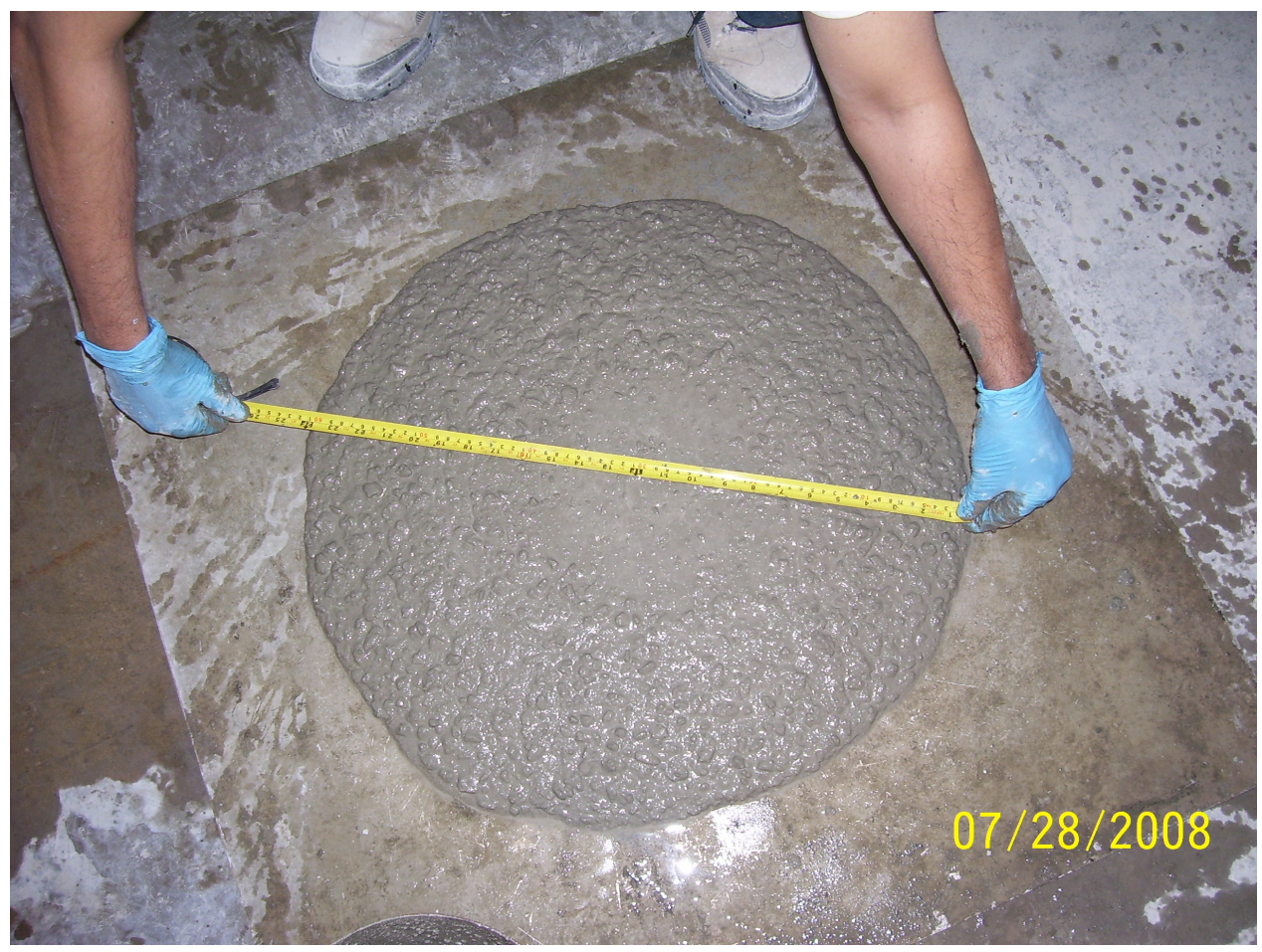

Figure (3-5): SCC slump test.

$$
\text { Slump Flow }=\frac{\left(d_{1}+d_{2}\right)}{2}
$$

$\boldsymbol{d}_{1}=$ The largest diameter of the circular spread of the concrete

$\boldsymbol{d}_{2}=$ The second measured diameter of the circular spread of the concrete at an angle of $90^{\circ}$ with the first measured diameter $d_{1}$

The values of the slump flow test are measured in $\mathrm{mm}$. $\mathrm{ACl} 237 \mathrm{R}-07$ refers to the common range of slump flow as 450 to $760 \mathrm{~mm}$. Higher slump flow indicates that the SCC can move farther and faster under its own weight from one side of casting.

\subsubsection{Compression test}

Compressive strength is one of the essential properties of concrete, and it can be defined as the maximum resistance of concrete cylinders to axial loading (Brzev and Pao, 2006). It depends on the quality of the concrete and its aggregates, and the time and quality of the curing. According to CSA A23.3 and many other concrete standards, $f_{c}^{\prime}$ refers to the compressive strength of concrete, measured in MPa. 


\subsubsection{Split cylinder test}

There are many ways to measure the tensile strength of concrete, such as the direct tension test, modulus of rupture test, and double-punch test (MacGregor and Bartlett, 2000) and (Collins and Mitchell, 1991). In this research, the results obtained are exclusively from the split cylinder test.

To study the tensile strength of the concrete, the spilt cylinder test was used (A23.2 -13C). The same concrete cylinder used in the compression test was placed on its side and a compression force was applied along a diameter of the concrete cylinder, as shown in Figure (3-6a), (MacGregor and Bartlett, 2000). Figure (3-6c) shows an element on the vertical diameter subjected to biaxial tension and compression stresses. Figure (3-6d) shows the distribution of both compression and tension stresses along the vertical circular face. There are two areas of high transverse compression on the far ends of the top and bottom of the vertical circular face of the concrete cylinder, but on the rest of the diameter, there is almost a uniform tension.

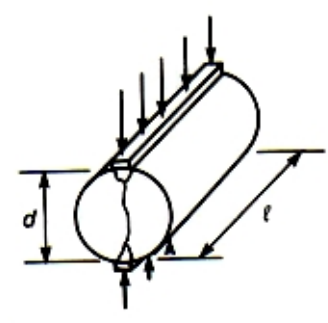

(a) Test procedure.

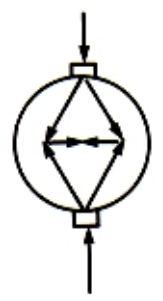

(b) Simplifled force system.
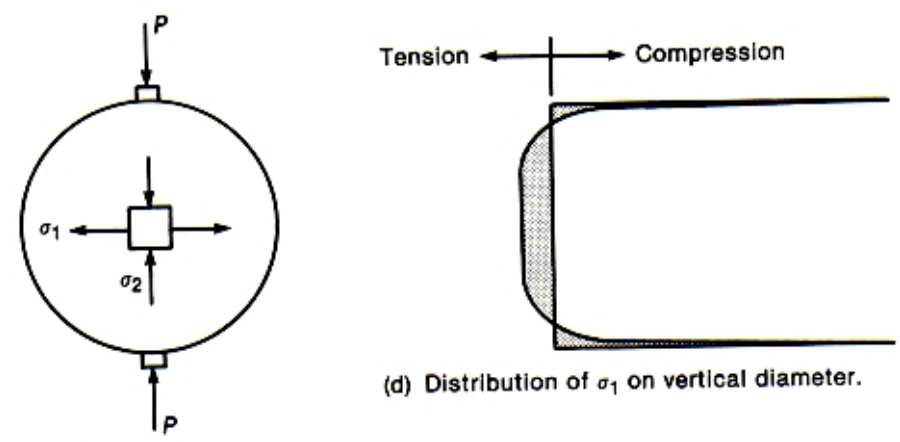

(d) Distribution of $\sigma_{1}$ on vertical diameter.

(c) Stresses on element.

Figure (3-6): Split cylinder test (MacGregor and Bartlett, 2000).

The splitting tensile strength, $f_{c t}$ from a split cylinder test is computed as follows (MacGregor and Bartlett, 2000) 


$$
f_{c t}=\frac{2 P}{\pi \ell d}
$$

Where $: \boldsymbol{P}=$ Maximum applied load in the test.

$$
\begin{aligned}
& \ell=\text { Length of specimen. } \\
& \boldsymbol{d}=\text { Diameter of specimen. }
\end{aligned}
$$

The crushed coarse aggregates increase the concrete tensile strength by $20 \%$ more than the concrete with rounded gravel (MacGregor and Bartlett, 2000). The tensile strength of concrete grows more quickly than the compressive strength. Therefore, shear strength and bond strength that are strongly affected by the tensile strength of concrete tend to grow more quickly than the compressive strength (Lew and Reichard, 1978).

\subsubsection{V-Funnel and L-Box tests}

These fresh SCC tests are used to evaluate and measure the deformability through the congested areas. Table (3-7) shows the fresh properties of NC and SCC, and Table (3-8) shows the compressive and split strength results for both NC and SCC.

Table (3-7): The fresh properties of NC and SCC used in this research.

\begin{tabular}{|c|c|c|c|c|}
\hline $\begin{array}{c}\text { Concrete } \\
\text { Type }\end{array}$ & $\begin{array}{c}\text { Slump } \\
(\mathrm{mm})\end{array}$ & $\begin{array}{c}\text { Slump Flow } \\
\text { Average of } \mathrm{d}_{1} \text { and } \mathrm{d}_{2} \\
(\mathrm{~mm})\end{array}$ & $\begin{array}{c}\text { V-Funnel flow } \\
\text { time }(\mathrm{sec})\end{array}$ & $\begin{array}{c}\text { L-Box } \\
\mathrm{H}_{2} / \mathrm{h}_{1}(\%)\end{array}$ \\
\hline NC & 80 & -- & -- & -- \\
\hline S.C.C & -- & 602 & 3.6 & 82.35 \\
\hline
\end{tabular}

Table (3-8): Compressive and split strength results for both NC and SCC.

\begin{tabular}{|c|c|c|c|}
\hline Concrete Type & $\begin{array}{c}\text { Compressive } \\
\text { Strength (MPa) @ 7 } \\
\text { Days }\end{array}$ & $\begin{array}{c}\text { Compressive } \\
\text { Strength (MPa) @ 28 } \\
\text { Days }\end{array}$ & $\begin{array}{c}\text { Split (Tensile) } \\
\text { Strength (MPa) } \\
\text { @ 28 Days }\end{array}$ \\
\hline \multirow{2}{*}{ NC } & $35.0 ; 34.5 ; 33.3 ; 34.4$ & $47.5 ; 42.8 ;$ & $4.7 ; 5.0 ;$ \\
& 34.3 & $44.1 ; 47.6$ & 5.9 \\
\hline Average of NC & $30.7 ; 31.4 ; 33.3 ; 31.5 ;$ & $43.0 ; 42.6 ;$ & 5.2 \\
\hline \multirow{2}{*}{ SCC } & 33.3 & $40.5 ; 42.2$ & $5.0 ; 4.1$ \\
& 32.0 & 42.9 & 3.5 \\
\hline Average of SCC & & & 4.2 \\
\hline
\end{tabular}




\subsubsection{Half-cell potential test and rapid chloride ion test}

The half-cell potential test was applied on the 16 beams subjected to accelerated corrosion to estimate the expected size of the corrosion activity of the uncoated reinforcing steel bars embedded in the concrete according to ASTM C $876-91$ (Reapproved 1999).The rapid chloride ion test was also implemented on both NCC and SCC specimens. of both The results tests are presented in Chapter 4.

\subsection{Accelerated Corrosion}

\subsubsection{Electrolyte cell setup and voltage daily monitoring}

The electrolyte cell is composed of the following, as shown in Figure (3-7):

1. Electrical power suppliers, to provide constant direct current.

2. Reinforced concrete beams (as anode) and metal mesh (as cathode).

3. Saline solution of $\mathrm{NaCl}(5 \%$ concentration by weight).

4. Connecting wires and plastic basin.

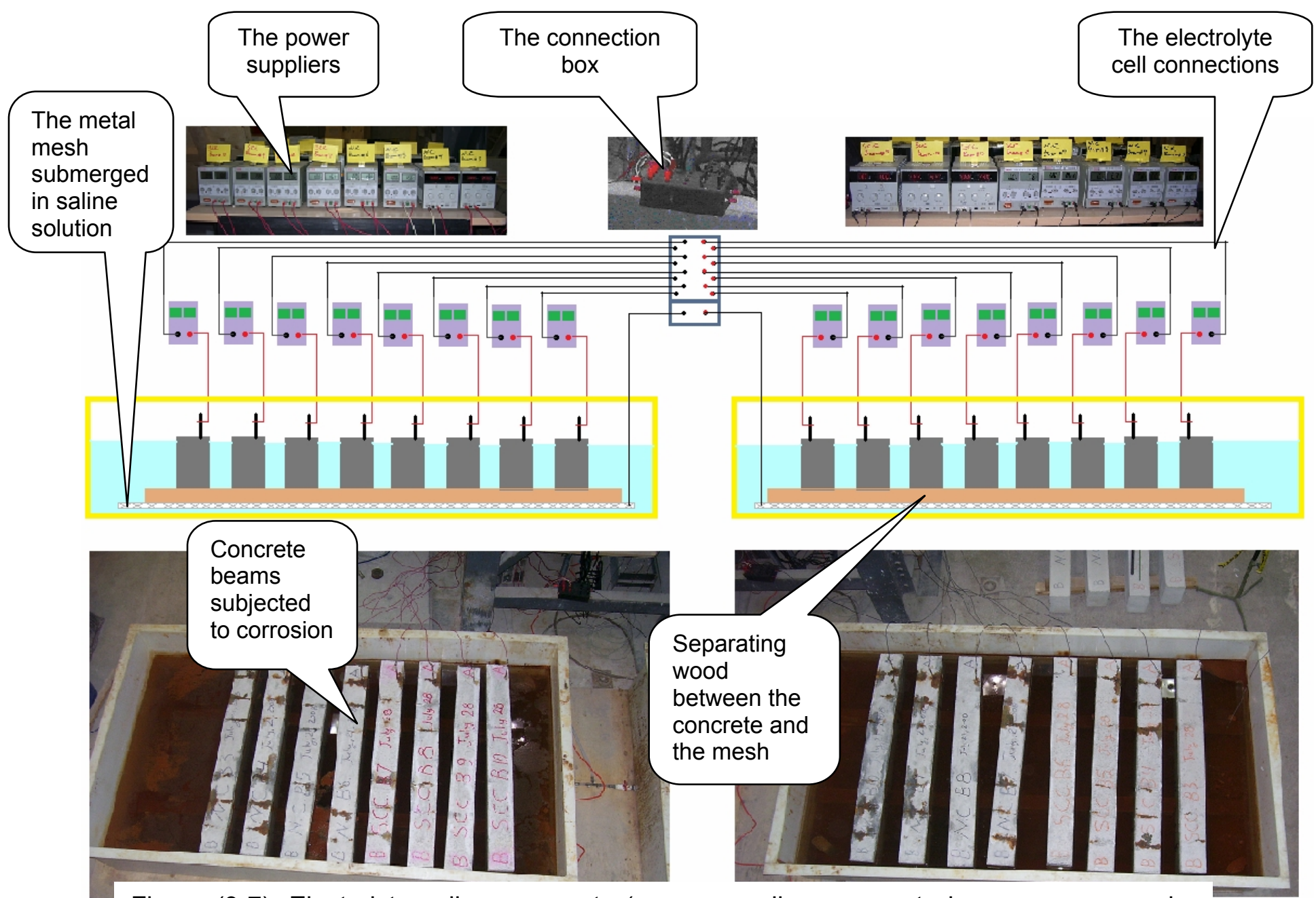

Figure (3-7): Electrolyte cell components (power suppliers, concrete beams as an anode, metal mesh as a cathode, saline solution, plastic basins, and connecting wires). 
Monitoring of the beams, connections, power suppliers, and drainage tubes took place throughout the experiment, and voltage readings were taken every 24 hours. Two plastic basins were used; each contained eight concrete beams. To achieve the greatest possible similarity of corrosion conditions, four beams from both types of concrete were placed in each plastic basin. During the accelerated corrosion, the electrical current was constant at 1 ampere, the lab temperature was constant at $21 \mathrm{C}^{\circ}$, and the salinity of the electrolyte solution was $5 \%$ concentration by weight.

After the corrosion time elapsed for each stage, beams were chosen to represent each stage of corrosion. Choosing the beams for each stage depended on the voltage readings and the structural condition with relation to the cracks and spalling. After considering crack condition, the beams that showed the highest electrical resistance through the highest voltage readings were chosen. To put it simply, the healthiest beams were selected in the beginning, and then by the end of each stage, the next healthiest beams were selected from those remaining ones. The readings of the voltage versus the constant electrical current are located in Appendix D.

\subsubsection{Drawing corrosion cracks pattern}

The cracks appearing on the surface of the concrete beams are one of the early consequences of corrosion. Since the concrete is relatively weak in tension, the cracks start to appear in the weaker sides of the concrete around the rebars at the cover side, instead of on the thick internal beam side.

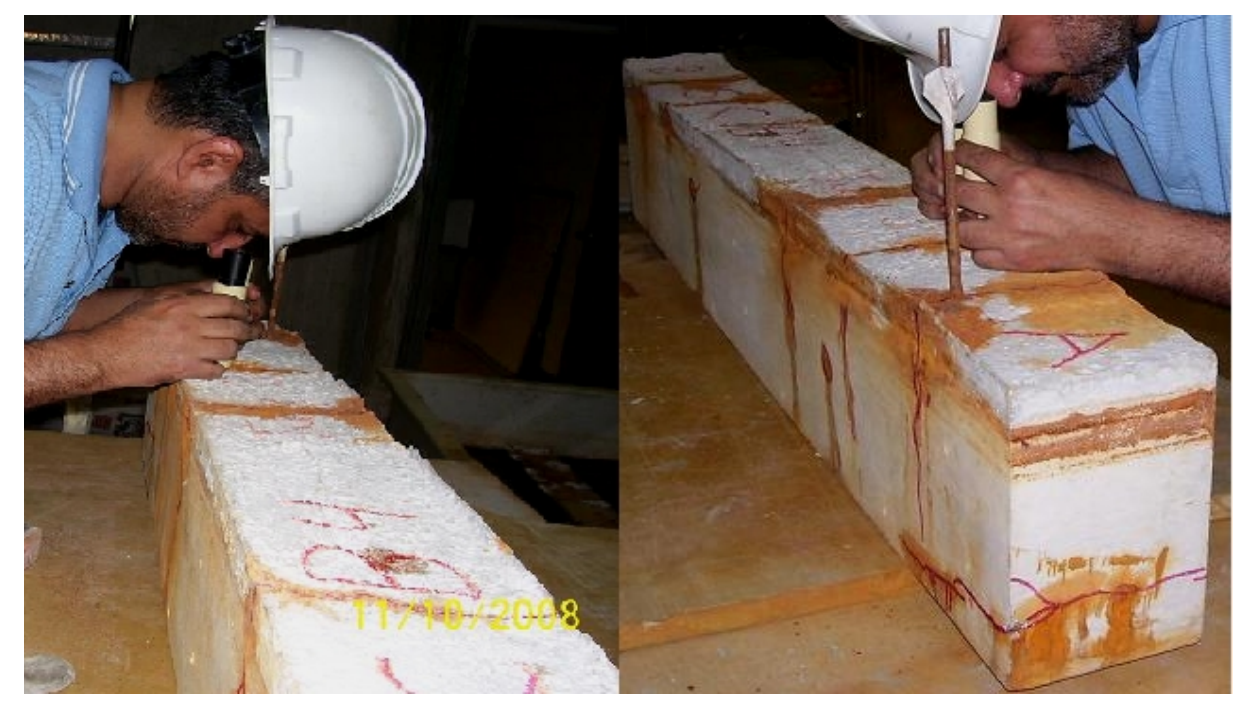

Figure (3-8): Measuring the crack widths on the corrosion cracks pattern. 
The crack patterns are important for the following reasons:

1. It refers to the location, size, and degree of corrosion of the corroded rebars that caused the cracks.

2. The expected cracks resulting from the loading test are an indicator of future structural response.

3. It shows the influence of the multi-stages of corrosion on crack shape and width, as well the total structural capacity.

1. It shows the effect of the congested rebars corrosion on its crack pattern.

Crack shapes and maximum crack width on each side of the beams were documented. The author measured the crack widths using a hand-held, illuminated optical microscope and an unmagnified comparator scale viewed under a high-powered magnifying glass, as shown in Figure (3-8).

As the corrosion process continued, the pressure of the corrosion products increased on weakened cover areas, resulting in spalling in some of the beams, as shown in Figure (3-9). The spalling occurred in the edges, corner areas, in the relatively congested area with the rebars, and in the area on the edges with no side support.

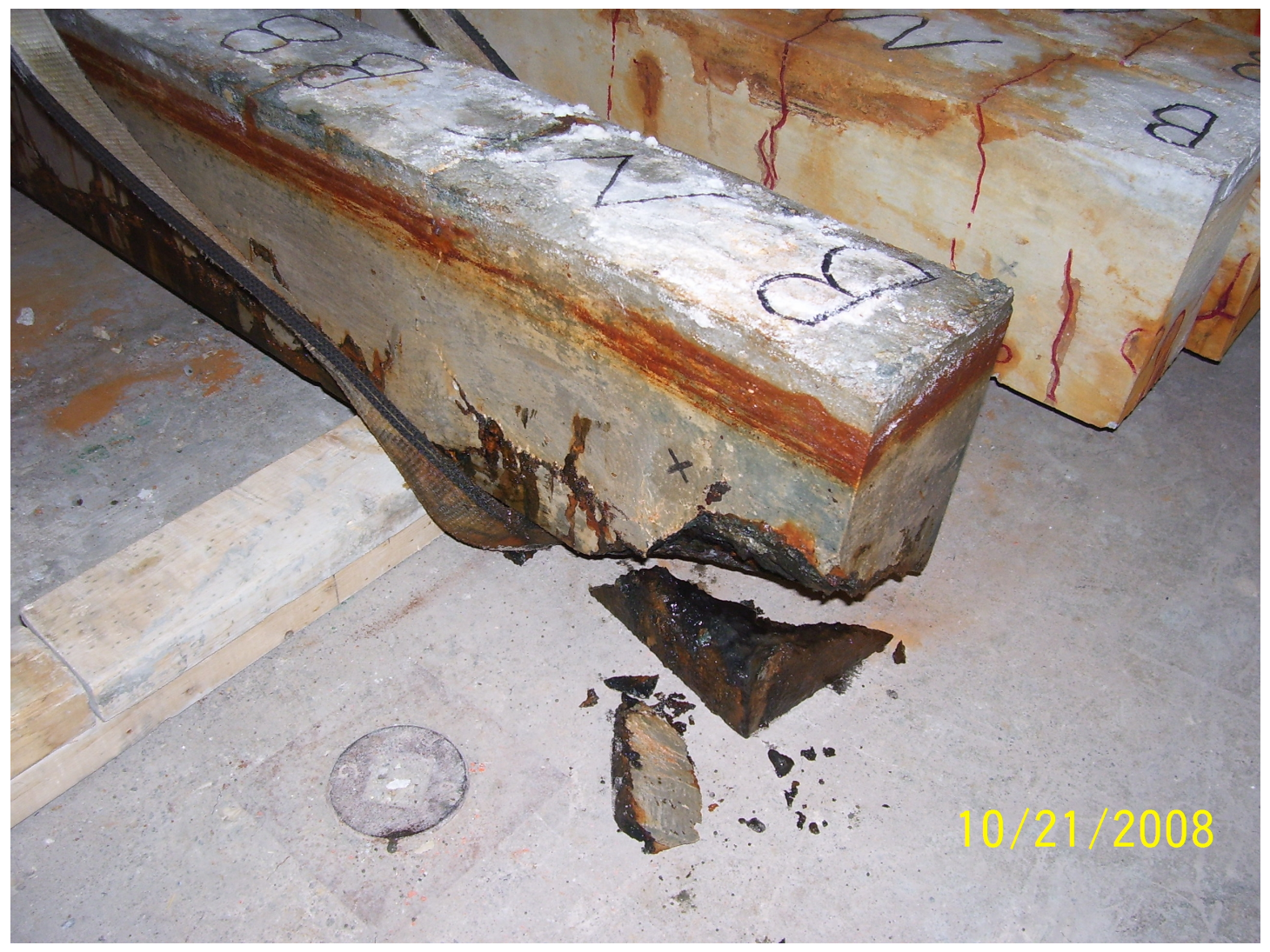

Figure (3-9): Spalling of concrete from the bottom of specimen NC B-8. 


\subsection{Loading test and LVDTs setting}

The loading test measures the structural capacity with relation to load vs. midspan deflection, shear strain, $\epsilon_{\mathrm{x}}$ and $\epsilon_{\mathrm{y}}$ and the deflection curvature all along the tested beams. The previous values, loading crack patterns and failure modes were subjected to comparison between the two types of concrete and the multi-stages of corrosion. The beams were placed as a simply supported with two loading points, considering the ratio of $\mathrm{a} / \mathrm{d}=2.5$ to maintain shear failure mode for the majority of beams.

\subsubsection{LVDT}

A set of nine linear variable differential transducers (LVDTs) were installed on the beam. The LVDT is an electrical transformer used for measuring linear displacement. To monitor the shear behaviour of NC and SCC beams at multi-stages of corrosion, the sets of LVDTs have to be arranged so that the data collected can be easily monitored and analyzed.

\subsubsection{LVDTs settings}

The beam loading, as shown in Figure (3-10a), produces shear stresses in the concrete. At the beginning, a square element of the reinforced concrete with vertical sides parallel to the direction of loading, is deformed by these stresses into a diamond shape, as illustrated in Figure (3-10b), where the deformation is greatly exaggerated for purposes on illustration. It can be concluded from Figure (3-10b) that shear strain is defined as the magnitude of the change in the value of the initial right angle of the element at the $X-Y$ origin. That is, (Rosettes strain gauge measurements, 2000)

$$
\gamma=\frac{\pi}{2}-\varphi
$$

Since shear strain is a change in angle, its natural units are radians, although it can also be expressed in terms of $(\mathrm{m} / \mathrm{m})$ and percentage. Based on Equation (3-3), the sign of the shear strain is positive when the initial right angle of the element is reduced $\left(\varphi<\frac{\pi}{2}\right)$. 
(a)

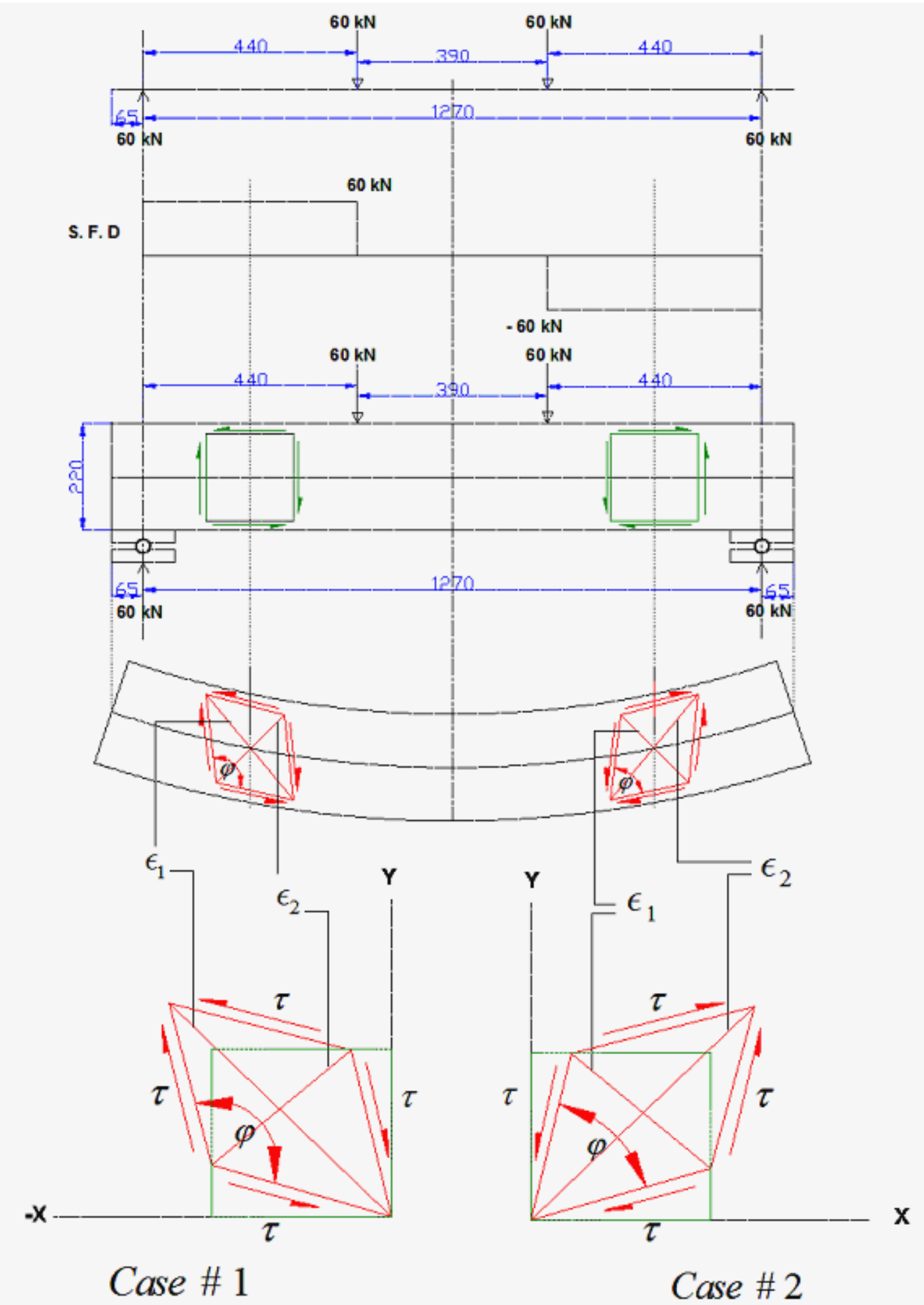

Figure (3-10): The effects of shear stress on two elements located on the concrete beam. The left element represents Case \#1, and the right element represents Case \#2. 
The change in the directions (sign) of the shear stresses in the above explanation causes the initial right angle to increase and results in a negative shear strain. Fortunately, shear and normal strains are related through mechanics principles, allowing LVDTs to provide a direct indication of shear strain. Proper placement and positioning of LVDTs on a strained surface produces a device signal that is directly proportional to the surface shear strain (Rosettes strain gauge measurements, 2000). Therefore, shear strain is positive if $\left(\varphi<90^{\circ}\right)$ or if the angle is reduced.

$$
\gamma_{x y}=\frac{\boldsymbol{\epsilon}_{1}-\boldsymbol{\epsilon}_{2}}{\operatorname{Sin} 2 \alpha}
$$

Compression LVDT means +ve ; and tension LVDT means -ve.

Case (1) in Figure (3-10) $\frac{-\boldsymbol{\epsilon}_{1}-\boldsymbol{\epsilon}_{2}}{\operatorname{Sin} 90} \Rightarrow-$ ve Shear Strain

Case (2) in Figure (3-10) $\quad \frac{\boldsymbol{\epsilon}_{1}-\left(-\boldsymbol{\epsilon}_{2}\right)}{\operatorname{Sin} 90} \Rightarrow+$ ve Shear Strain

By studying an array of two LVDTs oriented at different angles with respect to an X-Y coordinate system (as illustrated in Figure (3-11)), from fundamental mechanics of materials, the strains along the LVDT axes can be written as (Rosettes strain gauge measurements,

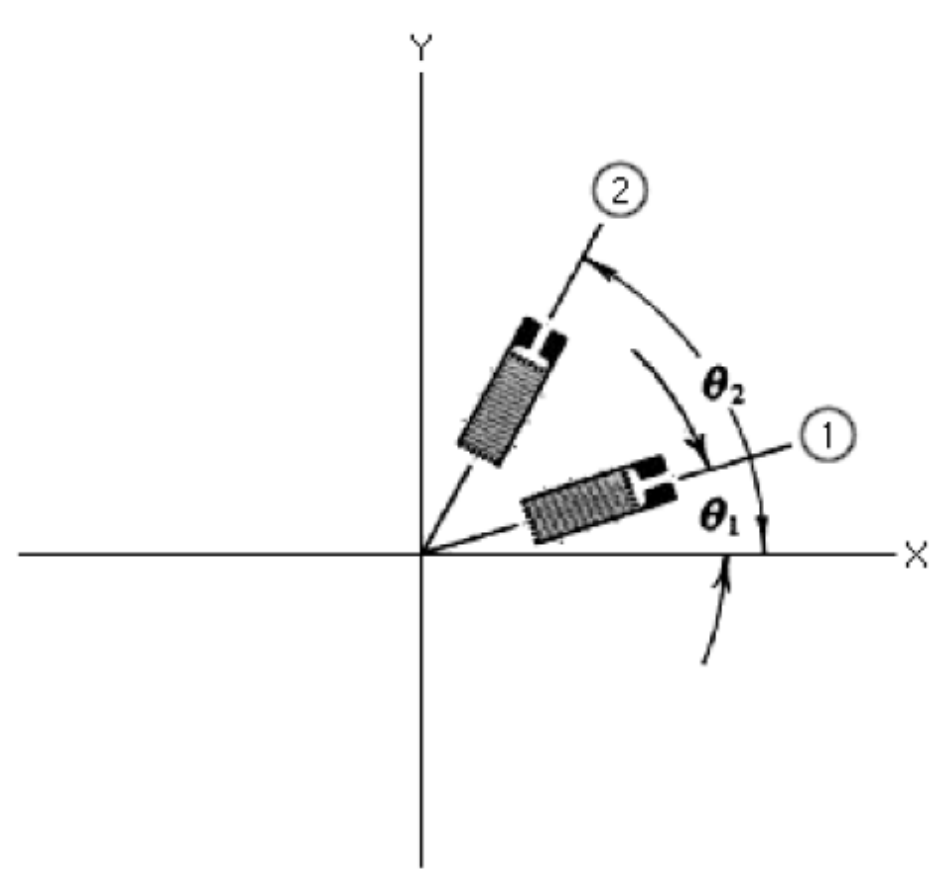

2000)

Figure (3-11): Arbitrarily oriented LVDTs in a biaxial strain field

(Rosettes strain gauge measurements, 2000). 


$$
\boldsymbol{\epsilon}_{1}=\frac{\boldsymbol{\epsilon}_{x}+\boldsymbol{\epsilon}_{y}}{2}+\frac{\boldsymbol{\epsilon}_{x}-\boldsymbol{\epsilon}_{y}}{2} \cos 2 \theta_{1}+\frac{\gamma_{x y}}{2} \sin 2 \theta_{1}
$$

In the same way,

$$
\boldsymbol{\epsilon}_{2}=\frac{\boldsymbol{\epsilon}_{x}+\boldsymbol{\epsilon}_{y}}{2}+\frac{\boldsymbol{\epsilon}_{x}-\boldsymbol{\epsilon}_{y}}{2} \cos 2 \theta_{2}+\frac{\gamma_{x y}}{2} \sin 2 \theta_{2}
$$

When $\theta_{1}=45^{\circ}$, then $\cos 2 \theta_{1}=0$, and $\sin 2 \theta_{1}=1$. Therefore, Equation (3-5) for $\epsilon_{1}$ can be written as following:

$$
\therefore \boldsymbol{\epsilon}_{1}=\frac{\boldsymbol{\epsilon}_{x}}{2}+\frac{\boldsymbol{\epsilon}_{y}}{2}+\frac{\gamma_{x y}}{2}
$$

It can be rewritten as following:

$$
\begin{aligned}
& \mathbf{2} \boldsymbol{\epsilon}_{1}=\boldsymbol{\epsilon}_{x}+\boldsymbol{\epsilon}_{y}+\gamma_{x y} \text {, and then, } \\
& \boldsymbol{\epsilon}_{x}+\boldsymbol{\epsilon}_{y}=\mathbf{2} \boldsymbol{\epsilon}_{1}-\gamma_{x y}
\end{aligned}
$$

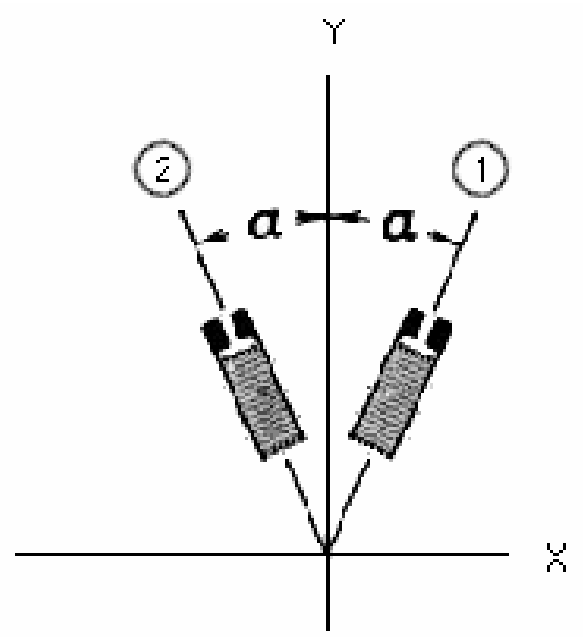

Figure (3-12): Defining $X$ axis as bisector of angle between gage axes (Rosettes strain gauge measurements, 2000). $\gamma_{x y}=\frac{\epsilon_{1}-\epsilon_{2}}{\operatorname{Sin} 2 \alpha}$ 
When $\theta_{2}=135^{\circ}$, then $\cos 2 \theta_{2}=0$, and $\sin 2 \theta_{2}=-1$. Therefore, Equation (3-5) for $\boldsymbol{\epsilon}_{2}$ can be written as following:

$$
\boldsymbol{\epsilon}_{2}=\frac{\boldsymbol{\epsilon}_{x}}{2}+\frac{\boldsymbol{\epsilon}_{y}}{2}-\frac{\gamma_{x y}}{2}
$$

And it can be rewritten as following:

$$
\boldsymbol{\epsilon}_{x}+\boldsymbol{\epsilon}_{y}=\mathbf{2} \boldsymbol{\epsilon}_{2}+\gamma_{x y}
$$

From Equation (3-5), when the angle between the LVDTs $\alpha=45$, then $\sin 2 \alpha=1$, as shown in Figure (3-12).

$$
\therefore \gamma_{x y}=\epsilon_{1}-\epsilon_{2}
$$

By substituting this in Equation (3-5-1-a)

$$
\begin{aligned}
& \boldsymbol{\epsilon}_{x}+\boldsymbol{\epsilon}_{y}=\mathbf{2} \boldsymbol{\epsilon}_{1}-\left(\boldsymbol{\epsilon}_{1}-\boldsymbol{\epsilon}_{2}\right) \Rightarrow \\
& \boldsymbol{\epsilon}_{x}+\boldsymbol{\epsilon}_{y}=\boldsymbol{\epsilon}_{1}+\boldsymbol{\epsilon}_{2}
\end{aligned}
$$

Similar substitution in Equation (3-6-2-a) results in

$$
\begin{aligned}
& \boldsymbol{\epsilon}_{x}+\boldsymbol{\epsilon}_{y}=\mathbf{2} \boldsymbol{\epsilon}_{2}+\left(\boldsymbol{\epsilon}_{1}-\boldsymbol{\epsilon}_{2}\right) \\
& \Rightarrow \boldsymbol{\epsilon}_{x}+\boldsymbol{\epsilon}_{y}=\boldsymbol{\epsilon}_{1}+\boldsymbol{\epsilon}_{2} \\
& \therefore \boldsymbol{\epsilon}_{x}=\boldsymbol{\epsilon}_{1}+\boldsymbol{\epsilon}_{2}-\boldsymbol{\epsilon}_{y}
\end{aligned}
$$

Results can be concluded as follows: the difference in normal strain measured by any two LVDTs arbitrarily oriented in a uniform strain field is proportional to the shear strain along an axis bisecting the LVDT axis, despite the included angle between the LVDTs. When the two LVDTs are $90^{\circ}$ aside, the denominator of Equation (3-4) becomes unity and the shear strain $\gamma_{x y}$ along the bisector is numerically equal to the difference in normal strains $\boldsymbol{\epsilon}_{1}-\boldsymbol{\epsilon}_{2}$. 


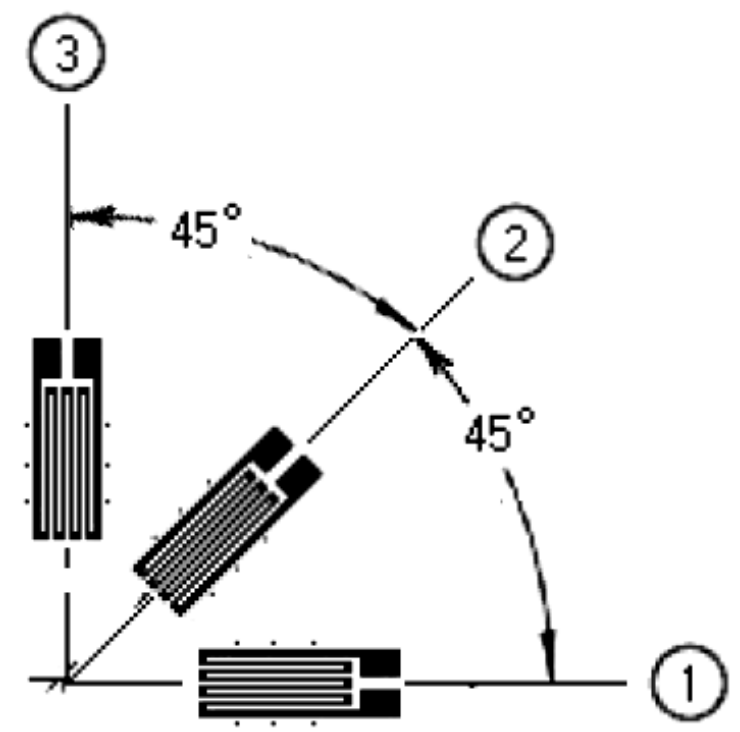

Figure (3-13): $45^{\circ}$ rosette LVDTs (Rosettes strain gauge measurements, 2000)

It is not possible to determine the maximum shear strain or the complete state of strain from any combination of LVDT outputs unless the orientation of the LVDT axes with respect to the principal axes is known. In general, when the directions of the principal axes are unknown, a three-LVDT $45^{\circ}$ rectangular rosette can be used, as shown in Figure (3-13) (Rosettes strain gauge measurements, 2000).

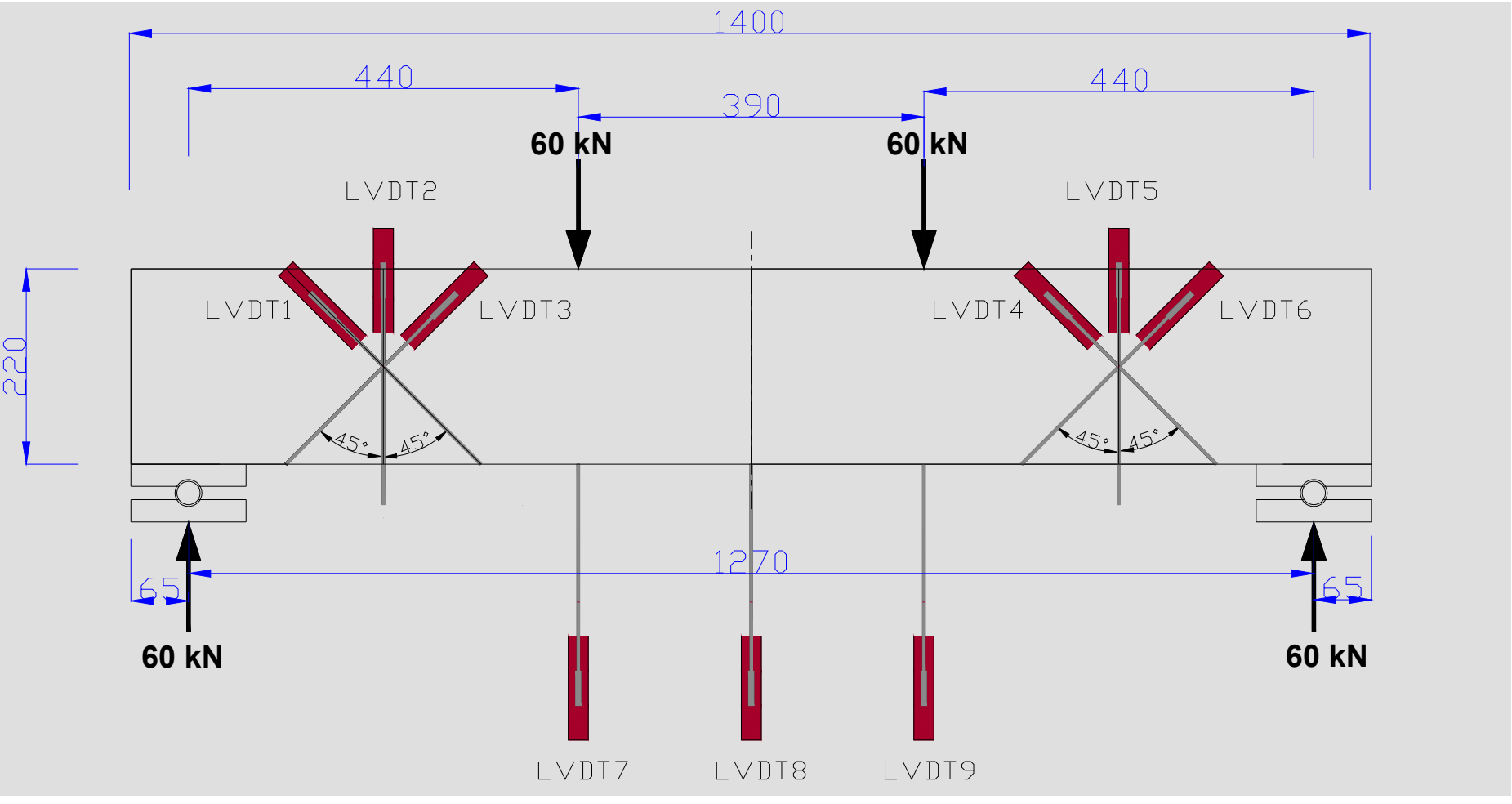

Figure (3-14): The LVDTs numbers and locations on the beam. 
In this research, in order to determine the shear strain $\gamma_{x y}$, longitudinal strain $\boldsymbol{\epsilon}_{x}$, and transversal strain $\epsilon_{y}$, the data obtained from LVDTs no.1-6 were divided by the length on the beam covered by these LVDTs. Since the depth of the beam is $220 \mathrm{~mm}$, LVDTs no. $1,3,4$, and 6 worked on a length of $(220 \mathrm{~mm} \times \sqrt{2}=311 \mathrm{~mm})$ while no.2 and 5 worked on a length of $220 \mathrm{~mm}$; no.7-9 were used to monitor the deflection in the midspan and under each load force directly.

Figure (3-14) shows the nine LVDTs installed on the concrete beams. The first three LVDTs on the left side intersected in the mid area between the left load and the left reaction, with a $45^{\circ}$ between the LVDTs. The second group of three LVDTs were located on the right side, similar to the location of the left, with the same angle between them. The last three LVDTs were vertically installed, but were turned upside down at the opposite directions of LVDTs no.2 and 5.

The data collected from the load cell and the LVDTs were analyzed as follows:

1. To calculate the shear strain $\gamma_{x y}$, Equation (3-4) was used to find out the values of shear strain. When $2 \alpha=90^{\circ} \therefore \gamma_{x y}=\boldsymbol{\epsilon}_{1}-\boldsymbol{\epsilon}_{2}$. By applying Equation (3-4) to the data collected from the LVDTs, the shear strain can be calculated as Shear Strain Left $\left(\gamma_{\text {left }}\right)=($ LVDT 3/311mm $)-($ LVDT 1/311mm $)$ Shear Strain Right $\left(\gamma_{\text {Right }}\right)=(L V D T 6 / 311 m m)-(L V D T \quad 4 / 311 m m)$

2. $\boldsymbol{\epsilon}_{x}$ Can be calculated by using Equation (3-8), since $\boldsymbol{\epsilon}_{x}=\boldsymbol{\epsilon}_{1}+\boldsymbol{\epsilon}_{2}-\boldsymbol{\epsilon}_{y}$

$$
\begin{aligned}
& \therefore \boldsymbol{\epsilon}_{x \text { left }}=(L V D T 1 / 311 \mathrm{~mm})+(L V T D 3 / 311 \mathrm{~mm})-(L V D T 2 / 220 \mathrm{~mm}) \\
& \therefore \boldsymbol{\epsilon}_{x \text { Right }}=(L V D T 4 / 311 \mathrm{~mm})+(L V T D 6 / 311 \mathrm{~mm})-(L V D T 5 / 220 \mathrm{~mm})
\end{aligned}
$$

3. $\boldsymbol{\epsilon}_{y}$ left and $\boldsymbol{\epsilon}_{y}$ right can be obtained from the data of (LVDT2) and (LVDT5) directly. So

$$
\boldsymbol{\epsilon}_{y \text { Left }}=L V D T 2 / 220 \mathrm{~mm} \text { and } \boldsymbol{\epsilon}_{y \text { Right }}=L V D T 5 / 220 \mathrm{~mm}
$$

4. Mid-span deflection $\Delta$ can be obtained from the data collected from LVDT no.8. Deflection vs. load is one of the important indicators of the RC beams' structural capacity. 
As illustrated in Figure (3-15), at small values of loading, the beam is uncracked and shows elastic uncracked behaviour, and the cross-sectional properties can be represented by a gross transformed moment of inertia $\left(I_{g}\right)$. At the cracking bending moment $\left(M_{c r}\right)$, the beam still behaves in elastic way until point $C$ on the diagram; by increasing the load after this point, the moment of inertia of the beam section at the midspan decreases. At point $S$ on the diagram, the bending moments in the mid-span of the beam usually exceed the cracking moment. After further loading, the tensile rebars start to yield at point $\mathrm{Y}$. The following stage showed a fully cracked beam section and the moment of inertia transformed from $\left(I_{g}\right)$ to the cracked transformed section $\left(I_{c r}\right)$. By adding a small amount of load after point $\mathrm{Y}$, point $\mathrm{F}$ on the diagram showed the failure point of the beam.

a)

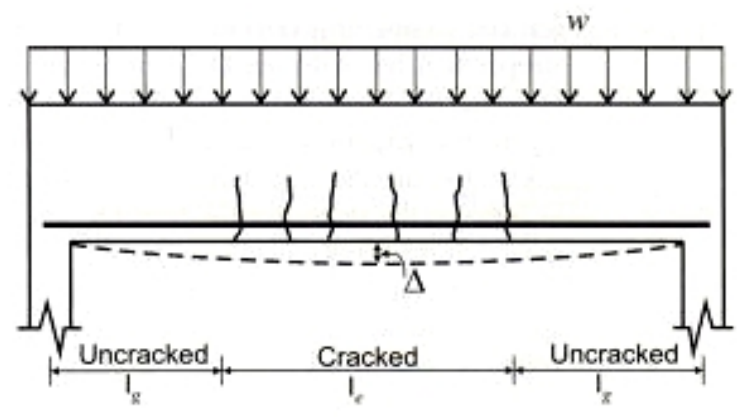

b)

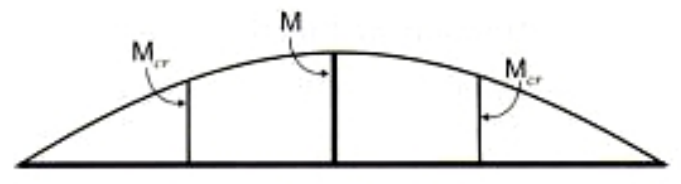

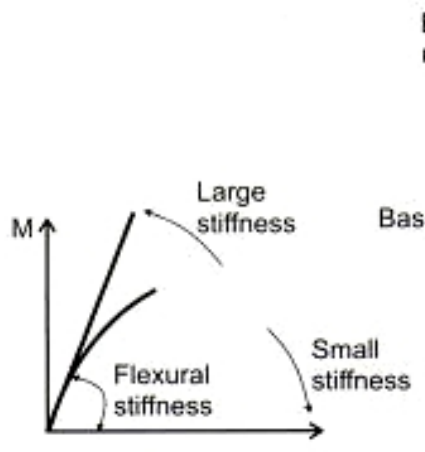

$\Delta$

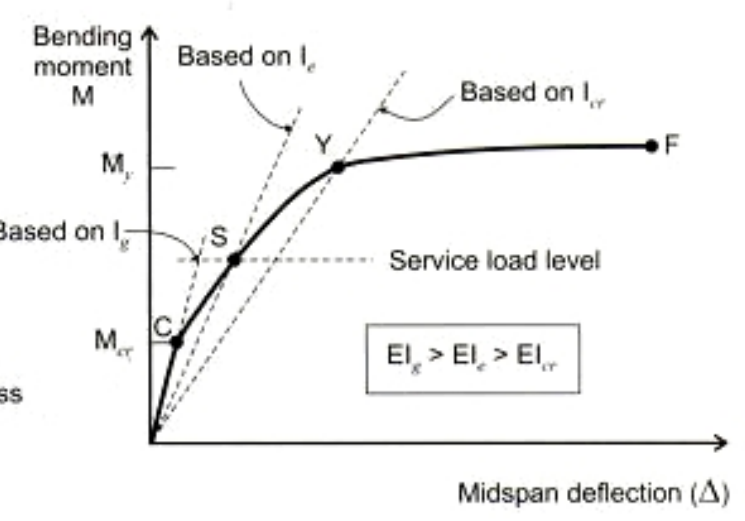

c)

d)

Figure (3-15): Deflection behavior for a reinforced concrete beam: a) beam elevation; b) bending moment diagram; c) flexural stiffness; d) bending moment deflection at the mid-span (Brzev and Pao, 2006). 
The radial lines on the diagram are in different slopes; the slope shows the size of the flexural stiffness of the member, as shown in Figure (3-15). Flexural stiffness is a resistance to deformation of a flexural member, so the beams with larger flexural stiffness develop smaller flexural stresses and deflections (Brzev and Pao, 2006).

5. Data collected from LVDTs no.7, 8, and 9 can be used to calculate the deflection on the mid-span and all along the beam.

\subsection{The loading crack pattern}

The cracks resulting from loading were monitored and marked on the concrete surface of the beams associated with the load values causing these cracks. The cracks normally started with few transverse cracks around the bottom side of the mid-span area, followed by diagonal cracks that propagated until failure. The corrosion cracks may affect the loading cracks depending on the location and width of the corrosion cracks. The cracks' shape, width, length, angle, and location give us a clear idea about the beam response to the increasing load. The failure mode is one of the most important pieces of information that we can obtain from the loading cracks pattern.

\subsection{Actual mass loss measuring}

At the end of the loading test, the concrete beams were demolished and the steel cages regained, and the concrete debris and corrosion residue were cleaned away. Muriatic "hydrochloric" acid was used to clean the corrosion residue from the corroded rebars. The epoxy-coated top rebars were free of corrosion due to the electrical isolation. Tables (4-6) and (4-7) show the mass loss details for the reinforcing steel elements. 


\section{Chapter 4}

\section{Results and discussions}

There are six categories of results presented and discussed in this chapter, as follows; half-cell potential test, rapid chloride permeability test, accelerated corrosion process, loading tests, crack patterns due to loading tests, and mass loss measuring. The discussion following each of these results analyzes the effect of using two different types of concrete and multi-stages of corrosion.

\subsection{Half-cell potential test}

The half-cell potential test, according to ASTM C 876 - 91 (Reapproved 1999), is designed to predict the amount of the corrosion activity in the uncoated reinforcing steel bars embedded in the concrete. The half-cell potential test was applied to concrete beams numbered 3 to 10, for both NC and SCC. Beams no. 1 and 2 were the control groups, and beams no. 3 to 10 were the beams subjected to corrosion after the half-cell potential test. Three points were selected on the left, bottom, and right side of each beam. The imaginary plane that includes stirrup no.1, which was composed of three points on the left, bottom, and right side. The second three points were located on the imaginary plane of the center line, and the imaginary plane that include stirrup no. 6 was composed of the third three points of left, bottom, and right side, as shown in Fig. (4-1).

As part of the requirements of the half-cell potential test, it is important to connect the positive side of the special voltmeter to the uncovered rebar. For this study, the connections of the voltmeter to the uncovered rebar were done through the long side of stirrup no. 1 of each beam. The readings of the voltmeter are shown in Table (4-1).

According to the half-cell potential test manual, the readings from the internal high impedance voltmeter are designed to break down into seven categories that enable a detailed analysis of the voltages. The categories are broken down from $A$ to $G$, with Category A being the highest possibility for corrosion. The categories and their voltages are shown in Table (4- 2) (Half-cell potential test manual, 2003).

The other references are to the ASTM's categorization of the voltmeter readings, as shown in Table (4-3) (ASTM C 876 - 91 Reapproved 1999). 
The half-cell potential test results showed the following:

- The readings for all of the beams are located above the case of sever corrosion and showed a higher than $90 \%$ chance for corrosion to occur. There are two main reasons for this high probability of corrosion:

1. The relatively high percentage of the humidity inside the concrete body of the tested beams.

2. The thin $30 \mathrm{~mm}$ concrete cover above the rebars, which was a result of the small size of the concrete beams used in this research and the majority of the other studies.

- A close value of the readings on each beam in the longitudinal and transverse directions, which was mainly because of the small size of the specimens, and the $1.2 \mathrm{~m}$ satisfactory spacing of the half-cell potential test readings, according to ASTM C 876 - 91 (Reapproved 1999).

\subsection{Rapid Chloride Permeability Test (RCPT)}

This test was done according to ASTM C1202-97. The RCPT evaluates the electrical conductivity of the concrete specimens to give a rapid indication of concrete resistance to the penetration of the chloride ions.

The results shown in Figure (4-2) are part of the group of concrete samples at age 64 days for NC, and 57 days for SCC. The results in Figure (4-3) show samples at age 137 days for NC, and 130 days for SCC. Samples no.1, 2, and 3 are SCC and samples no. 4,5 , and 6 are NC for both figures. The result of NC sample no.5 at age 64 days was cancelled due to leakage of $\mathrm{NaOH}$ and $\mathrm{NaCl}$.

Counter to expectations, the NC specimens' results showed less permeability than the SCC specimens. However, the results showed a strong compatibility with the daily voltage readings. The RCPT results were a relatively fast, inexpensive and useful indicator that helped evaluate and compare the chloride ion penetrability of the two types of concrete.

The results obtained from the second test, performed 73 days after the first one, showed an obvious development in the readings' value in relation to the readings from the first test. Continuous curing was the only thing applied to the concrete specimens used on the second test; they developed values of penetration that were much lower than in the first test. However, the results showed that the NC specimens retained superiority over the SCC ones, even in the second test. 


\subsection{Accelerated corrosion}

\subsubsection{Voltage readings}

The enforced constant current sent through the RC beams produces corrosion product, which affects both the electrical resistance of beams and the bond strength between rebars and concrete. The accumulated corrosion product around reinforcing steel bars increases the pressure on the concrete in all directions surrounding the rebars.

The internal pressure, in its earlier stages, strengthens the bond between the rebars and the concrete. But when this pressure increases, cracks in the concrete cover appear. These cracks reduce the magnitude of the electrical resistance of the RC beams due to the direct electrical contact through the cracks. The daily voltage readings showed that the average of the electrical resistance reduces as the corrosion processes continue.

The fluctuation in that resistance is related to the developed cracks in the concrete cover that sometimes open wider paths between the rebars and the saline solution, resulting in decreased resistance; or narrowing those paths to make direct contact more difficult, and increasing the beam resistance value. The relatively big drop in the value of the beam's electrical resistance is due to either developing very wide crack or spalling some pieces from the concrete cover. The case of sudden drop in beam resistance happens either in the advanced stages of corrosion or in the weak spots of the concrete cover in the early stages. According to Ohm's law, the resistance of the concrete beam determines the amount of current through the beam for a given electrical potential difference (voltage) across the beam (Ohms law, 1825-1830)

$$
R=\frac{V}{l}
$$

where

$R$ is the resistance of the beam, measured in ohms.

$V$ is the potential difference across the beam, measured in volts.

$I$ is the current through the beam, measured in amperes.

For a constant current of one ampere, the daily basis readings of voltage showed higher initial resistance of for the NC beams $(21.7-27.1 \mathrm{Ohm})$ than for the SCC beams 
(15.3 - 19.4 Ohm). Most of the beams of both types reached peak resistance within 5-6 days. The NC beams maintained higher resistance for the first 15 days, and the voltage readings of the second 15 days of the accelerated corrosion showed similar range of resistance for both types of concrete. The resistance values extended from 7-17 Ohm with a slightly higher average in the SCC beams' voltage readings than the NC beams' readings. The slight superiority of the SCC increased little more in the last 30 days of the accelerated corrosion, as shown in Figure (4-4).

The author suggests that the initial resistivity is more considerable in the evaluation of concrete resistance, and that these results were compatible with the Rapid Chloride Permeability Test results. Tables (D-1) and (D-2) in Appendix D show the voltage daily readings for both types of concrete beams in multi-stages of corrosion.

Figure (D-1) shows the resistance values of NC beams, while Figure (D-2) shows the resistance values of the SCC beams.

\subsubsection{Corrosion cracks patterns}

The number and width of the cracks resulting from accelerated corrosion increase with the increasing time of the corrosion process, and they appear as an indicator of the advancing process of corrosion. Spalling may be the next consequence of the advancing corrosion, especially in the congested rebars areas. It was noted that two types of cracks were generated from corrosion activities. The first kind are the transversal cracks originated from the stirrups. The second are the longitudinal cracks lines generated from the flexural reinforcing bars. The electrical isolation of the top epoxy-coated rebars in each beam allowed the top parts of the NC and SCC beams to be free of any longitudinal cracks. The monitored longitudinal cracks on the bottom side are one zigzag line instead of two, as expected. The congestion of the existing two rebars of $20 \mathrm{M}$ in a $150 \mathrm{~mm}$-wide beam caused combined effects on the concrete cover on the bottom side. The shape of the corrosion cracks showed a high correlation with the shape of the corroded rebars cage. The hand sketches show the location, shape, and width of the cracks on the beams affected by corrosion, especially the beams with the advanced stages of corrosion, which gained a dark color from the corrosion products dissipated in the electrolyte.

Table (4-4) concludes the maximum crack widths of the beams from both types of concrete and for the multi stages of the accelerated corrosion. The averages of corrosion crack widths were as follows: 
$\mathrm{NC}$ beams with $5 \%$ mass loss $0.478 \mathrm{~mm}$; SCC beams with $5 \%$ mass loss $0.595 \mathrm{~mm}$. NC beams with $10 \%$ mass loss $0.958 \mathrm{~mm}$; SCC beams with $10 \%$ mass loss $0.708 \mathrm{~mm}$. NC beams with $15 \%$ mass loss $4.921 \mathrm{~mm}$; SCC beams with $15 \%$ mass loss $2.179 \mathrm{~mm}$. NC and SCC beams with $20 \%$ mass loss spalling case were noticed. The noticed exponential increase in the crack width of the linearly increasing mass loss will influence the structural load capacity and failure mode later.

The NC beams with $5 \%$ mass loss showed a slightly lower value of crack width than the SCC beams of similar mass loss. NC beams of $10 \%$ showed a slight increase in the crack width compared with the similar SCC ones; the crack width measurements will continue to increase for the NC beams of $15 \%$ mass loss. Beams of $20 \%$ mass loss of both types showed cases of spalling, however, the SCC beams showed more cases of spalling than the comparable NC beams. The crack width values of the specimens used in this research corresponded to the results of daily voltage readings. The longitudinal cracks on all of the beams of both types of concrete showed wider crack space than the transverse cracks of the same beams over the four stages of corrosion. This is believed to be due to the bigger mass and diameter for the bottom flexural reinforcing steel than the mass of stirrups. The spacing between the two flexural rebars of $20 \mathrm{Mwas} 70 \mathrm{~mm}$, while the stirrups of $7.9 \mathrm{~mm}$ spacing were $175 \mathrm{~mm}$, so the combined action of the two close flexural rebars, with considering the bigger diameter and mass, resulted in wider crack width in the longitudinal direction. Figures $(A-1)$ to $(A-16)$ in appendix-A, show the corrosion cracks patterns for the beams subjected to corrosion from NCC and SCC. These figures contain the maximum crack width measured on each side of the beams.

\subsection{Loading tests and analysis}

In a comparison between NC and SCC beams with relation to pouring concrete during casting, the NC beams were cast using buckets and shovels to transfer concrete from both sides and the middle of the wooden form, and then vibrated for levelling. On the other hand, the SCC beams were cast by transferring the concrete through a handmade wooden funnel without using any vibration. Side-B was used for pouring the concrete for the SCC beams (side-A is the one closer to the long-sided stirrup). As a result of the method and direction of casting, it was noted that for NC beams, six beams failed from side-B, two beams failed from side-A, and the last two failed in the compression zone between the two load points. For the SCC beams, seven beams 
failed at side-A, two beams at side- $B$, and the last one failed in the compression zone between the two load points. Hassan (2008) refers to the variation of the properties along the beams when casting SCC from one side, which can cause poor quality of concrete at the far end due to improper compaction and distribution.

Loading test results are categorized into two types; a) the data obtained from the nine LVDTs and load cell, and $b$ ) the loading cracks patterns. These two kinds of results are combined and integrated with each other to describe the developments associated with the loading stages. The data from the LVDTs showed that the responses of the left and right sides are not similar; therefore, it was logical to install two sets of LVDTs, one on each side to monitor the shear strain $\gamma_{x y}$, the strain in x-direction $\boldsymbol{\epsilon}_{x}$, and the strain in y-direction $\boldsymbol{\epsilon}_{y}$. The data obtained from LVDT1 through LVDT9 vs. Load were used and analyzed as follows:

\subsubsection{Shear strain $\gamma_{x y}$}

As explained in chapter 3, Shear strain left $\gamma_{\text {left }}=(L V D T 3 / 311-L V D T 1 / 311)$ and Shear Strain Right $\left(\gamma_{\text {right }}\right)=(L V D T 6 / 311-L V D T 4 / 311)$. The graphs in Figures (B1-1) to (B1-5) in Appendix B represent the relationship between load (kN) and shear strain for beams in different stages of corrosion and of different types of concrete.

The graphs from load and shear strain $\gamma_{x y}$ were strongly correlated to the loading cracks patterns, and sometimes ended up explaining each other. Cracks were traced over the concrete surfaces as soon as they became visible, and the corresponding load levels were marked on the surface beside each crack, as illustrated in Figures $(C-1)$ to (C-20) in Appendix C.

Table (4-5) shows the ultimate loads at failure $P_{u}$, loads at first flexural crack $P_{f l}$, loads at first diagonal crack $P_{d}$, the effect of corrosion level on $P_{f l} / P_{u}$ and $P_{d} / P_{u}$, and the failure mode. Figure (4-5) and (4-6) show the effects of the corrosion stage on the percentage of $P_{f l} / P_{u}$ and $P_{d} / P_{u}$. Figure (4-7) shows the effects of stages of corrosion measured by mass loss on the average ratios of $\mathrm{P}_{\mathrm{fl}} / \mathrm{P}_{\mathrm{u}}$ and $\mathrm{P}_{\mathrm{d}} / \mathrm{P}_{\mathrm{u}}$ in a graph.

- Shear strain $\gamma_{x y}$ for NC and SCC beams with zero mass loss.

The beams represented in this stage of corrosion were NC B1, NC B2, SCC B1, and SCC B2. The information obtained from the graphs in Figure (B1-1) and Table (4-5) 
shows a variation from 152.08 - $180.18 \mathrm{kN}$ as a failure load $\left(P_{u}\right)$ for normal concrete beams and a variation from 143.3 - $190.52 \mathrm{kN}$ as a failure load for the SCC beams; while the average of failure load in both types of concrete beams was slightly above $166 \mathrm{kN}$. The loads at first flexural crack $\left(P_{\mathrm{fl}}\right)$ were $40-50 \mathrm{kN}$, and the loads at first diagonal cracks $\left(P_{d}\right)$ were $80-95 \mathrm{kN}$ for the NC beams, while the load at first flexural crack was $20 \mathrm{kN}$, and the loads at first diagonal cracks were $40-50 \mathrm{kN}$ for the SCC beams. The percentage of $P_{f l} / P_{u}$ is $(22.2 \%-32.8 \%)$ and $P_{d} / P_{u}$ is $52.6 \%-52.7 \%$ of the NC beams, while $P_{f l} / P_{u}$ is $10.49 \%-13.95 \%$ and $P_{d} / P_{u}$ is $26.24 \%-27.9 \%$ of the SCC beams. Therefore, the percentage of $\mathrm{P}_{\mathrm{fl}} / \mathrm{P}_{\mathrm{u}}$ and $\mathrm{P}_{\mathrm{d}} / \mathrm{P}_{\mathrm{u}}$ of the $\mathrm{NC}$ beams showed double of the same values of SCC beams.

This is believed to be due to:

1. Higher amounts of coarse aggregates in NC than in SCC will result in higher contribution of aggregate interlocking mechanism between the fracture surfaces. Lachemi et al. (2005) referred to experimental and theoretical studies that mentioned $30 \%$ to $90 \%$ of the aggregate interlock mechanism contributes to the post-cracking shear resistance of the concrete (Swamy and Andriopoulos 1974; Taylor 1970). Crushing strength of aggregates related to compressive strength of concrete and type of shear cracks and surface characteristics of crack planes all influence post-cracking shear strength.

2. Water/Binder (W/B ratio) in both NC and SCC is 0.4 , however the W/C ratio of $\mathrm{NC}$ is 0.53 and in SCC is 0.57 . This may affect the future structural behaviour of the beams that used these types of concrete.

3. Table (3-8), shows that the values of compressive strength at ages 7 and 28 days and the tensile strength of the NC specimens were higher than in the SCC ones.

4. Theoretically, there is no need to apply vibration on SCC because of its liquid nature. However, the lack of vibration may have left some air bubbles and air gaps that led to weaker structure compared to the vibrated concrete.

5. In addition to all of the above reasons, the NC beams were older than the SCC beams by one week. This may have had minor effects on relatively higher compressive and tensile strength.

The failure mode of both NC and SCC beams of zero mass loss showed initial response of flexural cracks. By increasing the load, diagonal cracks appeared and propagated in the shear zones in the beams to end the test with shear failure. From the beginning of 
the test up to the point of failure load, the deformations were elastic, but after this stage of loading, the values of shear strain increased as the plastic deformations developed in the beams. The beams of both types of concrete did not show symmetrical response in both sides. Therefore, as the graphs show, in most of the beams there was a variation of shear strain values in the stage of plastic deformation at one side more than the other side, followed by propagation of the cracks in the weaker side, then failure.

- Shear strain $\gamma_{x y}$ for NC and SCC beams with $5 \%$ mass loss.

The beams represented in this stage of corrosion were NC B3, NC B9, SCC B4, and SCC B10. The graphs in Figure (B1-2) and Table (4-5) show a variation of $189.18-$ $200.62 \mathrm{kN}$ as a failure load $\left(P_{\mathrm{u}}\right)$ for $\mathrm{NC}$ beams and a variation of $191.52-196.29 \mathrm{kN}$ as a failure load for SCC beams. The averages of the failure loads were similar for both types of concrete beams (193.9 for SCC and 194.9 for NC). The loads at first flexural crack $\left(P_{f f}\right)$ ranged from $20-30 \mathrm{kN}$, and the loads at first diagonal cracks $\left(P_{d}\right)$ were $50-130 \mathrm{kN}$ for the NC beams; for the SCC beams, the load at first flexural crack ranged from $10-20 \mathrm{kN}$, and the loads at first diagonal cracks were $20-90 \mathrm{kN}$. As shown in Table (4-5), the percentage of $P_{f} / P_{u}$ is $10.57 \%-14.95 \%$ and $P_{d} / P_{u}$ is $26.43 \%-64.8 \%$ of the NC beams, while $P_{f} / P_{u}$ is $5.09 \%-10.44 \%$ and $P_{d} / P_{u}$ is $10.18 \%-47.0 \%$ of the SCC beams.

The average failure load increased in the category with $5 \%$ mass loss to $116.8 \%$, compared to the beams with zero mass loss. It is obvious that the plain reinforcing steel bars used in the stirrups of the beams have lower bond strength than the deformed rebars. The beams with zero mass loss showed shear failure mode, but three out of the four beams with $5 \%$ mass loss showed flexural failure by crushing the concrete in the compression zone of these beams. That means the plain rebars showed less bond strength in the beams with zero mass loss than the similar stirrups of the beams with $5 \%$ mass loss. For this reason, the stirups of the $5 \%$ mass loss beams hold a higher load than the zero mass loss ones, which resulted in transferring the higher load to the flexural rebars that led to flexural failure in the compression zone of the beam. The failure appeared as a crushed crescent of the concrete between the two loads.

The failure mode of the majority of the beams with $5 \%$ mass loss started with flexural cracks, and then diagonal cracks appeared and propagated on both sides of the beam, followed by sudden concrete crashing in the compression zone of the beam section between the two beam loads. This development in the failure behaviour was 
the major difference when compared with the zero mass loss beams' behavior. Due to the use of the strut-tie model, an arch-shape of crushed concrete developed between the two points load as a result of high stresses, as shown in Figure (4-8). According to the strut-tie model, the area between the two points load is subjected to compression. When this compression stress exceeds the compressive strength of the concrete, concrete crushing in this zone is expected.

- Shear strain $\gamma_{x y}$ for NC and SCC beams with $10 \%$ mass loss.

The beams represented in this stage of corrosion were NC B7, NC B10, SCC B5, and SCC B8. The graphs in Figure (B1-3) and Table (4-5) show a variation of 120.31 $162.19 \mathrm{kN}$ as a failure load $\left(\mathrm{P}_{\mathrm{u}}\right)$ for $\mathrm{NC}$ beams and a variation of $127.2-163.74 \mathrm{kN}$ as a failure load for the SCC beams. Although there was a variation between the beams of the same type of concrete with relation to failure load, the average for NC beams was $141.25 \mathrm{kN}$ and $145.47 \mathrm{kN}$ for SCC beams. The load at first flexural crack $\left(P_{\mathrm{fl}}\right)$ was $20 \mathrm{kN}$, and the loads at first diagonal cracks $\left(P_{d}\right)$ were $40-70 \mathrm{kN}$ for the NC beams, while the load at first flexural crack was $10-30 \mathrm{kN}$, and the loads at first diagonal cracks were $40-50 \mathrm{kN}$ for the SCC beams. As shown in Table (4-5), the percentage of $\mathrm{P}_{\mathrm{fl}} / \mathrm{P}_{\mathrm{u}}$ is $12.33 \%-16.62 \%$ and $\mathrm{P}_{\mathrm{d}} / \mathrm{P}_{\mathrm{u}}$ is $33.24 \%-43.15 \%$ )of the NC beams, while $\mathrm{P}_{\mathrm{fl}} /$ $P_{u}$ is $6.1 \%-23.58 \%$ and $P_{d} / P_{u}$ is $24.4 \%-39.3 \%$ of the SCC beams.

The average failure load decreased in the category of $10 \%$ mass loss beams to $86 \%$ of the failure load of the group with zero mass loss.

The failure mode of beams with $10 \%$ mass loss started with flexural cracks around the mid-bottom area of the beams, followed by diagonal cracks that propagated until failure. The corrosion cracks showed higher influence on the loading cracks and failure mode.

- Shear strain $\gamma_{x y}$ for NC and SCC beams with $15 \%$ mass loss.

The beams represented in this stage of corrosion were NC B4, NC B5, SCC B6, and SCC B7. The graphs in Figure (B1-4) and Table (4-5) show a variation of 70.32 $73.65 \mathrm{kN}$ as a failure load $\left(P_{u}\right)$ for $\mathrm{NC}$ beams and a variation of $71.21-136.53 \mathrm{kN}$ for the SCC beams. Three of the beams showed close failure load, while the fourth beam (SCC B7) showed a higher value of failure load of $136.53 \mathrm{kN}$. The loads at first flexural crack $\left(P_{f l}\right)$ were $20-50 \mathrm{kN}$, and the loads at first diagonal cracks $\left(P_{d}\right)$ were $50-70 \mathrm{kN}$ for the NC beams, while the load at first flexural crack was $40-50 \mathrm{kN}$, and the loads 
at first diagonal cracks were $60-70 \mathrm{kN}$ for the SCC beams. As shown in Table (4-5), the percentage of $\mathrm{P}_{\mathrm{fl}} / \mathrm{P}_{\mathrm{u}}$ is $28.44 \%-69.45 \%$ and $\mathrm{P}_{\mathrm{d}} / \mathrm{P}_{\mathrm{u}}$ is $71.1 \%-95.0 \%$ of the NC beams, while $\mathrm{P}_{\mathrm{fl}} / \mathrm{P}_{\mathrm{u}}$ is $36.6 \%-56.17 \%$ and $\mathrm{P}_{\mathrm{d}} / \mathrm{P}_{\mathrm{u}}$ is $51.27 \%-84.25 \%$ of the SCC beams.

The average failure load decreased in the category of $15 \%$ mass loss beams to $52.79 \%$ of the failure load of the group with zero mass loss.

The corrosion impact severely affected the failure mode and the load capacity due to the huge changes associated with $15 \%$ mass loss, such as losing enormous amount of bond and cross-sectional area of the reinforcing steel bars, in addition to the effects of the corrosion cracks. NC and SCC beams with 15\% mass loss showed severe corrosion on one of the bottom longitudinal rebars, resulting in a wide crack on one of the ends of the beams that controlled the failure mode of this group. As a result of the big corrosion crack, a diagonal crack appeared and propagated in the shear zone, followed by failure. In addition to the main shear crack failure, the beams in this group showed compression cracks on the top side associated with the final failure cracks, starting from the loading point towards the top corner of the failing side. The author attributes this to the slip of the concrete body from the severely corroded rebars at the failing corner, as well as the relatively large deformation of the beams, especially the NC beams in this group. NC B4 and SCC B6 showed the first flexural crack above the longitudinal bottom rebars.

- Shear strain $\gamma_{x y}$ for NC and SCC beams with $20 \%$ mass loss.

The beams represented in this stage of corrosion were NC B6, NC B8, SCC B3, and SCC B9. The graphs in Figure (B1-5) and Table (4-5) show a variation of 45.1 $62.32 \mathrm{kN}$ as a failure load $\left(P_{u}\right)$ for $N C$ beams and a variation of $53.1-81.76 \mathrm{kN}$ for the SCC beams.

The NC and SCC beams at the stage of corrosion with $20 \%$ mass loss showed an excessive amount of corrosion with extreme deterioration in bond, cross-sectional area of the rebars, wider cracks and spalling. The concrete pieces spalled from the beams were reattached to the body of the beam using epoxy to maintain the same shape for all of the tested beams. The failure load varied from $45.1-62.32 \mathrm{kN}$ for NC beams, and $53.1-81.76 \mathrm{kN}$ for SCC beams. The loads at first flexural crack $\left(P_{\mathrm{fl}}\right)$ were $10-40 \mathrm{kN}$, and the loads at first diagonal cracks $\left(P_{d}\right)$ were $40-44 \mathrm{kN}$ for the NC beams, while the load at first flexural crack was $30-50 \mathrm{kN}$, and the loads at first 
diagonal crack were $30-80 \mathrm{kN}$ for the SCC beams. As shown in Table (4-5), the percentage of $\mathrm{P}_{\mathrm{fl}} / \mathrm{P}_{\mathrm{u}}$ is $16.04 \%-88.69 \%$ and $\mathrm{P}_{\mathrm{d}} / \mathrm{P}_{\mathrm{u}}$ is $64.18 \%-97.56 \%$ of the NC beams, while $\mathrm{P}_{\mathrm{fl}} / \mathrm{P}_{\mathrm{u}}$ is $56.49 \% 0^{-} 61.15 \%$ and $\mathrm{P}_{\mathrm{d}} / \mathrm{P}_{\mathrm{u}}$ is $56.49 \% 0^{-} 97.84 \%$ of the SCC beams.

The average failure load decreased in the category of $20 \%$ mass loss beams to $32.25 \%$ for NC and $40.49 \%$ for SCC beams at zero mass loss.

In this research, corrosion impact reached its peak at $20 \%$ mass loss, resulting in a decrease in the bond strength (which was the lowest among the groups), increase in the crack width, and many cases of spalling compared with the previous stages. The failure mode started with a few flexural cracks, followed by diagonal cracks that led to failure. The corrosion cracks showed a big influence on loading cracks and failure mode. The SCC beams in this category showed compression cracks on the top side of the final failure side, which happened on the same side as the spalling in the bottom corner.

\subsubsection{Longitudinal strain $\boldsymbol{\epsilon}_{x}$}

As explained in chapter 3 , the strain in $\mathrm{X}$-direction was calculated as $\boldsymbol{\epsilon}_{x \text { left }}=\frac{L V D T 1}{311 \mathrm{~mm}}+\frac{L V T D 3}{311 \mathrm{~mm}}-\frac{L V D T 2}{220 \mathrm{~mm}}$ for the left side $\boldsymbol{\epsilon}_{x \text { Right }}=\frac{L V D T 4}{311 \mathrm{~mm}}+\frac{L V T D 6}{311 \mathrm{~mm}}-\frac{L V D T 5}{220 \mathrm{~mm}}$ for the right side

The relationship between load $(\mathrm{kN})$ and X-direction strain $(\mathrm{mm} / \mathrm{mm})$ for the NC and SCC beams at each stage of corrosion is illustrated in Graphs (B2-1) to (B2-5) in Appendix B. The values of $\epsilon_{x}$ vs. load showed the changes in the deformation of the beams in the longitudinal direction as a response to the stresses resulting from loading. These values are useful for the following reasons:

1. To evaluate the bond stresses between the longitudinal rebars and the concrete, as well as the influence of aggregate interlock for each beam. Collins et al. (2007) referred to the direct effects of the crack widths that govern aggregate interlock capacity on the longitudinal strain at mid-depth $\boldsymbol{\epsilon}_{x}$.

2. The values of $\boldsymbol{\epsilon}_{x}$ were measured at the neutral axis, therefore $\boldsymbol{\epsilon}_{x}$ will vary according to the location from the neutral axis for the effects of compressive stresses above the neutral axes and tensile stresses below it. 
3. The values of $\boldsymbol{\epsilon}_{x}$ provided beside $\boldsymbol{\epsilon}_{c}^{\prime}$ that corresponds to $f_{c}^{\prime}$ allow us to calculate the strain on any height of the section, as illustrated in Figure (4-9). Due to the small values of $\boldsymbol{\epsilon}_{c}$ compared with $\boldsymbol{\epsilon}_{t}$, and the significant stiffness of concrete in compression, Collins et al. 2007) made a reasonable approximation by assuming $\boldsymbol{\epsilon}_{x}=\boldsymbol{\epsilon}_{t} / 2$.

Since the LVDTs reading is positive in compression and negative in tension, the majority of the beams showed very minor tensile deformation in the $\mathrm{x}$-direction before failure. The longitudinal strain $\boldsymbol{\epsilon}_{x}$ resulted in vertical cracks in the area between the two points load (the area of pure moment), while it developed with the transverse strain to produce diagonal cracks. The LVDTs installed on the left and right sides could only collect the data related to $\boldsymbol{\epsilon}_{x}$ on the left and right side that resulted from the deformation of the shear zones before and after cracking.

\subsubsection{Transverse strain $\epsilon_{y}$}

As explained in chapter 3 , the strain in $\mathrm{Y}$-direction was calculated as

$$
\boldsymbol{\epsilon}_{y \text { Left }}=\frac{L V D T 2}{220 \mathrm{~mm}} ; \boldsymbol{\epsilon}_{y \text { Right }}=\frac{L V D T 5}{220 \mathrm{~mm}}
$$

The relationship between load $(\mathrm{kN})$ and $\mathrm{Y}$-direction strain $(\mathrm{mm} / \mathrm{mm})$ for the $\mathrm{NC}$ and SCC beams at each stage of corrosion is illustrated in Graphs (B3-1) to (B3-5) in Appendix B. These values showed the changes in the deformation of the beams in the transverse direction as a response to the stresses resulting from loading. The transverse strain values, at ultimate failure load, showed the following variation in the beams of different stages of corrosion:

- NC beams with zero mass loss showed transverse strain values of -0.0036 to 0.0052 ;

- SCC beams with zero mass loss showed transverse strain values of -0.0018 to 0.0117 ;

- NC beams with $5 \%$ mass loss showed transverse strain values of -0.00063 to 0.0024 ;

- SCC beams with 5\% mass loss showed transverse strain values of 0 to -0.0041 ;

- NC beams with $10 \%$ mass loss showed transverse strain values of -0.0006 to 0.0027 ; 
- SCC beams with $10 \%$ mass loss showed transverse strain values of -0.00013 to 0.0013;

- NC beams with $15 \%$ mass loss showed transverse strain of -0.0002 to -0.01 ;

- SCC beams with $15 \%$ mass loss showed transverse strain of 0 to -0.0001 ;

- $\quad$ NC beams with $20 \%$ mass loss showed transverse strain values of 0 to -0.0016 ;

- SCC beams with $20 \%$ mass loss showed transverse strain values 0.00036 to $4.545 \times 10^{-5}$;

The transverse strain was influenced by the bond stresses between the stirrups, the surrounding concrete, and the effects of the aggregate interlock. Therefore, the beams with zero mass loss showed more flexibility with respect to transverse strain values than the beams with more mass loss. However, the NC and SCC beams did not show significant differences with respect to the transverse deformation vs. load for each stage of corrosion.

\subsubsection{Mid-span deflection $\Delta$}

Mid-span deflection values can be obtained directly from LVDT 8. Figures (B4-1) to (B4-5) in Appendix B represent the load vs. mid-span deflection.

The cracks resulting from load increase decreased the moment of inertia from the gross transformed moment of inertia $\left(I_{g}\right)$ to the cracked transformed moment of inertia $\left(I_{c r}\right)$. This reduction led to a nonlinear load deflection (Brzev and Pao, 2006).

By taking the slope of the load vs. mid-span deflection graphs at the elastic stage of the beams with zero mass loss as a reference, the observed slope of the graphs at the elastic stage for beams with $5 \%$ mass loss was slightly higher than the reference. The beams with $10 \%, 15 \%$, and $20 \%$ mass loss showed a descent in slope of the elastic stage with the increase in mass loss. The decreased flexural stiffness represented by the decrease of the slope is additional evidence of a lower structural capacity of the beams in the advanced stages of corrosion. Similarly, the gap between load at first flexural crack $\left(P_{f l}\right)$ and failure load $\left(P_{u}\right)$ also increased with $5 \%$ mass loss, then decreased with $10 \%, 15 \%$, and $20 \%$, as shown in Table (4-5) and Figure (4-5).

The slope of the NC beams with zero and $5 \%$ mass loss showed a slight superiority to the slope of the SCC beams. At $10 \%$ mass loss, the values of the slope were close to each other, while at $15 \%$ and $20 \%$ mass loss, the slope of the SCC beams was slightly higher than that of the NC beams, as shown in Figures (B4-1) to (B4-5). This is believed to be due to the influence of higher aggregate interlock at zero and $5 \%$ 
mass loss, but with the increase of the corrosion stage, the cracks broaden and the effect of aggregate interlock is decreased.

El Maaddawy et al. (2005) provide an analytical model to predict nonlinear flexural behavior of both corroded and newly constructed reinforced concrete. The model explains the effects of corrosion on the reduction in the steel area and the change in bond strength at the steel-to-concrete interface. They have found significant increase in mid-span deflections with the increase of corrosion.

Ballim et al. (2001) tested the simultaneous effects of load and corrosion conditions on the deflection of RC beams to simulate the conditions of real concrete structures in situ. The results showed that the mid-span deflection increased with beams in advanced stages of corrosion.

Castel et al. (2000) attributed the increase of mid-span deflections to the loss of bond between the tensile steel and the surrounding concrete. They considered the increase of the tensile stresses in the rebars to be a result of bond loss.

\subsubsection{Deflection along the beam}

Deflection along the beams was monitored through three LVDTs located in the bottom side of each beam. Figure (3-14) shows the locations of LVDTS 7, 8, and 9, which were installed to provide the data used to produce the graphs in Figure (B5-1) to (B5-19) in Appendix B. These graphs illustrate the deflection of each beam with the load increment stages. The shape of the curves may refer to the side of failure of the related beam, so the side that the curve leans towards is the side of failure.

\subsection{Loading crack patterns}

At the end of each loading test, crack pattern and stage of loading on each crack were documented, as shown in Figures (C-1) to (C-20) in Appendix C.

The beams with zero mass loss NC B1, NC B2, SCC B1, and SCC B2 showed the expected response of initial vertical cracks in the mid-span of the bottom side, followed by diagonal cracks, which extended and expanded until failure. NC B2 and SCC B1

showed higher load capacity and more diagonal cracks than the other two beams especially, on the side of the failure.

For the beams with 5\% mass loss NC B3, NC B9, SCC B4, and SCC B10, the point that attracted attention is that three of these beams showed clear development in the failure mode associated with the change from zero to $5 \%$ mass loss. The cracks in these 
three beams showed the case of the crushed concrete arch between the two load points. SCC B4 behaved in a different manner than the other beams, influenced by the severe corrosion located on side $A$ of the beam, which led to a diagonal crack ending in failure at that side. The beams in this stage of corrosion started to show continuity of the loading cracks with the corrosion cracks.

For the beams with $10 \%$ mass loss NC B7, NC B10, SCC B5, and SCC B8, the large amount of mass loss - with the associated bond loss - at this stage of corrosion showed a drop in the loading capacity and a change in the failure mode. It has been noted that the number of flexural and diagonal cracks is much smaller than the total number of cracks in the beams with zero and $5 \%$ mass loss. The author attributes this phenomenon to the lower influence of the reinforcing steel bars and higher influence of the remaining cracked concrete of the beams. In this stage of corrosion, the loading cracks were influenced by the corrosion cracks that were marked with red, followed by beam failure at the diagonal shear crack.

At $15 \%$ mass loss, NC B4, NC B5, SCC B6, and SCC B7 showed wider corrosion cracks, especially in the flexural rebars at one of the bottom ends of the beams that controlled the loading cracks and failure mode. The first flexural crack for NC B4 started from the longitudinal crack towards the upper side, which refers to the effect of the concrete separation between the concrete cover at the bottom side and the concrete of the beam body above the layer of the bottom flexural rebars. The concentrated corrosion on a bottom corners left that corner very weak compared to the rest of the beam, and the load increase led to diagonal shear crack. Additional load widened the shear crack until failure and caused a top longitudinal crack to appear on the top corner of the failure side. The slippery layer resulted from corrosion products, corrosion cracks, and the remaining weak rebars allowed the concrete box on the failure side to separate easily.

For the beams with $20 \%$ mass loss NC B6, NC B8, SCC B3, and SCC B9, the average of max-failure load was about $36 \%$ from the average max-failure load of the control beams with zero mass loss. The huge amount of corrosion, the widest crack noticed in this research, and the spalling of concrete in this category of beams influenced not only the lower average of the failure load but the failure mode as well. The loading cracks in this group were less significant than the previous groups with less mass loss. Irregularly, some of the flexural vertical cracks started not in the mid flexural zone but at the shear zones of the beam, as in the case of SCC B9 and NC B6. 
The crack patterns in NC and SCC beams were influenced more by the stage of corrosion than the type of concrete. In fact, the stage of corrosion had more effect on the structural condition, load capacity, crack pattern and failure mode than the type of concrete.

\subsection{Mass loss measuring}

Table (4-6) shows the weight of steel components of each beam before and after the corrosion activities, while Table (4-7) shows the comparison between the hypothetical and real mass loss of each beam. The results showed high correlation between the calculated and real mass loss for both NC and SCC, which proves the Faraday equation to have high credibility. These results showed that choosing the constant current along the accelerated corrosion process was much better than using constant voltage to calculate the time needed for targeting a specific amount of mass loss. In addition, all of the other parameters accompanying the corrosion process were suitable for achieving the targeted mass loss. These parameters were the electrolyte concentration, lab temperature, the values of the constants in Faraday's formula, the wire connection between the concrete beams as anode, the metal mesh as cathode, and the power supply. 


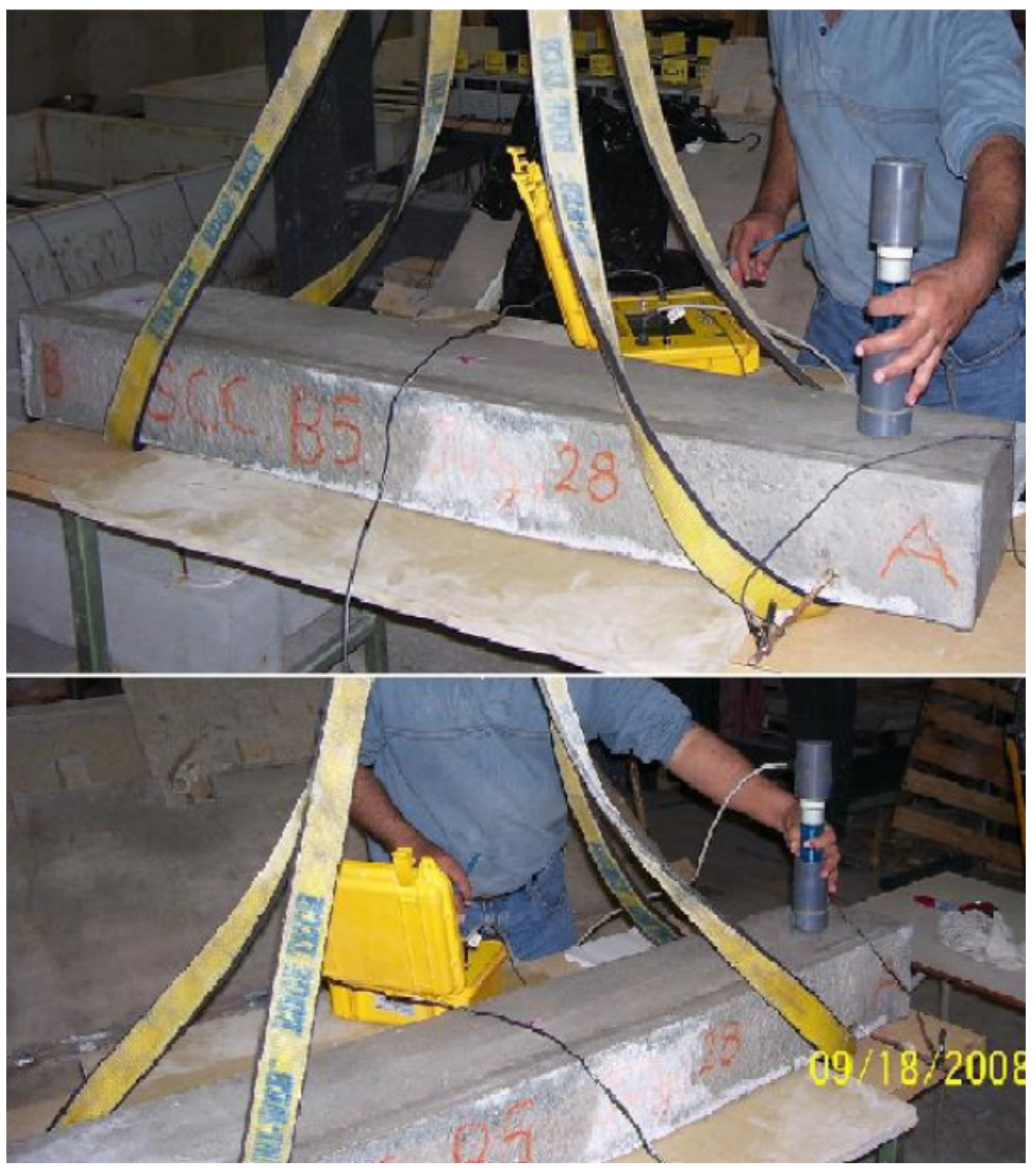

Figure (4-1): Half-cell potential test applied on NC and SCC beams numbered 3 - 10. 
Table (4-1): Half-cell potential test readings for the NC and SCC beams.

\begin{tabular}{|c|c|c|c|c|c|c|c|}
\hline \multirow{2}{*}{ Beam No } & & \multicolumn{3}{|l|}{ NC } & \multicolumn{3}{|l|}{ SCC } \\
\hline & & side A & Center & Side B & side $A$ & Center & Side B \\
\hline \multirow{3}{*}{ B\#3 } & Left Side & -0.963 & -0.986 & -0.987 & -0.995 & -1.006 & -1.007 \\
\hline & Bottom & -0.962 & -0.973 & -0.978 & -0.995 & -0.996 & -1.007 \\
\hline & Right Side & -0.963 & -0.984 & -0.985 & -0.996 & -1.007 & -1.004 \\
\hline \multirow{3}{*}{ B\#4 } & Left Side & -0.896 & -0.934 & -0.931 & -0.956 & -0.963 & -0.971 \\
\hline & Bottom & -0.892 & -0.927 & -0.932 & -0.948 & -0.945 & -0.945 \\
\hline & Right Side & -0.902 & -0.932 & -0.931 & -0.958 & -0.97 & -0.964 \\
\hline \multirow{3}{*}{ B\#5 } & Left Side & -0.893 & -0.909 & -0.906 & -0.478 & -0.435 & -0.473 \\
\hline & Bottom & -0.892 & -0.901 & -0.904 & -0.5 & -0.452 & -0.501 \\
\hline & Right Side & -0.895 & -0.909 & -0.911 & -0.491 & -0.458 & -0.497 \\
\hline \multirow{3}{*}{ B\#6 } & Left Side & -0.851 & -0.893 & -0.894 & -0.927 & -0.928 & -0.93 \\
\hline & Bottom & -0.854 & -0.888 & -0.891 & -0.903 & -0.914 & -0.919 \\
\hline & Right Side & -0.851 & -0.897 & -0.893 & -0.921 & -0.931 & -0.931 \\
\hline \multirow{3}{*}{ B\#7 } & Left Side & -0.814 & -0.855 & -0.862 & -0.896 & -0.916 & -0.915 \\
\hline & \begin{tabular}{|l|} 
Bottom \\
\end{tabular} & -0.828 & -0.849 & -0.86 & -0.903 & -0.913 & -0.917 \\
\hline & Right Side & -0.822 & -0.856 & -0.86 & -0.903 & -0.913 & -0.919 \\
\hline \multirow{3}{*}{ B\#8 } & Left Side & -0.652 & -0.654 & -0.652 & -1.002 & -1.006 & -1.007 \\
\hline & Bottom & -0.653 & -0.654 & -0.656 & -0.999 & -1.009 & -1.003 \\
\hline & Right Side & -0.658 & -0.652 & -0.663 & -1.006 & -1.009 & -1.006 \\
\hline \multirow{3}{*}{ B\#9 } & Left Side & -0.882 & -0.915 & -0.929 & -0.987 & -0.99 & -0.995 \\
\hline & Bottom & -0.885 & -0.917 & -0.924 & -0.988 & -0.987 & -0.989 \\
\hline & Right Side & -0.892 & -0.92 & -0.941 & -0.993 & -0.993 & -0.996 \\
\hline \multirow{3}{*}{ B\#10 } & Left Side & -0.882 & -0.91 & -0.912 & -0.962 & -0.975 & -0.981 \\
\hline & Bottom & -0.885 & -0.906 & -0.914 & -0.965 & -0.965 & -0.966 \\
\hline & Right Side & -0.886 & -0.907 & -0.914 & -0.966 & -0.972 & -0.974 \\
\hline
\end{tabular}

Table (4-2): Categories of half-cell potential test results (Half-cell potential test manual, 2003)

\begin{tabular}{|l|l|}
\hline$A=-0.420$ & A\&B $=90 \%$ chance corrosion is occurring in this area. \\
\cline { 1 - 1 }$B=-0.350$ & C\&D= Corrosion activity over this area is uncertain. \\
\cline { 1 - 1 }$C=-0.280$ & E- $G=90 \%$ chance that no corrosion activity is present over \\
\cline { 1 - 1 }$=-0.210$ & this area. \\
\hline$F=-0.070$ & \\
\hline$G=0.000$ &
\end{tabular}


Table (4-3): Categories of corrosion conditions of the copper/copper sulphate half-cell vs. the voltmeter reading ranges, ASTM C 876 - 91 (Reapproved 1999).

\begin{tabular}{|l|l|}
\hline $\begin{array}{l}\text { Copper/copper } \\
\text { sulphate }\end{array}$ & Corrosion condition \\
\hline$>-200 \mathrm{mV}$ & Low (10\% risk of corrosion) \\
\hline-200 to $-350 \mathrm{mV}$ & Intermediate corrosion risk \\
\hline$<-350 \mathrm{mV}$ & High (<90\% risk of corrosion) \\
\hline$<-500 \mathrm{mV}$ & Severe corrosion \\
\hline
\end{tabular}

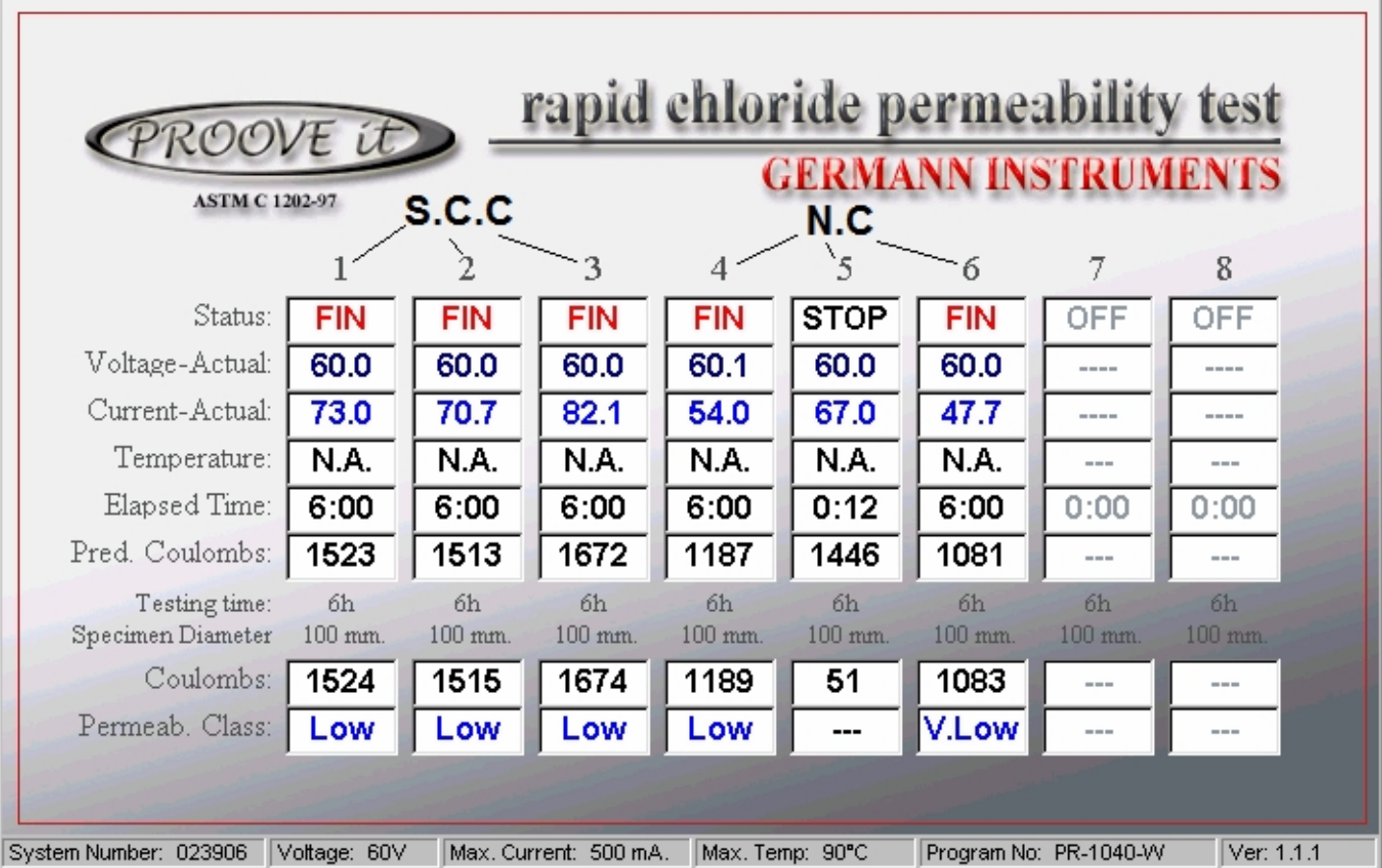

Figure (4-2): The RCPT results at age of 64 days for the NC samples, and 57 days for the SCC samples. 


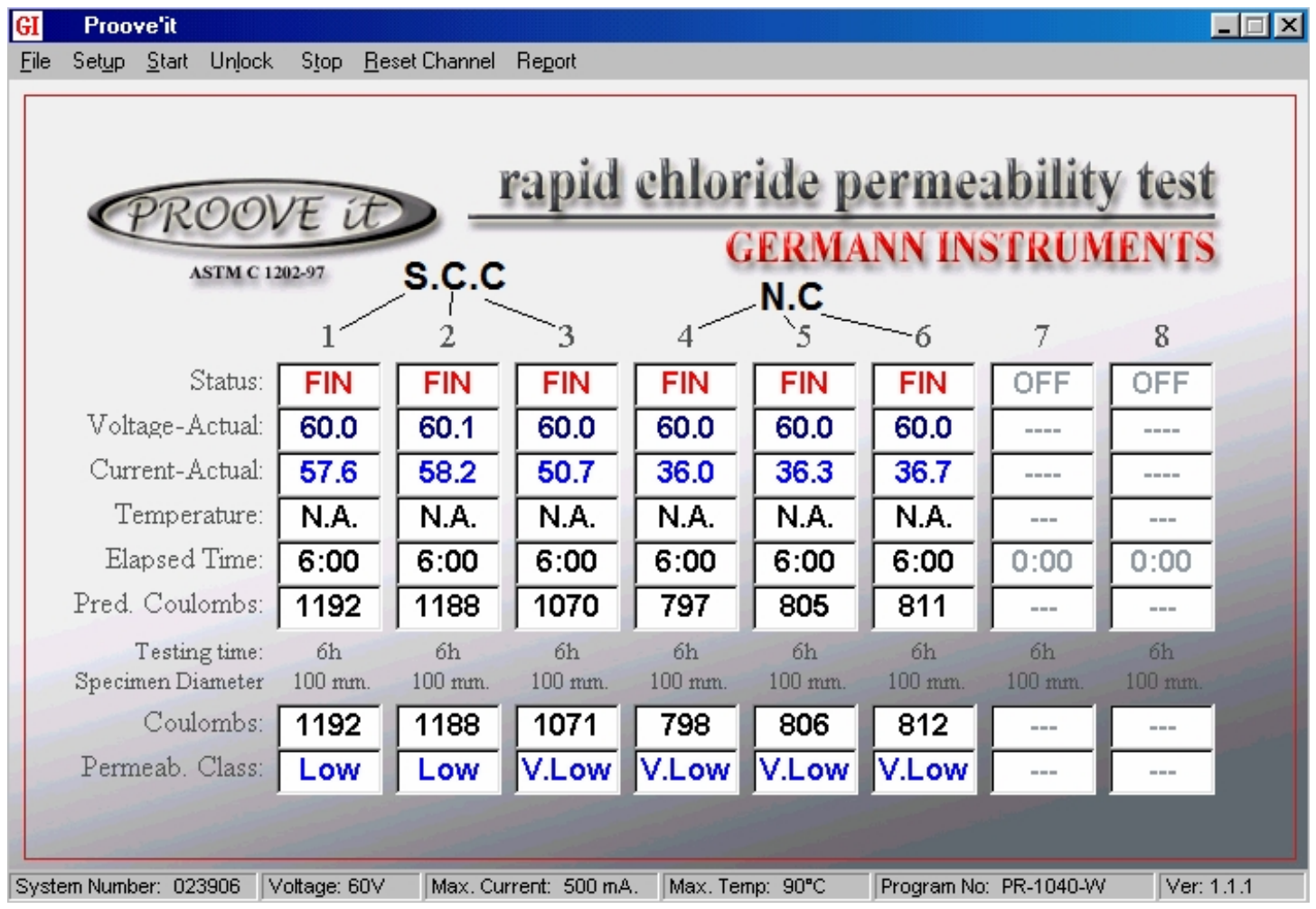

Figure (4-3): The RCPT results at age of 137 days for the NC samples, and 130 days for the SCC samples.

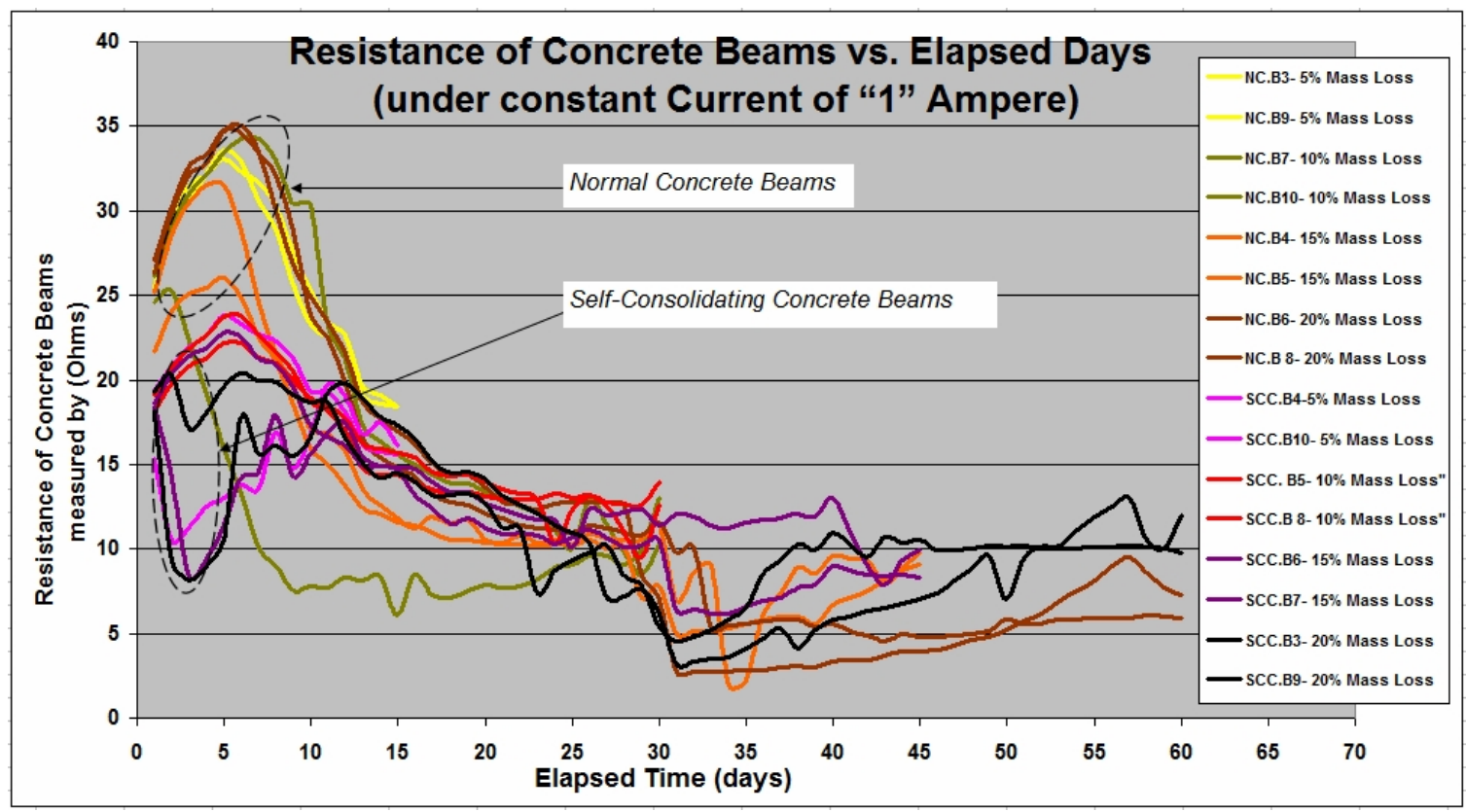

Figure (4-4): The electrical resistance values for both NC beams and SCC beams measured by (ohms) vs. the elapsed time by (days). 
Table (4-4): Max crack width on NC and SCC beams at multi-stages of corrosion.

\begin{tabular}{|c|c|c|c|c|c|c|c|}
\hline \begin{tabular}{|l|} 
Corrosion \\
Stage \\
(Mass \\
Loss)
\end{tabular} & $\begin{array}{l}\text { Type of } \\
\text { Concrete } \\
\text { and } \\
\text { beam } \\
\text { number }\end{array}$ & $\begin{array}{l}\text { Beam } \\
\text { Face }\end{array}$ & $\begin{array}{l}\text { Max. } \\
\text { Crack } \\
\text { width } \\
(\mathrm{mm})\end{array}$ & $\begin{array}{l}\text { Corrosion } \\
\text { Stage } \\
\text { (Mass } \\
\text { Loss) }\end{array}$ & $\begin{array}{l}\text { Type of } \\
\text { Concrete } \\
\text { and } \\
\text { beam } \\
\text { number }\end{array}$ & $\begin{array}{l}\text { Beam } \\
\text { Face }\end{array}$ & $\begin{array}{l}\text { Max. } \\
\text { Crack } \\
\text { width } \\
\text { (mm) }\end{array}$ \\
\hline \multirow{6}{*}{$5 \%$} & \multirow{6}{*}{ NC-B 3} & Top & 0.15 & \multirow{6}{*}{$5 \%$} & \multirow{6}{*}{ SCC-B 4} & Top & 0.45 \\
\hline & & Left & 0.13 & & & \begin{tabular}{|l|} 
Left \\
\end{tabular} & 1.4 \\
\hline & & Bottom & 0.33 & & & Bottom & 0.6 \\
\hline & & Right & 0.23 & & & \begin{tabular}{|l|} 
Right \\
\end{tabular} & 0.25 \\
\hline & & Side-A & & & & Side-A & 0.4 \\
\hline & & Side-B & & & & Side-B & \\
\hline \multirow{6}{*}{$5 \%$} & \multirow{6}{*}{ NC-B 9} & Top & 0.4 & \multirow{6}{*}{$5 \%$} & \multirow{6}{*}{ SCC-B 10} & Top & 0.3 \\
\hline & & \begin{tabular}{|l|} 
Left \\
\end{tabular} & 0.98 & & & \begin{tabular}{|l|} 
Left \\
\end{tabular} & 0.9 \\
\hline & & Bottom & 0.8 & & & Bottom & no crack \\
\hline & & Right & 0.9 & & & Right & 0.65 \\
\hline & & Side-A & & & & Side-A & 1 \\
\hline & & Side-B & 0.65 & & & Side-B & \\
\hline \multirow{6}{*}{$10 \%$} & \multirow{6}{*}{ NC-B 7} & \begin{tabular}{|l} 
Top \\
\end{tabular} & 0.3 & \multirow{6}{*}{$10 \%$} & \multirow{6}{*}{ SCC-B 5} & Top & 0.8 \\
\hline & & Left & 0.4 & & & Left & 0.4 \\
\hline & & Bottom & 0.2 & & & Bottom & 1.25 \\
\hline & & Right & 2 & & & \begin{tabular}{|l|} 
Right \\
\end{tabular} & 1.6 \\
\hline & & Side-A & 1 & & & Side-A & 1.2 \\
\hline & & Side-B & 1.2 & & & Side-B & 0.2 \\
\hline \multirow{6}{*}{$10 \%$} & \multirow{6}{*}{ NC-B 10} & Top & 1 & \multirow{6}{*}{$10 \%$} & \multirow{6}{*}{ SCC-B 8} & Top & 0.2 \\
\hline & & Left & 1.1 & & & \begin{tabular}{|l|} 
Left \\
\end{tabular} & 0.45 \\
\hline & & Bottom & 3 & & & Bottom & 1.25 \\
\hline & & Right & 0.2 & & & \begin{tabular}{|l|} 
Right \\
\end{tabular} & 0.35 \\
\hline & & Side-A & 1 & & & Side-A & 0.45 \\
\hline & & Side-B & 0.1 & & & Side-B & 0.35 \\
\hline \multirow{6}{*}{$15 \%$} & \multirow{6}{*}{ NC-B 4} & Top & 0.7 & \multirow{6}{*}{$15 \%$} & \multirow{6}{*}{ SCC-B 6} & Top & 0.5 \\
\hline & & Left & 0.3 & & & \begin{tabular}{|l|} 
Left \\
\end{tabular} & 5 \\
\hline & & Bottom & 0.4 & & & Bottom & 0.3 \\
\hline & & Right & 2.5 & & & \begin{tabular}{|l|} 
Right \\
\end{tabular} & 6 \\
\hline & & Side-A & & & & Side-A & 6 \\
\hline & & Side-B & & & & Side-B & 1 \\
\hline \multirow{6}{*}{$15 \%$} & & Top & 0.1 & & & Top & 0.8 \\
\hline & & \begin{tabular}{|l|} 
Left \\
\end{tabular} & 10 & & & \begin{tabular}{|l|} 
Left \\
\end{tabular} & 0.6 \\
\hline & NC-B 5 & Bottom & 5 & $15 \%$ & SCC-B 7 & Bottom & 0.8 \\
\hline & & \begin{tabular}{|l|} 
Right \\
\end{tabular} & 19 & & & \begin{tabular}{|l|} 
Right \\
\end{tabular} & 2.7 \\
\hline & & Side-A & 0.1 & & & Side-A & \\
\hline & & Side-B & 19 & & & Side-B & \\
\hline & & Top & 0.4 & & & Top & 0.6 \\
\hline & & \begin{tabular}{|l|} 
Left \\
\end{tabular} & 2 & & & \begin{tabular}{|l|} 
Left \\
\end{tabular} & \\
\hline $20 \%$ & NC-B 6 & Bottom & 6 & $20 \%$ & SCC-B 3 & Bottom & 0.2 \\
\hline & & Right & 3 & & & \begin{tabular}{|l|} 
Right \\
\end{tabular} & 3 \\
\hline & & Side-A & 0.3 & & & Side-A & \\
\hline & & Side-B & 3 & & & Side-B & 1.5 \\
\hline & & Top & 0.1 & & & Top & 0.3 \\
\hline & & Left & 0.2 & & & \begin{tabular}{|l|} 
Left \\
\end{tabular} & 5 \\
\hline $20 \%$ & NC-B 8 & Bottom & 2 & $20 \%$ & SCC-B 9 & Bottom & 6 \\
\hline & & Right & & & & \begin{tabular}{|l|} 
Right \\
\end{tabular} & 6 \\
\hline & & Side-A & 0.8 & & & Side-A & \\
\hline & & Side-B & 5 & & & Side-B & no crack \\
\hline
\end{tabular}


Table (4-5): Loads at failure, at first flexural crack, and at first diagonal crack with the effect corrosion level on $\mathrm{P}_{\mathrm{fl}} / \mathrm{P}_{\mathrm{u}}$ and $\mathrm{P}_{\mathrm{d}} / \mathrm{P}_{\mathrm{u}}$ and failure mode.

\begin{tabular}{|c|c|c|c|c|c|c|c|c|}
\hline & & \multicolumn{4}{|c|}{ Total applied load (kN) } & \multicolumn{2}{|c|}{ Ratio (\%) } & \\
\hline $\begin{array}{c}\text { Stage of } \\
\text { Corrosion }\end{array}$ & $\begin{array}{l}\text { Concrete } \\
\text { Type and } \\
\text { Beam } \\
\text { Number }\end{array}$ & \multicolumn{2}{|c|}{$\begin{array}{l}\text { at failure } \\
\left(\mathrm{P}_{\mathrm{u}}\right)\end{array}$} & $\begin{array}{l}\text { at first } \\
\text { flexural } \\
\text { crack } \\
\left(\mathrm{P}_{\mathrm{fl}}\right)\end{array}$ & $\begin{array}{l}\text { at first } \\
\text { diagonal } \\
\text { crack } \\
\left(\mathrm{P}_{\mathrm{d}}\right)\end{array}$ & $\mathrm{P}_{\mathrm{fl}} / \mathrm{P}_{\mathrm{u}}$ & $\mathrm{P}_{\mathrm{d}} / \mathrm{P}_{\mathrm{u}}$ & Failure Mode \\
\hline \multirow{4}{*}{ Zero } & NC B1 & 152.08 & \multirow{2}{*}{166.13} & 50 & 80 & $32.8 \%$ & $52.6 \%$ & \multirow{4}{*}{$\begin{array}{l}\text { Started with flexural cracks then with load } \\
\text { increase, diagonal cracks appeared and } \\
\text { propagated in the shear zones in the } \\
\text { beams to end the test with shear failure. }\end{array}$} \\
\hline & $\mathrm{NC} B 2$ & 180.18 & & 40 & 95 & $22.2 \%$ & $52.7 \%$ & \\
\hline & SCC B1 & 143.3 & \multirow{2}{*}{166.91} & 20 & 40 & $13.95 \%$ & $27.9 \%$ & \\
\hline & $\mathrm{SCC} B 2$ & 190.52 & & 20 & 50 & $10.49 \%$ & $26.24 \%$ & \\
\hline \multirow{4}{*}{$5 \%$} & $\mathrm{NC} B 3$ & 200.62 & \multirow{2}{*}{194.9} & 30 & 130 & $14.95 \%$ & $64.8 \%$ & \multirow{4}{*}{$\begin{array}{l}\text { Started with flexural cracks, then with } \\
\text { load increase, diagonal cracks appeared } \\
\text { and propagated on both sides of the beam } \\
\text { followed by sudden concrete crashing in } \\
\text { the compression zone of the beam section } \\
\text { between the two beam loads. }\end{array}$} \\
\hline & NC B9 & 189.18 & & 20 & 50 & $10.57 \%$ & $26.43 \%$ & \\
\hline & SCC B4 & 196.29 & \multirow{2}{*}{193.9} & 10 & 20 & $5.09 \%$ & $10.18 \%$ & \\
\hline & $\mathrm{SCC} B 10$ & 191.52 & & 20 & 90 & $10.44 \%$ & $47.0 \%$ & \\
\hline \multirow{4}{*}{$10 \%$} & NC B7 & 120.31 & \multirow{2}{*}{141.25} & 20 & $\underline{40}$ & $16.62 \%$ & $33.24 \%$ & \multirow{4}{*}{$\begin{array}{l}\text { Started with flexural cracks around the } \\
\text { mid-bottom area of the beams, followed } \\
\text { by diagonal cracks that propagated until } \\
\text { failure in shear plus split in the flexural } \\
\text { rebars. The corrosion cracks showed } \\
\text { higher influence on the loading cracks and } \\
\text { failure mode. }\end{array}$} \\
\hline & NC B10 & 162.19 & & 20 & 70 & $12.33 \%$ & $43.15 \%$ & \\
\hline & SCC B5 & 127.2 & \multirow{2}{*}{145.47} & 30 & 50 & $23.58 \%$ & $39.3 \%$ & \\
\hline & SCC B8 & 163.74 & & 10 & 40 & $6.1 \%$ & $24.4 \%$ & \\
\hline \multirow{4}{*}{$15 \%$} & NC B4 & 73.65 & \multirow{2}{*}{71.985} & 50 & 70 & $69.45 \%$ & $95.0 \%$ & \multirow{4}{*}{$\begin{array}{l}\text { The corrosion impact extremely affected } \\
\text { the failure mode and the load capacity. } \\
\text { Two of the beams started with a flexural } \\
\text { crack from above the flexural rebars } \\
\text { level. Then diagonal cracks appeared and } \\
\text { associated with cracks in the compression } \\
\text { zone located between the loading point } \\
\text { towards the outer corner of failure side. }\end{array}$} \\
\hline & NC B5 & 70.32 & & 20 & 50 & $28.44 \%$ & $71.1 \%$ & \\
\hline & SCC B6 & 71.21 & \multirow[b]{2}{*}{103.87} & 40 & 60 & $56.17 \%$ & $84.25 \%$ & \\
\hline & SCC B7 & 136.53 & & 50 & 70 & $36.6 \%$ & $51.27 \%$ & \\
\hline \multirow{4}{*}{$20 \%$} & NC B6 & 45.1 & \multirow{2}{*}{53.71} & 40 & 44 & $88.69 \%$ & $97.56 \%$ & \multirow{4}{*}{$\begin{array}{l}\text { The corrosion cracks showed a big } \\
\text { influence on the loading cracks and } \\
\text { failure mode. The failure mode started } \\
\text { with few flexural cracks, followed by } \\
\text { diagonal cracks that led to failure. The } \\
\text { SCC beams in this category showed } \\
\text { compression cracks on the top side of the } \\
\text { final failure side, which happened on the } \\
\text { same side of the spalling in the bottom } \\
\text { corner. Failure can be described by a split } \\
\text { in the flexural rebars in addition to } \\
\text { diagonal shear cracks of the failing side. }\end{array}$} \\
\hline & NC B8 & 62.32 & & 10 & 40 & $16.04 \%$ & $64.18 \%$ & \\
\hline & SCC B3 & 81.76 & \multirow[b]{2}{*}{67.43} & 50 & 80 & $61.15 \%$ & $97.84 \%$ & \\
\hline & SCC B9 & 53.1 & & 30 & 30 & $56.49 \%$ & $56.49 \%$ & \\
\hline
\end{tabular}




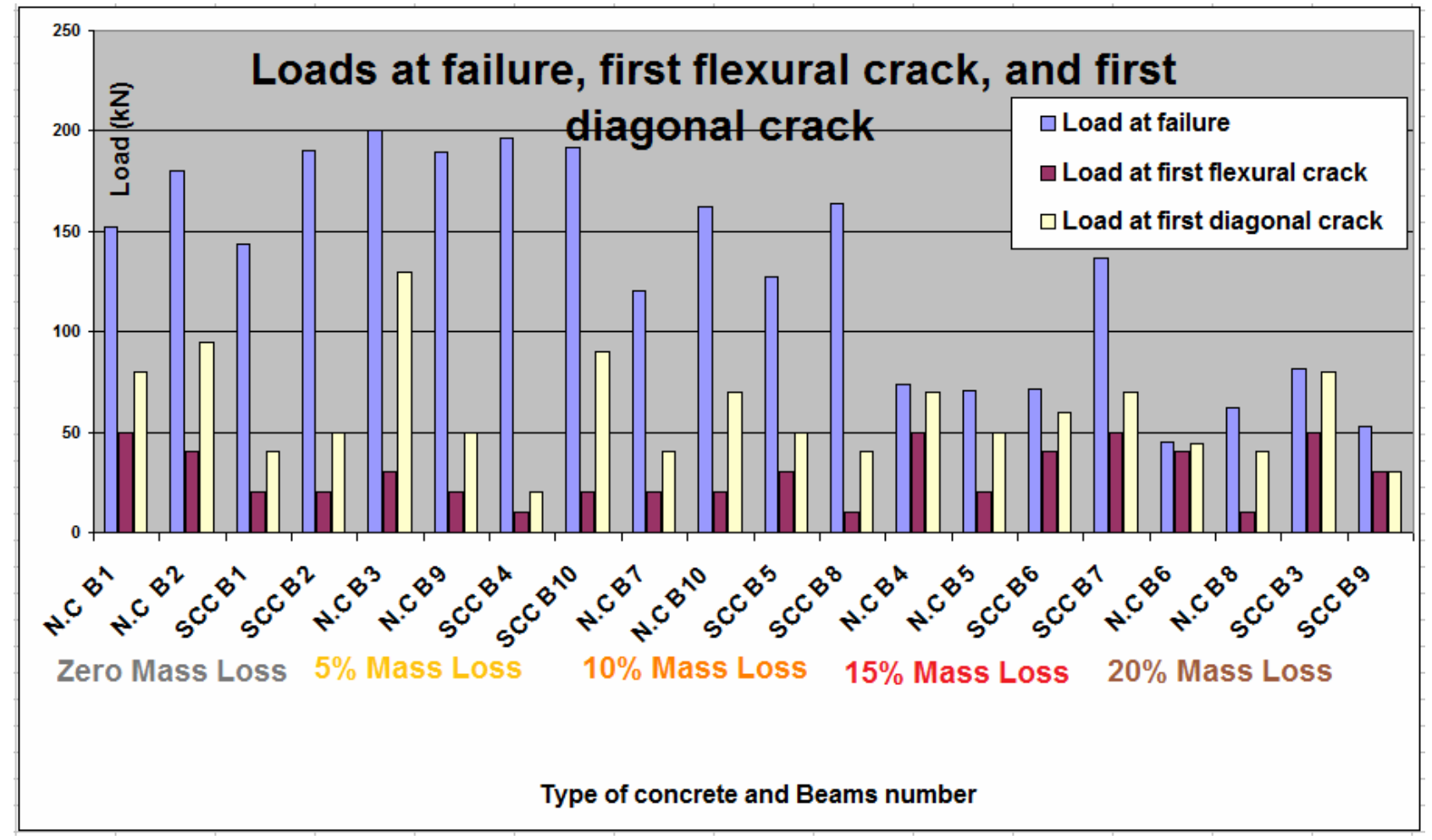

Figure (4-5): The effects of corrosion level on the load at failure, load at first flexural crack, and load at first diagonal crack.

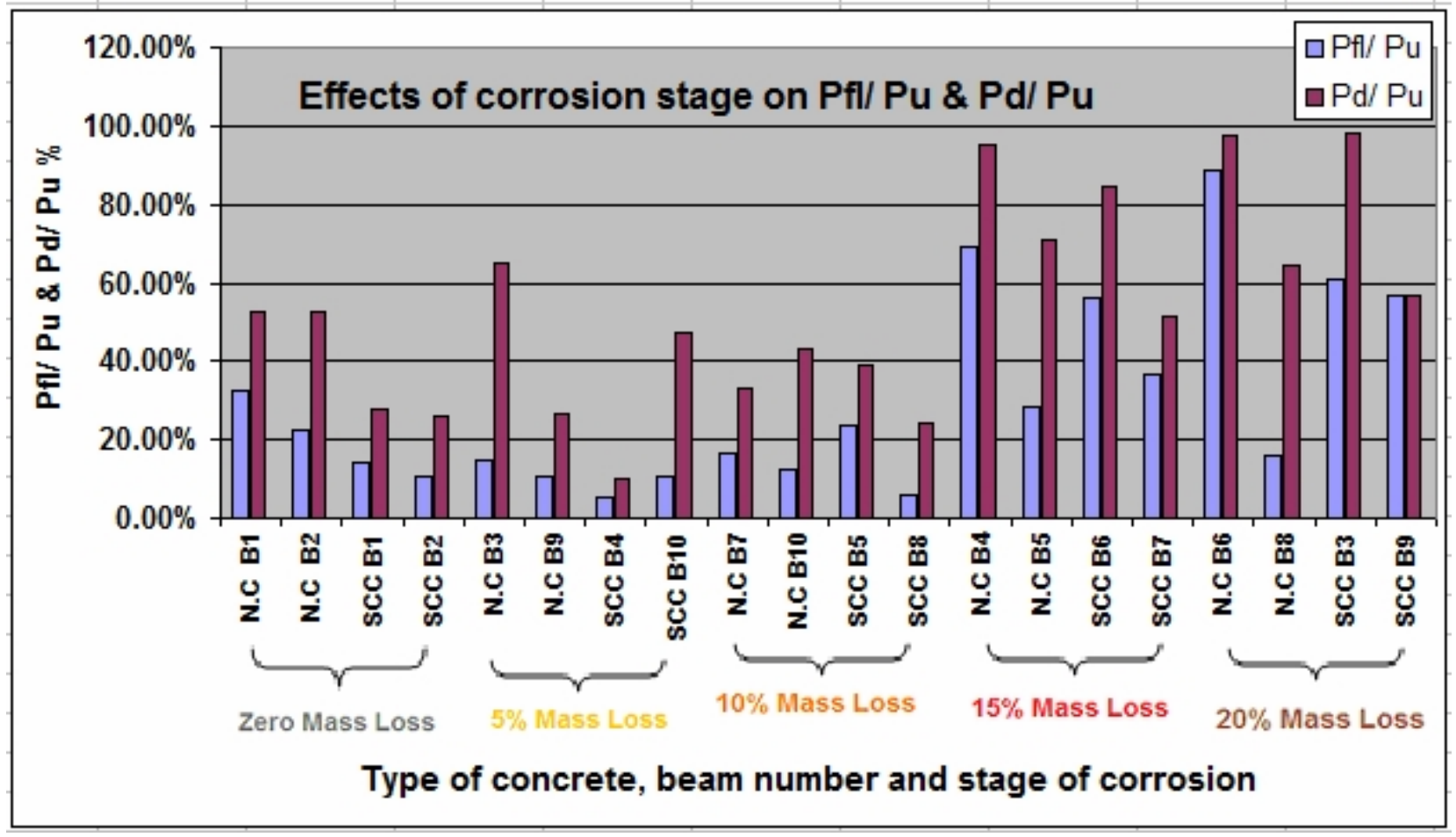

Figure (4-6): The effects of corrosion level on the percentage of $P_{f} / P_{u}$ and $P_{d} / P_{u}$. 


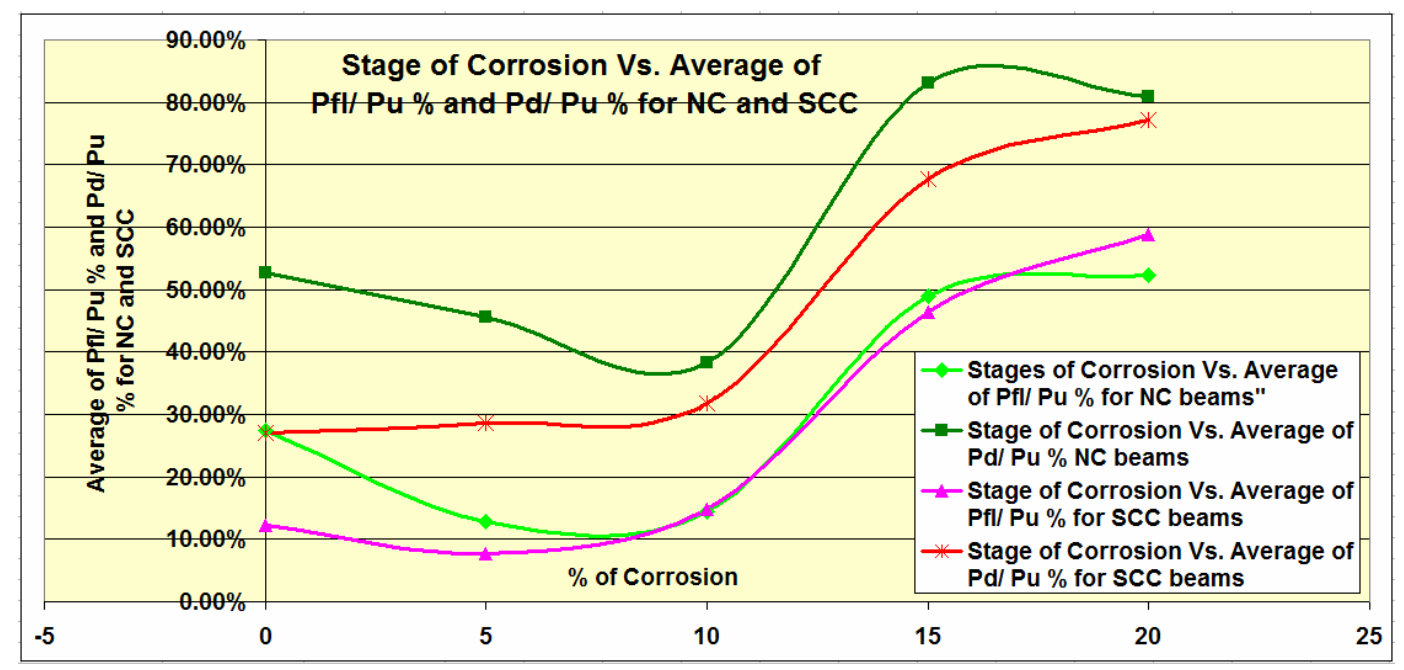

Figure (4-7): Effects of stages of corrosion measured by mass loss on the average ratios of $\mathrm{P}_{\mathrm{f}} / \mathrm{P}_{\mathrm{u}}$ and $\mathrm{P}_{\mathrm{d}} / \mathrm{P}_{\mathrm{u}}$.

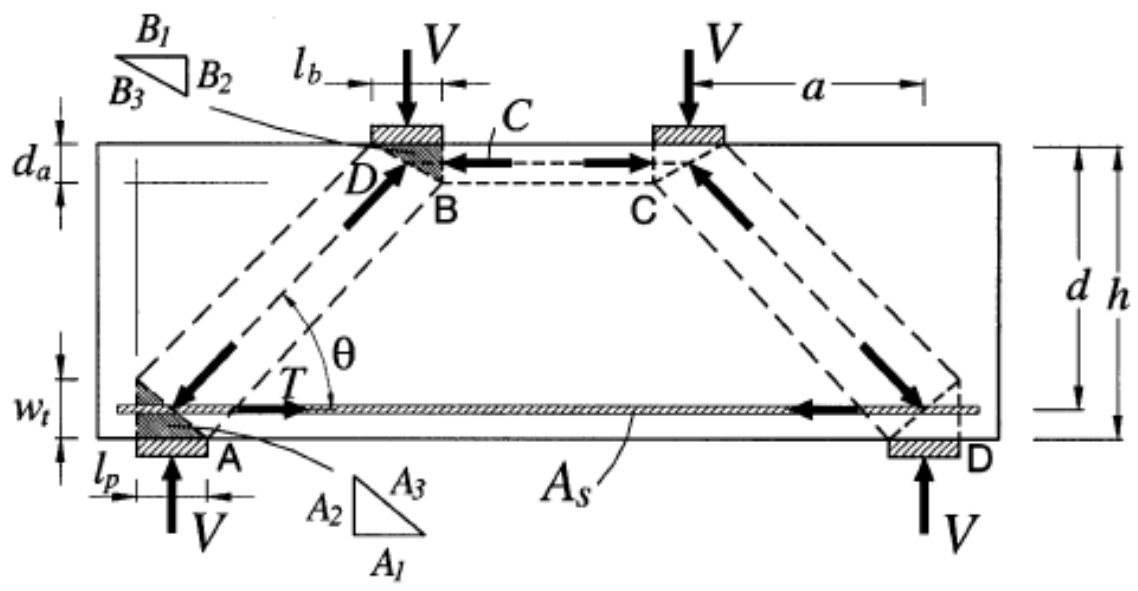

Figure (4-8): Strut and tie model shows the tension and compression zones in the beam (Russo et al. 2005).

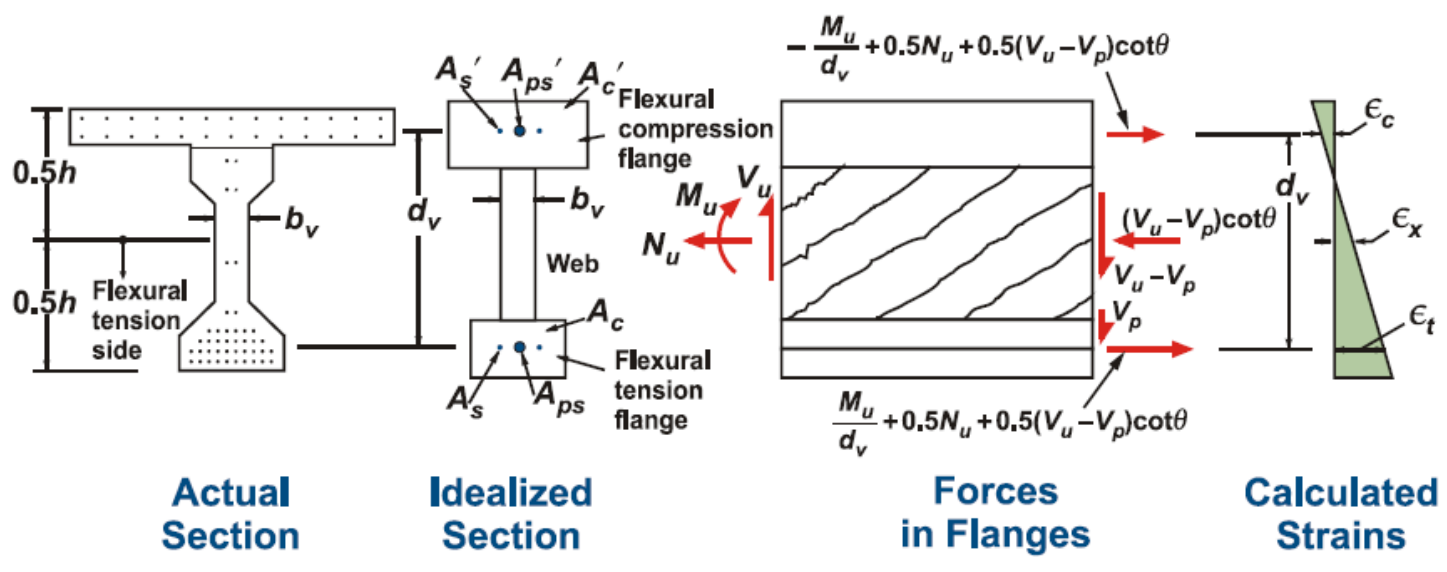

Figure (4-9): Calculation of longitudinal strain (Collins et al. 2007). 
Table (4-6): The weight of steel components of the steel cage for each NC and SCC beam, before and after corrosion.

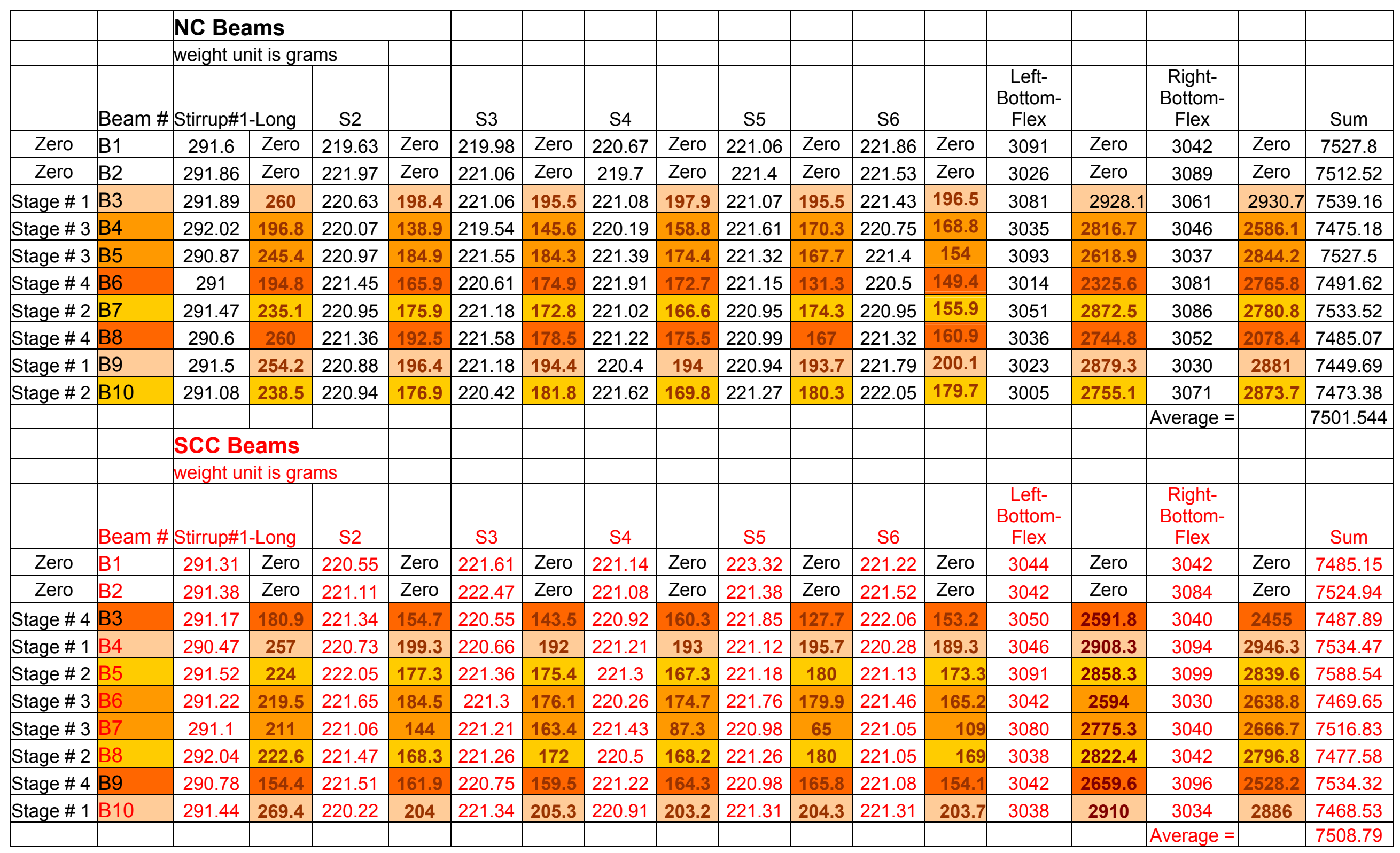


Table (4-7): The initial and final weight for the steel subjected to corrosion, the remaining percentage of that steel and the actual mass loss after finalizing the corrosion process.

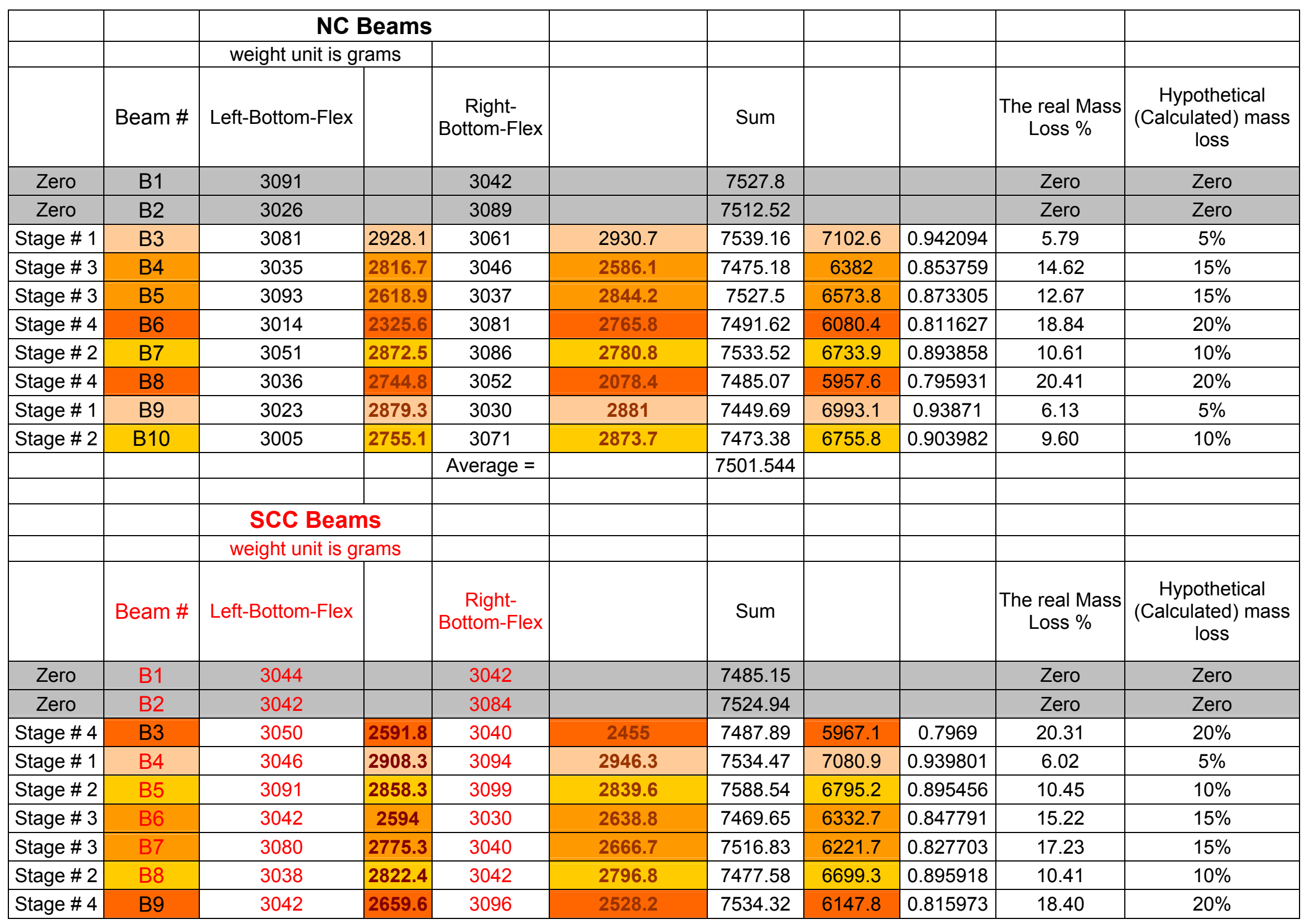




\section{Chapter 5}

\section{Conclusions and recommendations}

\subsection{Summary}

This laboratory study consisted of 20 beams using two different types of concrete. Each group of ten beams was similar in dimensions and reinforcing steel. The main difference was the type of concrete used in the construction of the beams. The first group was made of NC, and the second was made of SCC. Each group was divided into subgroups of two beams, based on the degree of corrosion. Accelerated corrosion was stimulated in the beams to achieve $5 \%, 10 \%, 15 \%$, and $20 \%$ mass loss in the reinforcing steel bars. The half-cell potential test was used for each beam to evaluate resistance to corrosion before applying the accelerated corrosion, and then the corrosion crack pattern was traced and plotted for all of the corroded beams. After finishing each corrosion stage, beams were subjected to a loading test in four points, taking into consideration the $\mathrm{a} / \mathrm{d}$ ratio $=2.5$ to get the highest probability of shear failure. The data collected from these tests were used to monitor and study the shear behaviour of the NC compared with the SCC beams, as well as the total structural failure mode. The data collected from nine LVDTs used on each beam with the loading cell were analyzed to get the shear strain $\gamma_{x y}$, the strain in x-direction $\boldsymbol{\epsilon}_{x}$, the strain in y-direction $\boldsymbol{\epsilon}_{y}$, the mid-span deflection, and deflection along the beams with loading variations. The loading crack patterns were traced and plotted to monitor and document the initial flexural crack and initial shear crack for each beam. The effects of multi-stages of corrosion on both NC and SCC were examined and documented through drawings of the corrosion crack patterns, then followed by an examining the influence of corrosion cracks on the loading cracks pattern initially and at final failure mode. The results showed a high correlation between the real mass loss of the corroded rebars and the calculated mass loss, which adds excellent creditability through the use of a specific value of constant current for a certain time, calculated through Faraday's equation. The results offered a better understanding of the effects of corrosion on the shear behaviour of SCC beams in comparison to NC beams. 


\subsection{Conclusions}

1. In relation to the SCC casting technique, the results of this research agree with the previous research findings by Hassan (2008) about the effects of pouring SCC from one side on the quality of the SCC at the farther side of the beams. Seven out of ten beams made of SCC failed in the loading test from Side-A, the side further from the pouring end. This is believed to be related to the nonuniform transfer of SCC aggregates along the direction of flowing concrete in the beam. The specimens used were only $1400 \mathrm{~mm}$ long, so this negative effect may increase with an increase in distance between the pouring point and the end of the far side of the concrete element.

2. The half-cell potential test results showed that the beams are located within the case of sever corrosion or above the chance of $90 \%$ of corrosion to be occurred. The relatively high percentage of humidity inside the concrete body of the tested beam and the relatively small concrete cover above the rebars are the two major reasons for the high probability of corrosion.

3. The corrosion crack pattern showed:

- A high correlation between the rebars cage shape and the shapes of the cracks with respect to the stage of corrosion measured by mass loss. The crack width increased with the increasing corrosion measured by mass loss.

- The corrosion cracks have a strong effect on the structural response to loading in general and especially on future cracks resulting from the load test.

- The linear increase of the mass loss resulting from corrosion had exponential effects on the reduction in the structural capacity and on the shape, width and number of cracks.

- The corrosion crack pattern on the congested rebars areas may not reflect the shape of the rebars cage for the close joint effect of the internal stresses that result from the congested rebars. The advanced stages of corrosion showed one longitudinal crack instead of two cracks at the bottom face, resulting in splitting in the covering layer, then spalling in some of the beams, especially in the bottom corners.

4. The beams with $5 \%$ mass loss failed at about a $16 \%$ higher load than the beams with zero mass loss. In addition, the mid-span deflection of beams with zero mass loss group were from $5 \mathrm{~mm}$ to $8 \mathrm{~mm}$, while the mid-span deflection of beams with $5 \%$ mass loss were from $12 \mathrm{~mm}$ to $15 \mathrm{~mm}$. This is believed to be 
due to the increase in bond strength of the plain rebars used in the stirrups at the corrosion stage of $5 \%$ mass loss.

5. There are catastrophic structural changes associated with increased corrosion mass loss. The data collected from the beams with $10 \%, 15 \%$, and $20 \%$ mass loss showed a rapid reduction in load capacity and relative beam stiffness "K," (which is the slope of load-deflection curve). Wider cracks, increasing losses in bond strength, and the rebars weakened by the corrosion process are the three major reasons for structural capacity reduction.

6. The loading crack patterns were valuable for the following reasons:

- They showed the changes of the failure mode associated with the increase of corrosion stage for both NC and SCC beams.

- They documented the load at failure $\left(P_{\mathrm{u}}\right)$, at first flexural crack $\left(P_{\mathrm{fl}}\right)$, and at first diagonal crack $\left(P_{d}\right)$. Therefore, the ratios of $P_{f l} / P_{u}$ and $P_{d} / P_{u}$ can be calculated for the purpose of comparison between the beams of different types of concrete and in multi-stages of corrosion.

- Both the observed and the internal corrosion cracks influenced the loading crack mode for all of the NC and SCC beams, and for those with $5 \%, 10 \%$, $15 \%$, and $20 \%$ mass loss stages of corrosion.

7. The anticipated mass loss through Faraday's equation calculations were close to the measured mass loss of the corroded NC and SCC beams. It proved that choosing the constant current along the accelerated corrosion is better than using constant voltage to calculate the time needed for targeting a specific amount of mass loss.

8. The slope of the graphs resulting from load vs. mid span deflection showed changes associated with increased stages of corrosion. At $5 \%$ mass loss, the slope showed a slight increase over the slope with zero mass loss. At 10\%, 15\% and $20 \%$ mass loss, the slope decreased clearly, which was a sign of the drop in the structural capacity of the beams at the advanced stage of corrosion.

9. To maintain accelerated corrosion for experimental purposes, the welding joints between the bottom flexural rebars and the stirrups maintained the connectivity throughout the forced accelerated corrosion experimental time. Keeping the joints connected showed high reliability, even with beams at high stages of corrosion. The alternative (normally used) ties failed to maintain connectivity 
between the cage elements due to the pressure resulting from the corrosion products that made isolation layers between the rebars.

10. During the forced accelerated corrosion stages, the NC beams showed higher initial resistance than their SCC counterparts. The first week of accelerated corrosion showed increases in the electrical resistance of both NC and SCC beams. In the first two weeks, the NC beams showed superior values of electrical resistance when compared to SCC beams. The next 30 days were needed to achieve $10 \%$ and $15 \%$ mass loss stages of corrosion, with close average values of electrical resistance in both NC and SCC beams. The last two weeks allotted to achieve the $20 \%$ mass loss stage of corrosion showed a slight increase in the values of electrical resistance in SCC beams compared with NC beams. There were some fluctuations in the values, especially in the period from the third week to the end of the accelerated corrosion process. The reason behind these inconsistencies is the continuous development and closing of micro and macro cracks in the concrete cover surrounded by the corroded rebars.

The author considers the initial electrical resistance value of the beams to be the most important indicator for evaluating the efficiency of both NC and SCC in regards to corrosion resistibility.

11. The Rapid Chloride Permeability Test results were compatible with the voltampere daily readings that showed the NC used in this research is higher in electrical resistance than the SCC.

12. It should be noted from Table (3-3) that SCC has a $20 \%$ lower percentage of coarse aggregates than NC, which might affect the final shear strength capacity. It is predicted that a higher percentage of coarse aggregates in the NC mixture results in higher shear strength of the NC beams. The expected increase in shear strength depends on the influence of the coarse aggregate interlock on the final shear strength of the NC and SCC.

13. Based on the information observed about the beams' failure mode, the degree of corrosion has a major effect on the change in failure mode of both NC and SCC beams. With the increase in the corrosion stage, the NC and SCC beams showed more brittle responses than the control beams with respect to the values of shear strain, longitudinal strain, and transversal strain. This is believed to be related to cracked concrete, and to the negative effects of corrosion on rebars mass and bond strength. 


\subsection{Recommendations}

1. To avoid non-uniform distribution of SCC aggregates and ingredients, SCC should be poured from different points.

2. More research is required to examine the corrosion mass loss that yields the peak value of bond stresses between reinforcing steel bars and surrounding concrete for different kinds of concrete, especially SCC.

3. Monitor the performance of structures built with the SCC to study and evaluate their long-term structural performance, durability and serviceability.

4. In similar concrete elements that need to be corroded (for research purposes), it is recommended to use a protection coat for stirrup no.1 or any steel rebar that may be used for transferring electrical current to the cage of rebars of any concrete unit. This helps avoid or minimize breakage of steel closer to the outer surface of the concrete, since the pitting corrosion happens in the advanced corrosion stages.

5. The thin concrete cover above the rebars of any concrete structure may lead to a high risk or high probability of corrosion in the rebars, so increasing the concrete cover is recommended.

6. It is useful to prevent excessive humidity in reinforced concrete structures after the curing period due to its negative effects on the rebar's probable future corrosion. The gain in the concrete strength due to curing in more advanced concrete age is negligible when compared with the harmful effects of rebars corrosion. In marine or hydraulic structures it is good to target the required concrete strength, then work on preventing incoming humidity from outside by applying humidity isolators on the outer concrete surfaces. This kind of treatment for reinforced concrete structures will protect them not only from the harmful effects of corrosion but also from the stresses resulting from cyclic freeze and thaw attacks.

7. In the RC structures with a high probability of corrosion attack, it is useful to avoid connectivity between rebars by using epoxy-coated rebars and putting thin electrical isolators between all of the contacting rebars. Moreover, if there are any expansion joints in these RC structures, they must be sealed completely so no water is allowed to spill from the decks toward the girders, beams, columns, and piers, especially salt water saturated with the chloride ions. 
8. In relation to shear, using the smallest available rebars size is recommended because the small steel size leads to a bigger number of stirrups, which can be distributed over more areas that are affected by shear stress. Although the shear stresses are inclined, vertical stirrups are much better to use for the following reasons :

- Vertical stirrups weigh less than the inclined ones because vertical stirrups are shorter.

- Because it is very hard to predict the exact shape or slope of the shear cracks, it is also hard to install the inclined stirrups to restrain the shear cracks.

- Most of the shear reinforcement is used not only to resist shear stresses or to prevent shear cracks, but also, to resist torsion. The inclined stirrups can resist the shear effectively, but to resist the torsion stresses the inclined stirrups are much less efficient.

- Vertical stirrups are used effectively in the beams resisting shear reversal, such as structures resisting seismic load, since the vertical reversals will cause cracking parallel to the inclined stirrups, causing it to become unable to resist the reversal shear stresses.

- Practically, it is much easier to install the vertical reinforcements than the inclined ones.

9. According to the results of this research, SCC is less resistant to corrosion attack, so the idea that SCC contains more fine admixtures and relatively more fine aggregates is not enough to prove that it resists corrosion attack better than NC.

10. Another recommendation is to develop additional kinds of SCC admixtures that meet SCC flowability requirements without adding an acidic environment or increasing the conductive minerals. The suggested admixtures to add to SCC are intended to provide an alkaline environment that isn't coming from a conductive mineral source, and has very small particles to close or fill all of the voids.

11. Performing extensive research on the early stages of corrosion with relation to their effects on the structural changes in RC structures is also recommended. 


\section{References}

- AASHTO LRFD, "Bridge Design Specifications and Commentary," 3rd Edition, American Association of State Highway Transportation Officials, Washington, D.C., 2004, 1264 pp.

- Abdul Hameed, M.; "A Study of Mix Design and Durability of Self-Compacting Concrete," MASc Thesis, December 2005.

- $\mathrm{ACl} 222 \mathrm{R}-01, \mathrm{ACl}$ Committee 222, "Protection of Metals in Concrete Against Corrosion," 2001.

- ACl 228.2R-98, (Reapproved 2004), "Non-destructive Test Methods for Evaluation of Concrete in Structures" Reported by ACI Committee 228.

- ACl 237R-07, Emerging Technology Series, "Self-Consolidating Concrete," Reported by ACI Committee 237. April 2007.

- $\mathrm{ACl}$ 445R-99, Reported by Joint ACl-ASCE Committee 445, "Recent Approaches to Shear Design of Structural Concrete," November 22, 1999.

- $\mathrm{ACl}$ Committee 318, "Building Code Requirements for Reinforced Concrete (ACl 318-63)," American Concrete Institute, Farmington Hills, Mich., 1963, 163 pp.

- $\mathrm{ACl}$ Committee 318, "Building Code Requirements for Structural Concrete (ACI 31805) and Commentary (318R-05)," American Concrete Institute, Farmington Hills, Mich., 2005, 430 pp.

- Adebar, P. E., and Collins, M. P., "Shear Strength of Members without Transverse Reinforcement," Canadian Journal of Civil Engineering, V. 23, No. 1, Feb. 1996, pp. $30-41$.

- Aldea, C. M.; Young, F.; Wang, K.; and Shah, S. P., "Effect of Curing Conditions on Properties of Concrete Using Slag Replacement," Cement and Concrete Research, V. 30 , No. 3, 2000, pp. 465-472.

- Amleh, L.; Mirza, S.; "Corrosion Influence on Bond between Steel and Concrete," ACI Structural Journal, V. 96, No. 3, May-June 1999.

- ASTM C 876 - 91 (Reapproved 1999) "Standard Test Method for Half-Cell Potentials of Uncoated Reinforcing Steel in Concrete1," 1999.

- ASTM C1202-97, "Standard Test Method for Electrical Indication of Concrete's Ability to Resist Chloride Ion Penetration," 1997.

- Avery, T., (2004), "Self-Compacting Concrete Powerful Tool for Complicated Pours," concrete monthly, news from the cement and concrete industries. 
- Baeckmann, W. V.; Bohnes, H.; Franke, G.; Funk, D.; Gey, C.; Grafen, H.; Heim, G.; Heinzelmann, V.; Horras, K.; Isecke, B.; Kampermann, H.; Leutner, B.; Paul, H. -U.; Paulekat, F.; Prinz, W.; Richter, B.; Rieger, G.; Schoneich, H. G.; Schwenk, W.; "Handbook of CATHODIC CORROSION PROTECTION" Copyright 1997 by Elsevier Science (USA). Gulf Professional Publishing is an imprint of Elsevier Science, Third edition, ISBN 0-88415-056-9.

- Ballim, Y., J. C Reid, and A R. Kemp. "Deflection of RC Beams Under Simultaneous Load and Steel Corrosion," Magazine of Concrete Research 53, no. 3 (2001): 171181.

- Bentz, E. C., "Sectional Analysis of Reinforced Concrete Members," PhD thesis, Department of Civil Engineering, University of Toronto, Toronto, Ontario, Canada, 2000, 198 pp.

- Bentz, E.C.; Vecchio, F.J.; and Collins, M.P.; "Simplified Modified Compression Field Theory for Calculating Shear Strength of Reinforced Concrete Elements," ACl Technical Journal. Technical paper, Title No.103-S65. ACI Structural Journal, V. 103, No. 4, July-August 2006.

- Broomfield, J.P.; "Corrosion of Steel in Concrete - Understanding, Investigation, and Repair," first published 1997 by E\&FN Spon, an imprint of Chapman \& Hall. This edition published in the Taylor \& Francis e-Library 2003. ISBN 0-203-47528-3 Master e-books ISBN.

- Brzev, S.; Pao, J.; "Reinforced Concrete design - A practical approach," ISBN 0-13039125-5, 2006.

- Castel, A; Francois, R.; and Arliguie, G. "Mechanical Behaviour of Corroded RC Beams - Pan 2: Bond and Notch Effects," Materials and Structures 33, no. 233 (2000): 545.

- Clemena, G. G.; Jackson, D. R.; and Crawford, G. C. (1992). "Benefits of Using Half-Cell Potential Measurements in Condition Surveys of Concrete Bridge Decks," Transportation Research Record No. 1347, Transportation Research Board, Washington, D.C., pp. 46-55.

- Collins, M.P.; Mitchell, D.; "Diagonal Compression Field Theory - A Rational Model for Structural Concrete in Pure Torsion," ACI Journal, Vol. 71, Title No. 71-28 Aug. 1974, pp. 396-408.

- Collins, M. P., "Towards a Rational Theory for RC Members in Shear," Journal of the Structural Division, ASCE, V. 104, No. 4, Apr. 1978, pp. 649-666. 
- Collins, M.P.; Mitchell, D.; "Prestressed Concrete Structures" Prentice Hall, Englewood Cliffs, N.J., 1991, 766 pp.1997 by Response Publication Canada. ISBN 0-9681958-0-6.

- Collins, M.P., "The Use of Rational Design Methods for Shear," sp157-16. Oakville, August 1993.

- Collins, M. P.; Mitchell, D.; Adebar, P.; and Vecchio, F. J., "A General Shear Design Method," ACI Structural Journal, V. 93, No. 1, Jan.-Feb. 1996, pp. 36-45.

- Collins, M. P., and Kuchma, D., "How Safe Are Our Large, Lightly Reinforced Concrete Beams, Slabs, and Footings?" ACI Structural Journal, V. 96, No. 4, JulyAug. 1999, pp. 482-490.

- Collins, M.P.; Bentz, E.C.; Sherwood, E.G.; XIE, L.; "An Adequate Theory for the Shear Strength of Reinforced Concrete Structures," University of Cambridge 23rd July, 2007.

- CSA Committee A23.3, (2006), "Design of Concrete Structures," Canadian Standards Association, Rexdale, Ontario.ISBN 1-896553-20-6

- Daczko, J. A., and Kurtz, M.A., 2001, "Development of High-Volume Coarse Aggregate Self-Compacting Concrete," Proceedings of the Second International Symposium on SCC, Oct. 23-25, Tokyo, Japan.

- Davy, H.; "Phil. Transact." 144, 151 (1824).

- EFNARC, "Specification and Guidelines for Self-Compacting Concrete," Feb. 2002, 32 pp., http://www.efnarc.org.

- El Maaddawy, Tamer; Soudki, Khaled; and Topper, Timothy, "Analytical Model to Predict Nonlinear Flexural Behaviour of Corroded RC Beams," ACI Structural journal 102, no. 4 (2005).Pages 550-559.

- Fang, W.; Jianxiong, C.; and Changhui, Y., 1999, "Studies of Self-Compacting High Performance Concrete with High Volume Mineral Additives," Proceedings of the First International RILEM Symposium on SCC, pp. 569-578.

- Gere, J.M.; and Timoshenko, S.P.; "Mechanics of Materials," 4th Ed.PWS Publishing, 1997, pp. 835-42.

- Ghezal, A., and Khayat, K. H., 2002, "Optimizing Self-Consolidating Concrete with Limestone Filler by Using Statistical Factorial Design Methods," ACl Materials Journal, V. 99, No. 3, May-June, pp. 264-272. 
- Groth, P.; "Fibre Reinforced Concrete - Fracture Mechanics Methods Applied on Self-Compacting Concrete and Energetically Modified Binders," Doctoral Thesis, January 2000.

- Hassan, A., "Performance of full scale self consolidating concrete structural elements in Shear, bond and under corrosion attack," PhD Dissertation, Civil Engineering Dept., Ryerson University, Sept. 2008.

- Higgins, C; Farrow III W, C.; Potisuk, T.; Miller, T.H.; Yim, S.C.; and Holcomb, G.R.; Stephen D. Cramer, Bernard S. Covino, Jr., Sophie J. Bullard, Margaret ZiomekMoroz, and Steven A. Matthes "Shear capacity assessment of corrosion damaged reinforced concrete beams," December 2003.

- Hoppe, E.J.; Lundy, L.J.; Schinekel, T.J.; "Implementation of an Automated Test Setup for Measuring Electrical Conductance of Concrete," http://www.virginiadot.org/vtrc/main/online_reports/pdf/07-r21.pdf,Virginia Transportation Research Council, Final Report VTRC 07-R21. February 2007.

- Jacobs, F., and Hunkeler, F., 2001, "SCC for the Rehabilitation of a Tunnel in Zurich/Switzerland," Proceedings of the Second International Symposium on SCC, K. Ozawa and M. Ouchi, Eds., Tokyo, pp. 707-714.

- Kani, G. N. J., "How Safe Are Our Large Concrete Beams?" ACl JOURNAL, Proceedings V. 64, No. 3, Mar. 1967, pp. 128-142.

- Khayat , K. H., and Aïtcin, P.-C., 1998, "Use of Self-Consolidating Concrete in Canada - Present Situation and Perspectives," Proceedings of the Workshop on Self- Compacting Concrete, Kochi, Japan.

- Khayat, K. H., and Manai, K., "Les Betons Autonivlants: Properties, Caracterisation et Applications," Colloque sur les Betons Autonivelants, Université de Sherbrooke, Quebec, Canada, Nov. 1996, 28 pp.

- Khayat, K. H., and Morin, R., 2002, "Performance of Self- Consolidating Concrete Used to Repair Parapet Wall in Montreal," Proceedings of the First North American Conference on the Design and Use of SCC, ACBM, Chicago, III., Nov. 12-13.

- Khayat, K.H.; Assaad, J.; and Daczko, J.; "Comparison of Field Oriented Test Methods to Assess Dynamic Stability of Self-Consolidating Concrete," ACl materials Journal, Technical paper, /March-April 2004.

- Korradi, M.; Khurana, R.; and Magarotto, R., "User Friendly self-Compacting Concrete in Precast Production," Degussa Construction Chemicals Admixture 
System Europe. Third International Symposium on Self-Compacting Concrete, Reykjavik, Iceland. 17-20 August 2003.

- Lachemi,M.; Hossain, K.M.A.; Lambros, V.; Nkinamubanzi, P.-C.; Bouzoubaa, N.; "Self-consolidating concrete incorporating new viscosity modifying admixtures," Cement and Concrete Research 34 (2004) 917-926.

- Lachemi, M.; Hossain, K.M.A.; and Lambros, V.; "Shear resistance of selfconsolidating concrete beams - experimental investigations," Can. J. Civ. Eng. Vol. 32, 2005. Canada

- Leierzapf, H.; "Werkstoffe und Korrosion" 36, 88 (1985).

- Lessard, M., Talbot, C., Phelan, W.S., Baker, D., (2002), "Self-Consolidating Concrete Solves Challenging Placement Problems at the Pearson International Airport in Toronto, Canada," 1st North American Conference on the Design and Use of Self-Consolidating Concrete (SCC). Rosemont, Illinois, pp. 12-13.

- Lew H. S.; and Reichard, T. W.; "Mechanical Properties of Concrete at Early Ages," ACI Journal, Proceedings, Vol. 75, No. 10, October 1978, pp. 533-542.

- Li, S., and Roy, D. M., "Investigation of Relations Between Porosity, Pore Structure, and Cl- Diffusion of Fly Ash and Blend Cement Pastes," Cement and Concrete Research, V. 16, No. 5, 1986, pp. 749-759.

- Lubell, A.; Sherwood, E.; Bentz, E. C.; and Collins, M. P., "Safe Shear Design of Large, Wide Beams," Concrete International, V. 26, No. 1, Jan. 2004, pp. 66-78.

- MacGregor, J.G.; Bartlett. F. M.; "Reinforced Concrete-Mechanics and Design," First Canadian Edition- 2000.

- Ouchi, M., 2001, "Current Condition of Self-Compacting Concrete in Japan," Proceedings of the Second International Symposium on SCC, K. Ozawa and M. Ouchi, eds., Tokyo, pp. 63-68.

- Ozawa, K.; Sakata, N.; Okamura, H.; "Evaluation of self-compatibility of fresh concrete using funnel test," Proc. JSCE 90 (23) (1994) 59-75.

- RILEM, 2000, "State-of-the-Art Report of RILEM Technical Committee 174-SCC, Self-Compacting Concrete," Report 23, A. Skarendahl and O. Peterson, eds., 154 pp.

- Roberge, Pierre R., "Handbook of Corrosion Engineering," New York, McGraw Hill, (2000). ISBN 0-07-076516-2. 
- Robert G. Kelly, John R. Scully, David W. Shoesmith , Rudolph G. Buchheit "Electrochemical Techniques in Corrosion Science and Engineering," ISBN: 0-82479917-8, Copt write 2003 by Marcel Dekker. Inc. pp 4-5.

- Rodriguez, J; Ortega, L. M; and Casel, J. "Load-carrying Capacity of Concrete Structures with Corroded Reinforcement," Construction Building Materials 11, no. 4 (1997), pp. 239-248.

- Rosettes strain gauge measurements, A Measurements Group Hypertext Publication, Also available in printed form as Measurements Group Tech Note TN512. "Plane-Shear Measurement with Strain Gages," ALS JOURNAL , According to: http://www.measurementsgroup.com/guide/tn/tn512/512index.htm $\quad\left(\begin{array}{lll}1 & \text { of } & 2\end{array}\right)$ [12/20/2000 9:20:22 AM].

- Russo, G.; Venir, R.; and Pauletta, M.; "Reinforced Concrete Deep Beams-Shear Strength Model and Design Formula," ACl Structural Journal, V. 102, No. 3, MayJune 2005.

- Schweitzer, Philip A. "Fundamentals of metallic corrosion: atmospheric and media corrosion of metals," Second Edition: CRC press 2007.

- Sharp, Stephen R., "Evaluation of two corrosion inhibitors using two application methods for reinforced concrete structures," Virginia Transportation Research Council, Charlottesville, Virginia, December 2004.

- Skarendahl, A., 2001, "Market Acceptance of Self-Compacting Concrete, the Swedish Experience," Proceedings of the Second International Symposium on SCC, K. Ozawa and M. Ouchi, eds., Tokyo, pp. 1-12.

- Swamy, R.N., and Andriopoulos, A.S. 1974. "Contributions of aggregate interlock and dowel forces to shear resistance of reinforced beams with web reinforcement. In Shear in Reinforced Concrete," American Concrete Institute, Detroit, Mich. ACI SP- 42, pp. 43-77.

- Taylor, H.P.J. 1970. "Investigation of the forces carried across cracks in reinforced concrete beams in shear by interlock of aggregates," British Cement Association (formerly Cement and Concrete Association), London, U.K.

- The half-cell potential test manual "James COR-MAP II system," NDT James Instruments INC. Non-Destructive Testing Systems. 2003.

- Timoshenko, S.P.; "History of Strength of Materials", Dover Publications, 1983.

- Vecchio, F. J., "Nonlinear Finite Element Analysis of Reinforced Concrete Membranes," ACI Structural Journal, V. 86, No. 1, Jan.-Feb.1989, pp. 26-35. 
- Vecchio, F., and Collins, M.P., "The Response of Reinforced Concrete to In-Plane shear and Normal Stresses," Publication No. 82-03, Department of Civil Engineering, university of Toronto, Mar.1982,332 pp.

- Vecchio, F.J., and Collins, M.P., "The Modified Compression Field Theory for Reinforced Concrete Elements Subjected to Shear," ACl journal, Vol.83, No.2, Mar.Apr. 1986, pp.219-231.

- Walraven, J. C., 2001, "State of the Art on Self-Compacting Concrete in the Netherlands," Proceedings of the Second International Symposium on SCC, K. Ozawa and M. Ouchi, eds., Tokyo, pp. 13-24.

- Walraven, Joost C., "Fundamental Analysis of Aggregates Interlock," Journal of the Structural Division, ASCE, Vol. 107, No. ST11, Nov. 1981, pp. 2245-2270.

- Wang, C.K.; Salmon. C.G.; "Reinforced Concrete design," Third Edition. 1979.

- Wee, T. H.; Suryavanshi, A. K.; and Tin, S. S., "Evaluation of Rapid Chloride Permeability Test (RCPT) Results for Concrete Containing Mineral Admixtures," $\mathrm{ACl}$ Materials Journal, V. 97, No. 2, Mar.-Apr. 2000, pp. 221-232.

- Wee, T. H.; Suryavanshi, A. K.; and Tin, S. S., "Influence of Aggregate Fraction in the Mix on the Reliability of the Rapid Chloride Permeability Test," Cement and Concrete Research, V. 21, No. 1, 1999, pp. 59-72.

- Whiting, D. (1981). "Rapid Determination of the Chloride Permeability of Concrete," FHWA-RD-81/119. Federal Highway Administration, Washington, D.C.

- Wong, P. S.-L., "User Facilities for Two-Dimensional Nonlinear Finite Element Analysis of Reinforced Concrete," MASc thesis, Department of Civil Engineering, University of Toronto, Toronto, Ontario, Canada, 2002, 213 pp.

- Yang, C. C., and Su, J. K., "Approximate Migration Coefficient of Interfacial Transition Zone and the Effect of Aggregate Content on the Migration Coefficient of Mortar," Cement and Concrete Research, V. 32, No. 10, 2002, pp. 1559-1565.

- Yang, C-C.; "Relationship between Migration Coefficients of Chloride lons and Charge Passed in Steady State," AACI Materials Journal, V. 101, No.2, March-April 2004. 


\section{Appendices}

\section{Appendix A: Corrosion crack pattern}

The following figures illustrate the corrosion crack pattern for the NC and SCC beams with $5 \%, 10 \%, 15 \%$, and $20 \%$ mass loss.

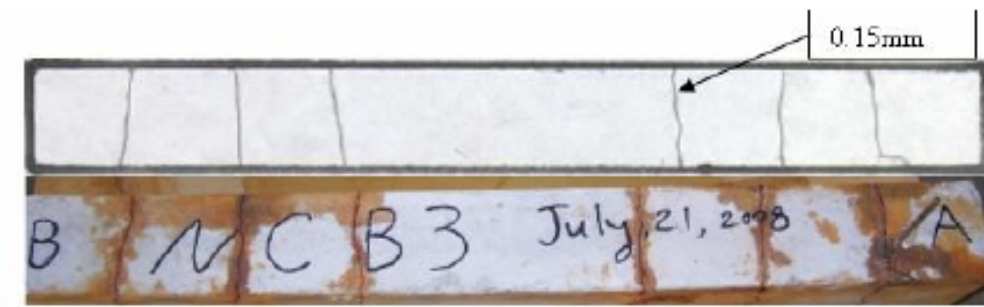

Top

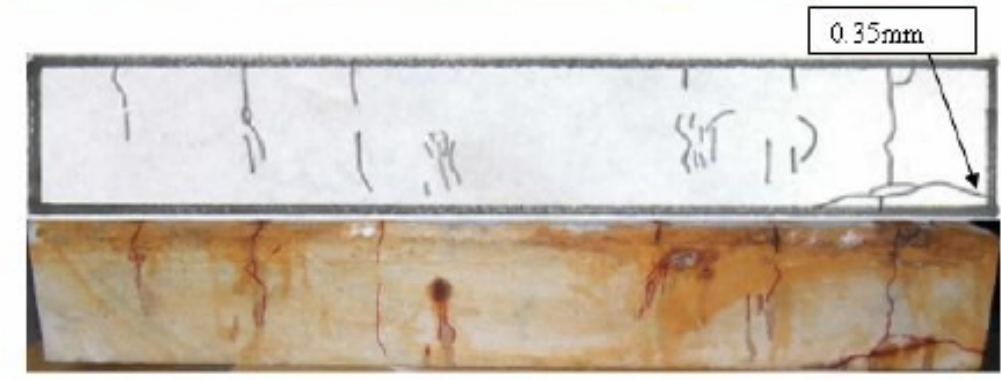

Left
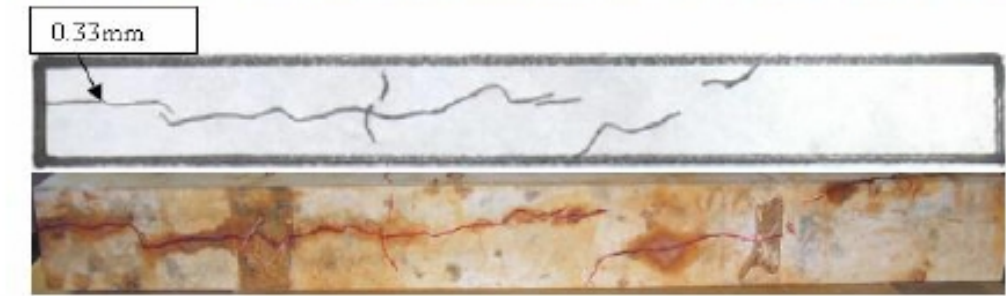

Bottom
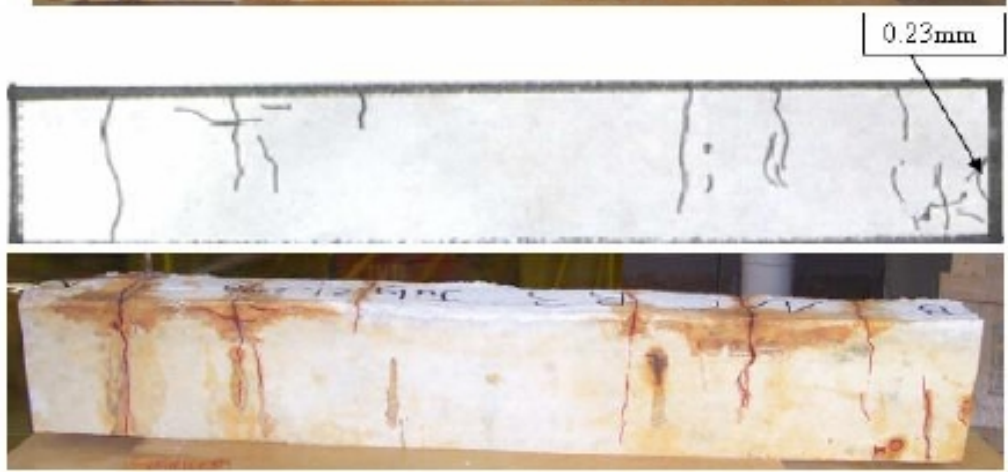

Right
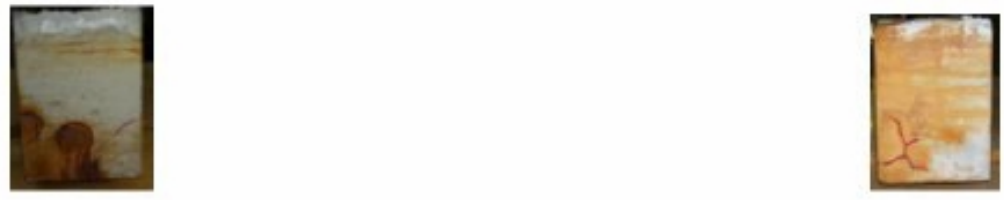

Side B

Side A

Figure (A-1): The crack pattern of NC beam no. 3. 5\% mass loss. 

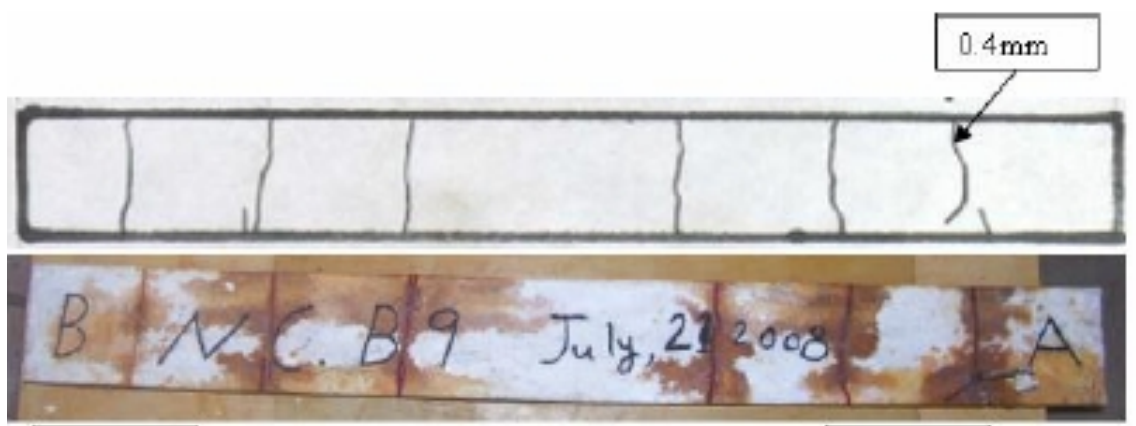

Top
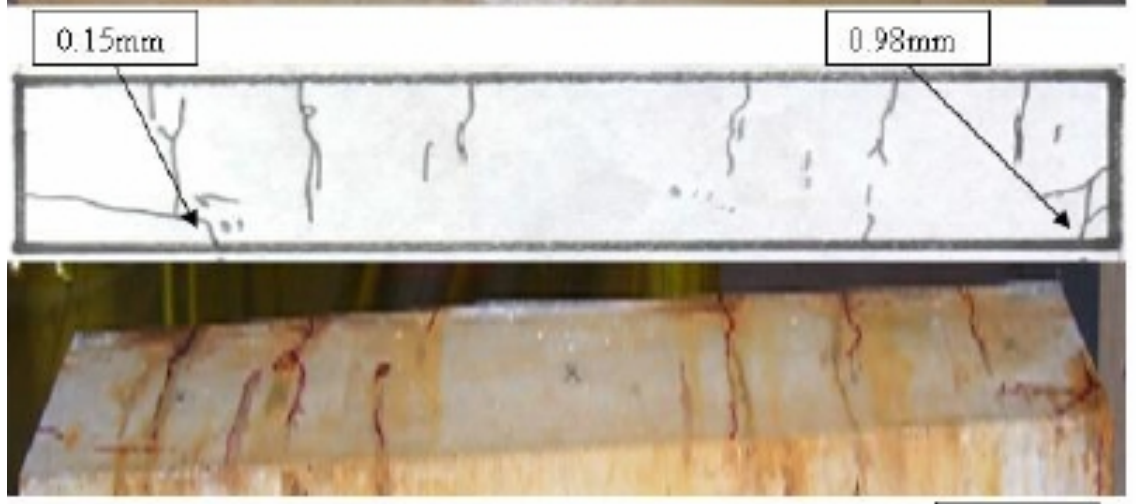

Left
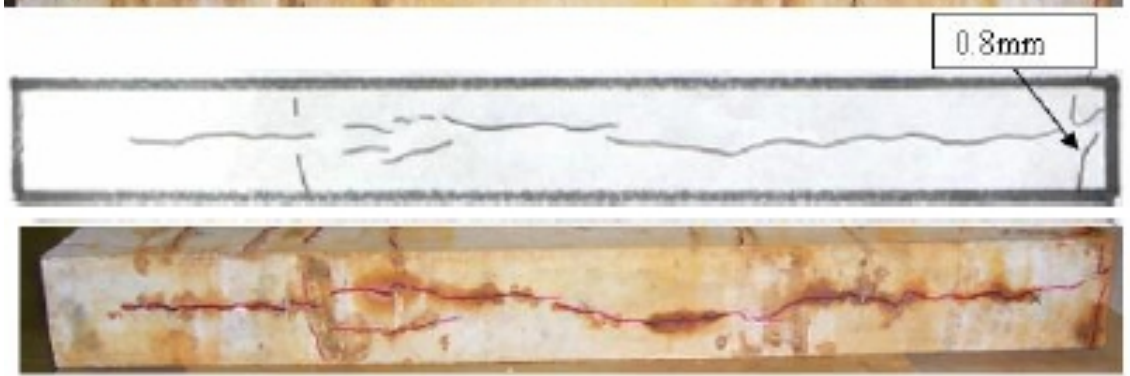

Bottom

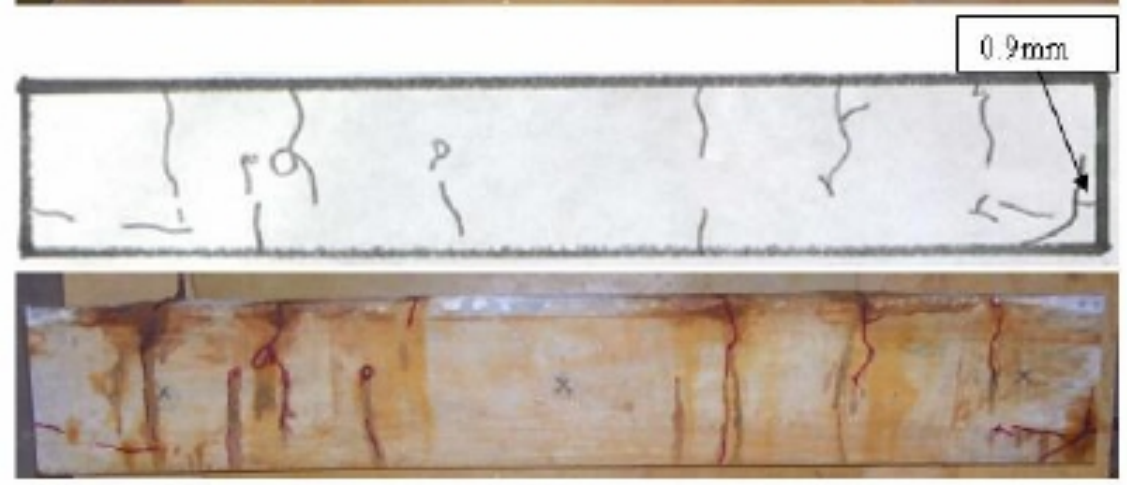

Right

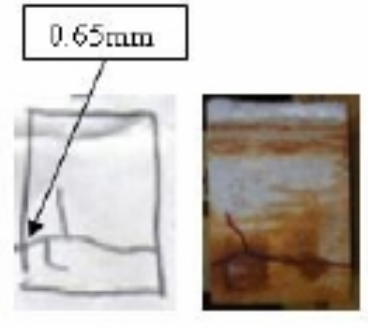

Side B

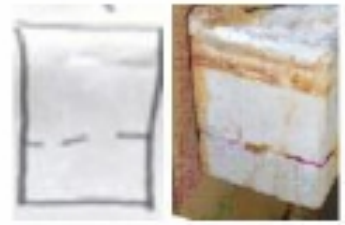

Side A

Figure (A-2): The crack pattern of NC beam no. 9. 5\% mass loss. 


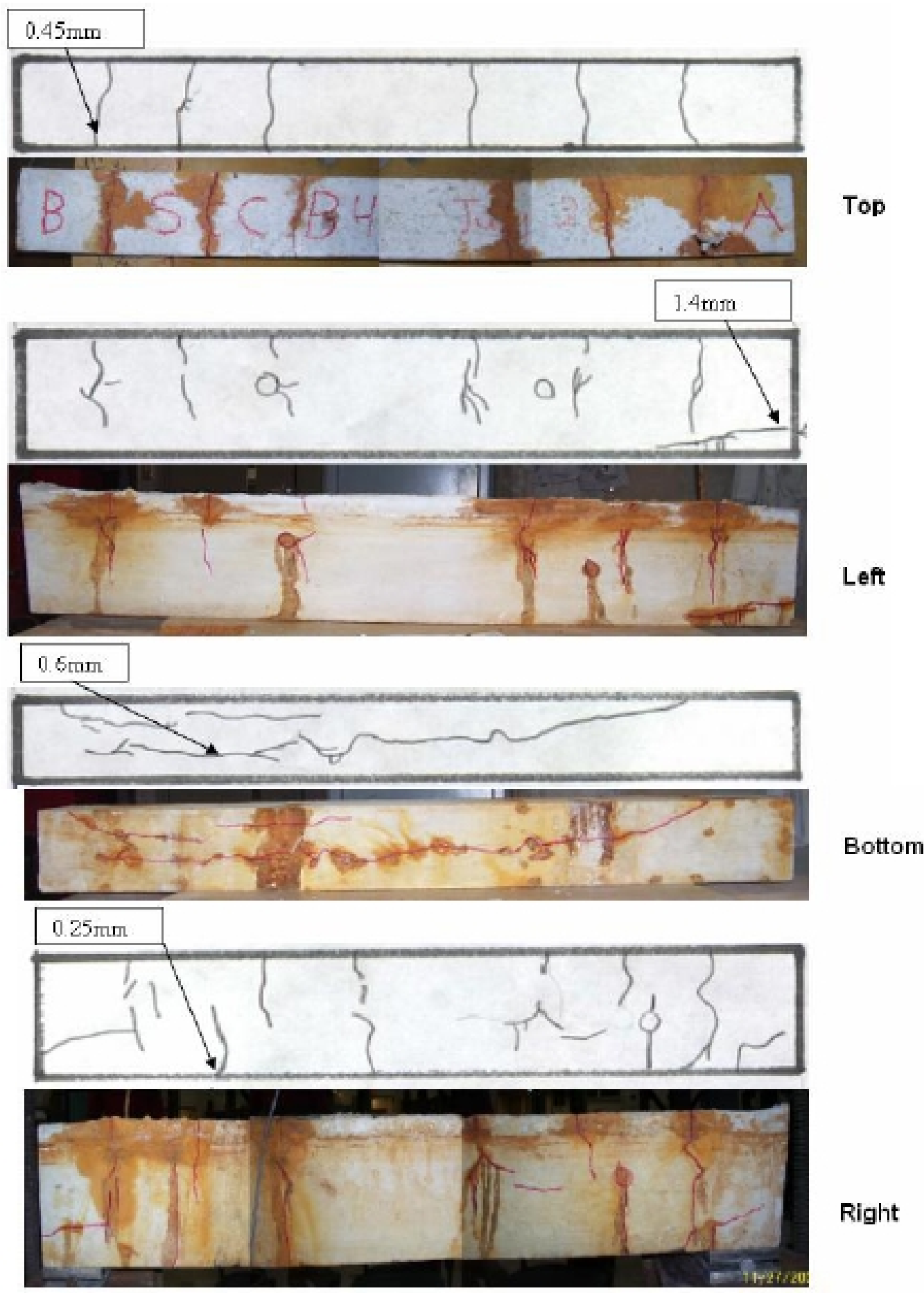

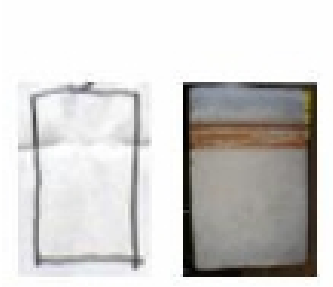

Side B

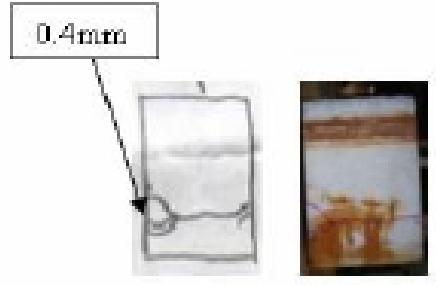

Side A

Figure (A-3): The crack pattern of SCC beam no. $4.5 \%$ mass loss. 

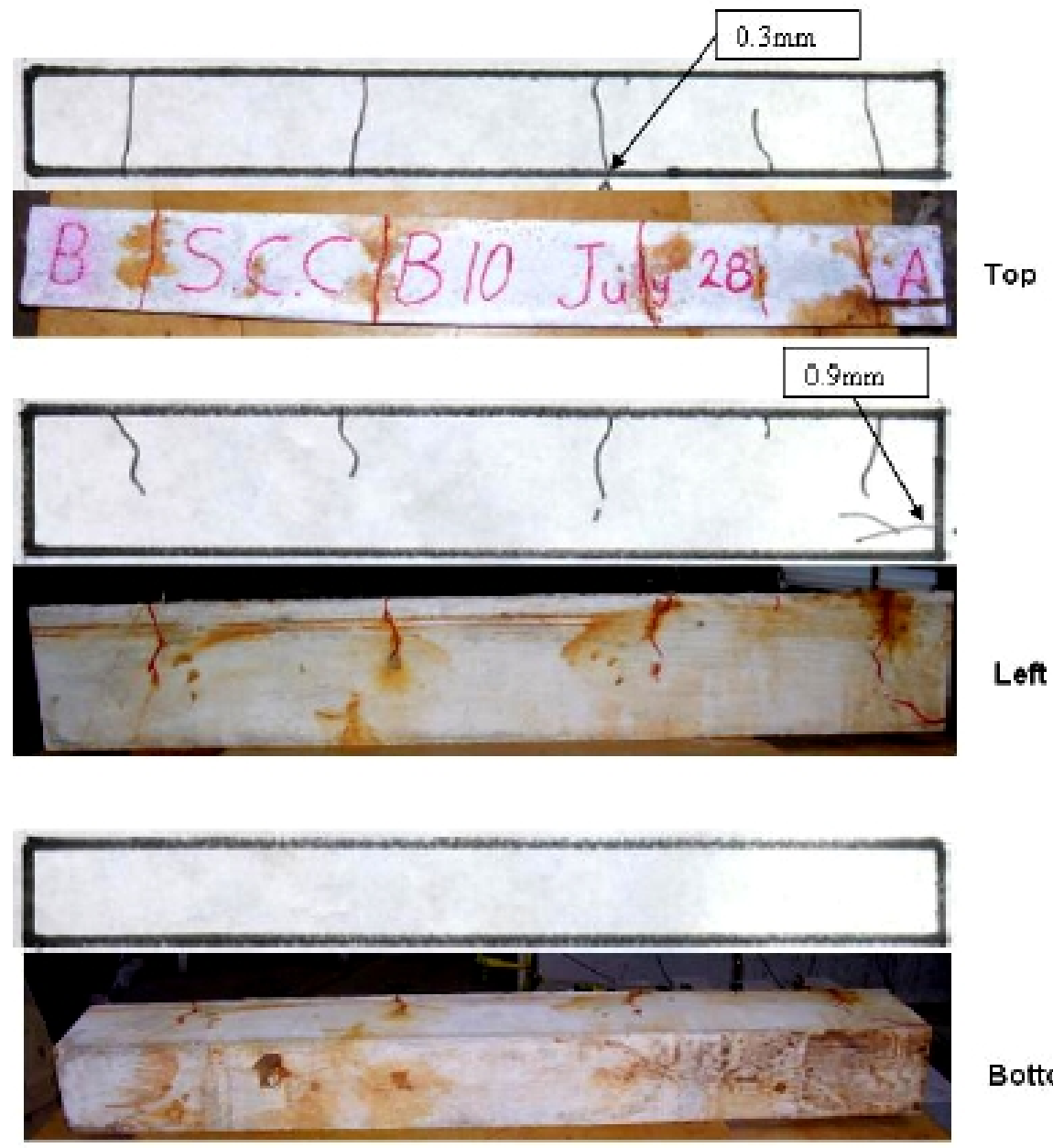

Bottom
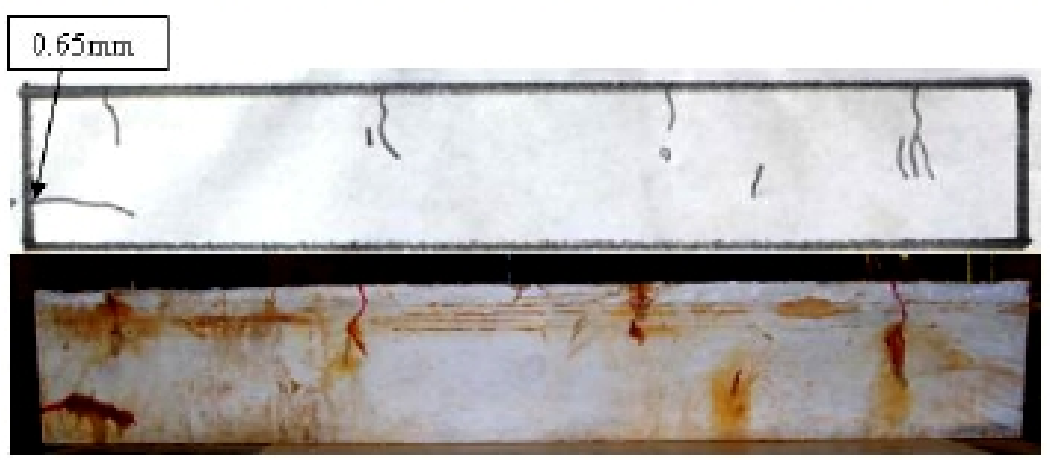

Right

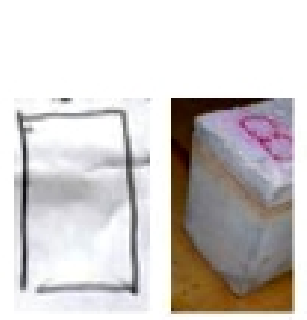

Side B

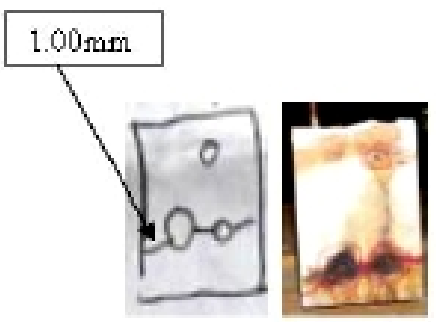

Side A

Figure (A-4): The crack pattern of SCC beam no.10. 5\% mass loss. 

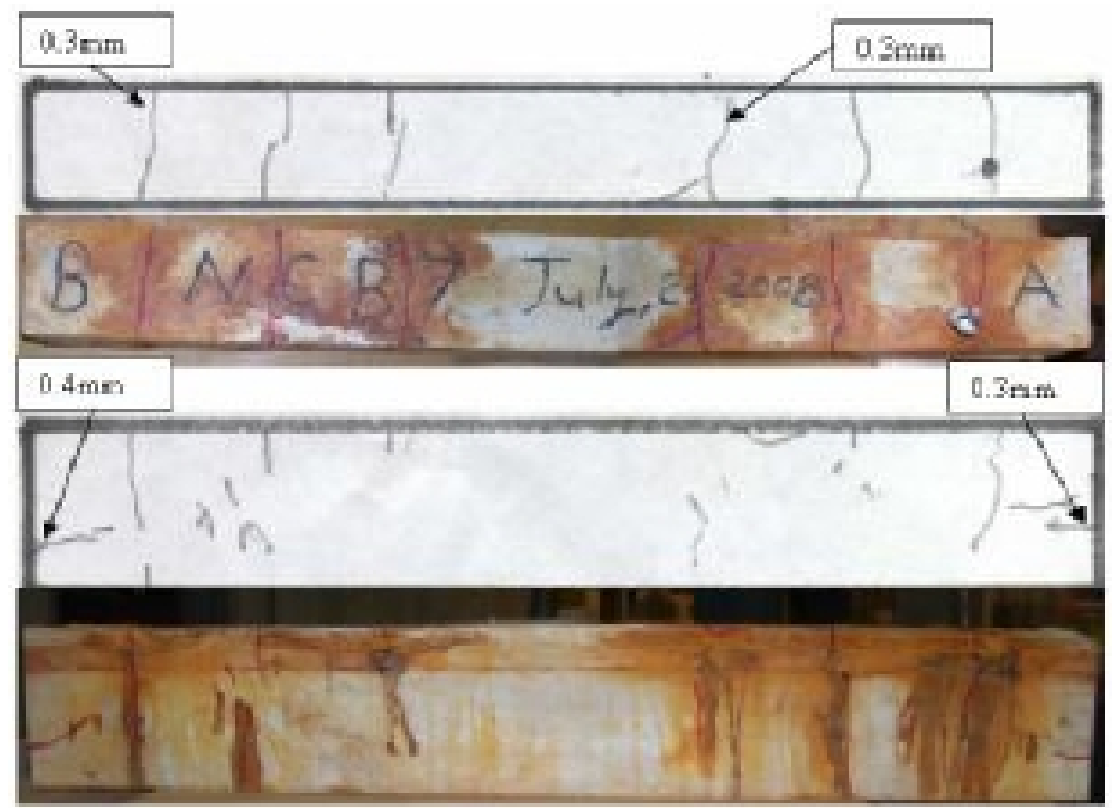

Top

Lef
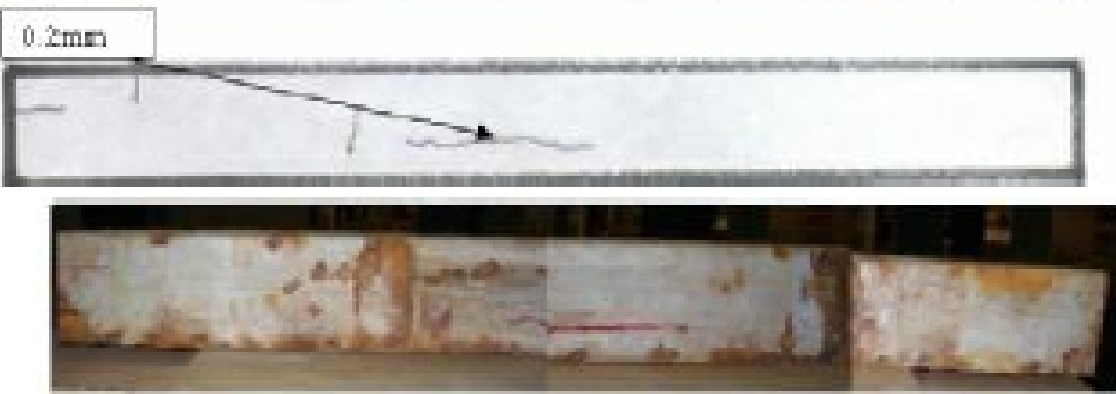

Bettom
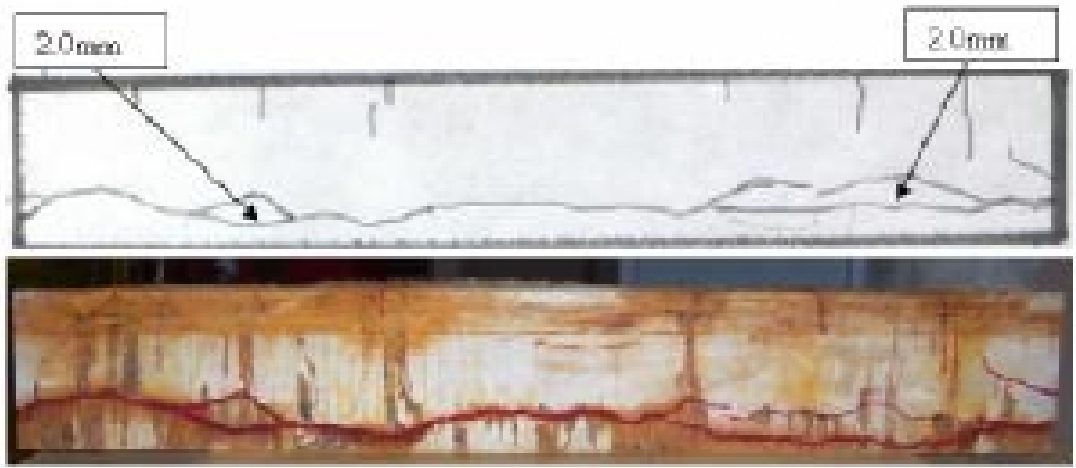

Right

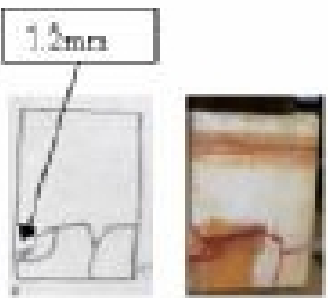

Side B

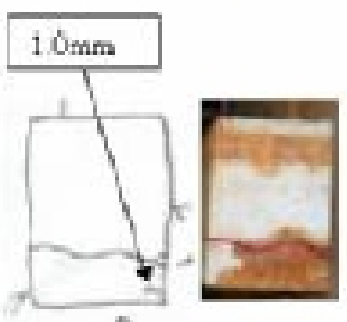

Side A

Figure (A-5): The crack pattern of NC beam no. 7. 10\% mass loss. 

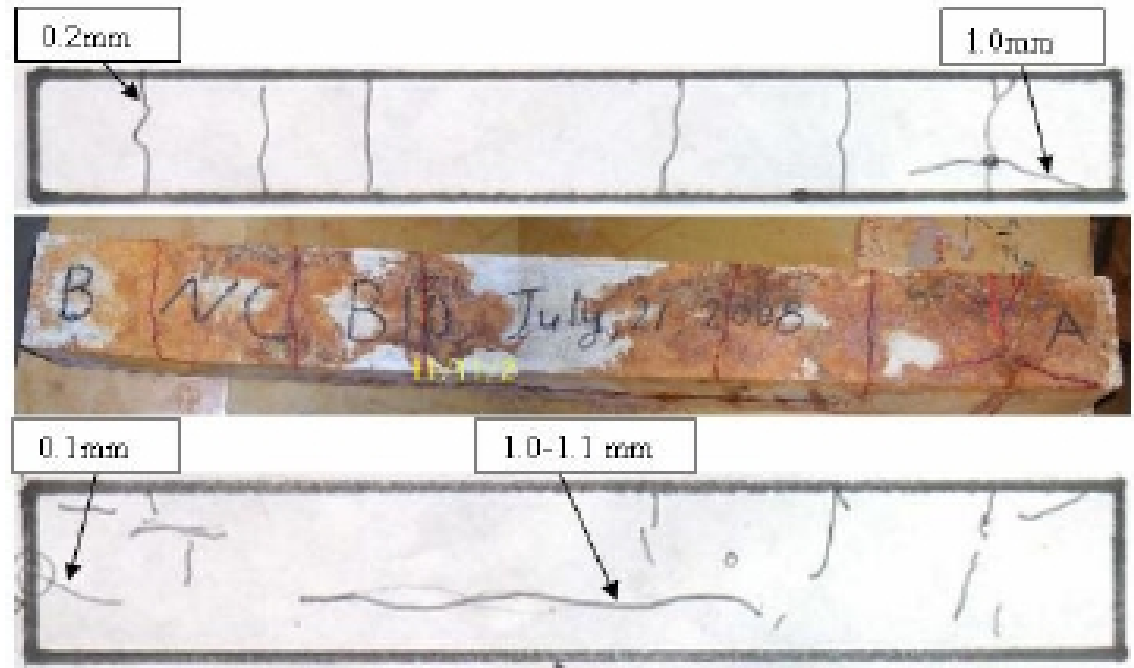

Top

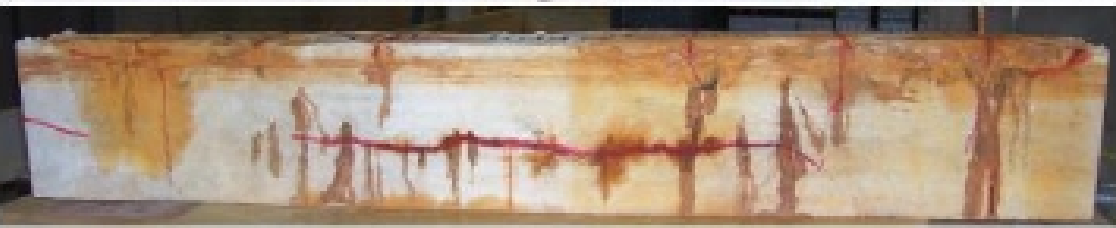

Left
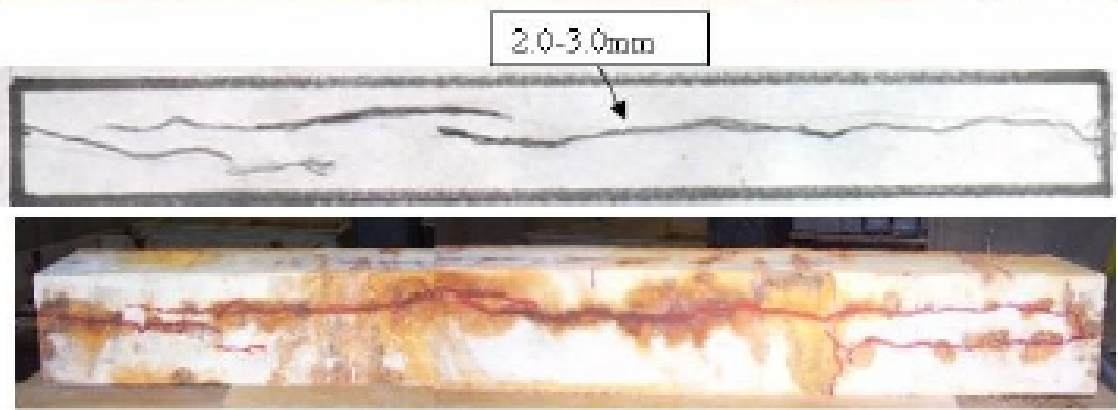

Bottom
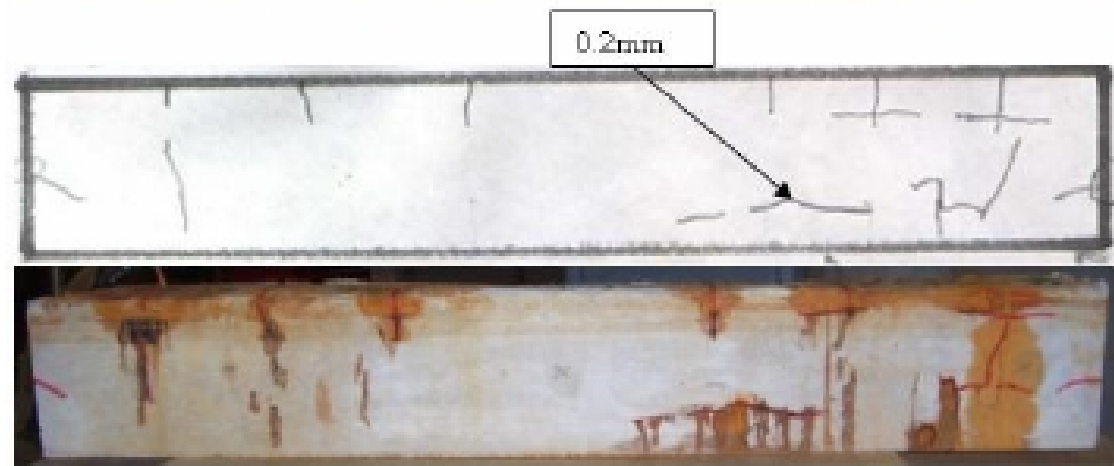

Right

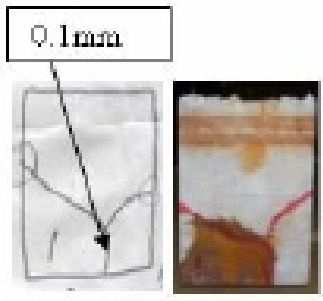

Side B

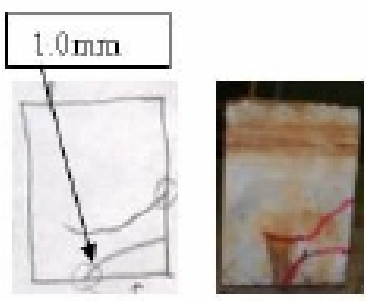

Side A

Figure (A-6): The crack pattern of NC beam no.10. 10\% mass loss. 


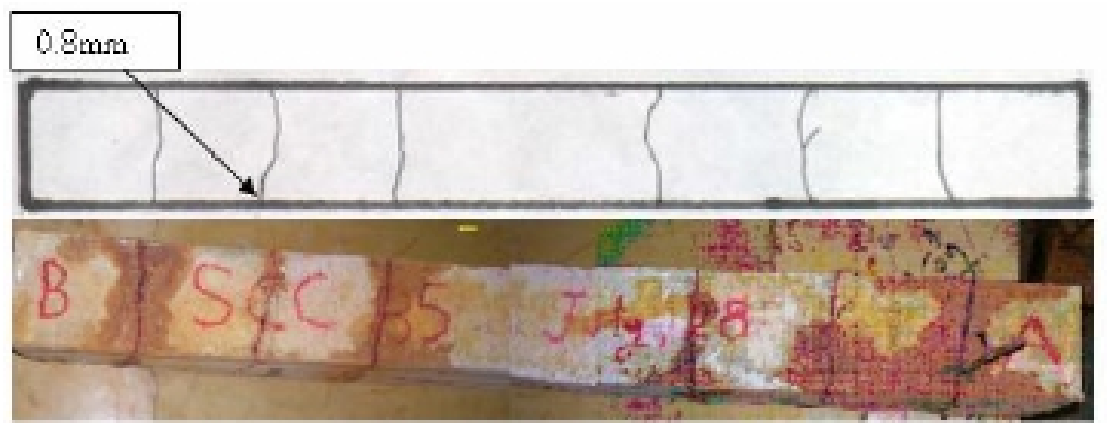

Top
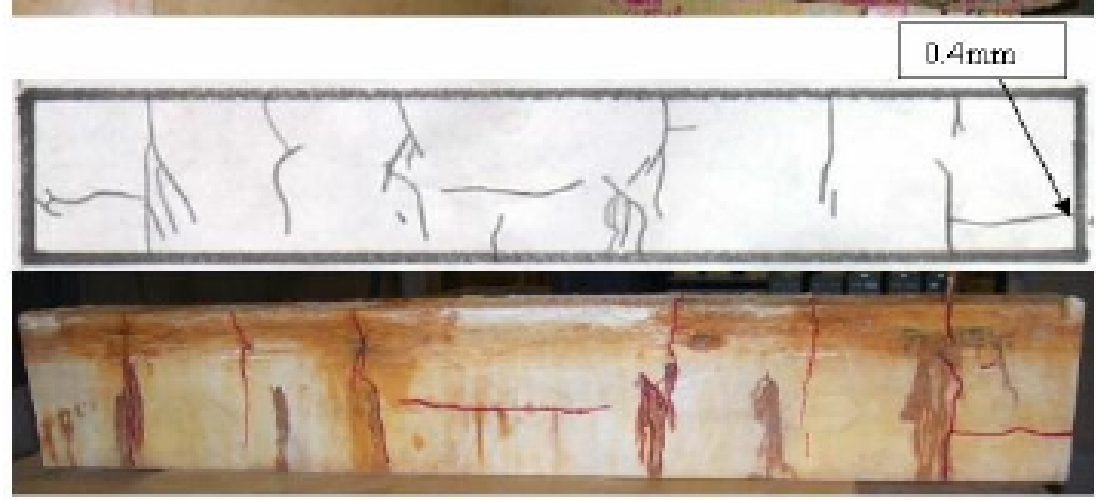

Left
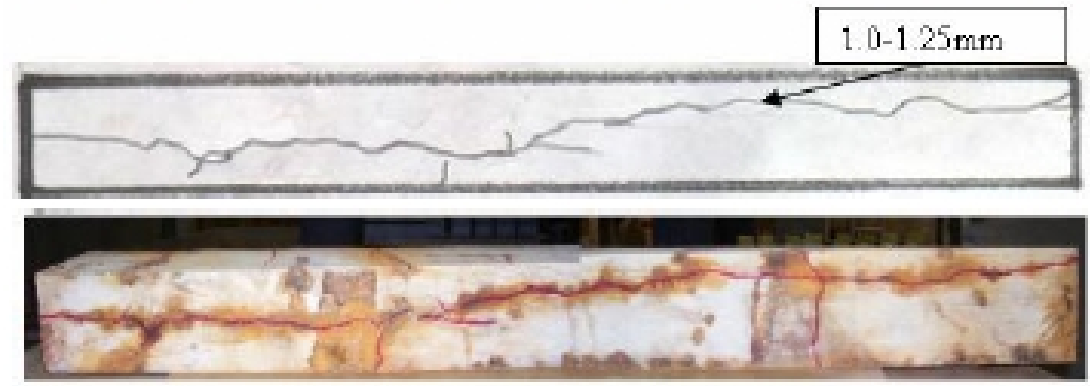

Bottom

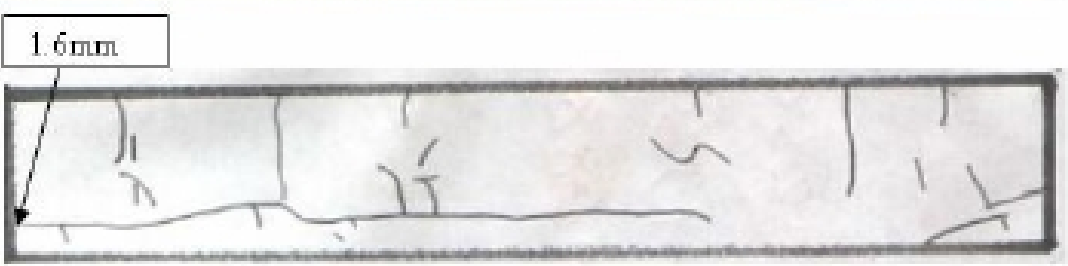

Right

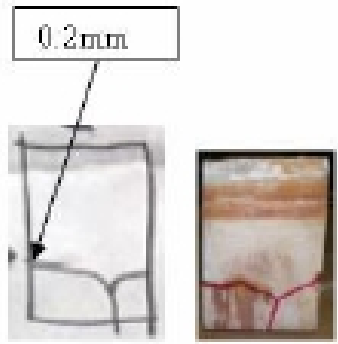

Side B

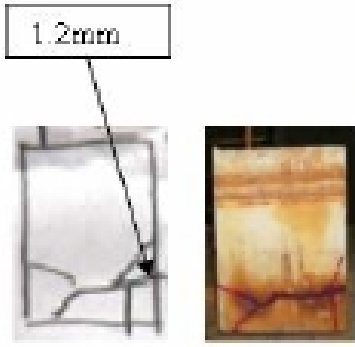

Side A

Figure (A-7): The crack pattern of SCC beam no.5. 10\% mass loss. 

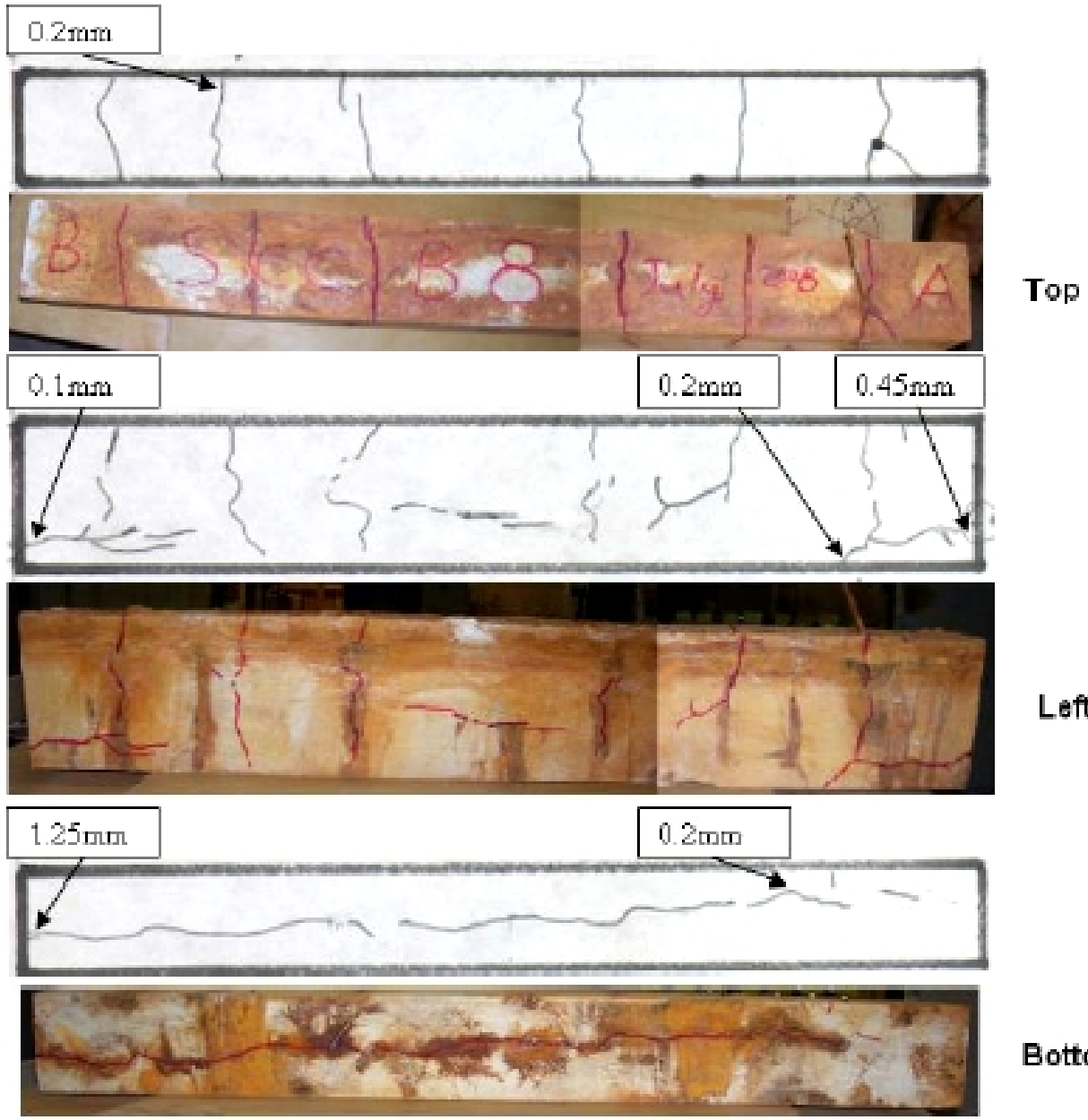

Bottom
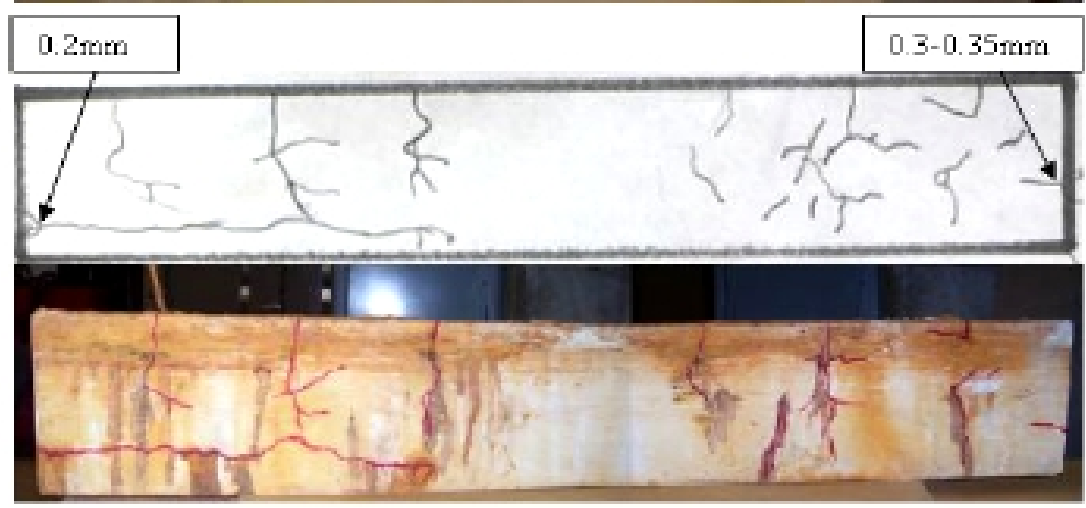

Right

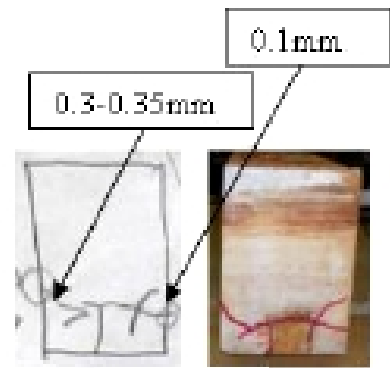

Side B

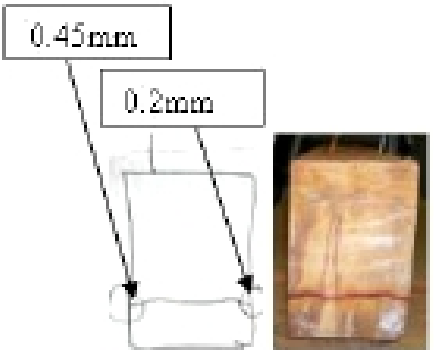

Side A

Figure (A-8): The crack pattern of SCC beam no.8. 10\% mass loss. 


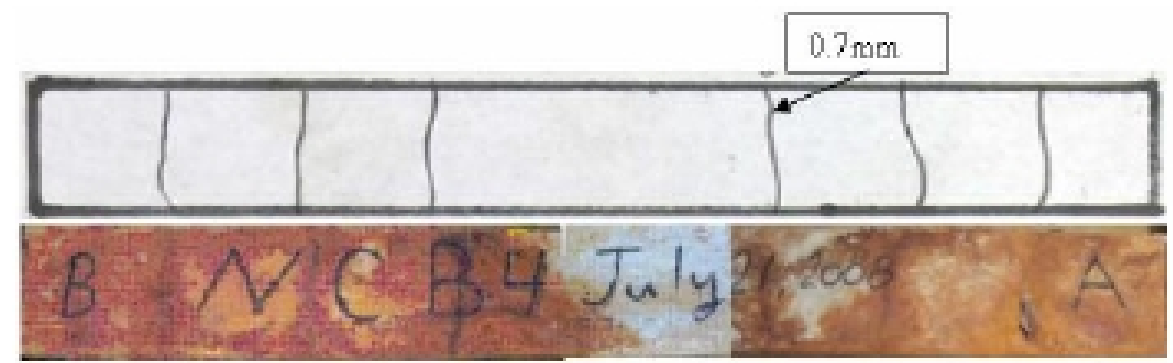

Top
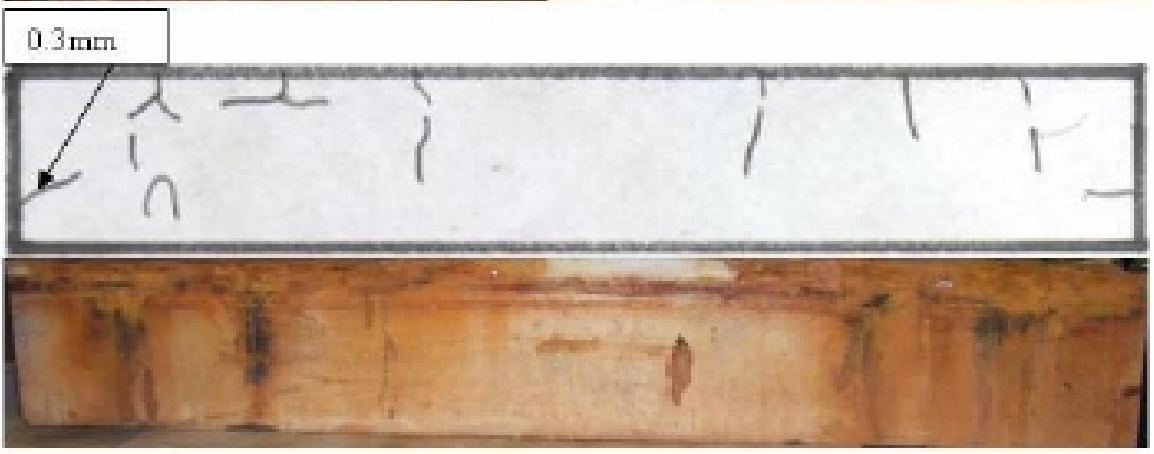

Left

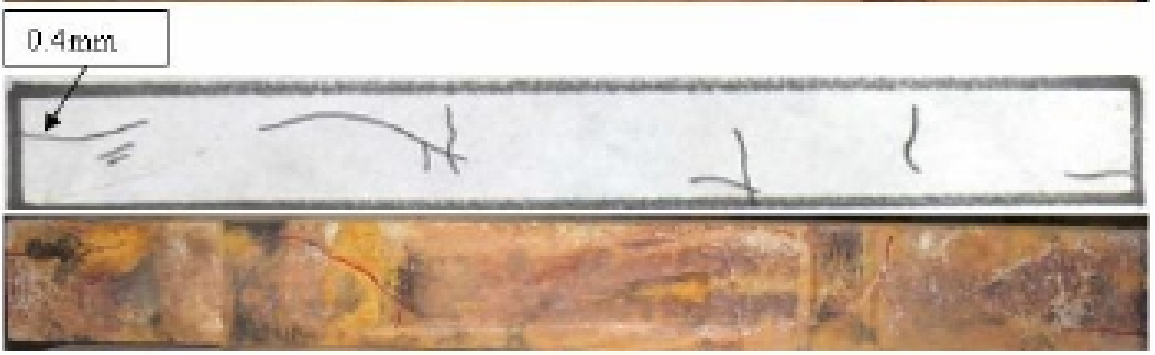

Bottom
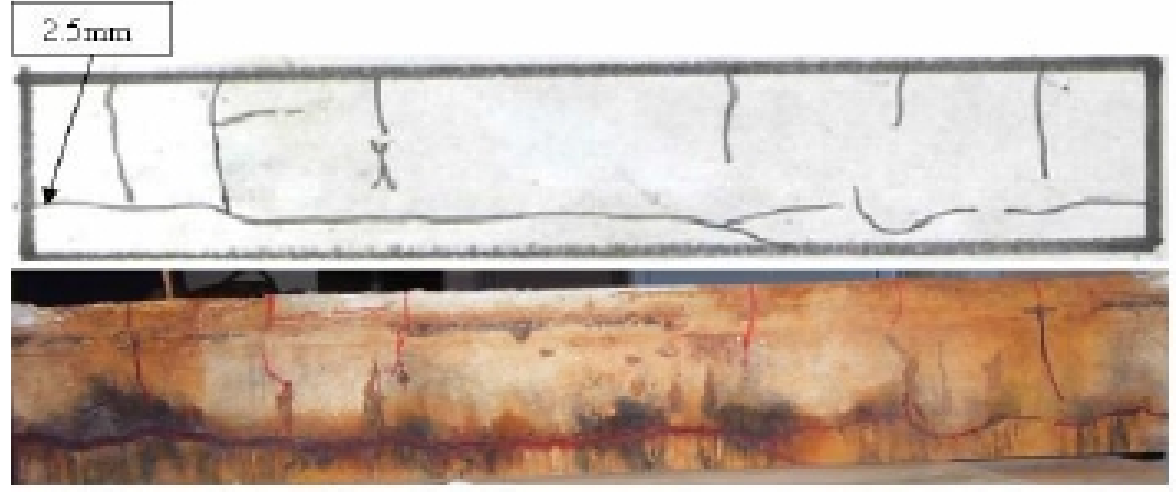

Right
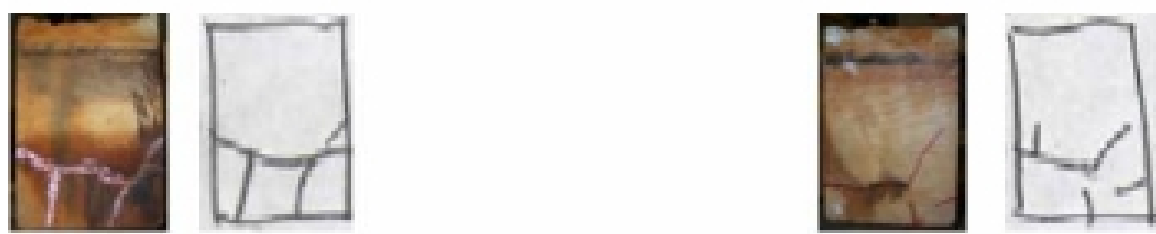

Side B

Side A

Figure (A-9): The crack pattern of NC beam no.4. $15 \%$ mass loss. 

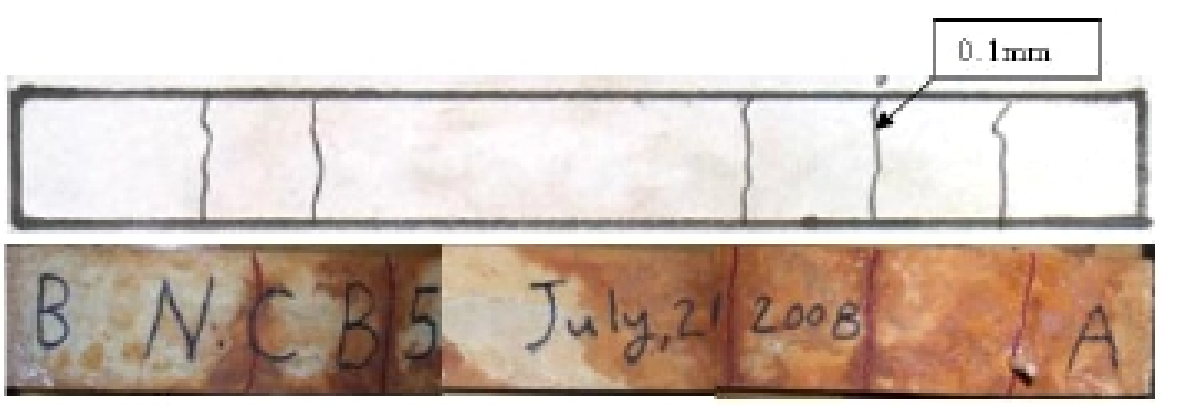

Top

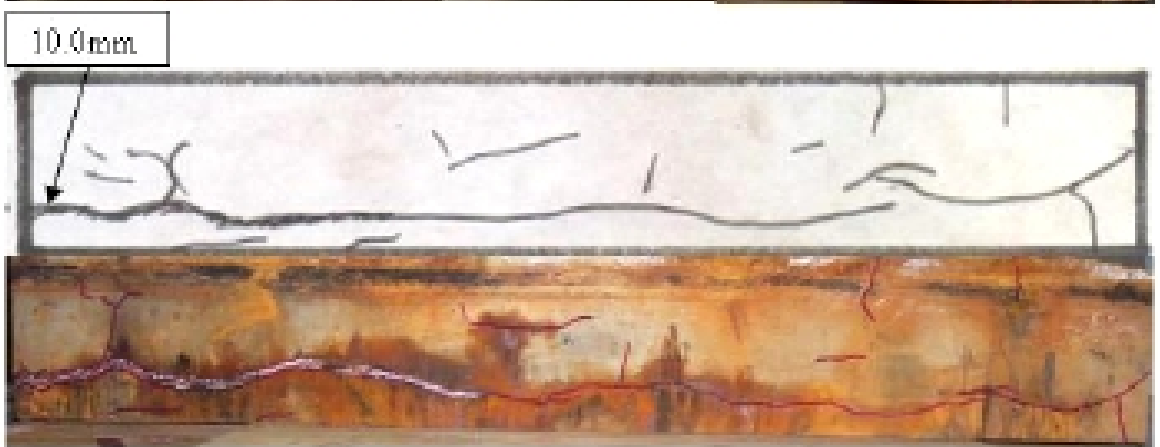

Left
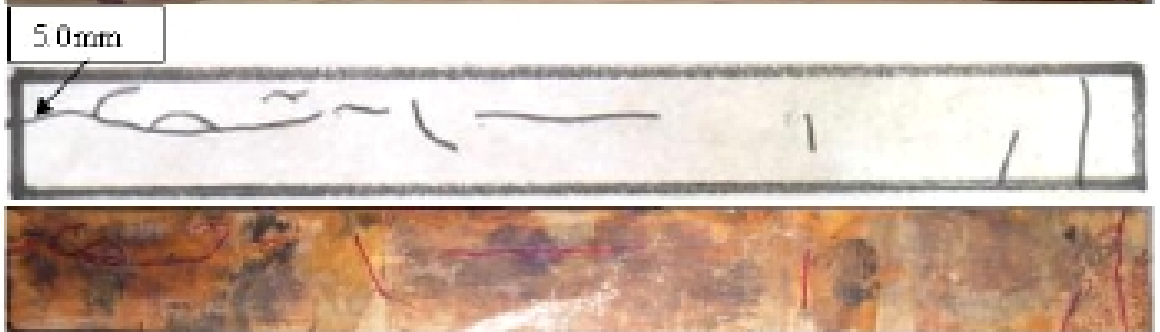

Bottom
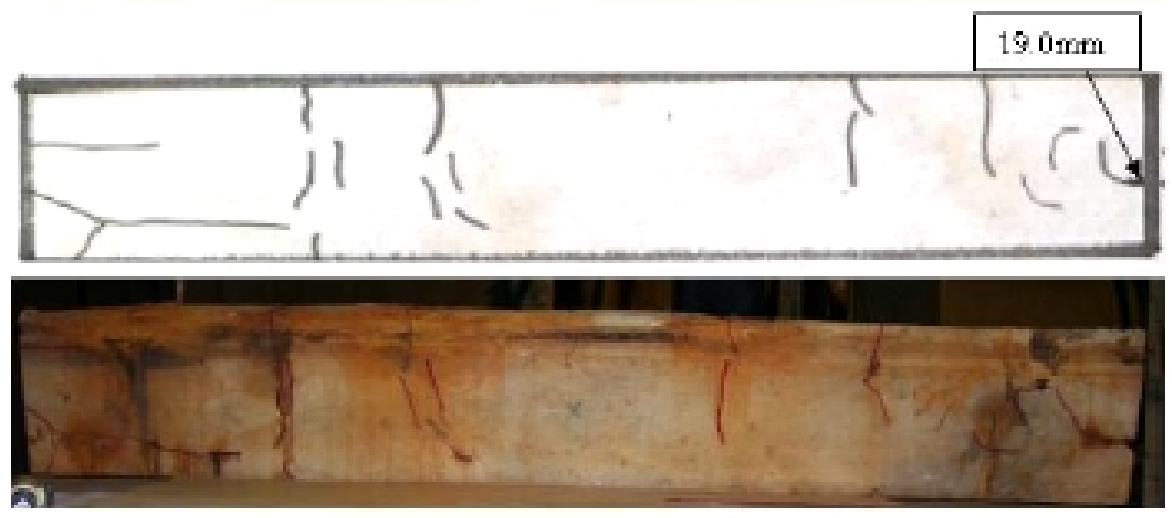

Right

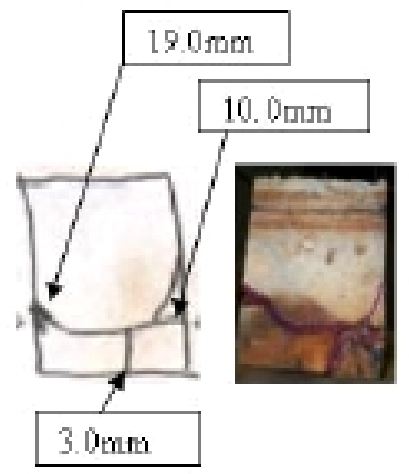

Side B

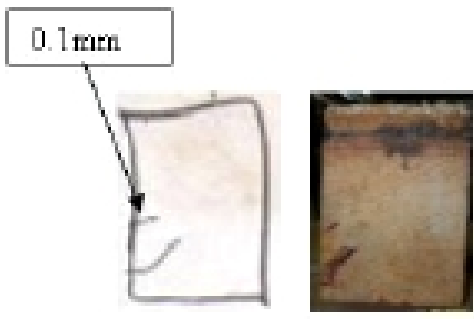

Side A

Figure (A-10): The crack pattern of NC beam no.5. 15\% mass loss. 


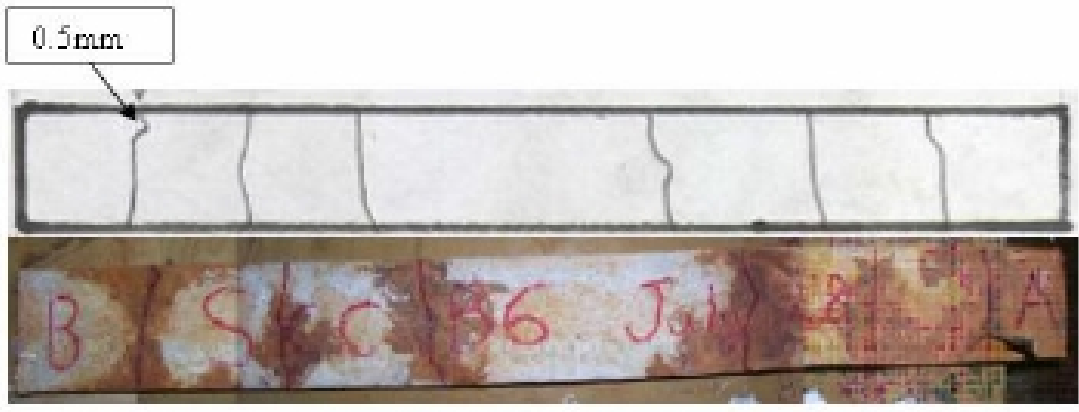

Top
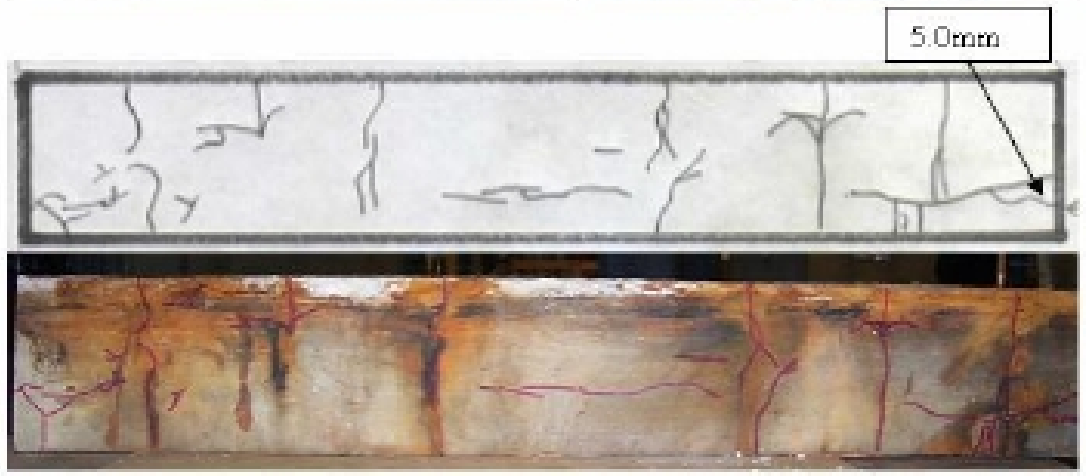

Left
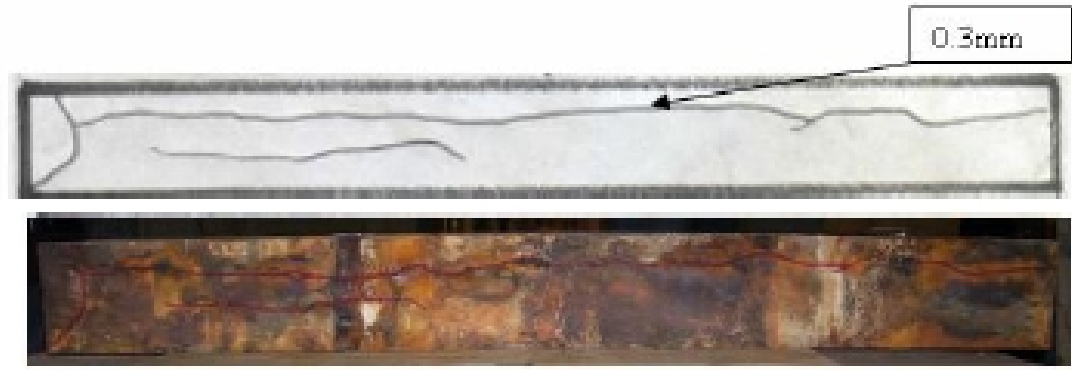

Bottom
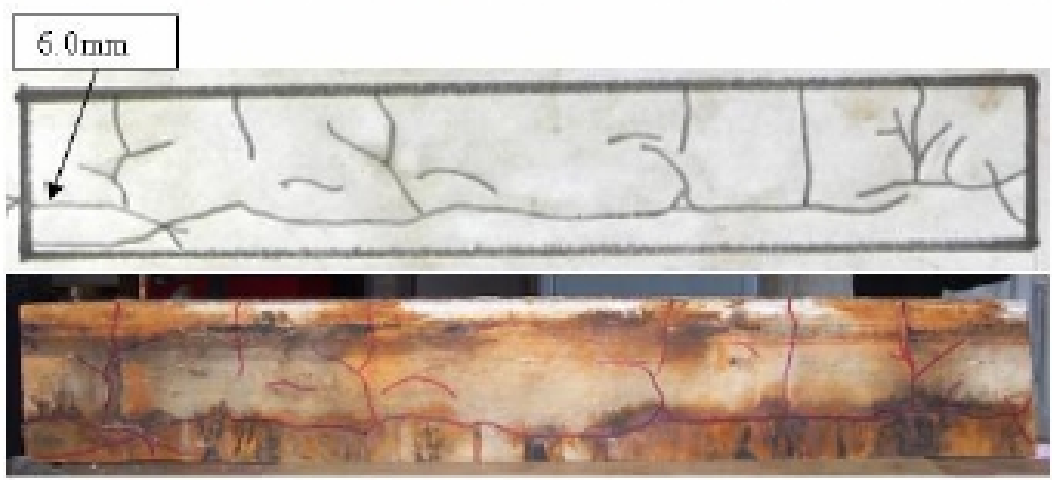

Right

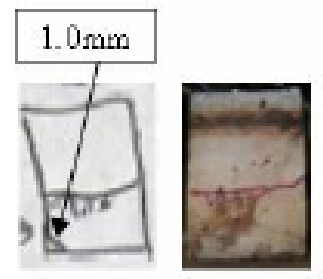

Side B

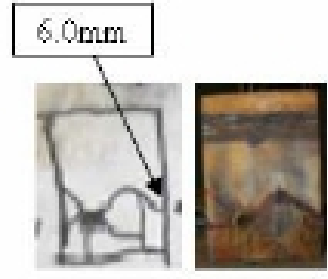

Side A

Figure (A-11): The crack pattern of SCC beam no.6. 15\% mass loss. 


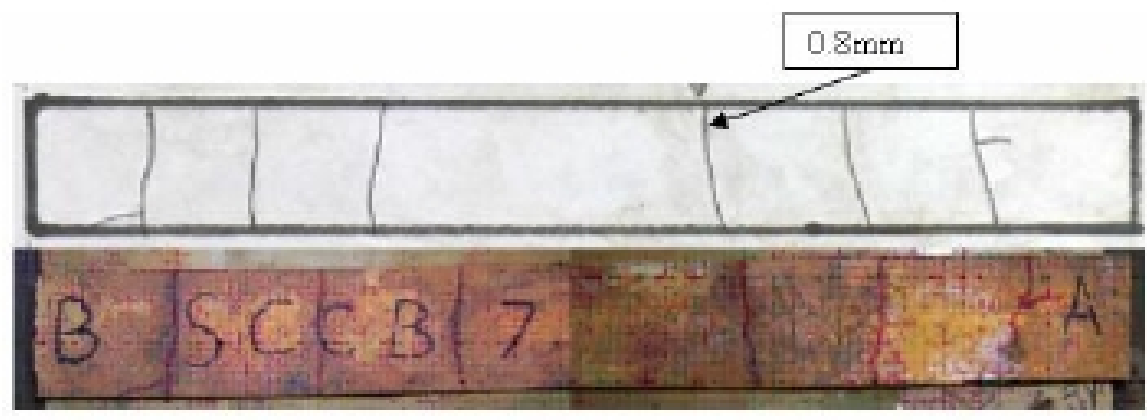

Top
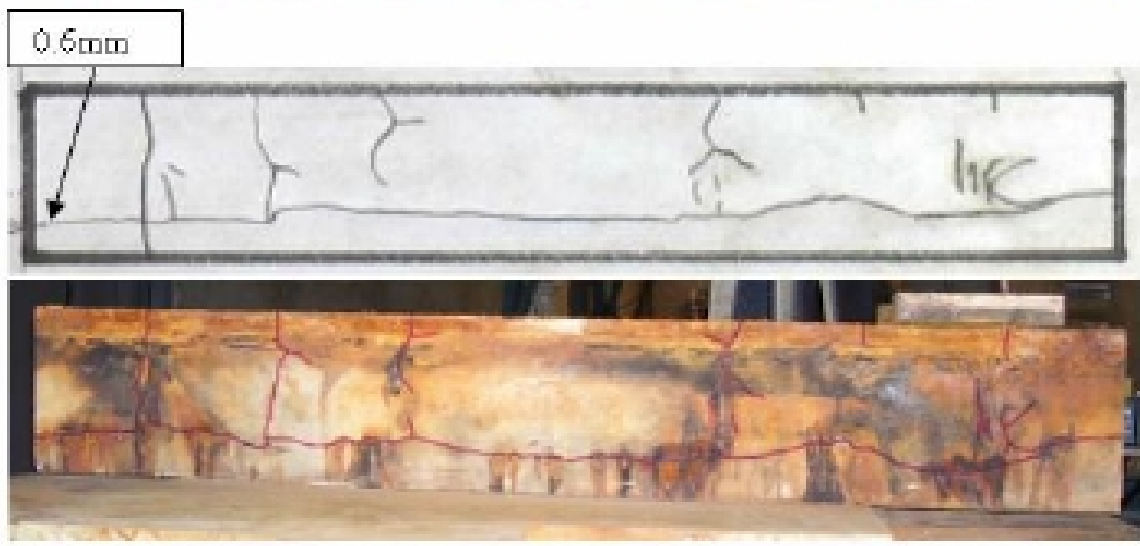

Left
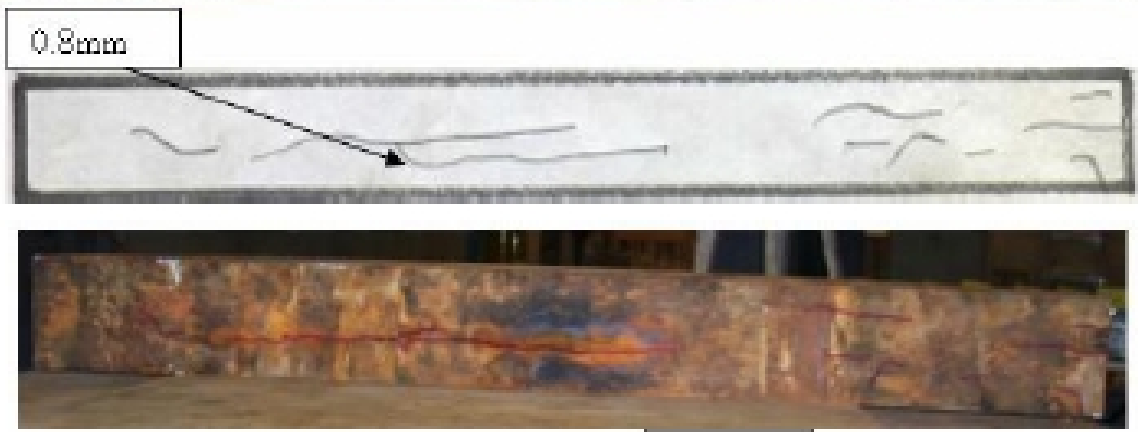

Bottom
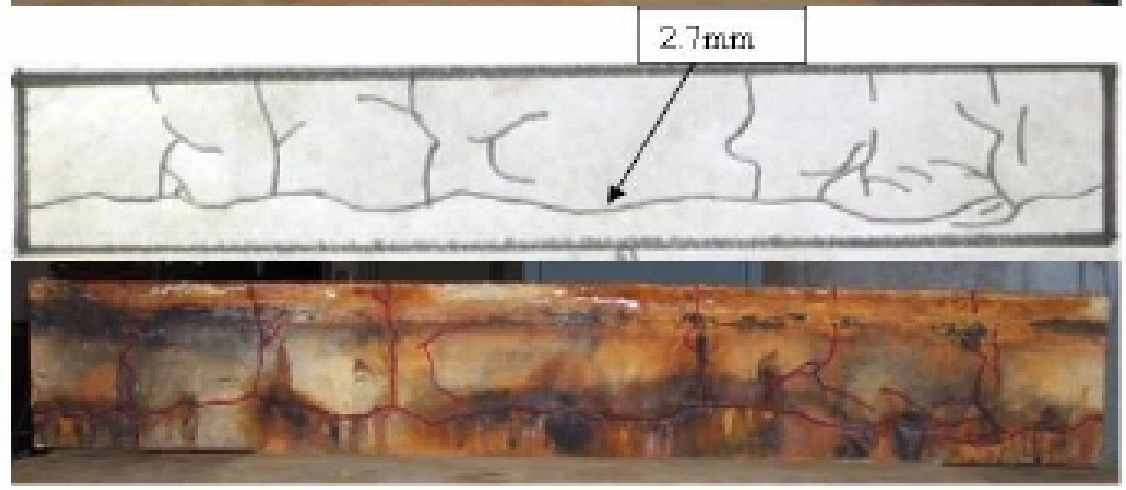

Right
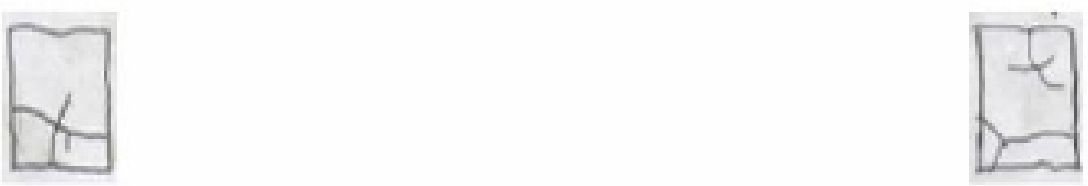

Side B

Side A

Figure (A-12): The crack pattern of SCC beam no.7. 15\% mass loss. 


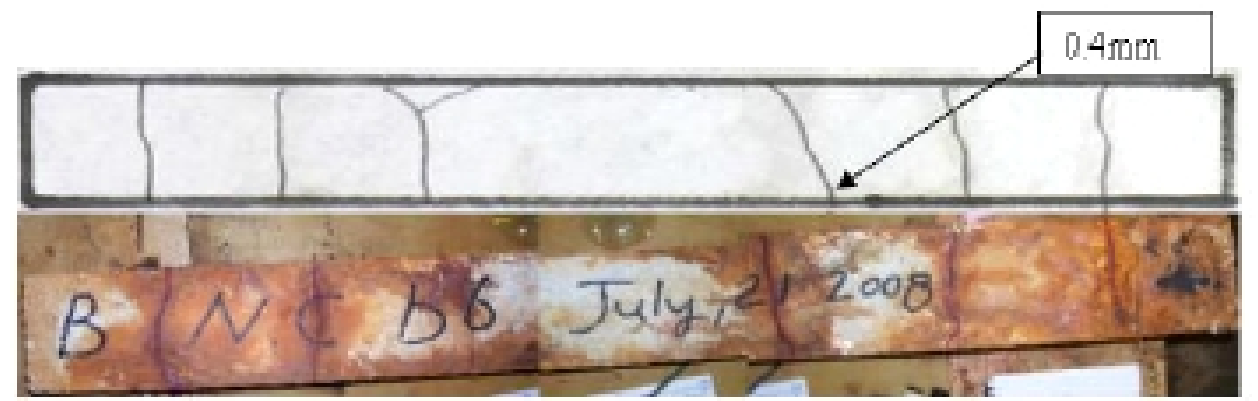

Top

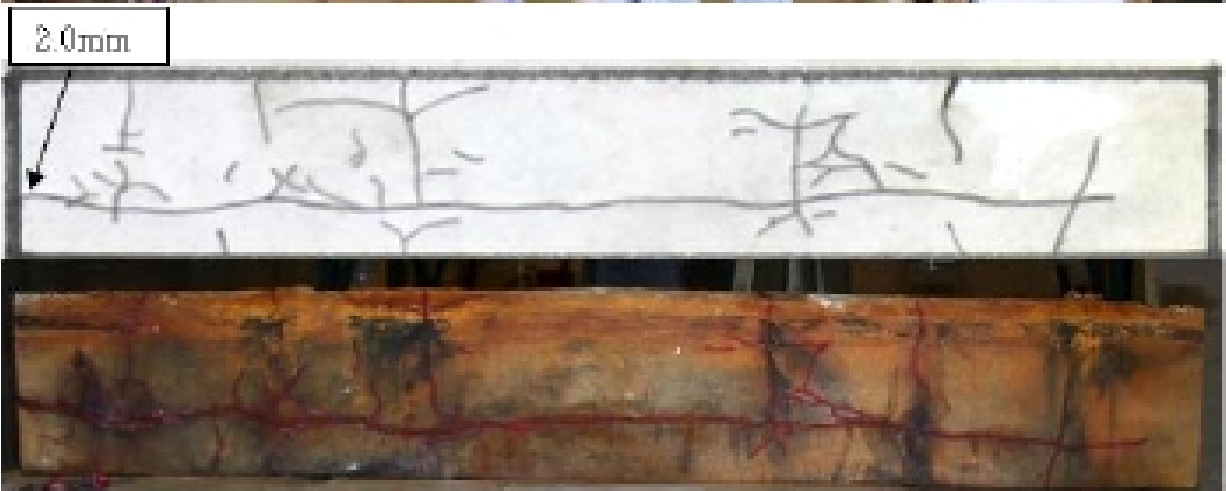

Left

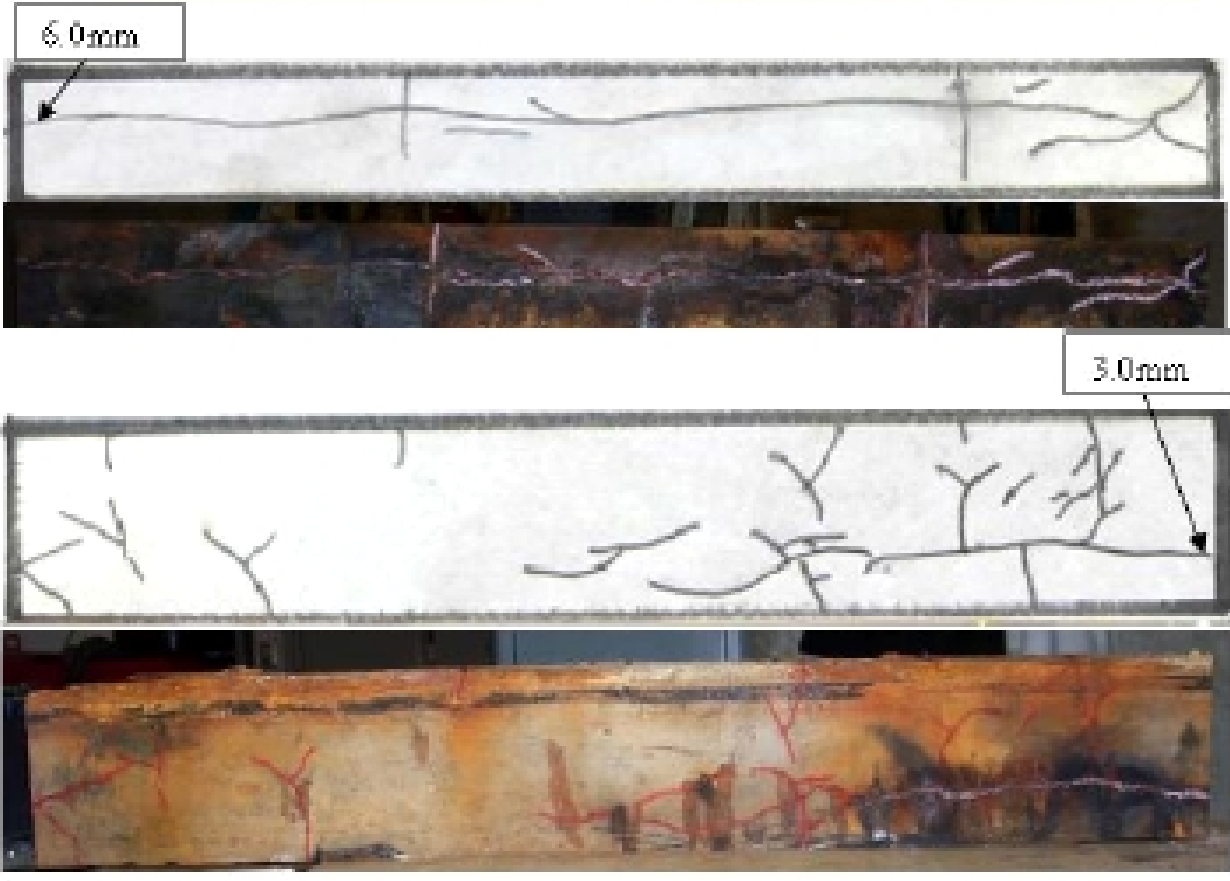

Bottom

Right

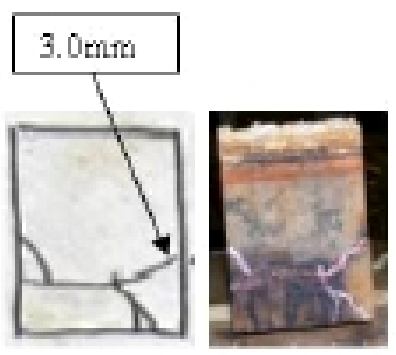

Side B

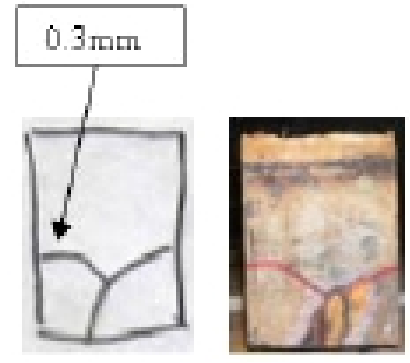

Side A

Figure (A-13): The crack pattern of NC beam no.6. $20 \%$ mass loss. 

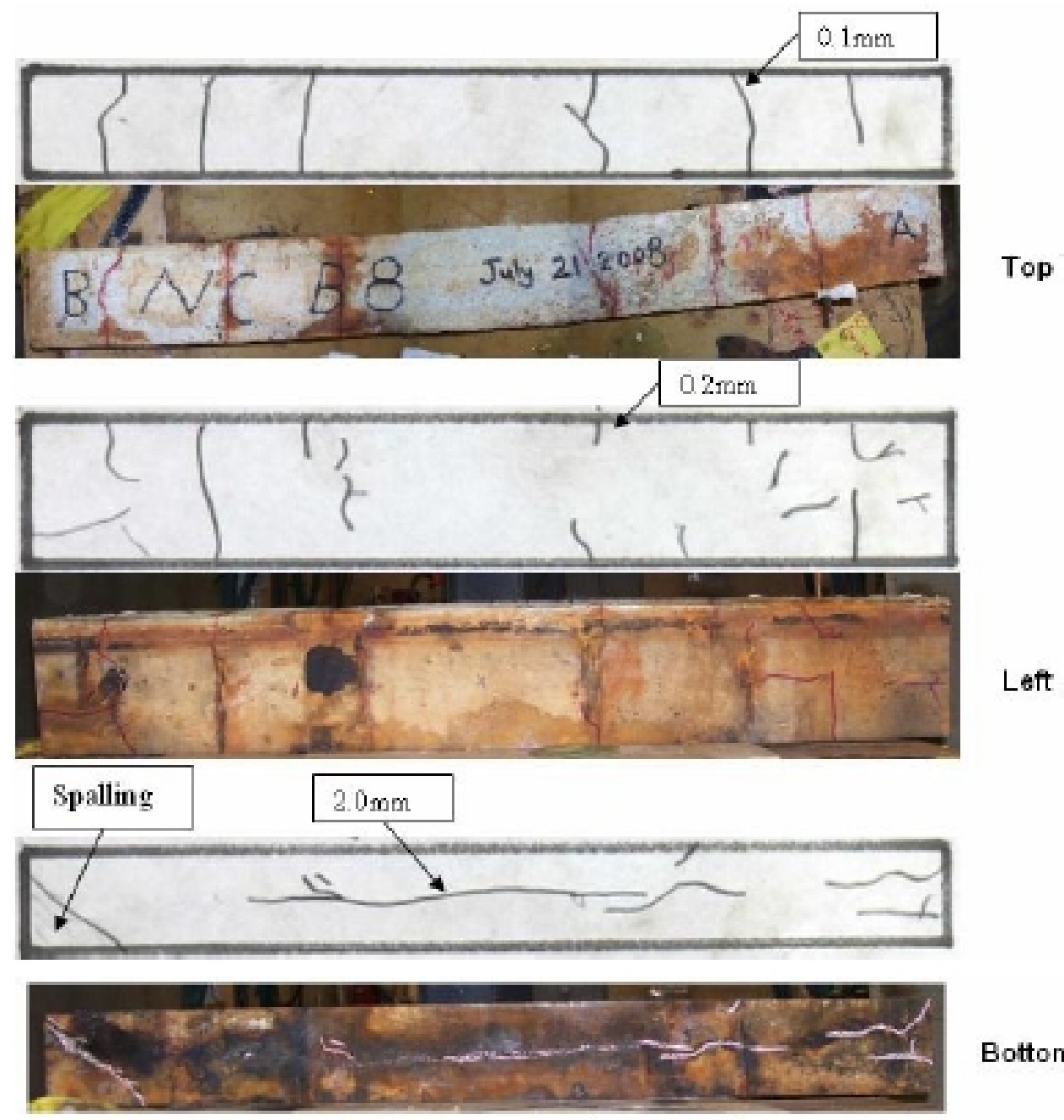

Bottom

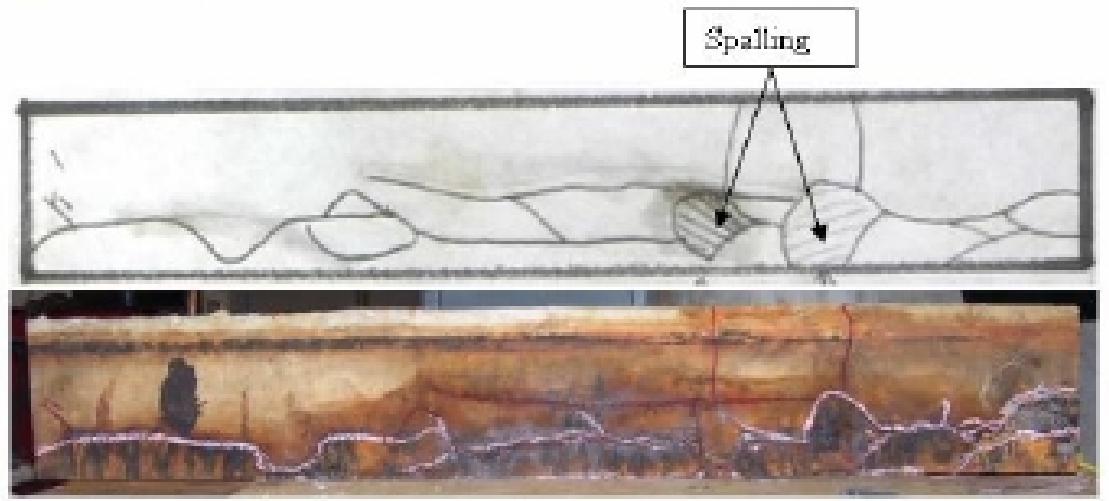

Right

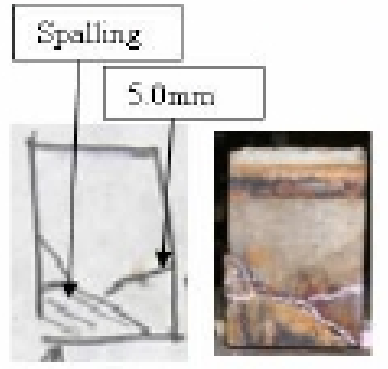

Side $\mathrm{B}$

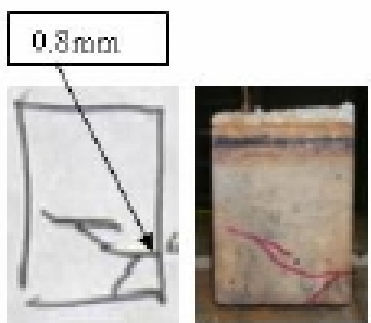

Side A

Figure (A-14): The crack pattern of NC beam no.8. $20 \%$ mass loss. 

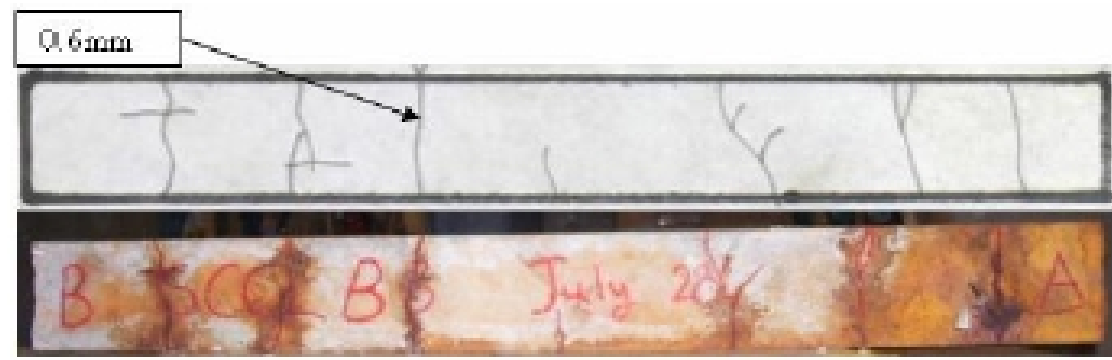

Top

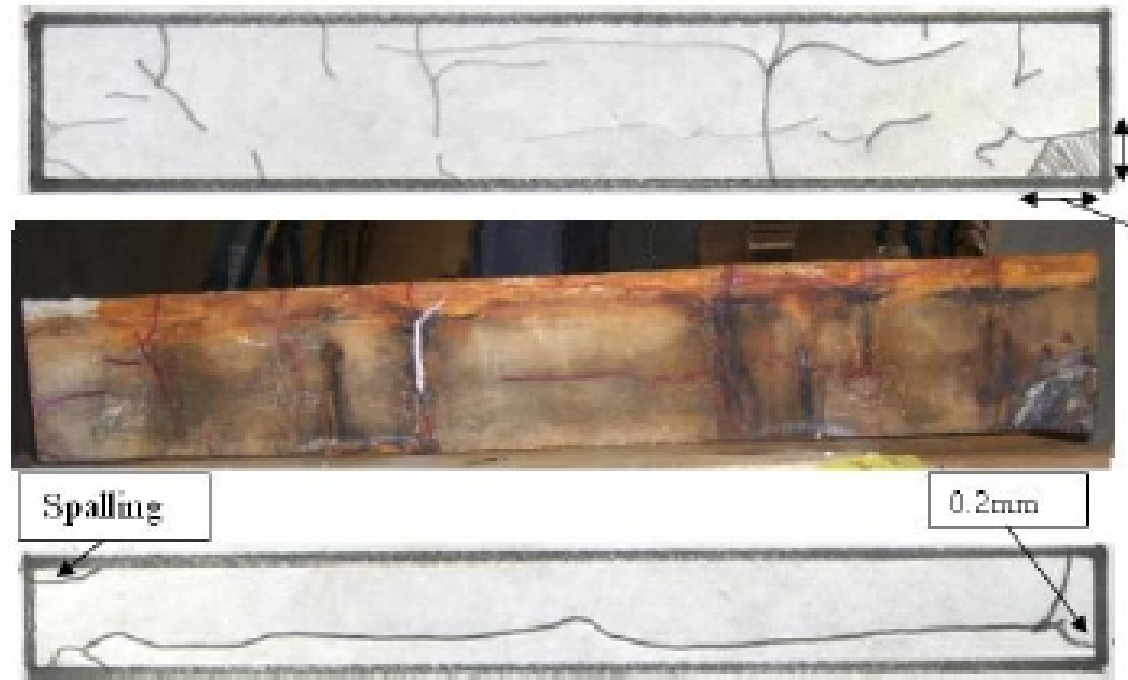

$87 \mathrm{~m} \pi$

$110 \mathrm{mrn}$

Left

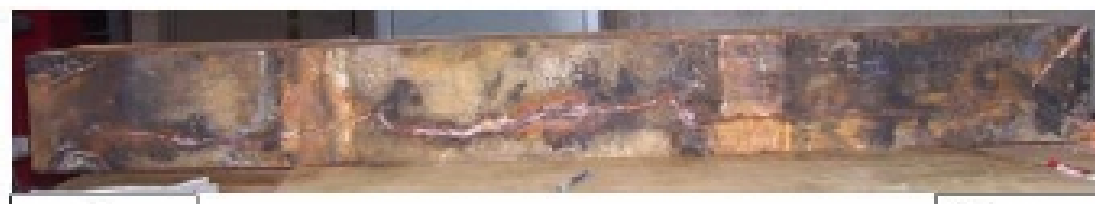

Bottom
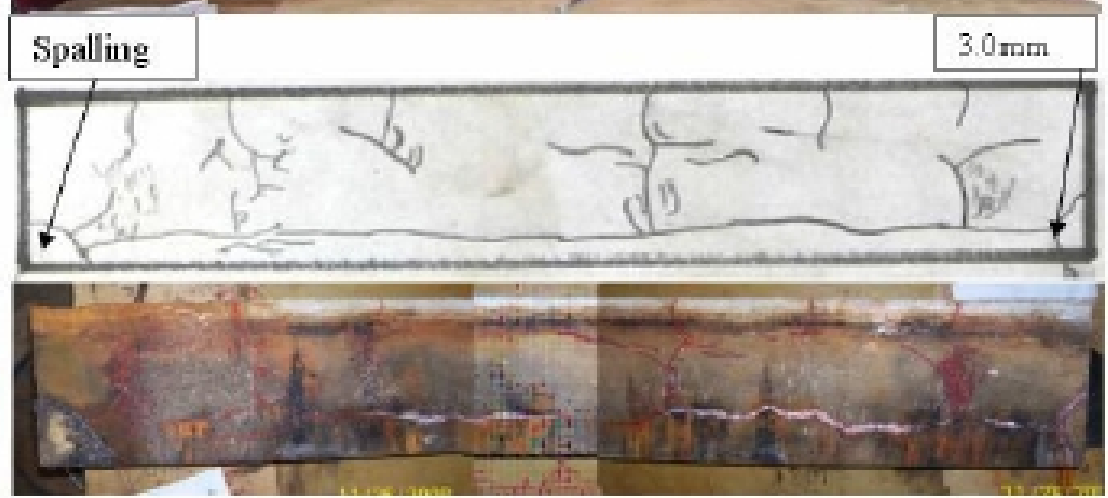

Right

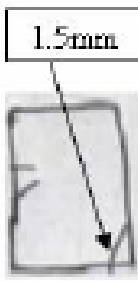

Side B

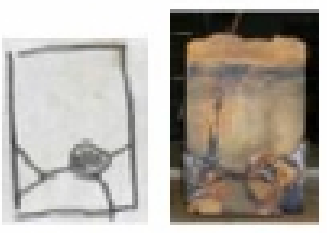

Side A

Figure (A-15): The crack pattern of SCC beam no.3. $20 \%$ mass loss. 

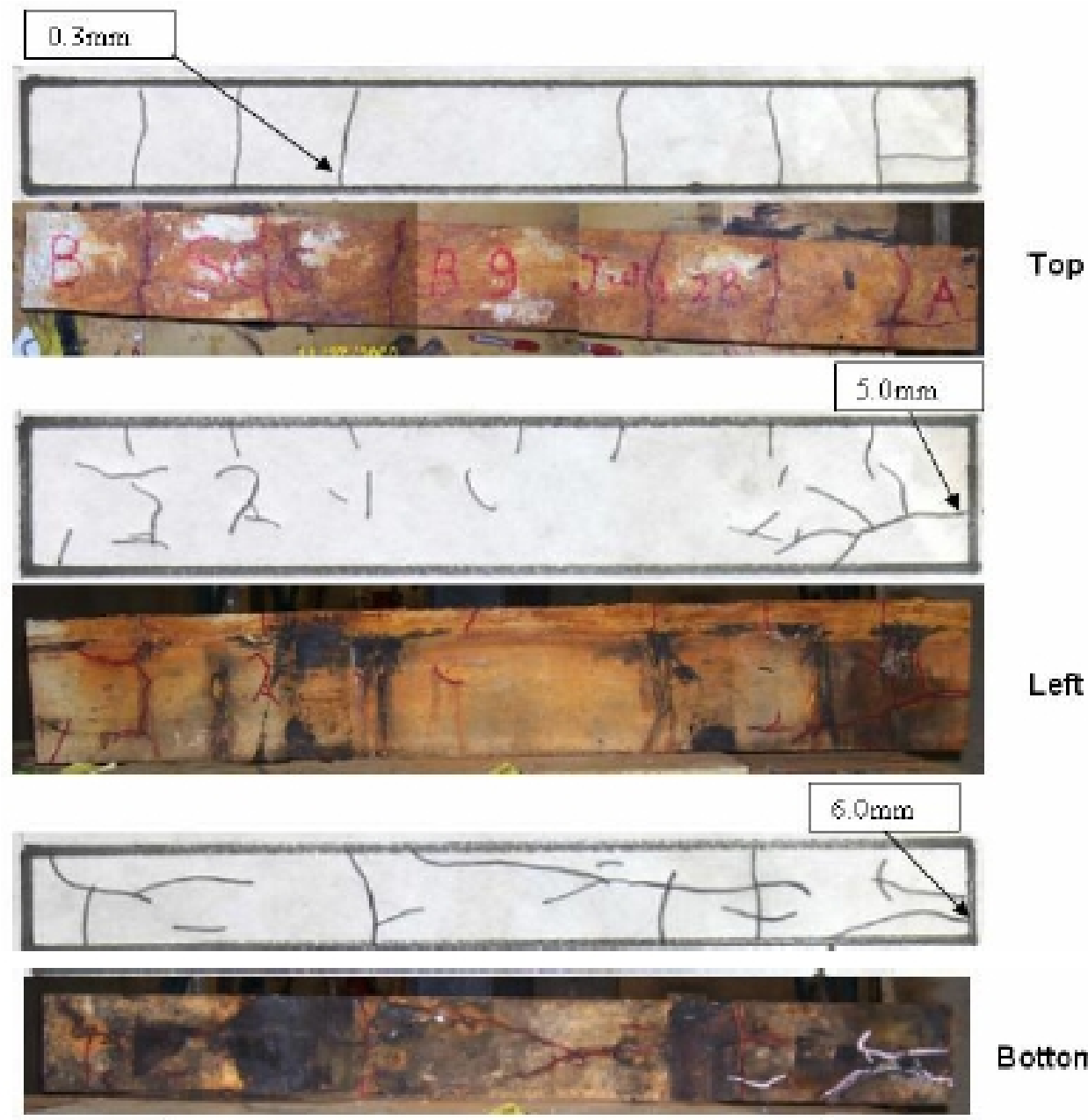

Bottom
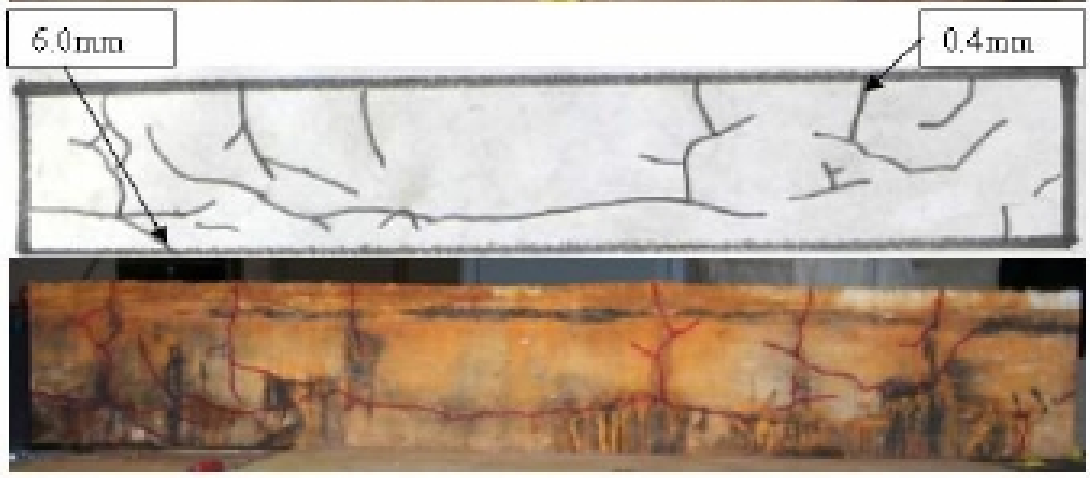

Right

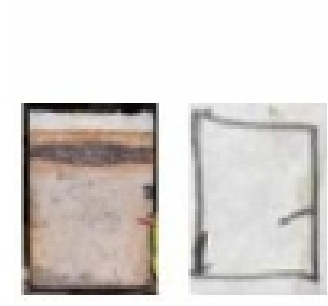

Side B

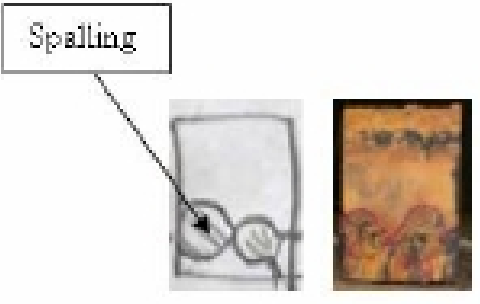

Side A

Figure (A-16): The crack pattern of SCC beam no.9. $20 \%$ mass loss. 


\section{Appendix B: Structural parameters}

1. The following figures illustrate the load vs. shear strain relationships. Each graph contains a comparison of these values for all of the beams belonging to that stage of corrosion.

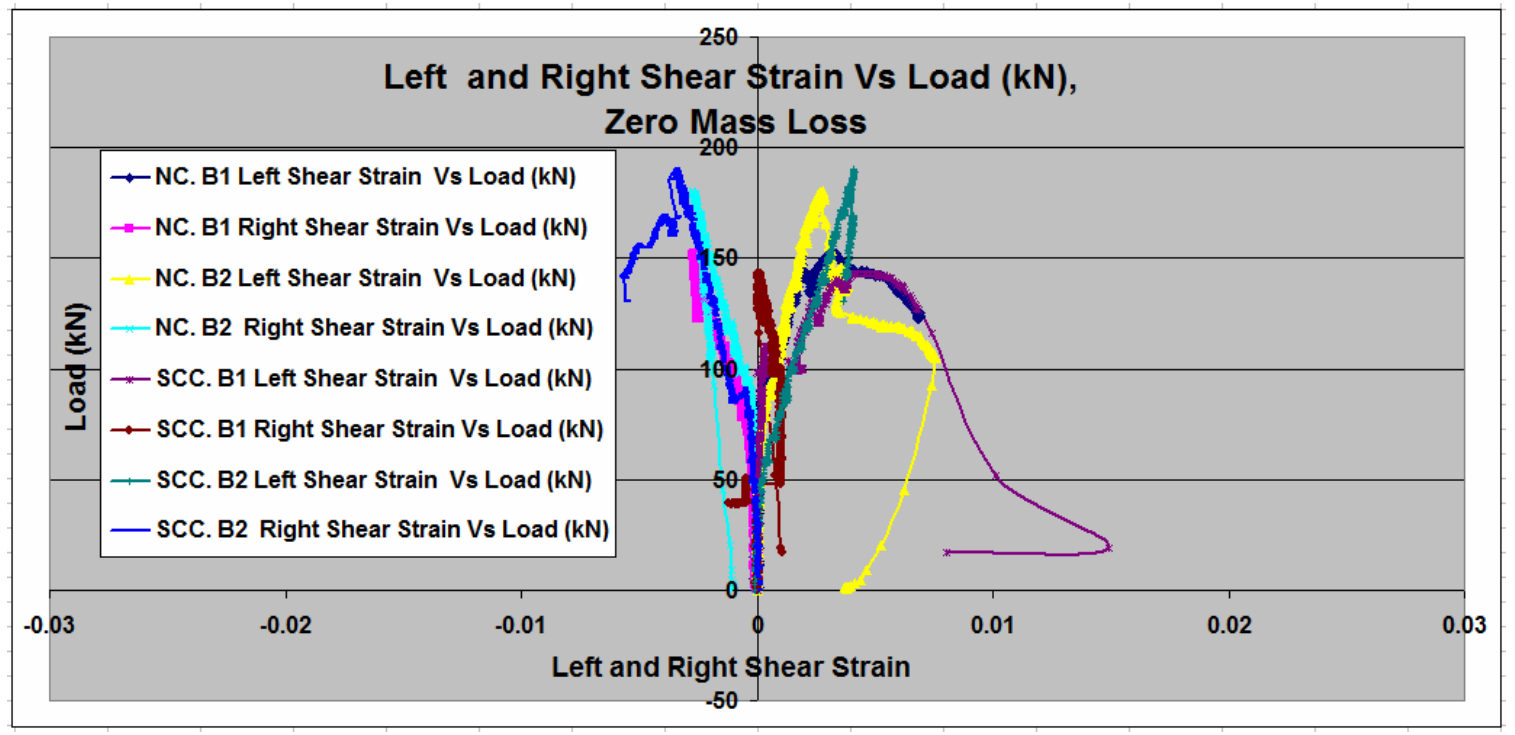

Figure (B1- 1): Shear strain for the left and right sides of the NC and SCC control beams with zero mass loss.

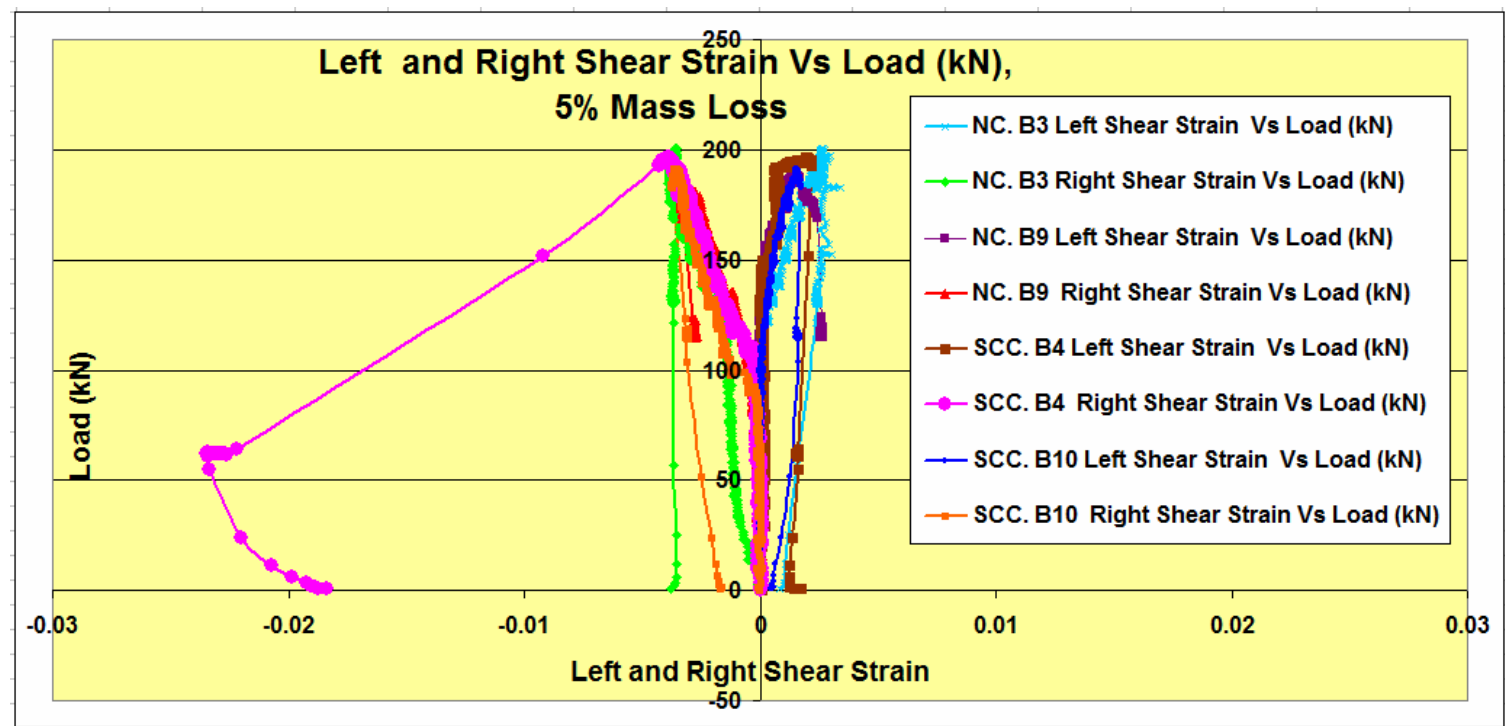

Figure (B1-2): Shear strain for the left and right sides of the $5 \%$ mass loss NC and SCC beams. 


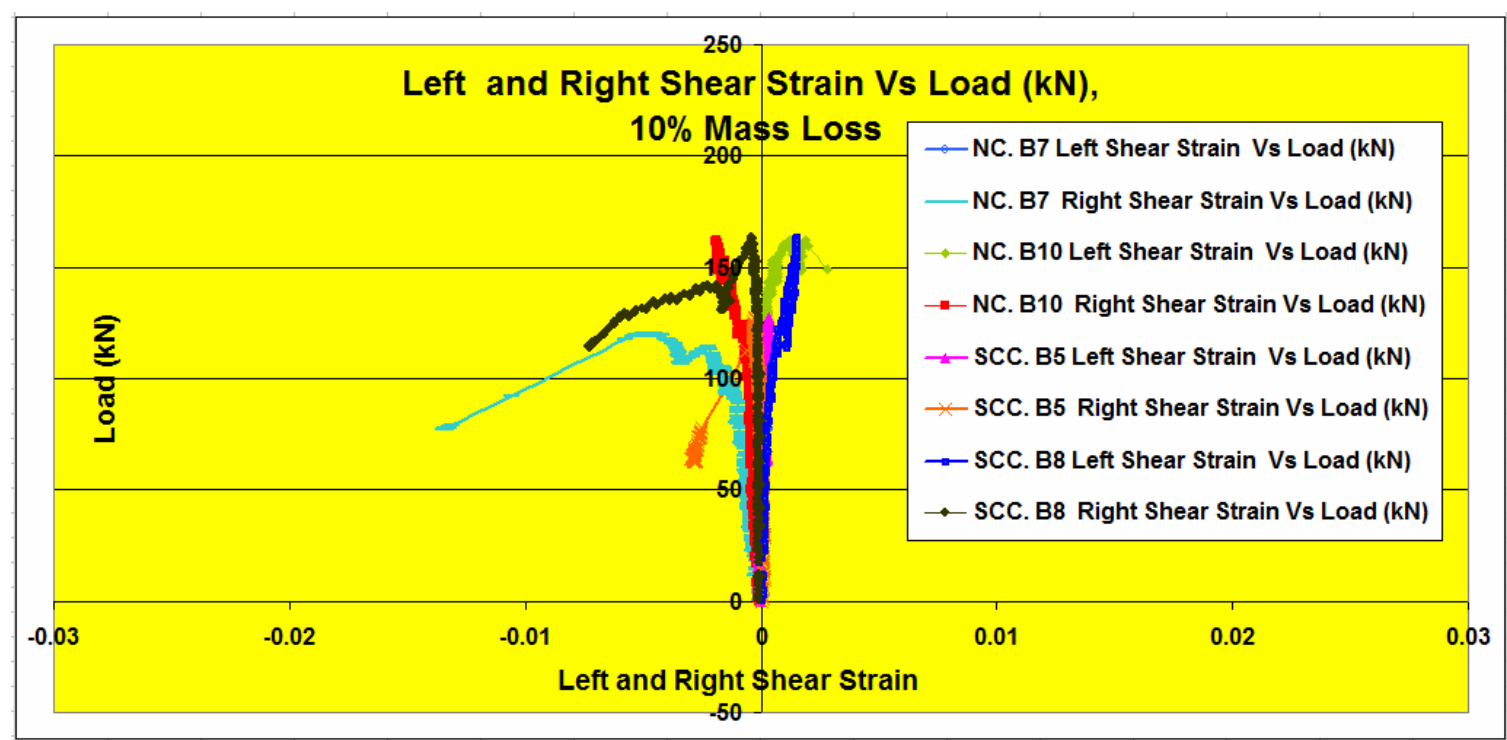

Figure (B1-3): Shear strain of the left and right sides of the $10 \%$ mass loss NC and SCC beams.

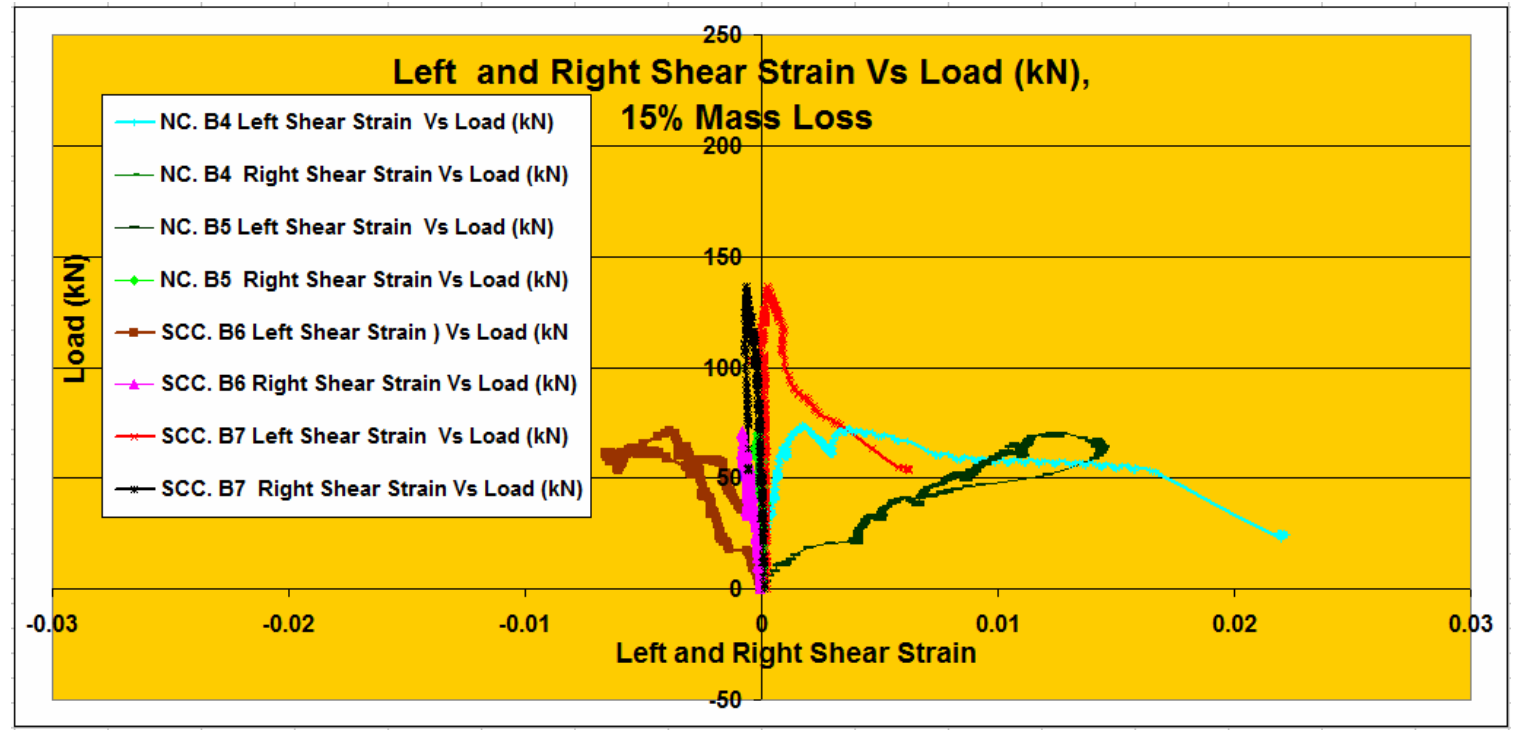

Figure (B1- 4): Shear strain for the left and right sides of the $15 \%$ mass loss NC and SCC beams. 


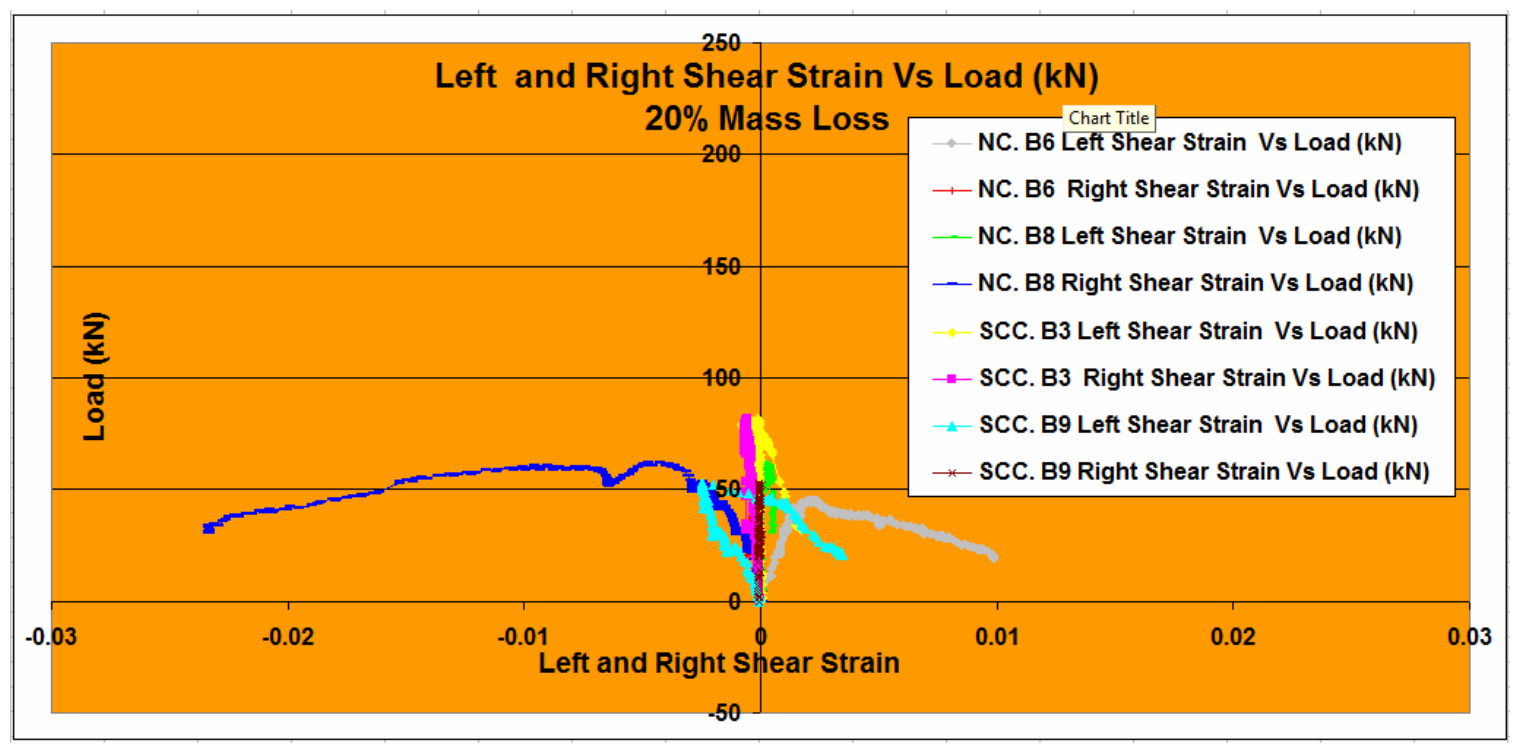

Figure (B1- 5): Shear strain for the left and right sides of the $20 \%$ mass loss NC and SCC beams. 
2. The following figures illustrate the load vs. $\boldsymbol{E}_{x}$ relationships. In each graph there is a comparison of these values for all of the beams belonging to that stage of corrosion.

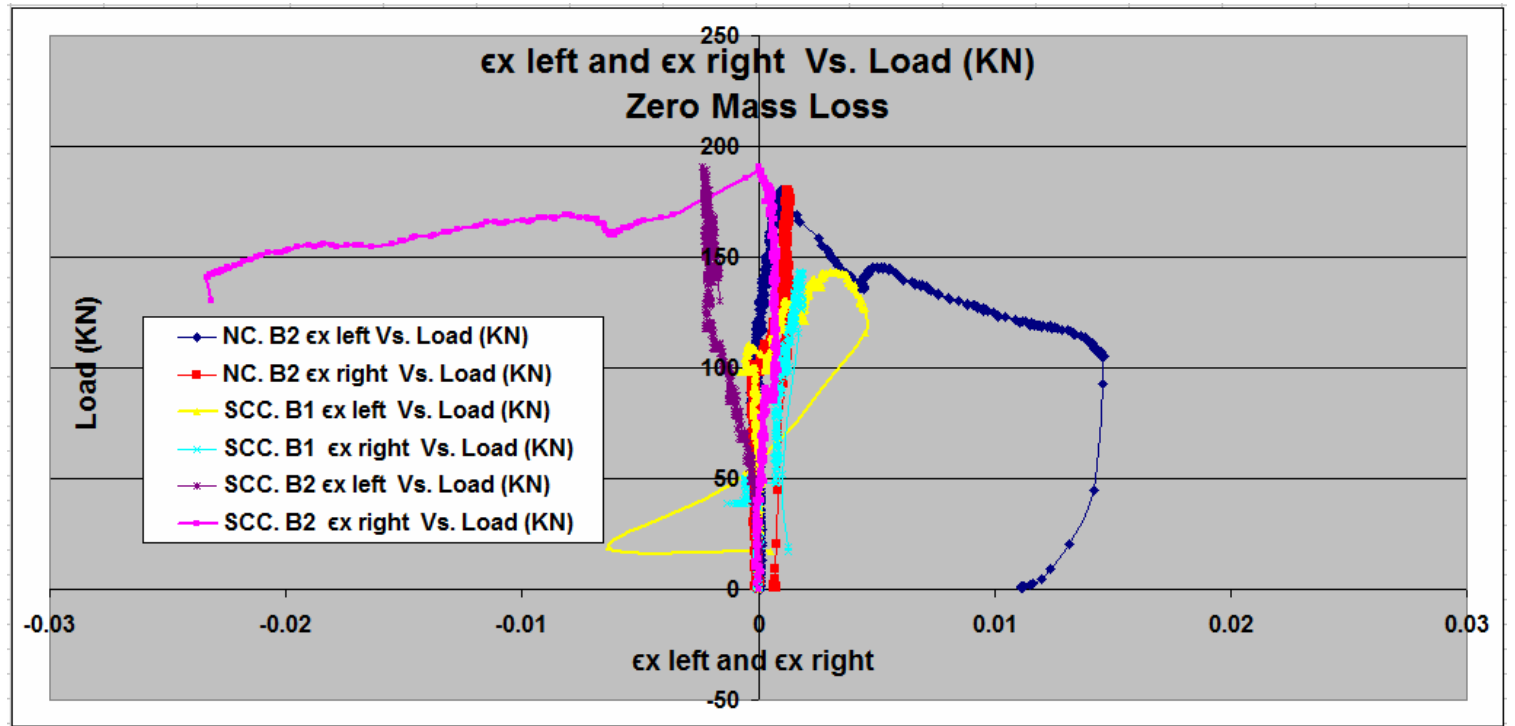

Figure (B2-1): $\quad \epsilon_{x \text { left }}$ and $\boldsymbol{\epsilon}_{x \text { right }}$ for the NC and SCC control beams with zero mass loss.

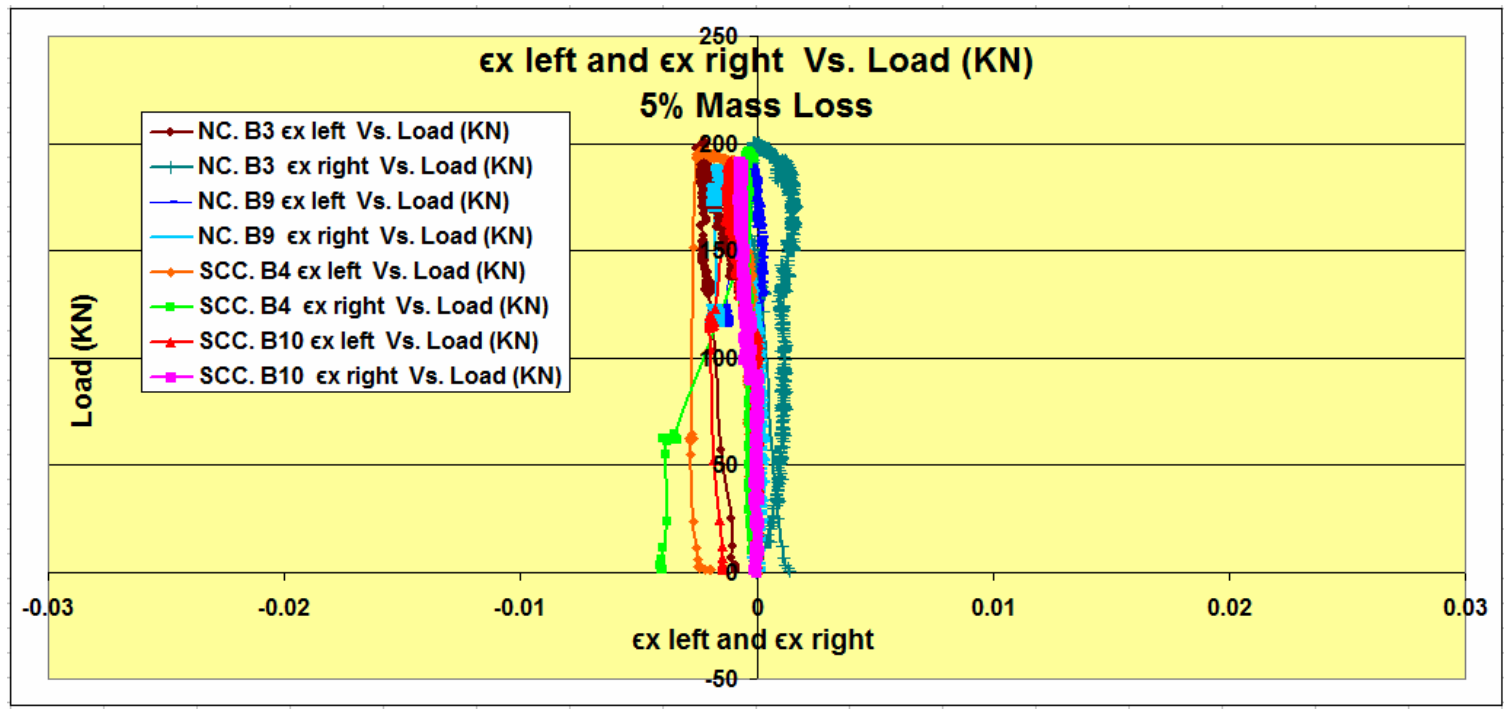

Figure (B2-2): $\quad \boldsymbol{\epsilon}_{x}$ left and $\boldsymbol{\epsilon}_{x \text { right }}$ for the $5 \%$ mass loss NC and SCC beams. 


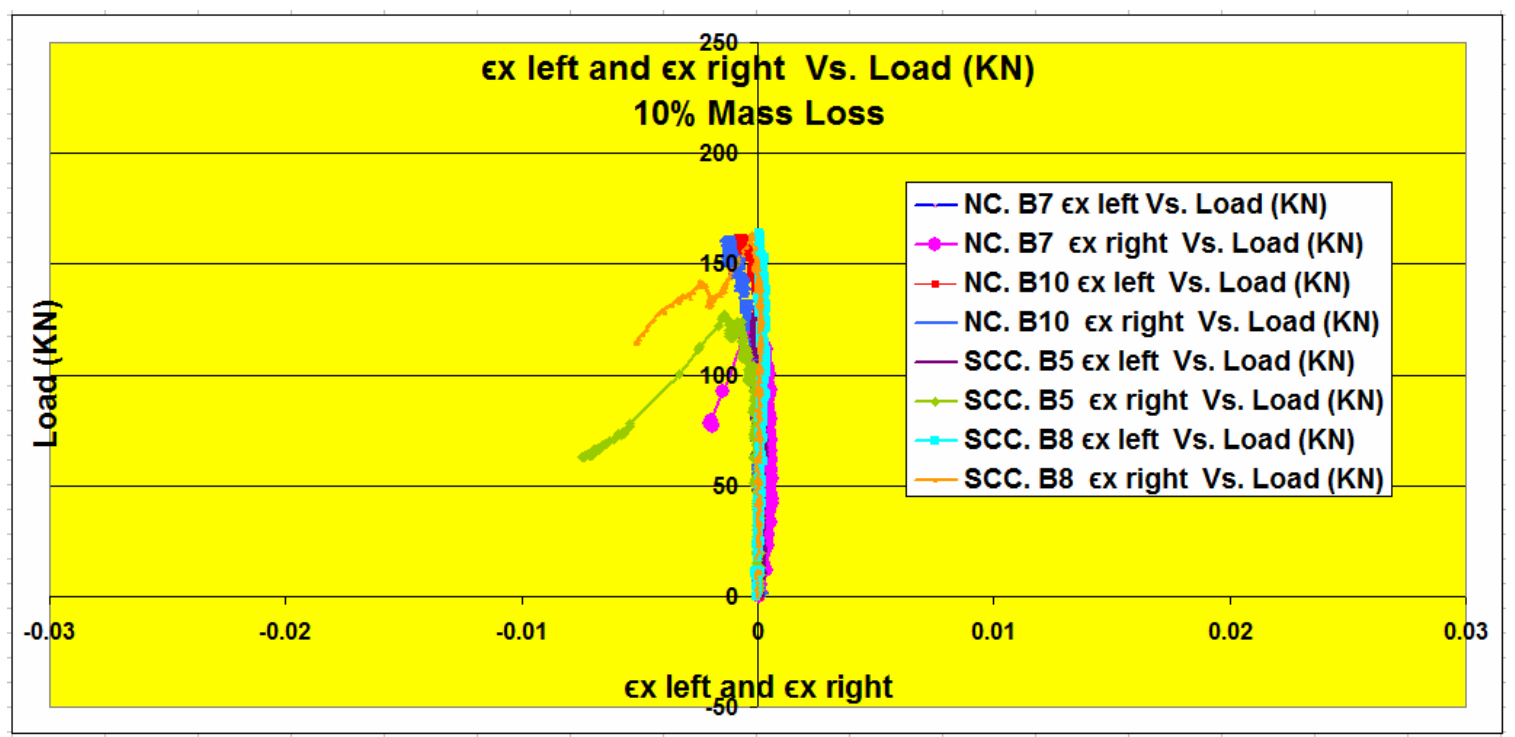

Figure (B2-3): $\quad \boldsymbol{\epsilon}_{x}$ left and $\boldsymbol{\epsilon}_{x \text { right }}$ for the $10 \%$ mass loss NC and SCC beams.

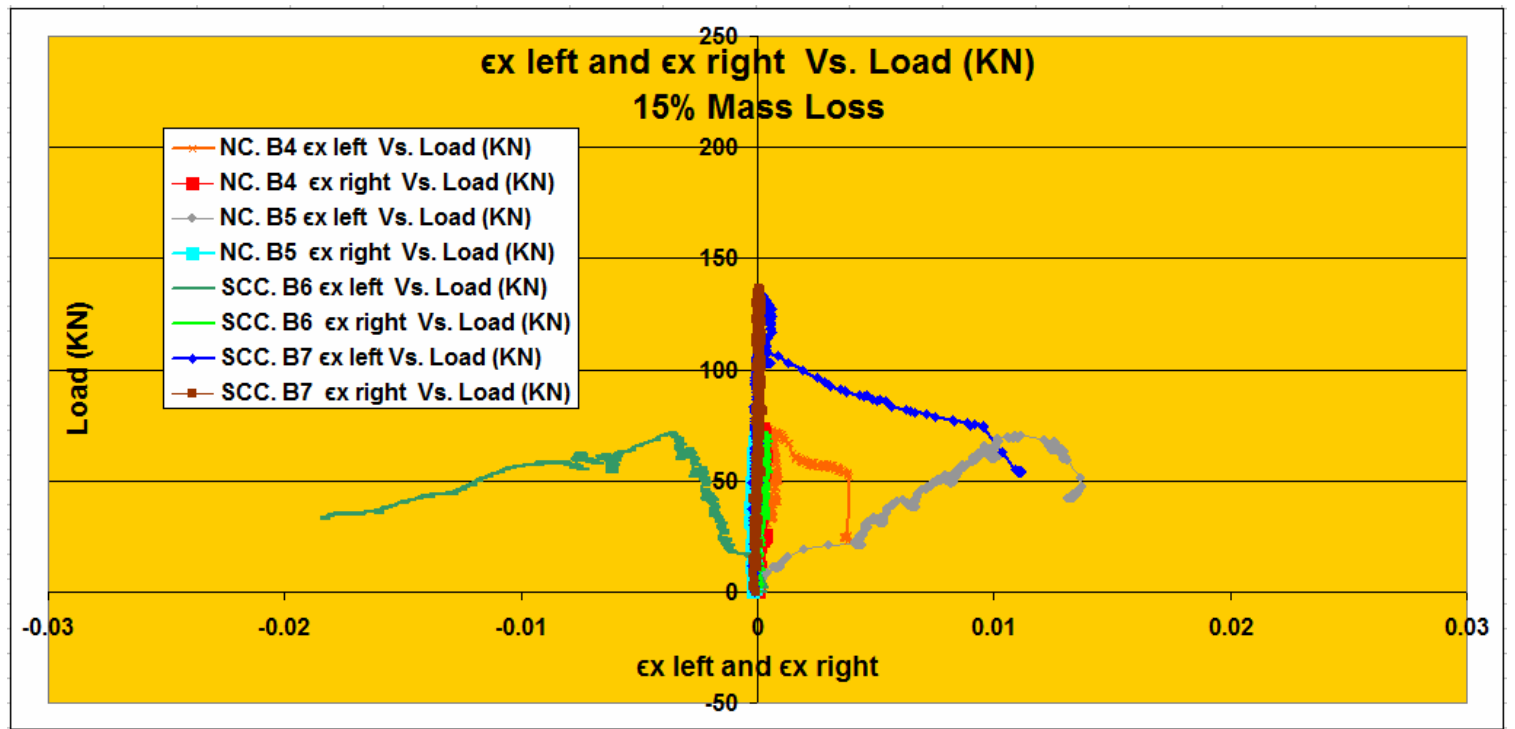

Figure (B2-4): $\quad \boldsymbol{\epsilon}_{x \text { left }}$ and $\boldsymbol{\epsilon}_{x \text { right }}$ for the $15 \%$ mass loss NC and SCC beams. 


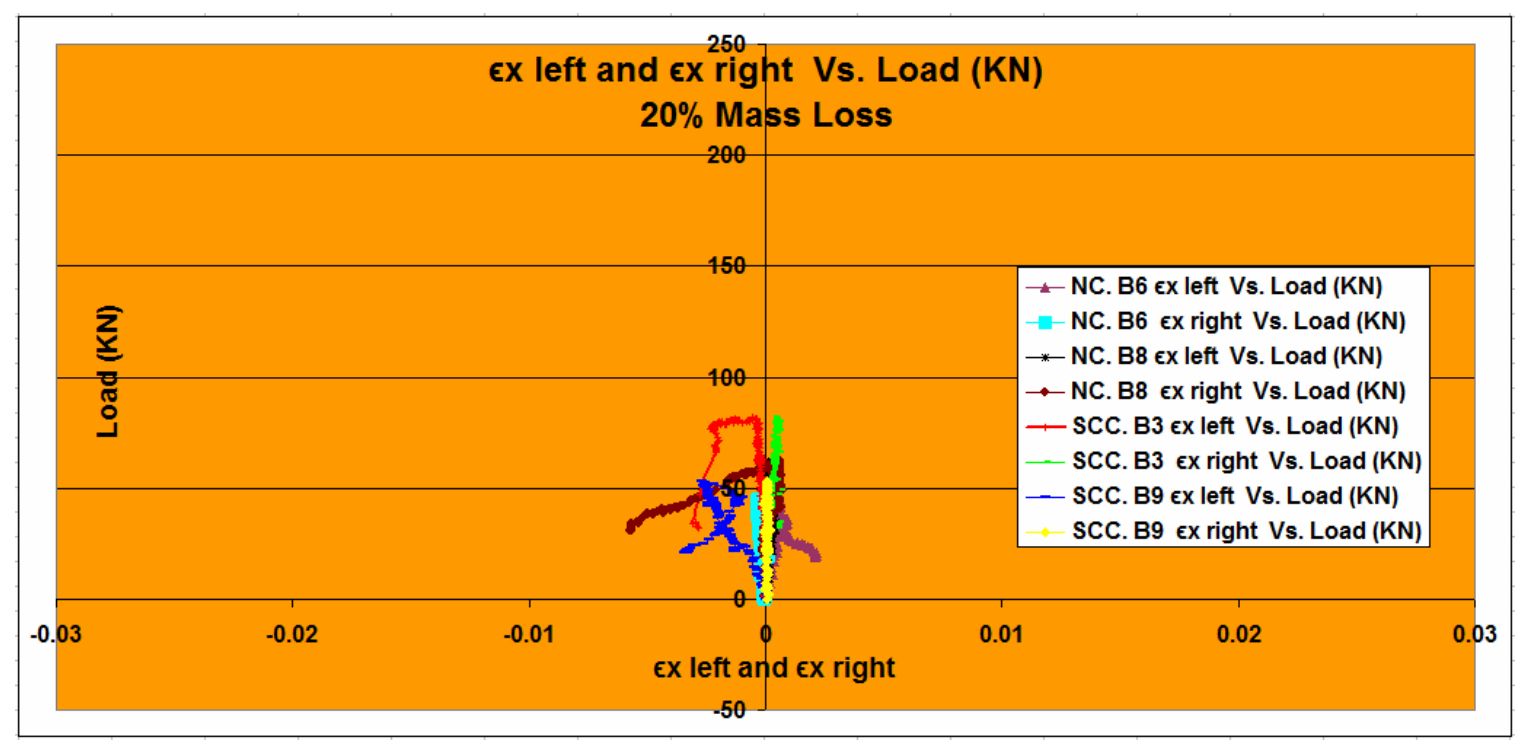

Figure (B2-5): $\quad \boldsymbol{\epsilon}_{x}$ left and $\boldsymbol{\epsilon}_{x}$ right for the $20 \%$ mass loss NC and SCC beams. 
3. The following figures illustrate the load vs. $\boldsymbol{\epsilon}_{y}$ relationships. In each graph there is a comparison of these values in all of the beams belonging to that stage of corrosion.

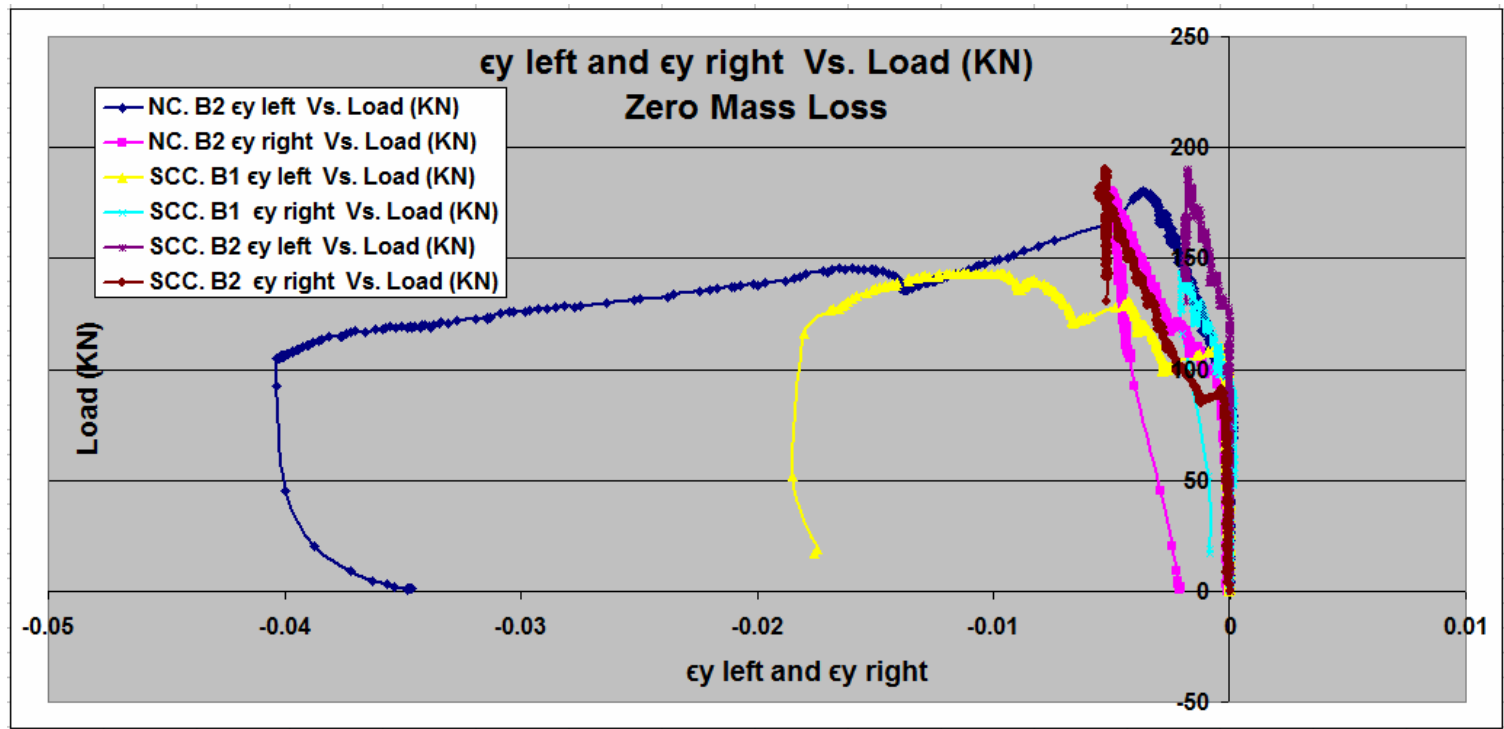

Figure (B3-1): $\quad \boldsymbol{\epsilon}_{y}$ left and $\boldsymbol{\epsilon}_{y}$ right for the control NC and SCC beams with zero mass loss.

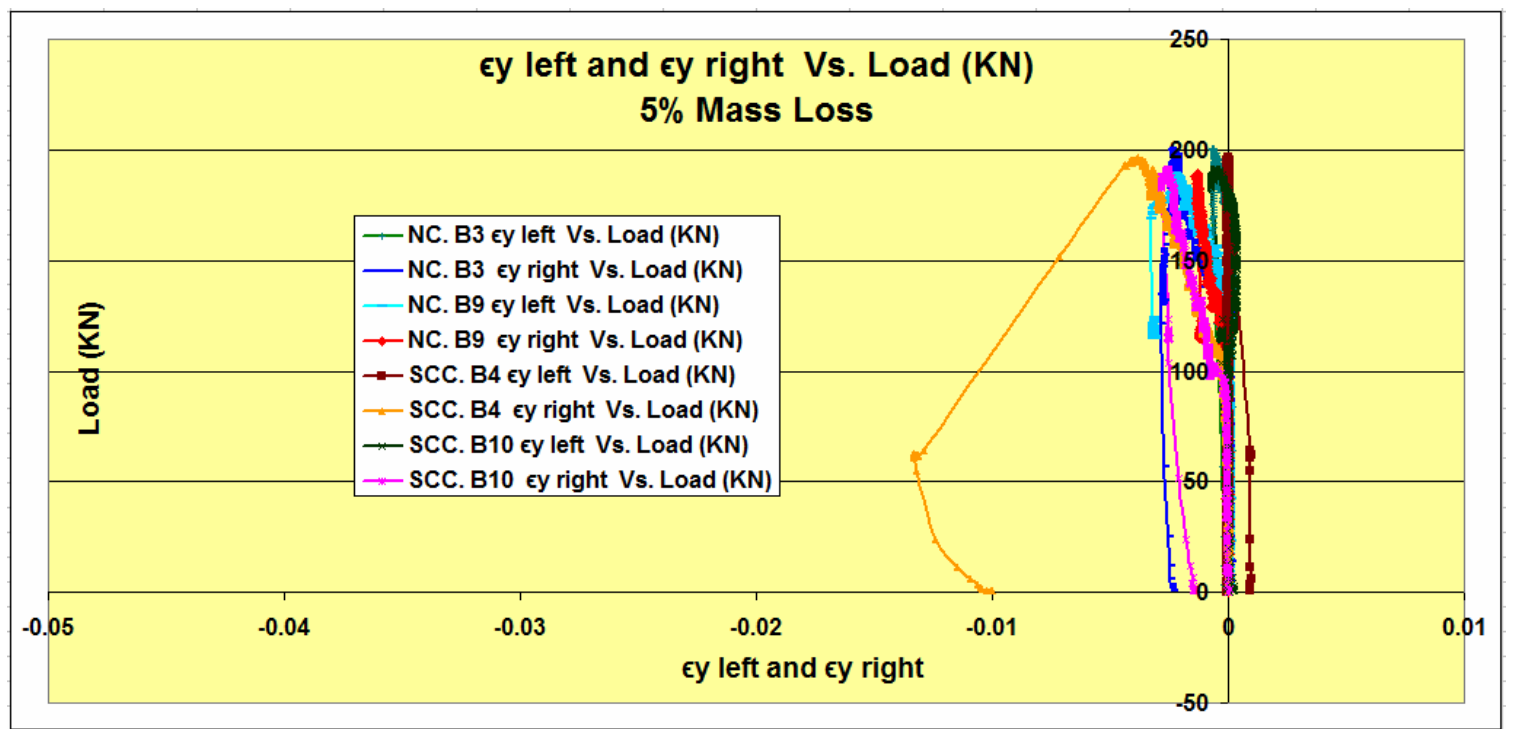

Figure (B3-2): $\quad \boldsymbol{\epsilon}_{y \quad \text { left }}$ and $\boldsymbol{\epsilon}_{y}$ right for the 5\% mass loss NC and SCC beams. 


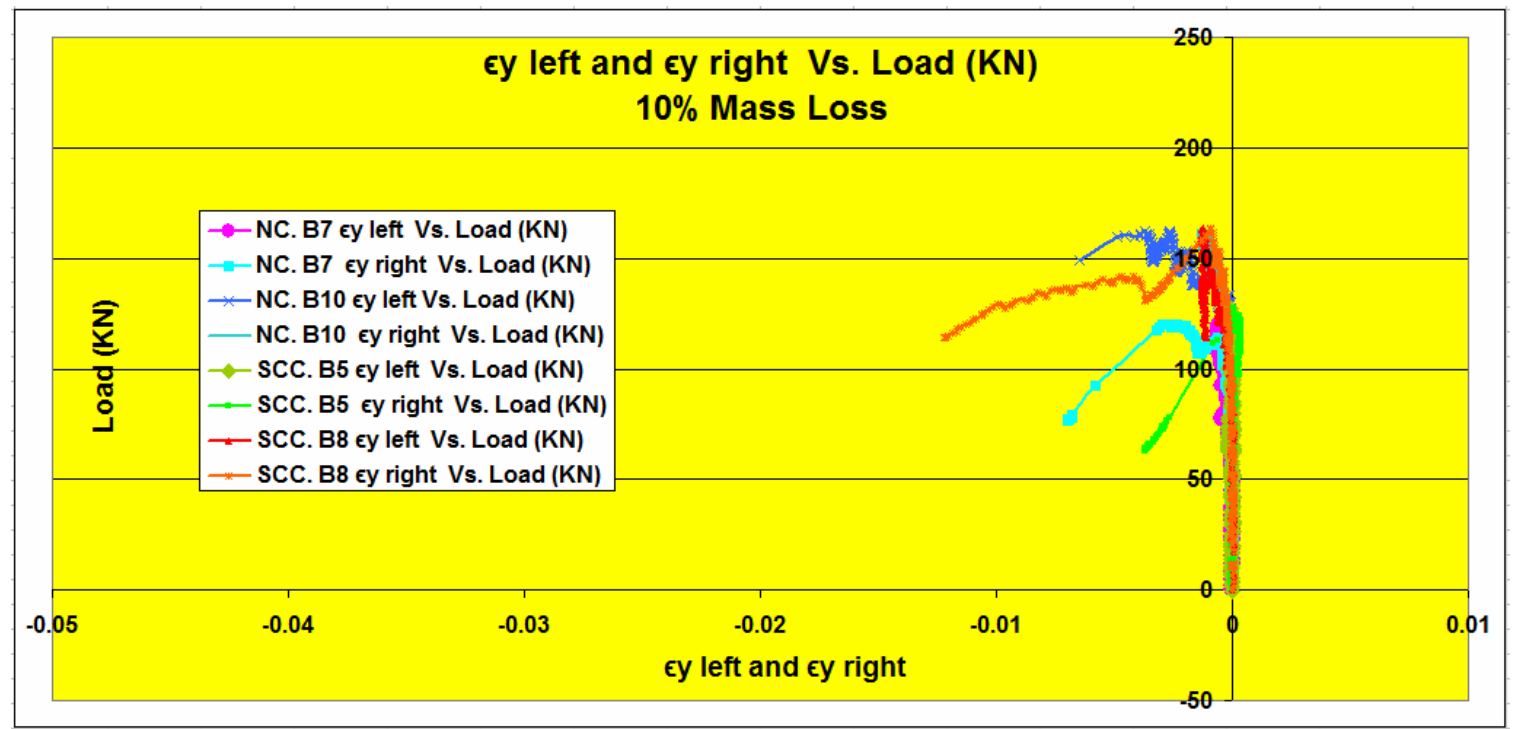

Figure (B3-3): $\quad \boldsymbol{\epsilon}_{y}$ left and $\boldsymbol{\epsilon}_{y \text { right }}$ for the $10 \%$ mass loss NC and SCC beams.

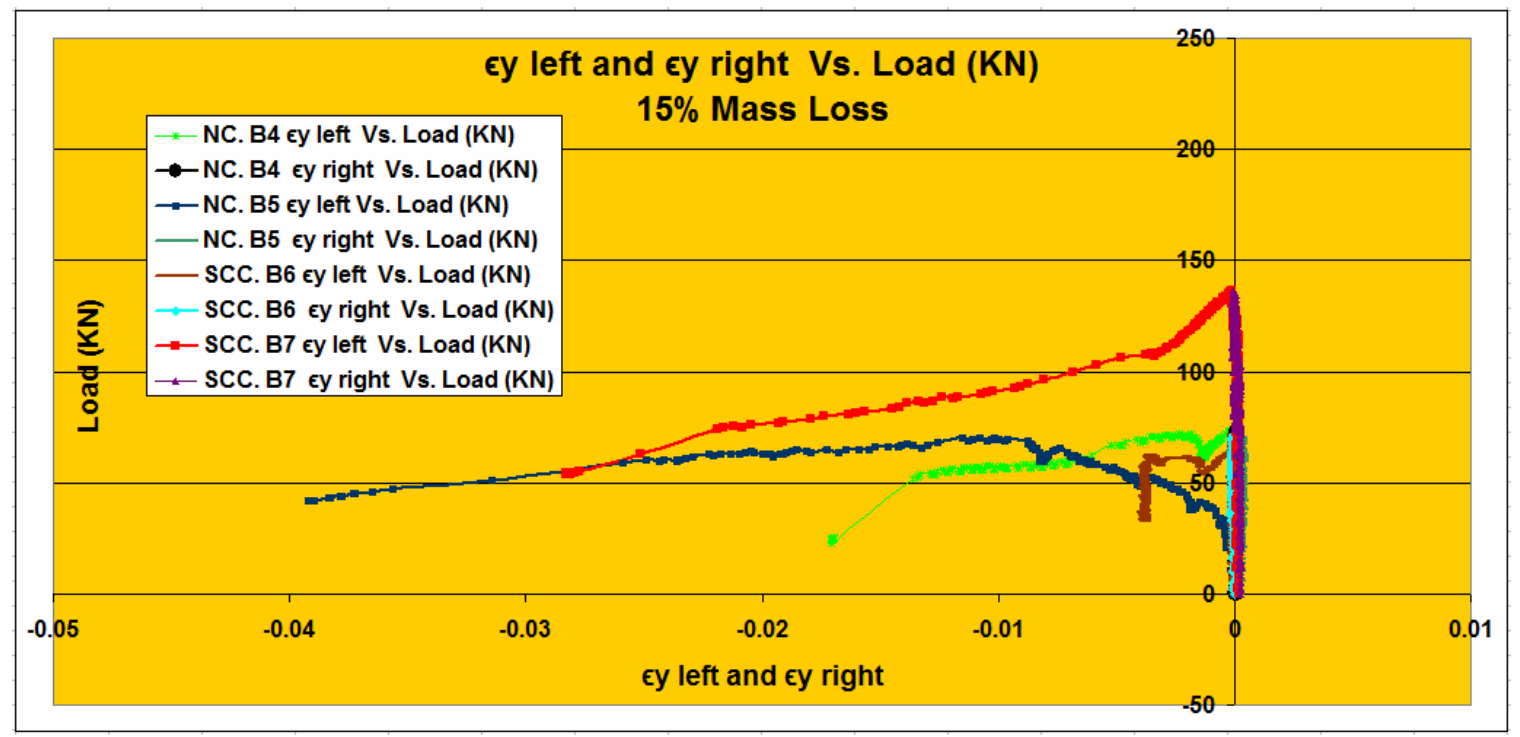

Figure (B3-4): $\quad \boldsymbol{\epsilon}_{y \quad \text { left }}$ and $\boldsymbol{\epsilon}_{y \text { right }}$ for the $15 \%$ mass loss NC and SCC beams. 


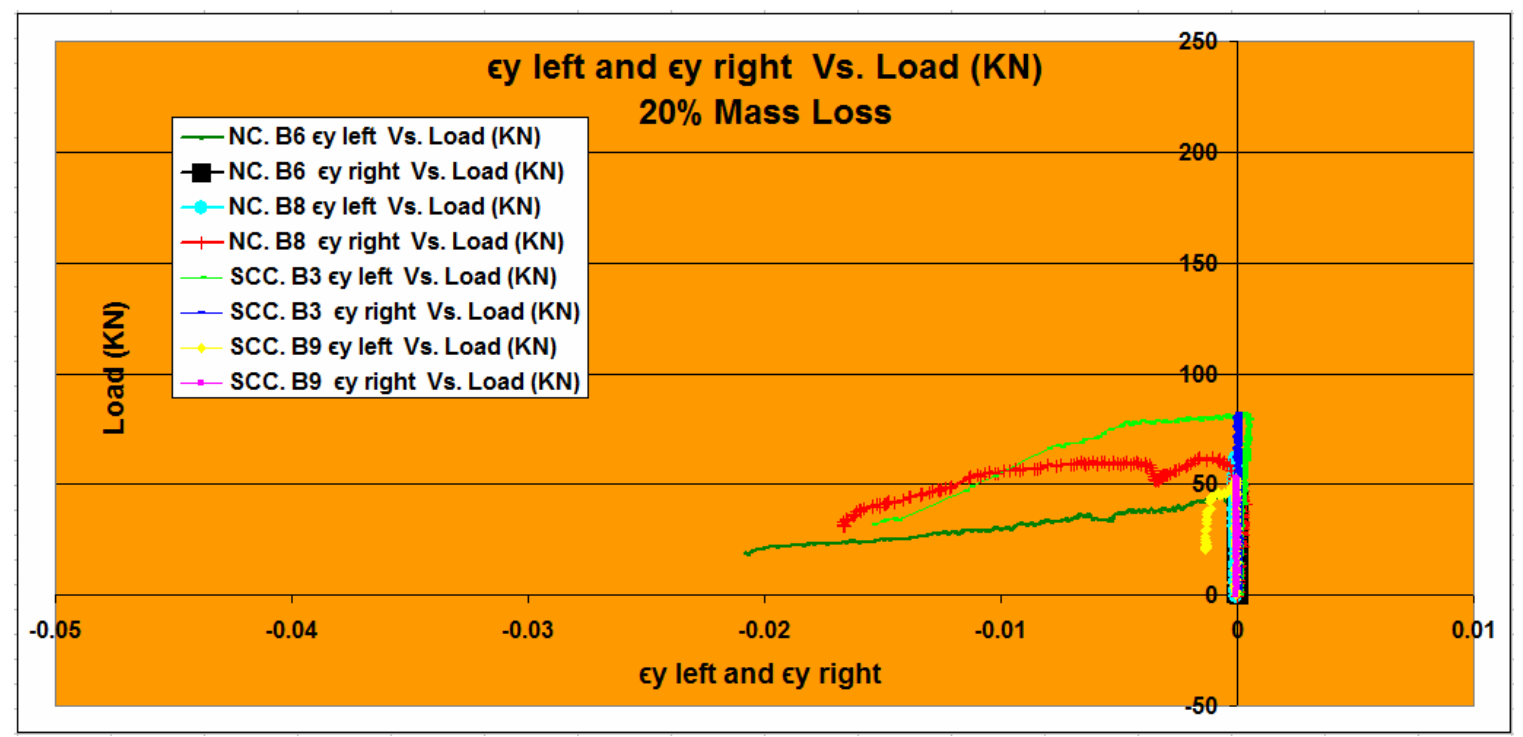

Figure (B3-5): $\quad \boldsymbol{\epsilon}_{y}$ left and $\boldsymbol{\epsilon}_{y \text { right }}$ for the $20 \%$ mass loss NC and SCC beams. 
4. The following figures illustrate the load vs. mid-span deflection $\Delta$ relationships. In each graph there is a comparison of these values for all of the beams belonging to that stage of corrosion.

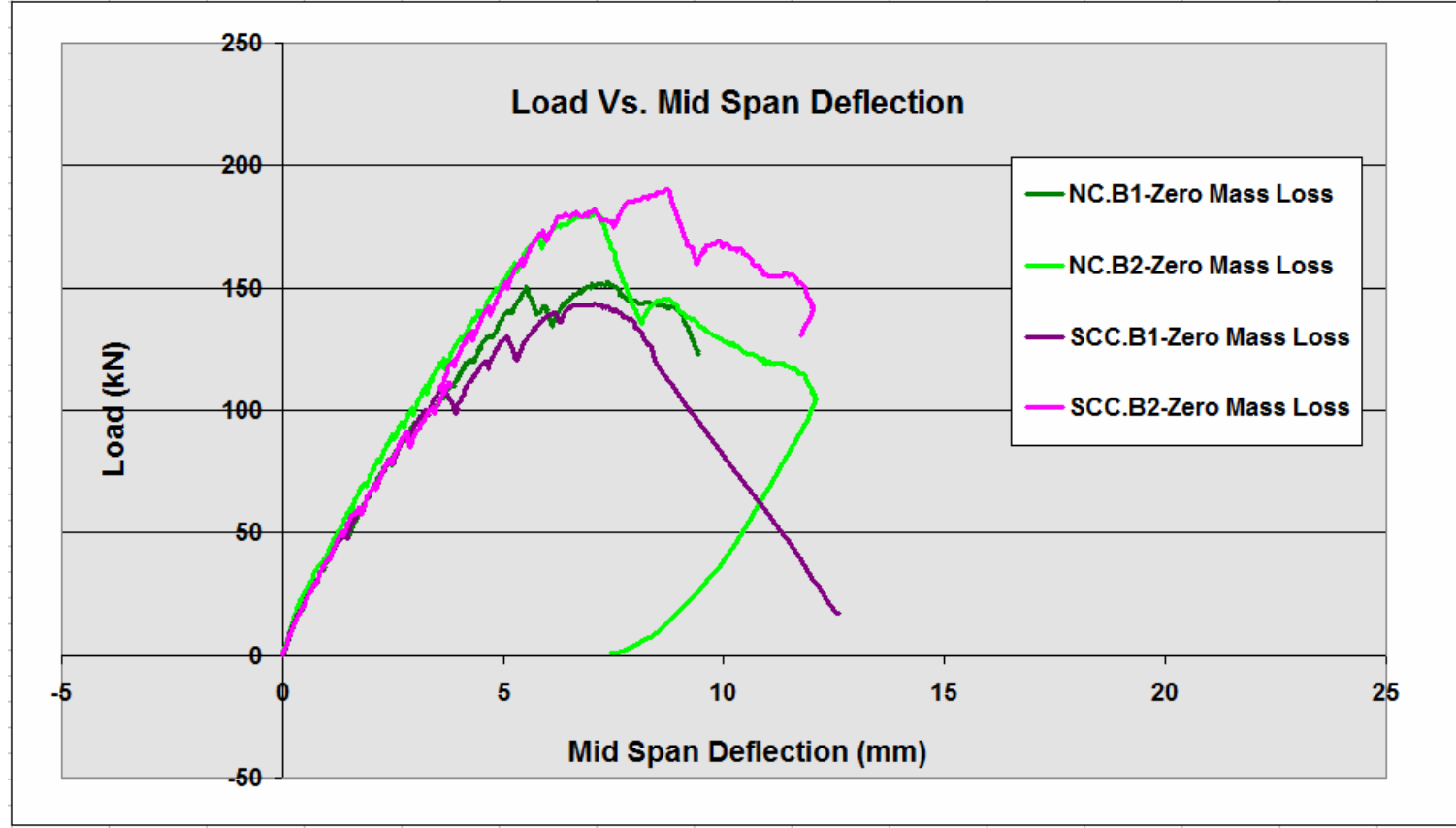

Figure (B4-1): Mid-span deflection $\Delta$ vs. load for the control NC and SCC beams with zero mass loss.

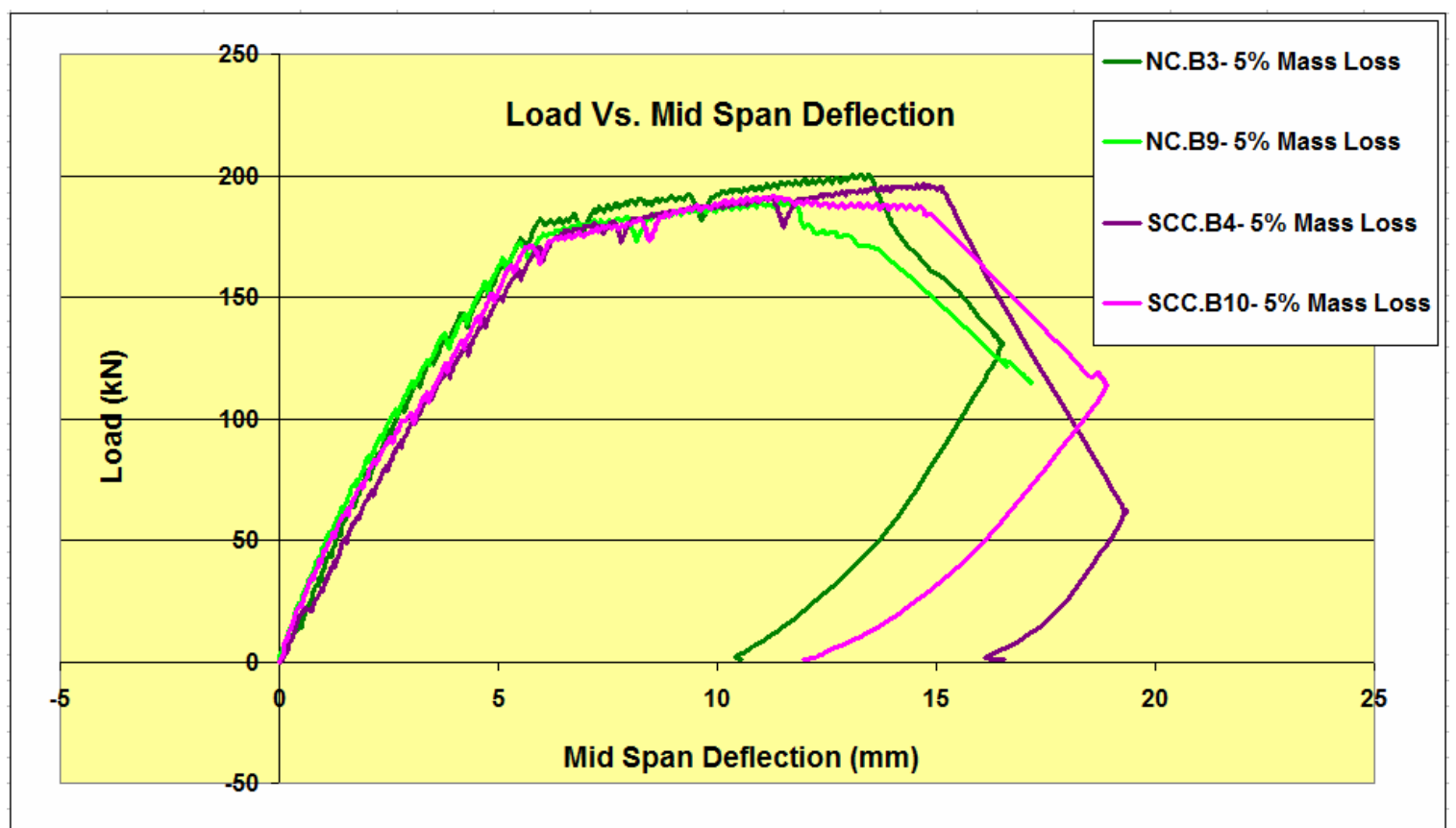

Figure (B4-2): Mid-span deflection $\Delta$ vs. load for the $5 \%$ mass loss NC and SCC beams. 


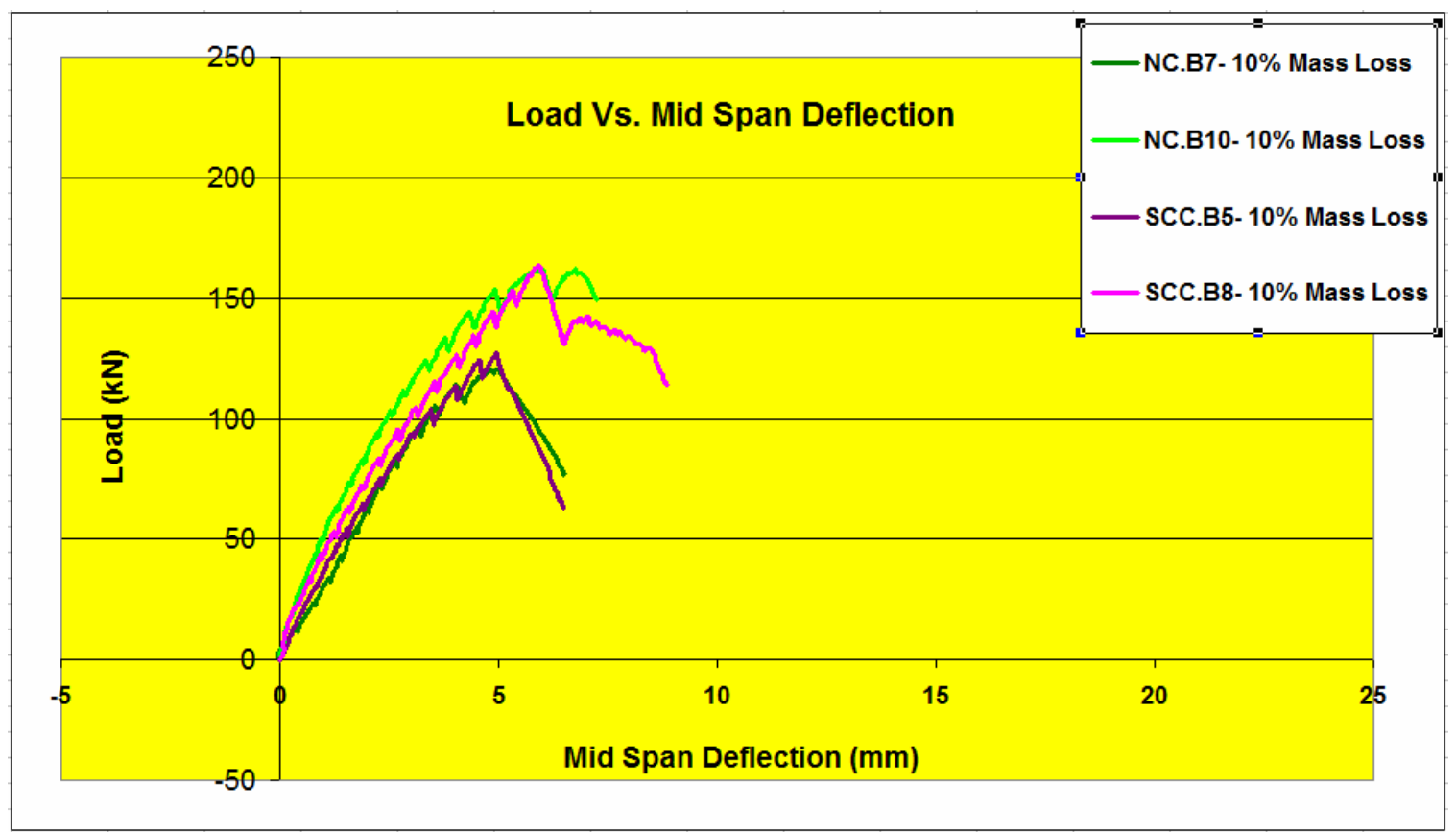

Figure (B4-3): Mid-span deflection $\Delta$ vs. load for the $10 \%$ mass loss NC and SCC beams.

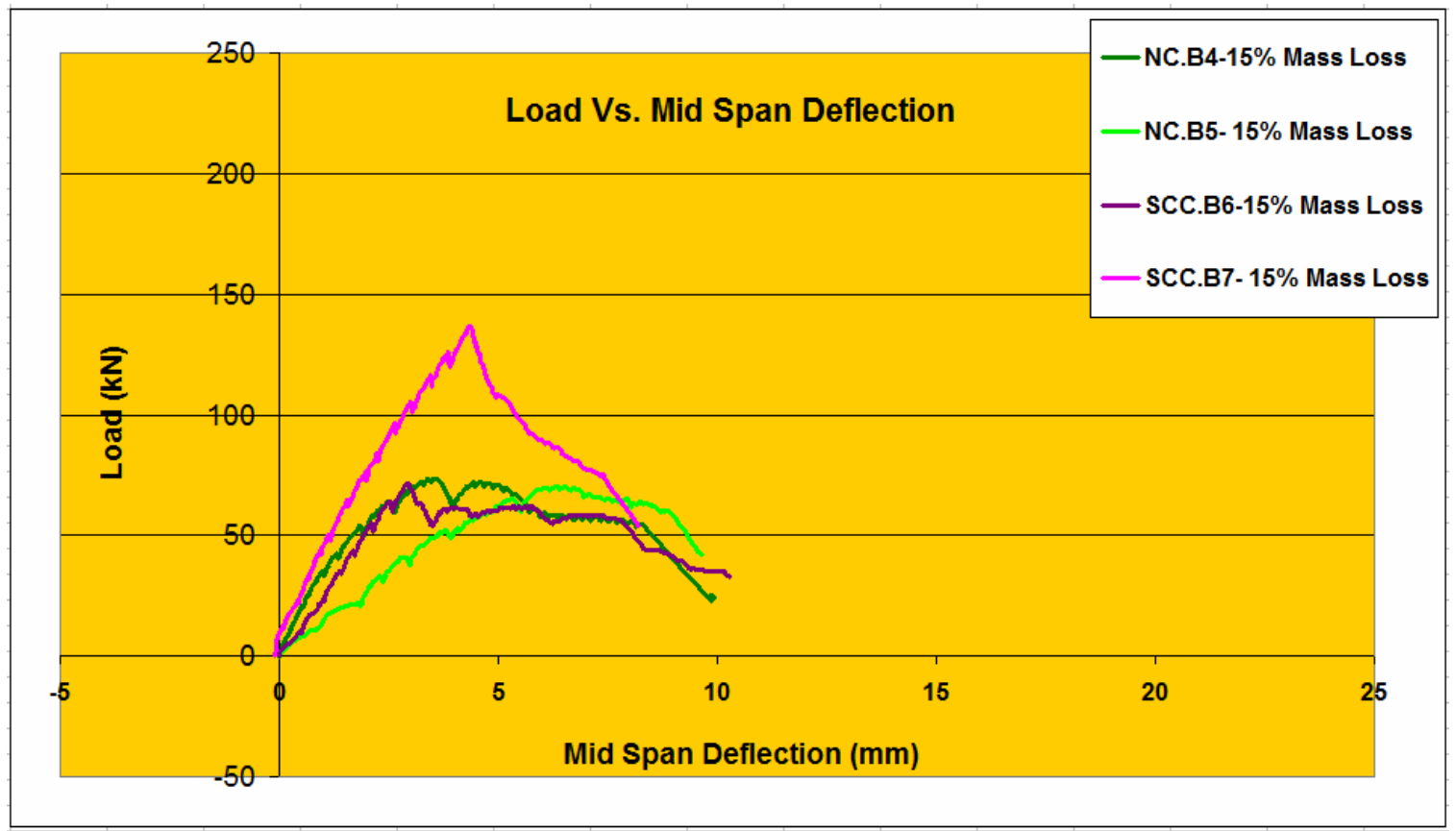

Figure (B4-4): Mid-span deflection $\Delta$ vs. load for the $15 \%$ mass loss NC and SCC beams. 


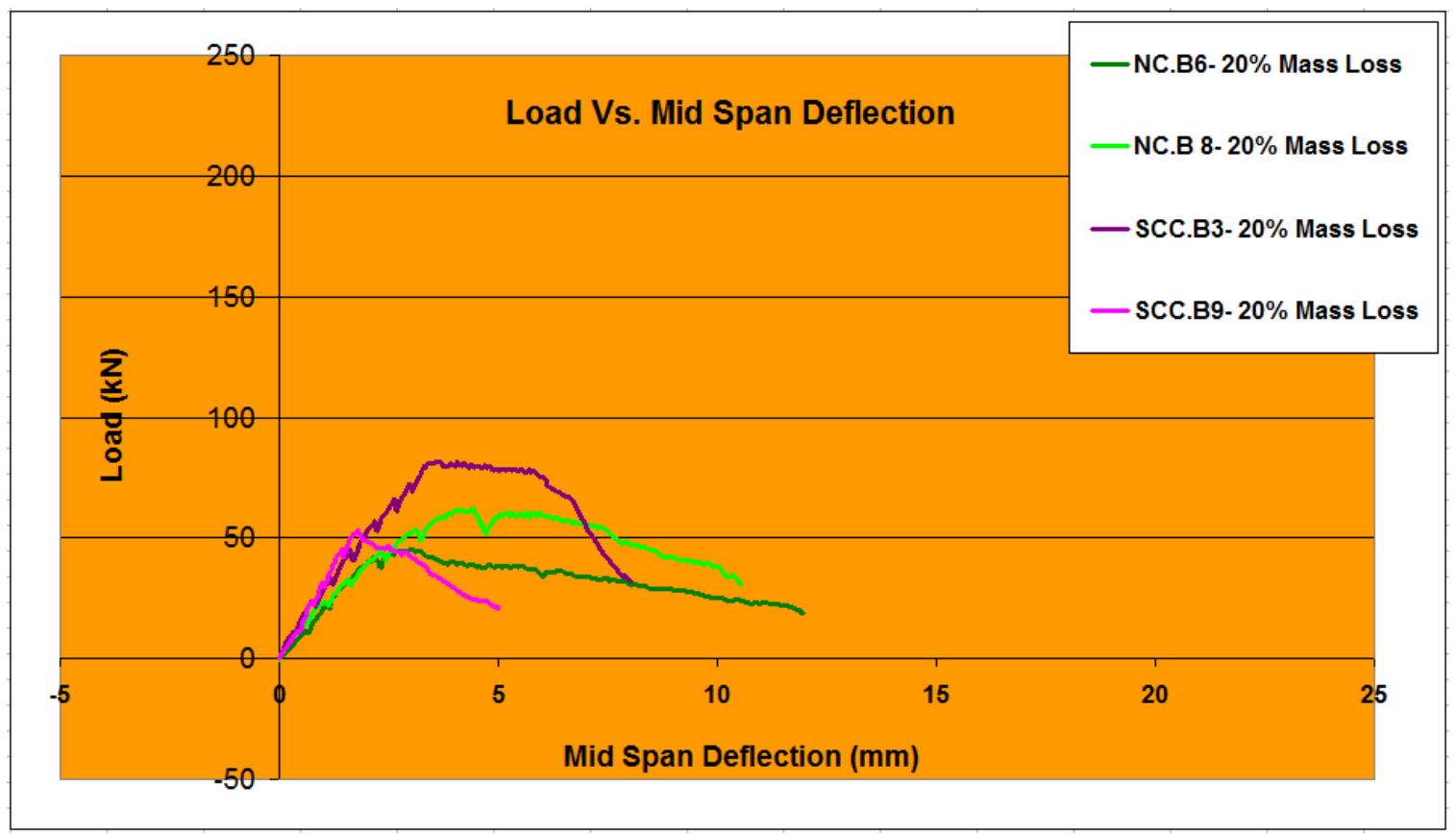

Figure (B4-5): Mid-span deflection $\Delta$ vs. load for the $20 \%$ mass loss NC and SCC beams.

1. The following figures illustrate the values of deflection along the beams during the loading stages for each beam.

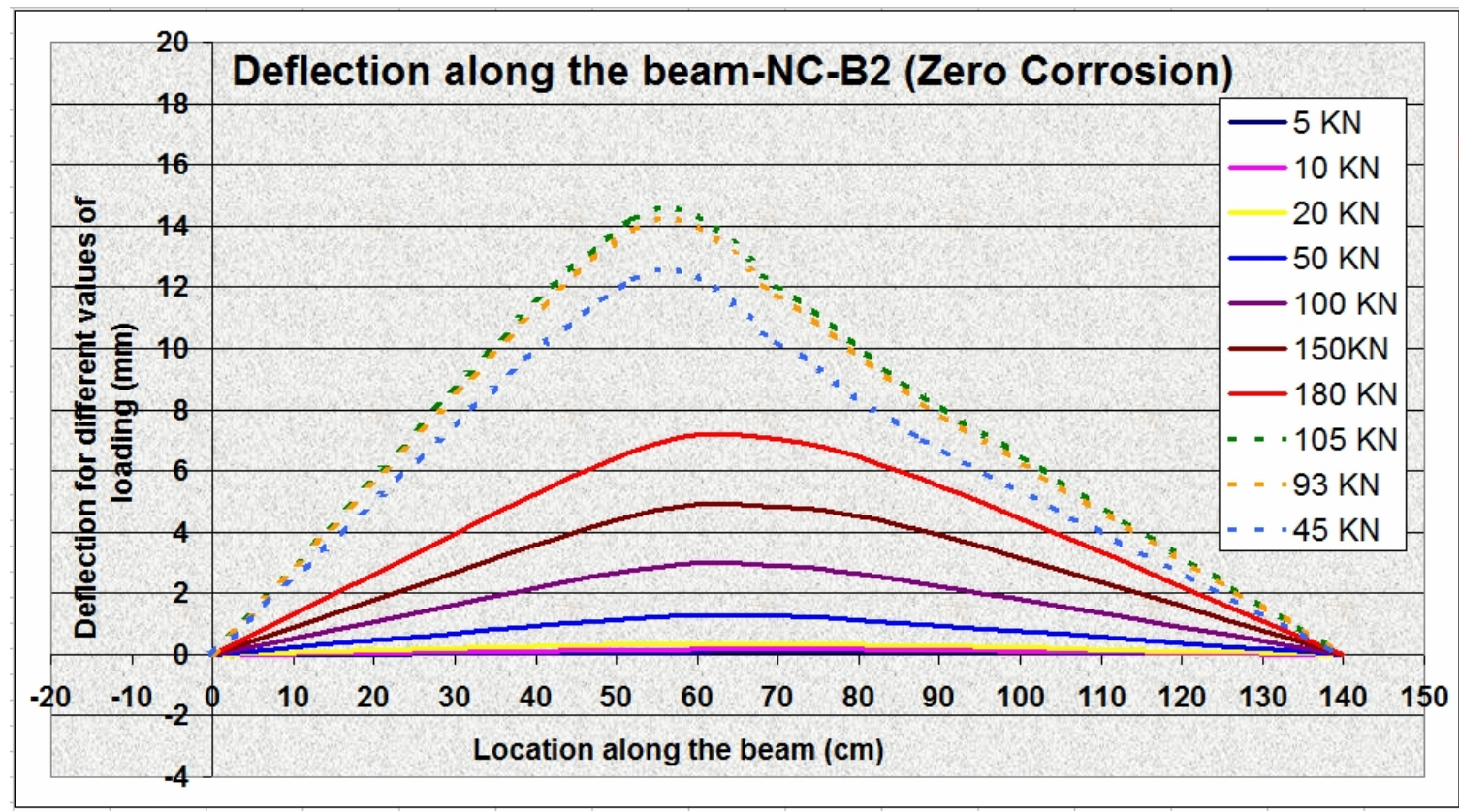

Figure (B5-1): The deflection along the beam for different values of loading for NC; B2 (zero mass loss). 


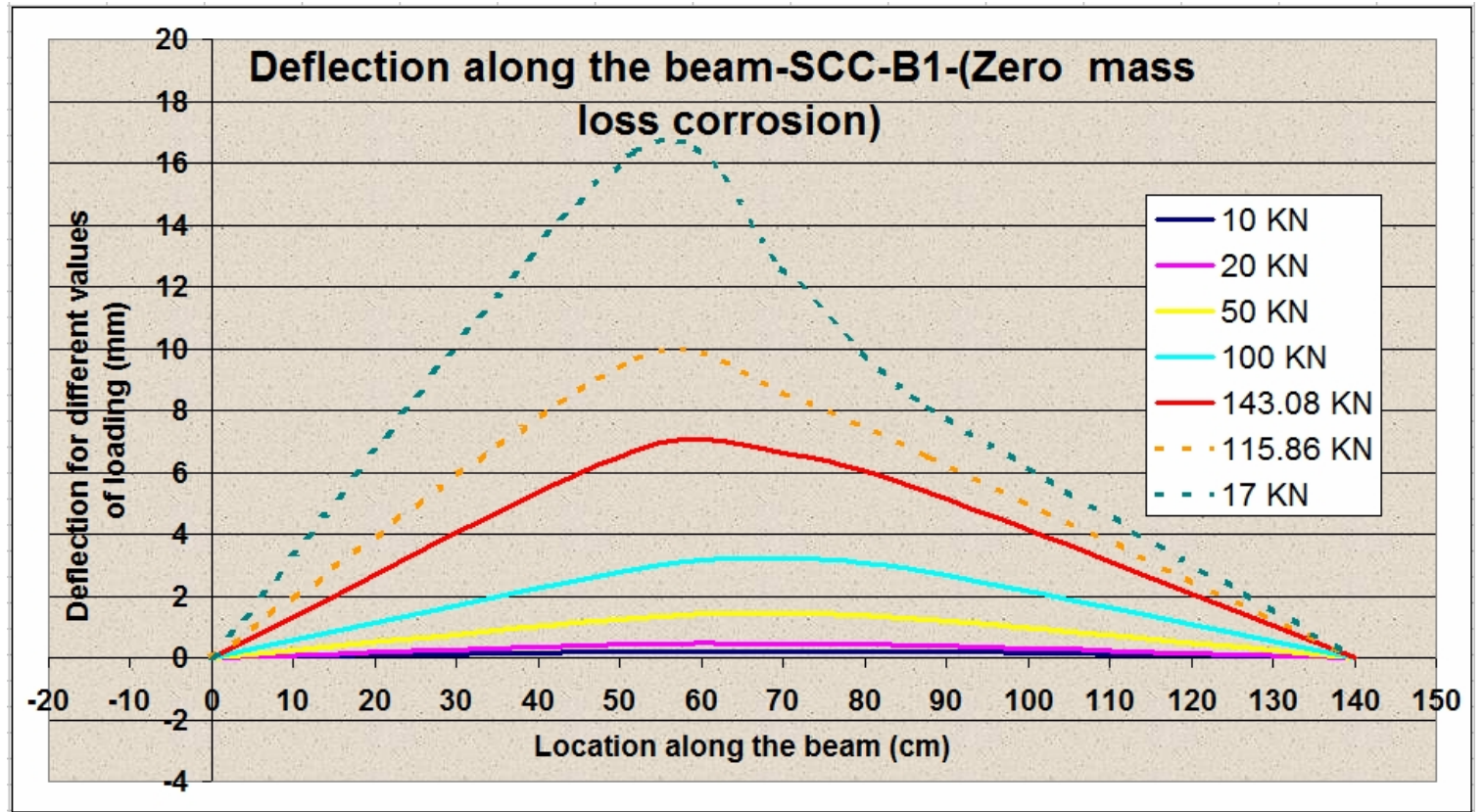

Figure (B5-2): The deflection along the beam for different values of loading for SCC; B-1 (zero mass loss).

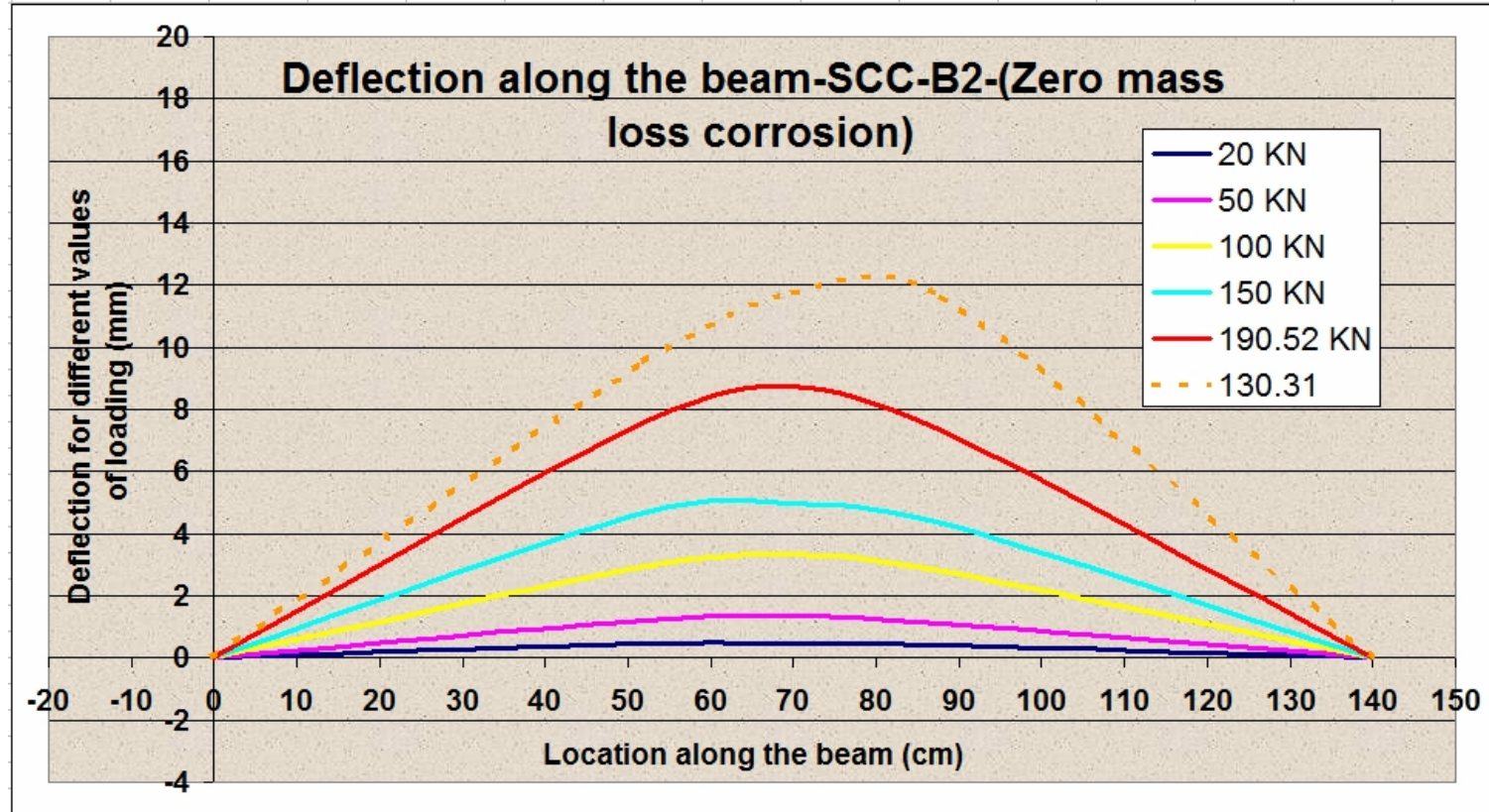

Figure (B5-3): The deflection along the beam for different values of loading for SCC; B-2 (zero mass loss). 


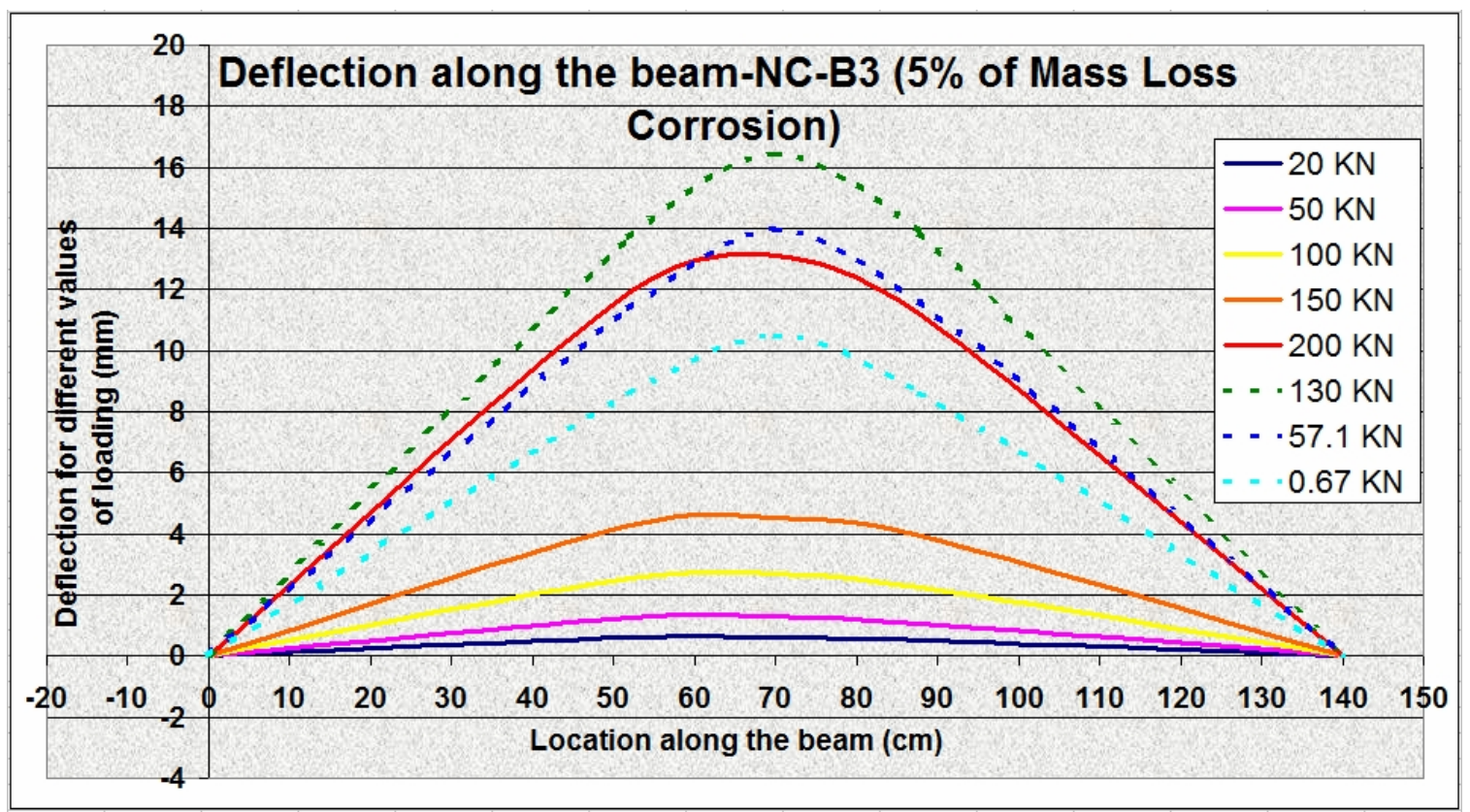

Figure (B5-4): The deflection along the beam for different values of loading for NC; B3 (5\% mass loss).

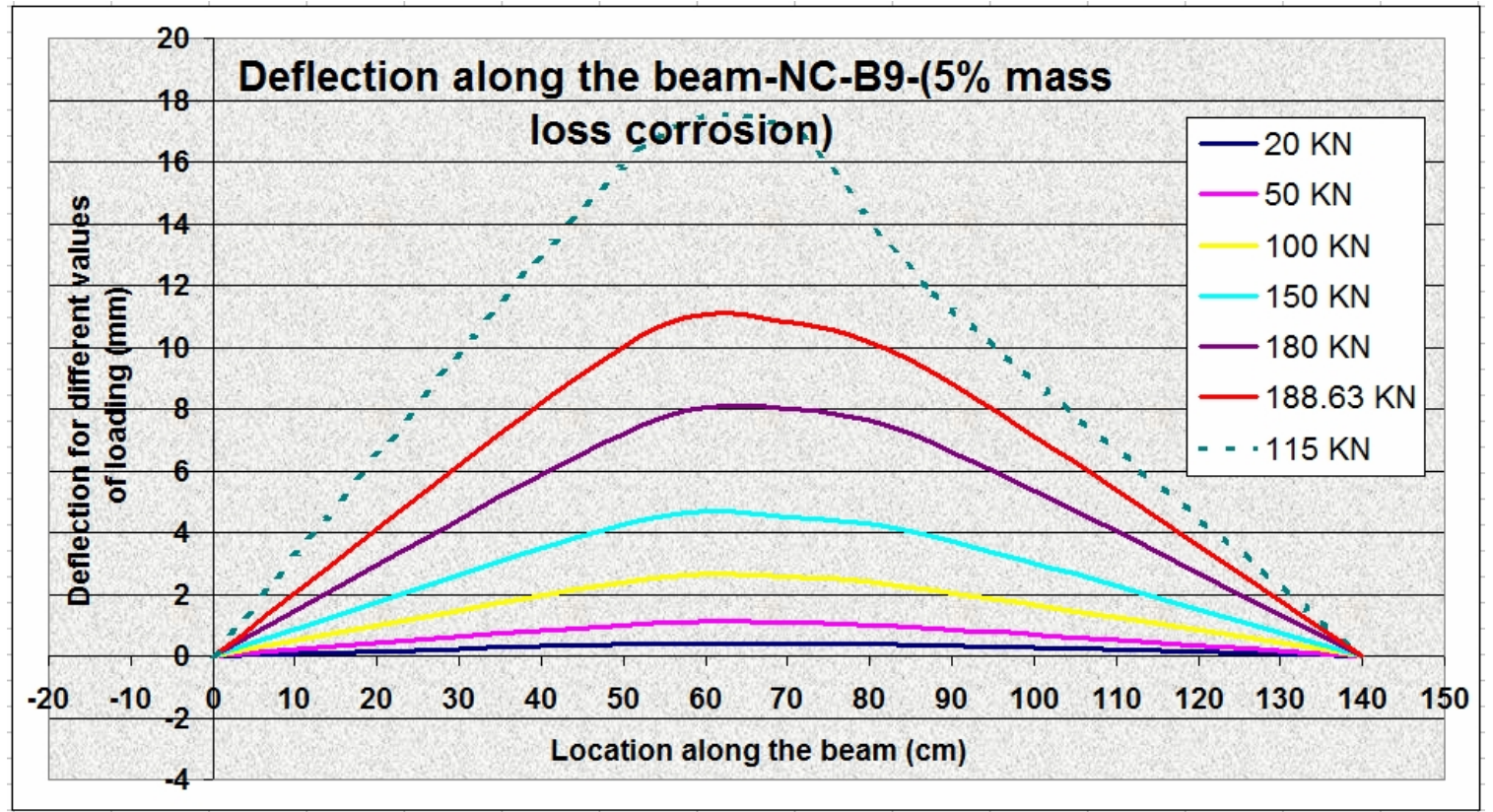

Figure (B5-5): The deflection along the beam for different values of loading for NC; B9 (5\% mass loss). 


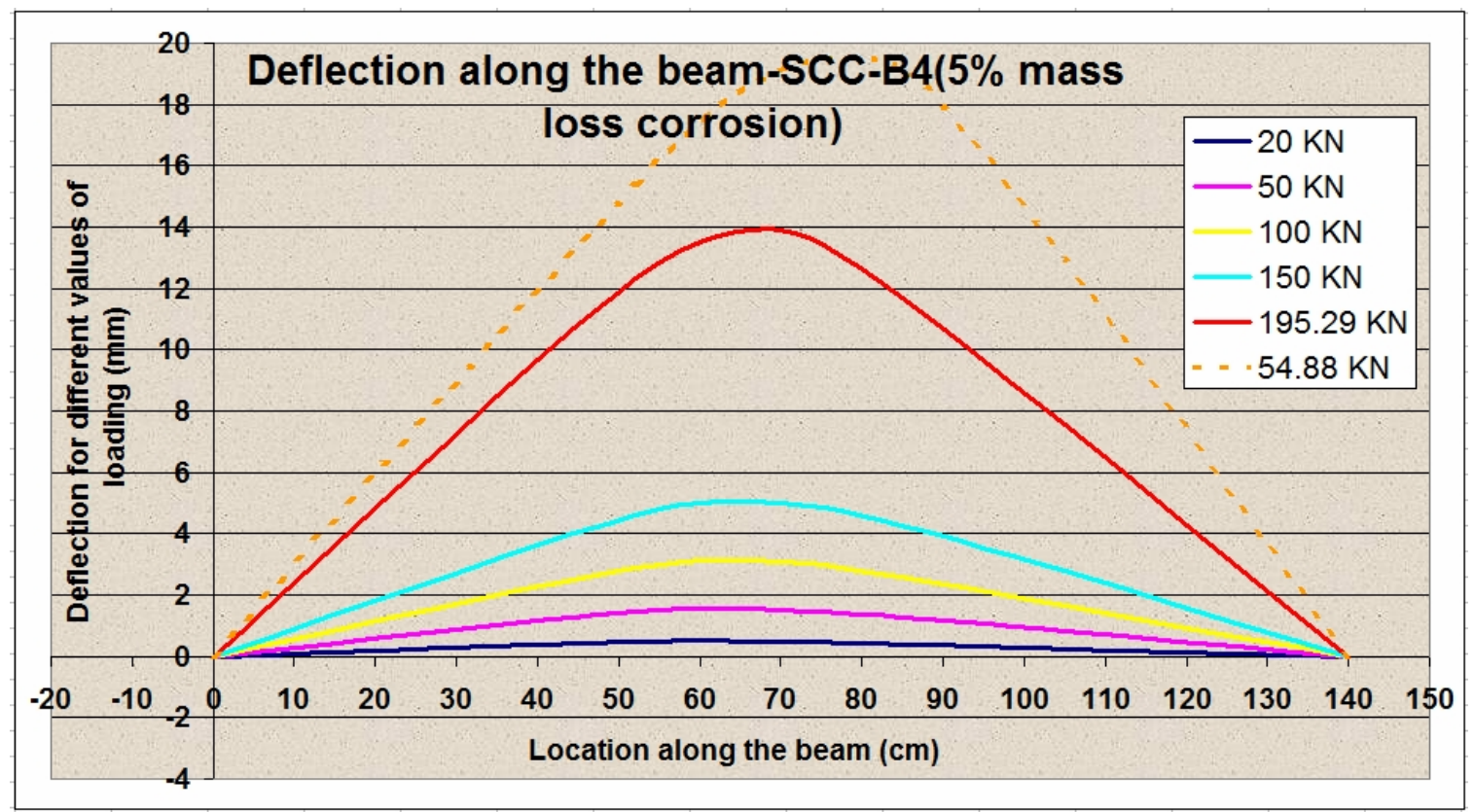

Figure (B5-6): The deflection along the beam for different values of loading for SCC; B-4 (5\% mass loss).

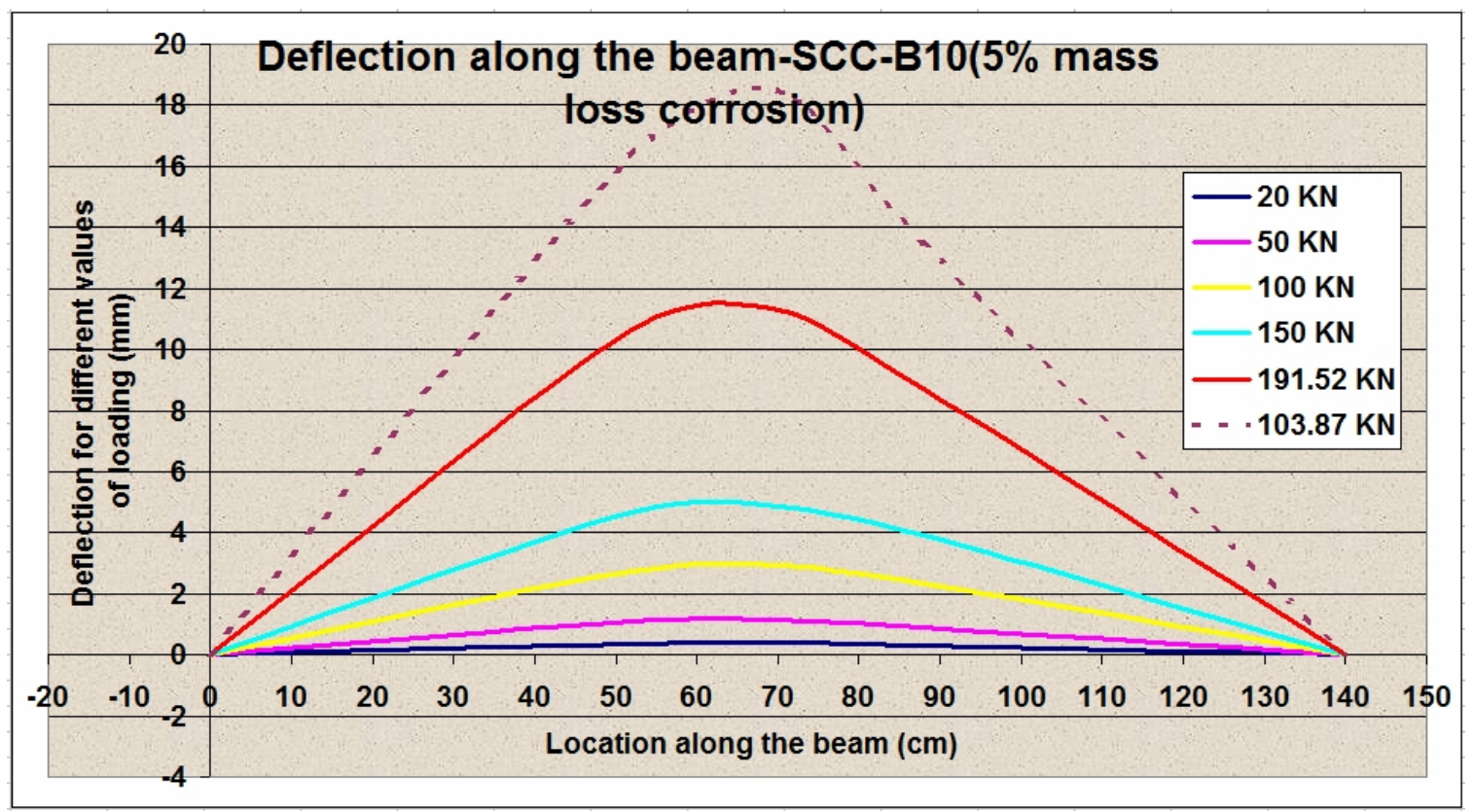

Figure (B5-7): The deflection along the beam for different values of loading for SCC; B-10 (5\% mass loss). 


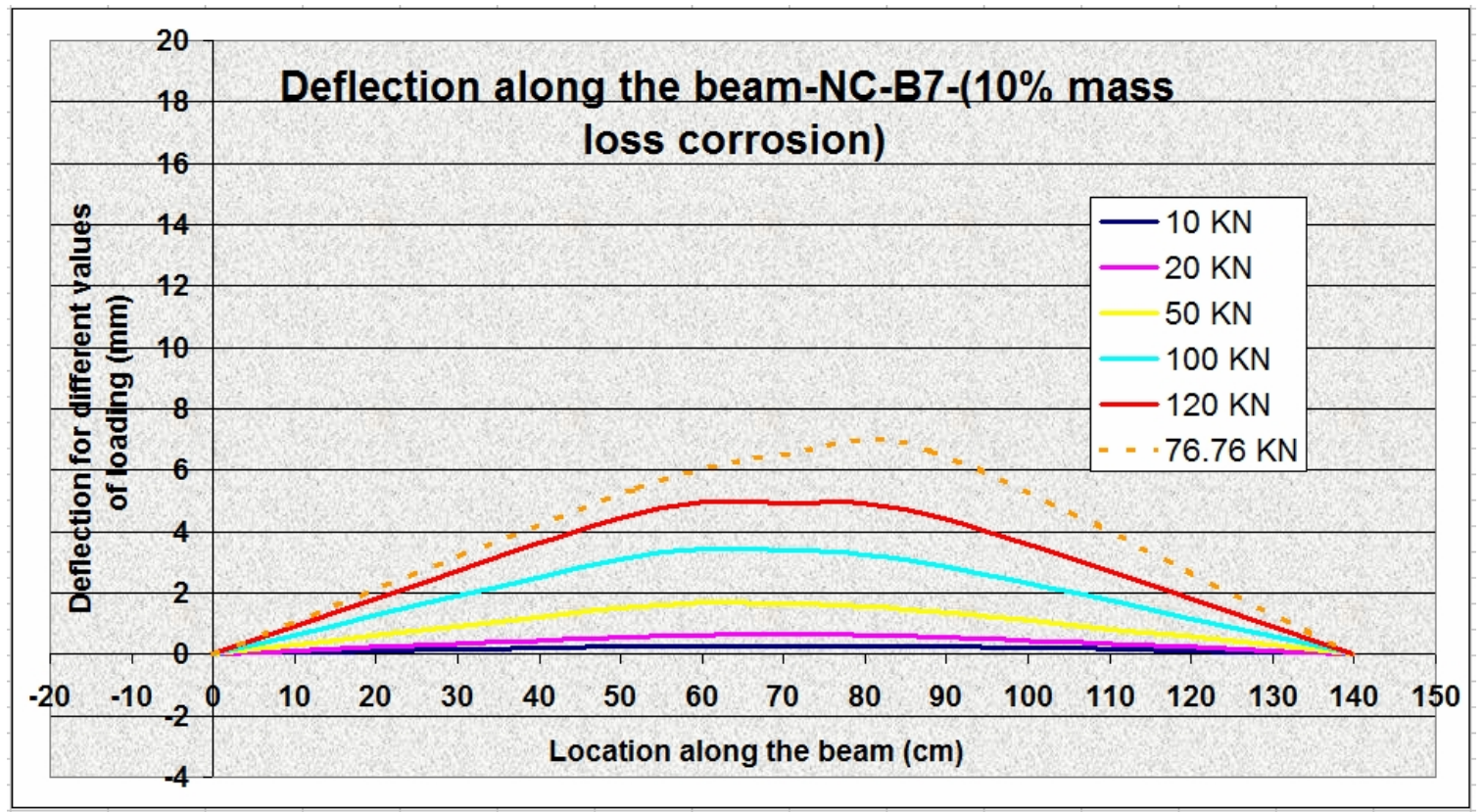

Figure (B5-8): The deflection along the beam for different values of loading for NC; B7 (10\% mass loss).

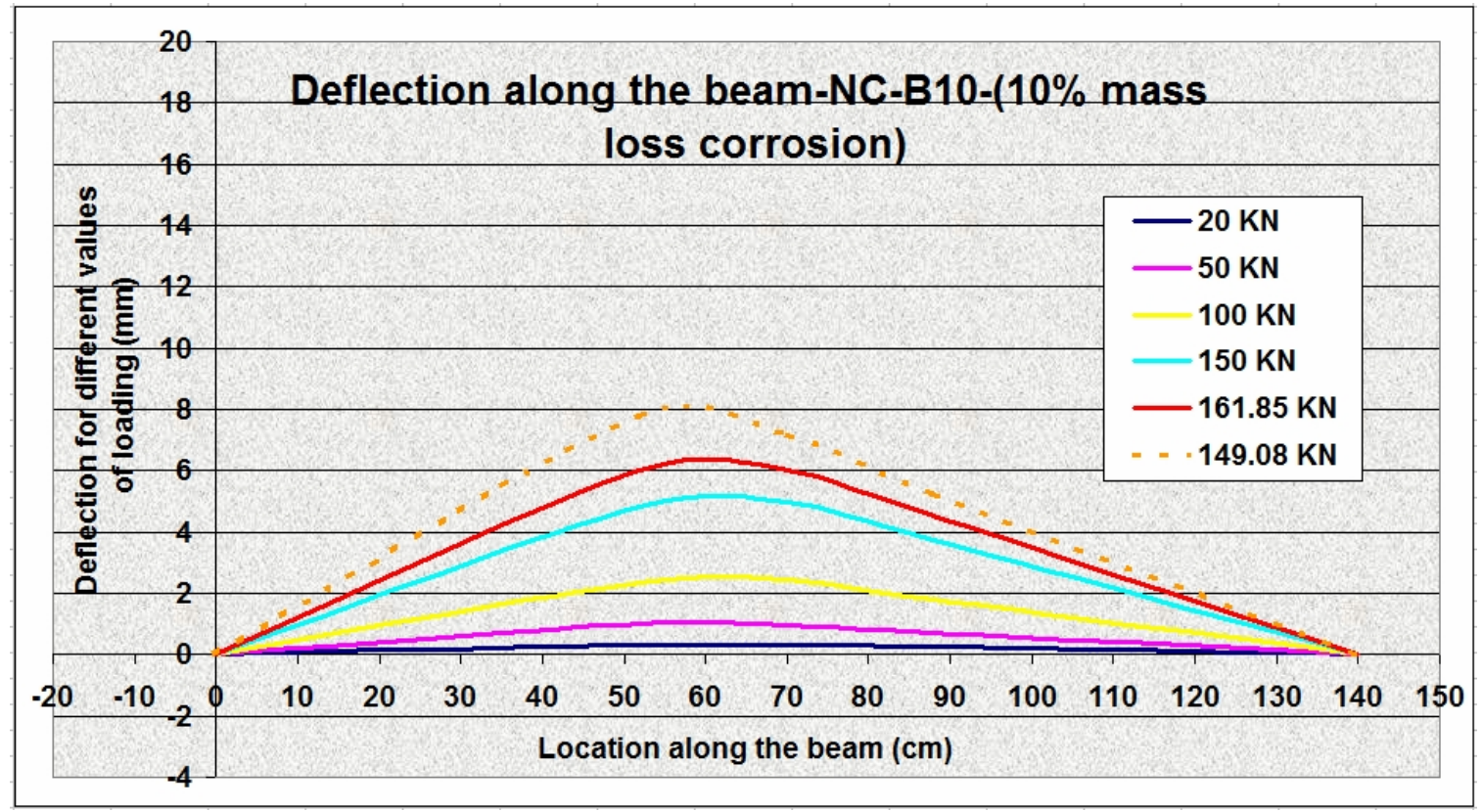

Figure (B5-9): The deflection along the beam for different values of loading for NC; B10 (10\% mass loss). 


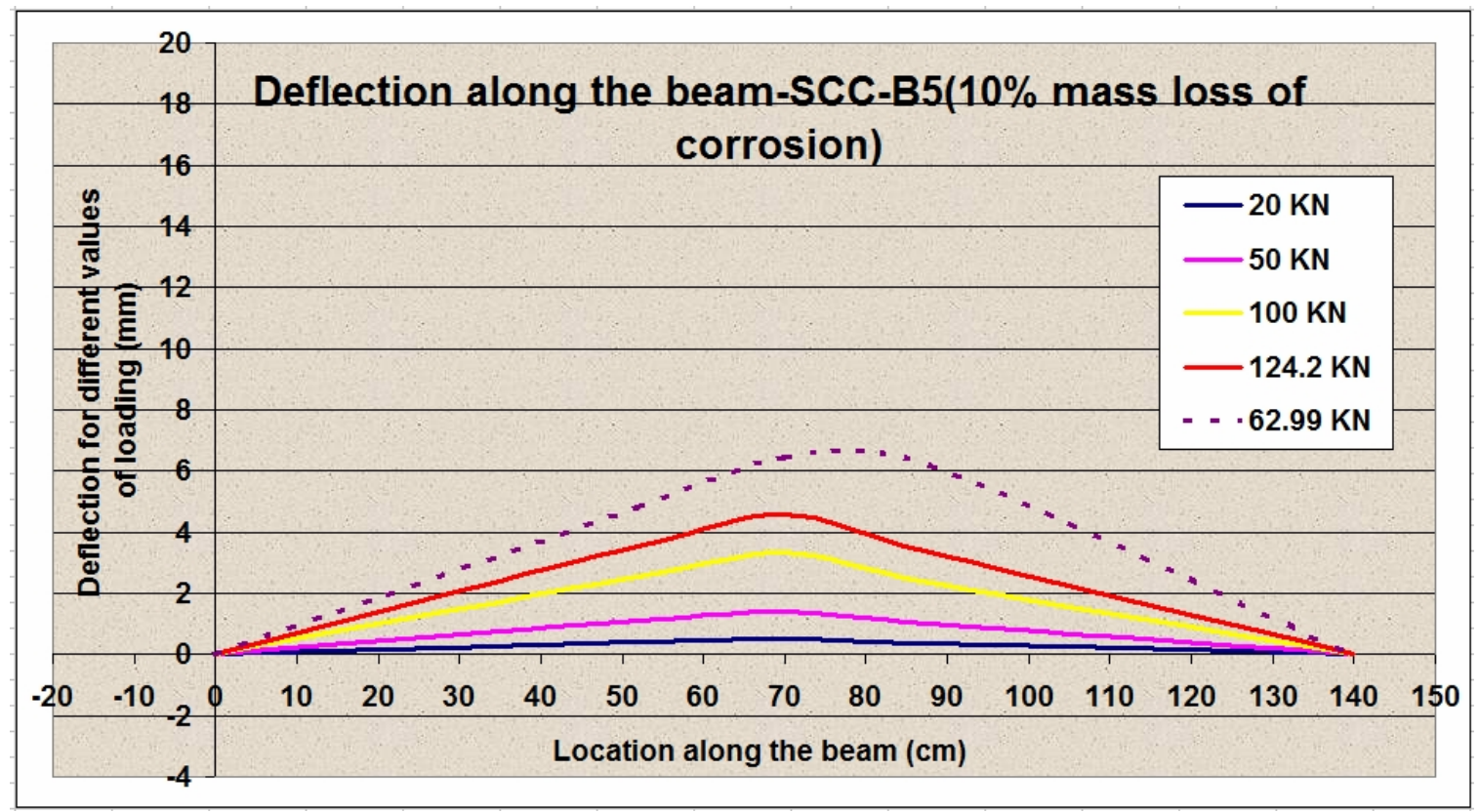

Figure (B5-10): The deflection along the beam for different values of loading for SCC; B-5 (10\% mass loss).

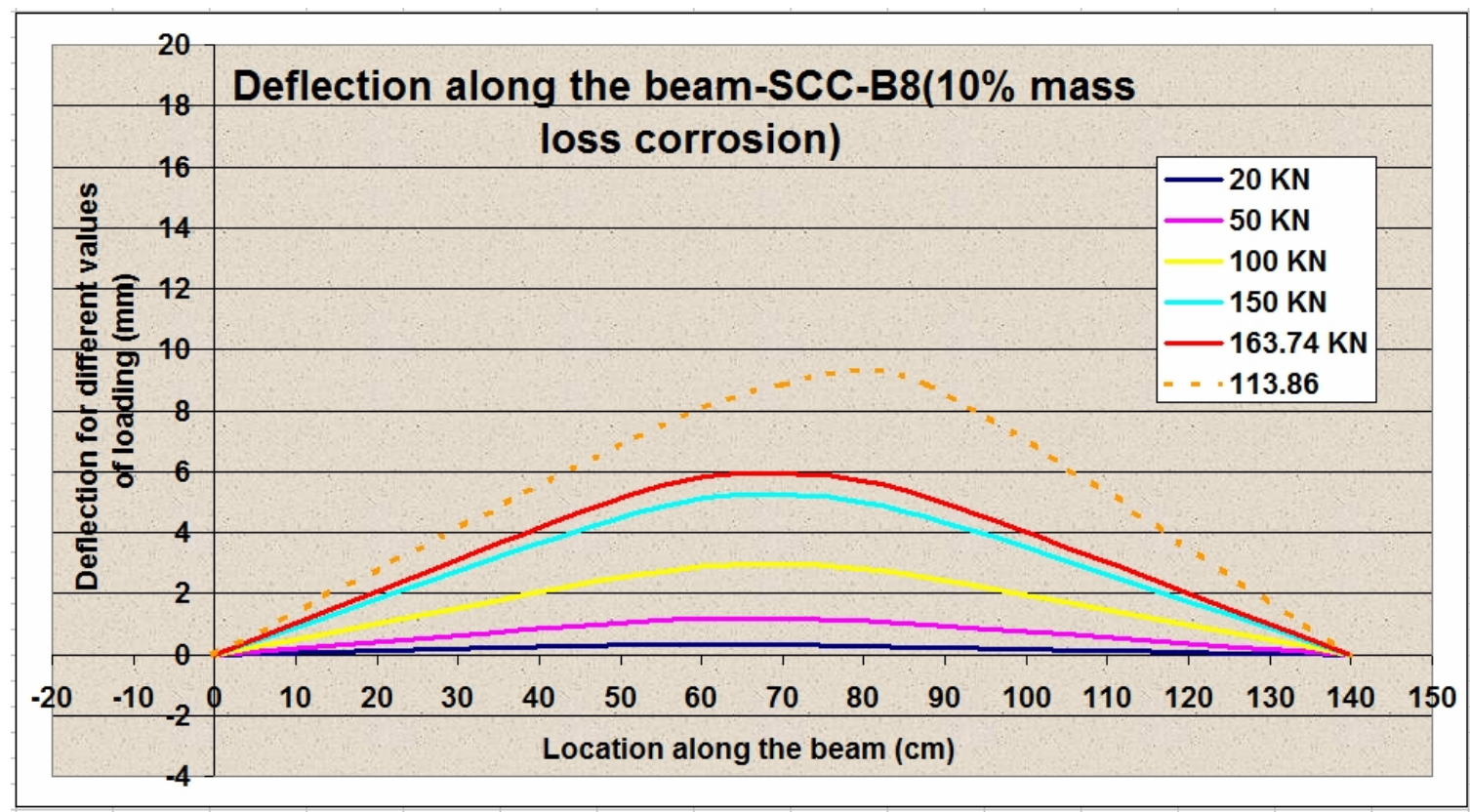

Figure (B5-11): The deflection along the beam for different values of loading for SCC; B-8 (10\% mass loss). 


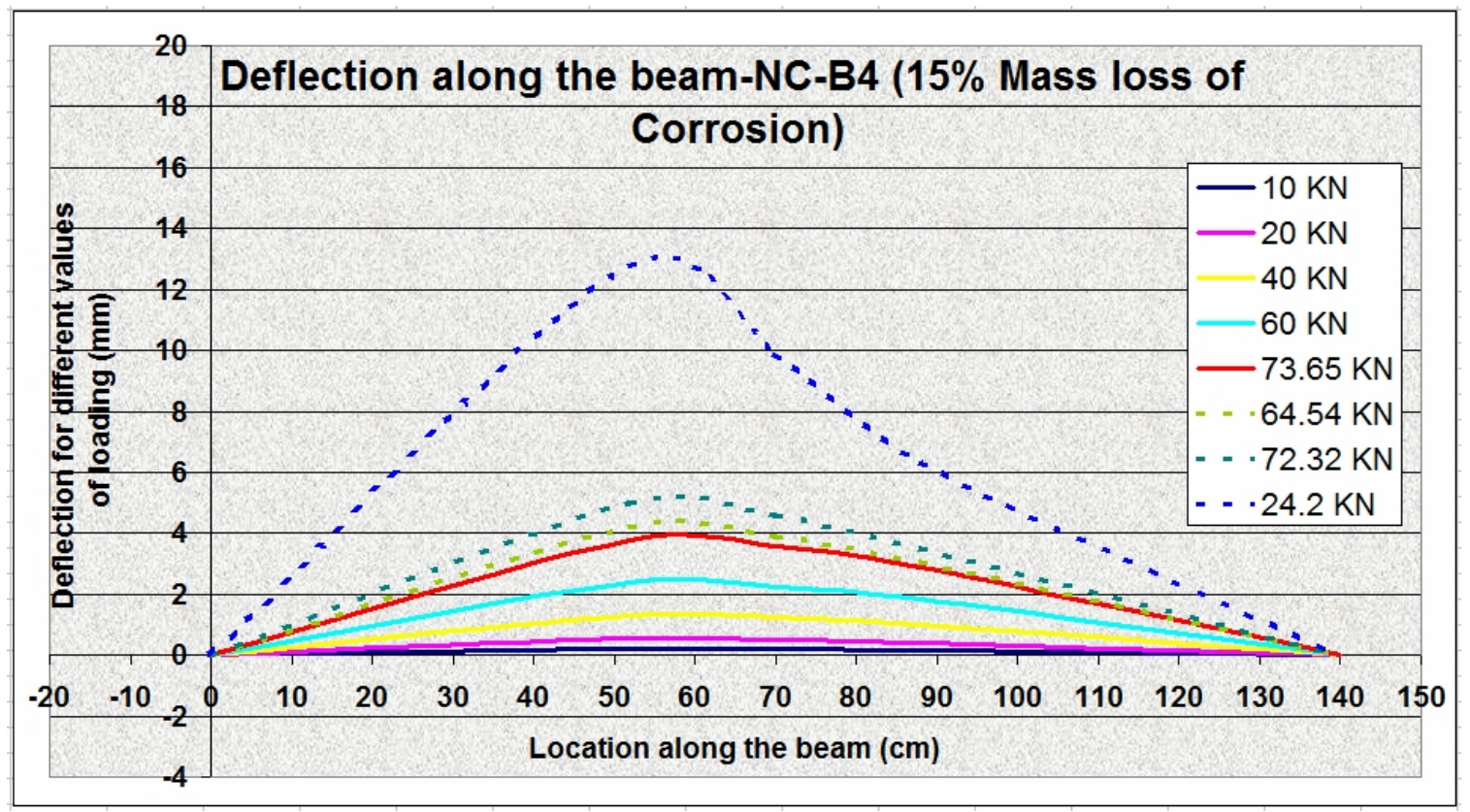

Figure (B5-12): The deflection along the beam for different values of loading for NC; B-4 (15\% mass loss).

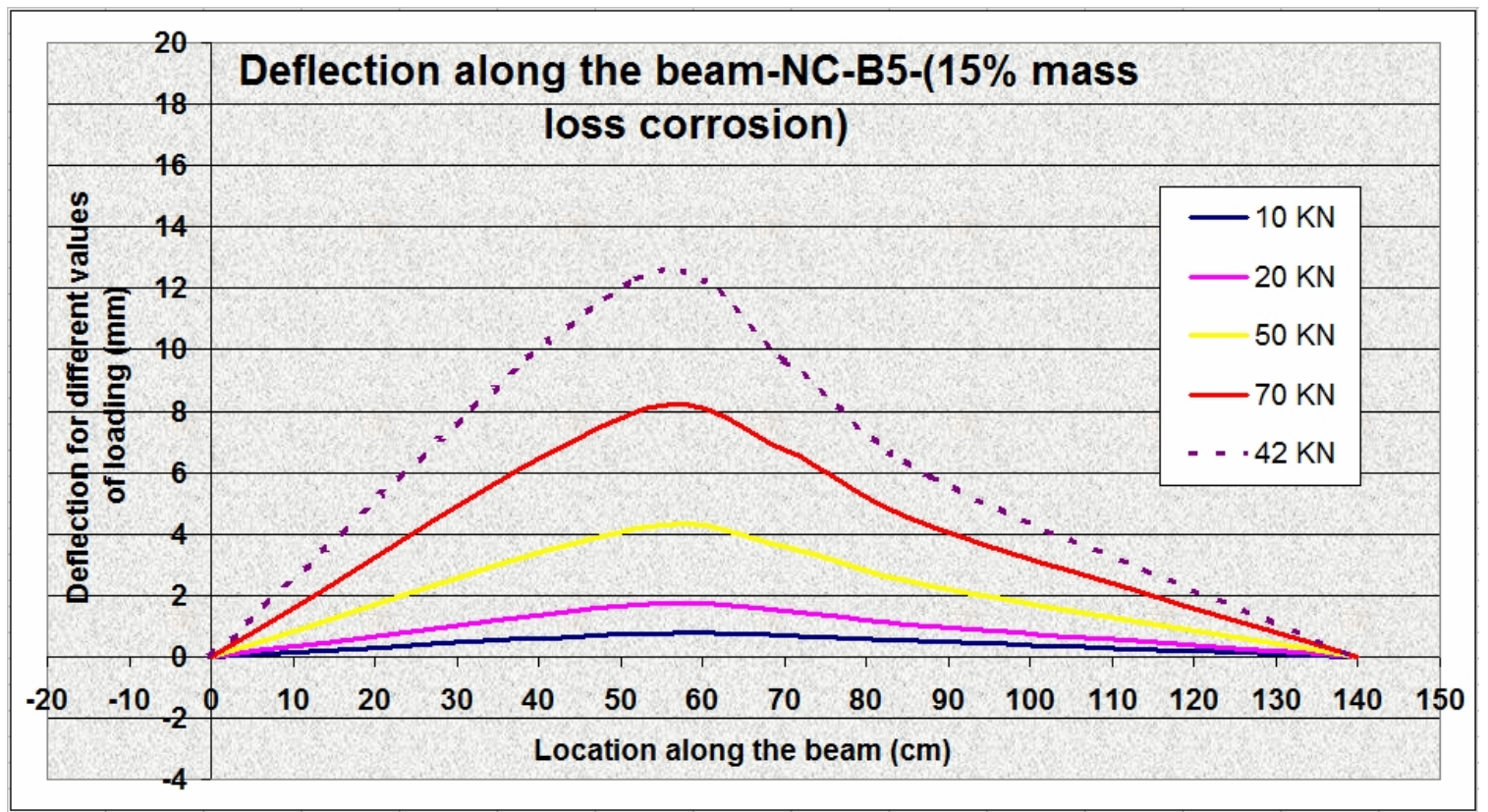

Figure (B5-13): The deflection along the beam for different values of loading for NC;

B-5 (15\% mass loss). 


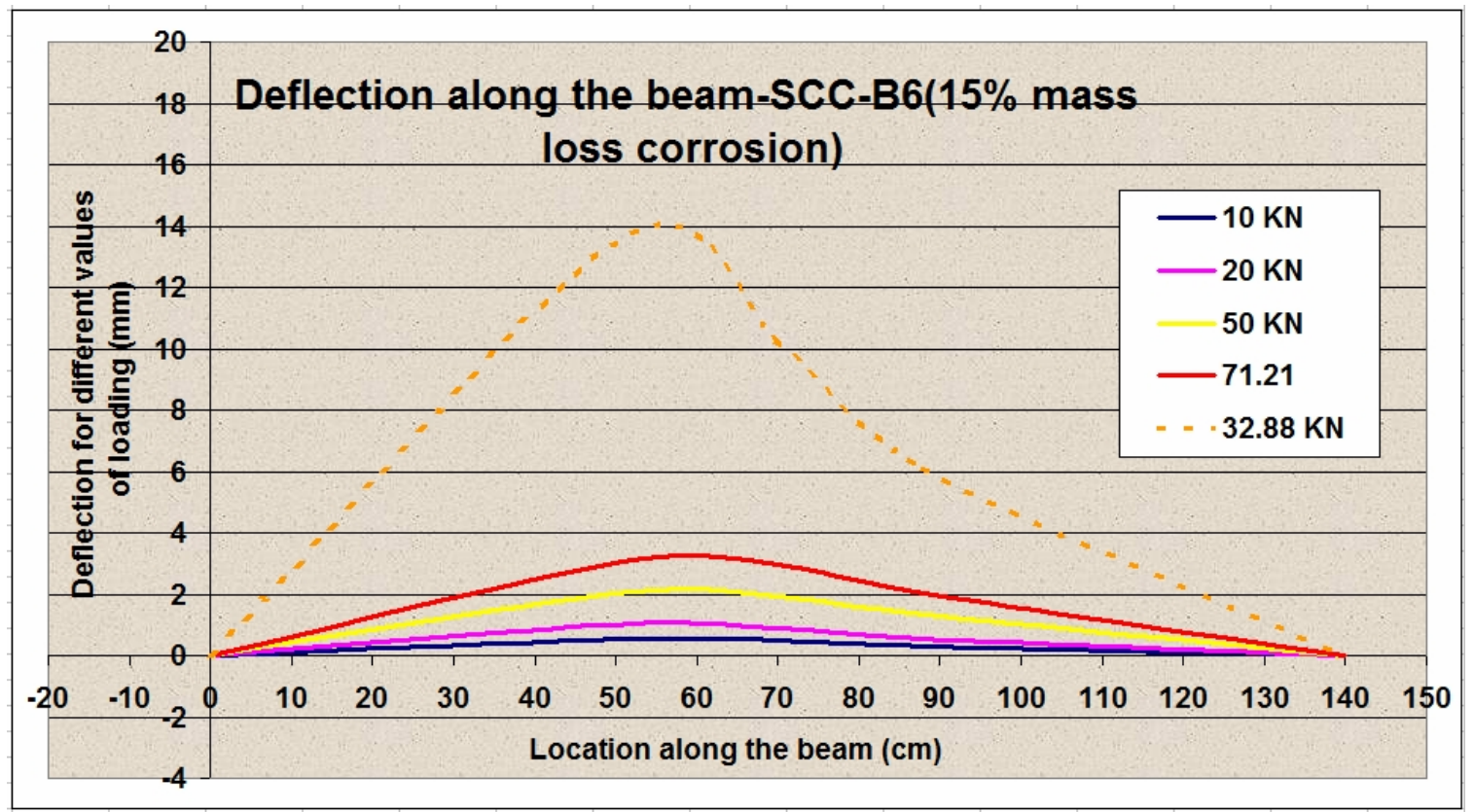

Figure (B5-14): The deflection along the beam for different values of loading for SCC; B-6 (15\% mass loss).

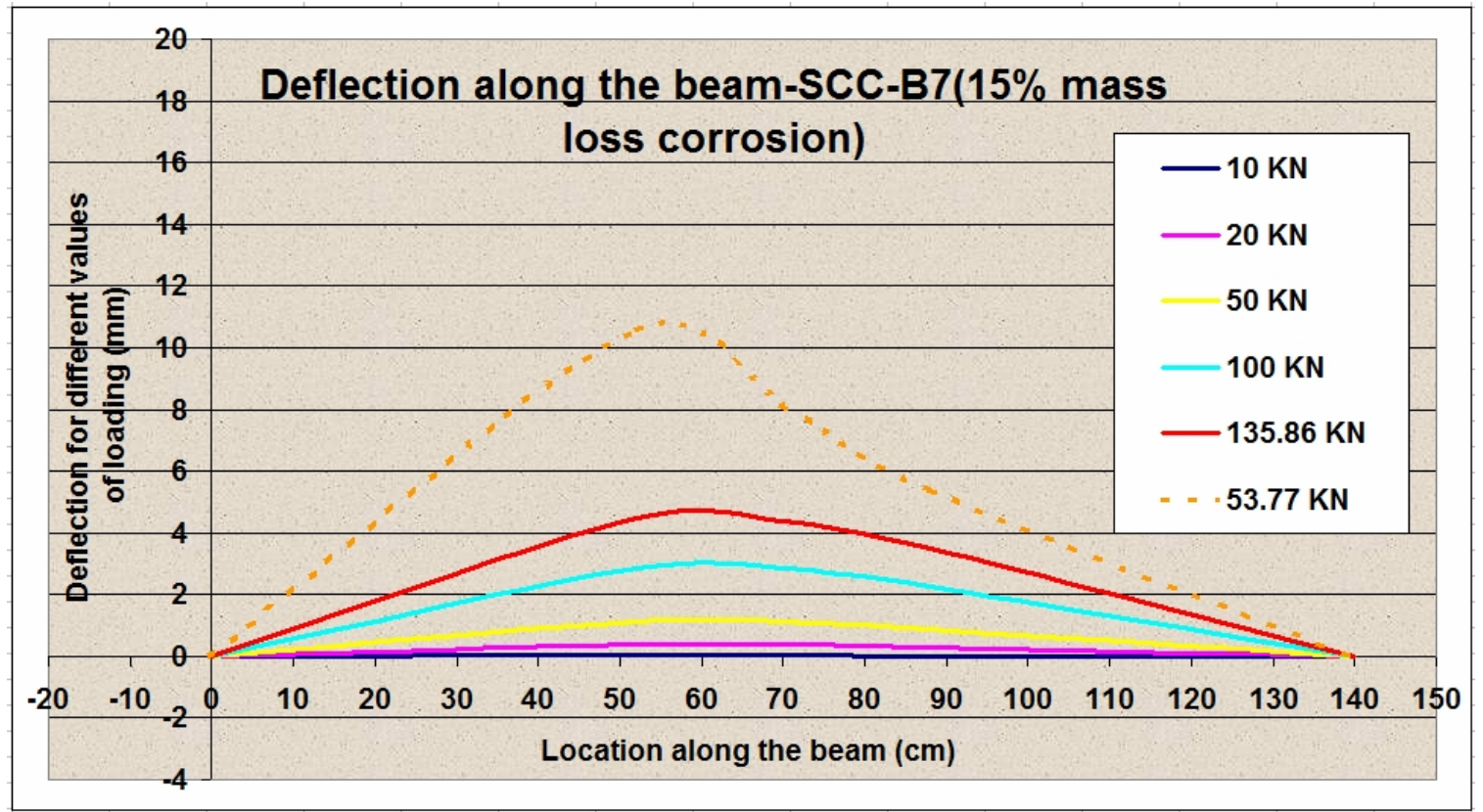

Figure (B5-15): The deflection along the beam for different values of loading for SCC; B-7 (15\% mass loss). 


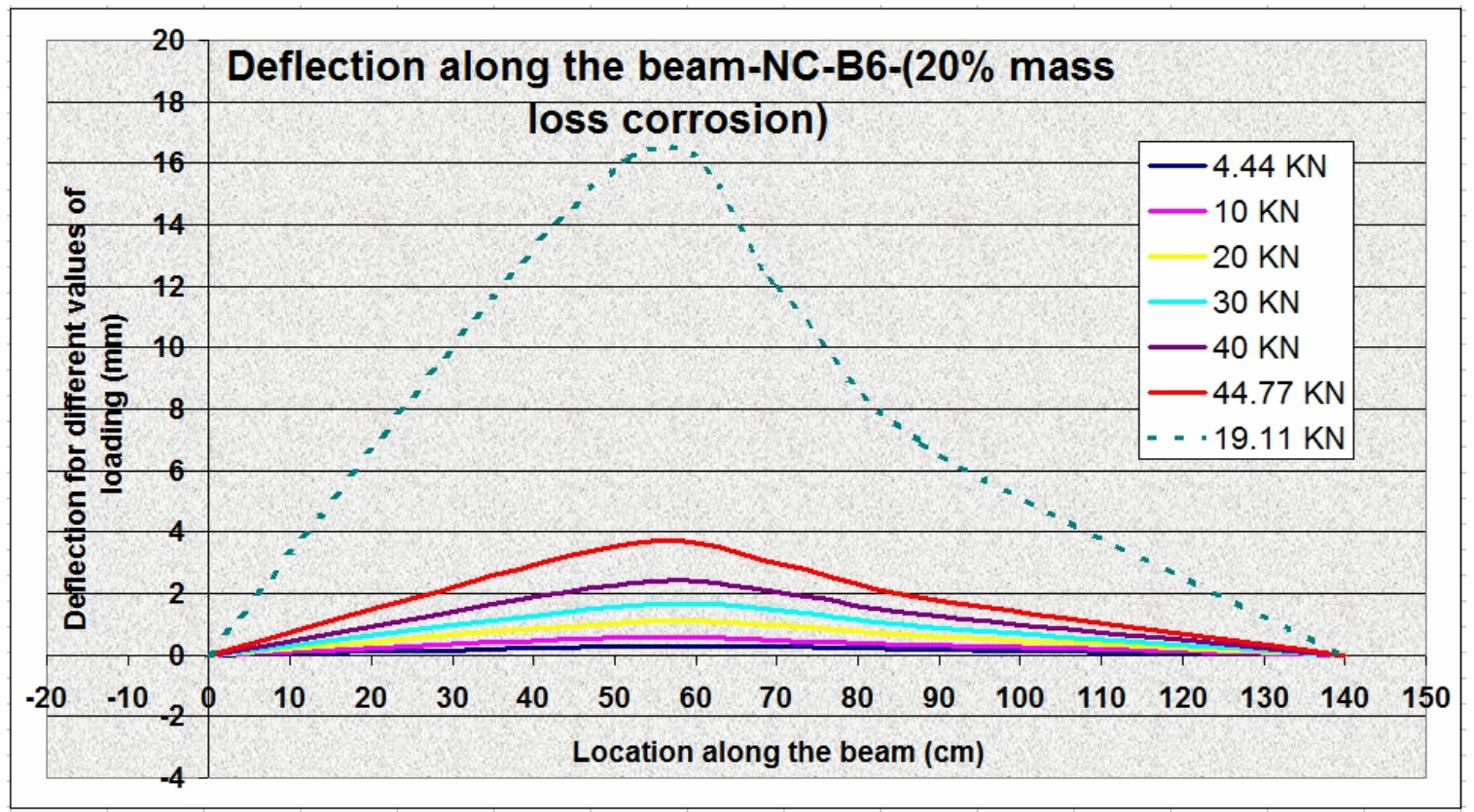

Figure (B5-16): The deflection along the beam for different values of loading for NC; B-6 (20\% mass loss).

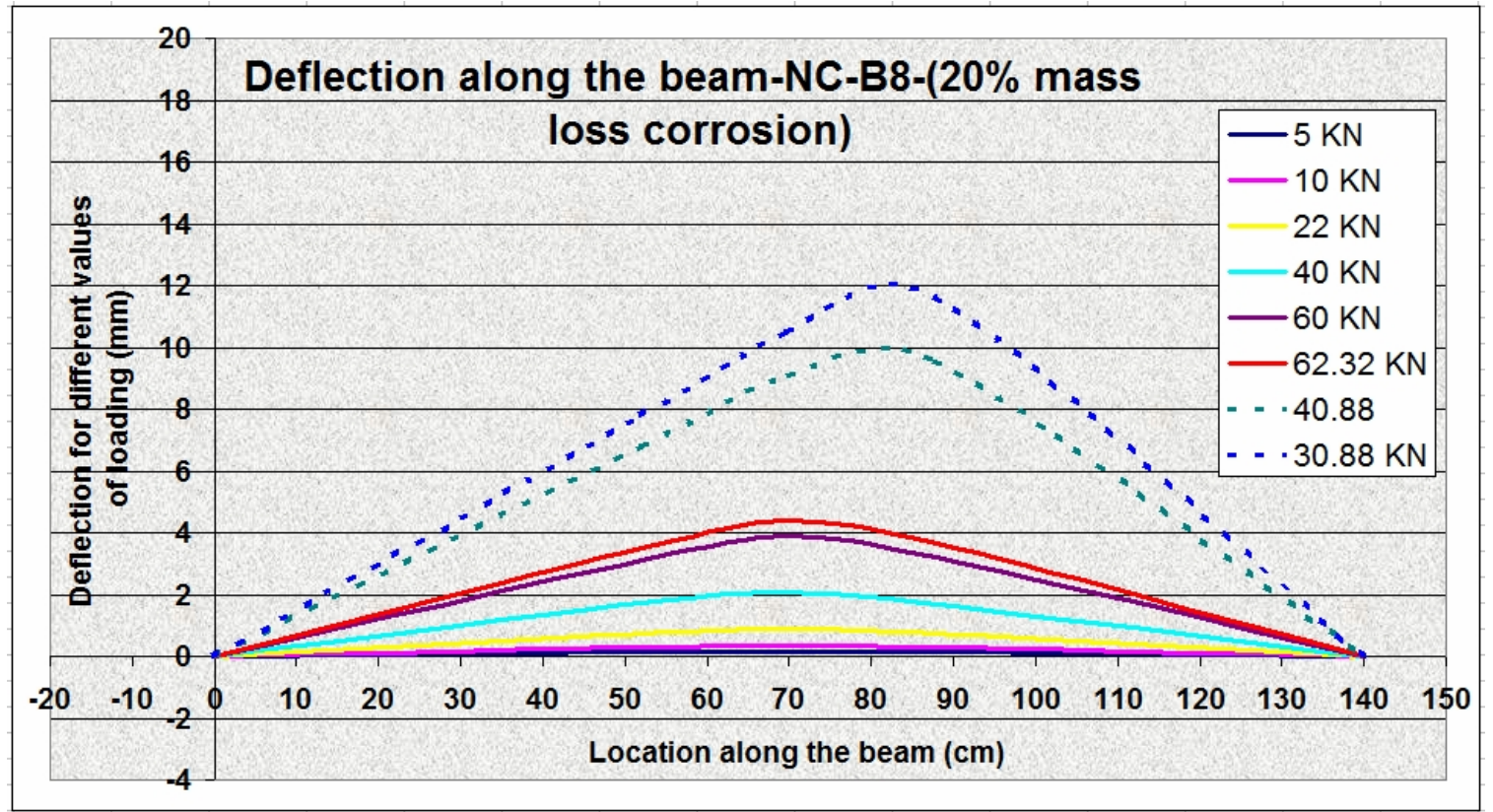

Figure (B5-17): The deflection along the beam for different values of loading for NC; B-8 (20\% mass loss). 


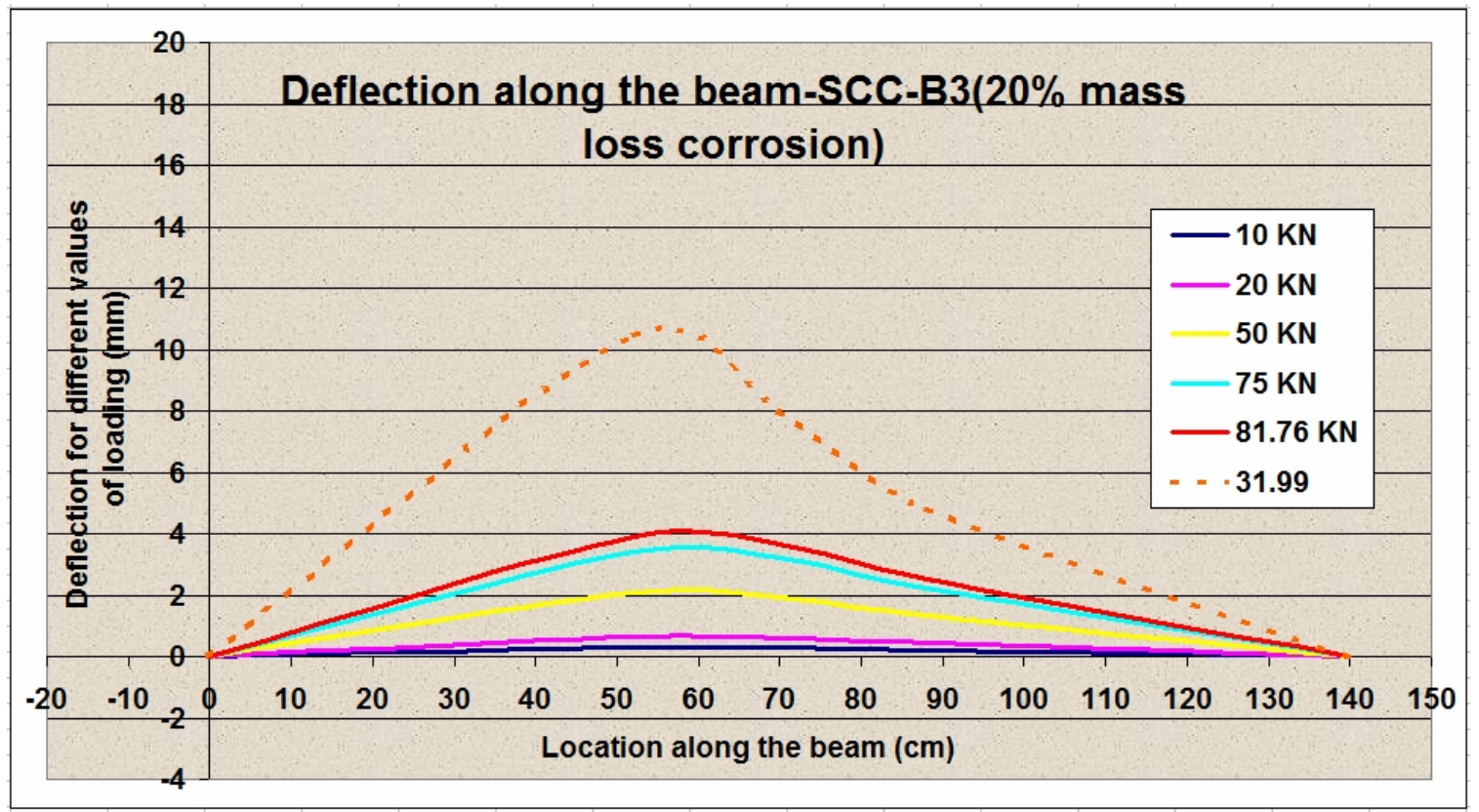

Figure (B5-18): The deflection along the beam for different values of loading for SCC; B-3 (20\% mass loss).

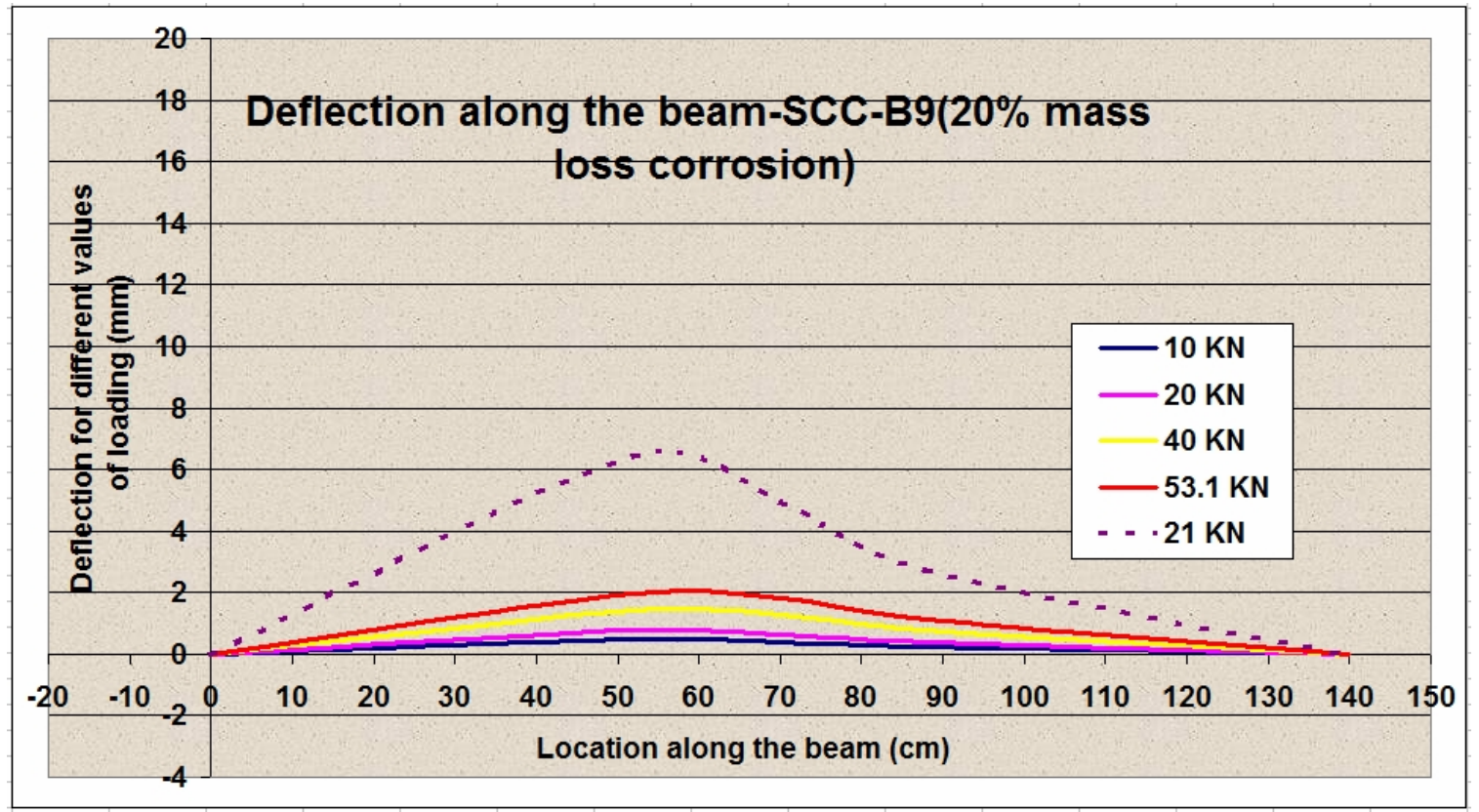

Figure (B5-19): The deflection along the beam for different values of loading for SCC; B-5 (10\% mass loss). 


\section{Appendix C: Loading crack pattern}

The following photos present the loading crack patterns for the beams with zero, $5 \%, 10 \%, 15 \%$, and $20 \%$ mass loss.

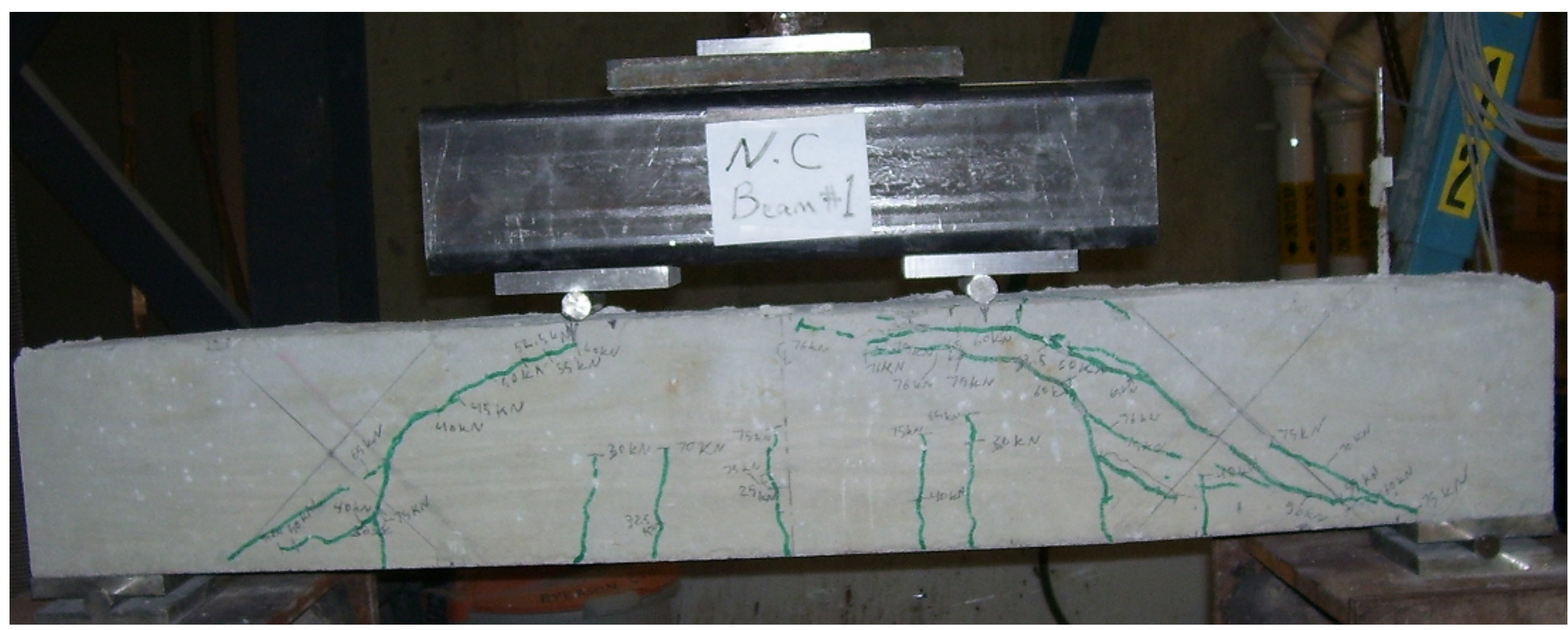

Figure (C-1): Loading cracks pattern for NC beam no.1 with zero mass loss. 


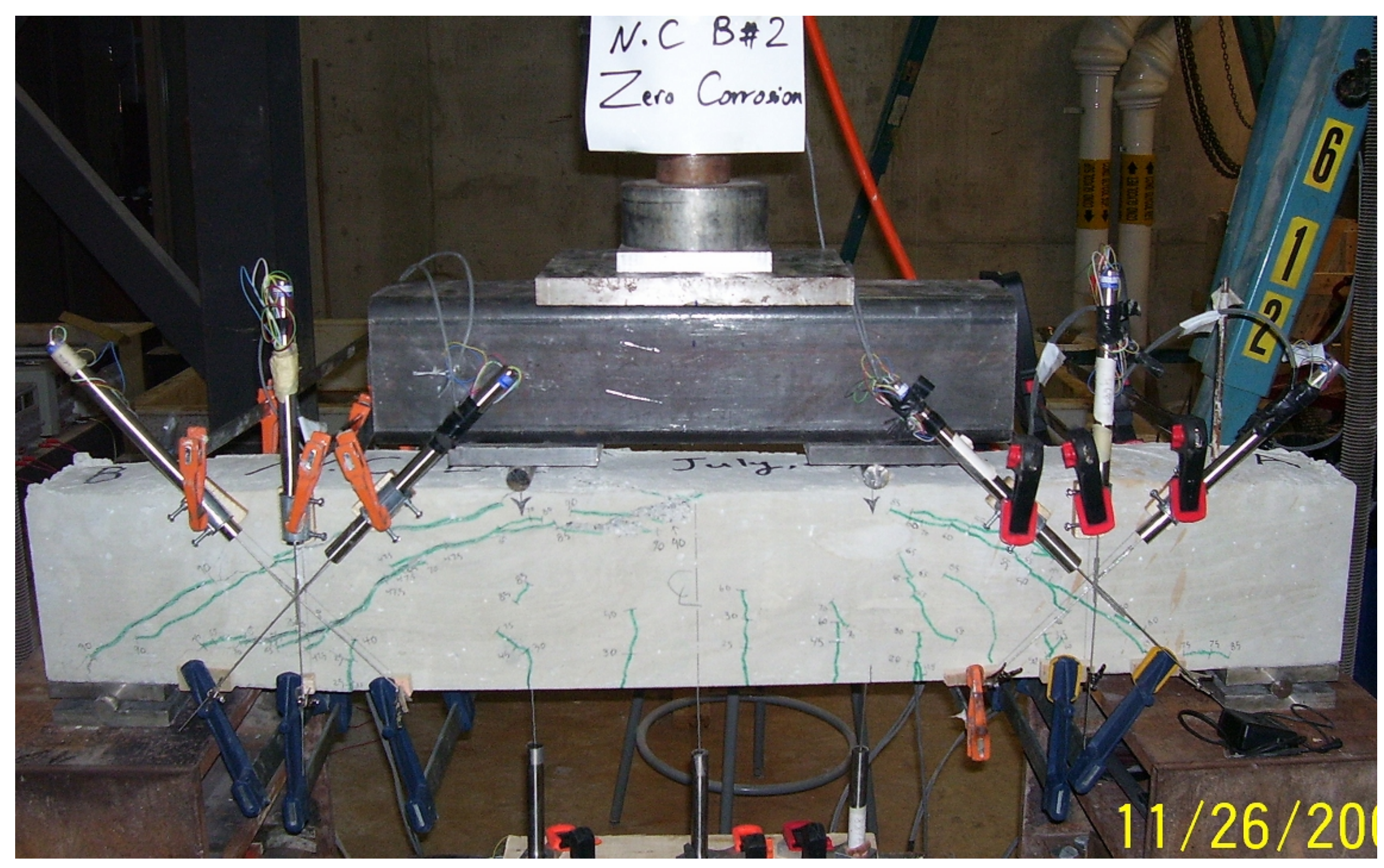

Figure (C-2): Loading cracks pattern for NC beam no.2 with zero mass loss. 


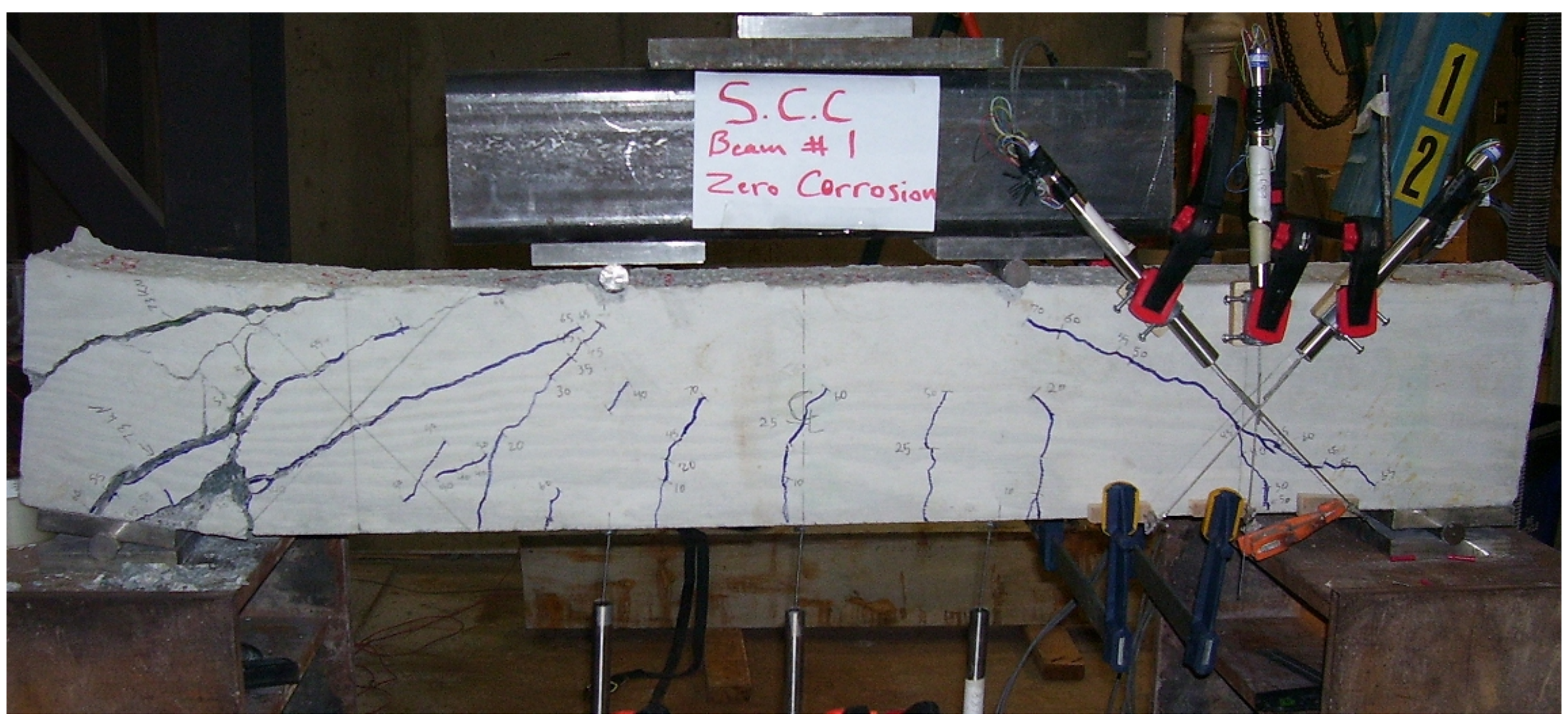

Figure (C-3): Loading cracks pattern for SCC beam no.1 with zero mass loss. 


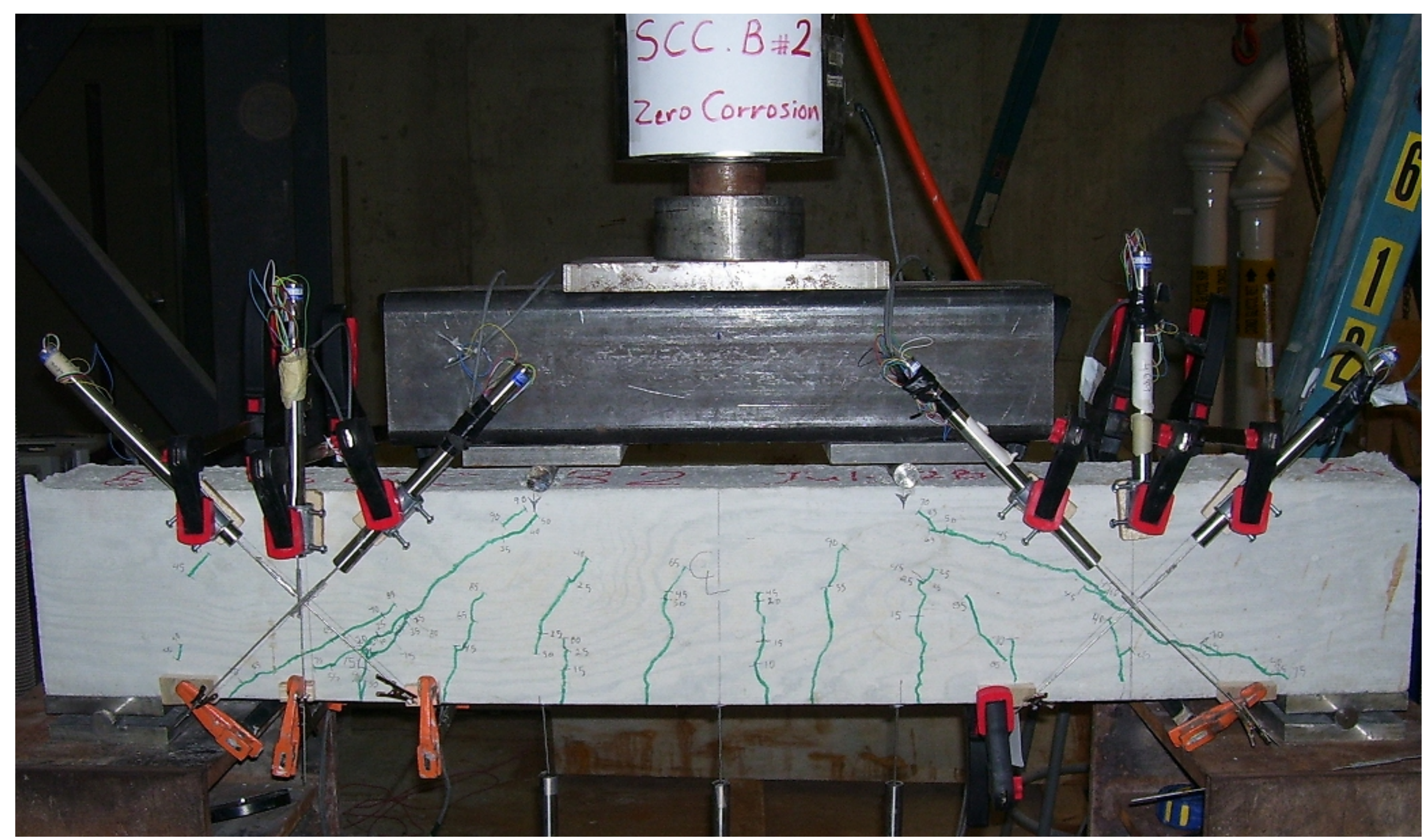

Figure (C-4): Loading cracks pattern for SCC beam no.2 with zero mass loss. 


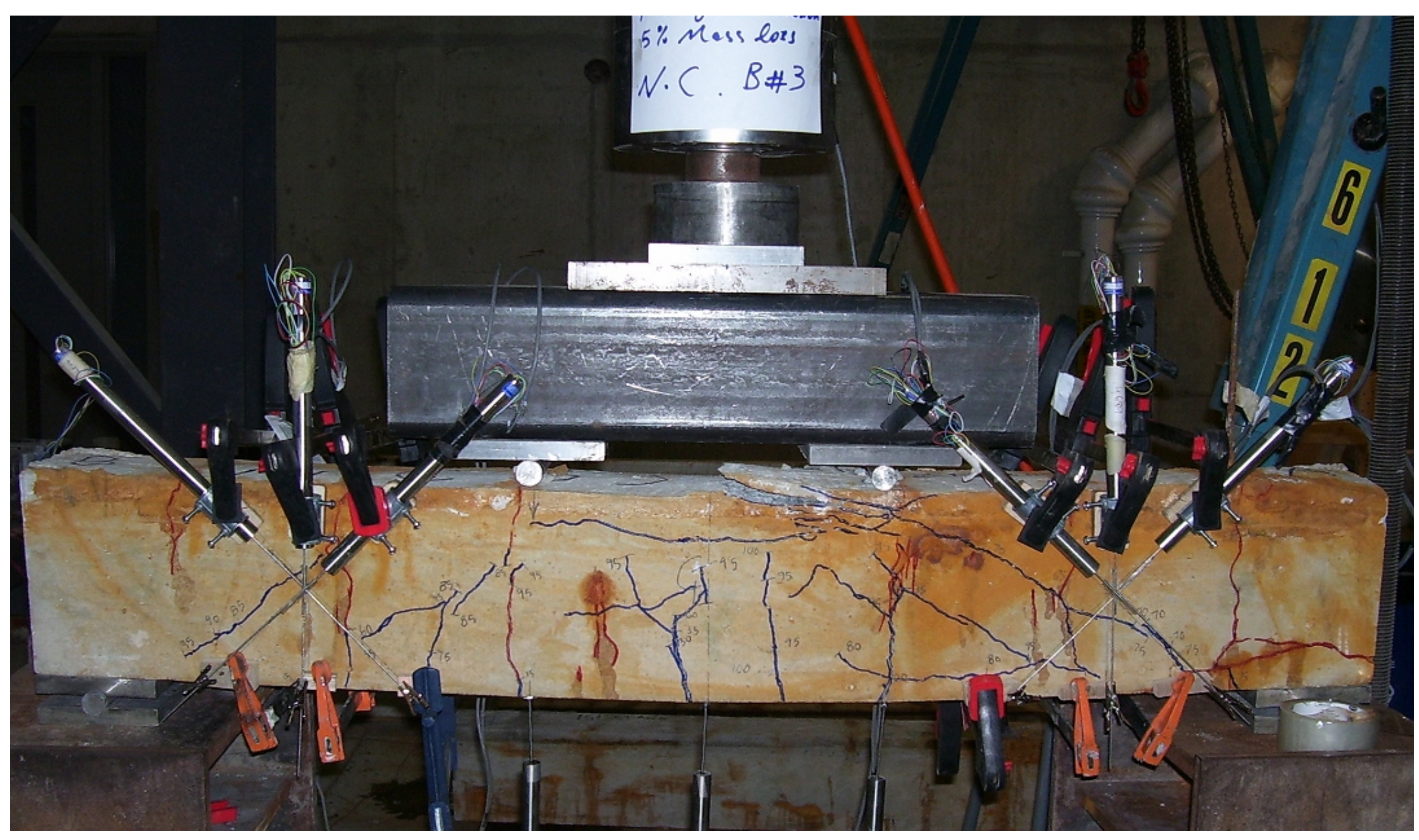

Figure (C-5): Loading cracks pattern for NC beam no. 3 with $5 \%$ mass loss. 


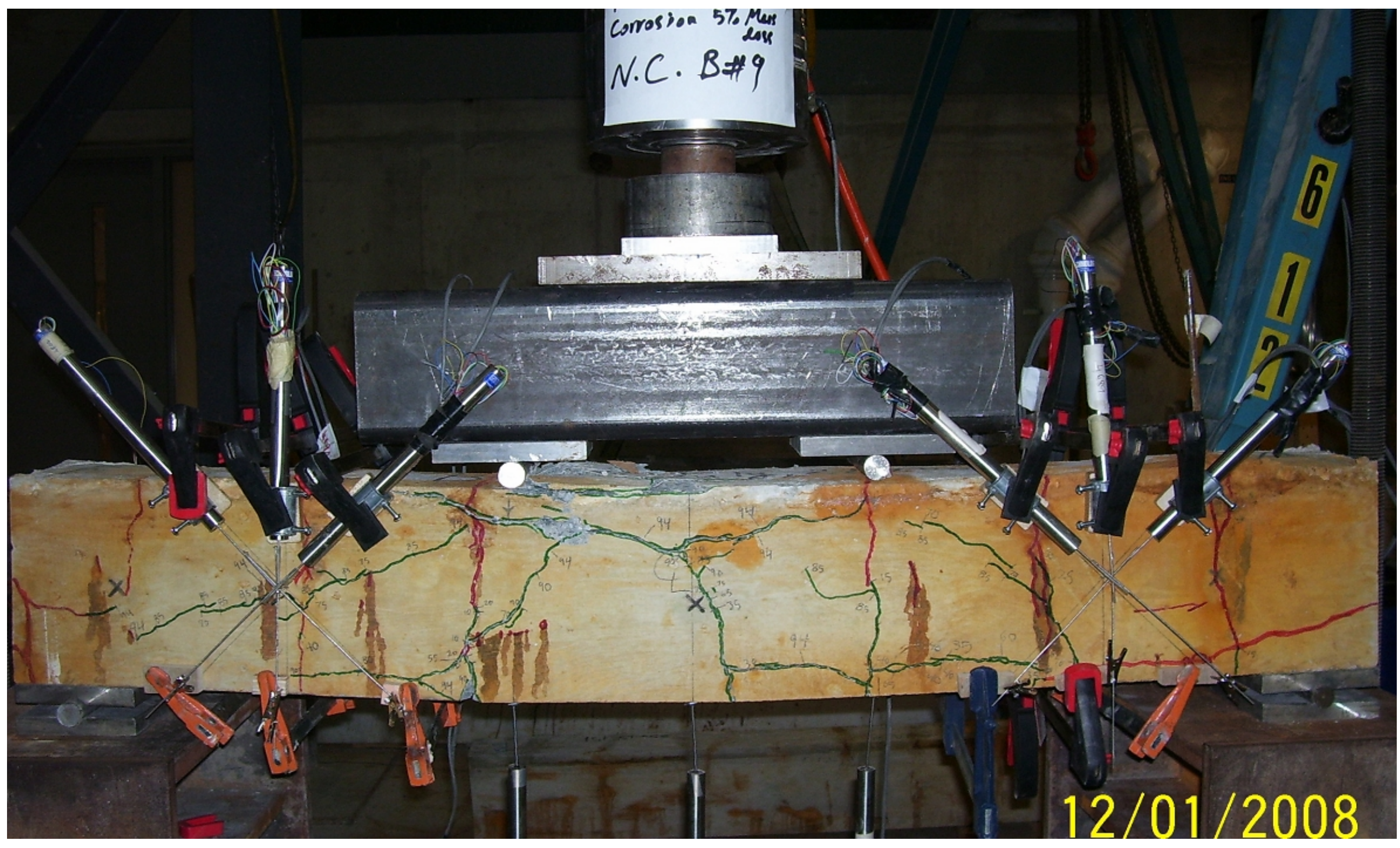

Figure (C-6): Loading cracks pattern for NC beam no.9 with $5 \%$ mass loss. 


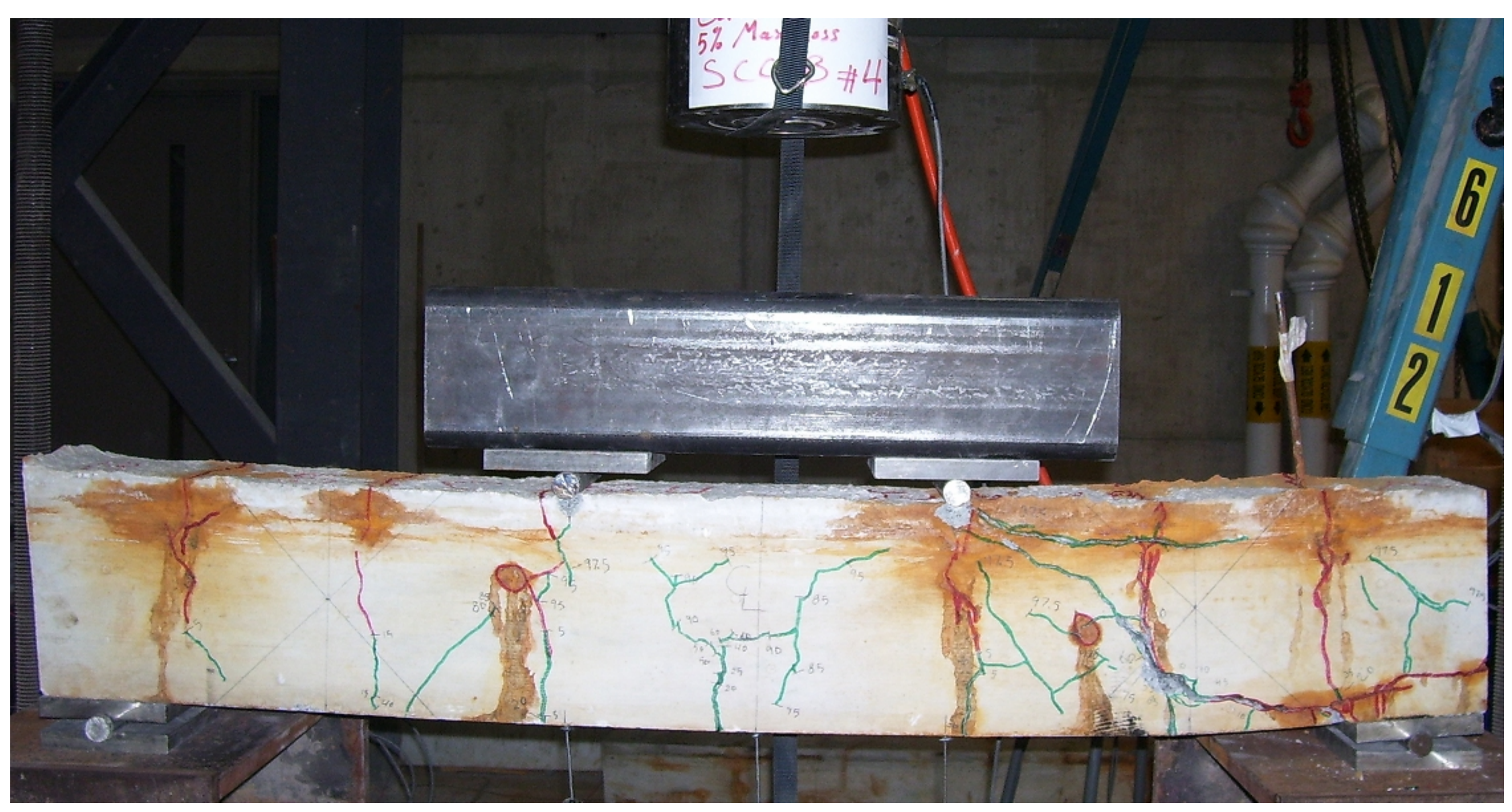

Figure (C-7): Loading cracks pattern for SCC beam no. 4 with $5 \%$ mass loss. 


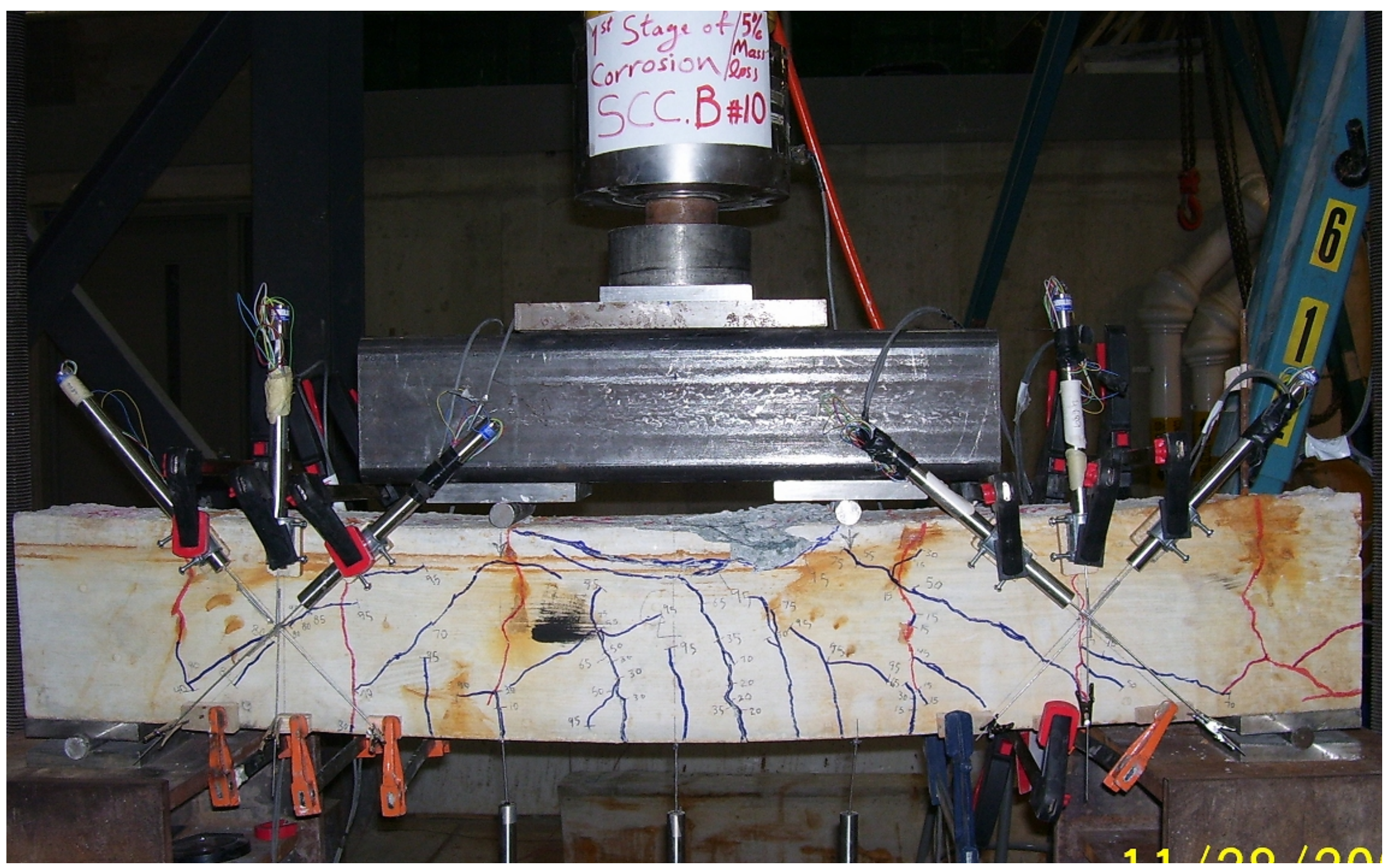

Figure (C-8): Loading cracks pattern for SCC beam no. 10 with $5 \%$ mass loss. 


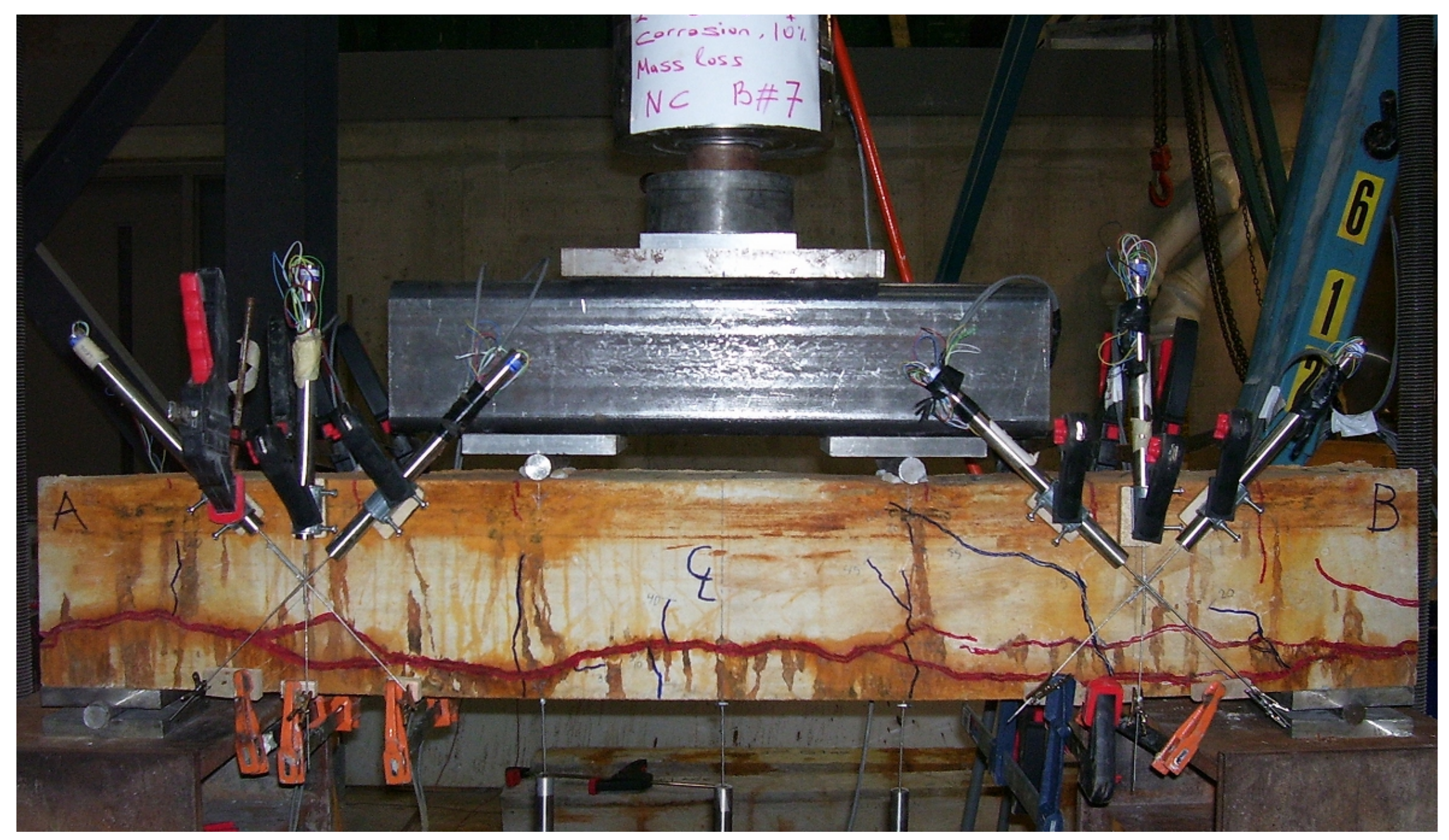

Figure (C-9): Loading cracks pattern for NC beam no.7 with $10 \%$ mass loss. 


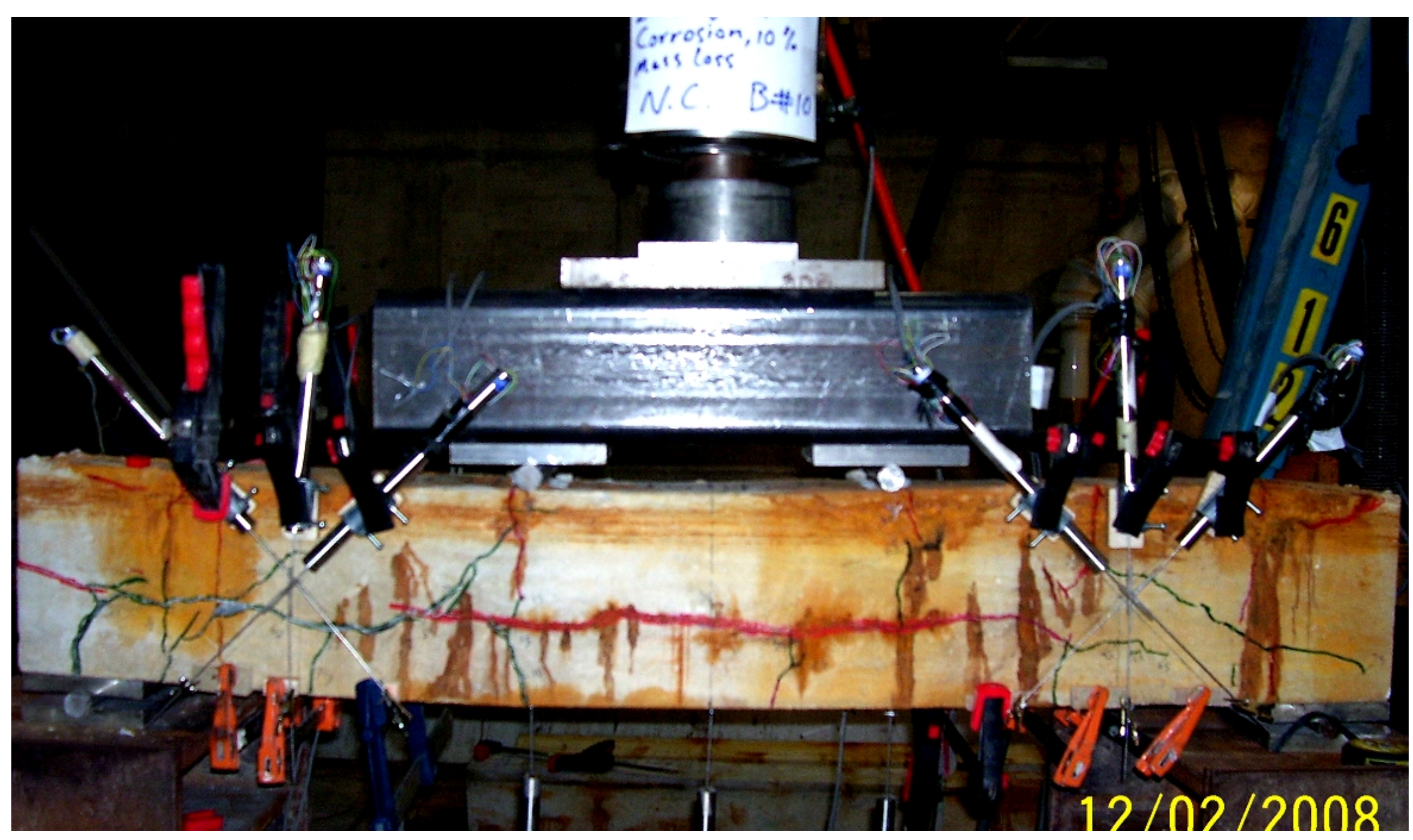

Figure (C-10): Loading cracks pattern for NC beam no.10 with $10 \%$ mass loss. 


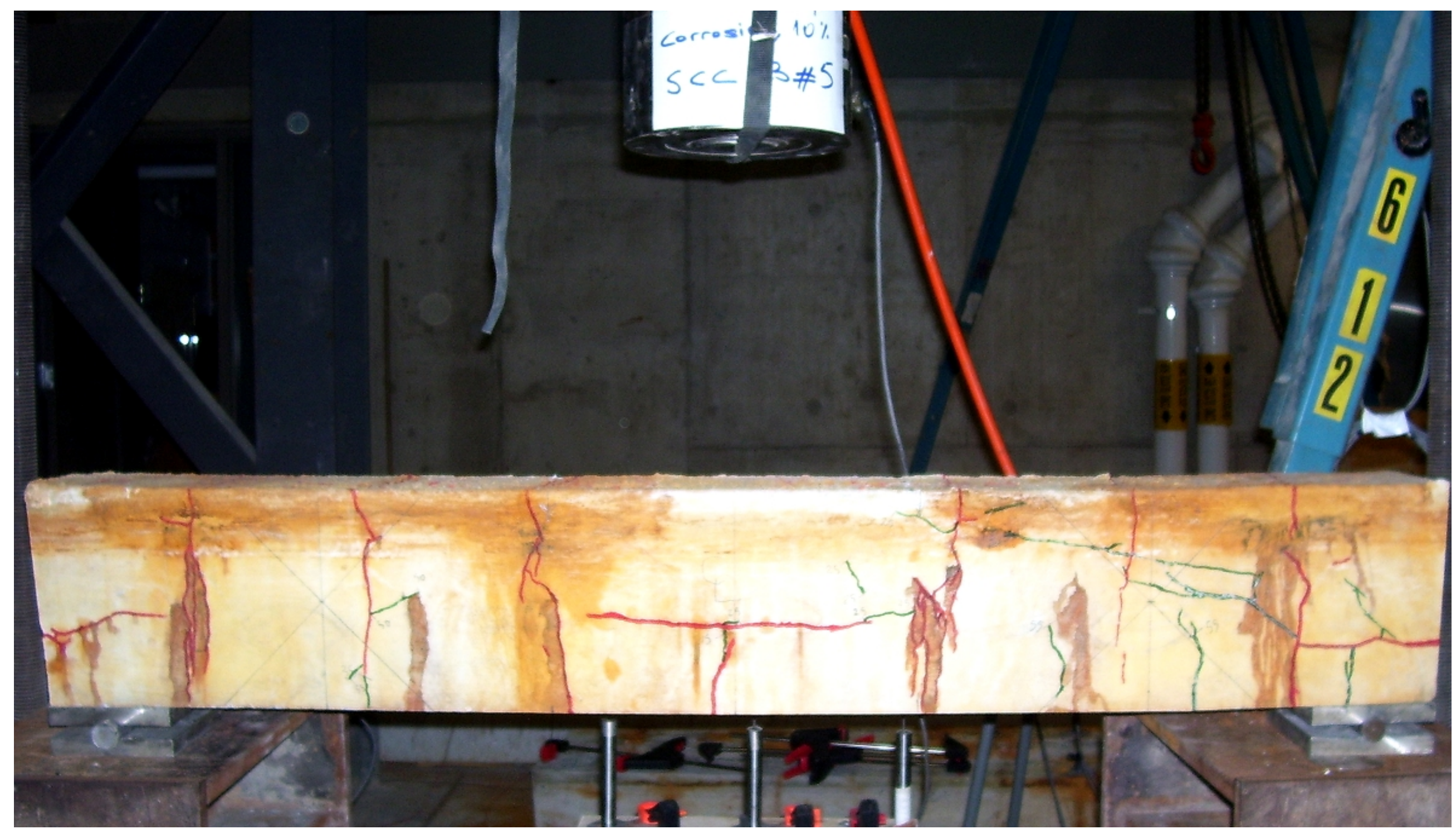

Figure (C-11): Loading cracks pattern for SCC beam no.5 with $10 \%$ mass loss. 


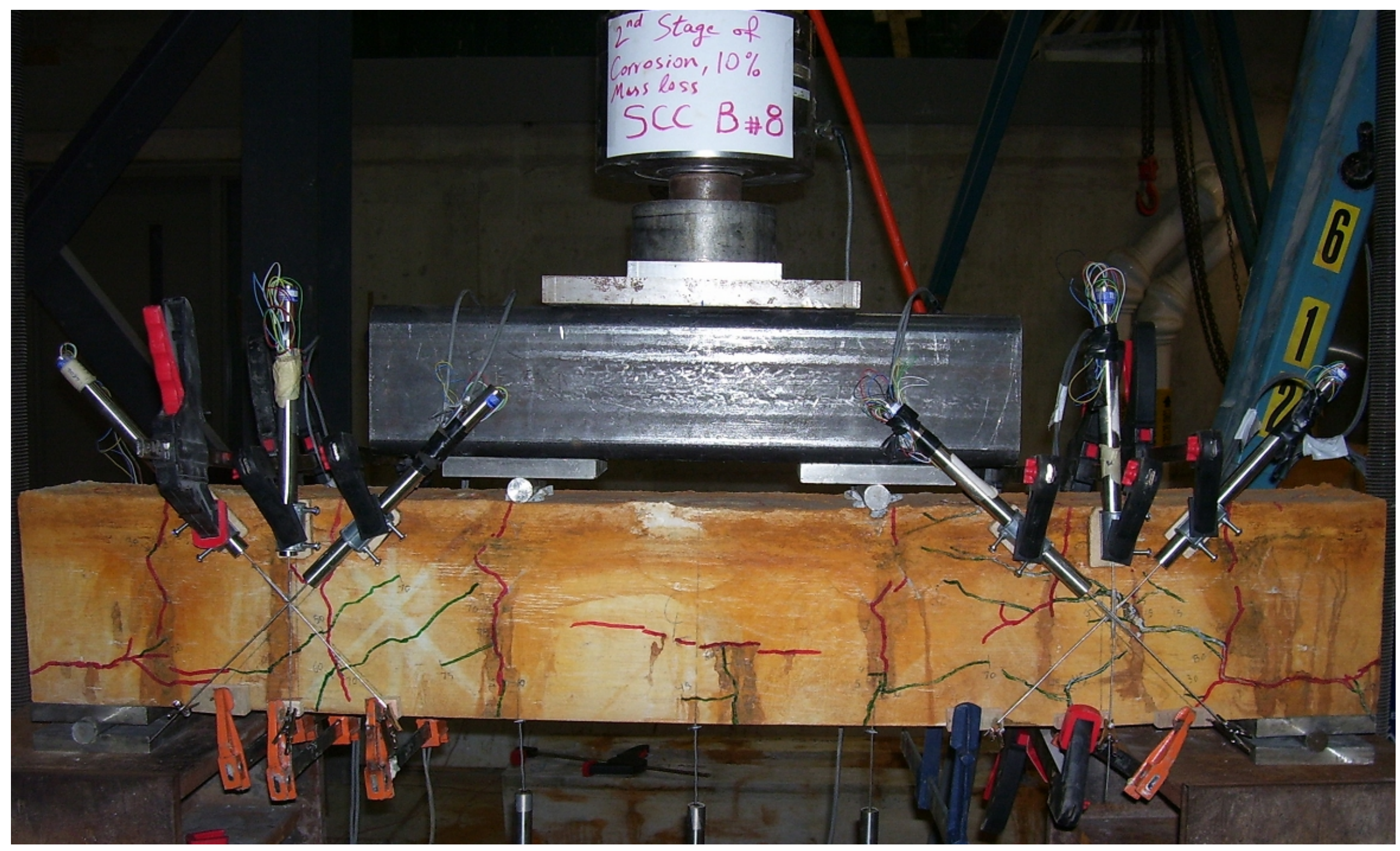

Figure (C-12): Loading cracks pattern for SCC beam no.8 with $10 \%$ mass loss. 


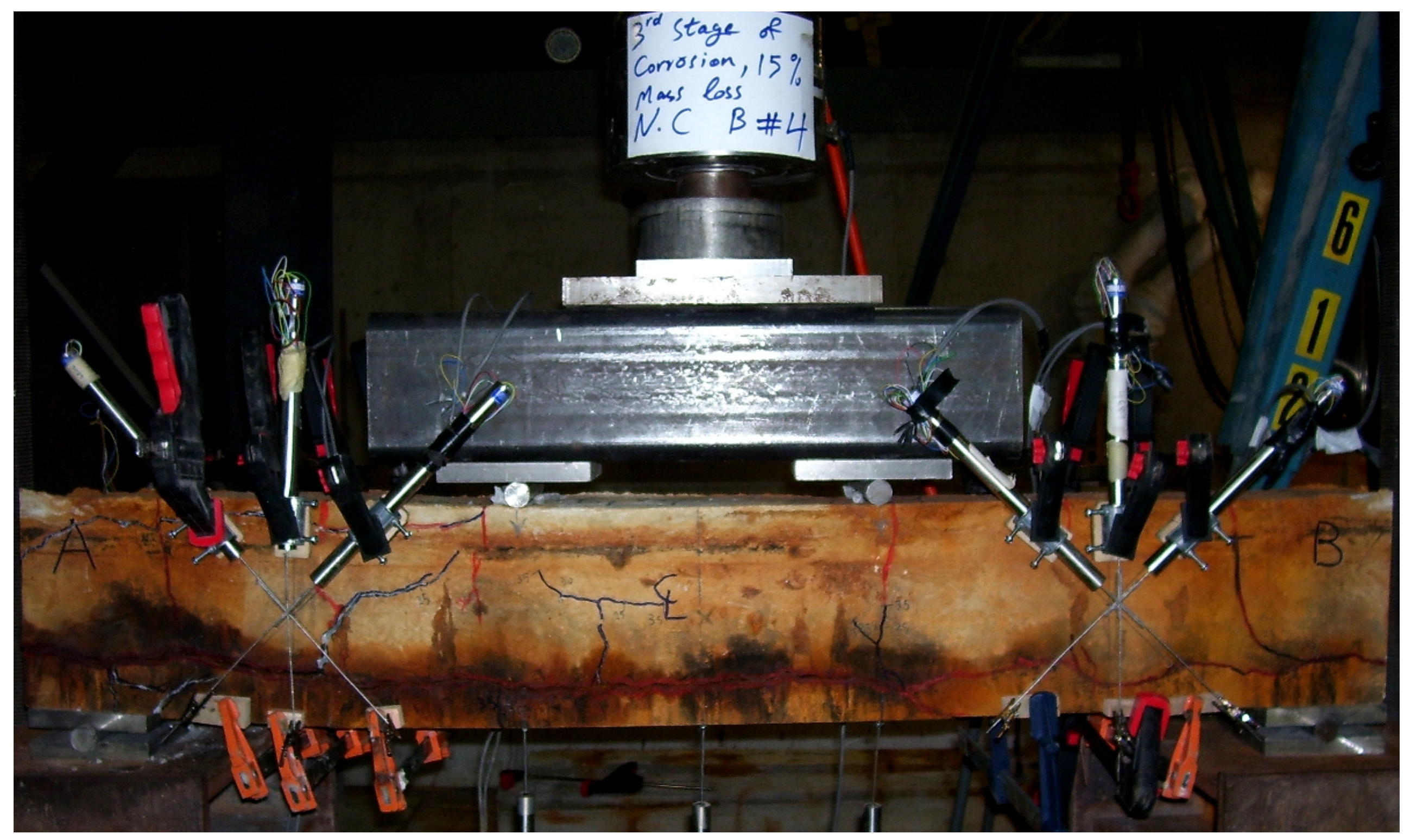

Figure (C-13): Loading cracks pattern for NC beam no.4 with $15 \%$ mass loss. 


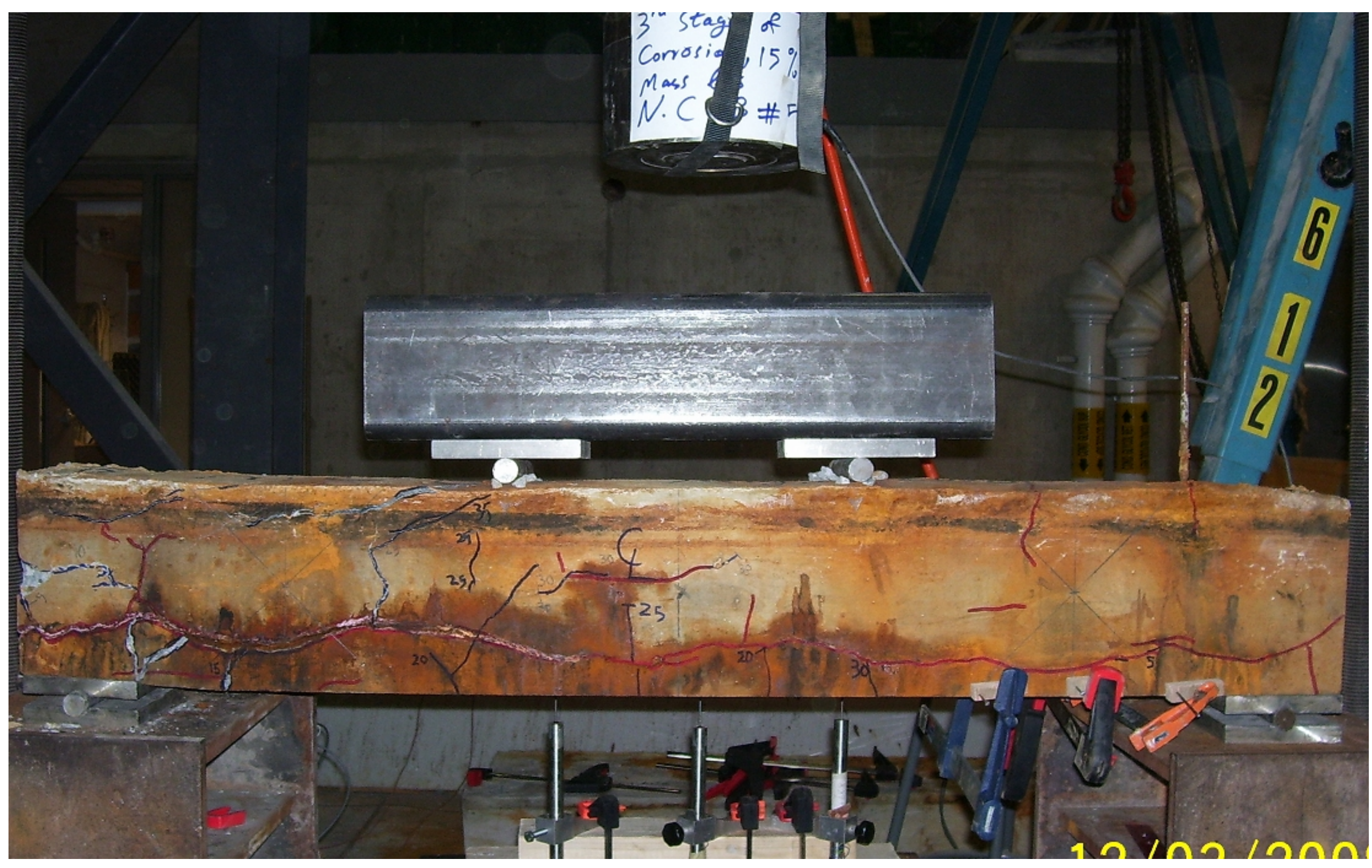

Figure (C-14): Loading cracks pattern for NC beam no.5 with $15 \%$ mass loss. 


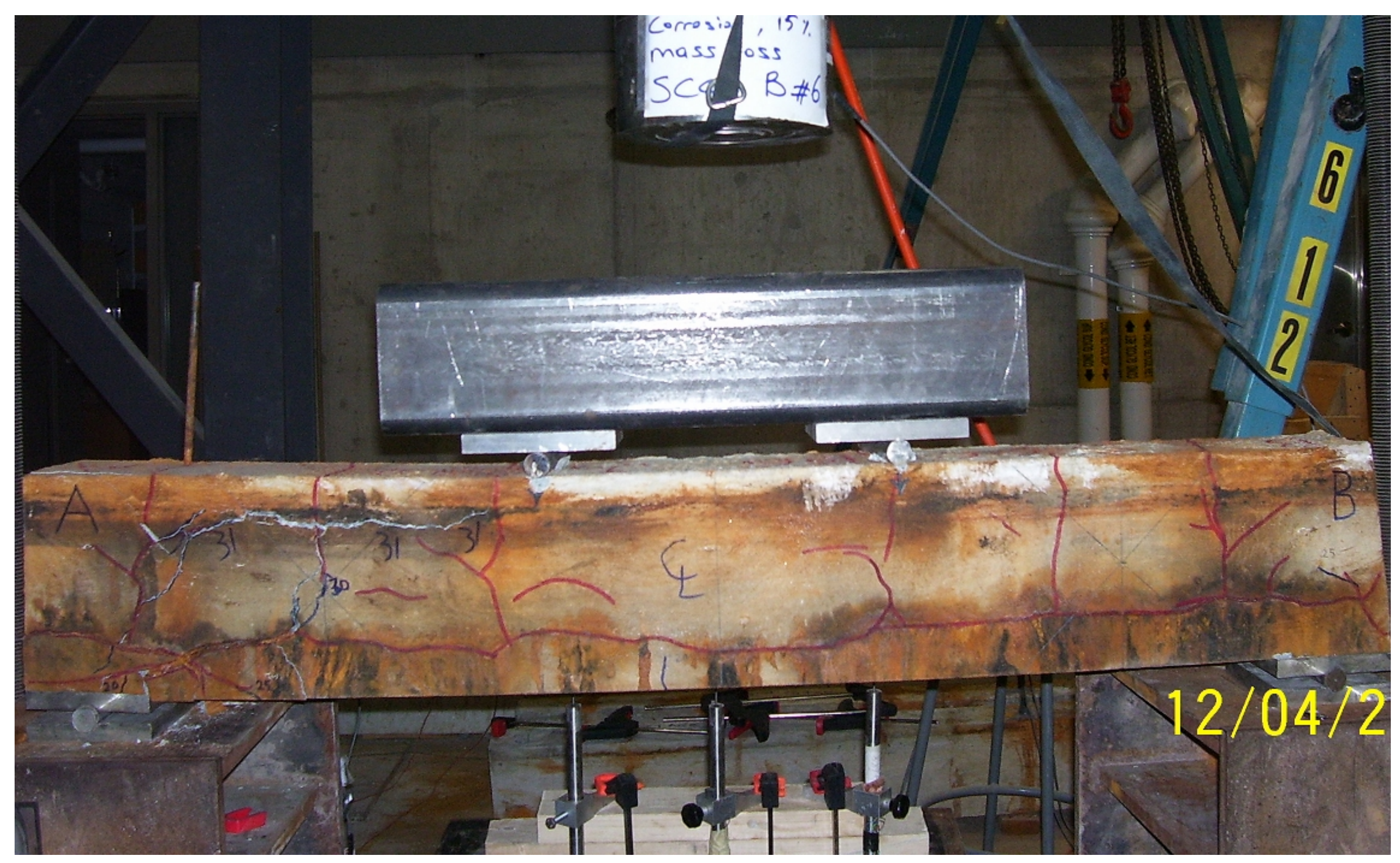

Figure (C-15): Loading cracks pattern for SCC beam no. 6 with $15 \%$ mass loss. 


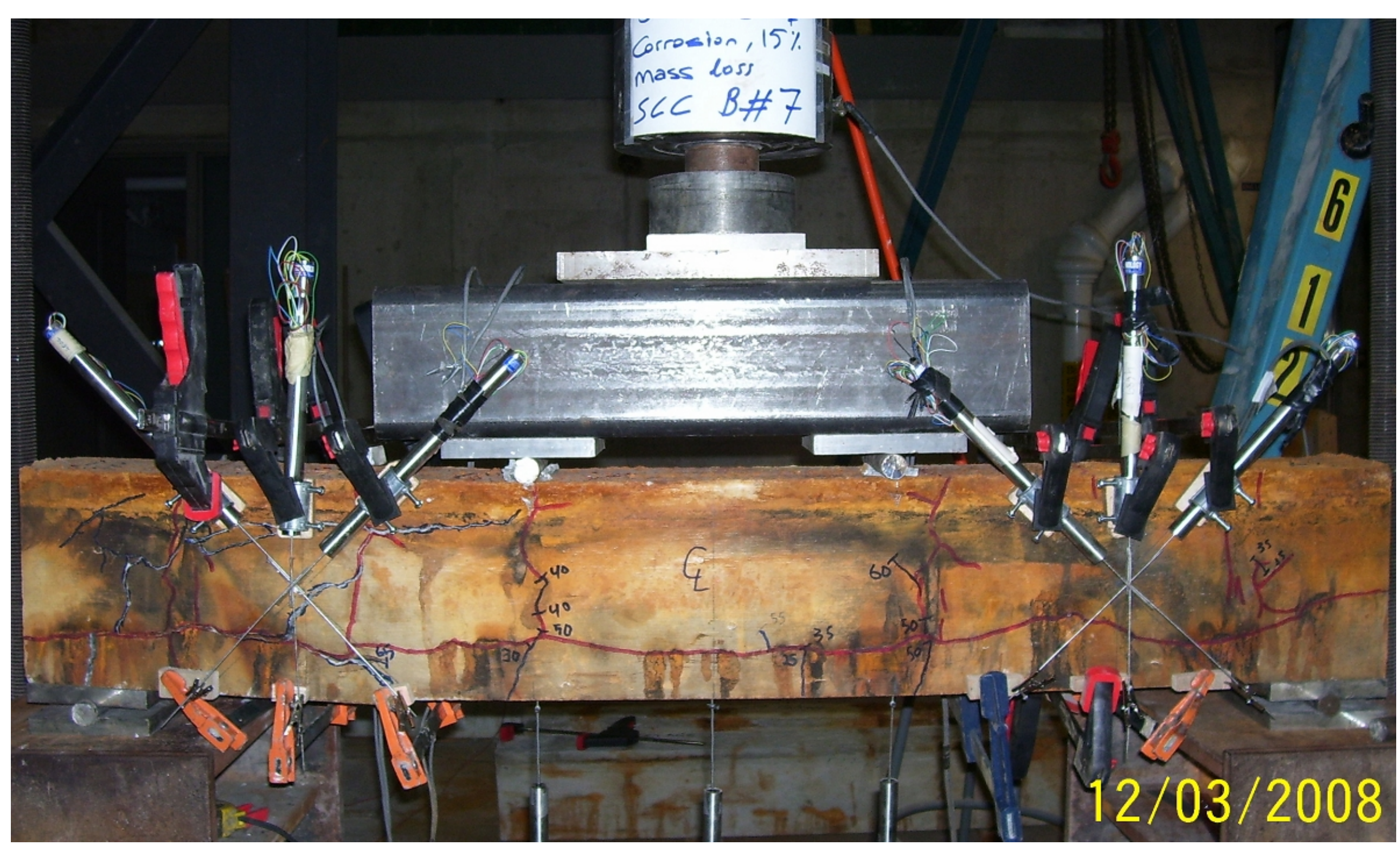

Figure (C-16): Loading cracks pattern for SCC beam no.7 with $15 \%$ mass loss. 


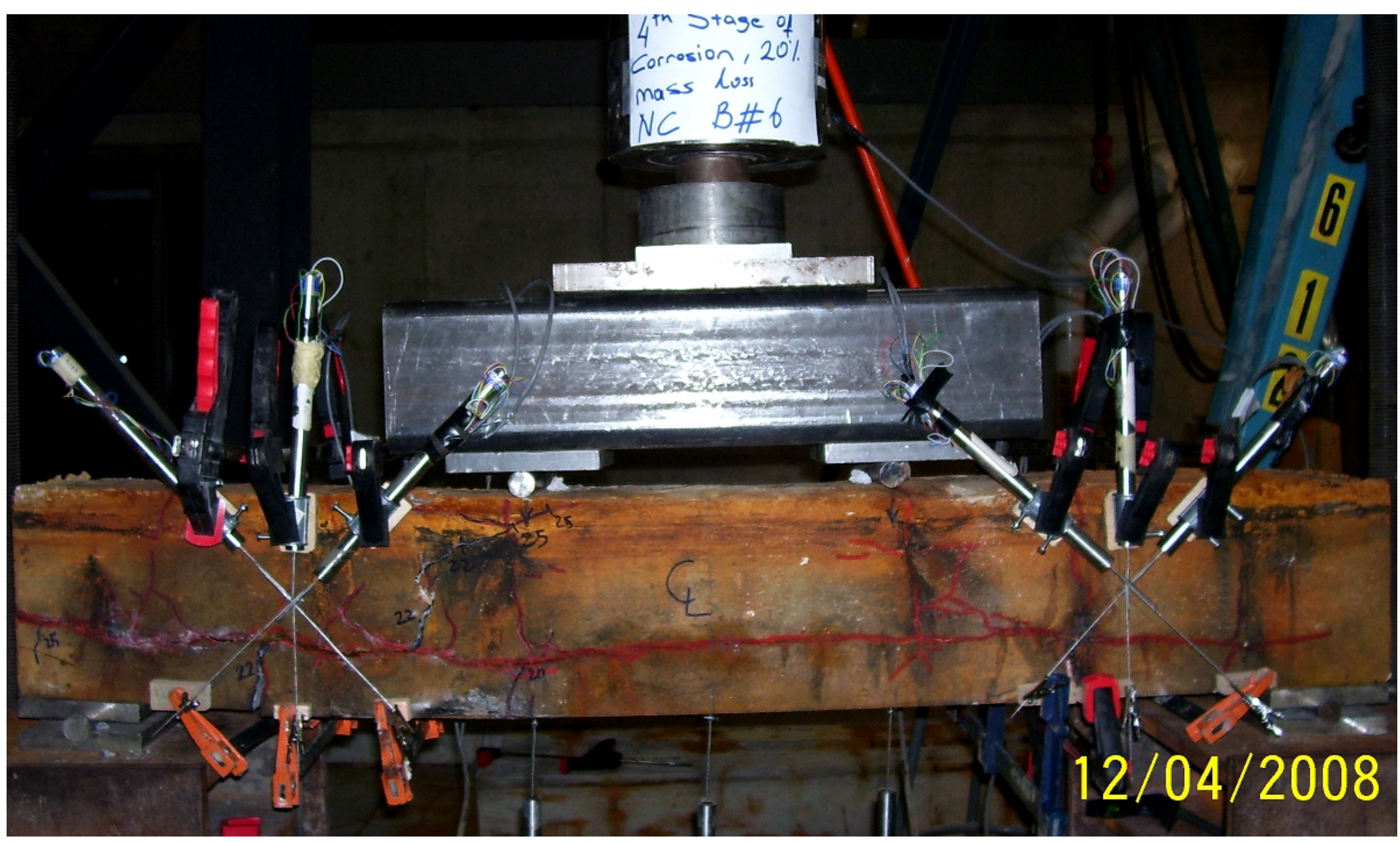

Figure (C-17): Loading cracks pattern for NC beam no. 6 with $20 \%$ mass loss. 


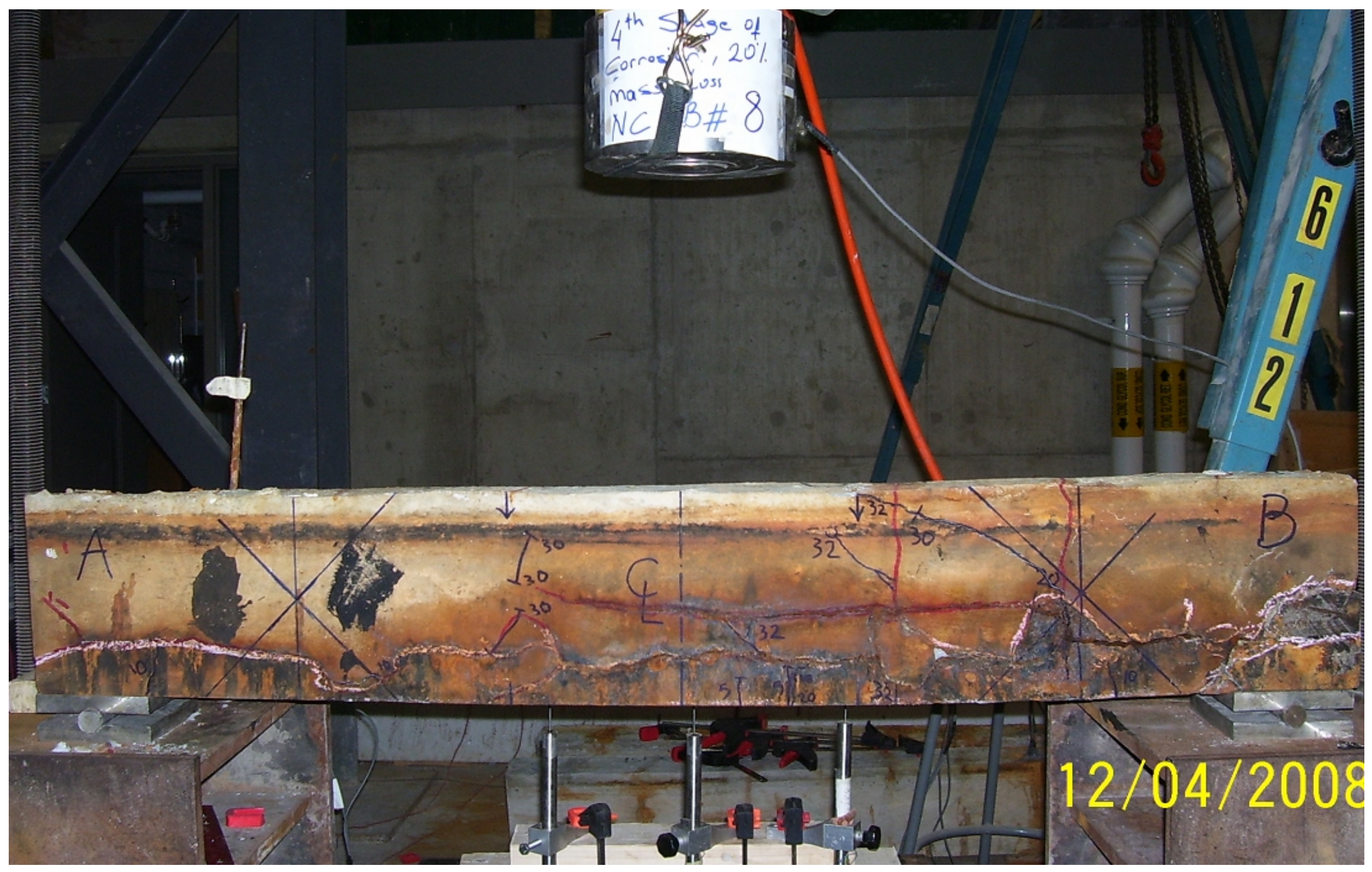

Figure (C-18): Loading cracks pattern for NC beam no.8, with $20 \%$ mass loss. 


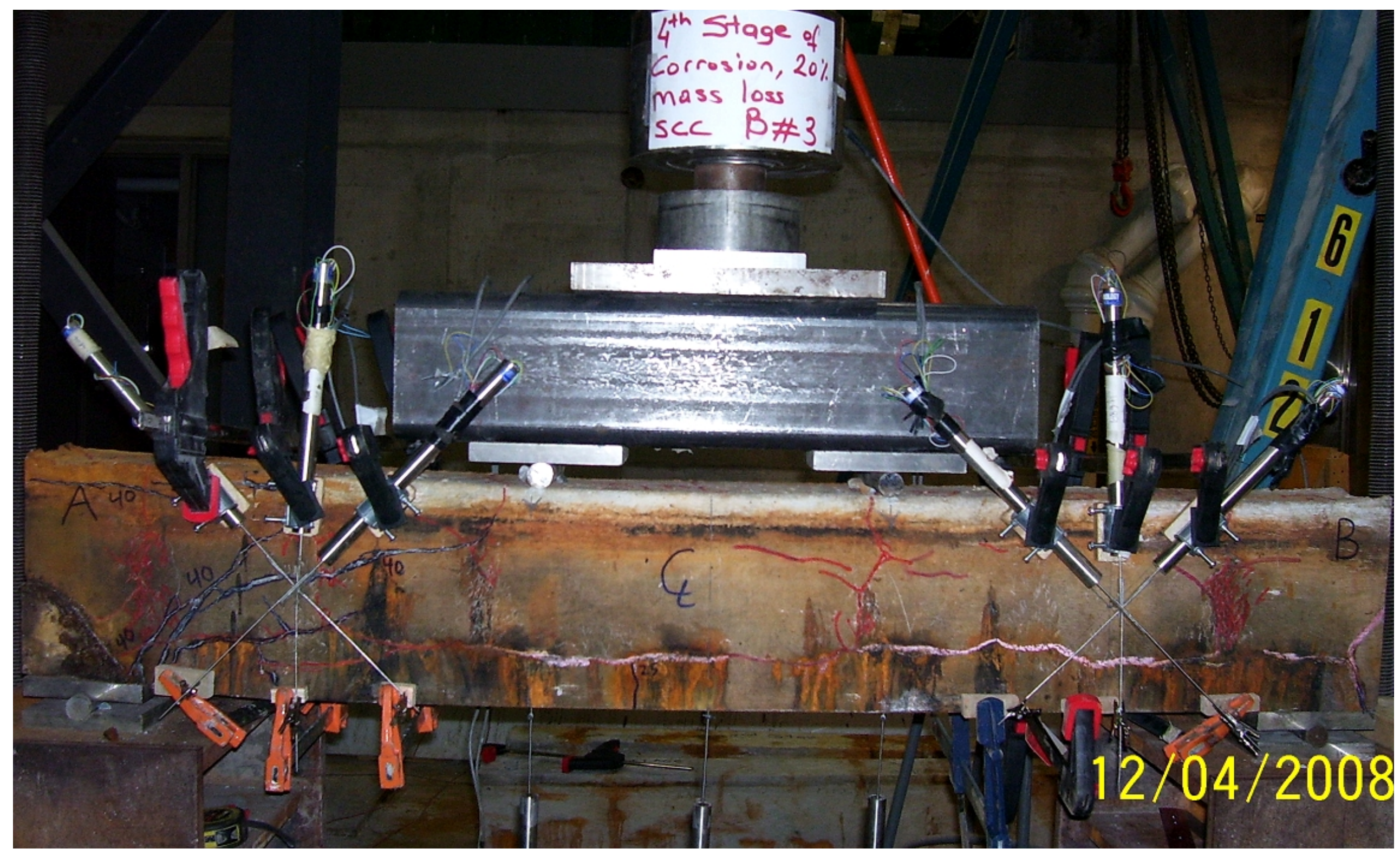

Figure (C-19): Loading cracks pattern for SCC beam no. 3 with $20 \%$ mass loss. 


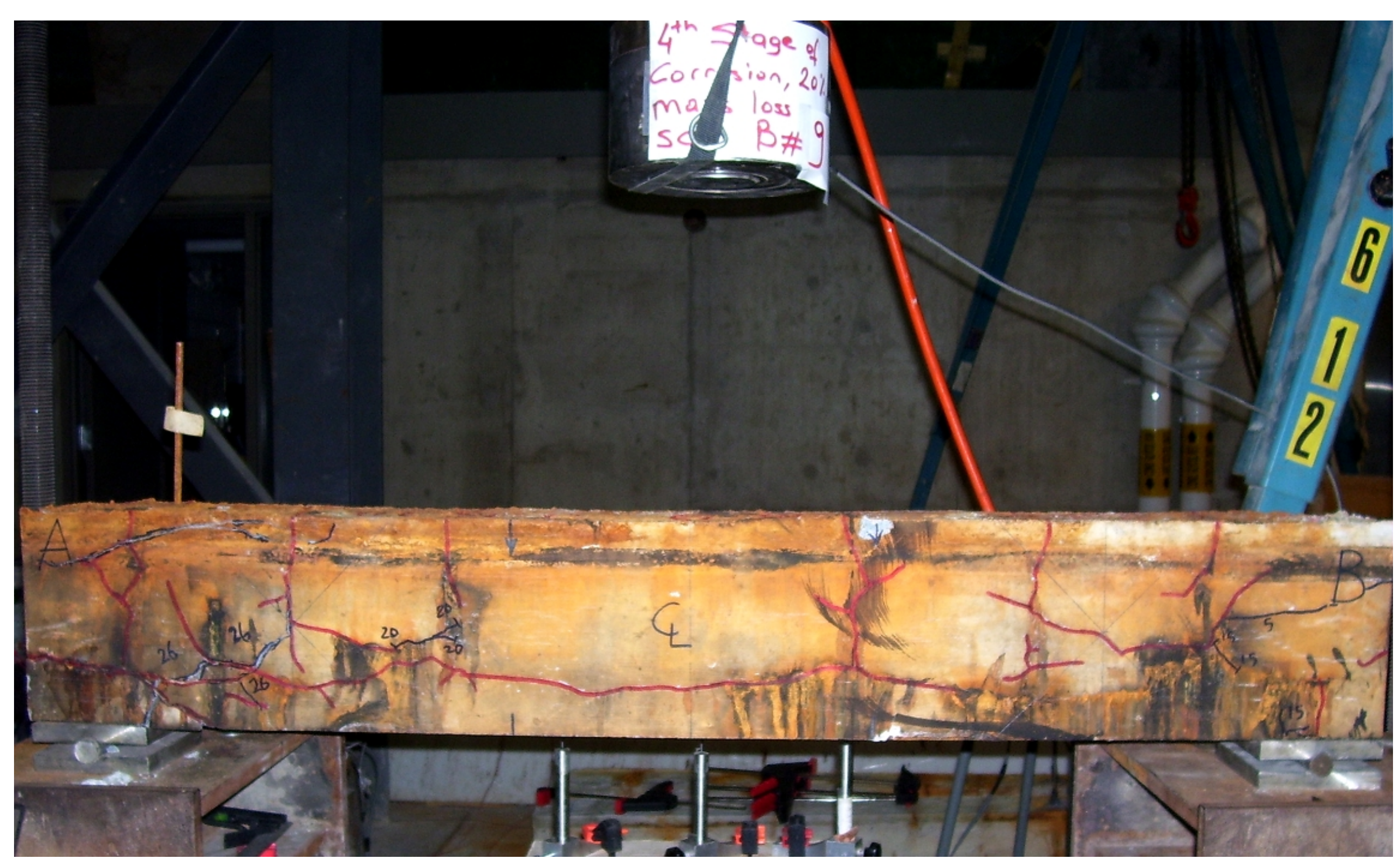

Figure (C-20): Loading cracks pattern for SCC beam no. 7 with $20 \%$ mass loss. 


\section{Appendix D: Voltage vs. constant current}

The following two tables show the daily voltage readings vs. constant current of 1 ampere.

Table (D-1): Voltage readings for the NC beams at different stages of corrosion.

\section{Normal Concrete}

\begin{tabular}{|c|c|c|c|c|c|c|c|c|c|}
\hline \multirow{2}{*}{ Day \# } & \multirow{2}{*}{ Date } & \multirow{2}{*}{\begin{tabular}{|l|} 
Stage-1 \\
B 3 \\
\end{tabular}} & \multirow{2}{*}{$\begin{array}{l}\text { Stage-3 } \\
\text { B } 4\end{array}$} & \multirow{2}{*}{$\begin{array}{l}\text { Stage-3 } \\
\text { B } 5\end{array}$} & \multirow{2}{*}{$\begin{array}{l}\text { Stage-4 } \\
\text { B } 6\end{array}$} & \multirow{2}{*}{$\begin{array}{l}\text { Stage-2 } \\
\text { B } 7\end{array}$} & \multirow{2}{*}{$\begin{array}{l}\text { Stage }-4 \\
\text { B } 8\end{array}$} & \multirow{2}{*}{$\begin{array}{l}\text { Stage-1 } \\
\text { B } 9\end{array}$} & \multirow{2}{*}{$\begin{array}{l}\text { Stage-2 } \\
\text { B } 10\end{array}$} \\
\hline & & & & & & & & & \\
\hline 1 & 22 Sept. 08 & $\begin{array}{l}25.4 \text { Vol. } \\
1 \text { Amp. }\end{array}$ & $\begin{array}{l}\text { 21.7 Vol. } \\
1 \mathrm{Amp} .\end{array}$ & $\begin{array}{l}25.2 \text { Vo } \\
1 \mathrm{Amp} .\end{array}$ & $\begin{array}{l}26.3 \text { Vol. } \\
1 \mathrm{Amp} \text {. }\end{array}$ & $\begin{array}{l}24.6 \mathrm{Vol} \\
1 \mathrm{Amp} .\end{array}$ & $\begin{array}{l}27.1 \text { Vol. } \\
1 \text { Amp. }\end{array}$ & $\begin{array}{l}26.4 \mathrm{Vol} \\
1 \mathrm{Amp} .\end{array}$ & $\begin{array}{l}26.1 \text { Vol. } \\
1 \mathrm{Amp} .\end{array}$ \\
\hline 2 & 23 Sept. 08 & $\begin{array}{l}\text { 29.0 Vol. } \\
1 \mathrm{Amp} .\end{array}$ & $\begin{array}{l}24.1 \mathrm{Vol} \\
1 \mathrm{Amp} .\end{array}$ & $\begin{array}{l}28.6 \mathrm{Vo} \\
1 \mathrm{Amp} .\end{array}$ & $\begin{array}{l}\text { l. } 29.9 \mathrm{Vol} . \\
1 \mathrm{Amp} .\end{array}$ & $\begin{array}{l}25.3 \text { Vol. } \\
1 \mathrm{Amp} .\end{array}$ & $\begin{array}{l}30.2 \mathrm{Vol} . \\
1 \mathrm{Amp} .\end{array}$ & $\begin{array}{l}29.8 \mathrm{Vol} \\
1 \mathrm{Amp} .\end{array}$ & $\begin{array}{l}29.1 \mathrm{Vol} \text {. } \\
1 \mathrm{Amp} .\end{array}$ \\
\hline 3 & 24 Sept. 08 & $\begin{array}{l}30.6 \text { Vol. } \\
1 \mathrm{Amp} .\end{array}$ & $\begin{array}{l}25.1 \mathrm{Vol} \\
1 \mathrm{Amp} .\end{array}$ & $\begin{array}{l}30.5 \mathrm{Vo} \\
1 \mathrm{Amp} .\end{array}$ & $\begin{array}{l}30.5 \text { Vol. } \\
0.95 \\
\text { Amp. }\end{array}$ & $\begin{array}{l}22.3 \text { Vol. } \\
1 \mathrm{Amp} .\end{array}$ & $\begin{array}{l}30.6 \text { Vol. } \\
0.94 \\
\text { Amp. }\end{array}$ & $\begin{array}{l}30.8 \mathrm{Vol} \\
0.98 \\
\text { Amp. }\end{array}$ & $\begin{array}{ll}30.6 & \text { Vol. } \\
0.99 & \\
\text { Amp. } & \end{array}$ \\
\hline 4 & 25 Sept. 08 & $\begin{array}{l}30.9 \text { Vol. } \\
0.96 \\
\text { Amp. } \\
30.9 \text { Vol }\end{array}$ & $\begin{array}{l}25.4 \text { Vol. } \\
1 \text { Amp. }\end{array}$ & $\begin{array}{l}30.2 \text { Vo } \\
0.96 \\
\text { Amp. } \\
30.2 \quad \text { Vo }\end{array}$ & $\begin{array}{l}30.5 \text { Vol. } \\
0.93 \\
\text { Amp. } \\
30.5 \text { Vol }\end{array}$ & $\begin{array}{l}19.2 \text { Vol. } \\
1 \text { Amp. }\end{array}$ & $\begin{array}{l}30.6 \mathrm{Vol} . \\
0.92 \\
\text { Amp. } \\
30.6 \mathrm{Vol}\end{array}$ & \begin{tabular}{|l}
$30.8 \mathrm{Vol}$ \\
0.96 \\
Amp. \\
$30.8 \mathrm{Vol}$
\end{tabular} & $\begin{array}{l}30.5 \mathrm{Vol} . \\
0.95 \\
\text { Amp. } \\
30.5 \mathrm{Vol}\end{array}$ \\
\hline 5 & 26 Sept. 08 & $\begin{array}{l}30.9 \text { Vol. } \\
0.92 \\
\text { Amp. }\end{array}$ & $\begin{array}{l}26.0 \text { Vol. } \\
1 \text { Amp. }\end{array}$ & $\begin{array}{l}30.2 \text { Vo } \\
0.96 \\
\text { Amp. }\end{array}$ & $\begin{array}{l}30.5 \text { Vol. } \\
0.88 \\
\text { Amp. }\end{array}$ & $\begin{array}{l}15.9 \text { Vol. } \\
1 \mathrm{Amp}\end{array}$ & $\begin{array}{l}30.6 \text { Vol. } \\
0.88 \\
\text { Amp. }\end{array}$ & $\begin{array}{l}30.8 \text { Vol } \\
0.93 \\
\text { Amp. }\end{array}$ & $\begin{array}{ll}\text { I. } & 30.5 \\
0.91 & \text { Vol. } \\
\text { Amp. } & \\
\text { Amp. }\end{array}$ \\
\hline 6 & 27 Sept. 08 & $\begin{array}{l}30.9 \text { Vol. } \\
0.94 \\
\text { Amp. }\end{array}$ & $\begin{array}{l}24.8 \text { Vol. } \\
1 \text { Amp. }\end{array}$ & $\begin{array}{l}28.8 \mathrm{Vo} \\
1 \mathrm{Amp} .\end{array}$ & $\begin{array}{l}30.4 \text { Vol. } \\
0.88 \\
\text { Amp. }\end{array}$ & $\begin{array}{l}13.3 \text { Vol. } \\
1 \text { Amp. }\end{array}$ & $\begin{array}{l}30.5 \text { Vol. } \\
0.87 \\
\text { Amp. }\end{array}$ & $\begin{array}{l}30.7 \text { Vol } \\
0.95 \\
\text { Amp. }\end{array}$ & $\begin{array}{l}30.5 \text { Vol. } \\
0.89 \\
\text { Amp. }\end{array}$ \\
\hline 7 & 28 Sept. 08 & $\begin{array}{l}30.6 \text { Vol. } \\
1 \mathrm{Amp} .\end{array}$ & $\begin{array}{l}22.5 \text { Vol } \\
1 \mathrm{Amp} .\end{array}$ & $\begin{array}{l}24.6 \mathrm{Vo} \\
1 \mathrm{Amp} .\end{array}$ & $\begin{array}{l}30.4 \text { Vol. } \\
0.91 \\
\text { Amp. }\end{array}$ & $\begin{array}{l}10.1 \text { Vol. } \\
1 \text { Amp. }\end{array}$ & $\begin{array}{l}30.5 \text { Vol. } \\
0.91 \\
\text { Amp. }\end{array}$ & $\begin{array}{l}30.7 \mathrm{Vol} \\
0.97 \\
\text { Amp. }\end{array}$ & $\begin{array}{ll}30.5 & \text { Vol. } \\
0.89 & \\
\text { Amp. } & \end{array}$ \\
\hline 8 & 29 Sept. 08 & $\begin{array}{l}28.9 \text { Vol. } \\
1 \mathrm{Amp} .\end{array}$ & $\begin{array}{l}21.1 \mathrm{Vol} \\
1 \mathrm{Amp} .\end{array}$ & $\begin{array}{l}21.5 \text { Vo } \\
1 \mathrm{Amp} .\end{array}$ & $\begin{array}{l}30.4 \text { Vol. } \\
0.95 \\
\text { Amp. }\end{array}$ & $\begin{array}{l}9.0 \mathrm{Vol} . \\
1 \mathrm{Amp} .\end{array}$ & $\begin{array}{l}30.0 \mathrm{Vol} \text {. } \\
1 \mathrm{Amp} \text {. }\end{array}$ & $\begin{array}{l}30.2 \mathrm{Vol} \\
1 \mathrm{Amp} .\end{array}$ & $\begin{array}{ll}30.4 & \text { Vol. } \\
0.92 & \\
\text { Amp. } & \end{array}$ \\
\hline 9 & 30 Sept. 08 & $\begin{array}{l}25.6 \text { Vol. } \\
1 \text { Amp. }\end{array}$ & $\begin{array}{l}18.5 \mathrm{Vol} \\
1 \mathrm{Amp} .\end{array}$ & $\begin{array}{l}19.6 \mathrm{Vo} \\
1 \mathrm{Amp} .\end{array}$ & $\begin{array}{l}28.8 \mathrm{Vol} . \\
1.01 \\
\text { Amp. }\end{array}$ & $\begin{array}{l}7.5 \text { Vol. } \\
1 \mathrm{Amp} .\end{array}$ & $\begin{array}{l}\text { I.26.8 Vol. } \\
1 \mathrm{Amp} \text {. }\end{array}$ & $\begin{array}{l}27.5 \mathrm{Vol} \\
1 \mathrm{Amp}\end{array}$ & $\begin{array}{l}30.4 \mathrm{Vol} . \\
1 \mathrm{Amp} .\end{array}$ \\
\hline 10 & 1 Oct. 08 & $\begin{array}{l}23.1 \text { Vol. } \\
0.99 \\
\text { Amp. }\end{array}$ & $\begin{array}{l}15.9 \mathrm{Vol} \\
1 \mathrm{Amp}\end{array}$ & $\begin{array}{l}17.1 \text { Vo } \\
1 \mathrm{Amp} .\end{array}$ & $\begin{array}{l}1.23 .9 \mathrm{Vol} \text {. } \\
1 \mathrm{Amp} \text {. }\end{array}$ & $\begin{array}{l}7.8 \text { Vol. } \\
1.01 \\
\text { Amp. }\end{array}$ & $\begin{array}{l}24.9 \mathrm{Vol} \text {. } \\
1 \mathrm{Amp} \text {. }\end{array}$ & $\begin{array}{l}25.3 \mathrm{Vol} \\
1 \mathrm{Amp} .\end{array}$ & $\begin{array}{ll}30.3 & \text { Vol. } \\
1.13 & \\
\text { Amp. } & \end{array}$ \\
\hline 11 & 2 Oct. 08 & $\begin{array}{l}22.5 \text { Vol. } \\
1 \mathrm{Amp} .\end{array}$ & $\begin{array}{l}15.0 \text { Vol. } \\
1 \mathrm{Amp} .\end{array}$ & $\begin{array}{l}16.8 \text { Vo } \\
1 \mathrm{Amp} .\end{array}$ & $\begin{array}{l}1.22 .4 \text { Vol. } \\
1 \mathrm{Amp} \text {. }\end{array}$ & $\begin{array}{l}7.7 \text { Vol. } \\
1 \mathrm{Amp} . \\
\end{array}$ & $\begin{array}{l}23.4 \text { Vol. } \\
1 \mathrm{Amp} .\end{array}$ & $\begin{array}{l}23.4 \mathrm{Vol} \\
1 \mathrm{Amp} .\end{array}$ & $\begin{array}{l}23.2 \mathrm{Vol} \text {. } \\
1 \mathrm{Amp} .\end{array}$ \\
\hline 12 & 3 Oct. 08 & $\begin{array}{l}21.7 \text { Vol. } \\
1 \mathrm{Amp} .\end{array}$ & $\begin{array}{l}13.7 \text { Vol. } \\
1 \mathrm{Amp} .\end{array}$ & $\begin{array}{l}15.8 \text { Vo } \\
1 \mathrm{Amp} .\end{array}$ & $\begin{array}{l}19.8 \mathrm{Vol} . \\
1 \mathrm{Amp} .\end{array}$ & $\begin{array}{l}8.3 \text { Vol. } \\
1 \mathrm{Amp} .\end{array}$ & $\begin{array}{l}\text { I.21.6 Vol. } \\
1 \mathrm{Amp} \text {. }\end{array}$ & $\begin{array}{l}22.6 \mathrm{Vol} \\
1 \mathrm{Amp} .\end{array}$ & $\begin{array}{l}21.2 \mathrm{Vol} . \\
1 \mathrm{Amp} .\end{array}$ \\
\hline 13 & 4 Oct. 08 & $\begin{array}{l}19.1 \text { Vol. } \\
1 \text { Amp. }\end{array}$ & $\begin{array}{l}12.4 \mathrm{Vol} \\
1 \mathrm{Amp} .\end{array}$ & $\begin{array}{l}13.5 \mathrm{Vo} \\
1 \mathrm{Amp} .\end{array}$ & $\begin{array}{l}16.5 \mathrm{Vol} . \\
1 \mathrm{Amp} .\end{array}$ & $\begin{array}{l}8.1 \text { Vol. } \\
1 \text { Amp. }\end{array}$ & $\begin{array}{l}18.7 \text { Vol. } \\
1 \mathrm{Amp} .\end{array}$ & $\begin{array}{l}19.6 \mathrm{Vol} \\
1 \mathrm{Amp} . \\
\end{array}$ & $\begin{array}{l}17.3 \mathrm{Vol} . \\
1 \mathrm{Amp} .\end{array}$ \\
\hline 14 & 5 Oct. 08 & $\begin{array}{l}18.6 \text { Vol. } \\
1 \mathrm{Amp} .\end{array}$ & $\begin{array}{l}12.1 \mathrm{Vol} . \\
1 \mathrm{Amp} .\end{array}$ & $\begin{array}{l}12.6 \mathrm{Vo} \\
1 \mathrm{Amp} .\end{array}$ & $\begin{array}{l}15.4 \mathrm{Vol} \text {. } \\
1 \mathrm{Amp} \text {. }\end{array}$ & $\begin{array}{l}8.4 \text { Vol. } \\
1 \text { Amp. }\end{array}$ & $\begin{array}{l}17.7 \mathrm{Vol} \text {. } \\
1 \mathrm{Amp} .\end{array}$ & $\begin{array}{l}19.1 \mathrm{Vol} \\
1 \mathrm{Amp} .\end{array}$ & $\begin{array}{l}16.4 \mathrm{Vol} . \\
1 \mathrm{Amp} .\end{array}$ \\
\hline 15 & 6 Oct. 08 & $\begin{array}{l}18.4 \text { Vol. } \\
1 \mathrm{Amp} .\end{array}$ & $\begin{array}{l}11.6 \text { Vol } \\
1 \mathrm{Amp} .\end{array}$ & $\begin{array}{l}11.8 \mathrm{Vo} \\
1 \mathrm{Amp} .\end{array}$ & $\begin{array}{l}\text { l. } 14.8 \mathrm{Vol} . \\
1 \mathrm{Amp} .\end{array}$ & $\begin{array}{l}6.1 \text { Vol. } \\
1 \mathrm{Amp} .\end{array}$ & $\begin{array}{l}17.0 \mathrm{Vol} . \\
1 \mathrm{Amp} .\end{array}$ & $\begin{array}{l}18.4 \mathrm{Vol} \\
1 \mathrm{Amp} .\end{array}$ & $\begin{array}{l}\text { I. } 15.6 \mathrm{Vol} . \\
1 \mathrm{Amp} .\end{array}$ \\
\hline
\end{tabular}




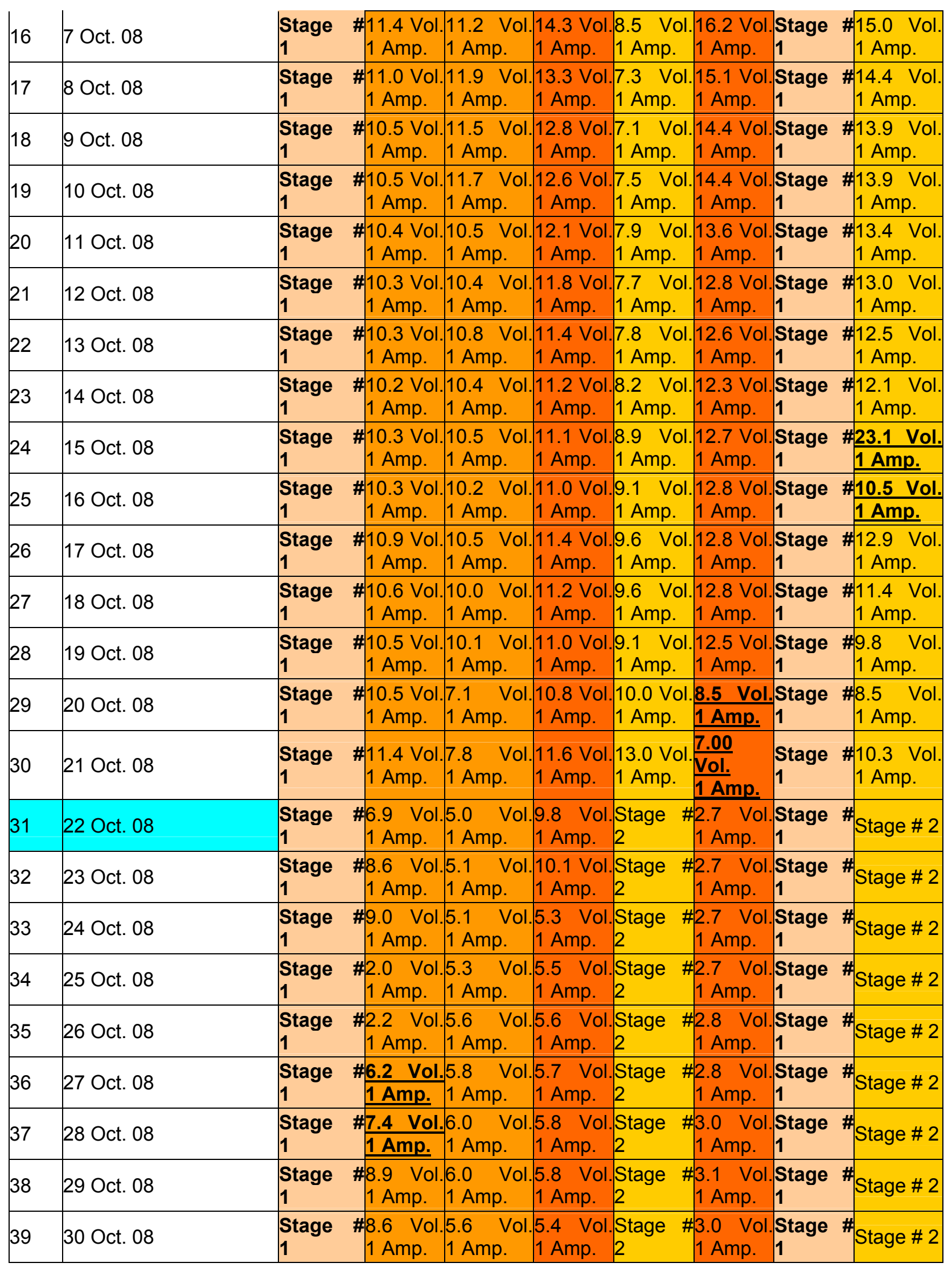




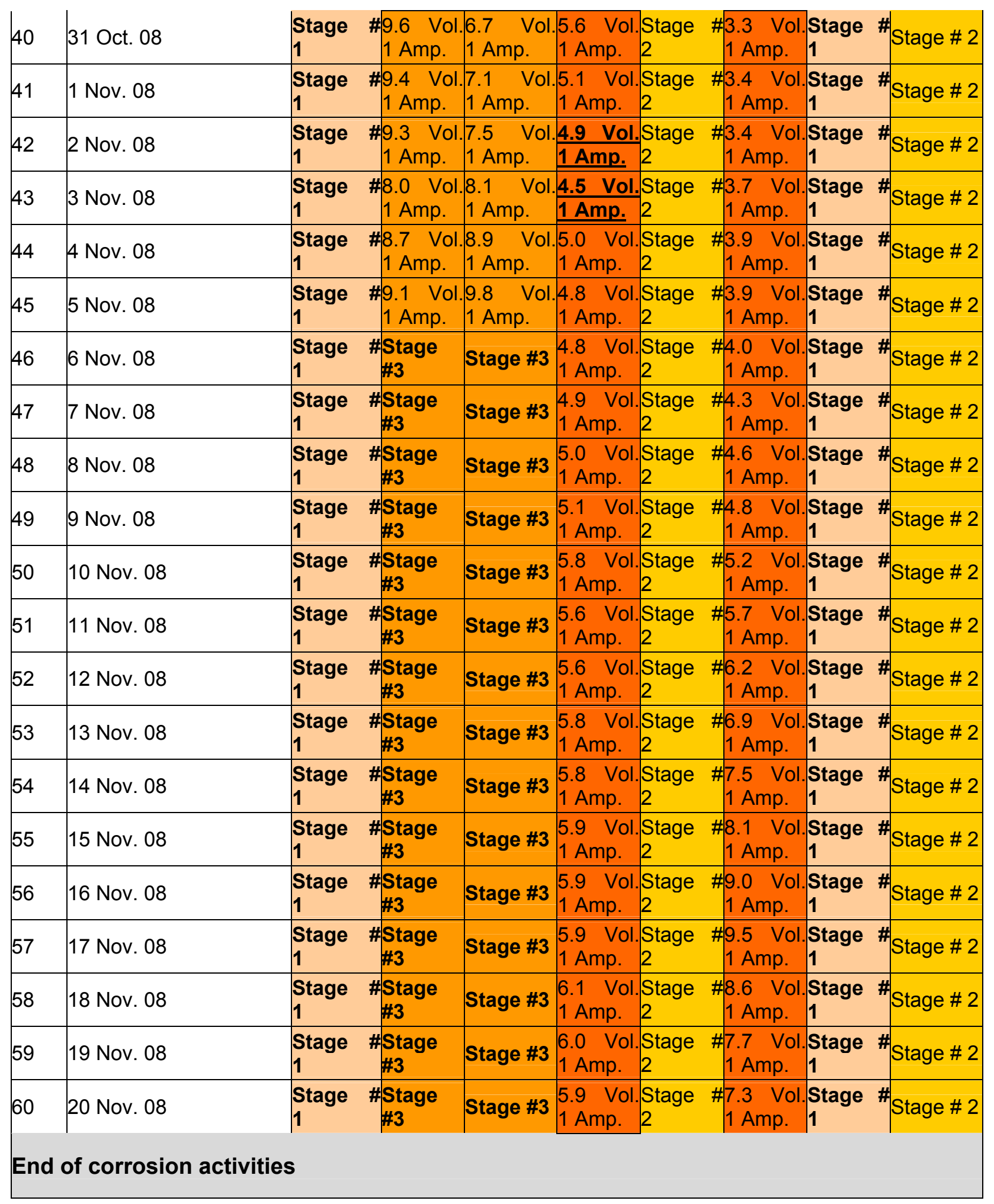


Table (D-2): Voltage readings for the SCC beams at different stages of corrosion.

\begin{tabular}{|c|c|c|c|c|c|c|c|c|c|c|}
\hline \multicolumn{11}{|c|}{ Self-Consolidating Concrete } \\
\hline \multirow{2}{*}{ Day \# } & \multirow{2}{*}{ Date } & \multirow{2}{*}{$\begin{array}{l}\text { Stage-4 } \\
\text { B } 3 \\
\end{array}$} & \multirow{2}{*}{$\begin{array}{l}\text { Stage-1 } \\
\text { B } 4\end{array}$} & \multirow{2}{*}{$\begin{array}{l}\text { Stage-2 } \\
\text { B } 5 \\
\end{array}$} & \multirow{2}{*}{$\begin{array}{l}\text { Stage-3 } \\
\text { B } 6 \\
\end{array}$} & \multirow{2}{*}{$\begin{array}{l}\text { Stage-3 } \\
\text { B } 7 \\
\end{array}$} & \multirow{2}{*}{$\begin{array}{l}\text { Stage-2 } \\
\text { B } 8\end{array}$} & \multirow{2}{*}{$\begin{array}{l}\text { Stage }-4 \\
\text { B } 9\end{array}$} & \multirow{2}{*}{\multicolumn{2}{|c|}{\begin{tabular}{|l|} 
Stage-1 \\
B 10 \\
\end{tabular}}} \\
\hline & & & & & & & & & & \\
\hline 1 & 22 Sept. 08 & $\begin{array}{l}19.3 \text { Vol. } \\
1 \text { Amp. }\end{array}$ & $\begin{array}{ll}19.4 \quad \text { Vo } \\
1 \mathrm{Amp} .\end{array}$ & $\begin{array}{l}19,1 \\
1 \text { Amp. }\end{array}$ & 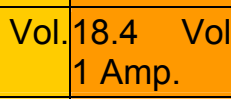 & $\begin{array}{l}18.6 \\
1 \mathrm{Amp}\end{array}$ & $\begin{array}{c}\text { Voll18.2 Vc } \\
1 \text { Amp. }\end{array}$ & ol.18.2 & Vol. 1 & $\begin{array}{l}15.3 \text { Vol. } \\
1 \text { Amp. }\end{array}$ \\
\hline 2 & 23 Sept. 08 & $\begin{array}{l}20.3 \text { Vol. } \\
1 \mathrm{Amp} .\end{array}$ & $\begin{array}{ll}20.8 & \text { Vo } \\
1 \mathrm{Amp} . & \\
\end{array}$ & $\begin{array}{l}20.7 \\
1 \text { Amp. }\end{array}$ & Vol. $\begin{array}{l}14.2 \text { Vol } \\
1 \mathrm{Amp} .\end{array}$ & $\begin{array}{l}20.4 \mathrm{Vol} \\
1 \mathrm{Amp} .\end{array}$ & \begin{tabular}{l|l}
19.8 \\
$1 \mathrm{Amp}$
\end{tabular} & $\begin{array}{l}9.3 \\
1 \text { Amp. }\end{array}$ & Vol. 1 & $\begin{array}{ll}10.5 \\
1 \mathrm{Amp} .\end{array}$ \\
\hline 3 & 24 Sept. 08 & $\begin{array}{l}17.1 \text { Vol. } \\
1 \mathrm{Amp} .\end{array}$ & $\begin{array}{l}21.9 \quad \text { Vo } \\
1 \mathrm{Amp} .\end{array}$ & $\begin{array}{l}21.8 \\
1 \mathrm{Amp}\end{array}$ & $\begin{array}{ll}\text { Vol. } 8.4 \quad \text { Vol } \\
1 \mathrm{Amp} .\end{array}$ & $\begin{array}{l}21.4 \mathrm{Vol} \\
1 \mathrm{Amp} .\end{array}$ & $\begin{array}{l}20.8 \quad \mathrm{Vc} \\
1 \mathrm{Amp} . \\
\end{array}$ & $\begin{array}{l}\text { ol. } 8.1 \\
1 \mathrm{Amp} .\end{array}$ & Vol.1 & $\begin{array}{l}11.3 \text { Vol. } \\
1 \mathrm{Amp} .\end{array}$ \\
\hline 4 & 25 Sept. 08 & $\begin{array}{l}18.1 \text { Vol. } \\
1 \mathrm{Amp} .\end{array}$ & $\begin{array}{l}22.6 \quad \text { Vo } \\
1 \mathrm{Amp} .\end{array}$ & $\begin{array}{l}22.6 \\
1 \mathrm{Amp} \\
\end{array}$ & \begin{tabular}{l|l} 
Vol. & $9.4 \quad$ Vol \\
$1 \mathrm{Amp}$.
\end{tabular} & $\begin{array}{l}21.8 \mathrm{Vol} \\
1 \mathrm{Amp} .\end{array}$ & $\begin{array}{l}21.2 \quad \mathrm{Vc} \\
1 \mathrm{Amp} .\end{array}$ & $\begin{array}{l}\text { ol. } 9.0 \\
1 \mathrm{Amp} .\end{array}$ & Vol. 1 & $\begin{array}{l}12.5 \text { Vol. } \\
1 \mathrm{Amp} .\end{array}$ \\
\hline 5 & 26 Sept. 08 & $\begin{array}{l}19.6 \text { Vol. } \\
1 \mathrm{Amp} .\end{array}$ & $\begin{array}{l}23.8 \quad \text { Vo } \\
1 \mathrm{Amp} .\end{array}$ & $\begin{array}{l}23.7 \\
1 \mathrm{Amp}\end{array}$ & $\begin{array}{l}\text { Vol. } \mid \begin{array}{ll}11.4 \quad \text { Vol } \\
1 \mathrm{Amp} .\end{array}\end{array}$ & $\begin{array}{l}22.8 \mathrm{Vol} \\
1 \mathrm{Amp} .\end{array}$ & $\begin{array}{l}22.2 \quad \mathrm{Vc} \\
1 \mathrm{Amp} .\end{array}$ & $\begin{array}{l}\text { ol. } 10.5 \\
1 \mathrm{Amp} .\end{array}$ & Vol. & $\begin{array}{l}13.0 \quad \text { Vol. } \\
1 \mathrm{Amp} .\end{array}$ \\
\hline 6 & 27 Sept. 08 & $\begin{array}{l}20.4 \text { Vol. } \\
1 \mathrm{Amp} .\end{array}$ & $\begin{array}{l}23.3 \text { Vo } \\
1 \mathrm{Amp} .\end{array}$ & $\begin{array}{l}23.8 \\
1 \text { Amp. }\end{array}$ & Vol. $\begin{array}{l}14.2 \quad \text { Vol } \\
1 \mathrm{Amp} .\end{array}$ & $\begin{array}{l}22.5 \mathrm{Vol} \\
1 \mathrm{Amp} .\end{array}$ & $\begin{array}{l}22.2 \quad V c \\
1 \mathrm{Amp} .\end{array}$ & $\begin{array}{l}\text { ol. } 17.8 \\
1 \text { Amp. }\end{array}$ & Vol. & $\begin{array}{ll}13.8 & \text { Vol. } \\
1 \text { Amp. }\end{array}$ \\
\hline 7 & 28 Sept. 08 & $\begin{array}{l}20.0 \text { Vol. } \\
1 \mathrm{Amp} .\end{array}$ & $\begin{array}{ll}22.7 & \text { Vo } \\
1 \mathrm{Amp} .\end{array}$ & $\begin{array}{l}22.6 \\
1 \mathrm{Amp} \\
\end{array}$ & $\begin{array}{l}\text { Vol. } \mid \begin{array}{ll}14.6 \quad \text { Vol } \\
1 \mathrm{Amp} .\end{array} \\
\end{array}$ & $\begin{array}{l}21.2 \mathrm{Vol} \\
1 \mathrm{Amp}\end{array}$ & $\begin{array}{l}21.3 \quad \mathrm{Vc} \\
1 \mathrm{Amp} . \\
\end{array}$ & $\begin{array}{l}\text { ol.15.7 } \\
1 \text { Amp. }\end{array}$ & Vol. & $\begin{array}{l}13.5 \quad \text { Vol. } \\
1 \mathrm{Amp} .\end{array}$ \\
\hline 8 & 29 Sept. 08 & $\begin{array}{l}19.9 \text { Vol. } \\
1 \mathrm{Amp} .\end{array}$ & $\begin{array}{l}22.3 \quad \text { Vo } \\
1 \mathrm{Amp} .\end{array}$ & $\begin{array}{l}21.7 \\
1 \mathrm{Amp} .\end{array}$ & $\begin{array}{l}\text { Vol. }\left[\begin{array}{l}17.9 \quad \text { Vol } \\
1 \mathrm{Amp} .\end{array}\right. \\
\end{array}$ & $\begin{array}{l}20.9 \mathrm{Vol} \\
1 \mathrm{Amp} \text {. }\end{array}$ & $\begin{array}{l}21.0 \quad \mathrm{Vc} \\
1 \mathrm{Amp} .\end{array}$ & $\begin{array}{l}\text { ol.16.1 } \\
1 \text { Amp. }\end{array}$ & Vol. & $\begin{array}{ll}16.9 & \text { Vol. } \\
1 \mathrm{Amp} .\end{array}$ \\
\hline 9 & 30 Sept. 08 & $\begin{array}{l}19.1 \text { Vol. } \\
1 \mathrm{Amp} .\end{array}$ & $\begin{array}{l}21.2 \quad \text { Vo } \\
1 \mathrm{Amp} .\end{array}$ & $\begin{array}{l}20.5 \\
1 \mathrm{Amp}\end{array}$ & Vol. 14.3 Vol & $\begin{array}{l}19.5 \mathrm{Vol} \\
1 \mathrm{Amp}\end{array}$ & $\begin{array}{l}20.0 \quad V c \\
1 \mathrm{Amp} .\end{array}$ & $\begin{array}{l}\text { 'ol. } 15.5 \\
1 \text { Amp. }\end{array}$ & Vol. & $\begin{array}{ll}14.7 & \text { Vol. } \\
1 \mathrm{Amp} . & \end{array}$ \\
\hline 10 & 1 Oct. 08 & $\begin{array}{l}18.7 \text { Vol. } \\
1 \mathrm{Amp} .\end{array}$ & $\begin{array}{ll}19.4 & \text { Vo } \\
1 \mathrm{Amp} . & \\
\end{array}$ & $\begin{array}{l}18.7 \\
1 \text { Amp. }\end{array}$ & $\begin{array}{l}\text { Vol. } \begin{array}{ll}15.6 \quad \text { Vol } \\
1 \mathrm{Amp} .\end{array} \\
\end{array}$ & $\begin{array}{l}17.3 \mathrm{Vol} \\
1 \mathrm{Amp}\end{array}$ & $\begin{array}{l}18.9 \quad \mathrm{Vc} \\
1 \mathrm{Amp} .\end{array}$ & $\begin{array}{l}\text { ol. } 16.6 \\
1 \mathrm{Amp} .\end{array}$ & Vol. & $\begin{array}{ll}16.8 & \text { Vol. } \\
1 \mathrm{Amp} .\end{array}$ \\
\hline 11 & 2 Oct. 08 & $\begin{array}{l}18.7 \text { Vol. } \\
1 \mathrm{Amp} .\end{array}$ & $\begin{array}{l}19.3 \quad \text { Vo } \\
1 \mathrm{Amp} .\end{array}$ & $\begin{array}{l}18.2 \\
1 \mathrm{Amp}\end{array}$ & $\begin{array}{l}\text { Vol. } 16.9 \text { Vol } \\
1 \mathrm{Amp} .\end{array}$ & $\begin{array}{ll}16.6 \\
1 \mathrm{Amp}\end{array}$ & $\begin{array}{l}\text { Vol } 18.4 \quad \text { Vc } \\
1 \text { Amp. }\end{array}$ & $\begin{array}{l}\text { ol.19.3 } \\
1 \text { Amp. }\end{array}$ & Vol. & $\begin{array}{ll}19.7 & \text { Vol. } \\
1 \mathrm{Amp} . & \end{array}$ \\
\hline 12 & 3 Oct. 08 & $\begin{array}{l}16.5 \text { Vol. } \\
1 \mathrm{Amp} .\end{array}$ & $\begin{array}{ll}18.1 \quad \text { Vo } \\
1 \mathrm{Amp} .\end{array}$ & $\begin{array}{l}17.0 \\
1 \mathrm{Amp}\end{array}$ & $\begin{array}{l}\text { Vol. } \begin{array}{l}17.5 \quad \text { Vol } \\
1 \mathrm{Amp} .\end{array} \\
\end{array}$ & $\begin{array}{l}16.1 \\
1 \mathrm{Amp} \\
\end{array}$ & $\begin{array}{l}\text { Voll17.7 Vc } \\
1 \text { Amp. }\end{array}$ & $\begin{array}{l}\text { ol. } 19.8 \\
1 \mathrm{Amp} .\end{array}$ & Vol. & $\begin{array}{ll}19.0 & \text { Vol. } \\
1 \mathrm{Amp} .\end{array}$ \\
\hline 13 & 4 Oct. 08 & $\begin{array}{l}15.1 \text { Vol. } \\
1 \mathrm{Amp} .\end{array}$ & $\begin{array}{ll}16.2 \quad \text { Vo } \\
1 \mathrm{Amp} .\end{array}$ & $\begin{array}{l}14.8 \\
1 \mathrm{Amp}\end{array}$ & $\begin{array}{l}\text { Vol. } \begin{array}{ll}15.5 \quad \text { Vol } \\
1 \mathrm{Amp} .\end{array} \\
\end{array}$ & $\begin{array}{l}14.9 \\
1 \text { Amp } \\
\end{array}$ & $\begin{array}{l}\text { Vol } 16.2 \text { Vc } \\
1 \text { Amp. }\end{array}$ & $\begin{array}{l}\text { ol.18.9 } \\
1 \mathrm{Amp} .\end{array}$ & Vol. & $\begin{array}{ll}16.8 & \text { Vol. } \\
1 \mathrm{Amp} .\end{array}$ \\
\hline 14 & 5 Oct. 08 & $\begin{array}{l}14.2 \text { Vol. } \\
1 \mathrm{Amp} .\end{array}$ & $\begin{array}{ll}15.8 \quad \text { Vo } \\
1 \mathrm{Amp} .\end{array}$ & $\begin{array}{l}14.4 \\
1 \mathrm{Amp}\end{array}$ & $\begin{array}{l}\text { Vol. } 14.9 \text { Vol } \\
1 \mathrm{Amp} .\end{array}$ & $\begin{array}{l}14.9 \\
1 \text { Amp } \\
\end{array}$ & $\begin{array}{l}\text { Voll15.9 Vc } \\
1 \text { Amp. }\end{array}$ & $\begin{array}{l}\text { ol. } 17.8 \\
1 \mathrm{Amp} .\end{array}$ & Vol. & $\begin{array}{ll}17.5 & \text { Vol. } \\
1 \mathrm{Amp} .\end{array}$ \\
\hline 15 & 6 Oct. 08 & $\begin{array}{l}14.5 \text { Vol. } \\
1 \mathrm{Amp} .\end{array}$ & $\begin{array}{l}15.6 \quad \text { Vo } \\
1 \mathrm{Amp} .\end{array}$ & $\begin{array}{l}14.4 \\
1 \mathrm{Amp}\end{array}$ & $\begin{array}{l}\text { Vol. } 14.7 \quad \text { Vol } \\
1 \mathrm{Amp} .\end{array}$ & $\begin{array}{l}14.8 \\
1 \mathrm{Amp}\end{array}$ & $\begin{array}{l}\text { Voll } 15.7 \quad \text { Vc } \\
1 \text { Amp. }\end{array}$ & $\begin{array}{l}\text { ol.17.3 } \\
1 \text { Amp. }\end{array}$ & Vol. & $\begin{array}{l}16.1 \text { Vol. } \\
1 \text { Amp. }\end{array}$ \\
\hline 16 & 7 Oct. 08 & $\begin{array}{l}14.0 \text { Vol. } \\
1 \mathrm{Amp} .\end{array}$ & Stage \# 1 & $\begin{array}{l}14.0 \\
1 \mathrm{Amp} . \\
\end{array}$ & $\begin{array}{l}\text { Vol. } \begin{array}{l}13.2 \text { Vol } \\
1 \mathrm{Amp} .\end{array} \\
\end{array}$ & $\begin{array}{l}14.7 \\
1 \text { Amp. } \\
\end{array}$ & $\begin{array}{l}\text { Voll15.4 Vc } \\
1 \text { Amp. }\end{array}$ & $\begin{array}{l}\text { ol. } 16.5 \\
1 \text { Amp. }\end{array}$ & Vol. & Stage \# 1 \\
\hline 17 & 8 Oct. 08 & $\begin{array}{l}13.2 \text { Vol. } \\
1 \mathrm{Amp} .\end{array}$ & Stage \# 1 & \begin{tabular}{|l|}
13.5 \\
1 Amp. \\
\end{tabular} & $\begin{array}{l}\text { Vol. } \mid \begin{array}{ll}12.4 \quad \text { Vol } \\
1 \mathrm{Amp} .\end{array} \\
\end{array}$ & \begin{tabular}{|l|l|}
14.0 \\
1 Amp \\
\end{tabular} & $\begin{array}{l}\text { Voll14.6 Vc } \\
1 \text { Amp. }\end{array}$ & $\begin{array}{l}\text { ol. } 15.2 \\
1 \text { Amp. }\end{array}$ & Vol. & Stage \# 1 \\
\hline 18 & 9 Oct. 08 & $\begin{array}{l}13.2 \text { Vol. } \\
1 \mathrm{Amp} .\end{array}$ & Stage \# 1 & $\begin{array}{l}13.3 \\
1 \text { Amp. }\end{array}$ & $\begin{array}{l}\text { Vol. } 11.5 \text { Vol } \\
1 \mathrm{Amp} .\end{array}$ & $\begin{array}{l}13.4 \\
1 \mathrm{Amp}\end{array}$ & $\begin{array}{l}\text { Voll14.3 Vc } \\
1 \text { Amp. }\end{array}$ & $\begin{array}{l}\text { ol.14.5 } \\
1 \text { Amp. }\end{array}$ & Vol. & Stage \# 1 \\
\hline 19 & 10 Oct. 08 & $\begin{array}{l}13.3 \text { Vol. } \\
1 \mathrm{Amp} .\end{array}$ & Stage \# 1 & \begin{tabular}{|l|}
13.4 \\
1 Amp. \\
\end{tabular} & $\begin{array}{l}\text { Vol. } \begin{array}{ll}11.8 \quad \text { Vol } \\
1 \mathrm{Amp} .\end{array} \\
\end{array}$ & \begin{tabular}{|l|l|}
13.4 \\
$1 \mathrm{Amp}$ \\
\end{tabular} & $\begin{array}{l}\text { Voll14.4 Vc } \\
1 \text { Amp. }\end{array}$ & $\begin{array}{l}\text { ol. } 14.6 \\
1 \text { Amp. }\end{array}$ & Vol. & Stage \# 1 \\
\hline 20 & 11 Oct. 08 & $\begin{array}{l}12.7 \text { Vol. } \\
1 \mathrm{Amp} .\end{array}$ & Stage \# 1 & \begin{tabular}{|l|}
13.1 \\
1 Amp.
\end{tabular} & $\begin{array}{l}\text { Vol. } 11.2 \text { Vol } \\
1 \mathrm{Amp} .\end{array}$ & $\begin{array}{l}12.7 \\
1 \text { Amp. }\end{array}$ & $\begin{array}{l}\text { Vol } 14.0 \quad \text { Vc } \\
1 \text { Amp. }\end{array}$ & $\begin{array}{l}\text { ol.14.1 } \\
1 \text { Amp. }\end{array}$ & Vol. & \\
\hline
\end{tabular}




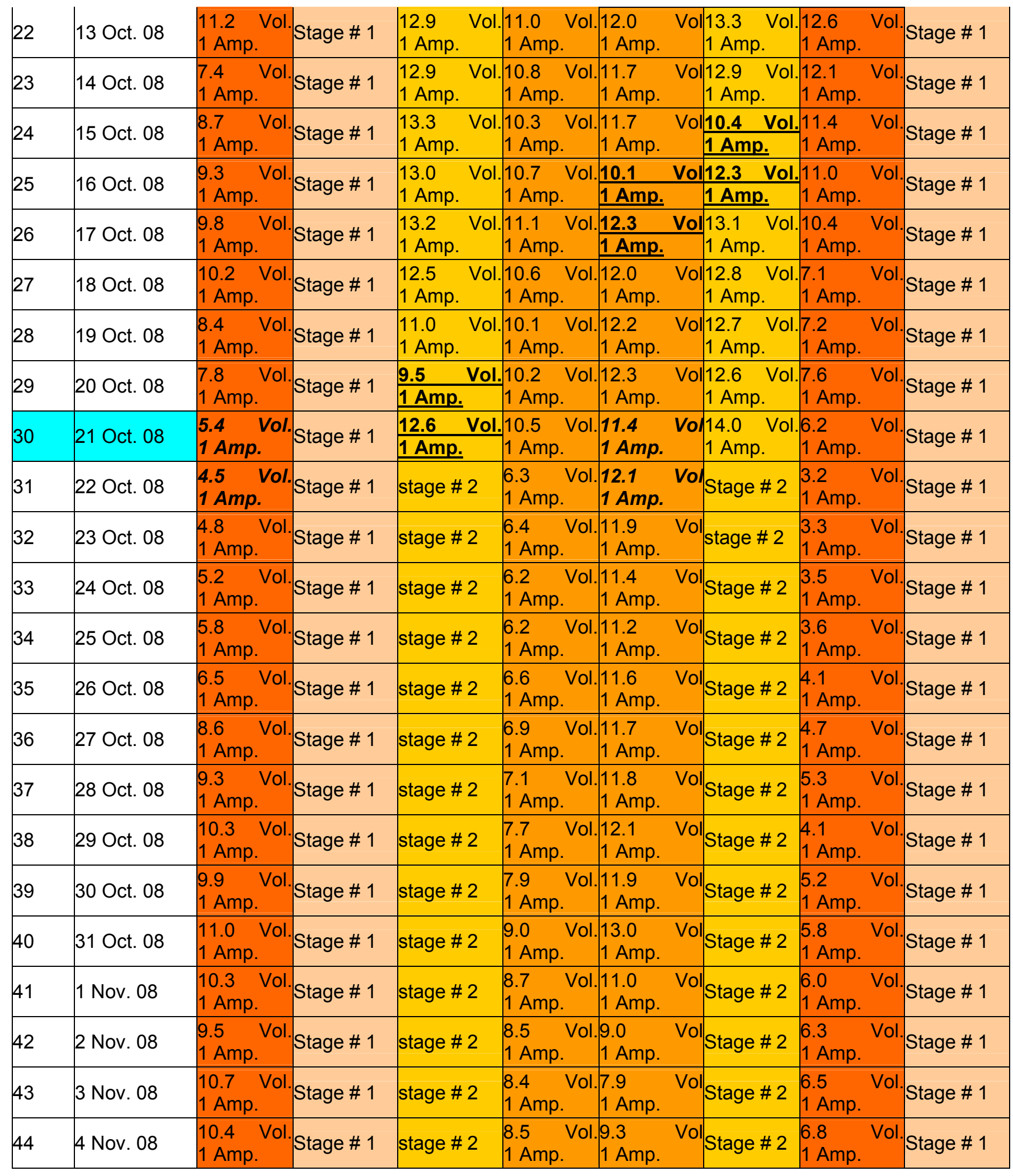




\begin{tabular}{|c|c|c|c|c|c|c|c|c|c|}
\hline 45 & 5 Nov. 08 & $\begin{array}{l}10.5 \text { Vol. } \\
1 \mathrm{Amp} .\end{array}$ & Stage \# 1 & stage \# 2 & $\begin{array}{l}8.3 \text { Vol } \\
1 \mathrm{Amp} .\end{array}$ & $\begin{array}{l}9.9 \\
1 \text { Amp. }\end{array}$ & Stage \# 2 & $\begin{array}{ll}7.0 & \text { Vol. } \\
1 & \text { Amp. }\end{array}$ & Stage \# 1 \\
\hline 46 & 6 Nov. 08 & $\begin{array}{l}9.9 \text { Vol. } \\
1 \text { Amp. }\end{array}$ & Stage \# 1 & stage \# 2 & Stage \# 3 & Stage \# 3 & Stage \# 2 & $\begin{array}{ll}7.4 & \text { Vol. } \\
1 \mathrm{Amp} . & \end{array}$ & Stage \# 1 \\
\hline 47 & 7 Nov. 08 & $\begin{array}{l}9.9 \text { Vol. } \\
1 \mathrm{Amp} .\end{array}$ & Stage \# 1 & stage \# 2 & Stage \# 3 & Stage \# 3 & Stage \# 2 & $\begin{array}{ll}8.1 & \text { Vol. } \\
1 & \text { Amp. } \\
\end{array}$ & Stage \# 1 \\
\hline 48 & 8 Nov. 08 & $\begin{array}{l}10.0 \text { Vol. } \\
1 \mathrm{Amp} .\end{array}$ & Stage \# 1 & stage \# 2 & Stage \# 3 & Stage \# 3 & Stage \# 2 & $\begin{array}{ll}8.8 & \text { Vol. } \\
1 & \text { Amp. } \\
\end{array}$ & Stage \# 1 \\
\hline 49 & 9 Nov. 08 & $\begin{array}{l}10.2 \text { Vol. } \\
1 \mathrm{Amp} .\end{array}$ & Stage \# 1 & stage \# 2 & Stage \# 3 & Stage \# 3 & Stage \# 2 & $\begin{array}{ll}9.6 & \text { Vol. } \\
1 & \text { Amp. }\end{array}$ & Stage \# 1 \\
\hline 50 & 10 Nov. 08 & $\begin{array}{l}10.1 \text { Vol. } \\
1 \mathrm{Amp} .\end{array}$ & Stage \# 1 & stage \# 2 & Stage \# 3 & Stage \# 3 & Stage \# 2 & $\begin{array}{ll}7.0 & \text { Vol. } \\
1 & \text { Amp. }\end{array}$ & Stage \# 1 \\
\hline 51 & 11 Nov. 08 & $\begin{array}{l}10.2 \text { Vol. } \\
1 \mathrm{Amp} .\end{array}$ & Stage \# 1 & stage \# 2 & Stage \# 3 & Stage \# 3 & Stage \# 2 & $\begin{array}{ll}9.4 & \text { Vol. } \\
1 & \text { Amp. } \\
\end{array}$ & Stage \# 1 \\
\hline 52 & 12 Nov. 08 & $\begin{array}{l}10.0 \text { Vol. } \\
1 \mathrm{Amp} .\end{array}$ & Stage \# 1 & stage \# 2 & Stage \# 3 & Stage \# 3 & Stage \# 2 & $\begin{array}{ll}10.1 & \text { Vol. } \\
1 & \text { Amp. }\end{array}$ & Stage \# 1 \\
\hline 53 & 13 Nov. 08 & $\begin{array}{l}10.0 \text { Vol. } \\
1 \mathrm{Amp} .\end{array}$ & Stage \# 1 & stage \# 2 & Stage \# 3 & Stage \# 3 & Stage \# 2 & $\begin{array}{ll}10.2 & \text { Vol. } \\
1 & \text { Amp. }\end{array}$ & Stage \# 1 \\
\hline 54 & 14 Nov. 08 & $\begin{array}{l}10.0 \text { Vol. } \\
1 \mathrm{Amp} .\end{array}$ & Stage \# 1 & stage \# 2 & Stage \# 3 & Stage \# 3 & Stage \# 2 & $\begin{array}{ll}11.1 & \text { Vol. } \\
1 & \text { Amp. }\end{array}$ & Stage \# 1 \\
\hline 55 & 15 Nov. 08 & $\begin{array}{l}10.1 \text { Vol. } \\
1 \mathrm{Amp} .\end{array}$ & Stage \# 1 & stage \# 2 & Stage \# 3 & Stage \# 3 & Stage \# 2 & $\begin{array}{l}11.9 \text { Vol. } \\
1 \mathrm{Amp} .\end{array}$ & Stage \# 1 \\
\hline 56 & 16 Nov. 08 & $\begin{array}{l}10.1 \text { Vol. } \\
1 \mathrm{Amp} .\end{array}$ & Stage \# 1 & stage \# 2 & Stage \# 3 & Stage \# 3 & Stage \# 2 & $\begin{array}{l}12.5 \text { Vol. } \\
1 \mathrm{Amp} .\end{array}$ & Stage \# 1 \\
\hline 57 & 17 Nov. 08 & $\begin{array}{l}10.2 \text { Vol. } \\
1 \mathrm{Amp} .\end{array}$ & Stage \# 1 & stage \# 2 & Stage \# 3 & Stage \# 3 & Stage \# 2 & $\begin{array}{ll}13.0 & \text { Vol. } \\
1 \mathrm{Amp} .\end{array}$ & Stage \# 1 \\
\hline 58 & 18 Nov. 08 & $\begin{array}{l}10.1 \text { Vol. } \\
1 \mathrm{Amp} .\end{array}$ & Stage \# 1 & stage \# 2 & Stage \# 3 & Stage \# 3 & Stage \# 2 & $\begin{array}{ll}10.5 & \text { Vol. } \\
1 & \text { Amp. }\end{array}$ & Stage \# 1 \\
\hline 59 & 19 Nov. 08 & $\begin{array}{l}10.0 \text { Vol. } \\
1 \mathrm{Amp} .\end{array}$ & Stage \# 1 & stage \# 2 & Stage \# 3 & Stage \# 3 & Stage \# 2 & $\begin{array}{ll}10.0 & \text { Vol. } \\
1 \mathrm{Amp} . & \end{array}$ & Stage \# 1 \\
\hline 60 & 20 Nov. 08 & $\begin{array}{l}9.8 \quad \text { Vol. } \\
1 \mathrm{Amp} .\end{array}$ & Stage \# 1 & stage \# 2 & Stage \# 3 & Stage \# 3 & Stage \# 2 & $\begin{array}{ll}12.0 & \text { Vol. } \\
1 \mathrm{Amp} .\end{array}$ & Stage \# 1 \\
\hline \multicolumn{10}{|c|}{ End of corrosion activities } \\
\hline
\end{tabular}




\section{Appendix E: 1. Beam design}

For the concrete beams without web reinforcement, the highest probability of shear failure will take place when the ratio is $a / d=2.5$. According to the graphs shown in Figures ( $\mathrm{E}-1 \mathrm{~b}$ and $\mathrm{c}$ ), the chance of getting shear failure will be less when $a / d$ is smaller or larger than 2.5 .

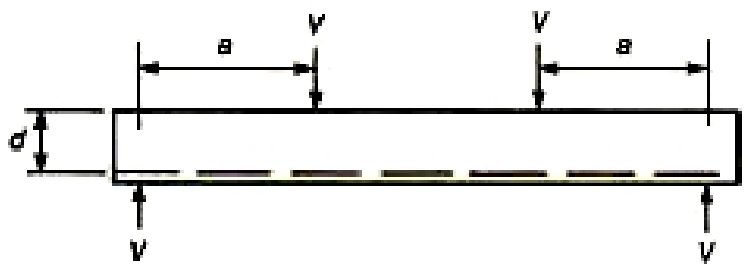

(a) Beam.

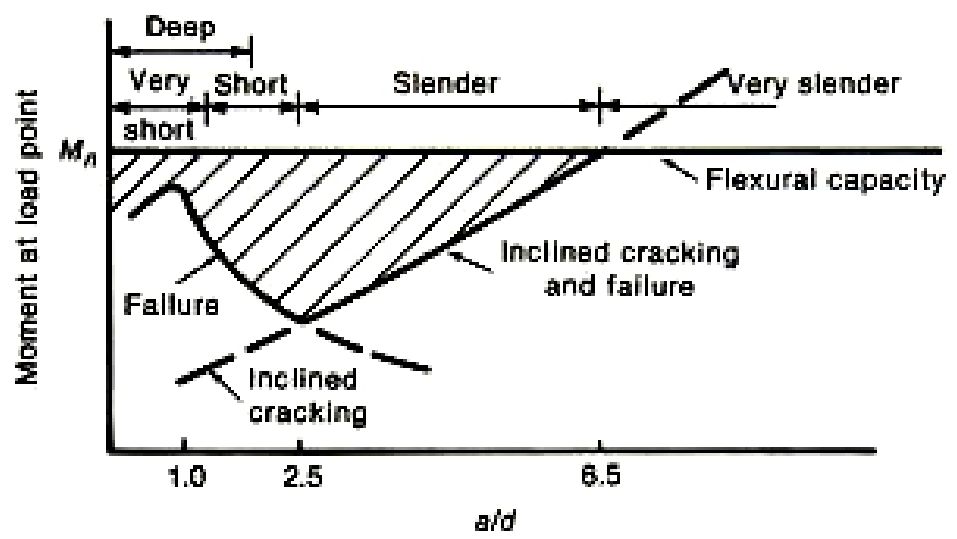

(b) Moments at cracking and tailure.

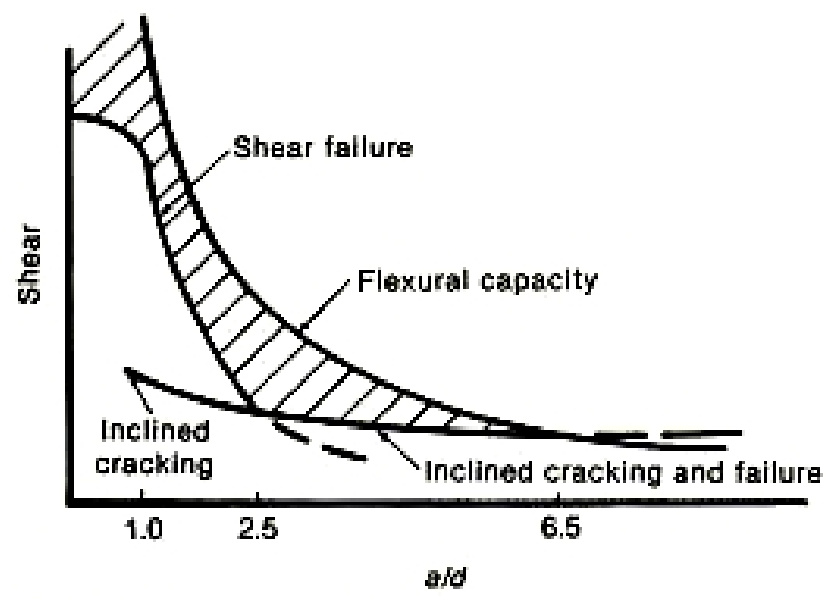

(c) Shear at cracking and failure.

Figure (E-1): Effect of $a / d$ ratio on the shear strength of beams without stirrups (MacGregor and Bartlett, 2000). 
The shaded areas in this figure show the reduction in strength due to shear. Stirrups are provided to ensure that the beam reaches full flexural capacity. The ratio of a/d can divide the beams into four categories; very short, short, slender, and very slender, as shown in Figure (E-1-b). The term "deep beam" is also used for the very short and short beams.

In this research, the beams were designed to monitor and examine gradual failure in shear apart from the sudden failure in shear for beams without stirrups.

Fig (E-1-b) shows that $a / d=2.5$ is located between two categories of beams. The first one is the short beam and the second is the slender beam.

"The short shears spans with $a / d=1$ to 2.5 develop inclined cracks and are able to carry additional loads after the redistribution of the internal forces, partly by arch action. The reason of the final failure for this kind of beam will be by a bond failure, a splitting failure, or a dowel failure along the tension reinforcement as illustrated in Figure (E-2a), or by damaging the area over the cracks in the compression zone, as illustrated in Figure (E-2b). The type mentioned second is known as compression shear failure. Because the inclined crack generally extends higher into the beam than a flexural crack, failure occurs at less than the flexural moment capacity." (MacGregor and Bartlett, 2000).

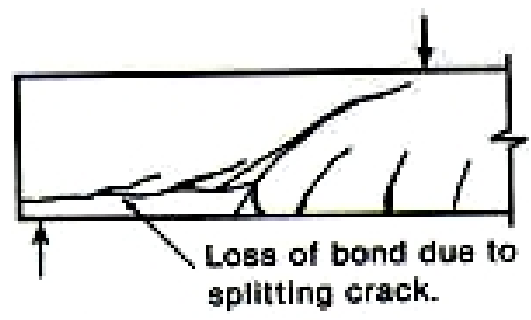

(a) Shear tension tailure.

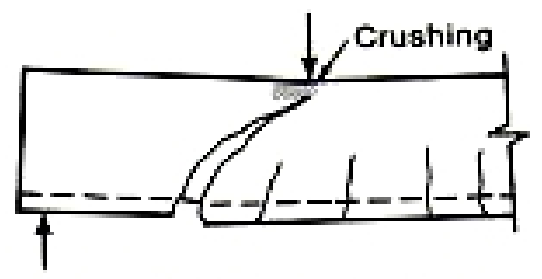

(b) Shear compression failure.

Figure (E-2): Shear failure in short beams, $\mathrm{a} / \mathrm{b}=1-2.5 \quad$ (MacGregor and Bartlett, 2000).
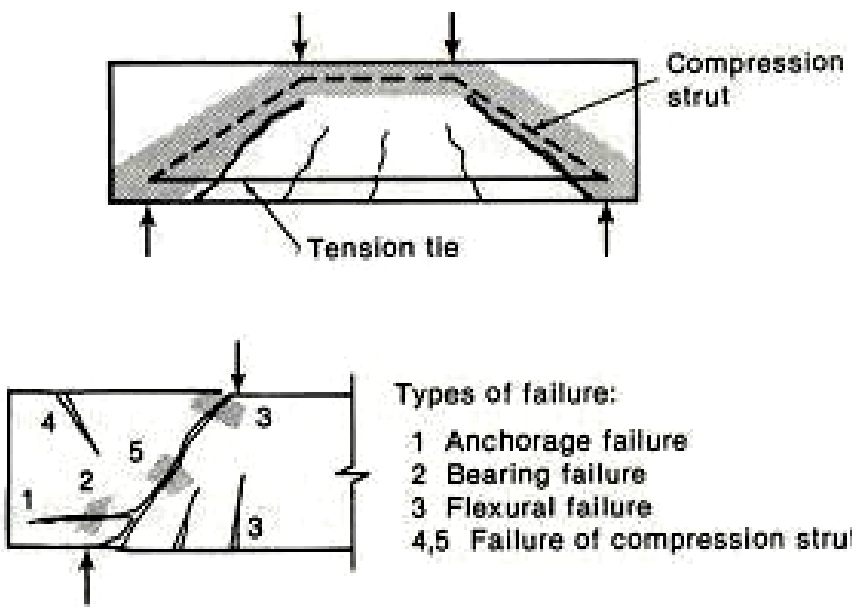

Types of failure:

1 Anchorage failure

2 Bearing failure

3 Flexural failure

4,5 Fallure of compression strut

Figure (E-3): Modes of failure in deep beams, $\mathrm{a} / \mathrm{d}=0.5$ to 2 (MacGregor and Bartlett, 2000). 
"In slender shear spans $a / d=2.5$ to 6 , the inclined cracks disrupt equilibrium to such an extent that the beam fails at the inclined cracking load as shown in Figure (E1b." (MacGregor and Bartlett, 2000).

The case of $a / d=2.5$ is located between the short shear spans and slender shear spans, so it will take from both properties and their failing behaviour. Wang and Salmon (1979) and MacGregor and Bartlett (2000) illustrate the beams' response to the loading and failure modes in Figures (E-1) to (E-3).

The beam design in this research is loaded at four points load. In this research, four points load means there are two equal concentrated loads and two other equal reaction forces on the left and right sides of the beam. For the calculation of the applied moment, taking in consideration the concentrated load as in Figure (E-1a) $\mathrm{M}=V a$ Figure $(E-1 c)$ is replotted, in terms of shear capacity, from Figure $(E-1 b)$ with the change that in Figure $(E-1 b)$ the $y$-axis is moment at load point, but the $y$-axis in Figure $(E-1 c)$ is shear. Figure (E-1c) shows the shear match with a flexural failure is the upper curved line. For the beams without web reinforcement, the shear failure line is the indicator of shear failing behaviour. The shear failure line is approximately constant for $a / d \geq 2$ Figures $(\mathrm{E}-1 \mathrm{~b})$ and $(\mathrm{E}-1 \mathrm{c})$ show that the selection of $a / d=2.5$ is going to lead to shear failure.

\section{E.2 Design calculations}

The total length of the beam $1400 \mathrm{~mm}$, and the supporting area is $130 \mathrm{~mm}$ from each side. Therefore, the clear span is $1140 \mathrm{~mm}$. If we choose $a=500 \mathrm{~mm}$, then $d=200 \mathrm{~mm}$, and the distance between the two load forces is $400 \mathrm{~mm}$

$\therefore$ Design of a rectangular beam with tension reinforcement:

Shear force $=60 \mathrm{KN}$ (from the reaction to $500 \mathrm{~mm}$ to the direction of the middle of the beam)

Moment $=26.4 \mathrm{kN} . \mathrm{m}$

$f_{c}^{\prime}=45 \mathrm{MPa}$

$f_{y}=400 \mathrm{MPa}$

The beam is to be built in a non-corrosive environment, and then forced for an accelerated corrosion with multiple stages. Therefore, the concrete cover selected to be $30 \mathrm{~mm} \mathrm{A23.3} \mathrm{(Table} \mathrm{2.6).} \mathrm{The} \mathrm{suggested} \mathrm{values} \mathrm{for} b=150 \mathrm{~mm}$ and $\mathrm{d}=220 \mathrm{~mm}$. 
For the design of reinforcement when beam width (b) and total depth (d) are known, the following steps must be followed.

1. Effective depth, $d$, Calculation:

$d=220-30-7.9-(19.5 / 2)=172.35 \mathrm{~mm} \approx 172 \mathrm{~mm}$;

$\therefore \frac{a}{d}=2.5 \Rightarrow a=430.625 \mathrm{~mm}$

$\therefore$ Assume $a=440 \mathrm{~mm}$

2. Shear force and bending moment calculations:

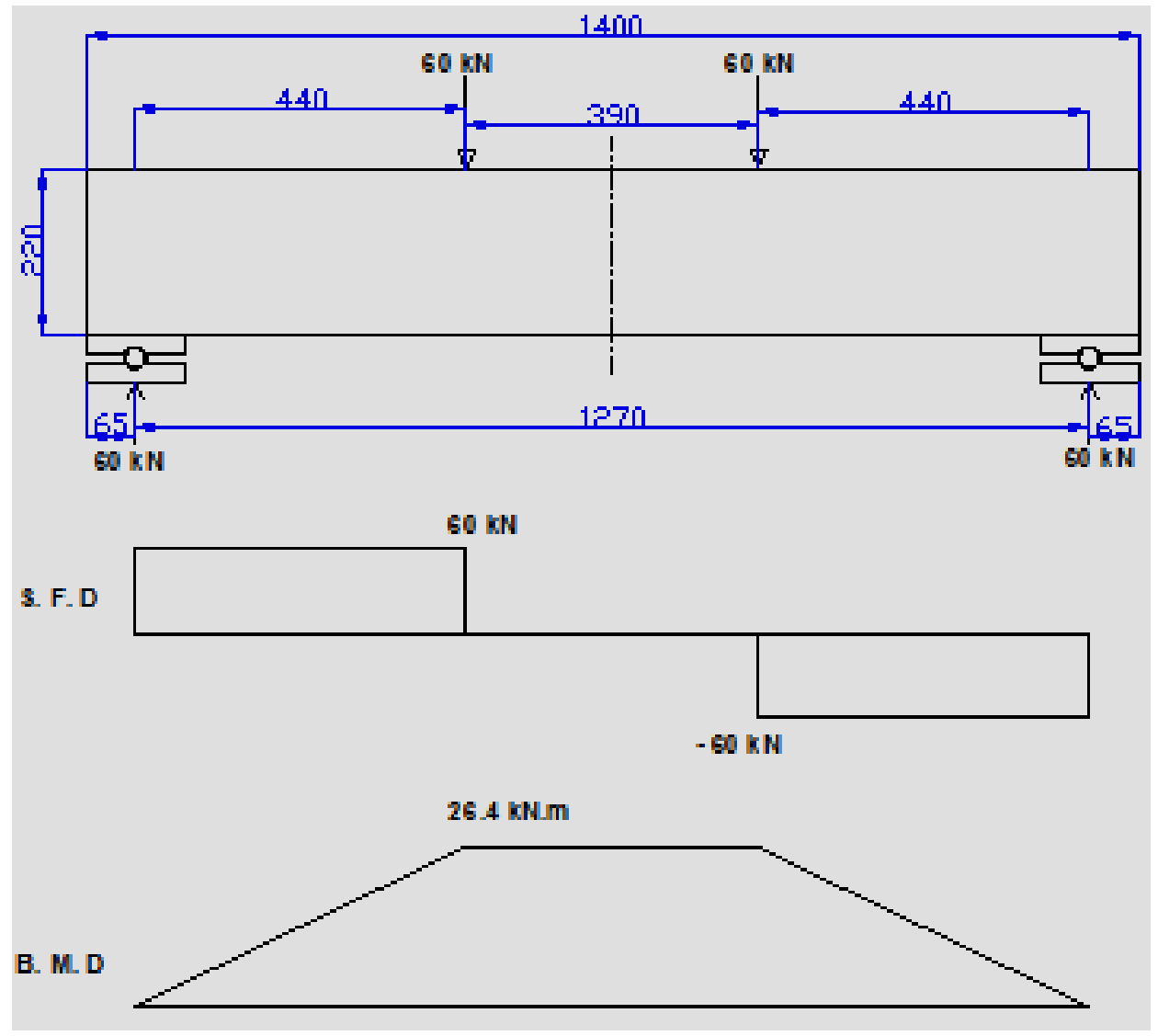

Figure (E-4) Shear force diagram and bending moment diagram.

Where:

S.F.D = Shear Force Diagram

B.M.D = Bending Moment Diagram $\left(M_{f}\right)=60 \times 0.44=26.4 \mathrm{kN} . \mathrm{m}$ 


\section{Flexural $A_{s}$ calculations}

Estimate effective depth d, assuming $20 \mathrm{M}$ bars for flexural reinforcement and 7.9M for stirrups.

Assume $a=0.3 d \Rightarrow j=0.85,(j=085-0.92)$ Table A-3 (MacGregor and Barttlet, 2000)

$$
j d=0.85 \times 172=146.2 \mathrm{~mm}
$$

$$
\begin{aligned}
& A_{s}=\frac{M_{f}}{\phi_{s} f_{y j d}} \\
&=\frac{26.4 \times 10^{6}}{0.85 \times 400 \times 146.2}=531.1 \mathrm{~mm}^{2} \\
& \frac{531.1}{300}=1.77 \text { bar } \Rightarrow \text { Use } 2 \text { bars } 20 \mathrm{M}
\end{aligned}
$$

4. Check whether $A_{s}>A_{s, \min }$

$$
\begin{aligned}
A_{s, \min } & =\frac{0.2 \sqrt{f_{c}^{\prime}} b_{t} h}{f_{y}} \\
& =\frac{0.2 \times \sqrt{45} \times 150 \times 220}{400}=110.68 \mathrm{~mm}^{2} \\
& 110.68 \mathrm{~mm}^{2}<531.1 \mathrm{~mm}^{2} \text { or } 600 \mathrm{~mm}^{2} \text { or } \therefore O K
\end{aligned}
$$

5. Compute a, and check if $f_{s}=f_{y}$

$$
\begin{array}{cr}
a=\frac{\phi_{s} A_{s} f_{y}}{\phi_{c} \alpha_{1} f_{c}^{\prime} b} & (\mathrm{E}-2){ }^{(\text {MacGregor and Bartlett, 2000-pp 126) }} \\
\alpha_{1}=0.85-0.0015 f_{c}^{\prime} \geq 0.67 & \text { A23.3 Eq. 10-1 } \\
\beta_{1}=0.97-0.0025 f_{c}^{\prime} \geq 0.67 & \text { A23.3 Eq. 10-2 } \\
\alpha_{1}=0.85-0.0015(45) & \\
& =0.7825 \geq 0.67 \\
a & =\frac{0.85 \times 600 \times 400}{0.6 \times 0.783 \times 45 \times 150}=64.33 \mathrm{~mm}
\end{array}
$$




$$
\begin{aligned}
& \frac{a}{d}=\frac{64.33}{172}=0.374<0.5 \\
& \therefore f_{s}=f_{y}
\end{aligned}
$$

Where: $f_{s}=$ Stress in tension reinforcement, $\mathrm{MPa}$.

$f_{y}=$ Specified yield strength of reinforcement, MPa.

$\therefore$ Tension failure (or ductile failure)

6. Compute $M_{r}$ (factored moment resistance of a section kN.m)

$$
\begin{aligned}
& \qquad \begin{aligned}
M_{r} & =\phi_{s} A_{s} f_{y}\left(d-\frac{a}{2}\right) \\
& =0.85 \times 600 \times 400 \times\left(172-\frac{64.33}{2}\right) \\
& =28.526 \mathrm{kN} . \mathrm{m}
\end{aligned} \\
& \begin{array}{l}
M_{f}=26.4 k N . m, M_{r} \\
\text { Since } M_{f}<M_{r}
\end{array}
\end{aligned}
$$

$\therefore$ Two bars of $20 \mathrm{M}$ are adequate to hold $M_{f}=26.4 \mathrm{kN} . \mathrm{m}$

\section{Shear Design}

1. Compute shear force envelope

$$
V_{f}=60 \mathrm{kN}
$$

2. Is the cross section large enough? A23.3 C1.11.3.4 gives the maximum shear resistance as

$V_{r, \max }=V_{c}+0.8 \lambda \phi_{c} \sqrt{f_{c}^{\prime}} b_{w} d$ $(E-4){ }^{(\text {MacGregor and Bartlett, 2000-pp209) }}$

Where $V_{c}=0.2 \lambda \phi_{c} \sqrt{f_{c}^{\prime}} b_{w} d$ A23.3.04 Eq. 11-6

Thus, recognizing the common factor $\lambda \phi_{c} \sqrt{f_{c}^{\prime}} b_{w} d$

$\lambda=1.0$ (for normal concrete)

$V_{r, \max }=1.0 \times 0.6 \times \sqrt{45} \times 150 \times 172=103.843 \mathrm{kN}$

Since $\mathrm{V}_{f}<\mathrm{V}_{r, \max }$ 
$\therefore$ Section is adequate

3. Are stirrups required by $\mathbf{A} 23.3 \mathrm{C} 1 . \mathbf{1 1 . 2 . 8 . 1}$ ? No stirrups are required if $V_{f} \leq 0.5 V_{c}$, where

$$
\begin{array}{lr}
V_{c}=0.2 \lambda \phi_{c} \sqrt{f_{c}^{\prime}} b_{w} d \quad \text { A23.3.04 Eq. 11-6 } \\
V_{c}=0.2 \times 1.0 \times 0.6 \times \sqrt{45} \times 150 \times 172=20.768 \mathrm{kN}
\end{array}
$$

Since $V_{f}=60 k N$ exceeds $V_{c} / 2=10.384 k N$, stirrups are required.

\section{Determine the maximum stirrup spacing.}

Based on the beam depth,

$$
\begin{array}{r}
0.1 \lambda \phi_{c} f_{c}^{\prime} b_{w} d=0.1 \times 1.0 \times 0.6 \times 45 \times 150 \times 172 \\
=69.66 \mathrm{kN}
\end{array}
$$

Since $V_{f}=60 k N$ is less than this, A23.3 Cl. 11.2.11(a) governs, and the maximum spacing is the smaller of $0.70 \mathrm{~d}=0.7 \times 172 \mathrm{~mm}$ or $600 \mathrm{~mm}$. Therefore, the maximum spacing according to the calculation is $120.4 \mathrm{~mm}$. Note: According to the tensile tests that are done to the samples of the stirrups plain steel bars, the magnitude of the steel used for the stirrups was slightly above $600 \mathrm{MPa}$, therefore $f_{y}=600 \mathrm{MPa}$ for the stirrups only.

Based on minimum $A_{v}$

$$
A_{v, \min }=\frac{0.06 \sqrt{f_{c}^{\prime}} b_{w S}}{f_{y}}
$$

Rearranging gives

$$
S \text { max }=\frac{f_{y} A_{v}}{0.06 \sqrt{f_{c}^{\prime}} b_{w}}
$$

